\title{
PHYSICAL AND THERMODYNAMIC PROPERTIES OF ALUMINOSILICATE MELTS AS A FUNCTION OF COMPOSITION
}

\author{
Dissertation \\ zur Erladung des Doktorgrades \\ der Mathematisch-Naturwissenschaftlichen Fakultäten \\ der Georg-August-Universität zu Göttingen
}

\author{
vorgelegt von \\ Katarzyna Falenty \\ Diplom-Geologin \\ aus \\ Wołomin, Polen
}

Göttingen, 2007 


\section{7}

Referentin/Referent:

Prof. Dr. Sharon Webb

(Georg-August Universität Göttingen)

Koreferentin/Koreferent:

Priv. Doz. Dr. Harald Behrens

(Leibniz Universität Hannover)

Tag der mündlichen Prüfung: 7. Dezember 2007 


\section{ACKNOWLEDGEMENTS}

Several people have been instrumental in allowing this project to be completed.

I would like to thank especially Prof. Dr. Sharon L. Webb for choosing the accurate and successful range of composition, for making this study possible, for her encouragement and patience at all stages of my work, for invaluable supervision, for help in producing the experimental results for this thesis, for very productive scientific discussions, for linguistic help. Her constructive criticism and comments from the initial conception to the end of this work are highly appreciated.

I am also very grateful to all members of Mineralogy Department (Uni Göttingen). Heinz Büttner is gratefully acknowledged for introducing me into torsion machine technique and CATMAN software, for his help and advice at the start of the project. My appreciation also goes to Peter Meier who helped with the glass samples preparation. I am also deeply indebted to Uschi Köhler for making wet chemistry and AAS analyses, for a lot of warmth and nice plants :) I would like to thank Gerrit Raschke for his help with the sample preparation and viscosity measurements. I am grateful to Johanna Rahn for density and viscosity measurements.

My thanks also goes to Dr. Burkhard Schmidt and Prof. Dr. Roland Stalder for an interest in the project and formal and informal conversations.

I thank to Dr. Andreas Kronz for the chemical analyses in the Geochemistry Department (Uni Göttingen). I would like to thank people from the Institute for Low Temperature Physics (Uni Göttingen) who willingly lent me their time and equipment to do the ultrasonic measurements and for valuable discussion and comments.

I am very grateful to Dr. Milada Zímová, Elke Schlechter, Aneta Kiebała and Magda Banaszak who supported me at this time and have found enough time and patient for nice chatting or sometimes to listen my grumbling.

Appreciation continues to Petra Wolfrath for the help with formalities; to D.G. Brouwer, K.H. Klapproth and E. Wartala for technical support.

A very special appreciation is due to my husband, Andrzej. Dziękuję Ci za zrozumienie, cierpliwość, codzienne wsparcie i skuteczne dodawanie wiary w siebie.

I am also thankful to my parents. Kochani Rodzice - dziękuję Wam za troskę, duchowe wsparcie i stały kontakt.

I thank Prof. Dr. Sharon L. Webb, Priv. Doz. Dr. Harald Behrens, Prof. Dr. Konrad Samwer, Prof. Dr. Gerhard Wörner, Prof. Dr. Roland Stalder and Dr. Burkhard Schmidt for being the members of the examination committee.

I would like to thank the DFG (Deutsche Forschungsgemeinschaft), not only for providing the funding which allowed me to undertake this research, but also for giving me the opportunity to attend conferences and meet so many interesting people. This work was supported by the grants WE1810/3-1 (2004-2006) and WE1810/3-3 (2006-2007).

Finally, I am very grateful to all others, who supported me during this PhD study... 



\section{ABSTRACT}

Viscosity $\eta$, heat capacity $C_{p}$, density $\rho$, compressibility $K$, thermal conductivity $\lambda$, thermal expansion $\alpha$, diffusivity $D$, surface tension of melts are all controlled by the melt structure. The structure of the melt is a function of composition, temperature and pressure.

Here viscosity $\eta$, heat capacity $C_{p}$, density $\rho$ and shear modulus $G$ have been determined in melts as a function of composition (with constant $\mathrm{SiO}_{2}$ content $=66.7 \mathrm{~mol} \%$ ) from peralkaline to peraluminous. All of these physical and thermodynamic parameters show that there is a change in structure close to the subaluminous composition, when there are not enough charge balancers to compensate the negative charge of $\mathrm{Al}$ in tetrahedral coordination (i.e. at $\gamma \sim 0.5^{1}$; $\gamma$ is $\left.\left(\mathrm{Na}_{2} \mathrm{O}+\mathrm{FeO}\right) /\left(\mathrm{Na}_{2} \mathrm{O}+\mathrm{Al}_{2} \mathrm{O}_{3}+\mathrm{FeO}+\mathrm{Fe}_{2} \mathrm{O}_{3}\right)\right)$.

For the first time in a controlled series of composition the configurational heat capacity $\mathrm{C}_{\mathrm{p}}{ }^{\text {conf }}$ was calculated. $\mathrm{C}_{\mathrm{p}}{ }^{\text {conf }}$ shows a deep minimum at $\gamma \sim 0.5$. Configurational entropy $\mathrm{S}^{\text {conf }}$ and $\mathrm{B}_{\mathrm{e}}$ parameter also have not been shown before for the melts with composition investigated in this study. The obtained results indicate a change in structure at $\gamma \sim 0.5$. Viscosities were determined in the range of $10^{8.8}-10^{12.6} \mathrm{~Pa} \mathrm{~s}$ using micropenetration technique. These data also indicate that there is a change in melt structure at $\gamma \sim 0.5$.

The lifetimes of bonds within the melt have been determined by mechanical spectroscopy $(0.001 \mathrm{~Hz}-1 \mathrm{~Hz})$. The Si-O and Al-O bond lifetimes could not be separately resolved from the slow $\alpha$-relaxation peak in mechanical spectrum, but the short lived $\mathrm{Na}-\mathrm{O}$ bond lifetimes were obtained from the $\beta$-relaxation peak.

The major advance in this study is the fact that the measured structural relaxation time deviates from the calculated Maxwell relaxation time. This is contrary to the accepted theory of silicate melt flow and structural relaxation. However, the present melts contain much more $\mathrm{Al}_{2} \mathrm{O}_{3}$ $(\mathrm{Al} / \mathrm{Si}=0.6)$ than all previous investigated melts $(\mathrm{Al} / \mathrm{Si}=0.2)$ and therefore the effect of the short lived $\mathrm{Al}-\mathrm{O}$ bonds on viscous flow is seen for the first time.

As the amount of $\mathrm{Al}$ increases, a probable structure is produced in which clumps of longlived Si-O clusters are surrounded by short-lived Al-O matrix. Thus flow occurs in the melt via Al-O bond breaking at a rate faster than the Si-O bond breaking. Flow occurs in a melt in which the Si-O clusters are not structurally relaxed. This is geologically important, as modelling of viscosity at high pressure, diffusion rates in melts, crystallization rates in melts, cooling rates of magma, flow rates of magma, modelling of the effect of crystals on magma viscosity all rely on the Maxwell equation which relates structural relaxation time to all rate processes involving the motion of $\mathrm{Si}$ and $\mathrm{O}$ atoms in melt. In $\mathrm{Al}_{2} \mathrm{O}_{3}$ rich melts (e.g. in phonolites) all these rate processes occur at least 10 times faster than models based on standard Maxwell relationship theory would predict.

\footnotetext{
1 “ " means "approximately equal to"
} 



\section{ZUSAMMENFASSUNG}

Viskosität $\eta$, Wärmekapazität $C_{p}$, Dichte $\rho$, Kompressionsmodul $K$, Wärmeleitfähigkeit $\lambda$, Wärmeausdehnung $\alpha$, Diffusion D und Oberflächenspannung der Schmelzen werden durch die Schmelzstruktur kontrolliert. Die Schmelzstruktur ist wiederum abhängig von der chemischen Zusammensetzung, Temperatur und Druck.

In dieser Arbeit wurden Viskosität $\eta$, Wärmekapazität $C_{p}$, Dichte $\rho$ und Schermodul $G$ als Funktion des Schmelzchemismus zwischen peralkaliner und peraluminöser Zusammensetzung (mit einem festgelegten $\mathrm{SiO}_{2}$-Gehalt von $66.7 \mathrm{~mol} \%$ ) untersucht. Alle oben genannten physikalischen und thermodynamischen Parameter zeigen eine Änderung der Struktur in der Nähe von subaluminöser Zusammensetzung, d.h. wenn nicht genug ladungsausgleichende Kationen vorhanden sind um die negative Ladung der Al-Tetraeder zu neutralisieren (bei $\gamma \sim 0.5^{1}$; $\gamma$ ist $\left.\left(\mathrm{Na}_{2} \mathrm{O}+\mathrm{FeO}\right) /\left(\mathrm{Na}_{2} \mathrm{O}+\mathrm{Al}_{2} \mathrm{O}_{3}+\mathrm{FeO}+\mathrm{Fe}_{2} \mathrm{O}_{3}\right)\right)$.

Zum ersten Mal wurden Konfigurations-Wärmekapazitäten $\mathrm{C}_{\mathrm{p}}{ }^{\text {conf }}$ in einer systematischen Zusammensetzungsserie ermittelt. $C_{p}{ }^{\text {conf }}$ zeigt ein Minimum bei $\gamma \sim 0.5$. Die Konfigurationsentropie $\mathrm{S}^{\text {conf }}$ und der $\mathrm{B}_{\mathrm{e}}$ Parameter wurden ebenfalls für Proben dieser Zusammensetzungen zum ersten Mal berechnet. Die Ergebnisse weisen auf eine Strukturänderung bei $\gamma \sim 0.5$ hin. Viskositäten wurden im Bereich $10^{8.8}-10^{12.6} \mathrm{~Pa} s$ mit der Mikropenetrationstechnik ermittelt. Diese Daten weisen ebenfalls auf Änderungen der Schmelzstruktur bei $\gamma \sim 0.5$ hin.

Die Lebenszeiten der Bindungen in der Schmelze wurden mit mechanischer Spektroskopie ermittelt $(1 \mathrm{~Hz}-0.001 \mathrm{~Hz})$. Die Lebenszeiten der Bindungen Si-O und Al-O konnten aus dem langsamen $\alpha$-Relaxionspeak im mechanischen Spektrum nicht getrennt aufgelöst werden, aber die kurzlebigen Na-O Bindungen konnten separat aus dem $\beta$-Relaxionspeak ermittelt werden.

Eine wesentliche Neuerung dieser Arbeit ist die Tatsache, dass die gemessene strukturelle Relaxationszeit von der berechneten Maxwell Relaxationszeit abweicht. Dies steht im Widerspruch zur allgemein anerkannten Theorie zum Fließen und Relaxation von Silikatschmelzen. Die hier untersuchten Schmelzen enthielten jedoch viel mehr $\mathrm{Al}_{2} \mathrm{O}_{3}(\mathrm{Al} / \mathrm{Si}=0.6)$ als früher untersuchte Schmelzen (Al/Si=0.2), wodurch hier zum ersten Mal der Einfluss der kurzlebigen Al-O Bindungen auf das viskose Fließen gesehen werden konnte.

Der relativ hohe Al Gehalt führt zur Bildung von langlebigen Si-O Clustern umgeben von einer weniger langlebigen Al-O Matrix. Daher wird das viskose Fließen durch das Brechen und Neubilden der Al-O Bindungen bestimmt. Die Schmelze fließt, obwohl die Si-O Cluster nicht vollständig relaxiert sind. Dies ist deshalb Bedeutung, da Modellierungen von Schmelzviskosität bei hohen Drücken, Viskositäten von teilkristallisierten Schmelzen, Diffusions- und Kristallisationsraten in Schmelzen, Abkühl- und Fließraten von Magma, alle auf der Maxwell Beziehung beruhen, die die strukturelle Relaxation mit allen Prozessen in Beziehung setzt, die auf der Bewegung von Si und $\mathrm{O}$ beruhen.

In $\mathrm{Al}_{2} \mathrm{O}_{3}$ reichen Schmelzen (z.B. Phonolithe) laufen alle diese Prozesse $10 \mathrm{Mal}$ schneller ab, als die Modelle vorhersagen, die auf der Standard Maxwell Beziehung beruhen.

\footnotetext{
1 “ " bedeutet "ungefähr gleich"
} 

TABLE OF CONTENTS

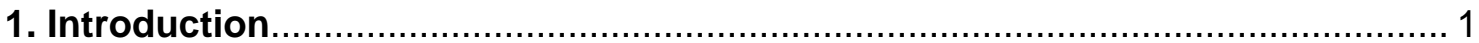

1.1. Viscoelasticity, flow and structural deformation.............................................

2. Structure of $\mathrm{Na}_{2} \mathrm{O}-\mathrm{Al}_{2} \mathrm{O}_{3}-\mathrm{SiO}_{2}$ and $\mathrm{Na}_{2} \mathrm{O}-\mathrm{Fe}_{2} \mathrm{O}_{3}-\mathrm{Al}_{2} \mathrm{O}_{3}-\mathrm{SiO}_{2}$ melts................... 11

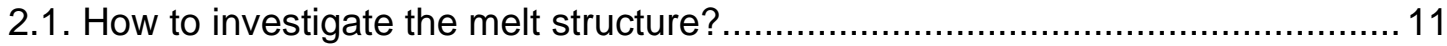

2.2. Step by step - theories developed through the years................................. 11

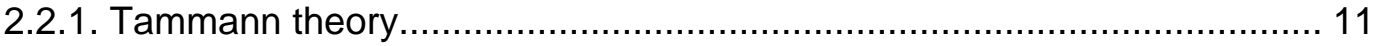

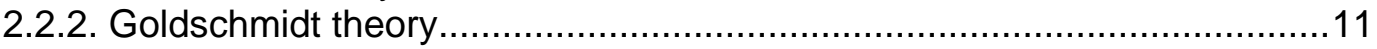

2.2.3. Zachariasen - Warren theory and fundamental groups of ions................12

2.2.4. Dietzel theory, field strength and bonds in the structure........................ 14

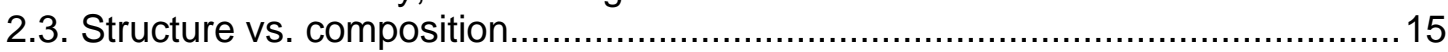

2.4. How to describe the melt structure? ........................................................ 19

2.5. Triclusters - new structural unit. Do they really exist?................................... 22

2.6. Flow mechanisms in aluminosilicates......................................................... 23

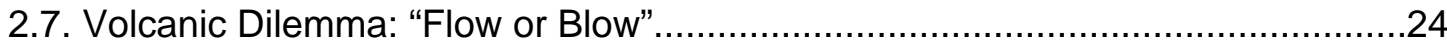

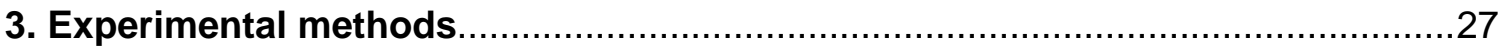

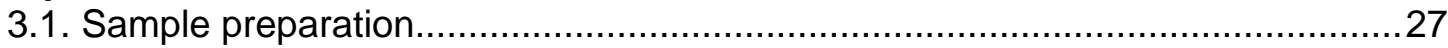

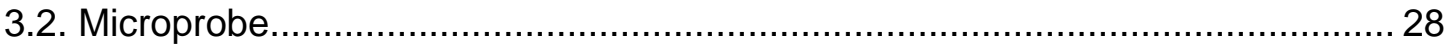

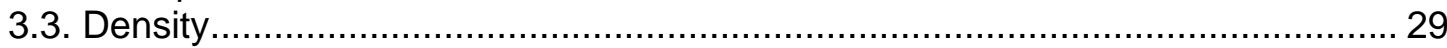

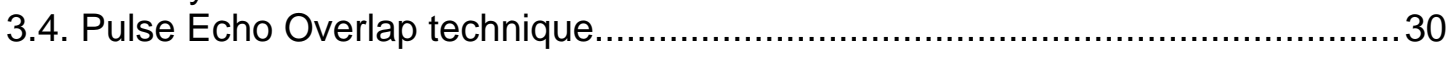

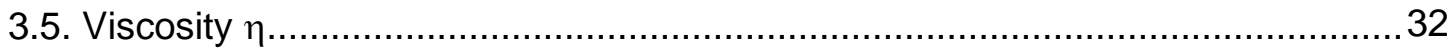

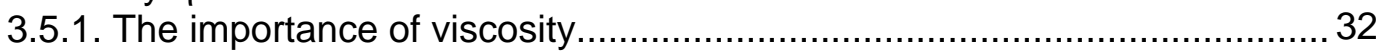

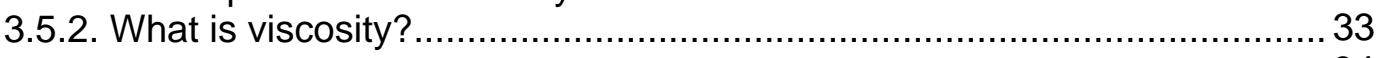

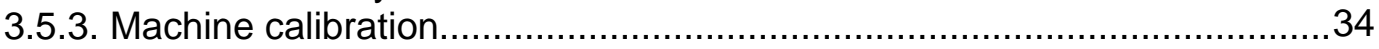

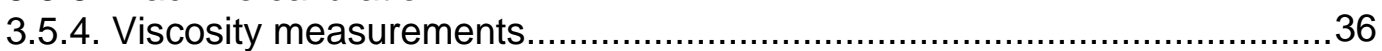

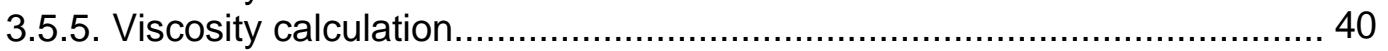

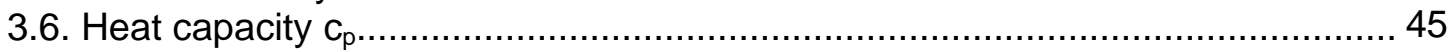

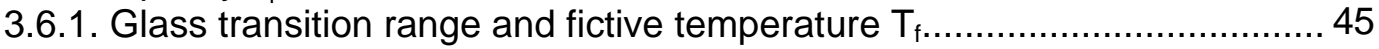

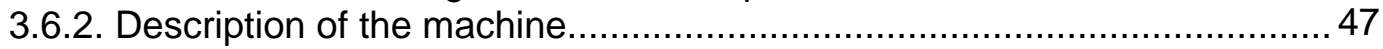

3.6.3. Calibration of the calorimeter........................................................... 48

3.6.4. Measurement and calculation of heat capacity and configurational heat

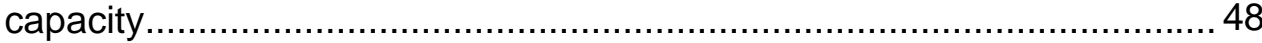

3.6.5. Calculation of the fictive temperature ............................................... 51

3.6.6. Dependence of the glass transition on the heating rates of the sample....51

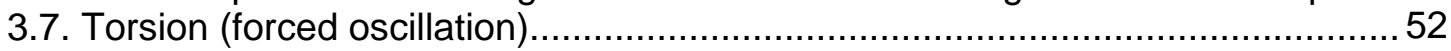

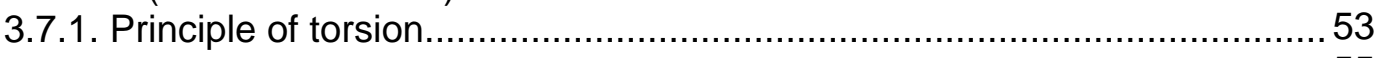

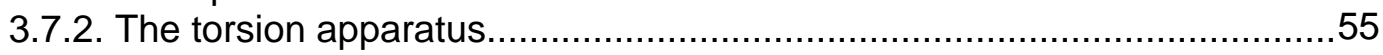

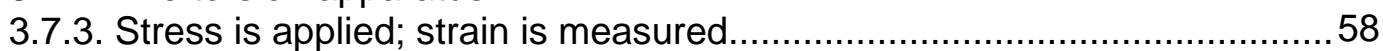

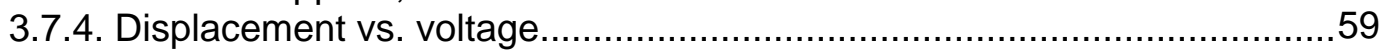

3.7.5. Frequency dependent measurements...............................................6 60

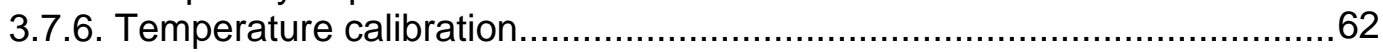

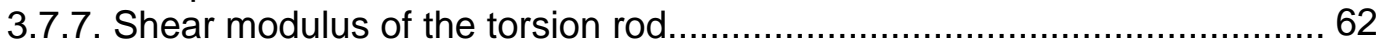

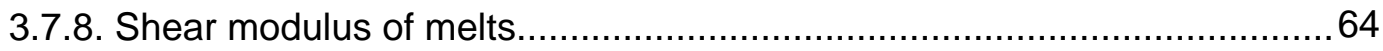

3.7.9. Shear modulus as a function of frequency ....................................... 70

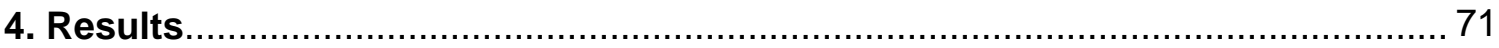

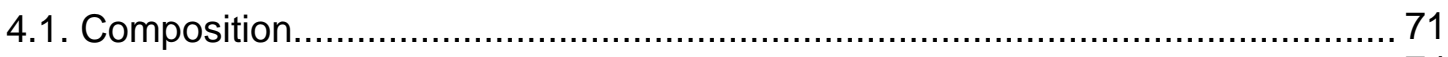

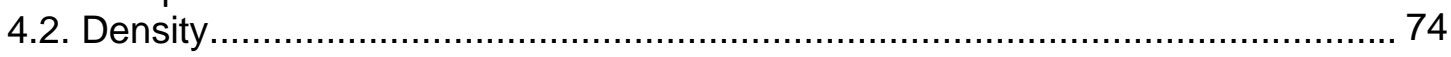

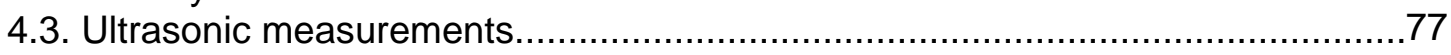

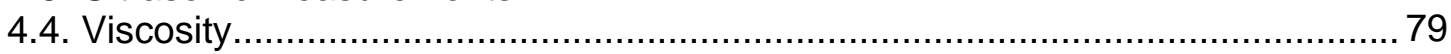

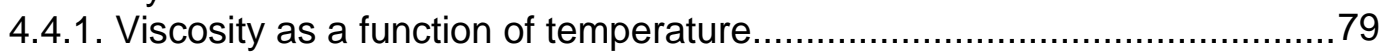

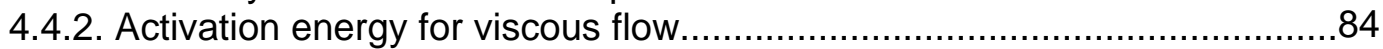




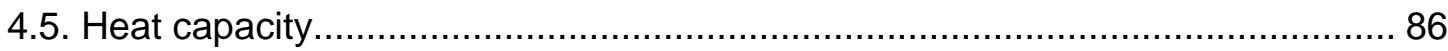

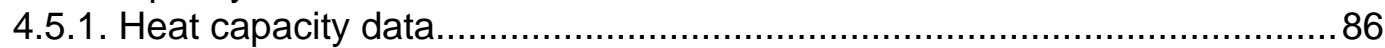

4.5.2. Glass transition temperature $T_{g}$ and fictive temperature $T_{f} \ldots \ldots \ldots \ldots \ldots \ldots . . . . .18$

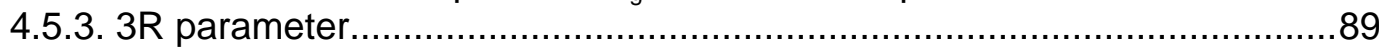

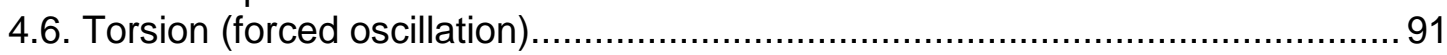

4.6.1. Real and imaginary shear modulus................................................... 91

4.6.2. Comparison of the shear moduli with literature data............................... 106

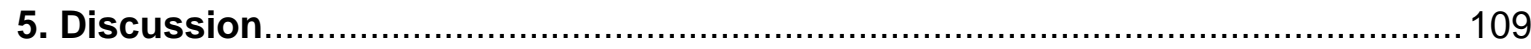

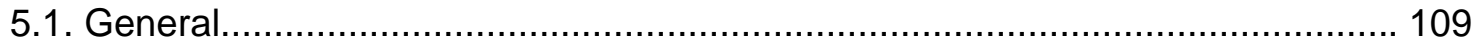

5.2. Density and partial molar volume at room temperature .................................. 109

5.3. Heat capacity and configurational heat capacity............................................ 111

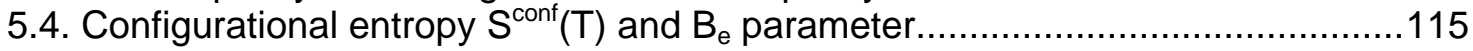

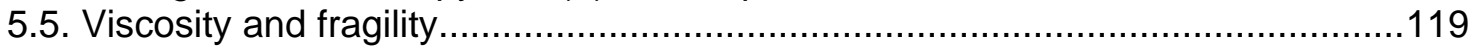

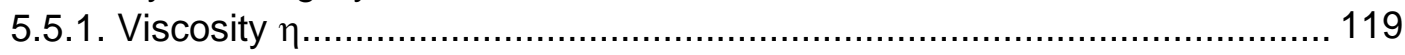

5.5.2. Frequency dependent shear viscosity $\eta \star(\omega)$ (from torsion)..................... 121

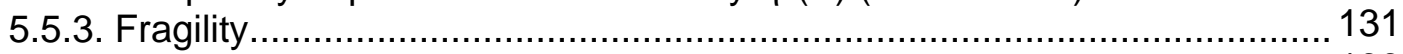

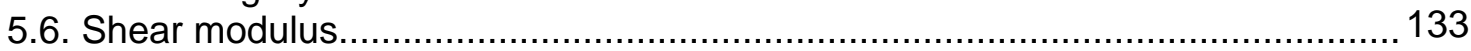

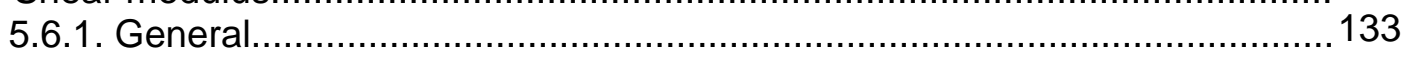

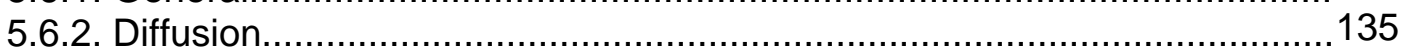

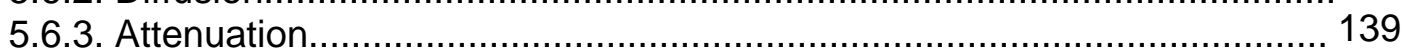

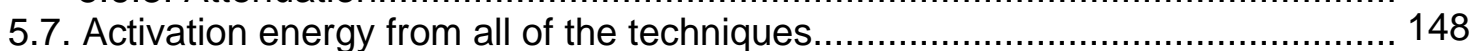

5.8. Fast relaxation time in aluminosilicate melts................................................. 149

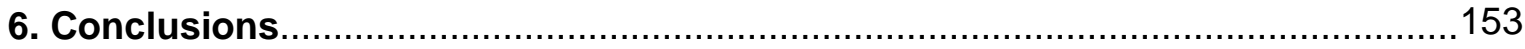

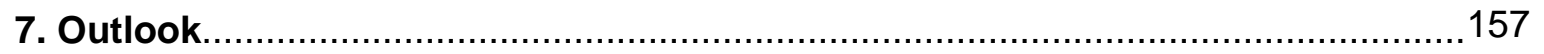

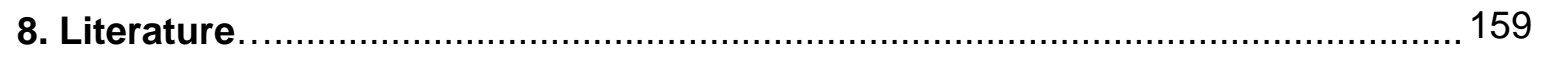

\section{Appendices}




\section{INTRODUCTION}

The mechanical and thermodynamic properties of Si-melts change dramatically with composition. This is because the structure of the melts is determined by composition; and the physical properties of melts depend on structure.

As shown in Figure 1, there is a complex dependence between structure, composition and physical and thermodynamic properties of the melts. Structure is a function of temperature, pressure, composition and time. The measured physical properties also depend on time. If the melt structure is in thermodynamic equilibrium, and also in equilibrium with the perturbation applied in the measurement of a physical property, the melt structure and the physical property are "relaxed". If the melt structure is in thermodynamic equilibrium but not in equilibrium with the perturbation applied to measurement of physical property, the property is "unrelaxed". If the melt structure is not in thermodynamic equilibrium, it is also "unrelaxed". Thus time - especially the time required for equilibrium to be reached - is important in the study of silicate melts.

The relationship between physical properties and structure in the $\mathrm{Na}_{2} \mathrm{O}-\mathrm{Al}_{2} \mathrm{O}_{3}-\mathrm{SiO}_{2}$ system has been investigated by a number of authors, especially Hunold \& Brückner (1980) who measured viscosity as a function of composition (Fig. 2) and temperature. The

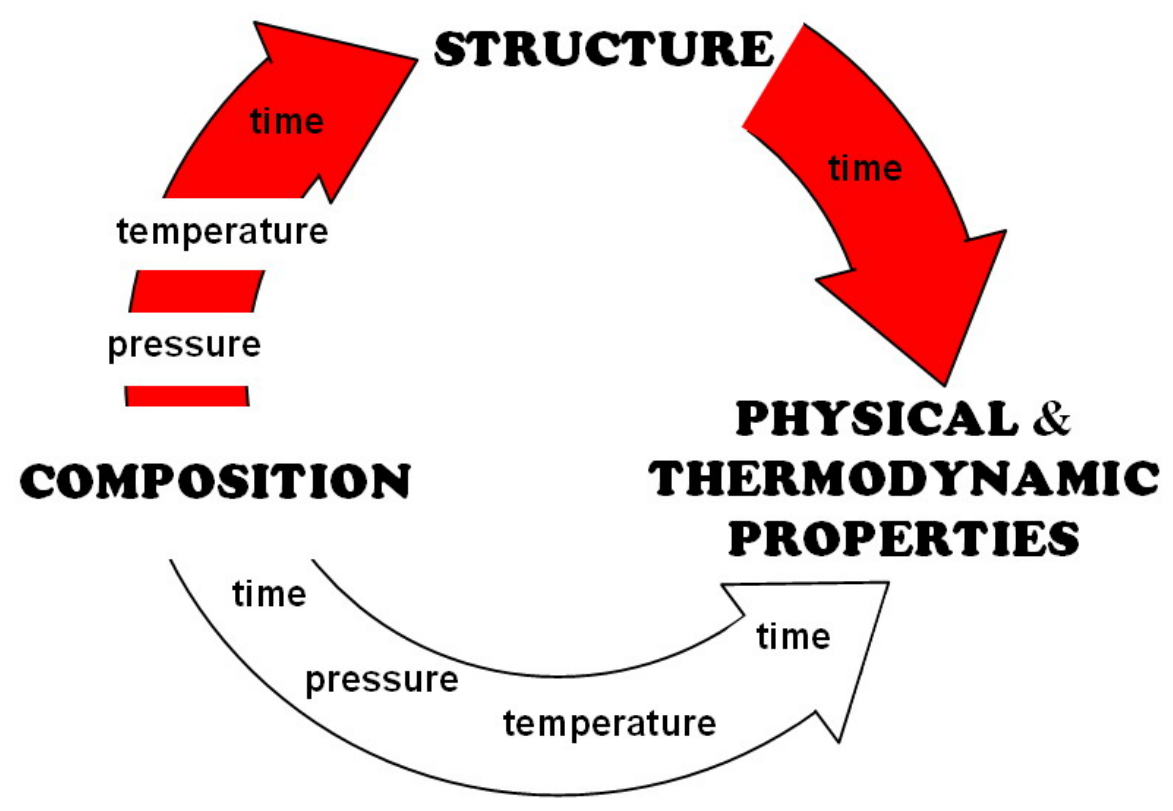

Fig. 1. Complex dependence between structure, composition, temperature, pressure, time and physical and thermodynamic properties of the melts. The red path of investigation has been chosen in this study. 
observation was a breaking point in trend at all temperatures as a function of composition at $\mathrm{Na}_{2} \mathrm{O} /\left(\mathrm{Na}_{2} \mathrm{O}+\mathrm{Al}_{2} \mathrm{O}_{3}\right) \sim 0.45$, indicating the presence of a structural change. Interest in this anomalous viscosity trend was awoken by the recent studies of Toplis et al. (1997a, b) and Webb et al. (2004). These studies show the same trend in viscosity as a function of composition as determined by Hunold \& Brückner (1980). A similar viscosity trend has been observed in the $\mathrm{LiO}_{2}-\mathrm{Al}_{2} \mathrm{O}_{3}-\mathrm{SiO}_{2}$ melts by Shelby (1978).

Here the structure of silicate melts is investigated as a function of the changes in their physical and thermodynamic properties with changing composition. It is known that there is a change in structure as a function of $\mathrm{Al}_{2} \mathrm{O}_{3}$ content in the $\mathrm{Na}_{2} \mathrm{O}-\mathrm{Fe}_{2} \mathrm{O}_{3}-\mathrm{Al}_{2} \mathrm{O}_{3}-\mathrm{SiO}_{2}$ system (see Webb, 2005b for a review) but the nature of this structural change is not fully understood. The heat capacity, shear modulus, shear viscosity and structural relaxation rate of a series $\mathrm{Na}_{2} \mathrm{O}-\mathrm{Fe}_{2} \mathrm{O}_{3}-\mathrm{Al}_{2} \mathrm{O}_{3}-\mathrm{SiO}_{2}$ melts have been measured; and are presented here together with the calculated structural relaxation time distribution, configurational entropy and configurational heat capacity as a function of composition. The combination of these measurements and calculated parameters has been used to address the question of the structure of these melts.

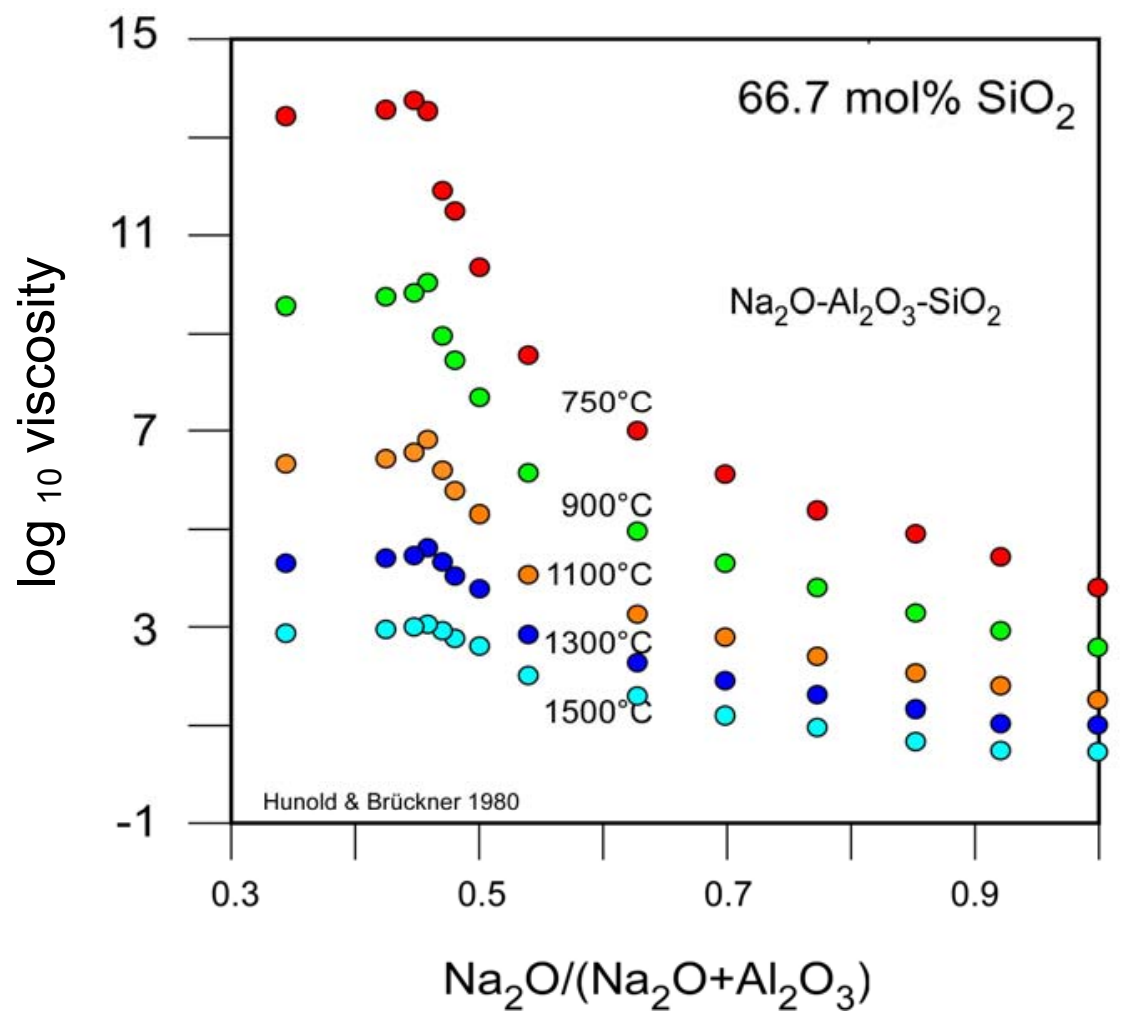

Fig. 2. $\log _{10}$ viscosity (Pa $\mathrm{s}$ ) as a function of composition for a series of sodium aluminosilicate melts at different temperatures showing the breaking point at $\mathrm{Na}_{2} \mathrm{O} /\left(\mathrm{Na}_{2} \mathrm{O}+\mathrm{Al}_{2} \mathrm{O}_{3}\right) \sim 0.45$. Redrawn after Hunold \& Brückner, 1980. 


\subsection{Viscoelasticity, flow and structural deformation}

The timescale of structural relaxation is traditionally determined by measurement of the viscoelasticity of the melt. Figure 3 illustrates the viscoelastic deformation of a melt due to the application of a step function of stress. First, the instantaneous recoverable elastic deformation occurs. This is followed by the time-dependent recoverable anelastic deformation. Finally, the time-dependent non-recoverable viscous deformation occurs. Thus, the deformation mechanism of the melt changes as a function of time. The structure of the melt is in equilibrium with temperature and pressure; but not in equilibrium with stress $\sigma$.

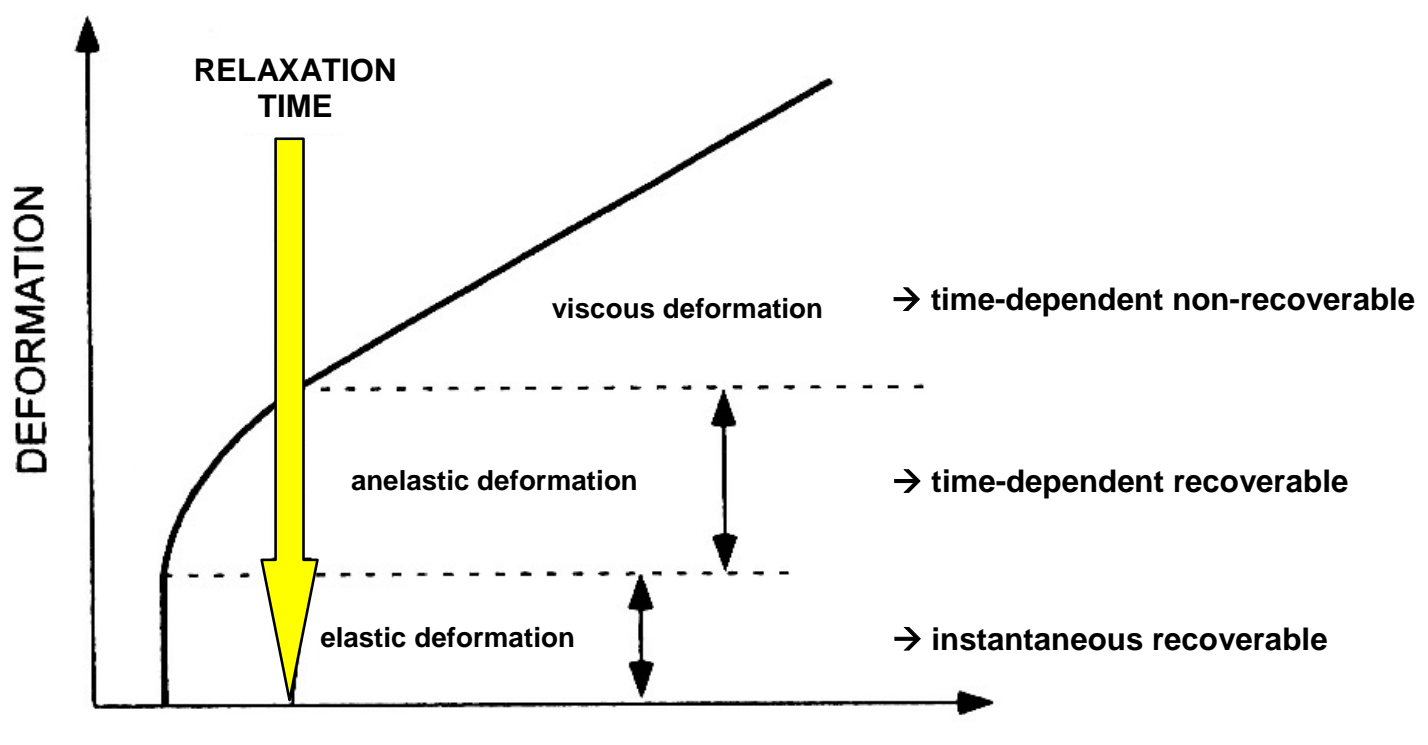

TIME

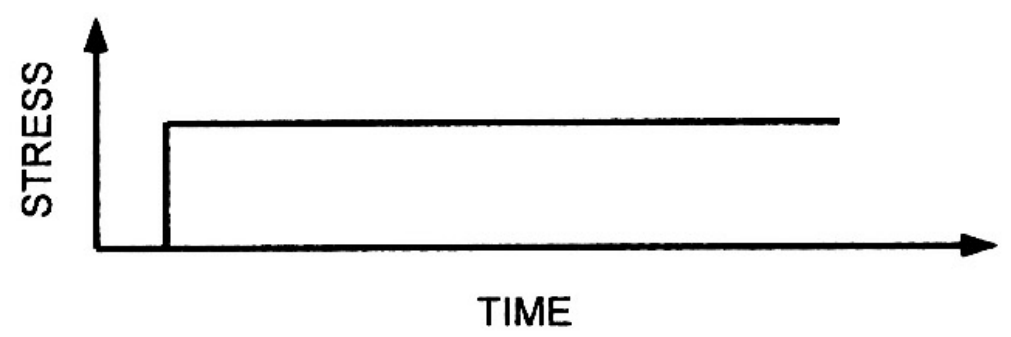

Fig. 3. A step function in stress as a function of time and the resulting time-dependent viscoelastic deformation of the melt. Redrawn after Webb, $2005 a$. 
Such stress - strain measurements result in the calculation of the shear modulus $\mathrm{G}$ (stress $\sigma$ divided by strain $\varepsilon$ ):

$$
G(t)=\frac{\sigma}{\varepsilon(t)}
$$

and shear viscosity $\eta$ (stress $\sigma$ divided by strain rate $\dot{\varepsilon}$ ):

$$
\eta(t)=\frac{\sigma}{\dot{\varepsilon}(t)}
$$

(see Fig. 4) as a function of time (Jaeger \& Cook, 1979). Similarly, a sinusoidal stress wave can be used to determine the shear modulus and viscosity as a function of frequency (Jackson, 1986; Webb, 1992a).

Here, both modulus and viscosity are linear - that is they are independent of the magnitude of the stress and strain. In general the $\varepsilon<10^{-5}$ is required for linearity of shear modulus (Jackson, 1986).

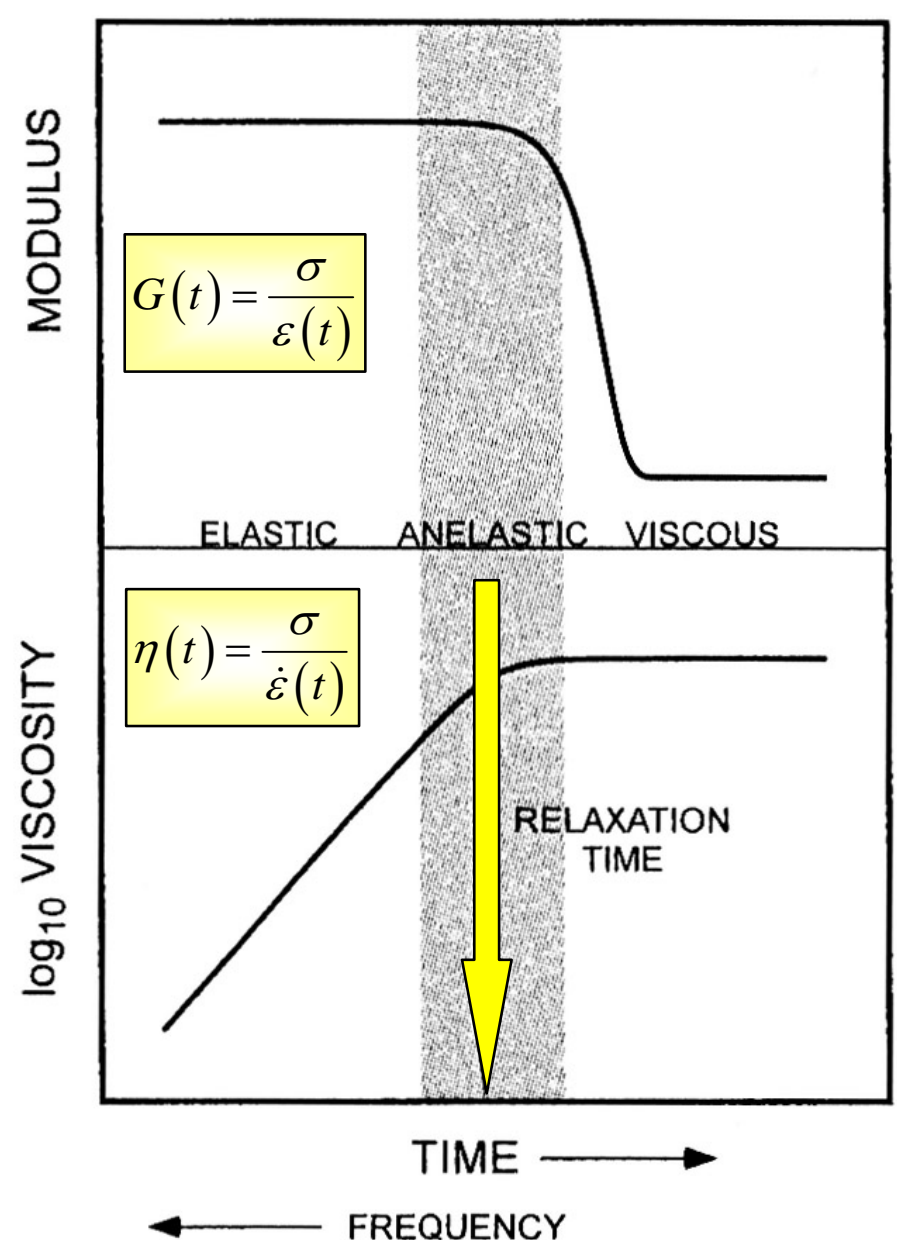

Fig. 4. The time-dependent shear modulus and shear viscosity calculated from the stress-strain plot of Figure 3. The shear modulus and shear viscosity determined by the application of a sinusoidal stress (e.g. torsion or ultrasonic measurements) are dependent upon frequency. Redrawn after Webb, 2005a. 
Relaxed liquids deform in the time dependent non-recoverable way what requires a continuous equilibration of the structure during the applying stress. Maxwell (1867) showed the structural relaxation time for a hard-sphere gas is:

$$
\tau_{M}=\frac{\eta_{0}}{G_{\infty}}
$$

where $\tau_{M}$ is Maxwell relaxation time, $\eta_{0}$ - the long timescale relaxed shear viscosity, $G_{\infty}$ - the instantaneous elastic shear modulus. The Maxwell relaxation time has been found by number of authors to successfully describe the structural relaxation rate in stress - strain measurements (Herzfeld \& Litovitz, 1959; Brawer, 1984; Rivers \& Carmichael, 1987; Webb, 1992a; Dingwell \& Webb, 1990; Dingwell, 1995; Stebbins, 1995). It has also been shown that the same relaxation time is applicable to perturbations in temperature (Narayanaswamy, 1971; DeBolt et al., 1976; Moynihan et al., 1976; Webb, 1992a,b; Moynihan, 1993).

Figure 5 illustrates the viscosity and calculated Maxwell relaxation time of $\mathrm{Na}_{2} \mathrm{Si}_{2} \mathrm{O}_{5}$ melt as a function of inverse temperature. A number of different techniques with different timescales of measurement (ultrasonic interferometry 1-30ns: Webb, 1992b; torsion 160-0.2s: Webb, 1991; Webb, 1992a; fibre elongation $10^{2}-10^{6} \mathrm{~s}$ : Webb \& Dingwell, 1990) are indicated. In all of these measurements, temperature is held constant and the timescale of measurement is varied. In the calorimetry and dilatometry (30-2s: Webb and Dingwell, 1995) measurements, the sample is heated at a constant rate, and thus the structure of the melt is changing as the experiment is performed.

In all cases, the frequency (or time) dependent behaviour was measured to occur in the vicinity of the calculated Maxwell relaxation time.

The NMR (nuclear magnetic resonance) datum (red point in Fig. 5) is the measured lifetime of Si-O bonds in this melt determined by Liu et al. (1988). This measurement led to the conclusion that the structural relaxation observed in all of these physical property measurements was the lifetime of Si-O bonds. Thus, in the stress strain measurements at short timescales only the stretching of Si-O bonds is determined; while at timescales longer than the lifetime of $\mathrm{Si}-\mathrm{O}$ bonds, the measured deformation includes the movement of $\mathrm{Si}$ and $\mathrm{O}$ atoms. 


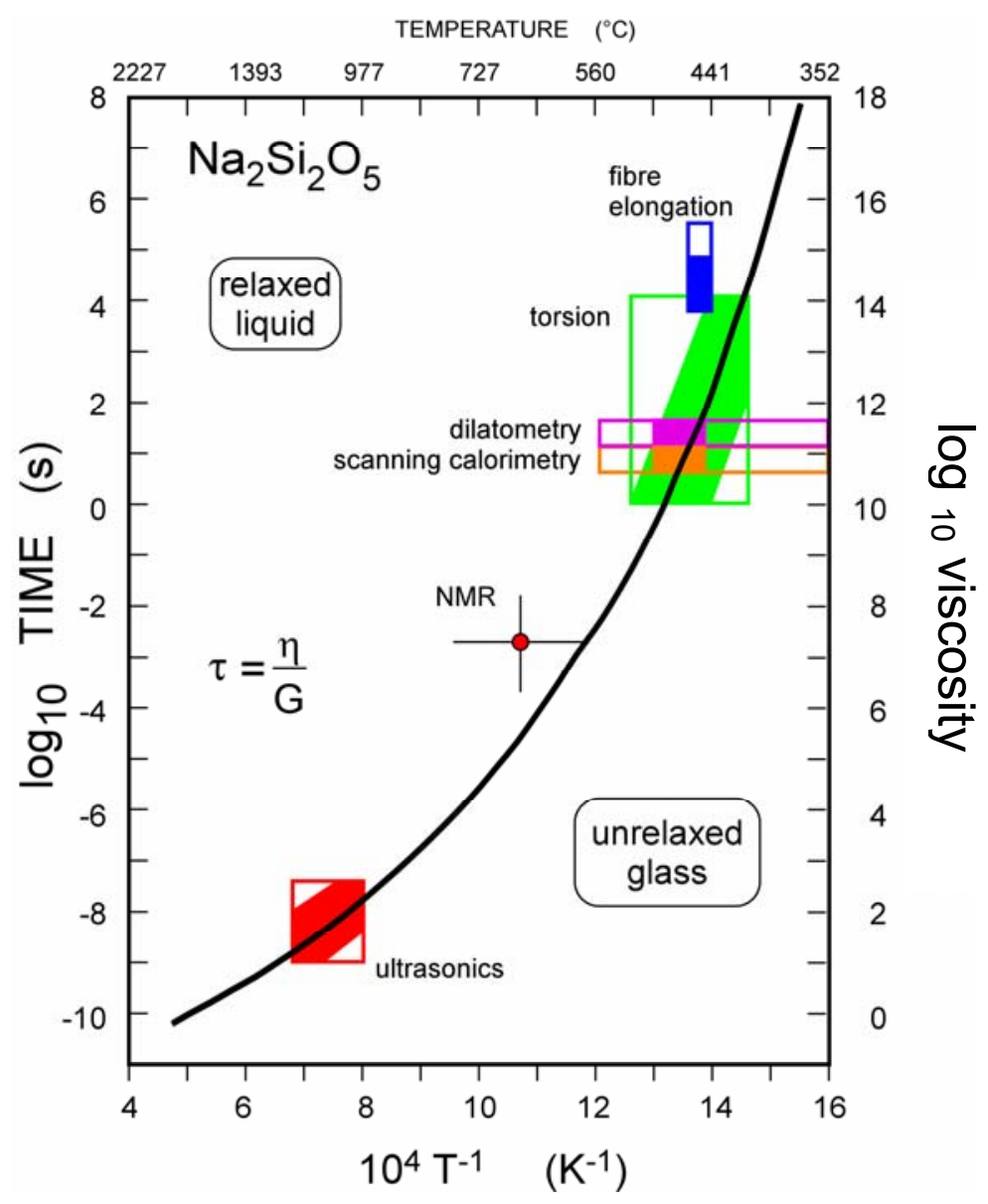

Fig. 5. The curve of relaxation time for $\mathrm{Na}_{2} \mathrm{Si}_{2} \mathrm{O}_{5}$ obtained from Maxwell relationship (1867) (see Eq. 3). Redrawn after Webb, 1992b.

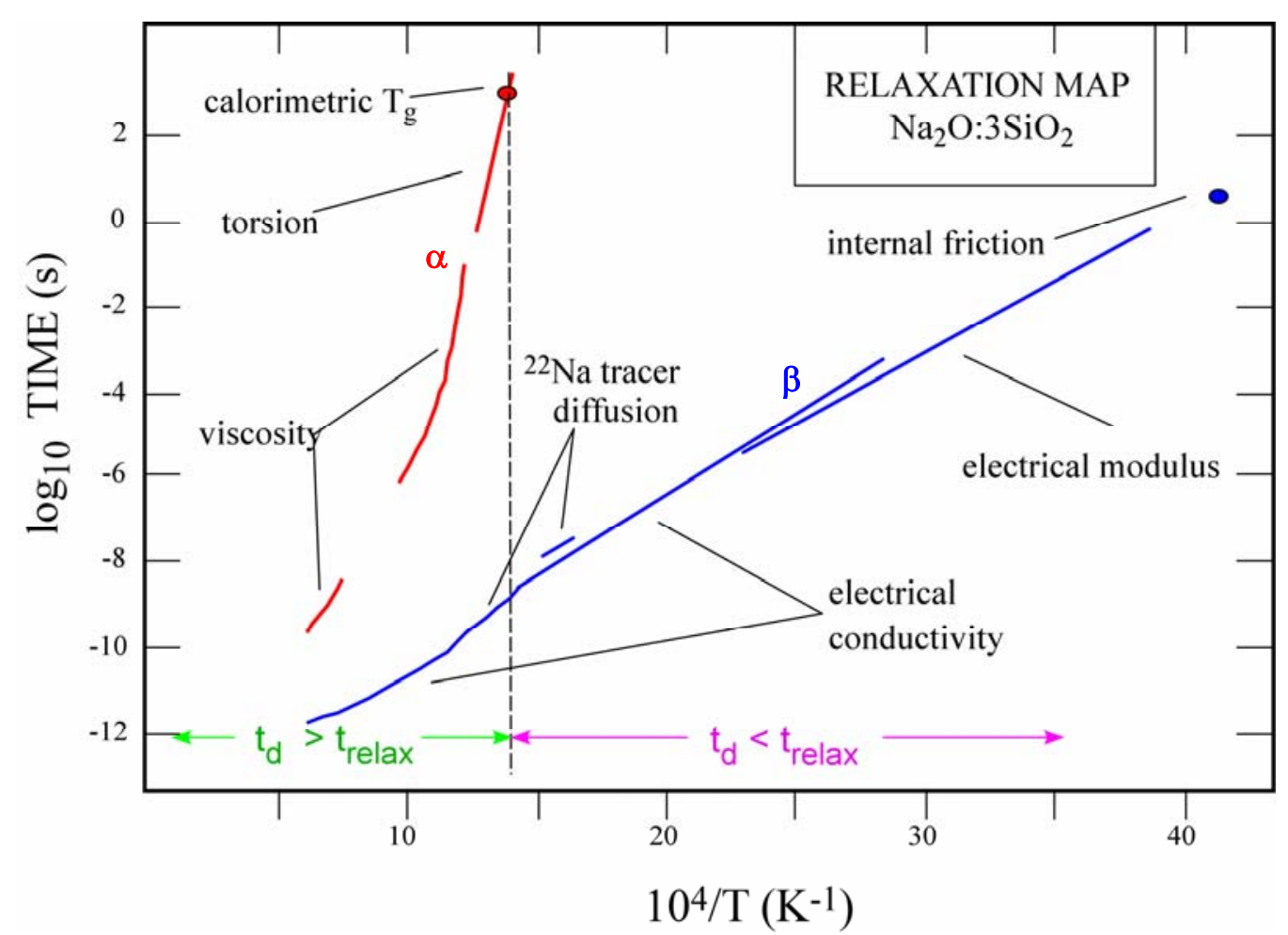

Fig. 6. $\alpha$-relaxation (slowest, characteristic for Si-O bonds) and $\beta$-relaxation (faster, for $\mathrm{Na}^{+}$ions) for $\mathrm{Na}_{2} \mathrm{Si}_{3} \mathrm{O}_{7}$ melt. Redrawn after Dingwell, 1990. 
The Maxwell relaxation time is the timescale on which the slowest part of the melt structure moves and thus is the glass transition of the melt - as shown in Figure 6 . The slowest structural relaxation in a material is called the $\alpha$-relaxation (Fig. 6). In order to relate melt structure to flow mechanisms and to physical properties, the lifetimes of not only the $\mathrm{Si}-\mathrm{O}$ but also the $\mathrm{Na}-\mathrm{O}$ and $\mathrm{Al}-\mathrm{O}$ bonds need to be measured. It was shown that relaxation process also occurs far away from the glass transition temperature towards lower temperature and it is called $\beta$ - or secondary relaxation (Zdaniewski et al., 1979; Dingwell \& Webb, 1990; Siewert \& Rosenhauer, 1997; Meyer et al., 2002). $\beta$-relaxation has in general lower activation energy than $\alpha$ and is explained as a diffusion of mono- and divalent ions in the melt. $\beta$-relaxation occurs in unrelaxed melt and after this process the melt structure still is not relaxed. $\alpha$-relaxation is the border between relaxed liquid and unrelaxed glass (see also Fig. 5).

The relationship between the lifetime of $\mathrm{Si}-\mathrm{O}$ bonds and viscosity in silicate melts has been discussed by a number of authors. The lifetime of the Si-O bonds in a silicate melt; or $\mathrm{Na}-\mathrm{O}$, or $\mathrm{Al}-\mathrm{O}$ bonds can be measured via a range of techniques. These include NMR (e.g. Stebbins, 1991; Stebbins \& McMillan, 1993; Stebbins \& Xu, 1997; Stebbins et al., 2001); diffusion measurements (e.g. Liu et al., 1988) and mechanical spectroscopy (e.g. Day and Rindone, 1962). The latter is the technique presented here. Mechanical spectroscopy or forced oscillation techniques - involve the determination of energy loss of a stress wave at the frequency at which part of the material structure moves. In the case of metals and ceramics (MacFarlane \& Rayne, 1967; Nagel \& Balogh, 1999; CarreñoMorelli et al., 2000) this may be the diffusion of atoms (Chakraborty, 1995; Brady, 1975; Freer, 1981; Brady, 1995); in the case of silicate melts it is the frequency (timescale) of motion of Si and O atoms (Bell \& Dean, 1970; Gaskell, 1970; Liu et al., 1988; Stebbins, 1991; Stebbins, 1995; Poe et al., 1997) or $\mathrm{Na}^{+}$and $\mathrm{K}^{+}$atoms (Day and Rindone, 1962). Previous forced oscillation measurements on silicate melts have shown the effect of the addition of $\mathrm{B}, \mathrm{P}$ and $\mathrm{F}$ on the frequency dependent energy loss spectrum (Bagdassarov et al., 1993). 


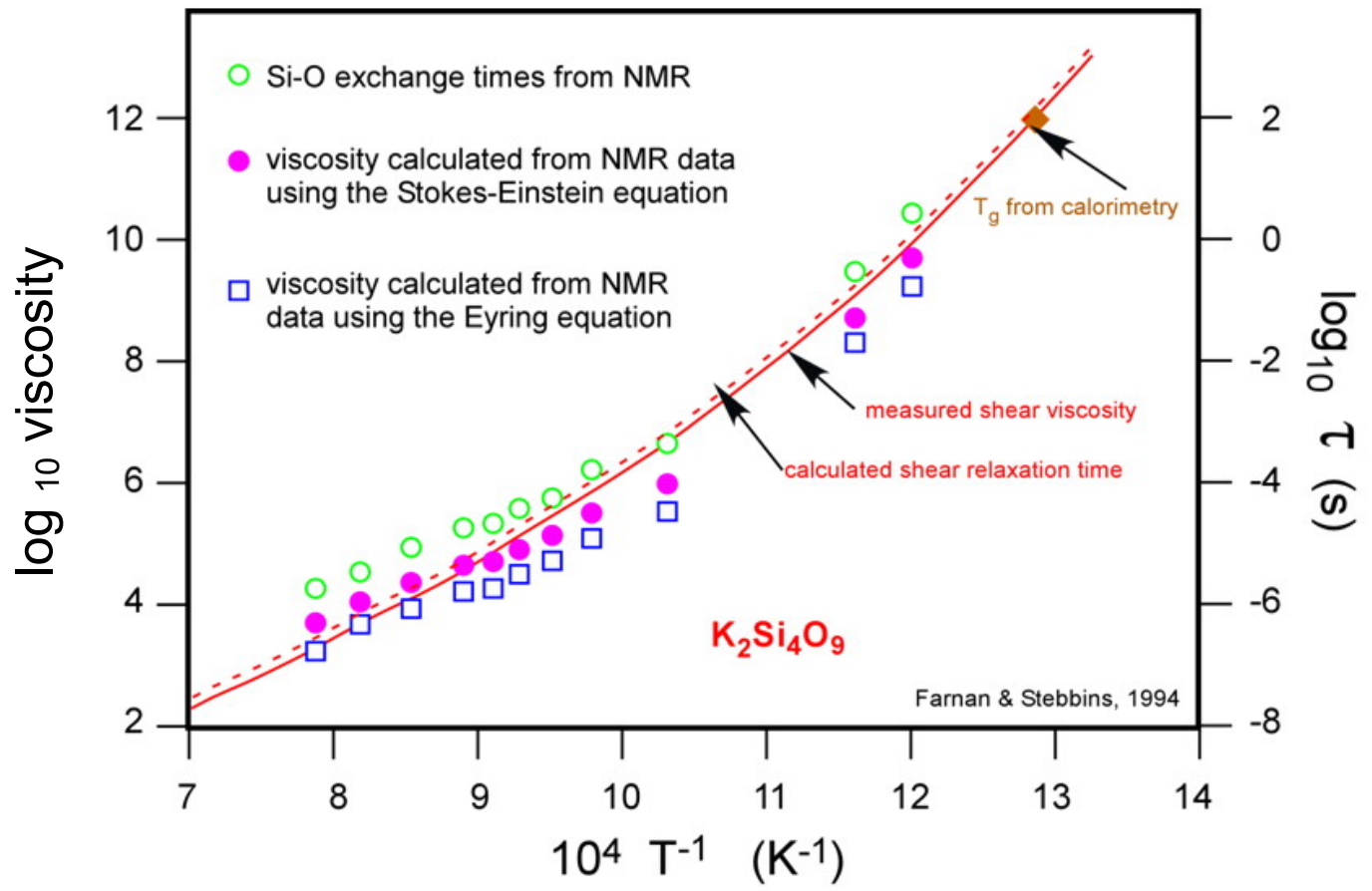

Fig. 7. Plot of the $\log _{10} \eta$ and relaxation time as a function of inverse temperature. Description in the text. Redrawn after Farnan \& Stebbins, 1994.

Farnan \& Stebbins (1994) showed that there is a close relationship between viscosity, relaxation time and diffusivity of the melts. In Figure 7 the solid curve shows the viscosity of $\mathrm{K}_{2} \mathrm{Si}_{4} \mathrm{O}_{9}$ (Farnan \& Stebbins, 1990a,b; Farnan \& Stebbins, 1994). The dotted curve is the calculated relaxation time using the Maxwell equation (Eq. 3). The open green circles are the lifetimes of $\mathrm{Si}-\mathrm{O}$ bonds from ${ }^{29} \mathrm{Si}$ NMR measurements; solid pink circles and open blue squares are the viscosity data calculated from NMR results but with two different equations $\left(\eta\right.$ is viscosity in $\mathrm{Pa} \mathrm{s}, k_{B}$ is Boltzmann's constant $1.380 \cdot 10^{-23} \mathrm{~J} \mathrm{~K}^{-1}, T$ is temperature in Kelvin, $D$ is self-diffusion coefficient in $\mathrm{m}^{2} \mathrm{~s}^{-1}, \lambda$ is translation distance; $r$ is the van der Waals radius of the molecule in meters):

- Eyring equation:

$$
\eta=\frac{k_{B} T}{\lambda D}
$$

- $\quad$ and Stokes-Einstein equation (Cruickshank Miller, 1924):

$$
\eta=\frac{k_{B} T}{6 \pi r D}
$$


They discovered that there is no difference between measured and calculated viscosity data and that correlation can be used in the future investigations. This relationship between viscosity, relaxation time and diffusivity is used to determine high pressure viscosity from diffusivity data (Reid et al., 2001; Reid et al., 2003)

In this study we address the changes in lifetimes of the $\mathrm{Si}-\mathrm{O}$ and $\mathrm{Al}-\mathrm{O}$ bonds; and the rate at which $\mathrm{Na}^{+}$ions move through $\mathrm{Na}_{2} \mathrm{O}-\mathrm{Al}_{2} \mathrm{O}_{3}-\mathrm{SiO}_{2}$ melts via forced oscillation measurements. As peralkaline and peraluminous melts have different structures, it is to be expected that the distribution of lifetimes of $\mathrm{Si}-\mathrm{O}$ and $\mathrm{Al}-\mathrm{O}$ bonds as well as the diffusion rate of $\mathrm{Na}^{+}$is different in the two composition extremes. 


\section{STRUCTURE OF $\mathrm{Na}_{2} \mathrm{O}-\mathrm{Al}_{2} \mathrm{O}_{3}-\mathrm{SiO}_{2}$ AND $\mathrm{Na}_{2} \mathrm{O}-\mathrm{Fe}_{2} \mathrm{O}_{3}-\mathrm{Al}_{2} \mathrm{O}_{3}-\mathrm{SiO}_{2} \mathrm{MELTS}$}

\subsection{How to investigate the melt structure?}

An investigation of the melt structure can be done by a range of methods. Because melts show short range order, medium range and extended medium range order the best methods are spectroscopic (Hawthorne, 1988; Beran \& Libowitzky, 2004), e.g. nuclear magnetic resonance (NMR) (Stebbins et al., 1995), infrared (IR), Raman spectroscopy, Mössbauer spectroscopy, X-ray absorption near-edge structure (XANES), extended X-ray absorption fine structure (XAFS), neutron scattering or X-ray diffraction (Meade et al., 1992; Zotov \& Delaplane, 2000; Hennet et al., 2005; Matsumura et al., 2007). These techniques allow measurement of the bond angles and distances between atoms, for determination of the coordination number of the central atom and for investigation the nearest and next nearest neighbours in the melt structure. Here changes in physical properties as a function of composition are used to infer possible changes in melt structure.

\subsection{Step by step - theories developed through the years}

\subsubsection{Tammann theory}

Many different theories describing the structure of glasses and melts have been developed during the past 100 years. The first theory was proposed by Tammann (Tammann, 1903; Tammann, 1923; Tammann, 1933), who said that glasses have exactly the same structure as the melt. He assumed that structure of liquids is largely retained and it stays intact during the cooling.

\subsubsection{Goldschmidt theory}

Second hypothesis by Goldschmidt defined a glass structure from his chemical investigations (Goldschmidt, 1926). Goldschmidt assumed that to create the glass there is needed a cation/anion ratio between 0.2 and 0.4 which is exactly in such glass forming compounds as $\mathrm{SiO}_{2}, \mathrm{P}_{2} \mathrm{O}_{5}$ or $\mathrm{B}_{2} \mathrm{O}_{3}$ and even $\mathrm{BeF}_{2}$ solidifying to a glass. 


\subsubsection{Zachariasen - Warren theory and fundamental groups of ions}

In 1932 Zachariasen (Zachariasen, 1932) proposed a new theory which was confirmed by Warren (1933) with the X-ray diffraction. They discovered that in the vitreous $\mathrm{SiO}_{2}$ glass the smallest unit is a $\mathrm{SiO}_{4}$ tetrahedron. The tetrahedra create a disordered three-dimensional network. The new idea was also introducing a "coordination number" term (an average number of nearest neighbours); for example, in the $\mathrm{SiO}_{4}$ tetrahedron in $\mathrm{SiO}_{2}$ glass this number equals 4; and for $\mathrm{B}_{2} \mathrm{O}_{3}$ glass coordination number for planar trigonal $\mathrm{BO}_{3}$ unit is 3 (Fig. 8).

Zachariasen - Warren theory assumes that oxides are trying to form the polyhedral groups as the smallest units building the structure and such two polyhedra may be linked just to one corner. On the other way, the polyhedron cannot have more than six corners. The anions like $\mathrm{O}^{2-}$ or $\mathrm{S}^{2-}$ create the bridges between pairs of polyhedra because they cannot be connected to more than two central atoms of polyhedra. Minimum three corners of a polyhedron need to be bonded with the neighbouring polyhedra through anion bridges (in silicate melts: bridging oxygens).

The bridges between polyhedra will be broken, if the large cation appears in the structure (e.g. $\mathrm{Na}^{+}$or $\mathrm{Mg}^{2+}$ ). Then oxygen from the additional oxide will go to a free corner of the separate tetrahedra and the cation will balance the negative charge of the tetrahedra and causes the breaking of the network by reason of its size (Vogel, 1965).
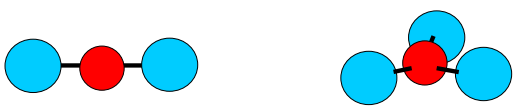

linear

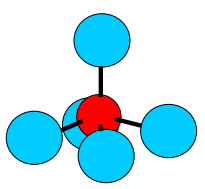

tetragonal pyramidal triangular

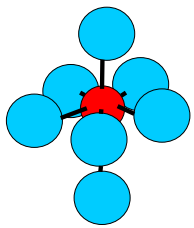

pentagonal bipyramidal

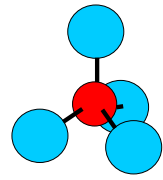

tetrahedral

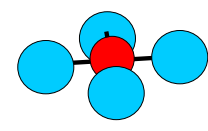

square planar

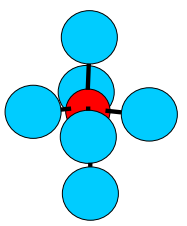

octahedral

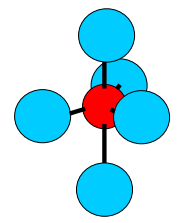

trigonal bipyramidal

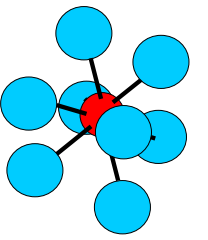

cubic

Fig. 8. Coordination number of the atom, congruous with the geometric shape. The number of blue surrounding atoms says about the value of coordination number of the central red atom. 
Zachariasen (1932) has classified the ions creating the glass structure into three groups: network formers (e.g. Si, B, P, Ge, As and Be with coordination number 3 or 4), network modifiers (like $\mathrm{Na}, \mathrm{K}, \mathrm{Ca}$ or $\mathrm{Ba}$ with coordination number higher than 6 ) and intermediate oxides (e.g. Al, Mg, $\mathrm{Zn}, \mathrm{Pb}, \mathrm{Be}, \mathrm{Nb}$ or Ta with coordination number between 4 and 8 ). The intermediate oxides can be either the network formers or network modifiers.

With the present stage of knowledge about the glass structure the scientists distinguish more groups (Fig. 9):

- network formers (e.g. $\mathrm{Si}^{4+}, \mathrm{Al}^{3+}, \mathrm{Fe}^{3+}$ or $\mathrm{Ti}^{4+}$ ) arranged in the tetrahedra and creating the network owing to the covalent bonding forces;

- network modifiers (alkali or alkaline-earth metal cations) - make with oxygen weaker covalent and metal bonds and they have mostly octahedral coordination. They connect with an oxygen and through that they generate non-bridging oxygens;

- charge balancers (alkali or alkaline-earth metal cations) compensating the negatively charged tetrahedrally coordinated units. There is loose exchange between network modifiers and charge balancer depending on the composition of the melt;

- bridging oxygens (BO) - oxygen atoms bonding two central atoms of the tetrahedra;

- non-bridging oxygens (NBO) - oxygen atoms bonding one central atom of tetrahedra with some other atom (e.g. network modifier).

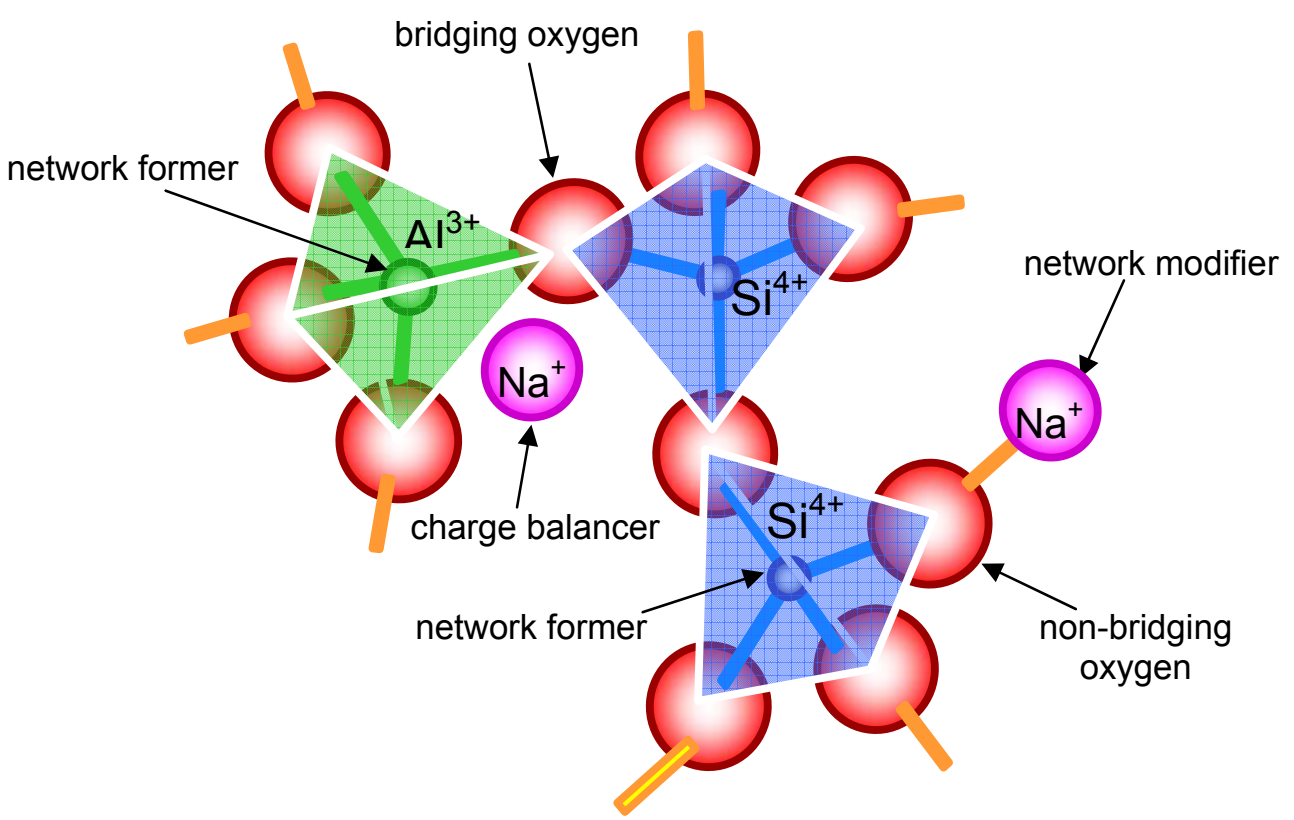

Fig. 9. Fundamental groups of ions in the melt. Description in the text. 


\subsubsection{Dietzel theory, field strength and bonds in the structure}

The next big step in the knowledge about structure of the glasses was made by Dietzel (1942). He developed the Goldschmidt's hypothesis and took into consideration also the field strengths of the ions. During the cooling, the central atoms are trying to keep the surrounding atoms in the closest possible packing. If the central atoms have the same value of field strength, then the homogenisation of the melt can not occur and melt divides into separate phases. In the case of the cations with different field strength, the oxygens will create a closest packing near to the atom with stronger field. The cation with the lowest field strength gets a higher coordination number and is bonded to the tetrahedron with negative charge, e.g. $\left[\mathrm{SiO}_{4}\right]^{4-}$.

Copolymerisation is possible when also separate structural units have similar chemical properties. The difference between donor - acceptor properties of two bonded elements determines the covalence (degree of ionicity) of the chemical bond. The strength of such bond can be described by a term $\gamma_{\Phi}$ :

$$
\gamma_{\Phi}=\frac{I_{n}}{r_{o r b}^{n+}}
$$

where $I_{n}$ is the ionisation potential of the $n^{\text {th }}$ electron and $r^{n+}$ orb is the orbital radius of an ion with a charge $n^{+}$(Godovikov, 1979). In other words, it says about Coulomb forces between the $\mathrm{n}^{\text {th }}$ electron and the atomic core with charge $n^{+}$(see Table 1).

In vitreous silicate melt only one type of bonds occurs, namely between network forming $\mathrm{Si}$ and the oxygens. Because $\mathrm{Si}^{4+}$ cations have the strongest $\gamma_{\Phi}$ parameter with oxygen, the structure of this melt should be very strong and that would explain the slowest (the longest) relaxation time. Smaller ionicity of the bond with oxygen show successively $\mathrm{Al}^{3+}$ and $\mathrm{Fe}^{3+}$.

$\mathrm{Al}^{3+}$ and $\mathrm{Fe}^{3+}$ are network formers in peralkaline melts and build the tetrahedra with one negative charge (Mysen et al., 1985c). Hierarchy of the charge balancer is the same for both: $\mathrm{K}^{+}, \mathrm{Na}^{+}, \mathrm{Ca}^{2+}, \mathrm{Fe}^{2+}, \mathrm{Mg}^{2+}$ (Mysen, 1987). To create a stable Al- and Fe-tetrahedra these units need to be charge balanced by cations with smaller $\gamma_{\Phi}$ value than respectively $\mathrm{Al}^{3+}$ and $\mathrm{Fe}^{3+}$. Hess and Wood (1982) also showed that compensation the Al-tetrahedra will first occur by cations with lower field strength and then together with increasing $\gamma_{\Phi}$. 
Tab. 1. Force characteristics $\gamma_{\Phi}$ of cation (Godovikov, 1979).

\begin{tabular}{|c|c|}
\hline Cation & $\gamma_{\phi}$ \\
\hline $\mathrm{Si}^{4+}$ & 225.6 \\
$\mathrm{Al}^{3+}$ & 128.7 \\
$\mathrm{Ti}^{4+}$ & 94.8 \\
$\mathrm{Fe}^{3+}$ & 86.3 \\
\hline $\mathrm{K}^{+}$ & 7.3 \\
$\mathrm{Ba}^{2+}$ & 11.5 \\
$\mathrm{Sr}^{2+}$ & 16.4 \\
$\mathrm{Na}^{+}$ & 18.5 \\
$\mathrm{Ca}^{2+}$ & 22.1 \\
$\mathrm{Li}^{+}$ & 28.5 \\
$\mathrm{Fe}^{2+}$ & 44.4 \\
$\mathrm{Mg}^{2+}$ & 61.1 \\
\hline
\end{tabular}

The experiments with glasses containing Fe-tetrahedra (Dingwell \& Virgo, 1988 a, b) showed that the most stable cation to compensate a negative charge of $\mathrm{Fe}^{3+}$ unit is $\mathrm{K}$, then $\mathrm{Na}, \mathrm{Ba}, \mathrm{Sr}, \mathrm{Ca}$ and the less stable in this group is $\mathrm{Mg}$.

In the fully polymerized melt (without NBO) the most important bonds are these between oxygen and network formers. The bonds between network former ions are covalent and their average bond valence is above $3 / 4$ of the valence unit, v.u.) and they are not willing to change the structure. Network modifiers create weak bonds (e.g. Na average bond valences below 0.2 v.u.) (Wispelaere et al., 2004). Na-O and Al-O bonds are longer than $\mathrm{Si}-\mathrm{O}$, but $\mathrm{Si}-\mathrm{O}$ energy bond is at about $20 \%$ higher than energy of $\mathrm{Al}-\mathrm{O}$ bond (Stein \& Spera, 1993).

\subsection{Structure vs. composition}

Structure of the aluminosilicates is strongly dependent on composition, what is shown on the plots as a breaking point in the trends of physical properties as a function of composition (Mysen \& Frantz, 1994; Mysen, 1995a). But bulk properties of the melt and glass depend on the presence of different structural units, their concentration and composition (Seifert et al., 1982).

Silicon is tetrahedrally coordinated network former and creates a $\mathrm{SiO}_{4}$ structural unit. $\mathrm{Si}$ is substituted by tetrahedrally coordinated $\mathrm{Al}^{3+}$ (Spiering \& Seifert, 1985); and tetrahedral Al copolymerizes with silicon (Riebling, 1966; Kushiro, 1976; Dickenson \& Hess, 1985).

Aluminium cation can have either a tetrahedral coordination and exists as a network former; or octahedral coordination with respect to oxygen and plays a modifier role (Mysen, 1981; Mysen et al., 1982). $\left[\mathrm{AlO}_{4}\right]^{-}$tetrahedron needs to be compensated by 
alkali or alkaline-earth metal cation with a positive charge of one. In peralkaline melts the ratio $\mathrm{Al} / \mathrm{Na}$ is smaller than one; it means that there is enough sodium to compensate the negative charge of the $\mathrm{Al}$ unit. The excess $\mathrm{Na}$ ions connect with non-bridging oxygens and play a network modifier role.

If there is enough mono- or divalent alkali or alkaline-earth metals in the melt to compensate negative charge of Al-tetrahedra, Al is tetrahedrally coordinated (Riebling, 1964; Riebling, 1966; Bottinga \& Weill, 1972, Mysen et al, 1980b; Wood \& Hess, 1980; Stebbins \& Farnan, 1992). Mysen et al. (1981a) and McMillan \& Piriou (1982) suggested, that when in the melt, there is a lack to charge balance of $\mathrm{Al}^{3+}, \mathrm{NBO}$ will form what extorts transformation of coordination state of $\mathrm{Al}^{3+}$ from tetrahedral to octahedral. MAS NMR measurements of highly peraluminous glasses have found $\mathrm{Al}$ in [IV] and [VI] coordination (Risbud et al., 1987; Bunker et al., 1991; Sato et al., 1991a; Sato et al., 1991b; Poe et al., 1992). The intermediate (fivefold) coordinated aluminium, has been also discovered (Poe et al., 1992).

The amount of ${ }^{[\mathrm{VI}]} \mathrm{Al}^{3+}$ was however not enough to account for all of the $\mathrm{Al}$ without a charge balancer and therefore the current melt structure is based on the idea of triclusters - introduced by Lacy (1963); and discussed by Toplis et al. (1997a, b). This is supported strongly by the viscosity data. Using the simple rules, network formers increase viscosity and polymerization, where network modifiers decrease viscosity and polymerization. One observes that viscosity stays almost constant in Figure 2 in the peraluminous field suggesting there are no new NBOs created upon the addition of $\mathrm{Al}_{2} \mathrm{O}_{3}$ and therefore $\mathrm{Al}$ cannot be octahedral or the amount of octahedral $\mathrm{Al}$ is too small to influence any structural change.

Iron plays a significant role in the magmatic systems. $\mathrm{Fe}$ is heterovalent and because of that is very important in melting and crystallization, depending on the conditions. However, coordination number of the iron ions can be dominant and has a big influence on the properties of the melt. From the other side, composition, temperature or pressure can control the properties of the iron, like coordination number or oxidation state (Johnston, 1964; Sack et al., 1980; Mysen, 1981; Kilinc et al., 1983; Dyar et al., 1985; Mysen et al., 1985a,b; Paul, 1990; Kress \& Carmichael, 1991; Baker \& Rutherford, 1996; Burkhard, 2000; Gaillard et al., 2001; Wilke et al., 2002; Botcharnikov et al., 2005; Wilke et al., 2006).

According to the Mössbauer spectroscopy it is known that in the melts iron can exist as $\mathrm{Fe}^{3+}$ and $\mathrm{Fe}^{2+}$ (Seifert et al., 1979; Virgo et al., 1981). $\mathrm{Fe}^{3+}$ may form the tetrahedra but $\mathrm{Fe}^{2+}$ can occur as a network modifier and a charge balancer. Then some sort of grouping must occur if a divalent Fe compensates two tetrahedra. One $\mathrm{Fe}^{2+}$ ion 
replaces two $\mathrm{Na}^{+}$ions. The higher field strength of the divalent iron leads to decreasing the free volume through the shortening of the bonds between $\mathrm{Fe}^{2+}$ and oxygen.

$\mathrm{Fe}^{2+}$ is taken to be octahedrally coordinated and to play a network modifier role (Seifert and Olesch, 1977a,b; Mysen \& Virgo, 1978; Nolet et al., 1979; Seifert et al., 1979; Mysen et al., 1980a; Spiering \& Seifert, 1985).

$\mathrm{Fe}^{3+}$ can be (1) octahedral - when not enough alkali or alkaline-earth metals exist to compensate a negative charge of the anion groups); or (2) tetrahedral - when there is enough alkali or alkaline-earth metals to play network balancer and network modifier roles (investigations of alkali silicate and soda lime silicate glasses by Bamford, 1960; Steele \& Douglas, 1965; Kurkjian \& Sigety, 1968; Levy et al., 1976; Hirao et al., 1979; DeGrave, 1980; Fenstermacher, 1980; Fox et al., 1982; Calas \& Petiau, 1983; Greaves at el., 1984; Brown et al., 1986; Wang \& Chen, 1987; Hannoyer et al., 1992; Wang et al., 1993; Wang et al., 1995). Virgo et al. (1982b) showed that $\mathrm{Fe}^{3+}$ tetrahedron does not copolymerize with Si-tetrahedron.

New investigations allow for precise estimation of the ratio between tetrahedrally and octahedrally coordinated cations of $\mathrm{Fe}^{3+}$ in melts. Weigel et al. (2006) determined that $95 \%$ of $\mathrm{Fe}^{3+}$ in the structure of $\mathrm{NaFeSi}_{2} \mathrm{O}_{6}$ form tetrahedra. The remaining $5 \%$ of iron can be five- or six-coordinated. This $5 \%$ of octahedral $\mathrm{Fe}^{3+}$ corresponds to $\sim 0.15 \mathrm{~mol} \%$ of the melt compositions investigated here.

Alkalis and alkaline earths plays a role of network modifiers and charge balancers of $\mathrm{Al}^{3+}$ in tetrahedral coordination in melts and glasses (Bottinga \& Weill, 1972; Mysen et al., 1981a; Secco et al., 1991; Neuville \& Mysen, 1996; Mysen, 1997). Sodium ion is very good charge balancer for Fe-tetrahedra (Russel \& Wiedenroth, 2004). Stabilization of Fe-tetrahedra with alkali cations increases with decreasing their field strength (Bingham et al., 2007). The effect of alkaline earths on Fe-tetrahedra is the inverse of that of the alkalis.

But what happens exactly in the melts going from peralkaline towards peraluminous composition?

In peralkaline glass or melt, there is enough network modifiers and charge balancers to compensate any negative charge of the structural units. With an increasing number of network modifiers the melt becomes more fluid because silicon-oxygen bonds being the bridges between silicon atoms need to be broken to connect new ion. Decreasing the amount of network modifiers up to their absence induces higher polymerization of the melt and follows that the viscosity increases. The structure gains free volume due to the polymerisation process and the energy needed to break stronger bonds between tetrahedra increases. The increase of the interstices causes that the "doorways" between tetrahedra become larger. That was shown in the experiments with 
the diffusion of $\mathrm{He}$ in aluminosilicate melts (Roselieb et al., 1992). There is very important dependence between empty and filled interstices because the size of filled interstices strongly depends on the size of empty interstices (Shelby, 1979).

In aluminosilicates with high Al content, the network is controlled by bonds between network formers and oxygen; and the identity of the network modifiers does not play any important role (Shelby, 1989).

Some polymerized units (regions) play an important role in the structure of the melts (Bottinga \& Weill, 1972; Burnham, 1975; Mysen, 1988). Lacy (1963) suggested that $\mathrm{AlO}_{6}$ octahedra are not stable and proposed the new structural unit of $\mathrm{AlO}_{6}$ tricluster. During formation of $\mathrm{AlO}_{6}$ tricluster no new NBO are formed. This theory has more or less followers but spectroscopic and diffraction measurements prove an existence of the denser and higher coordinated structural units in the network, which could be triclusters.

Gaskell \& Mistry (1979) in the $\mathrm{NaAlO}_{2}-\mathrm{SiO}_{2}$ system suggested the presence at least two different types of $3 \mathrm{D}$ connected units in aluminosilicate composition. Three dimensional $\mathrm{SiO}_{4}$ and $\mathrm{AlO}_{4}$ tetrahedra has been detected with the RDF X-ray technique by Taylor and Brown (1979a), and those results have been confirmed e.g. by Mysen et al. (1980b) with Raman spectroscopy. Dirken et al. (1997) have found clear separate Q3 units (see section 2.4.) with Si-O-Al bridging oxygen and Si-O-Si bridging oxygen in the structure of albite, but no Al-O-Al bridging oxygen or others. Stebbins \& Xu (1997) in the investigation of anorthite glass have found Q3 units with the same characteristics, but they have also distinguished NBO in peraluminous composition. That changes a theory that glass with peraluminous composition is fully polymerized. Stebbins \& Xu (1997) have also localized other very small feature in the structure of anorthite, what Xue \& Kanzaki (1999), using ${ }^{17} \mathrm{O}$ Q3 MAS NMR technique have interpreted as Al-O-Al bridging oxygen and showed that is possible to distinguish triclusters oxygen. However, it is possible to find $\mathrm{Si}-\mathrm{O}-\mathrm{Al} \mathrm{BO}$, but is rather difficult to interpret, whether is it $\mathrm{O}\left(\mathrm{Si}_{2} \mathrm{Al}\right)$ or $\mathrm{O}\left(\mathrm{SiAl}_{2}\right)$ triclusters oxygen (Xue \& Kanzaki, 1999).

Using a $\left\{{ }^{17} \mathrm{O}\right\}{ }^{27} \mathrm{Al}$ Heteronuclear Multiple Quantum Correlation technique luga et al. (2005) found a direct proof for the existence of triclusters in $0.50 \mathrm{Ca} \cdot 0.50 \mathrm{Al}_{2} \mathrm{O}_{3}$ glass. The authors have also observed ${ }^{[\mathrm{V}]} \mathrm{Al}$ in $\mathrm{Mg}$ - and $\mathrm{Ca}$-aluminosilicate glasses but this fact still needs better investigations to find full explanation of its influence on the melt structure (see also Toplis et al., 2000; Neuville et al., 2004; Neuville et al., 2006). It is quite difficult to precise, whether five-fold coordinated $\mathrm{Al}$ is network former or network modifier (Lee et al., 2006; Neuville et al., 2006).

Al-rich structural units balanced by Al-cations are more stable than these balanced by alkaline earths (Wood \& Hess, 1980; Bottinga \& Weill, 1972; Mysen et al., 1981a), what would explained high viscosity and activation energy of peraluminous melts. 
There is not enough data to fully explain the behaviour of trivalent iron ions in the melts with lack of charge balancer (Mysen et al., 1981b). But it is proved (Mysen et al., 1980a; Mysen et al., 1981a) that $\mathrm{Fe}^{3+}$ does not bond with Si-tetrahedra but build own clusters. Goldman (1983) and Mysen et al. (1984) proposed that $\mathrm{Fe}^{3+}$ occurs as threedimensionally interconnected $\mathrm{Fe}^{3+} \mathrm{O}_{2}$ units. Tetrahedrally coordinated $\mathrm{Fe}^{3+}$ polymerizes into own structural unit $\left[\mathrm{FeO}_{2}\right]^{-}$. Iron plays an important structural role but the importance of its influence depends on the interrelationship between polymerization degree, composition of the melt, amount of iron and its oxidation state (Waff, 1977). Because all the samples were prepared and measured in air conditions (or air-nitrogen conditions) it is obvious that $\mathrm{Fe}^{3+}$ can oxidize and change its oxidation state to $\mathrm{Fe}^{2+}$.

There is no clear model of the peraluminous melt structure. Two most popular hypotheses differ in the coordination number of $\mathrm{Al}$ ions. The first one assumes a change in Al-coordination from tetrahedral to octahedral, when there is not enough charge balancers. The second one bases on the structure with triclusters of Al- and Si-tetrahedra, where Al stays in tetrahedral coordination (Shelby, 2005). In spite of the considerable progress in experimental techniques the real arrangement of this unique structure still remains undetermined.

In the following discussion it is assumed that in melts with peraluminous composition, the $\mathrm{Al}$ and $\mathrm{Fe}^{3+}$ are in tetrahedral coordination and both occurs in triclusters. $\mathrm{Fe}^{3+}$ in octahedral coordination is negligible and has no large effect on the structure change.

\subsection{How to describe the melt structure?}

The local structure can be described by the structural units $\mathbf{Q}^{\mathrm{n}}$ for $\mathrm{SiO}_{4}$ tetrahedra and $\mathrm{Q}^{\mathrm{n}}(\mathrm{mAl})$ for Al-tetrahedra, where $n$ is the number of bridging bonds in the tetrahedron and $m$ is the number of aluminium atoms in the second coordination sphere of the silicon atoms (Mysen, 1990). Q species (e.g. Schramm et al., 1984) are commonly used in NMR measurements. It describes the connectivity of tetrahedra (polymerization of the melt). 

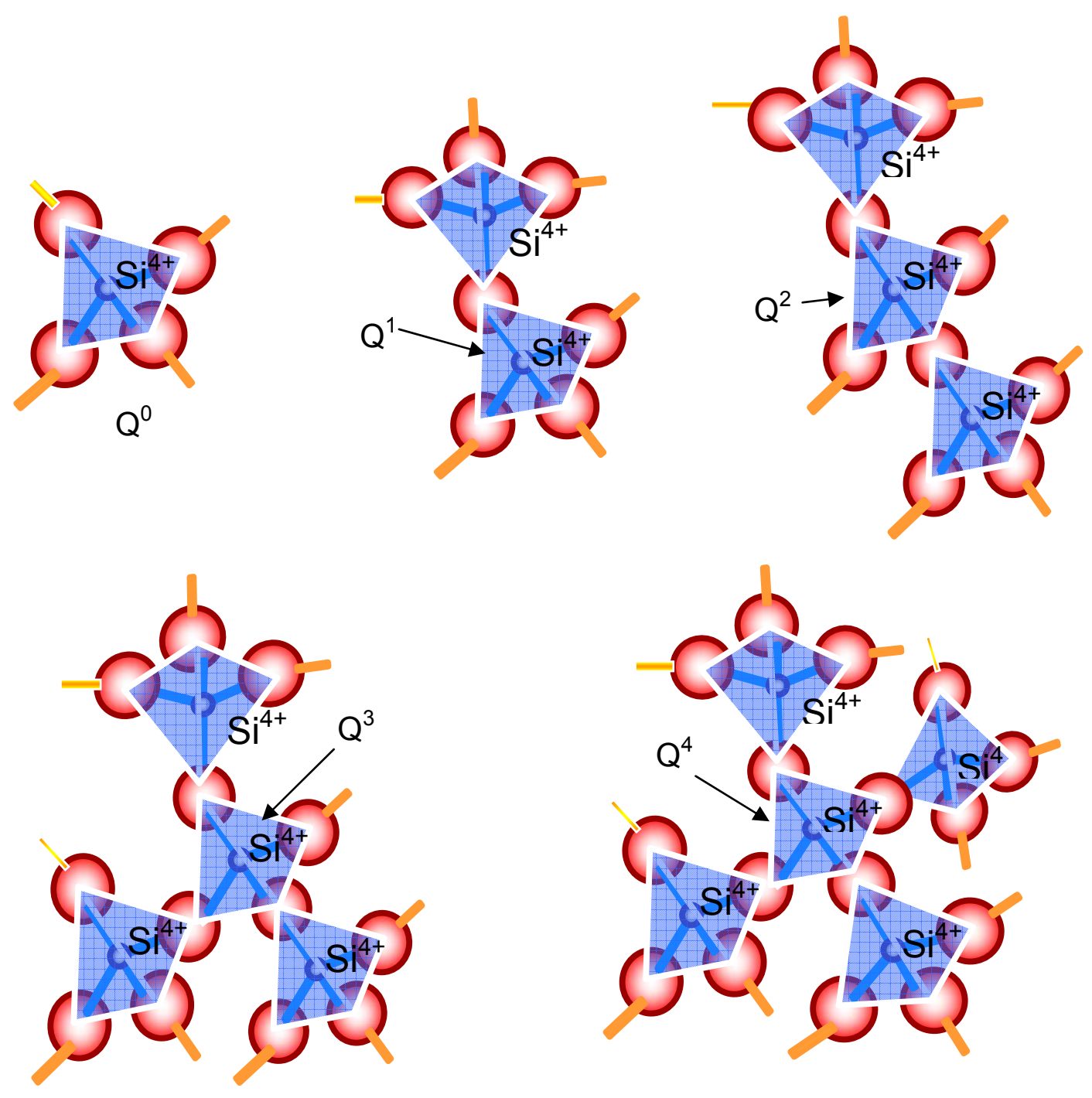

Fig. 10. Q species - distinguished as structural units in silicate melts. Description in the text.

$\mathrm{Q}^{0}$ is a separate $\mathrm{SiO}_{4}$ tetrahedron which has no connection with any other tetrahedron in the melt (Fig. 10). $Q^{1}$ unit is linked with one other tetrahedron, $Q^{2}-$ with two tetrahedra, $Q^{3}$ - with three tetrahedra and $Q^{4}$ is bonded to 4 other tetrahedra (Stebbins, 1995). The same nomenclature can be used for $\mathrm{Al}^{3+}$ tetrahedra (Mysen et al., 2003). $Q$ species show the arrangement of bridging and non-bridging oxygens in the melt structure. 
NBO/T parameter describes the number of non-bridging oxygens (NBO) per one tetrahedron $(T)$, e.g.:

$$
\frac{\mathrm{NBO}}{\mathrm{T}}=\frac{2\left(\mathrm{Na}_{2} \mathrm{O}-\mathrm{Al}_{2} \mathrm{O}_{3}\right)}{2 \mathrm{Al}_{2} \mathrm{O}_{3}+\mathrm{SiO}_{2}}
$$

(Mysen et al., 1981a; Mysen, 1987). NBO/T is generally used for peralkaline melts; otherwise the value will be negative. This historical problem has been solved by Gwinn \& Hess (1989) and more recently by Toplis et al. (1997a,b) using $\gamma$.

The GAMMA value $(\gamma)$ is the sum of network modifiers in the form of oxides $\left(X^{n+}\right)$ divided by the sum of all oxides in the melt (without $\mathrm{SiO}_{2}$ ):

$$
\gamma=\frac{\sum n X^{n+}}{\sum n X^{n+}+A l^{3+}},
$$

where $X^{n+}$ and $\mathrm{Al}^{3+}$ are a number of atoms in one mole of melt (Gwinn \& Hess, 1989; Toplis et al., 1997a,b; Webb et al., 2004). Gamma parameter avoids the negative values needed for NBO/T and also ignores silica content. In this work $\gamma$ is calculated as a ratio of the amounts of oxides (in mole fractions).

For Fe-free samples:

$$
\gamma=\frac{\mathrm{Na}_{2} \mathrm{O}}{\left(\mathrm{Na}_{2} \mathrm{O}+\mathrm{Al}_{2} \mathrm{O}_{3}\right)}
$$

and for Fe-bearing melts:

$$
\gamma=\frac{\mathrm{Na}_{2} \mathrm{O}+\mathrm{FeO}}{\left(\mathrm{Na}_{2} \mathrm{O}+\mathrm{Al}_{2} \mathrm{O}_{3}+\mathrm{FeO}+\mathrm{Fe}_{2} \mathrm{O}_{3}\right)}
$$

Using $\gamma$ parameter, the melts can be described as:

- peraluminous, when $0 \leq \gamma<0.5$;

- subaluminous, when $\gamma=0.5$;

- peralkaline, when $0.5<\gamma \leq 1$. 


\subsection{Triclusters - new structural unit. Do they really exist?}

The structure of the melt becomes more homogenous with increasing temperature (Bykov et al., 2003). There is also known that when the structure starts to flow, the anionic units of the network formers are connected in some structural groups with different degree of polymerisation and a short lifetime. In the Raman spectrum, at $1000 \mathrm{~cm}^{-1}$ vibrations of $\mathrm{Q}^{4}(\mathrm{mAl})$ (highly polymerised aluminosilicate anions) were observed (Bykov et al., 2003).

Tetrahedrally coordinated cations (such as $\mathrm{Si}^{4+}, \mathrm{Al}^{3+}, \mathrm{Ti}^{4+}$ or $\mathrm{Fe}^{3+}$ ) create tetrahedra which can be copolymerised or grouped in some special units (Kuryaeva, 2004). In Fefree melts alumina and silica tetrahedra can group in triclusters. In high peraluminous melts is not ruled out the existing of the triclusters with three Al-tetrahedra. In Fe-bearing melts the tricluster can contain also $\mathrm{Fe}^{3+}$ tetrahedra.

When for Fe-free glasses $\gamma=0.5$, the number of $\mathrm{Na}$ ions is exactly the same as the number of aluminium ions, and theoretically non-bridging oxygens do not exist. All $\mathrm{Na}$ ions compensate the negative charge of the Al-tetrahedra. With a further decrease in $\mathrm{Na}$ content there is not enough sodium to charge balance the Al-tetrahedra and a new structure must be formed. The new structure called "tricluster" is created (Isard, 1959; Day \& Rindone, 1962; Lacy, 1963; Lacy, 1965; Terai, 1969; Taylor \& Rindone, 1970; Shelby, 1978; Hunold \& Brückner, 1980; Toplis et al. 1997a; Kuryaeva, 2004).

Lacy (1963) described possible structures based on Zachariasen - Warren theory. His tricluster (Fig. 11) consists two Si-tetrahedra and one Al-tetrahedron (or one Sitetrahedron and two Al-tetrahedra) sharing one oxygen has been argued for by Toplis et al. $(1997 a, b)$.

Formation of such structure can arise according to the equations:

$$
\begin{aligned}
2 \mathrm{NaAlO}_{2}+\mathrm{SiO}_{2} \rightarrow & \mathrm{NaAl}_{2} \mathrm{SiO}_{5.5}+\mathrm{NaO}_{0.5} \\
& \text { tricluster } \quad \mathrm{NBO} \\
\mathrm{NaAlO}_{2}+2 \mathrm{SiO}_{2} \rightarrow & \mathrm{AlSi}_{2} \mathrm{O}_{5.5}+\mathrm{NaO}_{0.5} \\
& \text { tricluster } \quad \mathrm{NBO}
\end{aligned}
$$

(Kuryaeva, 2004).

In the measurements of the physical properties of aluminosilicate glasses the sharp breaks in their properties are observed at the moment when the composition changes from peralkaline to peraluminous and, as a consequence of that, the triclusters form. The triclusters can also occur in the peralkaline melt, but their sparse presence does not influence significantly on the physical properties of the melt (Toplis et al., 1997a). 


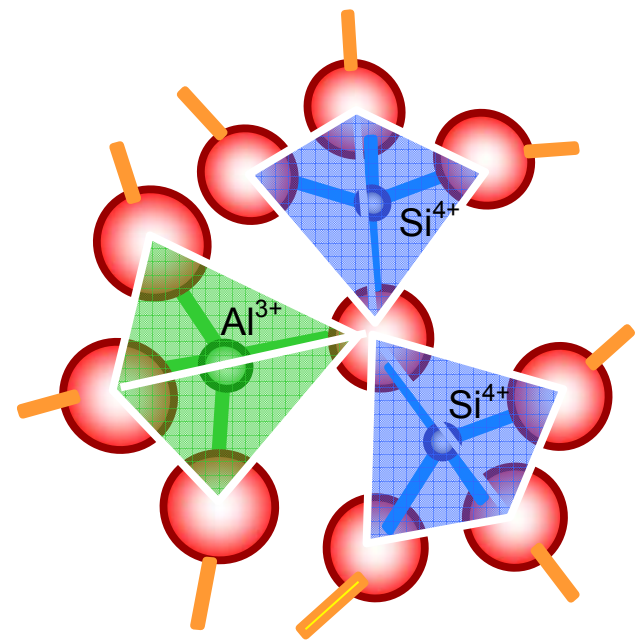

Fig. 11. Scheme of the tricluster - structural unit in the peraluminous melts proposed by Lacy, 1963.

NMR measurements (Kubicki \& Toplis, 2002) have not found triclusters, but calculations show the tricluster peak lies under large Si-O-Al and Al-O-Al peaks. While NMR data give us information about the structure of the melt, viscosity studies have shown the effect of structure on physical properties. Toplis et al. (1997a,b) and Webb et al. (2004) have shown that for melts with constant $\mathrm{mol}^{2} \mathrm{SiO}_{2}$ content, the viscosity of peralkaline melts increases as the amount of $\mathrm{NBO}$ formed by $\mathrm{Na}^{+}$is reduced, and the amount of $\mathrm{Al}^{3+}$ increases. In the peraluminous composition range, the viscosity decreases very slowly as the amount of charge-balancing $\mathrm{Na}^{+}$is reduced and the number of triclusters increases. This results in a maximum in $\eta$ at $\gamma \sim 0.5$.

\subsection{Flow mechanisms in aluminosilicates}

It has long been assumed that the structure of peraluminous $\mathrm{Na}_{2} \mathrm{O}-\mathrm{Al}_{2} \mathrm{O}_{3}-\mathrm{SiO}_{2}$ melts must be different to that of peralkaline melts. NMR studies of peralkaline $\mathrm{Na}_{2} \mathrm{O}-\mathrm{Al}_{2} \mathrm{O}_{3}-\mathrm{SiO}_{2}$ melts have found that $\mathrm{Na}^{+}$prefers to form non-bridging oxygens bonded to $\mathrm{Si}^{4+}$; and also acts as a charge balancer for the tetrahedrally coordinated $\mathrm{Al}^{3+}$ (Mysen, 1987; Allwardt et al., 2003). Mysen et al. (2003) concluded from their ${ }^{29} \mathrm{Si} \mathrm{NMR}$ and Raman spectroscopic study on peralkaline glasses that the dominant fraction $(>70 \%)$ of $\mathrm{Al}^{3+}$ resides in fully polymerized $\mathrm{Q}^{4}$ units. Lacy (1963) discussed the various possible structures of peraluminous melts in terms of geometry and energy and concluded that triclusters of two $\mathrm{Si}^{4+}$-tetrahedra and one $\mathrm{Al}^{3+}$-tetrahedron sharing one apical oxygen was the most probable structure for the $\mathrm{Al}^{3+}$ tetrahedra without a $\mathrm{Na}^{+}$charge balancer. Thus, the mechanism by which peralkaline and peraluminous $\mathrm{Na}_{2} \mathrm{O}-\mathrm{Al}_{2} \mathrm{O}_{3}-\mathrm{SiO}_{2}$ melts flow must be slightly different. 
Flow in peralkaline melts probably begins by

(1) the network modifying $\mathrm{Na}^{+}$diffusing away from its $\mathrm{Si}^{4+}$ tetrahedron, followed by

(2) the bonding of the free $\mathrm{O}$ to a neighbouring $\mathrm{Si}$ to create $\mathrm{a}^{[\mathrm{V}]} \mathrm{Si}$ (as seen to exist in NMR measurements, Stebbins, 1991; Stebbins \& McMillan, 1993),

(3) the breaking apart of an Si-O-Si bond, and

(4) the replacement of the $\mathrm{Na}^{+}$to the $\mathrm{O}$ with only one bond to a Si.

This flow process is that proposed by Farnan \& Stebbins (1994), McMillan et al. (1994) and Stebbins (1995).

The proposed flow mechanism for peraluminous composition $\mathrm{Na}_{2} \mathrm{O}-\mathrm{Al}_{2} \mathrm{O}_{3}-\mathrm{SiO}_{2}$ melts involves the creation of a tricluster and is

(1) the charge balancing $\mathrm{Na}^{+}$diffuses away from its $\mathrm{Al}^{3+}$ tetrahedron and the $\mathrm{Al}-\mathrm{O}$ bond is broken,

(2) a $\mathrm{AISi}_{2} \mathrm{O}_{5.5}$ tricluster is formed,

(3) the breaking apart of the Si-O-Si bond, and

(4) the replacement of the charge balancing $\mathrm{Na}^{+}$to the $\mathrm{Al}^{3+}$ tetrahedron and the $\mathrm{O}$ to the $\mathrm{Si}^{4+}$ with only 3 oxygens (Toplis et al., 1997a; Webb et al., 2004; Webb, 2005a).

This change of structure can be observed as a breaking point on the plots of viscosity, $T_{g}$, density or shear modulus. The base structure of Fe-free and Fe-bearing glasses appears to be similar. In peralkaline melts the major role is played by the modifying ions because they decide about the connectivity in the structure. In peraluminous melts the Si-O-Al bonds are more important.

Isostructural with aluminosilicate structure appears to be a galliosilicate structure, where the same behaviour of the trends was observed (Lapp \& Shelby, 1986; Piguet \& Shelby, 1985; Piguet et al., 1985).

\subsection{Volcanic Dilemma: "Flow or Blow"}

The volcanic explosions can look differently depending on the composition of the magma. More interesting is that even magma with the same composition can behave in different ways, what is caused by its various thermodynamic properties. Numerous experiments and computer modelling contributed to increase the significance of glass transition in volcanic processes and to prove that crossing the glass transition can be a reason for brittle failure (Dingwell, 1996).

The base of this hypothesis is enough high strain rate of the magma deformation to change the melt into a pseudo-plastic (shear thinning) liquid, which finishes in a brittle 
failure. But the present observations exclude such high strain rate of the magma in the conduits, where glass transition zone with brittle failure can not be reached.

The laboratory investigations showed that fragmentation of the sample being at temperature and pressure of volcanic eruption is easy when the fast decompression is used. In the condition of prompt decompression, high bubble density or high temperature are not required (Dingwell, 1996), what changes the present scientific point of view on the volcanic eruption.

One needs to discuss the aspect of hydration and dehydration of magma. Formation of the bubbles leads to depleting the magma of the water. Migrating upwards bubbles supply the sharp vertical volume, pressure and viscosity gradient. Ascending magma, crossing such a low pressure region, is suddenly decompressed, reaches the conditions of the glass transition region with brittle failure and explodes (Fig. 12).

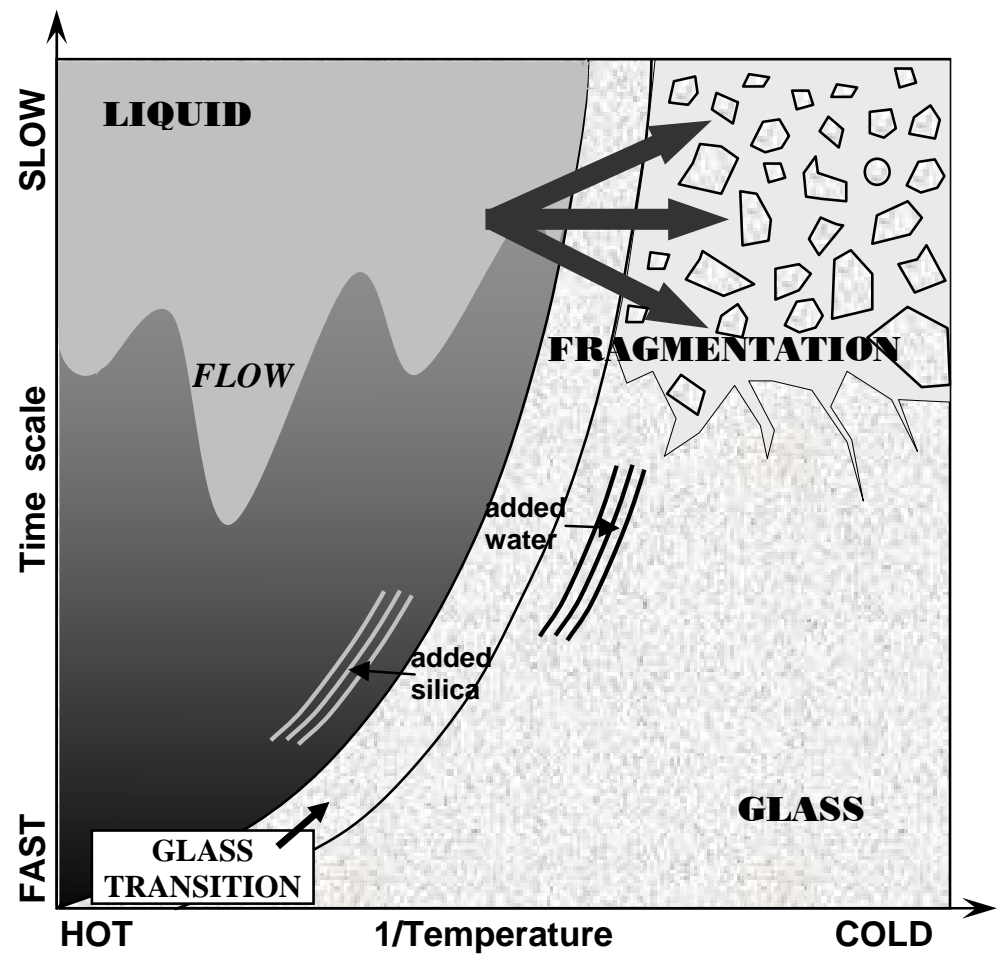

Fig. 12. The glass transition as a function of time and inverse temperature. Redrawn after Dingwell, 1996. 


\section{EXPERIMENTAL METHODS}

\subsection{Sample preparation}

Fourteen sodium-aluminosilicate samples with constant value of $66.7 \mathrm{~mol} \% \mathrm{SiO}_{2}$ and one sodium-silicate melt were investigated. Melts G9-G14 had $3 \mathrm{~mol} \% \mathrm{Al}_{2} \mathrm{O}_{3}$ replaced by $\mathrm{Fe}_{2} \mathrm{O}_{3}$ (sample $\mathrm{G} 8$ has only $1 \mathrm{~mol} \% \mathrm{Fe}_{2} \mathrm{O}_{3}$, otherwise it will crystallize). The batch composition is presented in the Table 2. The compositions of the final melts were determined by microprobe analysis (see Tables 8 and 9 - section 4.1.).

The ratio between $\mathrm{Al}$ and $\mathrm{Na}$ determines whether the melt is peralkaline or peraluminous. In this study the $\gamma$ value for the most peralkaline melt is 1.00 and for the most peraluminous is 0.40 . More peraluminous melts in this series crystallise upon cooling to a glass and thus and thus cannot be investigated using the techniques presented here.

The oxides $\mathrm{SiO}_{2}(99.9 \%), \mathrm{Al}_{2} \mathrm{O}_{3}(99.997 \%), \mathrm{Fe}_{2} \mathrm{O}_{3}(99.99 \%)$ and the carbonate $\mathrm{Na}_{2} \mathrm{CO}_{3}(99.5 \%)$ were used to make the samples. The chemicals were dried overnight before weighing. Weighing was done with the accuracy of $\pm 3 \mathrm{mg}$. These chemicals were shaken together in a plastic bottle to homogenise the powders before further preparation. The mixture was decarbonised at $800^{\circ} \mathrm{C}$ (at a heating rate of $300^{\circ} \mathrm{C} / \mathrm{h}$ for 12 hours) in a Nabertherm LHT 04/17 furnace. This process allows escaping $\mathrm{CO}_{2}$ from the carbonate and avoids the creation of $\mathrm{CO}_{2}$ bubbles in the final melt.

Tab. 2. Nominal compositions (in mol\%) of the investigated 15 samples.

\begin{tabular}{|c|c|c|c|c|c|c|c|}
\hline & $\begin{array}{c}\mathbf{S i O}_{\mathbf{2}} \\
\mathbf{m o l} \%\end{array}$ & $\begin{array}{c}\mathbf{N a}_{\mathbf{2}} \mathbf{O} \\
\mathbf{m o l} \%\end{array}$ & $\begin{array}{c}\mathbf{A l}_{\mathbf{2}} \mathbf{O}_{\mathbf{3}} \\
\mathbf{m o l} \%\end{array}$ & $\begin{array}{c}\mathbf{F e}_{\mathbf{2}} \mathbf{O}_{\mathbf{3}} \\
\mathbf{m o l} \%\end{array}$ & $\begin{array}{c}\mathbf{G A M M A} \\
\text { (Eq. 9\&10) }\end{array}$ & $\begin{array}{c}\text { Temperature } \\
\text { of melting }\end{array}$ & $\begin{array}{c}\text { Time of } \\
\text { melting }\end{array}$ \\
\hline $\mathbf{G 0}$ & 66.7 & 33.3 & - & - & 1.00 & $1200^{\circ} \mathrm{C}$ & 3 hours \\
\hline $\mathbf{G 1}$ & 66.7 & 13.3 & 20.0 & - & 0.40 & $1650{ }^{\circ} \mathrm{C}$ & 42 hours \\
$\mathbf{G 2}$ & 66.7 & 15.0 & 18.3 & - & 0.45 & $1650{ }^{\circ} \mathrm{C}$ & 30 hours \\
$\mathbf{G 3}$ & 66.7 & 15.6 & 17.7 & - & 0.47 & $1650{ }^{\circ} \mathrm{C}$ & 12 hours \\
$\mathbf{G 4}$ & 66.7 & 16.7 & 16.7 & - & 0.50 & $1650{ }^{\circ} \mathrm{C}$ & 13 hours \\
$\mathbf{G 5}$ & 66.7 & 17.7 & 15.6 & - & 0.53 & $1650{ }^{\circ} \mathrm{C}$ & 5 hours \\
$\mathbf{G 6}$ & 66.7 & 18.3 & 15.0 & - & 0.55 & $1600{ }^{\circ} \mathrm{C}$ & 6 hours \\
$\mathbf{G 7}$ & 66.7 & 20.0 & 13.3 & - & 0.60 & $1550^{\circ} \mathrm{C}$ & 4 hours \\
\hline \hline $\mathbf{G 8}$ & 66.7 & 13.3 & 19.0 & 1.0 & 0.40 & $1650^{\circ} \mathrm{C}$ & 20 hours \\
$\mathbf{G 9}$ & 66.7 & 15.0 & 15.3 & 3.0 & 0.45 & $1650^{\circ} \mathrm{C}$ & 13 hours \\
$\mathbf{G 1 0}$ & 66.7 & 15.6 & 14.7 & 3.0 & 0.47 & $1600{ }^{\circ} \mathrm{C}$ & 10 hours \\
$\mathbf{G 1 1}$ & 66.7 & 16.7 & 13.7 & 3.0 & 0.50 & $1315^{\circ} \mathrm{C}$ & 8 hours \\
$\mathbf{G 1 2}$ & 66.7 & 17.7 & 12.6 & 3.0 & 0.53 & $1300{ }^{\circ} \mathrm{C}$ & 5 hours \\
$\mathbf{G 1 3}$ & 66.7 & 18.3 & 12.0 & 3.0 & 0.55 & $1350{ }^{\circ} \mathrm{C}$ & 3 hours \\
$\mathbf{G 1 4}$ & 66.7 & 20.0 & 10.3 & 3.0 & 0.60 & $1380{ }^{\circ} \mathrm{C}$ & 6 hours \\
\hline
\end{tabular}


Melting the final composition of three (for Fe-free glasses) or four oxides (for Febearing glasses) took place in thin walled platinum crucible in the $\mathrm{MoSi}_{2}$ Nabertherm furnace. Heating rate was $600^{\circ} \mathrm{C} / \mathrm{h}$. The melting temperature depended on the composition of the samples (see Table 2) but the technical limitation of the furnace does not allow exceeding $1650^{\circ} \mathrm{C}$. However, at higher temperature, platinum from the crucibles $\left(\mathrm{PtRh}_{10}\right)$ could start to contaminate the melts. The Nabertherm Company guarantees the resistance of the insulation and heating elements up to $1870^{\circ} \mathrm{C}$ but the melting temperature of platinum is $1768^{\circ} \mathrm{C}(2041 \mathrm{~K})$.

Peralkaline samples needed lower melting temperature and shorter time of melting than the peraluminous samples. Some melts had to be mechanical stirred (up to 12 hours) to accelerate a homogenization and to remove the bubbles. Attention was also paid to the time and temperature of the melting, because too long at too high temperature causes the escape of sodium from the melt.

Cooling rate from the highest temperature to $700^{\circ} \mathrm{C}$ was always $600^{\circ} \mathrm{C} / \mathrm{h}$ but between $700^{\circ} \mathrm{C}$ and $500^{\circ} \mathrm{C}$ was much slower (only $60^{\circ} \mathrm{C} / \mathrm{h}$ ) to reduce the creation of internal stress upon cooling through the glass transition. If the sample is not well annealed, later, during drilling out with a diamond drill from the high crucible and polishing, the samples will break.

There were two kinds of samples (with plane parallel surfaces) prepared: (1) for viscosity measurements (discs, $\sim 8 \mathrm{~mm}$ in diameter, $3-4 \mathrm{~mm}$ thick) used also to determine the composition, density and ultrasonic shear modulus; and (2) for torsion measurements (cylinders, $\sim 8 \mathrm{~mm}$ in diameter, $25-30 \mathrm{~mm}$ long). Long cylinders did not need the polishing but faces of the small discs were polished with $1 \mu \mathrm{m}$ jewellers' rouge.

\subsection{Microprobe}

The ratio between oxides building the structure plays an important role in glass properties. Therefore, a precise measurement of the samples' composition was necessary.

Samples were analyzed by microprobe JEOL JXA $8900 \mathrm{RL}$ in the Geochemistry Department Georg-August University Göttingen using a $15 \mathrm{kV}$ voltage, with a defocused $10 \mu \mathrm{m}$ beam diameter and with $12 \mathrm{nA}$ current. Results, presented further in the text (see Tables 8 and 9), are an average of 10 analyses of each glass. Errors are $1 \sigma$ values. 
Polished samples, with a diameter $\sim 8 \mathrm{~mm}$ were (directly or fixed in the epoxy tablets) mounted on a gold coated holder. In order to avoid unwanted charging samples have been coated in a high vacuum chamber with a carbon film with a standard $25 \mathrm{~nm}$ thickness. Contact between coated sample and gold holder was assured by a silver based paint. Samples were measured in WDS (Wavelength-Dispersive Spectrometers) mode. To quantify the precisely the amount of each oxide $\left(\mathrm{SiO}_{2}, \mathrm{Al}_{2} \mathrm{O}_{3}, \mathrm{Na}_{2} \mathrm{O}\right)$, two standards were used, namely albite and anorthite crystals. Consequently $\mathrm{Fe}_{2} \mathrm{O}_{3}$, for iron bearing samples, was calibrated with hematite. Characteristically for microprobe analyses where only cations are analysed, the amount of oxygen was stoichiometrically calculated for each oxide.

\subsection{Density}

Definition of density $\rho$ is expressed as follows:

$$
\rho=\frac{m}{V}
$$

where $m$ is the mass of the sample and $V$ is its volume. The densities of the glasses at room temperature were determined using the Archimedean technique in ethanol (Fig. 13). Samples were weighed in air as well as in the ethanol. The immersion of the glass into the ethanol causes that the force of buoyancy works on the sample. Buoyancy is the weight of the ethanol displaced by the volume of the sample; in other words, the tendency of the sample to float in the liquid. The density $\rho$ of the sample was calculated from measured values:

$$
\rho=\frac{w_{a} \cdot \rho_{l}}{w_{a}-w_{l}}
$$

where $\rho$ is density of the sample, $\rho_{l}$ is density of the liquid (in this case - of ethanol), $w_{a}$ is the weight of the sample in air, $w_{l}$ is the weight of the sample in liquid (ethanol). Density of ethanol at the temperature of measurement was taken from the table of Sartorius (Sartorius, 2004). 


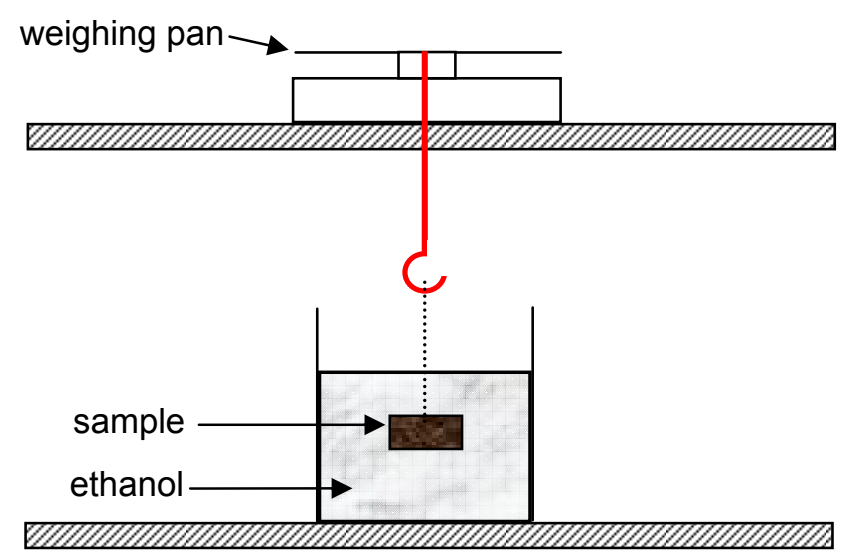

Fig. 13. Scheme of the buoyancy method to measure density of the sample.

Density of the glass is dependent on its thermal history. All the samples were quenched with the same cooling rate (between melting temperature and $700^{\circ} \mathrm{C}$ with the rate $600^{\circ} \mathrm{C} / \mathrm{h}$ and between $700^{\circ} \mathrm{C}$ and $500^{\circ} \mathrm{C}$ : only $60^{\circ} \mathrm{C} / \mathrm{h}$ to remove of the internal stress).

\subsection{Pulse Echo Overlap technique}

The shear modulus of the glasses was measured at room temperature using the ultrasonic Pulse Echo Overlap method (in the Institute for Low Temperature Physics, Georg-August-University Göttingen) (Fig. 14) (May, 1958; McSkimin, 1961; McSkimin \& Aadreatch, 1962; Papadakis, 1966; Truell et al., 1969; King \& Stephens, 1975; Papadakis, 1976). A pulse of high frequency waves (here $f=5 \mathrm{MHz}$ ) was sent into the sample via a $5 \mathrm{~mm}$ diameter $\mathrm{Y}$-cut quartz piezoelectric transducer which was glued to the sample using a very thin layer of honey. The echo pattern of the multiple reflected pulses was recorded (Fig. 15). After each travel through the sample reflections became weaker as the energy carried by the wave is gradually used for atom vibration motions.

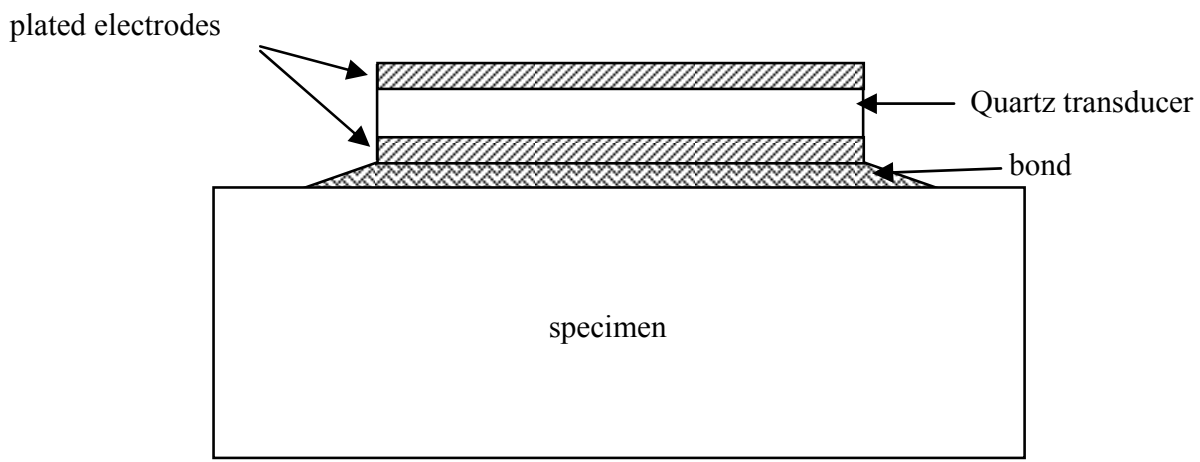

Fig. 14. Schema of specimen, bond and transducers with plated electrodes, use in the Pulse Echo Ovelap technique (redrawn after Truell et al., 1969). 


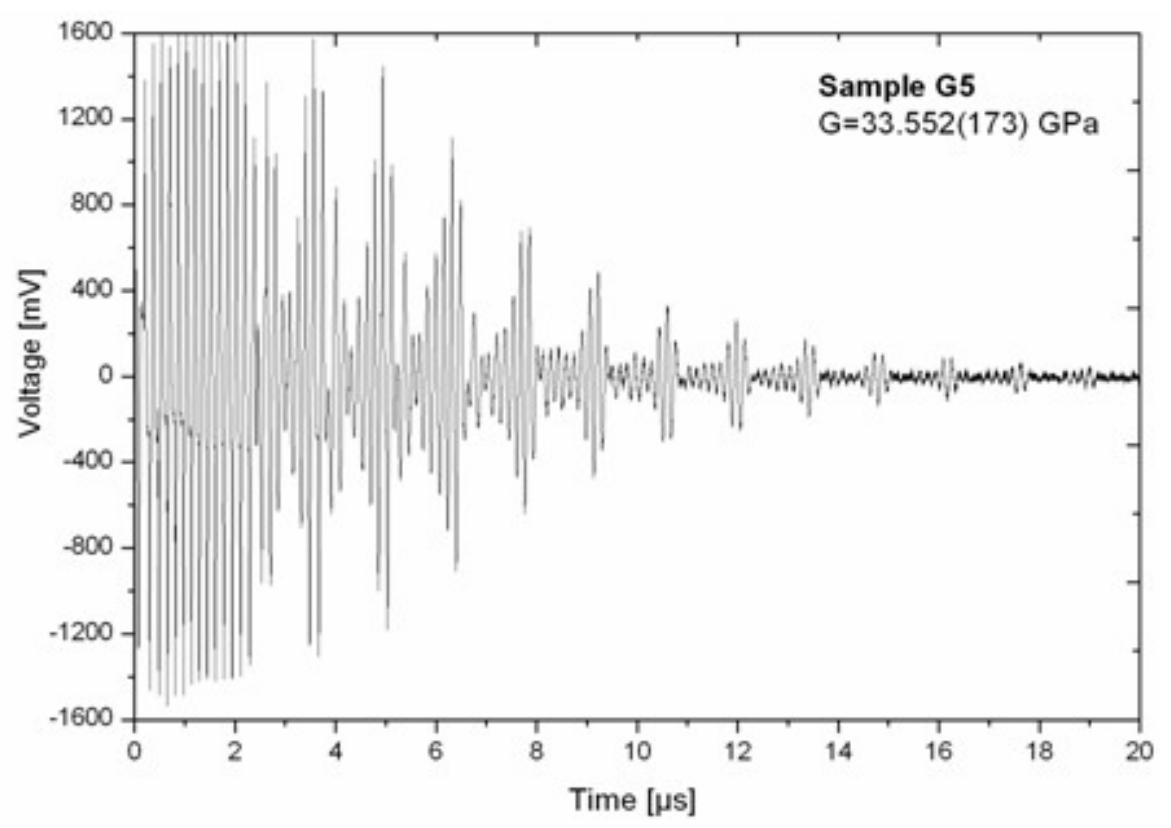

Fig. 15. Typical pulse echo pattern. Here for sample G5.

To determine the wave speed $V_{s}$, one has to measure two parameters: (1) thickness of the sample $d$, which multiplied by $2 n$ gives the total distance that the wave travels from the initial point of the measurement and back (where $n$ is the order of reflection) and (2) time delay $t$ between sending a signal and receiving its reflection from a distant surface:

$$
V_{s}=\frac{d}{t}
$$

Wave speed $V_{s}$ for each echo pulse was determined from linear relationship between $\Delta d$ and $\Delta t$ (Fig. 16).

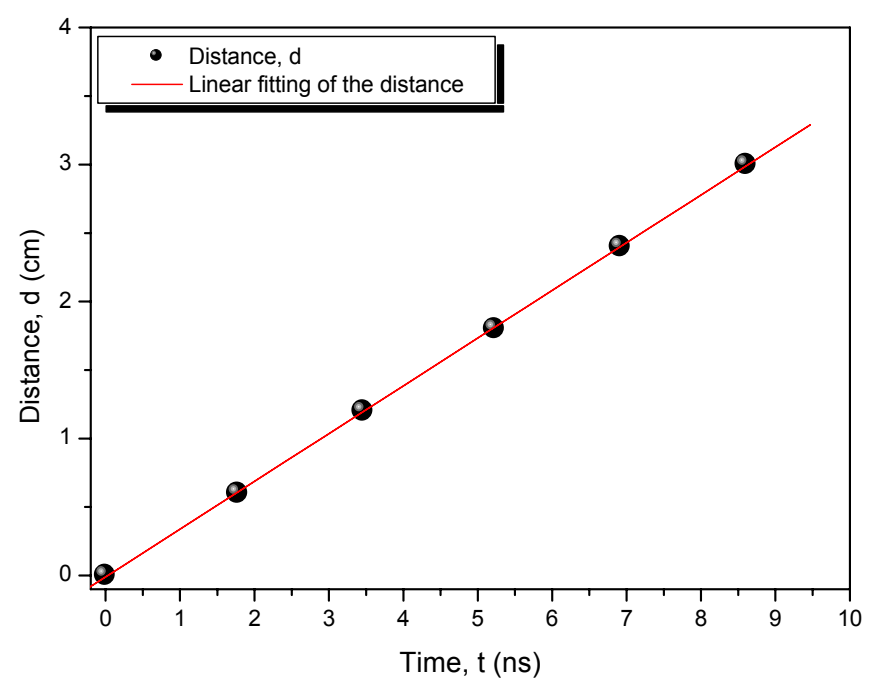

Fig. 16. Linear relationship of the echo pulses travelling through the sample as a function of time. 
With known density and wave speed, the shear modulus $G$ of the sample is calculated:

$$
G=\rho V_{s}^{2}
$$

Shear modulus $G$ can be determined from linear relationship between $V_{s}$ and $\rho$ (Fig. 17).

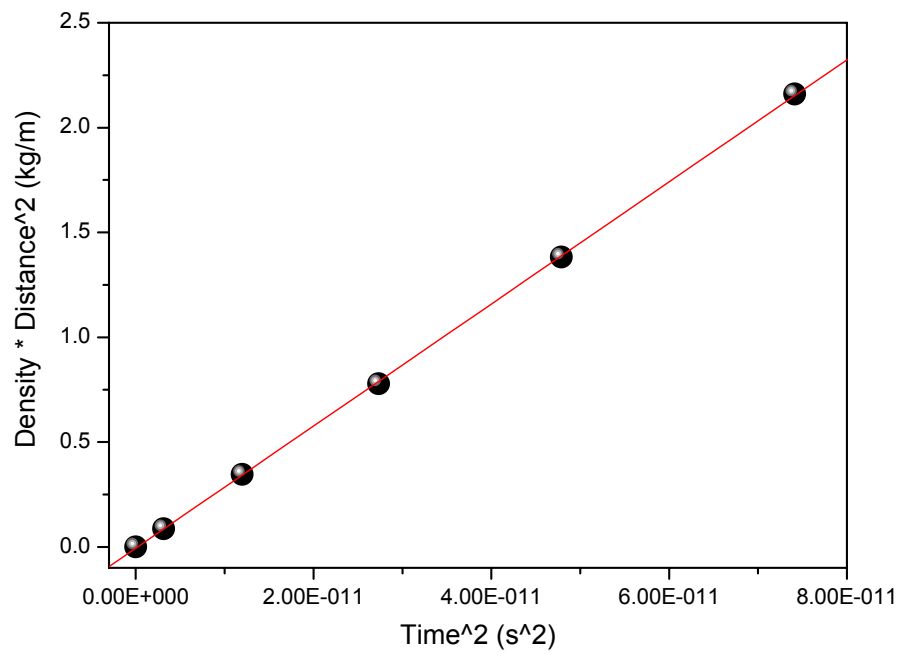

Fig. 17. Linear relationship between ultrasonic wave speed $V_{s}$ and density of the sample $\rho$.

The error associated with choosing the correct part of the pulsed signal is at most $\pm 0.6 \mathrm{GPa}$. The lengths of the samples were between $2.10 \pm 0.01$ and $3.70 \pm 0.01 \mathrm{~mm}$. The sample thickness was measured with a micrometer.

\subsection{Viscosity $\eta$}

\subsubsection{The importance of viscosity}

Viscosity is a very important parameter in the glass industry, as well as in geology. It gives information about structural changes and different flow mechanisms in the melt as a function of composition, temperature and pressure. This intrinsic property indicates the resistance of the melt to flow; to change in form.

Temperature effects on viscosity. If one adds some energy to the system (for example: in the form of heat) viscosity decreases - the melt becomes more fluid. Viscosity is inversely related to temperature: with decreasing temperature the viscosity increases. 
Viscosity controls the dynamics of the melt in geological settings. On this parameter many reactions depend, like crystallization and differentiation of the magma, diffusion within the melt or magma eruptions. Viscosity, therefore, has a huge importance in volcanology. Highly viscous lavas erupt very explosively. These eruptions occur less frequently because greater pressure is required to push magma outside of the volcano. When magma has low viscosity, the volcanoes are more likely to erupt. In these cases even a little force pushes magma to the surface.

Viscosity measurements play an important role in geology but determination of the viscosity value in very extreme conditions (like in the mantle) is technically difficult. On the contrary, laboratory measurements exclude the possibility to measure an influence of all the factors working in the nature simultaneously (e.g. time, temperature, pressure or volatiles). However, all the measurements can be used to investigate the dynamics of the internal melt structure.

\subsubsection{What is viscosity?}

Viscosity $\eta$ of the melt is the ratio of applied shear stress $\sigma$ to the shear strain rate $\dot{\varepsilon}$ (see Fig. 18):

$$
\eta=\frac{\sigma}{d v / d t}=\frac{\sigma}{\dot{\varepsilon}},
$$

where $\eta$ is viscosity in Pa s, $\sigma$ is shear stress and $\dot{\varepsilon}$ is strain rate. The applied stress $\sigma$ is directly proportional to the strain rate $\dot{\varepsilon}$, what means that with increasing stress, glass deforms faster.

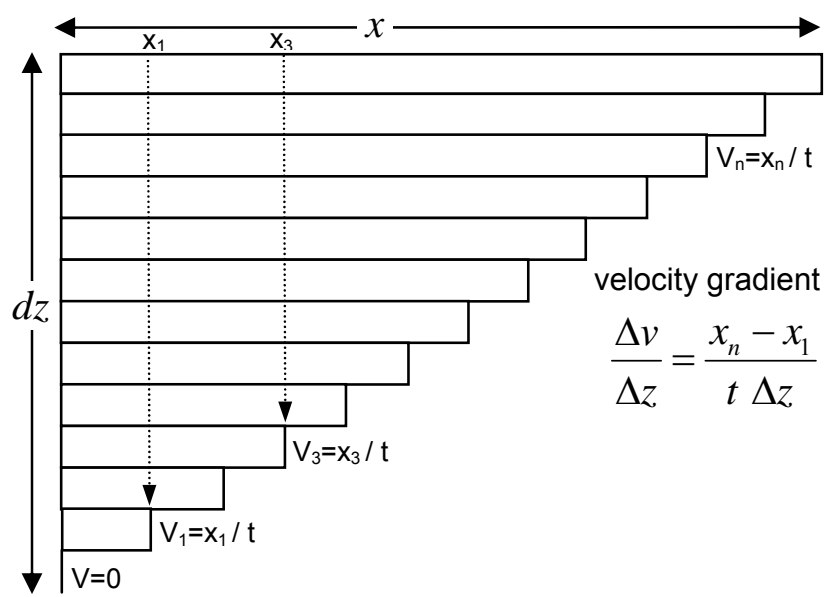

Fig. 18. Diagram for viscosity measurement. Viscosity is the ratio between stress $\sigma$ and shear strain rate $\dot{\varepsilon}$ 
Liquids, which obey the linear relationship between stress and strain rate with intercept zero, are known as Newtonian (Fig. 19); that is, the viscosity is independent of the magnitude of $\sigma$ and $\dot{\varepsilon}$ (see Fig. 26). Non-Newtonian and Bingham liquids behave differently. A Bingham liquid is a material which under low stresses is as a rigid body; only when high stress is applied, the material starts to flow. Non-Newtonian liquids do not have linear relationship between shear stress and strain rate and can be pseudo-plastic or dilatant (Fig. 19). In pseudo-plastic liquids (also called shear thinning fluids) the viscosity decreases with the strain rate. The opposite liquids are dilatant (shear thickening fluids) their viscosity increases with the higher strain rate.

Newtonian, non-Newtonian and Bingham flow behaviours of glasses and melts have been well explained by Yue \& Brückner (1994).

This classification of viscosity types does not include the basic ideas of viscoelastic materials, where the time dependence of physical properties needs to be taken into account. So that the definition of a Newtonian liquid is extended to include time dependence - that is that at high $\dot{\varepsilon}, \sigma$ decreases - but that there is a linear relationship between $\sigma$ and $\varepsilon$ - that is the same $\eta$ is determined for different magnitudes of $\sigma$ and $\varepsilon$, but is dependent upon $\dot{\varepsilon}$.

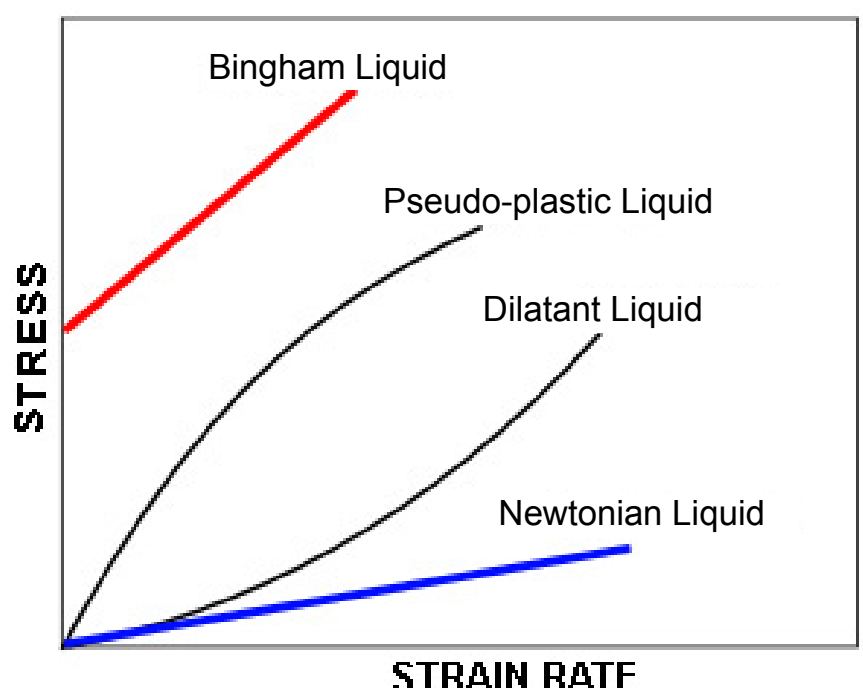

Fig. 19. Classification of the liquids behaviour as a function of dependence between stress and strain rate.

\subsubsection{Machine calibration}

Viscosity here was measured using the micropenetration technique in a NETZSCH Dilatometer TMA 402. The NETZSCH Dilatometer was calibrated with respect to both temperature and deformation distance. The melting temperatures of three elements were 
Tab. 3. Melting temperatures of the three elements used to calibration the NETZSCH Dilatometer, given by NETZSCH Company and measured in the calibration.

\begin{tabular}{|c|c|c|}
\hline & $\begin{array}{c}\text { Melting point } \\
\text { (Firma NETZSCH) }\left({ }^{\circ} \mathbf{C}\right)\end{array}$ & Measured melting point $\left({ }^{\circ} \mathrm{C}\right)$ \\
\hline $\mathbf{Z n}$ & 419.6 & 418.51 \\
$\mathbf{A l}$ & 660.3 & 658.92 \\
$\mathbf{A g}$ & 961.8 & 950.51 \\
\hline
\end{tabular}

obtained, namely $\mathrm{Ag}, \mathrm{Al}$ and $\mathrm{Zn}$. The melting temperatures of these elements have been measured electrically and are shown in the Table 3.

The calibration experiments were done for different heating rates: $1 \mathrm{~K} \mathrm{~min}^{-1}$, $2 \mathrm{~K} \mathrm{~min}^{-1}, 5 \mathrm{~K} \mathrm{~min}^{-1}$ and $10 \mathrm{~K} \mathrm{~min}^{-1}$. For all of the elements the melting temperature points at 4 defined heating rates were used to fit a straight line. The intercept of this line and the $Y$ axis gives a temperature $T_{0}$ used for the further steps of calibration (Fig. 20).

Three $T_{0}$ temperatures created a straight line which is the temperature calibration equation (Fig. 21):

$$
T_{\text {calibr }}=1.01677 \cdot T_{\text {measured }}-7.25686
$$
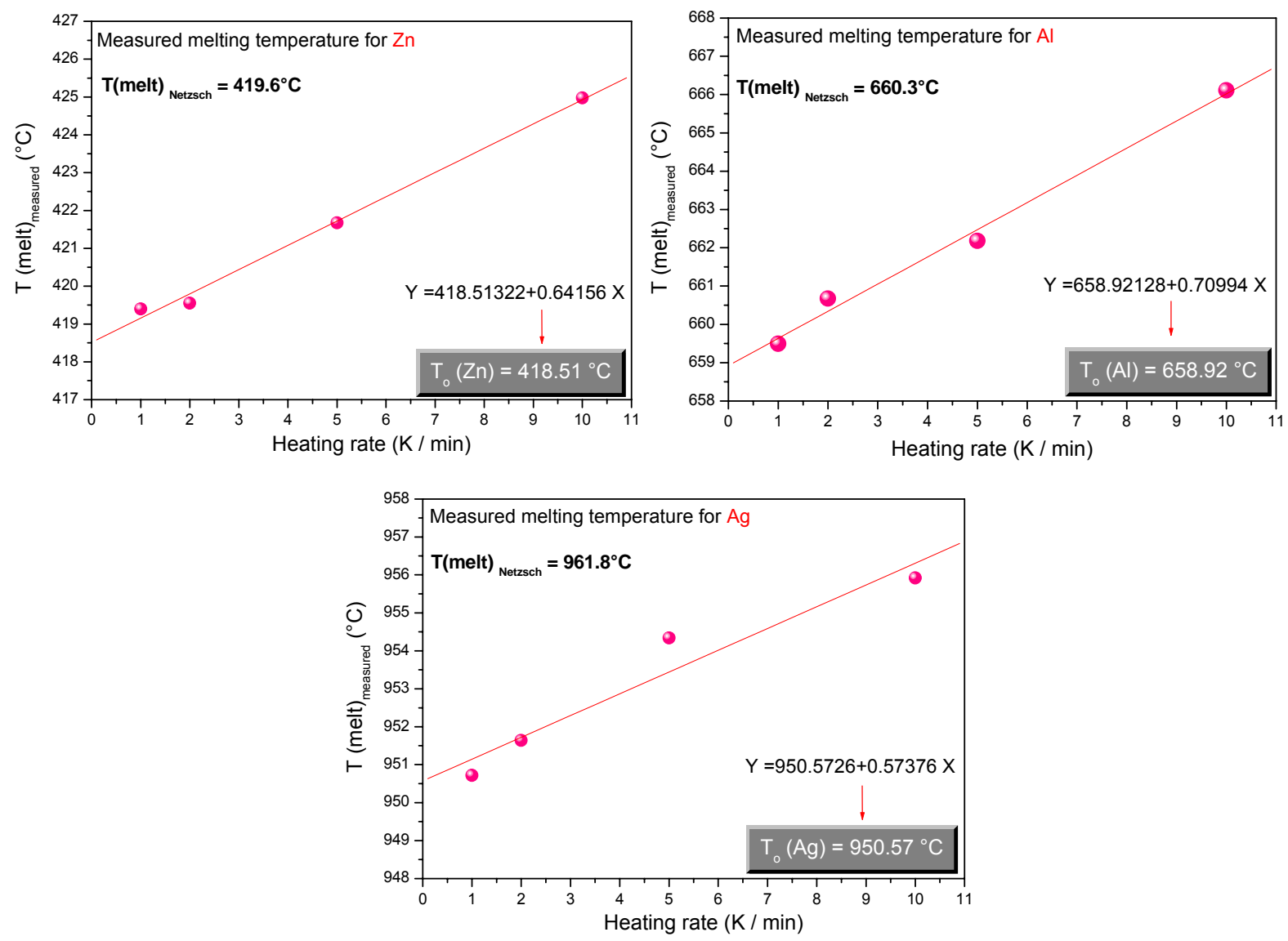

Fig. 20. Estimating a melting temperature $T_{0}$ for $\mathrm{Zn}, \mathrm{Ag}$ and $\mathrm{Al}$ used to calibrate a NETZSCH Dilatometer. 


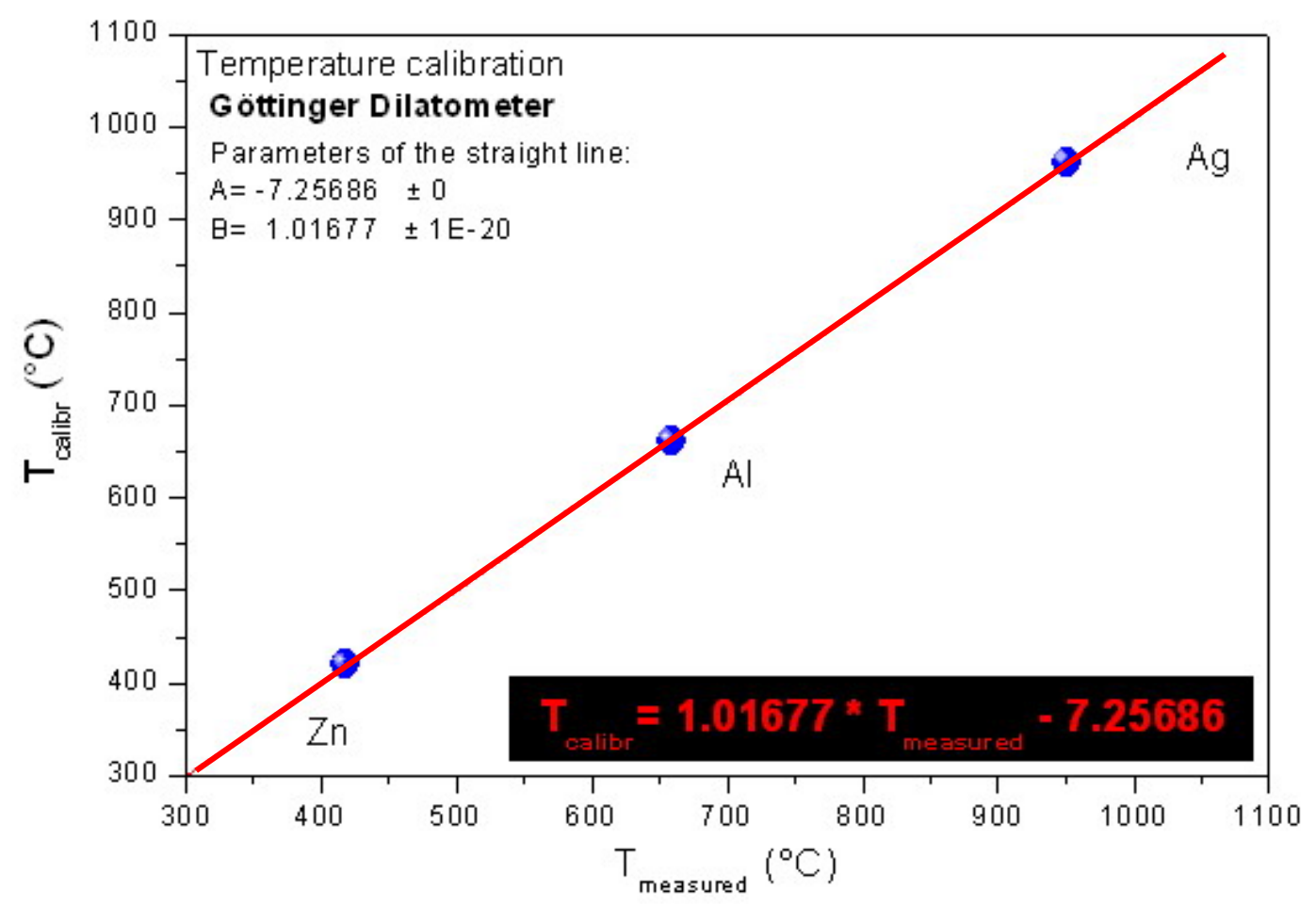

Fig. 21. Plot presenting a calibration equation for NETZSCH Dilatometer.

Viscosity of standard DGG1 (Deutsche Glastechnische Gesellschaft) was measured to check the electronic calibration of displacement measurement. At all temperatures the measured viscosity was within $\pm 0.6 \log \mathrm{Pa} s$ of the standard viscosity. The error assumed in all of the following viscosity measurements is $\pm 0.6 \log \mathrm{Pa}$ s.

\subsubsection{Viscosity measurements}

Viscosity measurements were done for all of the samples given in the Table 2 . The viscosities of the melts were measured by the micropenetration technique with a NETZSCH TMA 402 Dilatometer (Fig. 22).

The samples were $8 \mathrm{~mm}$ cylinders of melt, 3-4 mm thick. The faces of the cylinders were ground parallel to each other and the face which was used for the viscosity measurements was polished with $1 \mu \mathrm{m}$ jewellers' rouge. The samples were checked with the microscope to exclude the presence of bubbles and crystals.

Viscosity $\eta$ was determined from the rate at which a single crystal sphere made of sapphire (with diameter between 2006 and $2009 \mu \mathrm{m}$ ) was pushed into the surface of the melt using a force $F$ (Fig. 22b);

$$
\eta=\frac{0.1875 F t}{r^{0.5} l^{1.5}},
$$


where $r$ is radius of the sphere $(\mu \mathrm{m}), t$ - time $(\mathrm{s}), l$ - indent distance, 0.1875 value is a geometric factor (Pocklington, 1940; Douglas et al., 1965; Brückner \& Demharter, 1974; Dingwell et al., 1992). The applied force was induced by different masses between 8.253 and $1175.6 \mathrm{~g}$ under the acceleration of gravity $\left(9.81 \mathrm{~m} \mathrm{~s}^{-2}\right)$. Force was applied to the top of the weight pan and through the long ceramic rod (Fig. 22b) the sphere is pushed into the melt. The upper part of the ceramic rod has a ferromagnetic insert which sits in a linear voltage displacement transducer (LVDT). Moving this ferromagnetic rod in the LVDT induces a change in voltage, electronically converted into a displacement of the rod and a change in indent distance.

The indent distance must be less than $200 \mu \mathrm{m}$ as the truncation of the viscosity equation assumes that $l<r$. Measurements have shown that the viscosity equation does not fit the indent data as a function of time for $l>350 \mu \mathrm{m}$.

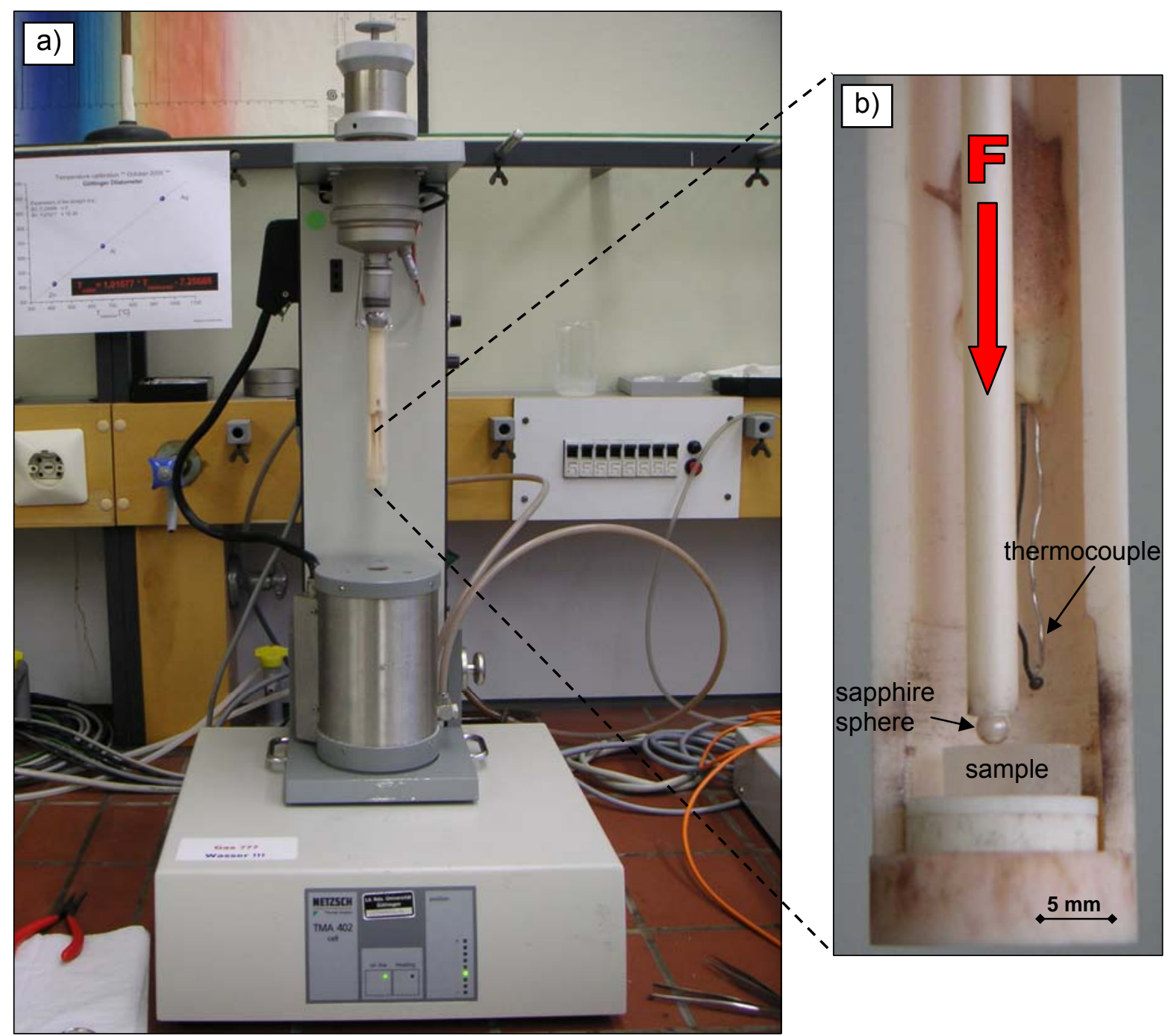

Fig. 22. NETZSCH TMA 402 Dilatometer in the Mineralogy Department, University of Göttingen; used to measure viscosity of the investigated samples. 
Controlling the heating and cooling rate and collecting the data was possible with a NETZSCH computer programme. The measurement has been consisted of five segments.

In the first segment the sample was heated from the room temperature up to the needed temperature with a constant rate of $10^{\circ} \mathrm{C} \mathrm{min}^{-1}$.

The length of the second segment (pink line in Figure 23) was dependent on the structural relaxation time $\tau_{M}$ of the sample. At high viscosity conditions (e.g. $10^{11.2} \mathrm{~Pa} \mathrm{~s}$ ), the sample was held at constant temperature for at least 1 hour in order to allow the melt structure to equilibrate with respect to the applied temperature, before the force is applied. The timescale of structural equilibration is calculated from the Maxwell equation (Eq. 3): $\tau_{M}=\eta_{0} / G_{\infty}$.

$G_{\infty}$ is the infinite frequency shear modulus (assuming that $\mathrm{G}_{\infty} \sim 10 \mathrm{GPa}$ from Dingwell \& Webb, 1990); and that at least $100 \tau_{M}$ are required for structural relaxation to be achieved (Dingwell \& Webb, 1989). The indent rate of the sapphire sphere is measured in a melt structure that has not relaxed with respect to the applied stress for $100 \tau_{M}$. This is taken into account in the data analysis and the unrelaxed data are not included in the calculation of viscosity. For viscosities higher than $10^{13} \mathrm{~Pa}$ s the relaxation time needs to be very long (over 27 hours) and for this reason such measurements were not done.

Because $\tau_{M}$ is directly proportional to the viscosity it was possible to compute the value of relaxation time from a roughly approximated viscosity (see Table 4). Equilibration time less than 15 minutes was not used.

Tab. 4. Maxwell relaxation times $\tau_{M}$ calculated for each viscosity from the Eq. 3 and equilibration time used in the experiments.

\begin{tabular}{|c|c|c|c|}
\hline $\begin{array}{c}\text { Viscosity } \\
\log 10 \eta(\mathrm{Pa} \mathrm{s})\end{array}$ & $\tau_{M} \begin{array}{c}\tau_{M}{ }^{*} 100 \\
(\mathrm{~s})\end{array}$ & $\begin{array}{c}\text { Equilibration time used } \\
\text { during the measurement }\end{array}$ \\
\hline 8.0 & $(\mathrm{~s})$ & 1 & $15 \mathrm{~min}$ \\
8.5 & 0.01 & 3 & $15 \mathrm{~min}$ \\
9.0 & 0.03 & 10 & $15 \mathrm{~min}$ \\
9.5 & 0.1 & 320 & $15 \mathrm{~min}$ \\
10.0 & 0.32 & 100 & $15 \mathrm{~min}$ \\
10.5 & 1 & 316 & $15 \mathrm{~min}$ \\
11.0 & 3.16 & 1000 & $30 \mathrm{~min}$ \\
11.5 & 10 & 3162 & $1.5 \mathrm{~h}$ \\
12.0 & 31.62 & 10000 & $3 \mathrm{~h}$ \\
12.5 & 100 & 31623 & $9 \mathrm{~h}$ \\
\hline
\end{tabular}




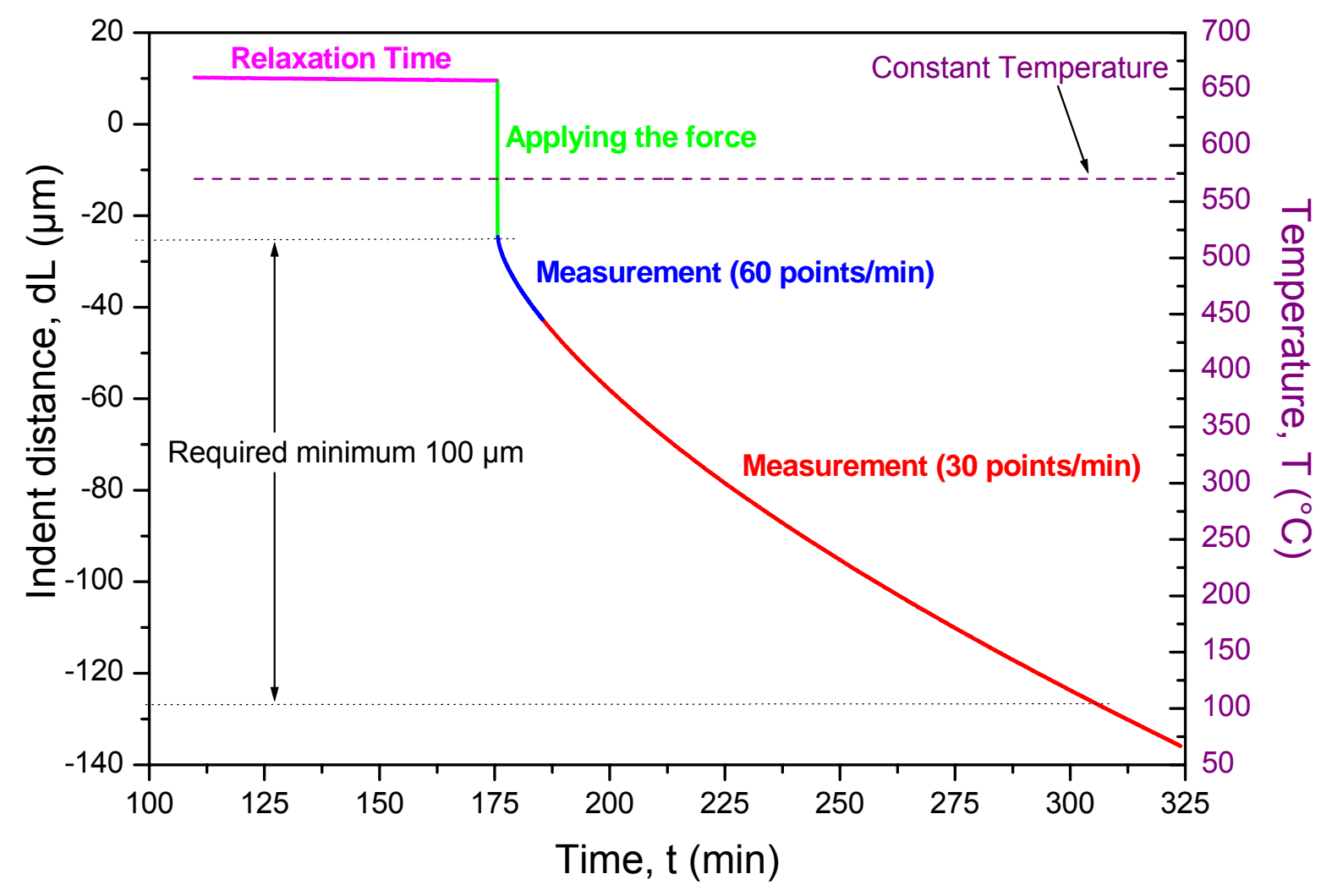

Fig. 23. Schematic view of the viscosity measurement. Description in the text.

Third segment of the experiment is a proper viscosity measurement at a certain temperature and occurs at the same temperature as the second segment. In this part the force is applied (see green line in Figure 23); it means the mass is placed on the top of the weight pan. The mass forces the sapphire sphere into the melt. At the first moment after applying force $\mathrm{F}$, the sapphire sphere comes into intimate contact with the sample.

It is easy to observe that viscosity line in the first stage has larger curvature and with deeper indent distance of sapphire sphere the curvature decreases. In the first 10 minutes of this segment the computer programme has counting 60 measuring points per minute to increase the accuracy of the fitting in the calculation (blue part of line in Figure 23).

The fourth segment starts with decreasing the counting rate to 30 points per minute (red part of line in Figure 23). The length of this segment depends on the viscosity of the sample and used weight. The main rule was that the indent distance has to exceed $100 \mu \mathrm{m}$ for precise calculation of viscosity. For viscosity $10^{8.76} \mathrm{~Pa} \mathrm{~s}$ (sample G0) and used weight $18.06 \mathrm{~g}$ the measurement time was 11 minutes. On the contrary, for high viscosity $\left(10^{12.19} \mathrm{~Pa} \mathrm{~s}\right.$ for sample $\mathrm{G} 13$ ) and used the maximum weight $1175.6 \mathrm{~g}$ the measurement was done over 7.5 hours. The average measurement time was $1.5-2$ hours and with the indent distance between 80 and $300 \mu \mathrm{m}$. 
During the last fifth segment the furnace was cooled down. This process was started automatically (after earlier programming) or manually (after reaching required at least $100 \mu \mathrm{m}$ of the indent distance).

\subsubsection{Viscosity calculation}

During the experiment with NETZSCH Dilatometer the computer programme saves three different parameters: (1) temperature $\mathrm{T}\left({ }^{\circ} \mathrm{C}\right)$ measured with a thermocouple inside the furnace ( $4 \mathrm{~mm}$ far away from the sample); (2) the increasing indent distance $\mathrm{dL}(\mu \mathrm{m})$ caused by the sapphire sphere forced into the glass; and (3) time $t$ (s) of the measurement. From the obtained data three plots were prepared.

First plot presents an indent distance $\mathrm{dL}(\mu \mathrm{m})$ as a function of time (Fig. 24). To this characteristic curve a new curve with known parameters was fitted in the ORIGIN Microcal programme, according to the equation

$$
y=(k \cdot x)^{n}
$$

where $y$ is the indent distance $\mathrm{dL}(\mu \mathrm{m}), x$ is time (s), $k$ is the factor of the fitting and $n$ is an exponent fixed as 0.6667 . This fitting equation is based on the formula of Douglas et al. (1965):

$$
l=\left(\frac{9 F}{32 \sqrt{2 R} \eta}\right)^{n} \cdot t^{n}
$$

where $l$ is the indent distance $\mathrm{dL}(\mu \mathrm{m}), F$ is used force $(F=m \cdot g), R$ is the radius of the sapphire sphere $(\mu \mathrm{m}), \eta$ is viscosity (Pa s) and $t$ - time (s), and $n=1 / 1.5=0.667$. 


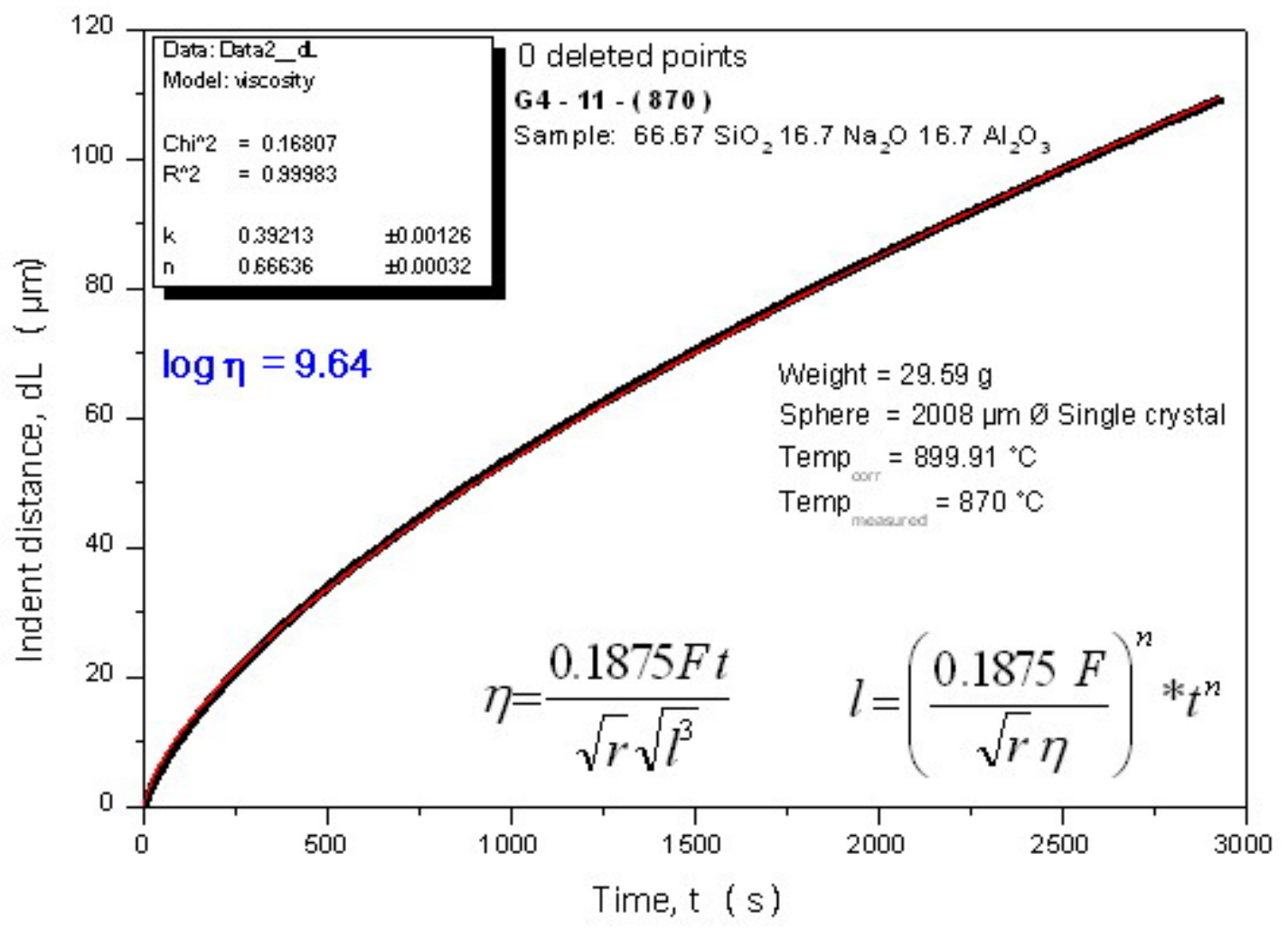

Fig. 24. Plot of the indent distance $\mathrm{dL}$ (in $\mu \mathrm{m}$ ) as a function of time (in s). Red line is the fit to the data (black line) using Eq. 20. The viscosity is calculated from not linear red curve.

Because the viscosity value must be calculated from the viscous part of the deformation plot, the first points of the plot (with lowest $\mathrm{dL}$ and the earliest in the time) need to be deleted. These points represent the unrelaxed part of the melt deformation. Removing them allows getting better estimation of $n$ parameter. Once $n \sim 0.667$ the parameter $k$ is automatically calculated.

With the known $k$ value, viscosity $\eta$ will be calculated:

$$
\eta=\frac{9 F}{32 \sqrt{2 R}} \cdot \frac{1}{k}
$$

(Douglas et al., 1965). The correct temperature for this viscosity needs to be calculated, according to the calibration (Eq. 18). 


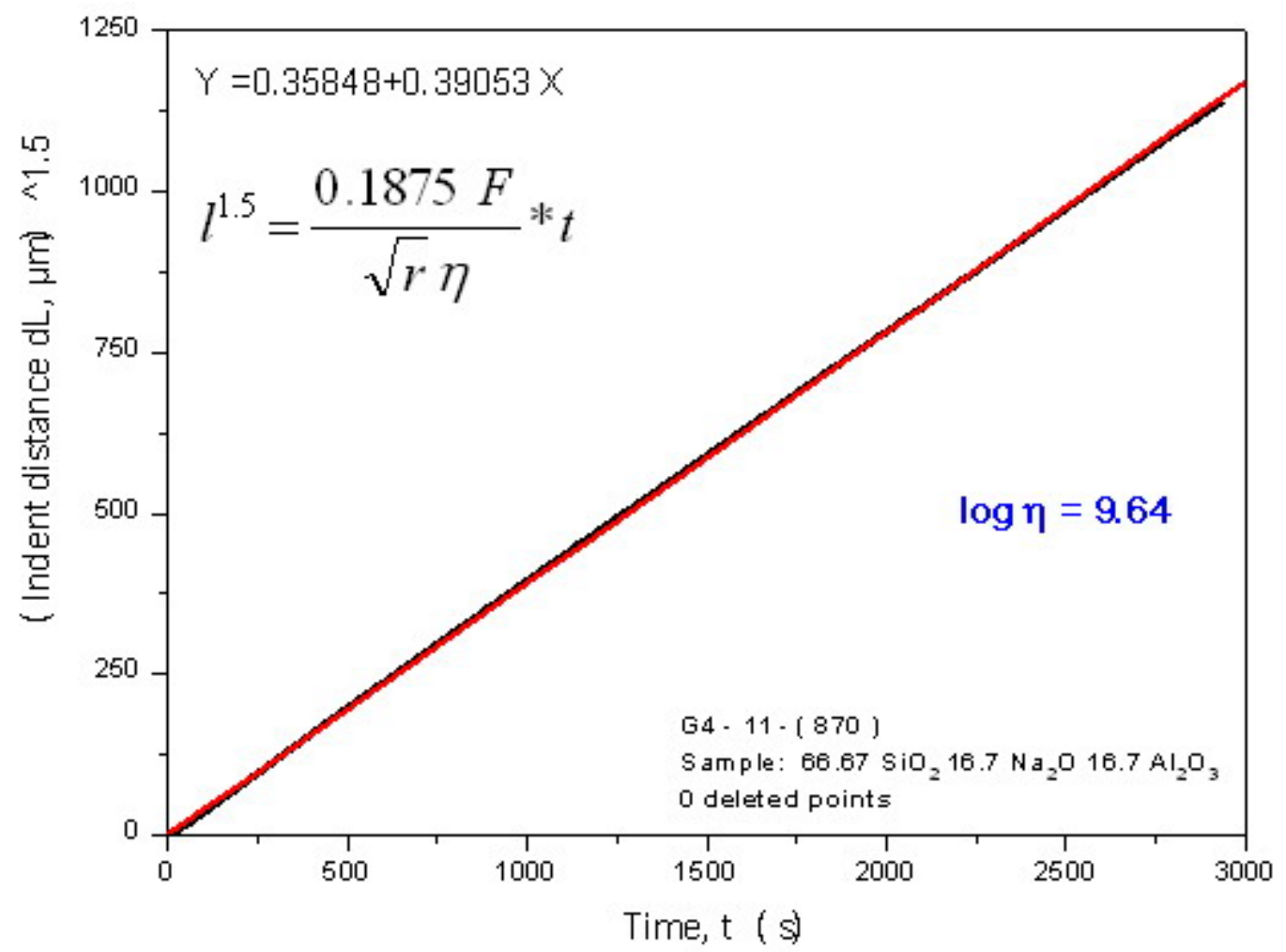

Fig. 25. Plot of the indent distance to the 1.5 (dL1.5) as a function of time. Viscosity is calculated from a straight line.

A second plot, $d L^{1.5}$ as a function of time is created to compare and to confirm the viscosity calculated from the first plot. Figure 25 shows the linear relationship between $d L^{1.5}$ and time. The line is described with an equation:

$$
y=a+k \cdot x .
$$

Parameter $k$ is used again to calculate viscosity.

A third plot shows the relationship of already calculated viscosity $\eta$ as a function of time (Fig. 26). The vertical initial part of the curve shows the viscosity of still unrelaxed glass. Because the investigated samples are Newtonian, after applying a constant stress, the viscosity of fully relaxed structure is constant and time independent. This behaviour is also used to indicate the absence of the sample crystallisation during the experiment. 


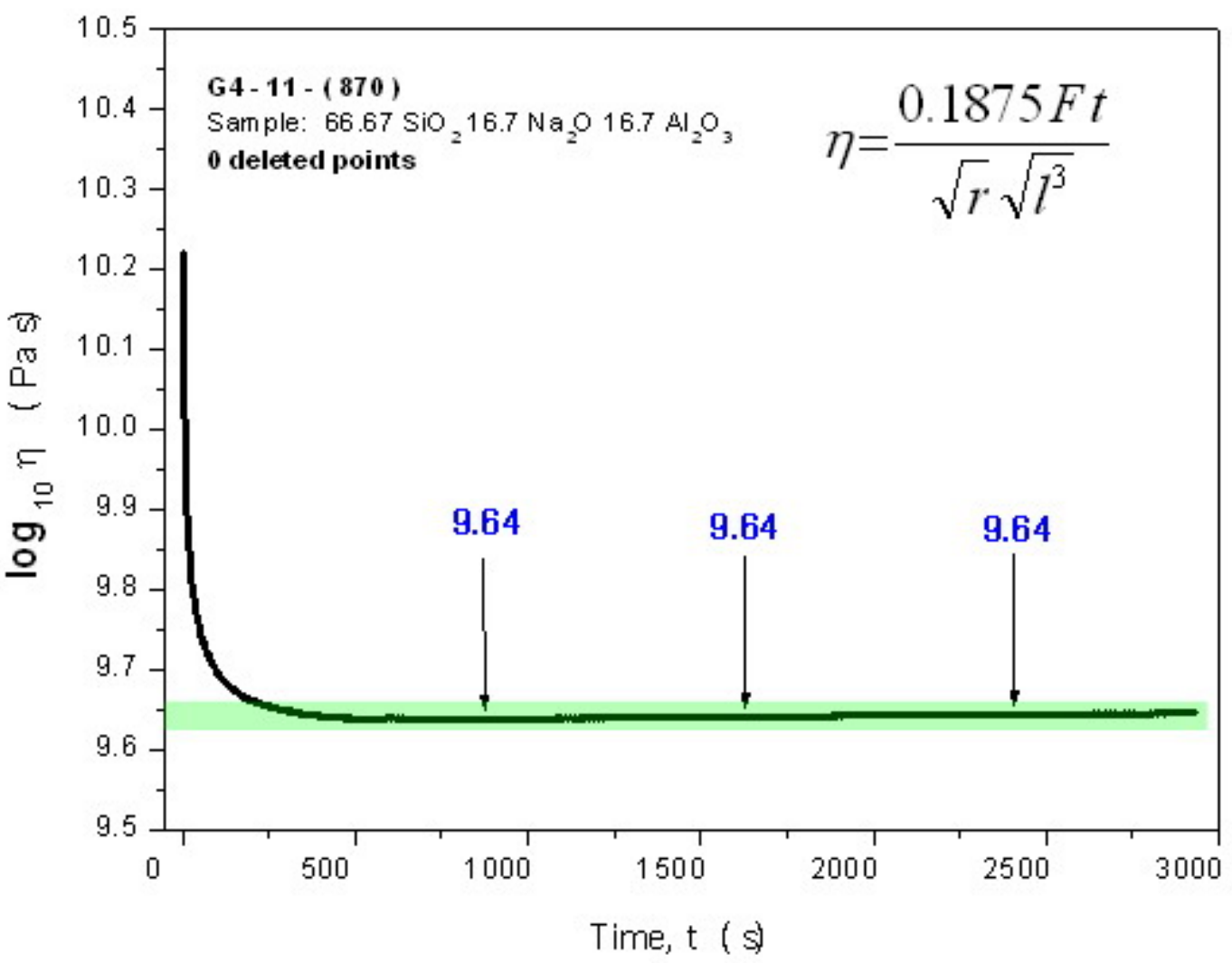

Fig. 26. Plot of the $\log _{10}$ viscosity as a function of time. Viscosity is independent of time.

Viscosities for all samples were measured in the range between $10^{8}$ and $10^{13} \mathrm{~Pa} \mathrm{~s}$. For each sample 10-13 measurements were done at different temperatures with steps of 10 or $15^{\circ} \mathrm{C}$. In Figure 27 data are presented as a function of inverse temperature $10^{4} / T\left(\mathrm{~K}^{-1}\right)$. A two-parameter Arrhenian equation:

$$
y=A+B \cdot x
$$

was fit to the data; as:

$$
\eta=\eta_{0} \exp \left(\frac{E_{a}}{R T}\right) \Rightarrow \log _{10} \eta=A_{0}+\frac{E}{R T \ln 10}=A+\frac{B}{T}
$$

(Richet \& Bottinga, 1995; Webb, 2005a), where $R$ is the gas constant (8.314474 $\left.\mathrm{J} \mathrm{K}^{-1} \mathrm{~mol}^{-1}\right), A_{0}$ is the adjustable pre-exponential factor $\left(A_{0}=\log _{10} \eta_{0}\right), E_{a}$ is activation energy (energy required to start the flow mechanism of the melt, Glasstone et al., 1941). 


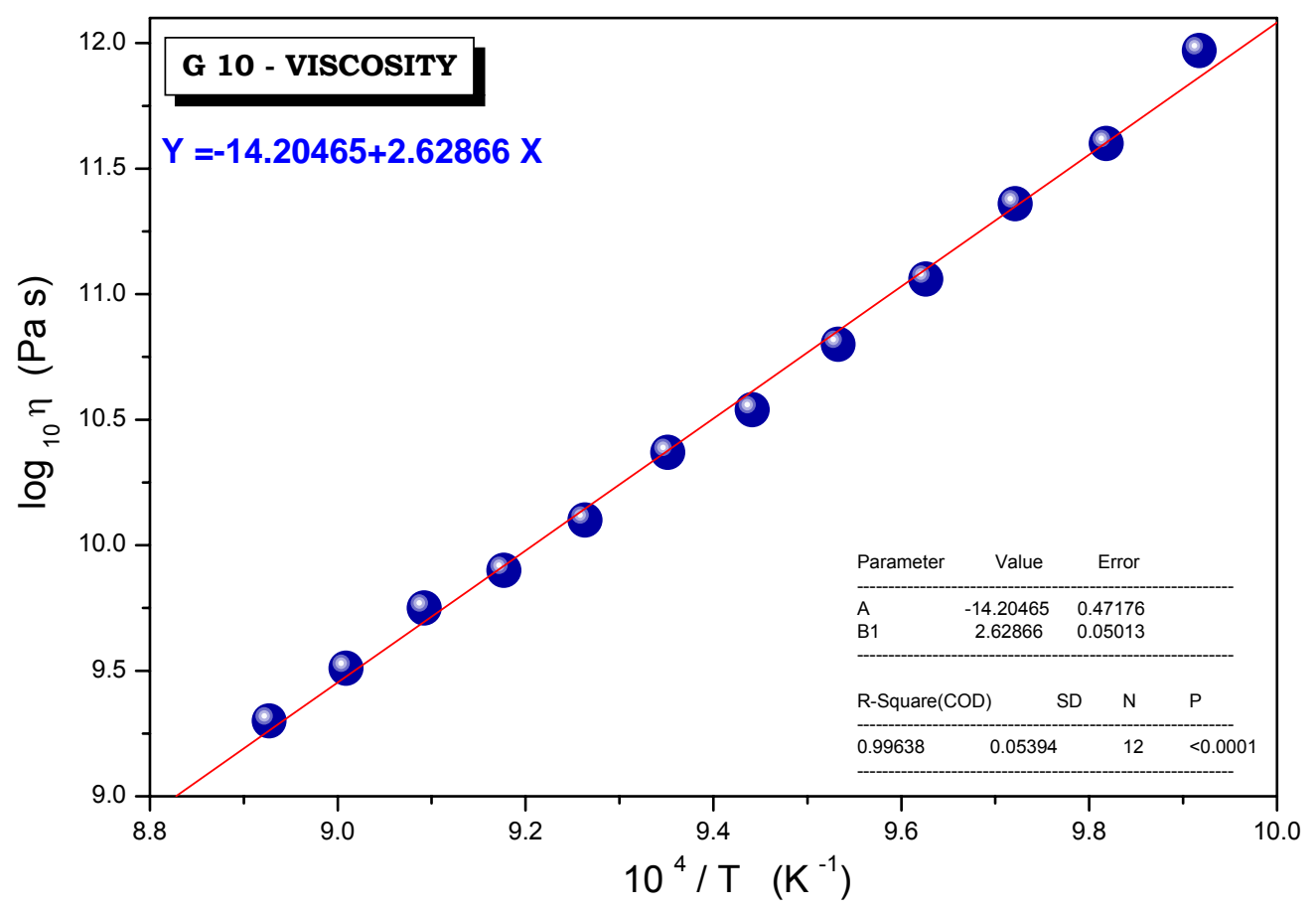

Fig. 27. $\log _{10}$ viscosity (here as an example: sample $\mathrm{G} 10$ ) as a function of inverse temperature.

The Arrhenian equation is exponential. If the temperature range of measurement is less than $\sim 500^{\circ} \mathrm{C}$ an Arrhenian line is required to describe the $\eta$ data. If the temperature range is large, the curvature in $\log _{10} \eta$ as a function of inverse temperature becomes large enough to the Vogel-Fulcher-Tamman (VFT) equation is used:

$$
\eta=\eta_{0} \exp \left(\frac{E_{a}}{R\left[T-T_{0}\right]}\right) \Rightarrow \log _{10} \eta=A_{V F T}+\frac{E}{R\left[T-T_{0}\right] \ln 10}=A_{V F T}+\frac{B_{V F T}}{T-C}
$$

(Fulcher, 1925; Tamman and Hesse, 1926; Angell, 1991a; Richet and Bottinga, 1995; Hess and Dingwell, 1996; Webb, 2005a), where $A_{V F T}$ is the constant value of viscosity at infinite temperature, $B_{V F T}$ is pseudo-activation energy and $C$ is VFT temperature. $T$ is absolute temperature (K). Russel et al. (2003) estimated value $A_{V F T}$ of $-4.31 \pm 0.74$, Giordano et al. (2006) respectively for large data sets as $-4.07 \pm 1$, Toplis (1998) calculated that as $-2.6 \pm 1$. 
Using $B$ parameter from Eq. 24 , it is possible to calculate activation energy $E_{a}$ :

$$
E_{a}=B \cdot R \cdot \ln 10
$$

Here, the Arrhenian equation was used to fit all data.

At the end, viscosity data were presented as a function of the composition ( $\gamma$ value) to illustrate the dependence of the viscosity on peraluminosity or peralkalinity of the melt.

\subsection{Heat capacity $c_{p}$}

The calorimetric techniques base on the measurement of heat. Heat capacity is the energy required to increase the temperature of the sample. In the case of the melts there are two heat capacities as a function of temperature: (1) that of the glass in which flow does not occur, and (2) that of the melt in which energy is also used for flow. The difference between the two $c_{p}$ values is the configurational heat capacity $c_{p}{ }^{\text {conf }}$.

The configurational properties of melts can have a large influence on the thermal and volume properties. The configurational heat capacity has a great importance in thermodynamic calculations involving silicate melts and can (through the configurational entropy $S_{\text {conf }}$ ) be related to viscosity (Richet \& Bottinga, 1985; Richet \& Neuville, 1992). Changes in the melt structure can be explained by variation of the configurational entropy $S_{\text {conf }}$ as a function of composition. Calorimetry is an excellent method to observe the change in enthalpy of the sample as a function of temperature.

\subsubsection{Glass transition range and fictive temperature $T_{f}$}

Differential scanning calorimetry (DSC) is ideal to observe a glass transition from glass to liquid and to calculate the fictive temperature of the melt - the characteristic parameters for each glass composition.

The glass transition occurs across a range of temperature, not at one strictly fixed temperature. It is a change in behaviour between liquid and glass. The width of glass transition region depends on the relaxation time of the glass and the heating rate. The 
kinetic nature of the glass transition is strongly connected with heating / cooling rate and is traditionally explained on the enthalpy or volume versus temperature plots.

As pointed out by Narayanaswamy (1971), if the structure of a melt determines the physical properties, the change in physical properties with temperature through the glass transition will follow the same curve as structure does; and thus all physical properties should show the same trend through glass transition. As shown by Webb (1992b) this is the case for enthalpy and volume.

At high temperature the melt structure is fully relaxed. With decreasing temperature the melt needs more time (longer relaxation time) to stay in equilibrium with the new temperature (Fig. 28). The viscosity also increases and with further cooling at some stage the structural units can no longer rearrange to the equilibrated structure because relaxation time is too short. Structure becomes "frozen", constant and it does not change with further decrease in temperature. Enthalpy of new structure is higher than would be in the equilibrium state and the curve has a decreasing slope, up to the state when could become fully temperature independent. This supercooled liquid with "frozen" structure is called a glass. The temperature region between the high temperature at which structure of the supercooled liquid is still in equilibrium and the temperature at which structure does not change anymore, is called glass transition (Brawer, 1985; Scherer, 1986; Goldstein \& Simha, 1976; O’Reilly \& Goldstein, 1981; Angell \& Goldstein, 1986).

Glass transition region depends on heating / cooling rate. With slower temperature changes, the structure has more time to follow the equilibrium line towards lower temperature and the glass transition will appear at lower temperatures. Such glass will have lower enthalpy than the glass with faster cooling rate.

Crystallization, upon cooling, results in a large drop in enthalpy and the creation of a long range order in the crystal. If the melt is cooled fast enough, the crystalline state does not occur and the change in enthalpy goes slowly then the liquid becomes supercooled. If the lines of the glass and supercooled liquid will be extrapolated, they will cross at the point indicating fictive temperature $T_{f}$ of the glass. $T_{f}$ characterises the last equilibrium state of the supercooled liquid.

Because glass transition occurs over a range of temperature, for simplifying this very important parameter (especially in the industry), $T_{g}$ was defined as the temperature, at which the shear viscosity equals $10^{12} \mathrm{~Pa} \mathrm{~s}$. By $\log _{10} \eta=10^{12} \mathrm{~Pa}$ s the relaxation time of the melt last 100 seconds (Scherer, 1990). The value of $T_{g}$ is in general, for normal laboratory quench rates comparable with $T_{f}$. 


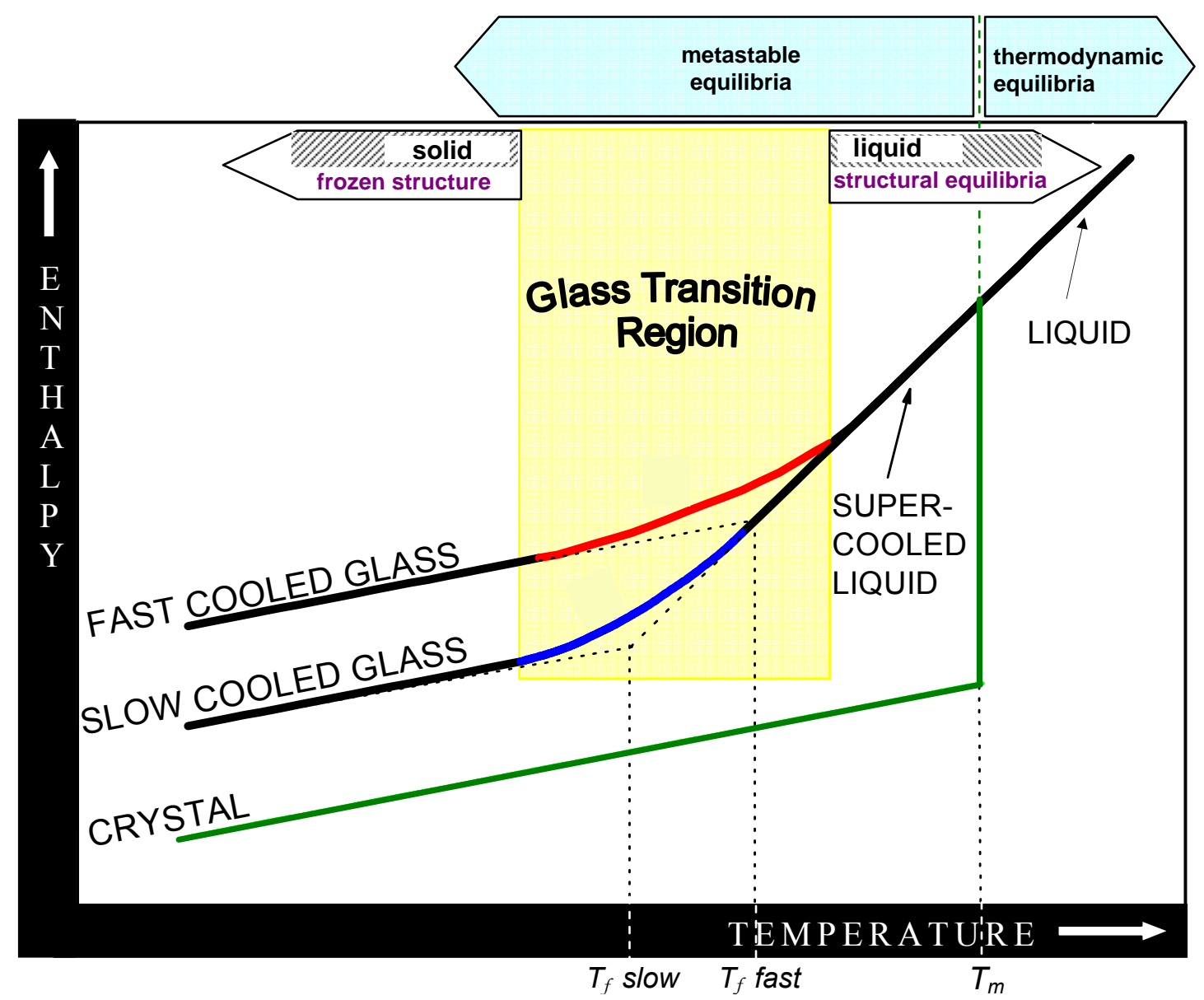

Fig. 28. Effect of temperature on the enthalpy of a glass forming melt for fast and slow cooled glasses and for crystalline material. Red part of the line indicate the glass transition region for fast cooled glass; blue line - glass transition region for slow cooled glass. Other descriptions in the text.

\subsubsection{Description of the machine}

The DSC measures the heat capacity $c_{p}$ of the sample against an empty crucible. In this project the heat flux DSC was used (Fig. 29).

The operating conditions for the Netzsch DSC 404C calorimeter are from room temperature up to $1000^{\circ} \mathrm{C}$. The heat flow is measured with $\mathrm{Pt} / \mathrm{Rh}$ thermocouples. Two crucibles composed of platinum are placed on the raised platform in the Pt10\%Rh head designed to be used at high temperature and that all is surrounded by the furnace. All the measurements were done in the atmosphere of argon gas to keep the sample dry and to remove superfluous volatiles. The reference crucible remains empty. Sample is placed in the second crucible. The computer programme measures the difference in voltage between the sample and reference thermocouple. Then voltage value is compared with the known equivalent of heat capacity. The result is a plot of differential heat capacity between sample and reference as a function of temperature. 

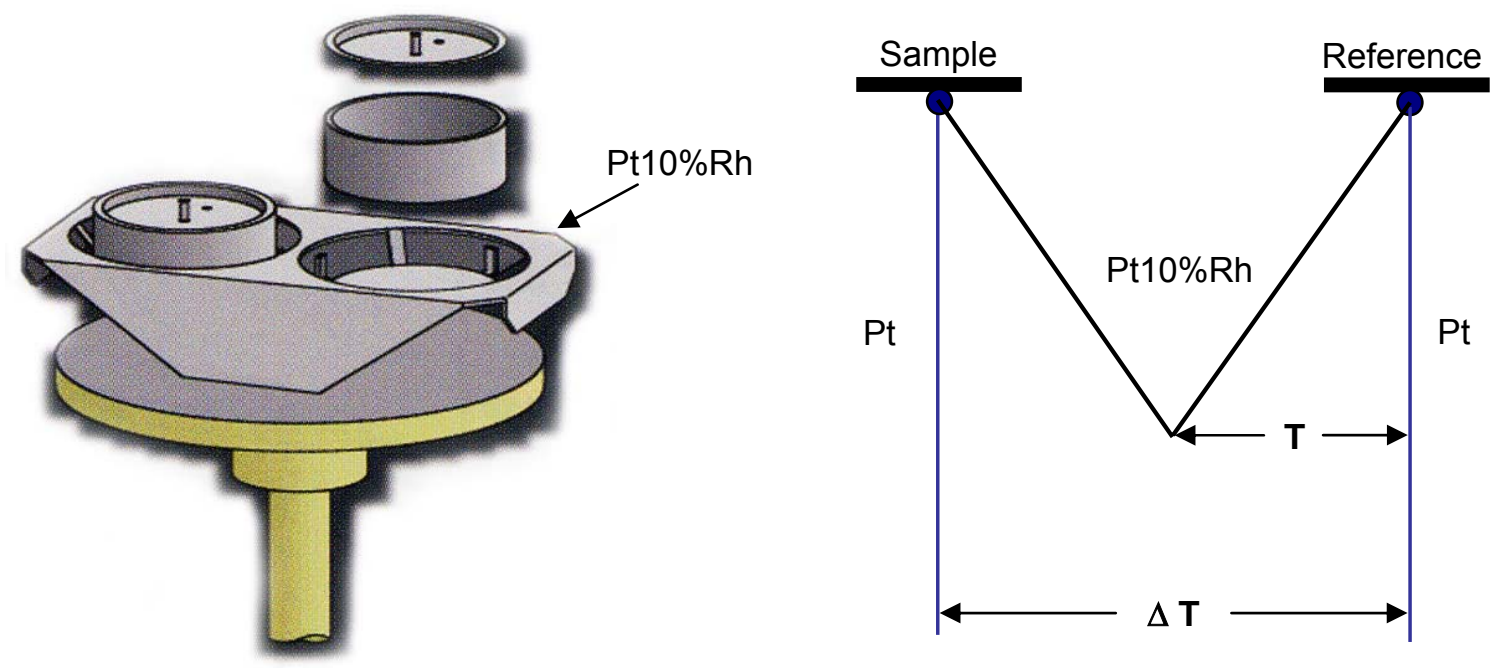

Fig. 29. Schematic presentation of the differential scanning calorimeter (DSC). Description in the text.

\subsubsection{Calibration of the calorimeter}

Calibration consists on two measurements: (1) when both crucibles are empty and (2) when reference crucible is empty and sample crucible contains a standard. The standard used to calibrate the machine is polycrystalline $\mathrm{Al}_{2} \mathrm{O}_{3}$ disk $(6 \mathrm{~mm}$ diameter, $\sim 1 \mathrm{~mm}$ thick) with the weight of $55.56 \mathrm{mg}$. The $\mu \mathrm{V}$ reading from two empty crucibles is subtracted from that with one empty + one crucible with the $\mathrm{Al}_{2} \mathrm{O}_{3}$ standard in order to remove the effect of the crucible containing the standard. The resulting $\mu \mathrm{V}$ values as a function of temperature are compared with the heat capacity of $\mathrm{Al}_{2} \mathrm{O}_{3}$ given by Robie et al. (1978). Data of Robie et al. (1978) are in excellent agreement with those of Krupka et al. (1979). The sensitivity curve needed to convert $\mu \mathrm{V} \mathrm{g}^{-1}$ to $\mathrm{J} \mathrm{g}^{-1} \mathrm{~K}^{-1}$ is then used in the subsequent measurements of the heat capacity of the melts.

\subsubsection{Measurement and calculation of heat capacity and configurational heat capacity}

The sample is prepared as a coarse-grained powder $(\sim 0.2-0.3 \mathrm{~mm}$ in diameter $)$ and in the crucible is placed about $55 \mathrm{mg}$ the material. Sample and reference are heated up with the same heating rate as the last cooling rate. Only first measurement is worthless because last cooling rate of the sample is unknown. 
Fig. 30 shows a typical curve obtained from heat capacity measurement. Marked black part of the heat capacity data was used to fit a curve described by Maier-Kelley equation:

$$
C_{p}=a+b \cdot T+c \cdot T^{-2}
$$

(Maier \& Kelley, 1932; Scherer, 1990) being an extrapolation of heat capacity of the glass, where $a, b$ and $c$ are parameters of the line; and $T$ is temperature in $\mathrm{K}$. Small values of the parameter $b$ describe the simple melts which can be expressed by harmonic oscillation theory.

Range of temperature chosen to this fitting was between $200^{\circ} \mathrm{C}$ (to exclude all disturbance of the lower temperature) and the temperature at which the glass transition starts (strong bend of the curve).

In the glass transition range the heat capacity curve goes up to reach the maximum at the temperature of structural relaxation of the melt. Melt becomes a supercooled liquid. When the curve becomes temperature independent (horizontal), the measurement needs to be broken off to prevent complete melting of the sample. End of the curve indicates a heat capacity of the melt.

Difference between heat capacity of the melt $c_{p l}$ and heat capacity of the glass $c_{p g}$ at the glass transition (measured at the same temperature) is the configurational heat capacity $c_{p}{ }^{\text {conf }}$ and is the energy difference - integrated over all atoms - between the minima of the potential energy wells explored when flow begins (Richet et al., 1986; Richet \& Toplis, 2001):

$$
c_{p}^{\text {conf }}=c_{p l}-c_{p g} .
$$

To a first approximation, the $c_{p g}$ can be calculated from a linear summation of the heat capacities of the oxide components. Similarly, if there are no anomalous changes in melt structure upon heating through the glass transition, the $c_{p l}$ will be a linear sum of the contributions of the component oxides (e.g. Richet \& Bottinga, 1985). Determination of $c_{p l}$ as a function of composition illustrates the presence of such anomalous structures (see section 4.5.). 


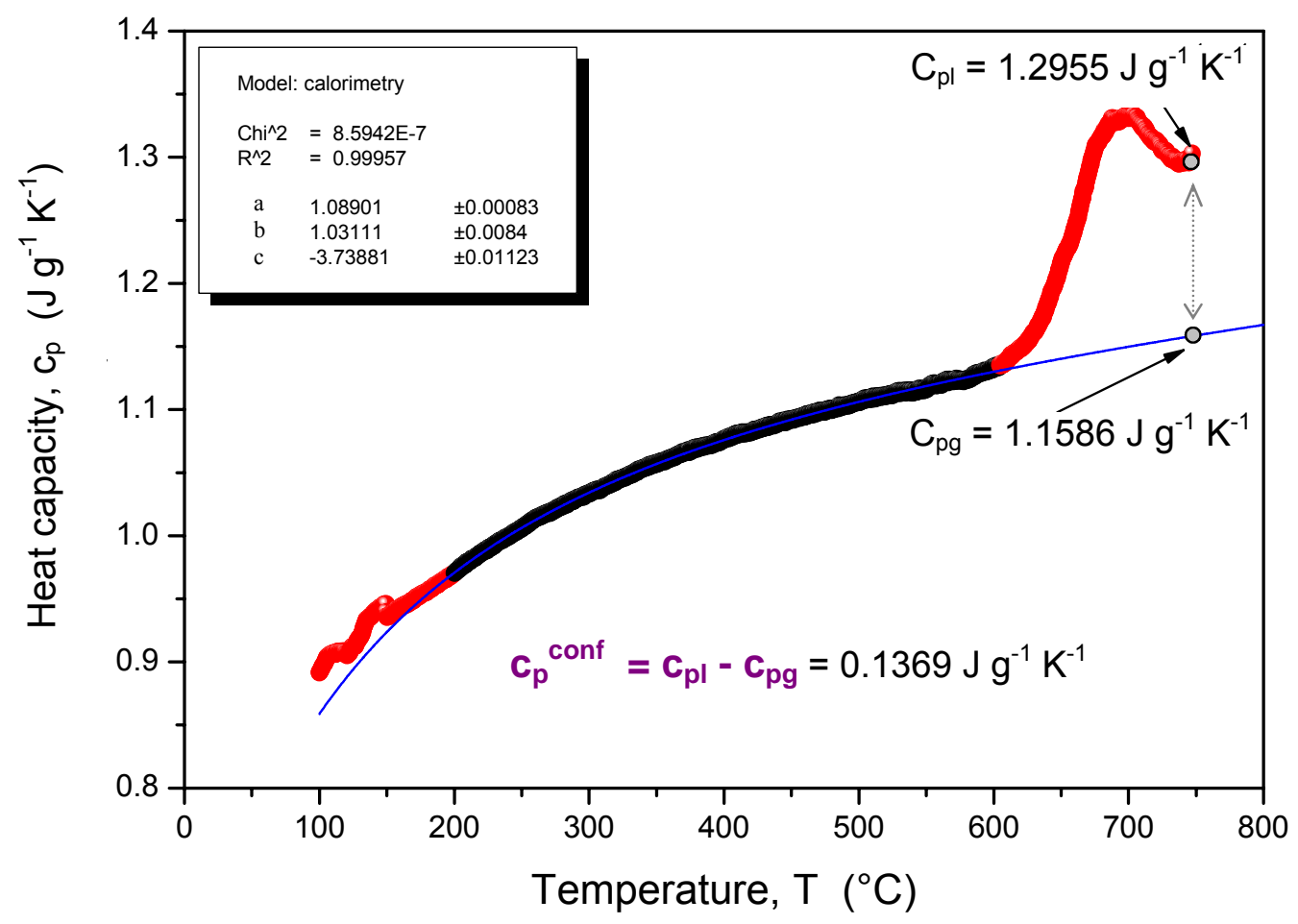

Fig. 30. Heat capacity $c_{p}\left(\mathrm{~J} \mathrm{~g}^{-1} \mathrm{~K}^{-1}\right)$ as a function of temperature $\left({ }^{\circ} \mathrm{C}\right)$. Blue line is a fit to the three parameter Maier - Kelley equation (Eq. 28). Description in the text.

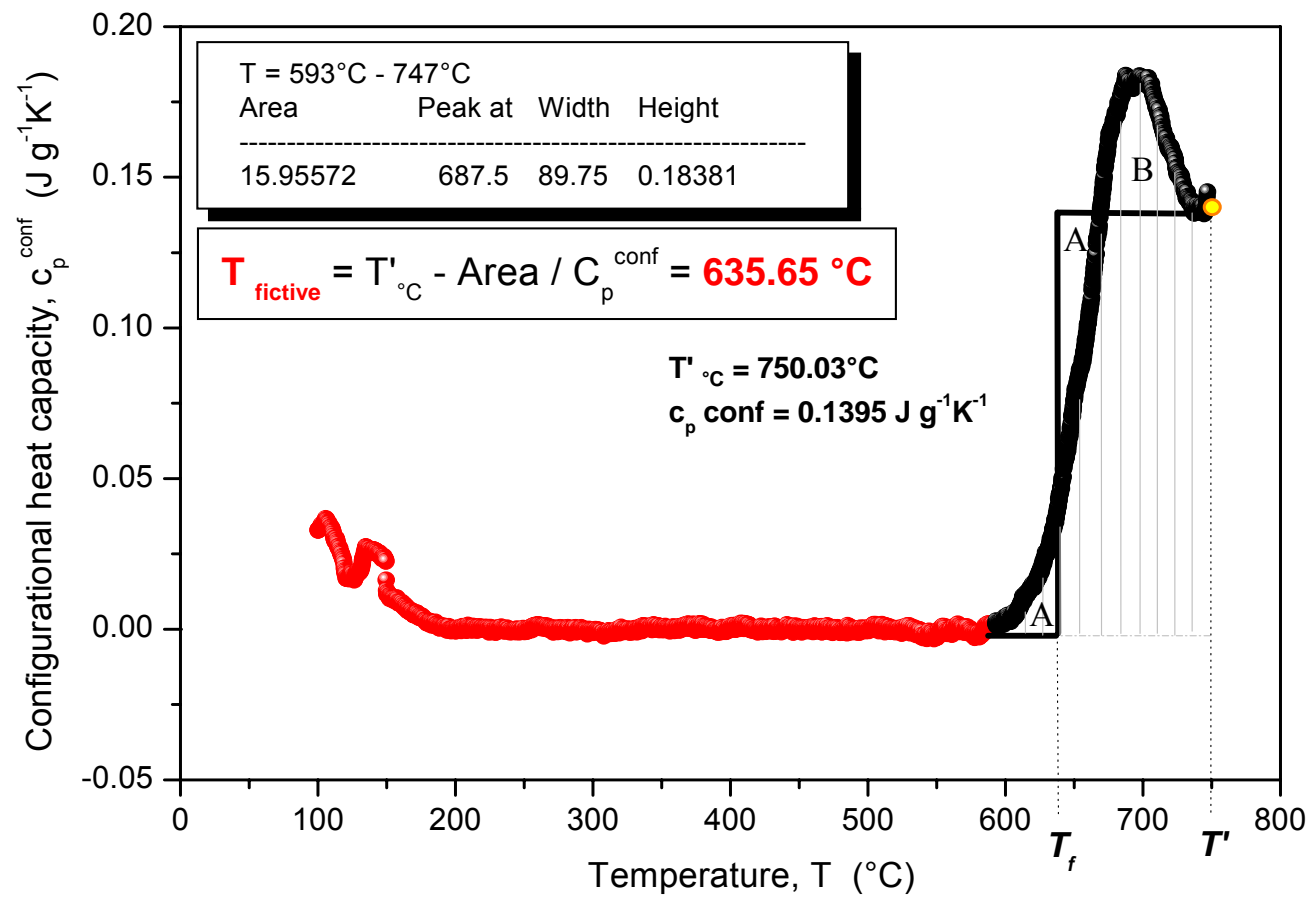

Fig. 31. Configurational heat capacity $c_{p}{ }^{\text {conf }}\left(\mathrm{J} \mathrm{g}^{-1} \mathrm{~K}^{-1}\right)$ as a function of temperature $\left({ }^{\circ} \mathrm{C}\right)$. Description in the text. 


\subsubsection{Calculation of the fictive temperature}

Next step of calculation is determination of the fictive temperature of the melt.

Fictive temperature describes the temperature at which the melt structure would be in equilibrium and decreases for decreasing cooling rate (see Fig. 32). Using the options of ORIGIN programme, points of fitted line are subtracted from heat capacity data and a plot of configurational heat capacity is obtained (Fig. 31). Area below the peak (marked as grey vertical lines) is calculated and used to determine fictive temperature according to the equation:

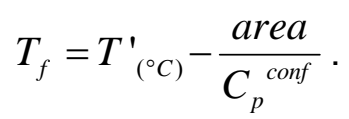

The areas $A$ and $B$ in Figure 31 are related to the equation:

$$
\int_{T \gg T_{g}}^{T_{f}{ }^{\prime}}\left(c_{p e}-c_{p g}\right) d T=\int_{T \gg T_{g}}^{T \ll T_{g}}\left(c_{p}-c_{p g}\right) d T
$$

where $c_{p e}$ is the equilibrium heat capacity of the glass, $c_{p g}$ is the heat capacity of the glass (Moynihan, 1995). Area A matches up to the left side of the equation (Eq. 31), and area B corresponds to the right side. Areas $A$ and $B$ should be equal.

\subsubsection{Dependence of the glass transition on the heating rates of the sample}

Sample is heated up at different heating rates: $5^{\circ} \mathrm{C} / \mathrm{min}, 10^{\circ} \mathrm{C} / \mathrm{min}, 15^{\circ} \mathrm{C} / \mathrm{min}$, $20^{\circ} \mathrm{C} / \mathrm{min}$ and $25^{\circ} \mathrm{C} / \mathrm{min}$. With reference to the kinetic of the melt and structural relaxation time, different heating rates contribute to change the position of the peak's maximum as a function of temperature (Fig. 32): at lower temperature when heating rate is slow and at higher temperature for faster heating rate. 

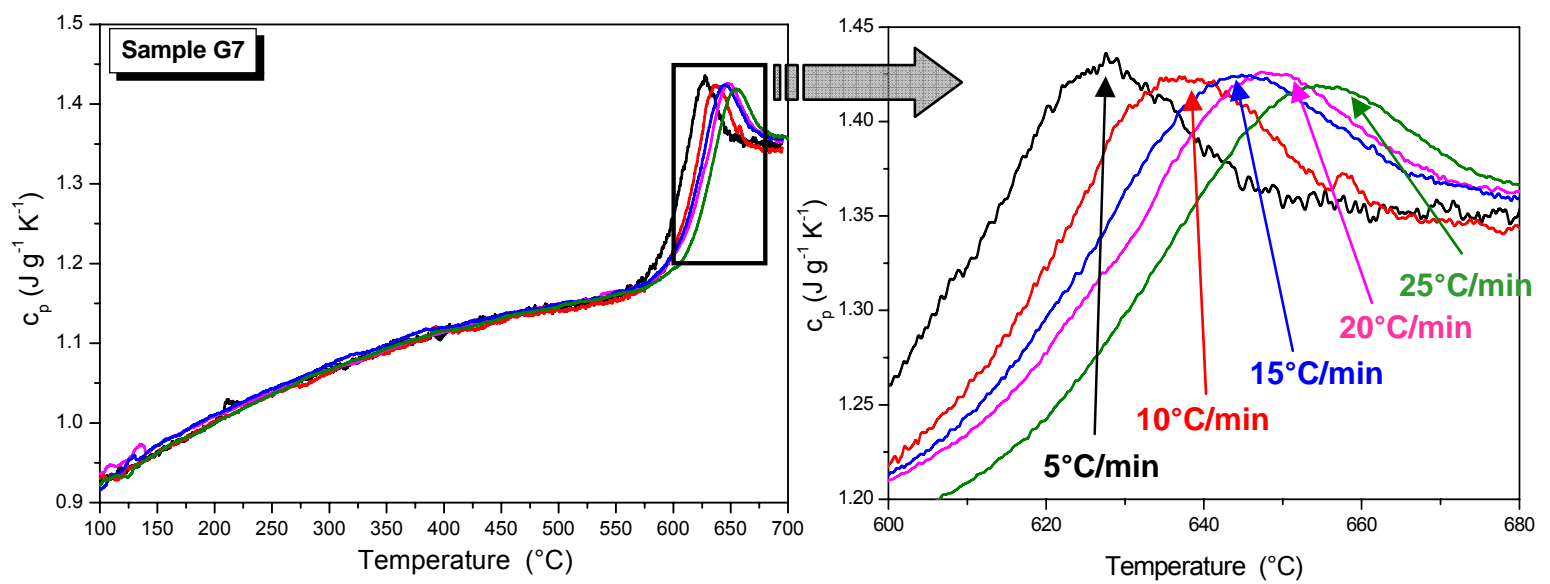

Fig. 32. Different heating rate of the sample causes shift of the peak's position. Description in the text.

\subsection{Torsion (forced oscillation)}

There are few studies of the frequency - dependence of the shear modulus and viscosity of silicate melts. This technique was first developed for crystalline materials, and is also used for molten samples. The studies on melts have shown:

(1) the melt structure cannot be described by a single flow mechanism with a single timescale of bond exchange. This is to be expected based upon the statistical nature of the melt structure;

(2) it is possible to resolve the creation of new structures involved in the flow process. The data of Bagdassarov et al. (1993) show that there is an effect on flow due to the presence of boron and fluorine, but it is only in the case of phosphorus that a second peak in the structural relaxation (flow) spectrum is seen. These data were obtained on single melt compositions and so it was not possible to determine the actual effect due to the added components.

Day \& Rindone (1962) have performed forced oscillation measurements on $\mathrm{Na}_{2} \mathrm{O}-\mathrm{Al}_{2} \mathrm{O}_{3}-\mathrm{SiO}_{2}$ melts, but were interested in the movement of $\mathrm{Na}$ atoms and therefore worked between -50 and $0^{\circ} \mathrm{C}$.

The determination of the shear modulus and the imaginary part of the shear modulus in the present study will be used to interpret the changes in bond strength and flow units in the melt. 


\subsubsection{Principle of torsion}

Forced oscillation shear deformation is based on the principle of torsion of a fixed cylindrical rod. Here a torque is applied and the resulting deformation (angle of twist, $\varphi$ ) of the rod is measured as a function of frequency.

Torsion measurements have been performed on the samples G0-G14 (see Table 2). In these measurements, the stress $\sigma$, and $\operatorname{strain} \varepsilon$ are determined as a function of frequency from $1 \mathrm{mHz}$ to $1 \mathrm{~Hz}$, and the real $G^{\prime}(\omega)$ and imaginary $G^{\prime \prime}(\omega)$ components of the shear modulus are calculated from these measurements.

The relationship between applied stress $\sigma$ and measured strain $\varepsilon$ gives shear modulus $G$ :

$$
G=\frac{\sigma}{\varepsilon}
$$

The cylinder in Figure 33 is held fixed at surface $A$ and the angle of twist $\varphi$ (in radians) is measured at surface $B$;

$$
\varphi=\frac{B B^{\prime}}{r}
$$

where $r$ is the distance from the centre of the cylinder. The shear strain $\varepsilon_{r}$ at a distance $r$ from the centre of the rod is given by $\varphi$; and for small values of $\varepsilon_{r}$

$$
\varepsilon_{r}=\tan \varepsilon_{r}=\frac{\varphi r}{L}
$$

for $L$ - the length of the rod (Riley et al. 1999). At the centre of the rod $\varepsilon_{0}=0$. The shear stress $\sigma$ is calculated from the applied torque $\tau_{\varphi}$ :

$$
\sigma_{r}=\frac{\tau_{\varphi} r}{J}
$$




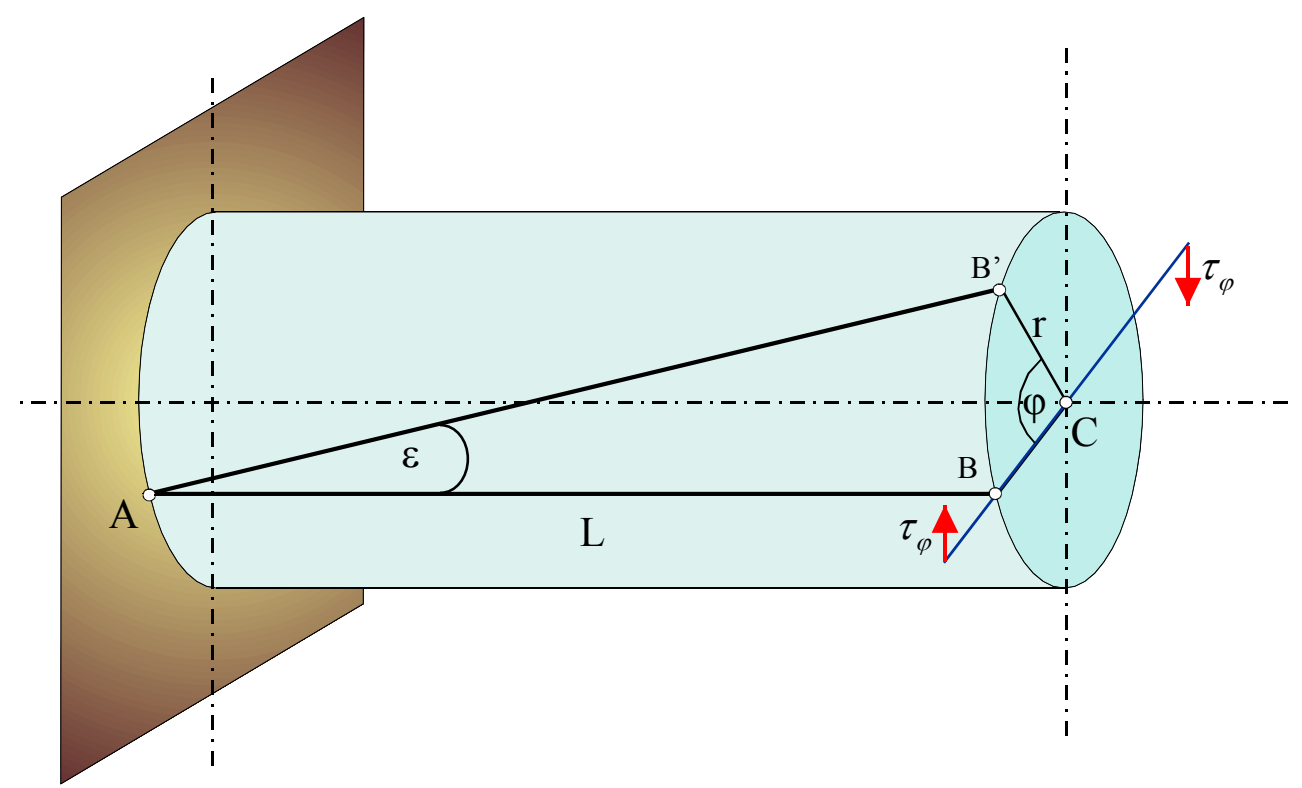

Fig. 33. Schematic diagram of the torsion experiment with characteristic quantities described in the text.

for $J$ - the polar second moment of area which is:

$$
J=\frac{\pi r^{4}}{2}
$$

(Sass et al., 1966). The shear modulus of the cylinder can then be calculated as a function of frequency:

$$
G^{*}(\omega)=\frac{\sigma}{\varepsilon}=\frac{2 \tau_{\varphi} L}{\pi r^{4} \varphi}
$$

The real component of modulus is the elastic deformation, while the imaginary component is a measure of the energy loss in the melt. A peak in the loss modulus indicates the motion of part of the melt structure.

The shear viscosity can be also calculated as a function of frequency:

$$
\eta^{*}(\omega)=\frac{\sigma}{\dot{\varepsilon}}=\frac{2 \tau_{\varphi} L}{\pi r^{4} \dot{\varphi}}
$$


As the shear modulus and shear viscosity are both frequency dependent and are a function of stress, strain and strain rate:

$$
\eta^{*}(\omega)=\frac{\sigma}{\dot{\varepsilon}}=\frac{G^{*}(\omega)}{i \omega}=\frac{G^{\prime}(\omega)}{i \omega}+\frac{i G^{\prime \prime}(\omega)}{i \omega}=\eta^{\prime}(\omega)+i \eta^{\prime \prime}(\omega)
$$

(Webb, 1991). Thus the real component of viscosity is the frequency independent (Newtonian) viscosity and the real component of shear modulus is the frequency independent elastic modulus. Torsional deformation can be used to determine $G$ and $\eta$ of a material, both in the time domain - for a constant applied torque, and in the frequency domain - for a sinusoidally oscillating torque.

\subsubsection{The torsion apparatus}

The torsion apparatus was calibrated by measuring the shear modulus of a long rod of polycrystalline $\mathrm{Al}_{2} \mathrm{O}_{3}$ (Al23 Degussit $\AA$ ). This rod is $8 \mathrm{~mm}$ in diameter and fixed at one end (see Fig. 34). The shear modulus of the alumina rod is determined by static measurements in which the torque $\tau_{\varphi}$ is created by a force $F$ applied to the rod:

$$
\tau_{\varphi}=F \cdot a,
$$

where $a$ is the distance from the centre of the rod to the applied force (Fig. 35). The force was created by applying different masses. In the successive measurements one pair of the weights were hung on threads which went from the end of the arm of length $a_{a v}=100.85 \mathrm{~mm}$ to a pulley at the edge of the top plate. The resulting twist in the alumina rod was determined by the inductive transducers at channels $1 \& 2$. There are aluminium rods fixed to the alumina rod at the points: channel 1 and channel 2 . These rods end in iron plates which sit within pairs of inductive transducers. As the $\mathrm{Al}_{2} \mathrm{O}_{3}$ rod twists, the angle of twist is magnified by the length of the aluminium rod and the motion of the iron plate results in a voltage change at the inductive transducers. 


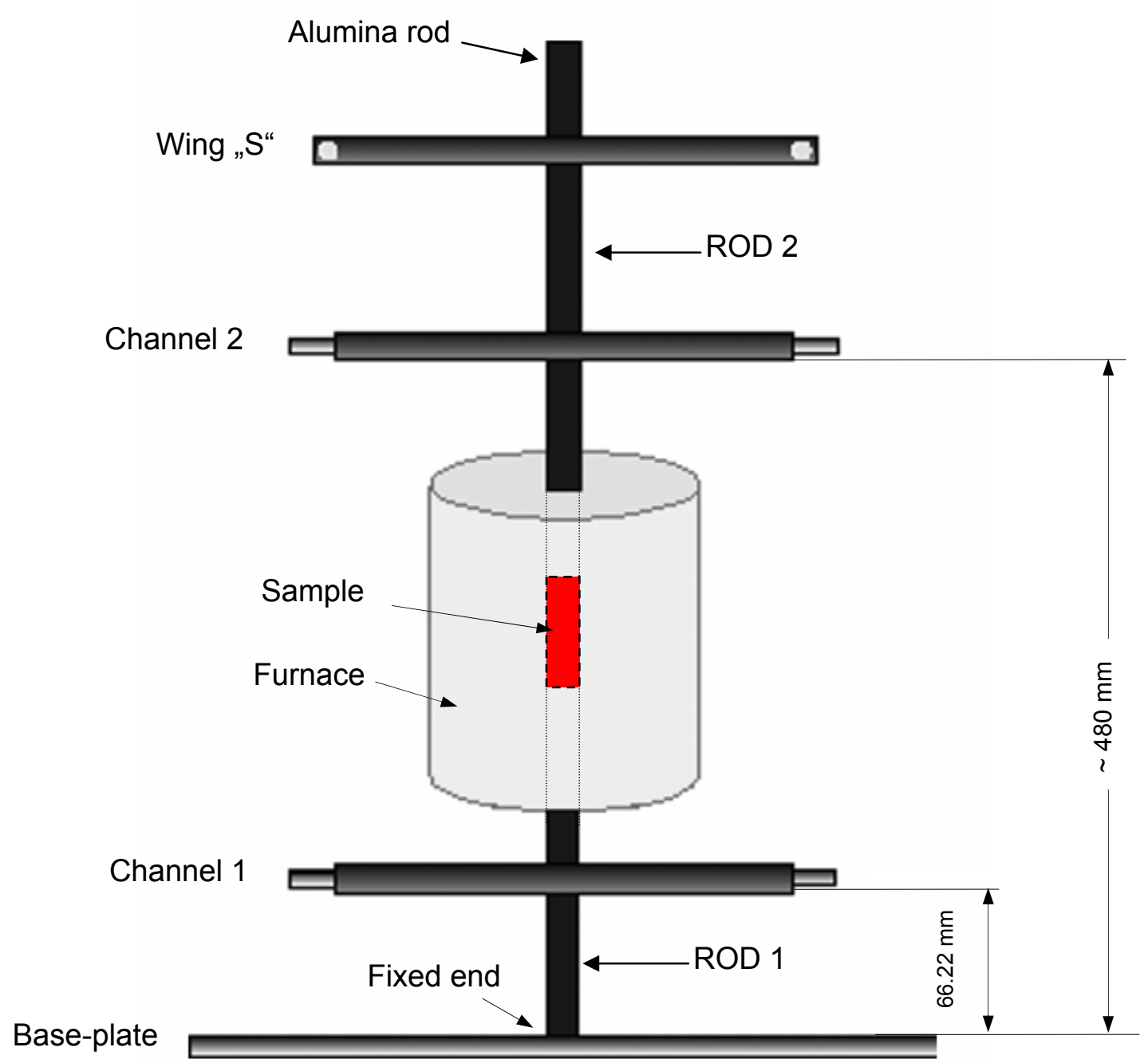

Fig. 34. Scheme of the torsion machine and furnace. The angles of twist are measured at channels 1 and 2; and the torque is applied at "wing S". The equipment is calibrated using a rod of polycrystalline $\mathrm{Al}_{2} \mathrm{O}_{3}$. The sample assembly with a cylinder of melt between two $\mathrm{Al}_{2} \mathrm{O}_{3}$ rods is shown here.

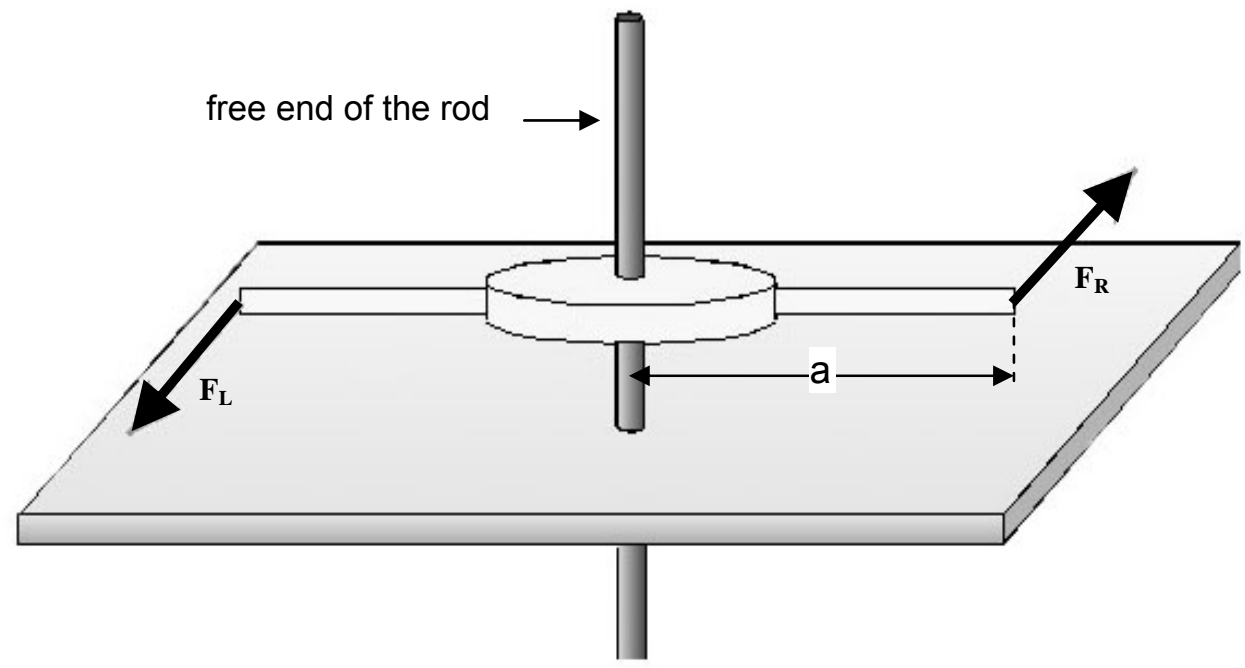

Fig. 35. Scheme of the wing on the top of alumina rod used to determine the shear modulus of the rod. $F_{L}$ - force applied to the left side of the arm; $F_{R}$ - force applied to the right side of the arm; $a$ the distance from the centre of the rod to the applied force (left arm: $100.75 \mathrm{~mm}$; right arm 100.95 $\mathrm{mm})$. 
Tab. 5. Table used to calculation of the torque (here: for $\mathrm{Al}_{2} \mathrm{O}_{3}$ in $600^{\circ} \mathrm{C}$ ). Description - in the text.

\begin{tabular}{|c|c|c|c|c|c|c|c|}
\hline \multirow{5}{*}{ 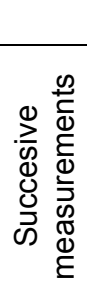 } & \multicolumn{2}{|c|}{ THE LEFT ARM } & \multicolumn{2}{|c|}{ THE RIGHT ARM } & & & \\
\hline & \multirow{3}{*}{$\begin{array}{l}\text { Applied } \\
\text { weight }\end{array}$} & \multirow{3}{*}{$\begin{array}{c}\text { (2) } \\
\text { multiplied } \\
\text { by length of } \\
\text { the left arm }\end{array}$} & \multirow{3}{*}{$\begin{array}{c}\text { Applied } \\
\text { weight }\end{array}$} & \multirow{3}{*}{$\begin{array}{c}\text { (4) } \\
\text { multiplied } \\
\text { by length of } \\
\text { the right arm }\end{array}$} & \multirow{3}{*}{$\begin{array}{c}\text { TORQUE } \tau_{\varphi} \\
\Sigma \\
(3)+(5)\end{array}$} & \multirow{2}{*}{\multicolumn{2}{|c|}{$\begin{array}{c}\mathrm{Al}_{2} \mathrm{O}_{3}-600^{\circ} \mathrm{C} \\
\text { VOLTAGE }\end{array}$}} \\
\hline & & & & & & & \\
\hline & & & & & & Channel 1 & Channel 2 \\
\hline & [g] & {$[\mathrm{g} \cdot \mathrm{mm}]$} & [g] & {$[\mathrm{g} \cdot \mathrm{mm}]$} & {$[N \cdot m]$} & [V] & [V] \\
\hline (1) & (2) & (3) & (4) & (5) & (6) & $(7)$ & (8) \\
\hline 1 & 0 & 0 & 0 & 0 & 0 & $5.00 \mathrm{E}-04$ & 4.00E-04 \\
\hline 2 & 1.747 & 176.051 & 1.750 & 176.642 & 0.0035 & 0.0117 & 0.0452 \\
\hline 3 & 3.512 & 353.784 & 3.508 & 354.173 & 0.0071 & 0.0235 & 0.0895 \\
\hline 4 & 5.256 & 529.572 & 5.231 & 528.019 & 0.0106 & 0.038 & 0.1387 \\
\hline 5 & 6.957 & 700.877 & 7.125 & 719.289 & 0.0142 & 0.0531 & 0.1899 \\
\hline 6 & 8.690 & 875.518 & 8.912 & 899.636 & 0.0178 & 0.0647 & 0.2375 \\
\hline 7 & 10.598 & 1067.738 & 10.598 & 1069.888 & 0.0214 & 0.0806 & 0.2886 \\
\hline 8 & 12.329 & 1242.167 & 12.338 & 1245.561 & 0.0249 & 0.0934 & 0.3358 \\
\hline 9 & 14.110 & 1421.552 & 14.077 & 1421.083 & 0.0284 & 0.1064 & 0.3846 \\
\hline 10 & 15.823 & 1594.208 & 15.882 & 1603.288 & 0.0320 & 0.1225 & 0.4333 \\
\hline 11 & 17.593 & 1772.515 & 17.589 & 1775.640 & 0.0355 & 0.1345 & 0.4848 \\
\hline 12 & 19.507 & 1965.310 & 19.548 & 1973.320 & 0.0394 & 0.1496 & 0.5381 \\
\hline
\end{tabular}

The calculations of torque are shown in the Table 5. In columns (2) and (4) the weight is summed up that each sum is larger by only one next weight. Columns (3) and (5) shows the sum of the weights multiplied suitable by left $(100.75 \mathrm{~mm})$ and right $(100.95$ $\mathrm{mm}$ ) length of arm of the force, what gives the torque of left and right side. The sum of these partial $\tau_{\varphi}$ gives the total torque (column 6). The measurements of the voltages on channels $1 \& 2$ are in columns (7) and (8) respectively. The voltage from channel 1 and channel 2 as a function of torque (see Tab. 5) are shown in Fig. 36. There is a linear relationship between voltage (measured twist of the $\mathrm{Al}_{2} \mathrm{O}_{3}$ rod) and torque. The parameters $f_{m} 1$ and $f_{m} 2$ which are needed in the following calculations were determined from straight line fits to the data.

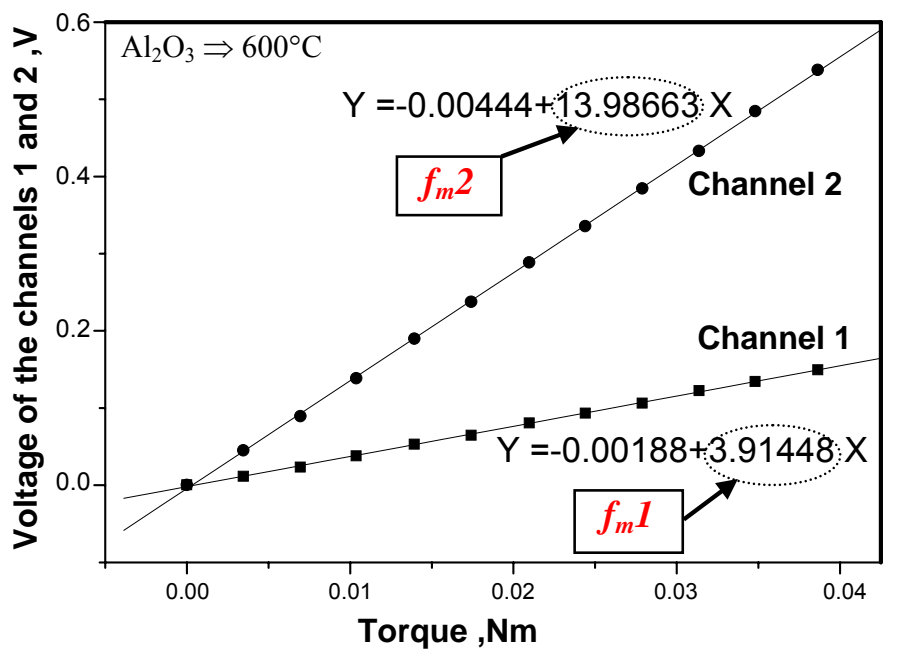

Fig. 36. Plot of the relationship between calculated torque and measured voltage - both parameters come from the weight analysis. $f_{m} 1$ - factor for the channel $1 ; f_{m} 2$ - factor for the channel 2. 


\subsubsection{Stress is applied; strain is measured}

A torque is applied to the rod by an electromagnetic system ("wing S" in Fig. 34; see also Fig. 37). The angle of twist of the rod is measured from the motion of $10 \mathrm{~cm}$ long aluminium arms attached to the rod $66.22 \mathrm{~mm}$ and $\sim 480 \mathrm{~mm}$ above the fixed end. Fe-plates are attached to these $\mathrm{Al}$ arms and move between pairs of inductive transducers (see Fig. 38). The circuits connecting the pairs of inductive transducers delete sway in the rod and only measure twist of the rod. The pick-ups which were used are inductive transducers of $\operatorname{Tr} 10 \mathrm{D}$ type (Fig. 39). They are waterproof front and made of hardened steel. Each pick-up has one coil. The transducers are connected to the half-bridge and work under the alternating current.

Movement of the Fe-plate between the inductive plates reduces the air-gap and results in a change in voltage output of the transducer circuit. The motion of the Fe-plate was also determined manually using a micrometer. For the $2 \mathrm{~mm}$ and $4 \mathrm{~mm}$ air-gaps, respectively at channel 1 and 2 , there is a linear relationship between transducer voltage and angle of twist.

The sensitivity of the inductive transducers depends on the distance between two pick-ups and on the distance between pick-ups and metal plate. When $\Delta \mathrm{L}$ (distance between pick-ups) is too large, then dependence between $\Delta \mathrm{L}$ and an outgoing signal is not linear.

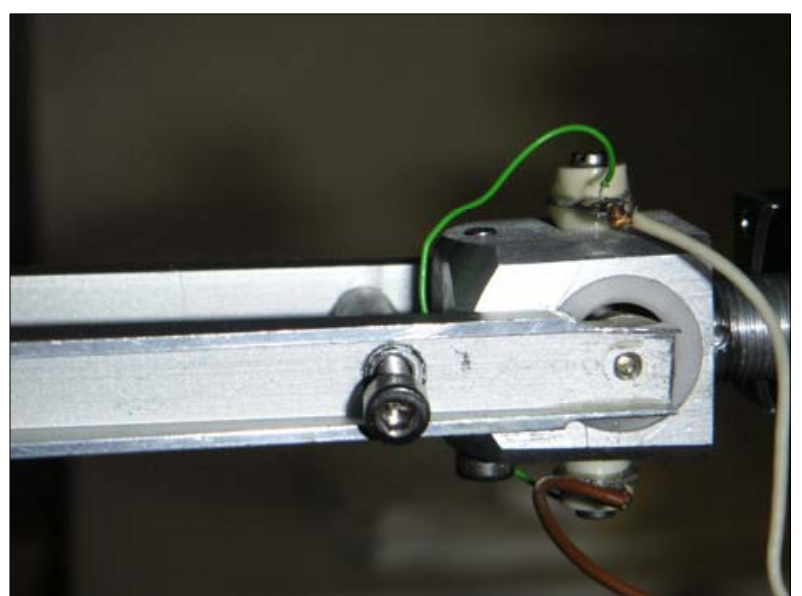

Fig. 37. Electromagnetic system of torsion machine which applied stress to the rod.

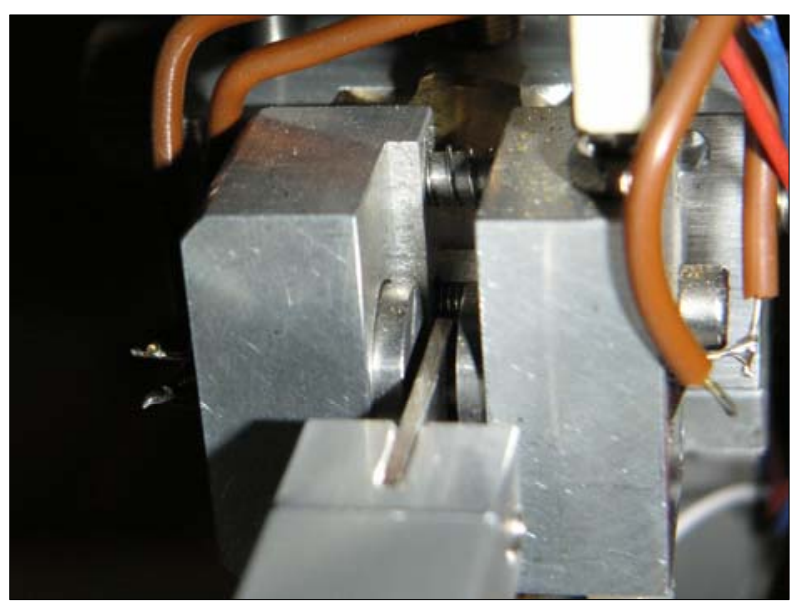

Fig. 38. Pair of inductive transducers with a iron plate between. 


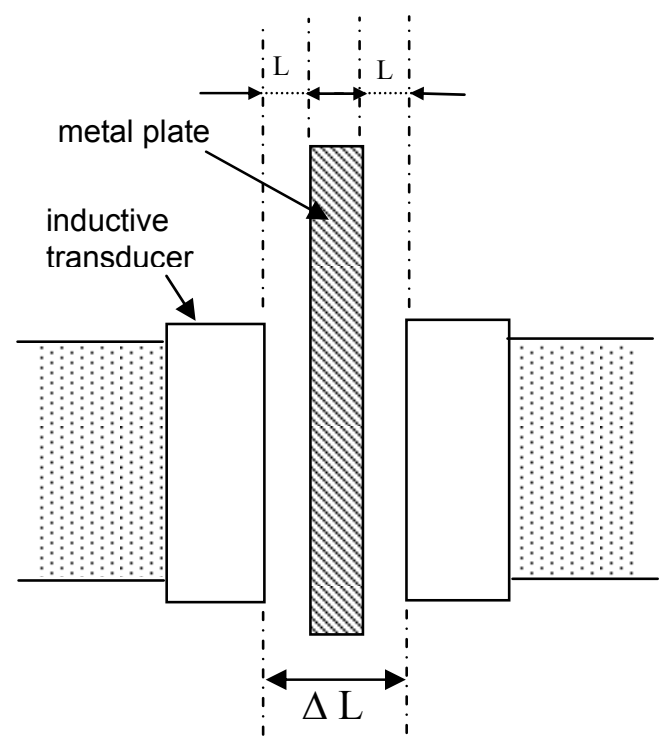

Fig. 39. Scheme of the linear voltage displacement transducer with the metal plate. $\mathrm{L}$ - distance between metal plate and pick-ups changes; $\Delta \mathrm{L}$ - distance between pick-ups: different but constant for the both wings.

\subsubsection{Displacement vs. voltage}

In order to determine the angle of twist $\varphi$ (Eq. 33), the relationship between displacement and voltage at channel 1 and channel 2 needs to be measured. As seen in Fig. 40, there is a linear relationship between displacement and the measured voltage for both channels (1\&2), described by the two factors: $f_{d} 1$ (channel 1 ) and $f_{d} 2$ (channel 2 ). These are used to calculate $B B_{1}^{\prime}$ (for channel 1) and $B B_{2}^{\prime}$ (for channel 2) according to equation:

$$
B B_{i}^{\prime}=\frac{V_{i} \cdot r}{f_{d i} \cdot R}
$$

where: $V_{i}$ is voltage of the channel 1 or 2 (see Fig. 42), $f_{d i}$ - factor of the channel 1 or 2 from the displacement analysis (see Fig. 40), $r$ - radius of the rod and $R$ - length of the alumina wings.

The displacements BB' need to be used to calculate the angles of twist $\varphi$ for both channels following equations (33) and (41). 

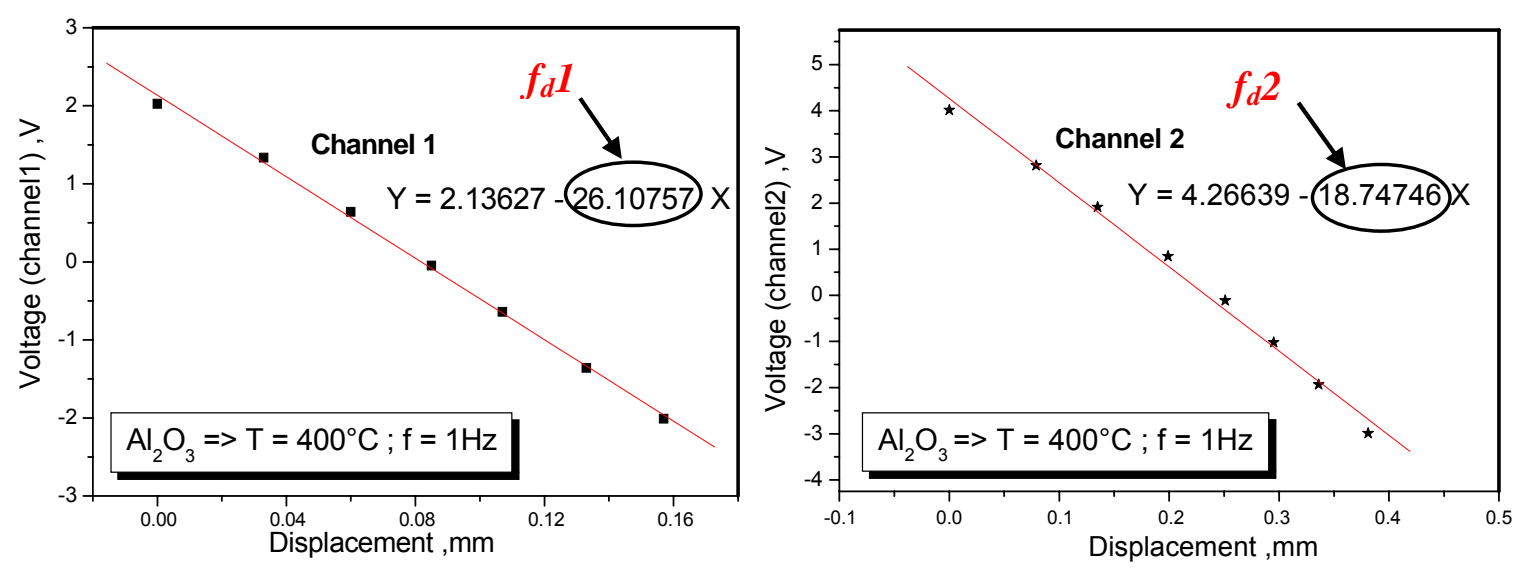

Fig. 40. Plot of the relationship between measured displacement and measured voltage - both parameters come from the displacement analysis. $f_{d} 1$ - factor for the channel $1 ; f_{d} 2$-factor for the channel 2.

\subsubsection{Frequency dependent measurements}

Once the static calibration has been performed the frequency dependence of shear modulus can be determined by applying a sinusoidal torque to the " $\mathrm{S}$ " wing. Magnets glued to the ends of the wing sit within loud speaker coils which are attached to the rigid frame near the ends of this wing. A sinusoidal electrical signal is sent to the electromagnetic coils. This signal attracts and repels the magnets attached to wing $S$ which is attached to the free end of the torsion rod - thus producing a sinusoidal torque. This torque is described by:

$$
y=a+b t+c \sin (\omega t+\delta),
$$

for: $a, b$-drift parameters, $c$ - amplitude of the sinusoid (in $\mathrm{V}$ ), $\delta$ - shift of the sinusoid from starting time (in radians), $t$ - time (in s), $\omega$ - angular frequency $(\omega=2 \pi f)$.

Comparing the voltages from the channels 1 and 2 and the voltage of the spool, there were calculated the factors of the voltage marked as $f_{v} 1$ and $f_{v} 2$ (see Fig. 41) and used in the next calculations. 


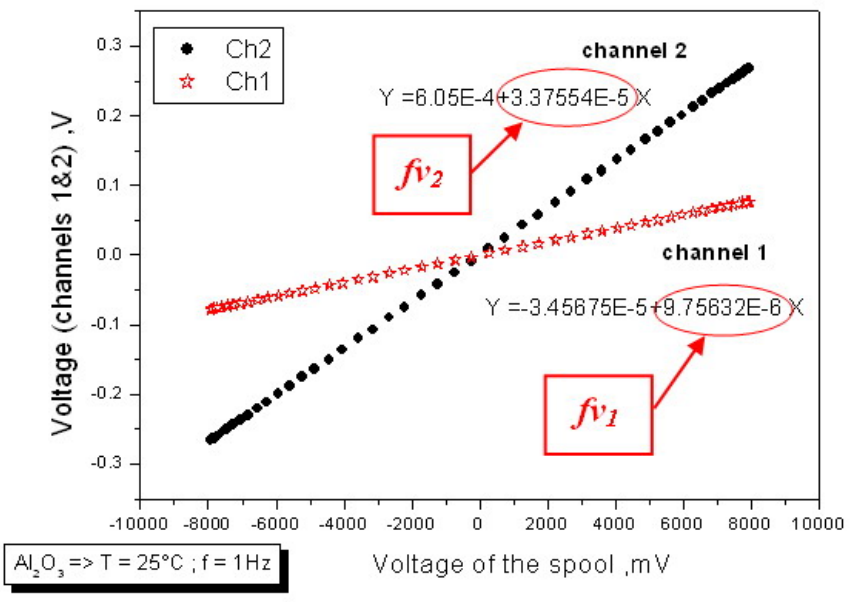

Fig. 41. Plot of the relationship of the voltages from the channels 1 and 2 and voltage of the spool. $f_{v} 1$ - factor for the channel $1 ; f_{v} 2$ - factor for the channel 2.

The final operation of the whole investigation is to calculate the shear modulus $G$ for the alumina rod using all parameters defined above what means that $G$ is equal:

$$
G=\frac{f_{v} \cdot V_{s} \cdot l}{f_{m} \cdot \varphi \cdot J},
$$

where $G$ is in $\mathrm{GPa}, l$ is the length of the rod from the place of fixing in the base-plate of torsion machine to the channels 1 or 2 , remaining symbols were explained in text.

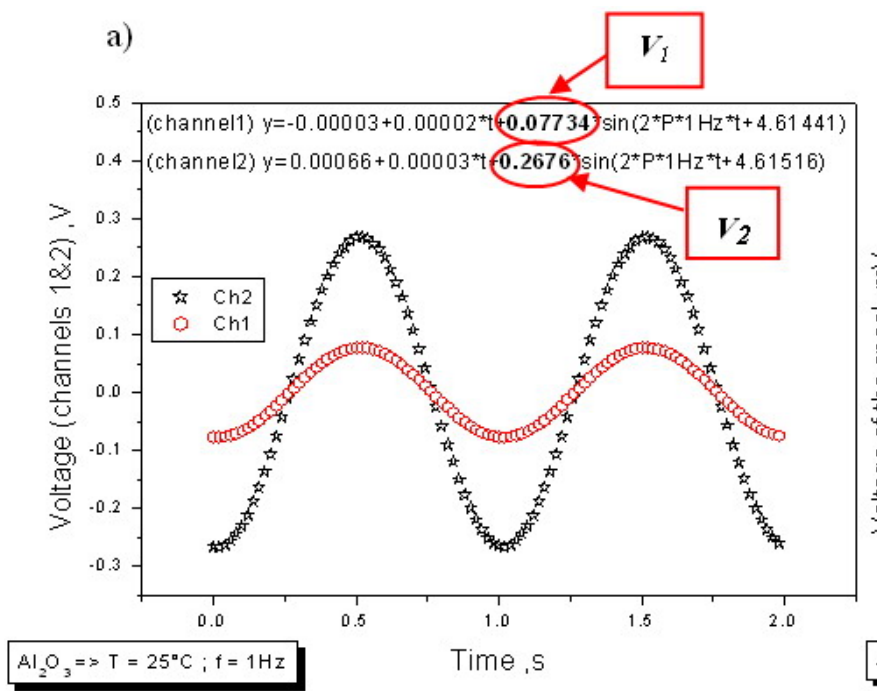

b)

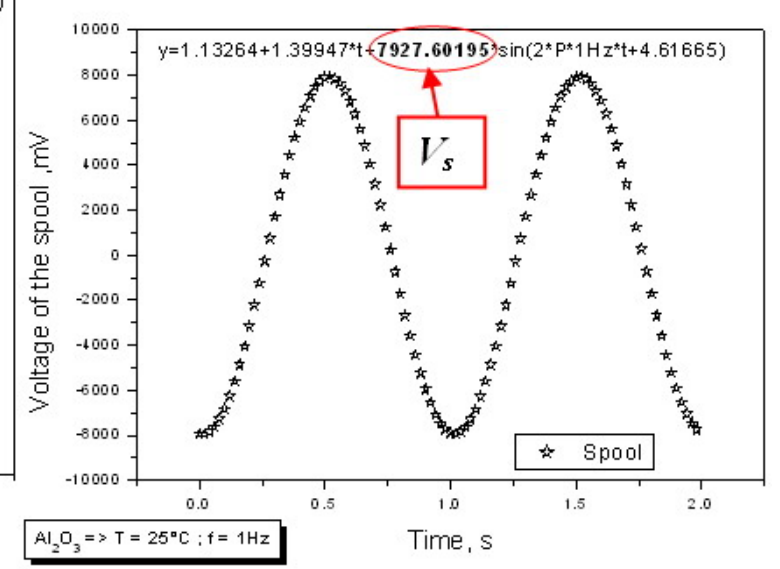

Fig. 42. Plots of the relationship: voltage vs. time. a) Sinusoidal signal measured from the channels 1 and 2, where V1 and V2 correspond with the voltage of these channels; b) Sinusoidal signal of the spool, where $V_{s}$ corresponds with the voltage of the spool. 


\subsubsection{Temperature calibration}

The angle of twist together and the applied torque are used to calculate shear modulus, as a function of temperature (see in Fig. 43).

The alumina wing of channel 1 (lower) measures only the deformation of rod at room temperature $\left(22-25^{\circ} \mathrm{C}\right)$, because the rod from the base-plate to the channel $1(66.22$ $\mathrm{mm}$ ) is not heated. The alumina wing of channel 2 (upper) measures not only the angular deformation of rod in room temperature, but also deformation this part of rod which is exposed to the high temperature inside the furnace. The deformation measurements at channel 1 are that of an $\mathrm{Al}_{2} \mathrm{O}_{3}$ rod at room temperature. There is small influence of the high temperature of furnace and therefore the shear modulus appears to be temperature dependent (Fig. 43). The alumina wing of channel 2 measures the deformation of the rod which sits in a temperature gradient from the temperature of interest in the centre of the furnace to room temperature.

\subsubsection{Shear modulus of the torsion rod}

At $1000^{\circ} \mathrm{C}$ the time delay $\delta$ is the same for both channels; showing that the alumina rod displays elastic behaviour over the entire the temperature range. The effective shear modulus of the alumina rod can then be calculated as a function of frequency from:

$$
G=\frac{\text { stress calculated from angle of twist at Channel } 1}{\text { strain calculated from angle of twist at Channel } 2} .
$$

The forced oscillation technique was tested by measuring the shear modulus of different materials:

- borosilicate glass rod with the composition given in the Table 6 and with $\rho=2.224 \pm 0.003 \mathrm{~g} \mathrm{~cm}^{-3}$. The shear modulus of the borosilicate glass at room temperature was found to be $26.04 \pm 1.64 \mathrm{GPa}$; with an ultrasonic value of $26.37 \pm 0.25 \mathrm{GPa}$;

- nickel with the result $77.54 \pm 0.05 \mathrm{GPa}$ (and literature data is $76 \mathrm{GPa}$ );

- shear modulus of brass was measured as $40.05 \pm 0.17 \mathrm{GPa}$ (where literature data is $40 \mathrm{GPa})$;

- and also the frequency-dependent deformation of a $0.33 \mathrm{Na}_{2} \mathrm{O}-0.67 \mathrm{SiO}_{2}$ melt (sample G0 - NS2). 


\begin{tabular}{|c|c|c|}
\hline Borosilicate Glass & $\mathbf{w t} \%$ & $\mathbf{m o l} \%$ \\
\hline $\mathrm{SiO}_{\mathbf{2}}$ & 81.060 & 83.650 \\
$\mathbf{A l}_{\mathbf{2}} \mathbf{O}_{\mathbf{3}}$ & 2.010 & 1.222 \\
$\mathrm{Fe}_{\mathbf{2}} \mathbf{O}_{\mathbf{3}}$ & 0.051 & 0.020 \\
$\mathbf{B}_{2} \mathbf{O}_{\mathbf{3}}$ & 12.730 & 11.338 \\
$\mathbf{C a O}$ & 0.016 & 0.018 \\
$\mathrm{Na}_{\mathbf{2}} \mathbf{O}$ & 3.010 & 3.011 \\
$\mathbf{K}_{\mathbf{2}} \mathbf{O}$ & 1.125 & 0.741 \\
\hline
\end{tabular}

Tab. 6. Composition (in wt $\%$ and $\mathrm{mol} \%$ ) of the borosilicate glass used to test of the torsion machine determined by microprobe (JEOL JXA $8900 \mathrm{RL}$ ) .

The shear modulus of the $\mathrm{Al}_{2} \mathrm{O}_{3}$ rod determined at room temperature from channel 1 is $162.07 \pm 0.74 \mathrm{GPa}$ and $162.04 \pm 0.08 \mathrm{GPa}$ from channel 2 . Straight line fits to the temperature dependent shear modulus data give apparent $d G / d T$ values of $-5.87(24) \mathrm{MPa} \mathrm{K}^{-1}$ and $-11.84(21) \mathrm{MPa} \mathrm{K}^{-1}$ for channels 1 and 2 respectively.

The room temperature shear modulus measured here is in good agreement with the literature data for the shear modulus of polycrystalline $\mathrm{Al}_{2} \mathrm{O}_{3}$ calculated from single crystal data which is 162.5-163.2 GPa (Ahrens, 1995; see also Ohno et al., 1976). Goto et al. (1989) measured the shear modulus of single crystal $\mathrm{Al}_{2} \mathrm{O}_{3}$ from room temperature to $1000^{\circ} \mathrm{C}$ and found $\mathrm{dG} / \mathrm{dT}=-24 \mathrm{MPa} \mathrm{K}{ }^{-1}$. The channel 1 shear modulus changes with furnace temperature despite it being outside the furnace. This is because the lower part of the rod heats up from $38^{\circ} \mathrm{C}$ to $51^{\circ} \mathrm{C}$, with furnace temperature changing from $400^{\circ} \mathrm{C}$ to $1000^{\circ} \mathrm{C}$. The shear modulus obtained at channel 2 is for a rod that is outside the furnace at the top and bottom, and sits within a temperature gradient within the furnace.

As most of the torsion measurements were conducted in the temperature range $450-800^{\circ} \mathrm{C}$, we have chosen to use a temperature independent value of $\mathrm{G}_{\mathrm{Al} 2 \mathrm{O} 3}=160 \mathrm{GPa}$ in our measurements. As the melts have a much lower shear modulus $\mathrm{G}_{\text {melt }} \sim 30 \mathrm{GPa}$, than the alumina rods, this assumption introduces an error $\pm 1 \mathrm{GPa}$ in the calculated melt shear modulus. 


\begin{tabular}{|c|c|c|}
\hline & $\begin{array}{c}\text { Channel } 1 \\
\mathrm{G} \mathrm{Al}_{2} \mathrm{O}_{3} \\
(\mathrm{GPa})\end{array}$ & $\begin{array}{c}\text { Channel } 2 \\
\mathrm{G} \mathrm{Al}_{2} \mathrm{O}_{3} \\
(\mathrm{GPa})\end{array}$ \\
\hline $25^{\circ} \mathrm{C}$ & 161.98 & 162.04 \\
\hline $100^{\circ} \mathrm{C}$ & 161.58 & 159.83 \\
\hline $200^{\circ} \mathrm{C}$ & 161.78 & 157.79 \\
\hline $300^{\circ} \mathrm{C}$ & 160.97 & 155.81 \\
\hline $400^{\circ} \mathrm{C}$ & 160.23 & 154.13 \\
\hline $500^{\circ} \mathrm{C}$ & 160.26 & 152.00 \\
\hline $600^{\circ} \mathrm{C}$ & 160.20 & 150.34 \\
\hline $700^{\circ} \mathrm{C}$ & 159.86 & 149.33 \\
\hline $800^{\circ} \mathrm{C}$ & 159.86 & 148.15 \\
\hline $900^{\circ} \mathrm{C}$ & 159.57 & 147.31 \\
\hline $1000^{\circ} \mathrm{C}$ & 159.38 & 145.83 \\
\hline
\end{tabular}

Tab. 7. Table of measured shear modulus of the alumina rod from room temperature to $1000^{\circ} \mathrm{C}$.

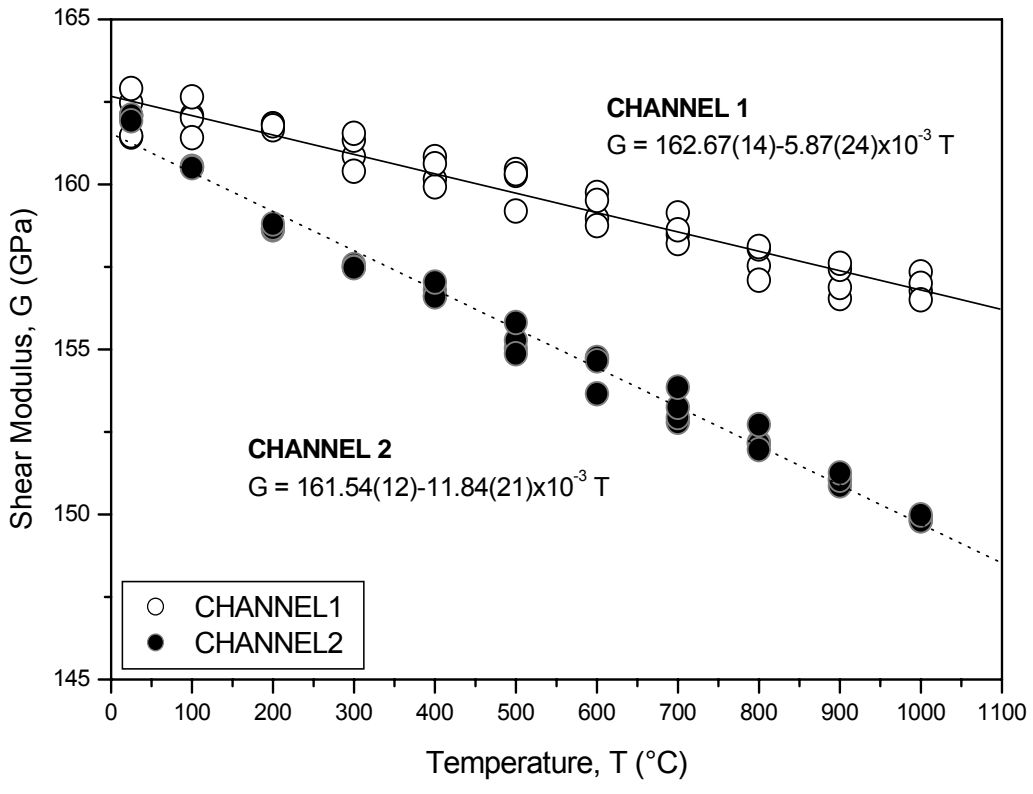

Figure 43. Measured shear modulus of the alumina rod from room temperature to $1000^{\circ} \mathrm{C}$. The $\mathrm{G}_{\mathrm{Al} 2 \mathrm{O} 3}$ used in calculation of shear modulus of the melt was set to be $160 \mathrm{GPa}$.

\subsubsection{Shear modulus of melts}

The shear modulus of the silicate melts was determined with a set: $\sim 30 \mathrm{~mm}$ long, $8 \mathrm{~mm}$ diameter glass cylinder glued to an upper and lower $\mathrm{Al}_{2} \mathrm{O}_{3}$ torsion rod. The lower rod is $\sim 295 \mathrm{~mm}$ long and the upper rod is $\sim 155 \pm 20 \mathrm{~mm}$ long (depending on the length of the sample). Preliminary experiments found it difficult to achieve a good physical connection between the melt cylinder and the alumina torsion rod. First attempts were done with a platinum tube (the method of Bagdassarov et al., 1993). This uses a Pt-foil wrapped around the melt cylinder and alumina rods to hold everything together at high temperature. The result was only bubble filled interfaces between the rods and the melt sample (see Fig. 44). Thus we reverted to the simpler method (Webb, 1992a) of wrapping the glass cylinder and alumina rods in paper to hold the assembly together at high temperature.

It was also necessary to use a glue to bond the rods to the melt. This glue is a Si-rich Fe-bearing melt (composition of obsidian from Little Glass Butte, Oregon), which has a viscosity slightly higher than the most peraluminous melts used. After initial attempts in which the alumina rod melted into the rhyolite melt; a recipe for temperature and duration of contact between rhyolite melt and alumina rod was found; and we now successfully $(85 \%)$ glue the rods to the glass sample in the first attempt. 


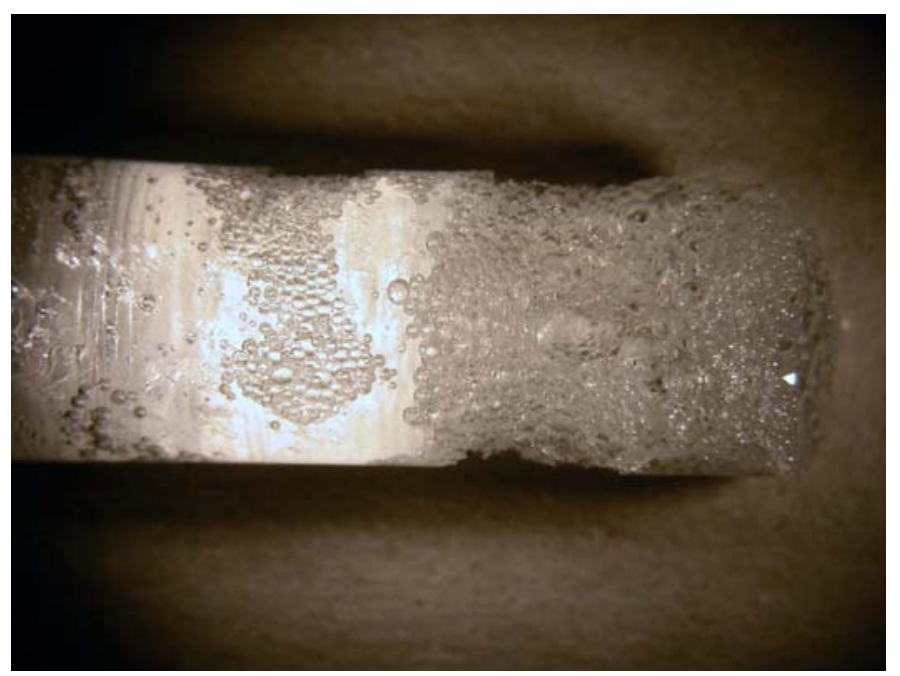

Fig. 44. Bubble filled sample produced by wrapping both glass and $\mathrm{Al}_{2} \mathrm{O}_{3}$ torsion rod in Pt-foil and going to high temperature.

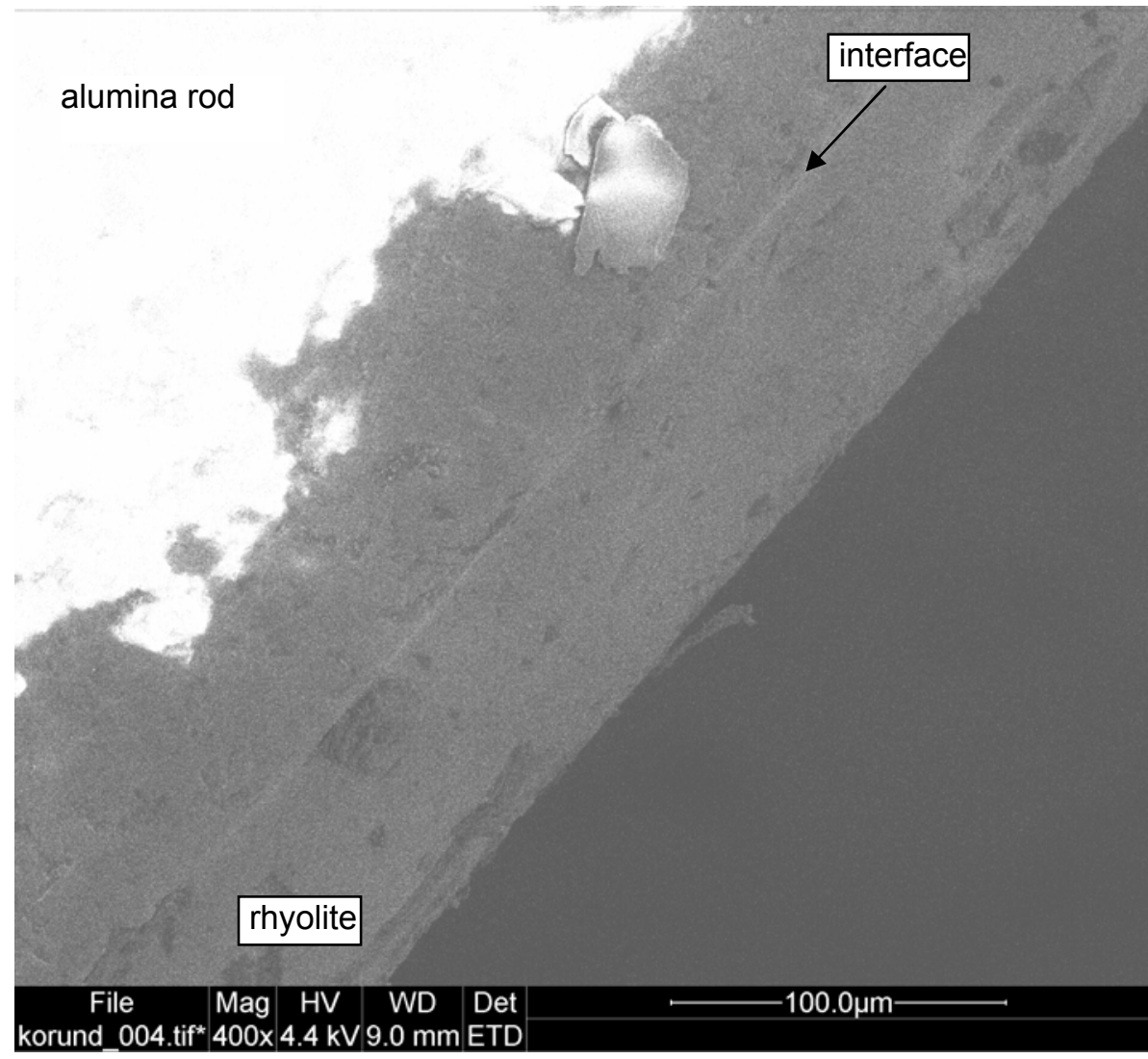

Fig. 45. Rhyolite infiltration $\sim 50 \mu \mathrm{m}$ into the alumina torsion rod measured with Quanta 200F FESEM (Crystallography Department, Georg-August-University Göttingen).

A thin film of this rhyolite melt is allowed to react with the $\mathrm{Al}_{2} \mathrm{O}_{3}$ rod (Fig. 45). The sample is then melted onto the rhyolite at the ends of the two torsion rods to produce a good contact between the $\mathrm{Na}_{2} \mathrm{O}-\mathrm{Al}_{2} \mathrm{O}_{3}-\mathrm{SiO}_{2}$ melt and the torque rod (Webb, 1991). The 
presence of this thin film of highly viscous melt will introduce an error of $\sim 1 \mathrm{GPa}$ into the calculated shear modulus of the melt. Thus the measured shear moduli have an error of $\pm 1.5 \mathrm{GPa}$ due to the use of a temperature independent shear modulus for the torsion rods, and the use of a glue between the sample and the torsion rods.

The temperature inside the furnace has been measured with a type $S$ thermocouple, and it was found to be constant $\pm 3^{\circ} \mathrm{C}$ across the $40 \mathrm{~mm}$ where the melt sample stands (Fig. 46). Therefore, only $10 \mathrm{~mm}$ of the alumina rod is at temperature of the measurement, $\sim 66 \mathrm{~mm}$ are at $\sim$ room temperature (lower part) and the remaining length is in a thermal gradient from room temperature to the temperature of interest. The upper part of the rod is partially at high temperature (up to the upper channel) and then is in a thermal gradient to the room temperature.

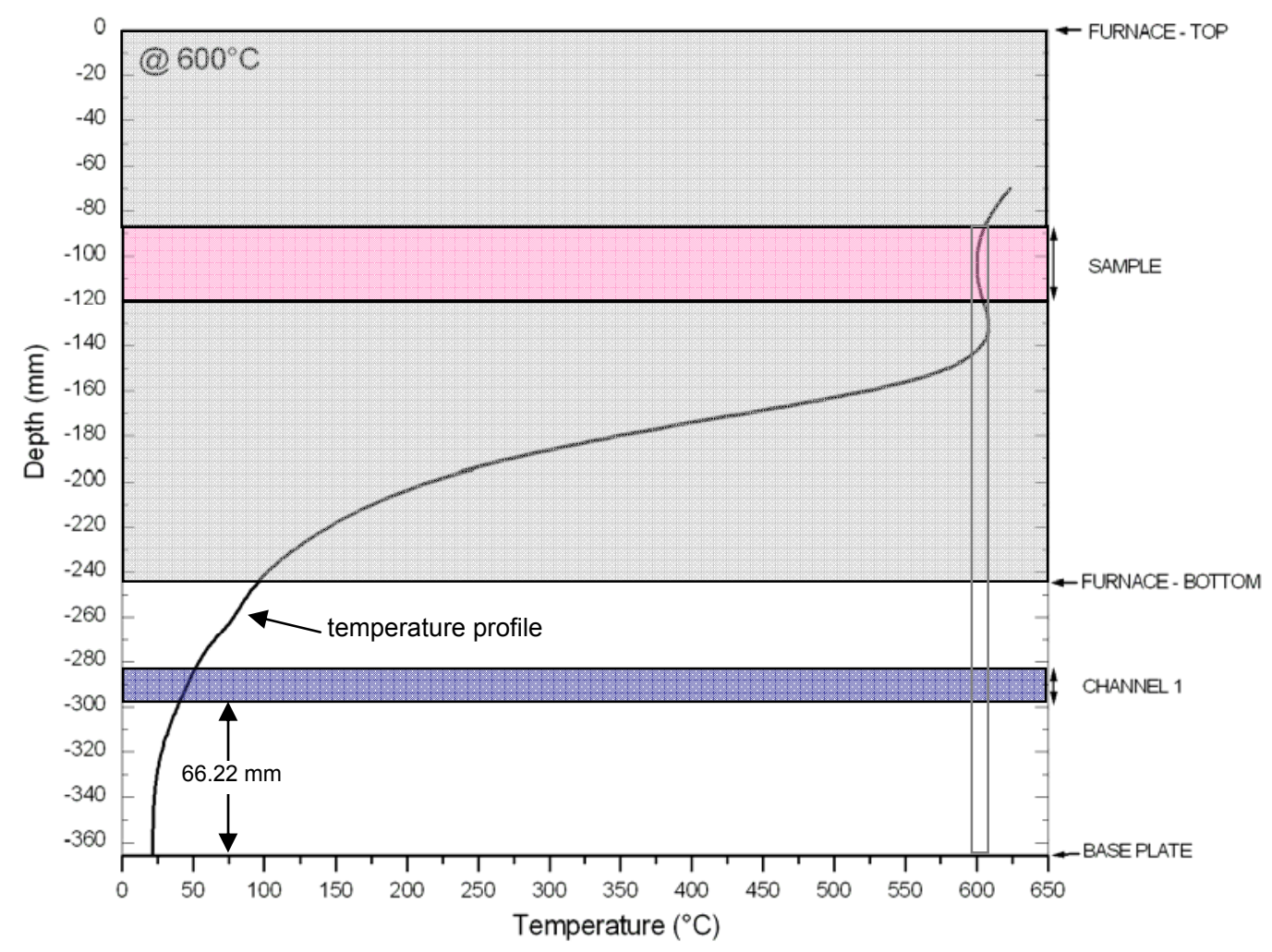

Fig. 46. Temperature profile in the torsion machine. Location of the furnace, sample and the channel 1 and base plate has been marked.

The deformation analysis for the compound torsion rod $\left(\mathrm{Al}_{2} \mathrm{O}_{3}+\right.$ melt $\left.+\mathrm{Al}_{2} \mathrm{O}_{3}\right)$ as a function of frequency is made in the same manner as for the simple alumina rod but the calculation is slightly different. For the measurement of the frequency dependent deformation of the melt, the torque is created by the application of a sinusoidal signal to a pair of loudspeaker coils. The angle of twist at channel 2, together with the time lag between applied stress $\sigma$ and resulting strain $\varepsilon$ are due to the elastic deformation of the 
two alumina rods plus the viscoelastic deformation of the melt. Voltage signals coming from the wing " $S$ " and both channels were also calculated the same way as in previous case. As a result one obtains a sinusoidal angle of twist for channels as a function of time for given frequency (see Fig. 47).

Based upon the earlier calibrations, the voltage from the transducers at channel 1 gives the applied stress, and the voltage at channel 2 gives the deformation of the compound torsion rod. The time delay $-\Delta \delta$ between the applied stress and the resulting strain is also calculated;

$$
\Delta \delta=\left|\delta_{1}-\delta_{2}\right|
$$

With known voltages of the channels $\mathrm{V} 1$ and $\mathrm{V} 2$ as well as time delay $\Delta \delta$ it is possible to calculate the angle of twist $\varphi$ for both channels:

$$
\varphi_{1}=\frac{V_{1}}{f_{d 1}(R+r)} \quad \text { and } \quad \varphi_{2}=\frac{V_{2}}{f_{d 2}(R+r)}
$$

where $R$ is the length of the wings (in $\mathrm{mm}$ ) and $r$ - radius of the rod (in $\mathrm{mm}$ ).

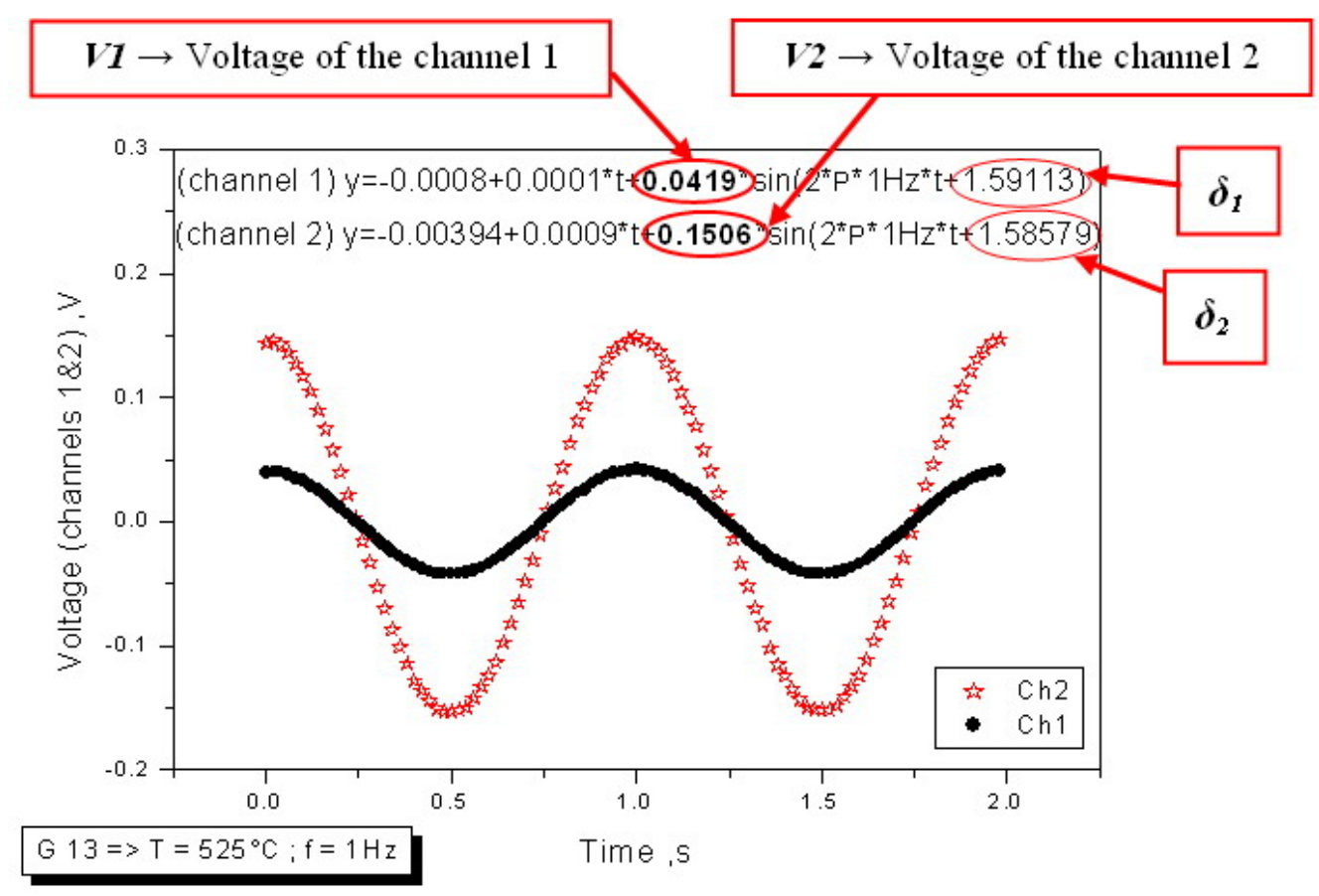

Fig. 47. Plot of the relationship voltage vs. time showed as a sinusoidal signal (measured from the channels 1 and 2), where $\mathrm{V} 1$ and $\mathrm{V} 2$ correspond with the voltage of these channels; $\delta_{1}$ and $\delta_{2}$ describe the shift of the sinusoids. 
Here it was unnecessary to do a weight analysis because the torque $\tau$ is calculated using the angle of twist of the channel $1 \varphi_{1}$ :

$$
\tau_{\text {from } \varphi 1}=\frac{\varphi_{1} G_{A l 2 O 3} \pi r^{4}}{2 l_{\text {rod } 1}}
$$

where $G_{\mathrm{Al} 2 \mathrm{O} 3}$ is the shear modulus of the alumina rod (160 GPa), $r$ - radius of the rod (in $\mathrm{mm}$ ) and $l_{\text {rod } 1}$ - length of the rod from the base-plate to the first channel (in $\mathrm{mm}$ ).

The angle of twist $\varphi_{2}$ of the compound rod must be separated into the components due to the twist of the lower alumina rod $-\varphi_{\text {rod } 1}$, the melt $-\varphi_{\text {melt }}$ and the upper alumina rod $-\varphi_{\text {rod } 2}$. This is shown graphically in Figure 48. Here, the measured parameters are $\delta$ - the delay between the applied stress and the resulting strain of the compound rod; and $\varphi_{2}$ - the deformation measured at channel 2. This must now be separated into the contribution from the elastic twist of rod 1 and rod 2:

$$
\varphi_{\text {rod } 1}=\frac{\tau 2 l_{r o d 1}}{G_{A l 2 O 3} \pi r^{4}} \quad \text { and } \quad \varphi_{\text {rod } 2}=\frac{\tau 2 l_{\text {rod } 2(c h 2)}}{G_{A l 2 O 3} \pi r^{4}}
$$

for $l_{\text {rod 2 (ch2) }}$ - length of the upper $\mathrm{Al}_{2} \mathrm{O}_{3}$ rod from the melt to the channel 2 (in $\mathrm{mm}$ ), where $\tau$ is calculated from Eq. 47. The complex shear modulus of the melt is:

$$
G^{*}(\omega)_{m e l t}=\frac{\tau 2 l_{\text {melt }}}{\pi r^{4} \varphi_{\text {melt }}}
$$

for $l_{\text {melt }}$ - the length of the melt (in $\mathrm{mm}$ ), where $\varphi_{\text {melt }}$ is:

$$
\varphi_{\text {melt }}=\sqrt{\left[\left(\varphi_{2} \sin \delta\right)^{2}+\left(\varphi_{2} \cos \delta-\varphi_{\text {rod } 1}\right)^{2}\right]}-\varphi_{\text {rod } 2}
$$


The complex shear modulus can be separated into the real and imaginary components by:

$$
\begin{gathered}
G(\omega)_{\text {im }}=G(\omega)_{\text {melt }} \sin \gamma=G^{\prime \prime}(\omega) \\
G(\omega)_{\text {real }}=G(\omega)_{\text {melt }} \cos \gamma=G^{\prime}(\omega),
\end{gathered}
$$

where

$$
\gamma=\tan \gamma=\frac{\varphi_{2} \sin \delta}{\varphi_{2} \cos \delta-\varphi_{\text {rod } 1}} .
$$

Finally, the shear modulus of the melt can be described by:

$$
G(\omega)_{\text {melt }}=\sqrt{G^{\prime \prime}(\omega)^{2}+G^{\prime}(\omega)^{2}} .
$$

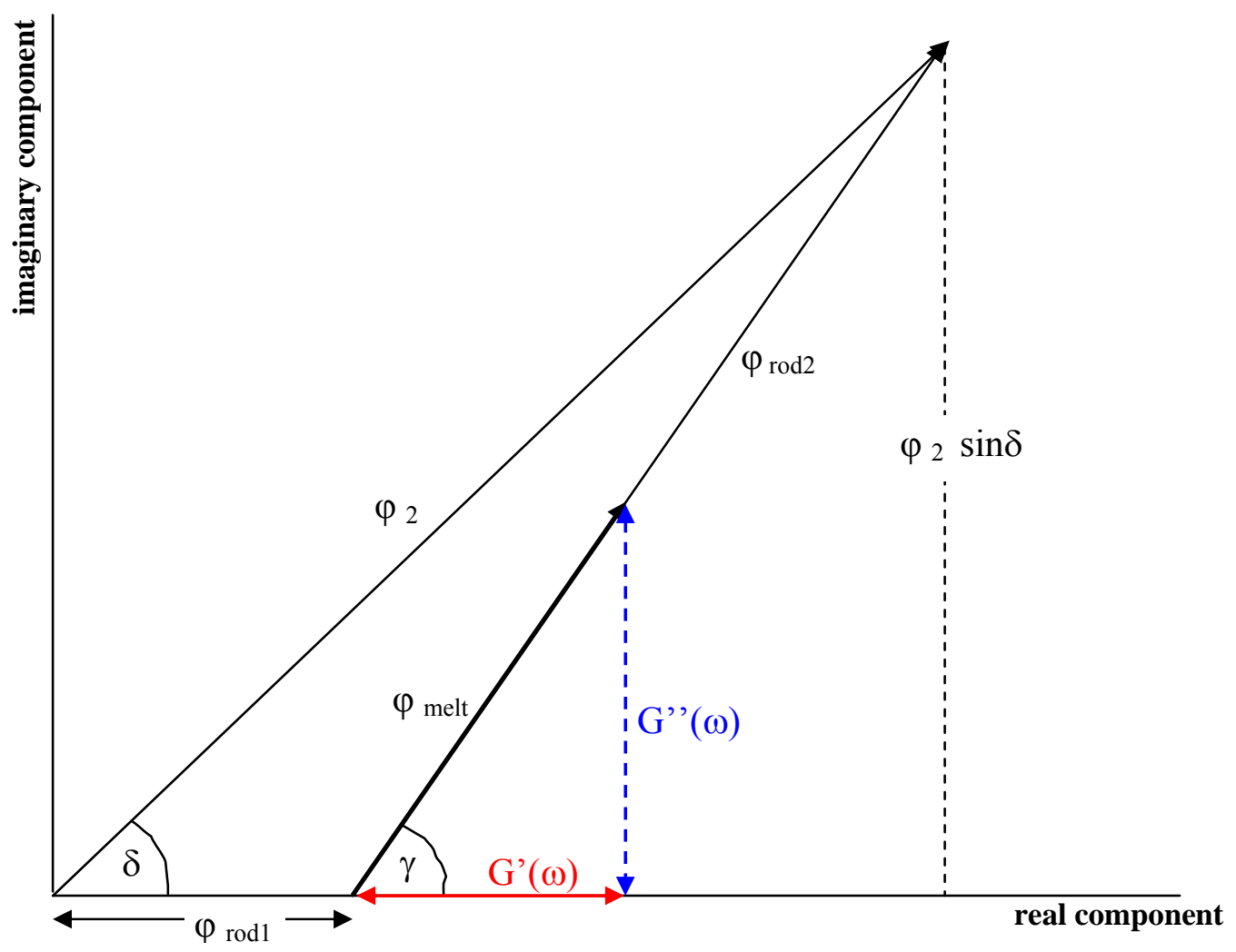

Fig. 48. Plot of trigonometrical relationships for the deformation of the compound torsion rod. 


\subsubsection{Shear modulus as a function of frequency}

This viscoelastic deformation is presented as the real and imaginary components of the shear modulus as a function of frequency. A maximum in imaginary part of the shear modulus (energy loss) appears at the frequency at which part of the melt structure moves. This maximum is accompanied by an increase in the real (storage) part of the modulus. One expects 3 peaks to occur in sodium aluminosilicate melts (Fig. 49). Based on a simplistic model of the rates of motion of the different atoms in the melt there should be the slowest motion of $\mathrm{Si}-\mathrm{O}$ (with the longest bond lifetime); the fast motion of $\mathrm{Na}^{+}$ atoms (based upon diffusion data $\mathrm{Na}-\mathrm{O}$ bonds are assumed to have the shortest lifetime) and at frequencies between these two there should be a peak for the lifetime of Al-O bonds.

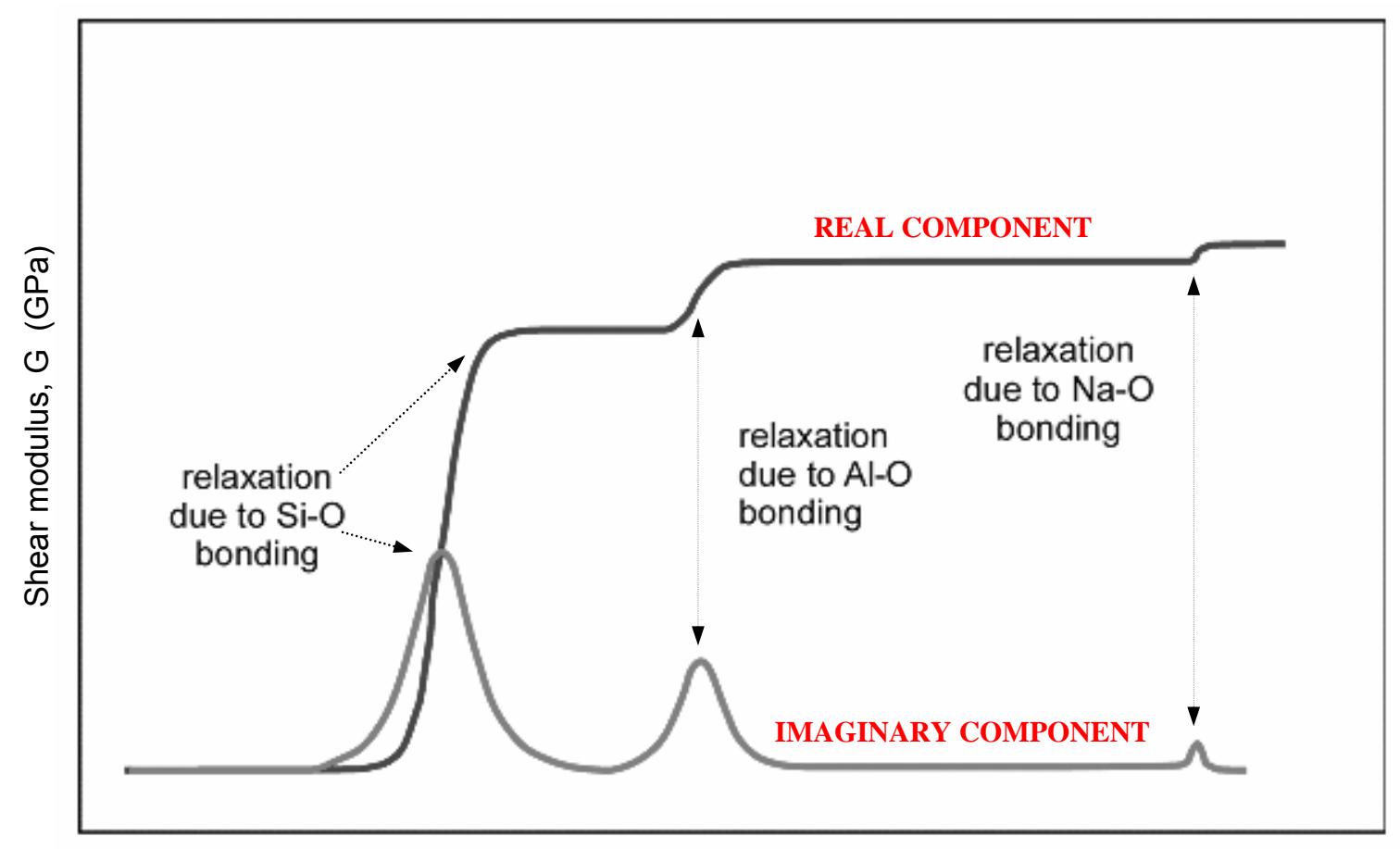

$\log 10 \omega \tau_{\mathrm{M}}$

Fig. 49. The known change in modulus with $\log _{10} \omega \tau_{\mathrm{M}}$ for the motion of $\mathrm{Si}$ and $\mathrm{O}$ atoms in silicate melts together with theoretically expected loss modulus associated with the motion of $\mathrm{Al}^{3+}$ and $\mathrm{Na}^{+}$ atoms in the present melts. 


\section{RESULTS}

\subsection{Composition}

The compositions of investigated samples were determined by microprobe and are presented in the Table 8. $\mathrm{Fe}^{2+}$ was measured with the $\mathrm{KMnO}_{4}$ titration method (Heinrichs \& Herrmann, 1998; Herrmann, 1975). $\mathrm{Fe}_{2} \mathrm{O}_{3 \text { total }}$ was determined by spectral photometry and did not differ from microprobe data.

Tab. 8. Measured glass compositions in wt\%. The compositions were determined by microprobe (JEOL JXA $8900 \mathrm{RL}$ ): $15 \mathrm{kV}$ voltage, $10 \mu \mathrm{m}$ beam diameter, $12 \mathrm{nA}$ current. Data are the average of 10 analyses of each glass. Errors are $1 \sigma$ values. Anorthite, albite and hematite were used as standards.

\begin{tabular}{|c|c|c|c|c|}
\hline \multirow{2}{*}{$\begin{array}{c}\text { melt } \\
\text { number }\end{array}$} & \multicolumn{4}{|c|}{$\mathbf{w t} \%$} \\
\cline { 2 - 5 } & $\mathbf{S i O}_{\mathbf{2}}$ & $\mathbf{N a}_{\mathbf{2}} \mathbf{O}$ & $\mathbf{A l}_{\mathbf{2}} \mathbf{O}_{3}$ & $\mathbf{F e}_{\mathbf{2}} \mathbf{O}_{3}$ total \\
\hline \hline $\mathrm{G} 0$ & $65.9 \pm 0.3$ & $34.1 \pm 0.2$ & - & - \\
\hline $\mathrm{G} 1$ & $58.1 \pm 0.2$ & $12.9 \pm 0.2$ & $29.8 \pm 0.2$ & - \\
G2 & $61.2 \pm 0.2$ & $12.8 \pm 0.2$ & $26.0 \pm 0.2$ & - \\
G3 & $59.4 \pm 0.2$ & $14.2 \pm 0.1$ & $26.6 \pm 0.2$ & - \\
G4 & $58.2 \pm 0.3$ & $14.9 \pm 0.2$ & $26.5 \pm 0.1$ & - \\
G5 & $60.1 \pm 0.2$ & $15.5 \pm 0.4$ & $24.1 \pm 0.2$ & - \\
G6 & $59.1 \pm 0.5$ & $16.6 \pm 0.3$ & $24.1 \pm 0.2$ & - \\
G7 & $59.4 \pm 0.5$ & $18.5 \pm 0.1$ & $21.8 \pm 0.2$ & - \\
\hline \hline G8 & $56.3 \pm 0.3$ & $12.2 \pm 0.1$ & $28.5 \pm 0.1$ & $2.54 \pm 0.05$ \\
G9 & $56.9 \pm 0.8$ & $12.9 \pm 0.2$ & $23.5 \pm 0.5$ & $7.12 \pm 0.24$ \\
G10 & $59.1 \pm 0.5$ & $13.0 \pm 0.2$ & $20.8 \pm 0.3$ & $6.86 \pm 0.16$ \\
G11 & $58.7 \pm 1.5$ & $14.0 \pm 0.4$ & $20.0 \pm 1.0$ & $7.08 \pm 0.32$ \\
G12 & $59.2 \pm 1.2$ & $15.1 \pm 0.2$ & $18.2 \pm 0.8$ & $7.49 \pm 0.33$ \\
G13 & $59.1 \pm 0.5$ & $16.0 \pm 0.2$ & $17.7 \pm 0.3$ & $7.20 \pm 0.12$ \\
G14 & $60.5 \pm 0.3$ & $17.5 \pm 0.1$ & $14.7 \pm 0.1$ & $6.98 \pm 0.10$ \\
\hline
\end{tabular}

Tab. 9. Calculated glass compositions in mol\%. The compositions of $\mathrm{SiO}_{2}, \mathrm{Na}_{2} \mathrm{O}$ and $\mathrm{Al}_{2} \mathrm{O}_{3}$ were determined by microprobe (Tab. 8); $\mathrm{FeO}$ was measured with a titration method, $\mathrm{Fe}_{2} \mathrm{O}_{3}$ was measured by microprobe and confirmed by spectral photometry (data are the average of 10 analyses of each glass). Errors are $1 \sigma$ values. Anorthite, albite and hematite were used as standards. NBO/T value was determined follow the Eq. 7; and $\gamma$ from the Eqs. 9\&10. Eatoms $/ \mathrm{mol}$ presents the number of atoms (times Avogadro's Number) in one mol of melt. $\mathrm{Fe}^{2+} / \mathrm{Fe}_{\text {total }}$ is an atomic ratio between $\mathrm{Fe}^{2+}$ and the total amount of iron in the sample.

\begin{tabular}{|c|c|c|c|c|c|c|c|c|c|}
\hline \multirow{2}{*}{$\begin{array}{c}\text { melt } \\
\text { number }\end{array}$} & \multicolumn{5}{|c|}{$\mathrm{mol} \%$} & \multirow{2}{*}{ NBO/T } & \multirow[b]{2}{*}{$\gamma$} & \multirow{2}{*}{$\frac{\sum \text { atoms }}{\mathrm{mol}}$} & \multirow{2}{*}{$\frac{\mathrm{Fe}^{2+}}{\mathrm{Fe}_{\text {total }}}$} \\
\hline & $\mathrm{SiO}_{2}$ & $\mathrm{Na}_{2} \mathrm{O}$ & $\mathrm{Al}_{2} \mathrm{O}_{3}$ & $\mathrm{FeO}$ & $\mathrm{Fe}_{2} \mathrm{O}_{3}$ & & & & \\
\hline G0 & $66.6 \pm 0.3$ & $33.4 \pm 0.3$ & - & - & - & 0.502 & 1.00 & 3.000 & - \\
\hline$\overline{\mathrm{G} 1}$ & $65.9 \pm 0.2$ & $14.2 \pm 0.1$ & $\overline{19.9 \pm 0.1}$ & - & - & -0.108 & 0.42 & 3.398 & - \\
\hline G2 & $68.8 \pm 0.2$ & $14.0 \pm 0.2$ & $17.2 \pm 0.1$ & - & - & -0.062 & 0.45 & 3.344 & - \\
\hline G3 & $66.9 \pm 0.2$ & $15.5 \pm 0.1$ & $17.7 \pm 0.1$ & - & - & -0.043 & 0.47 & 3.357 & - \\
\hline G4 & $65.9 \pm 0.2$ & $16.4 \pm 0.2$ & $17.7 \pm 0.1$ & _ & _ & -0.026 & 0.48 & 3.354 & _ \\
\hline G5 & $67.3 \pm 0.3$ & $16.8 \pm 0.4$ & $15.9 \pm 0.2$ & - & _ & 0.018 & 0.51 & 3.318 & _ \\
\hline G6 & $66.1 \pm 0.3$ & $18.0 \pm 0.3$ & $15.9 \pm 0.2$ & - & - & 0.043 & 0.53 & 3.318 & - \\
\hline G7 & $65.8 \pm 0.3$ & $20.0 \pm 0.2$ & $14.2 \pm 0.1$ & - & - & 0.123 & 0.58 & 3.284 & - \\
\hline$\overline{\mathrm{G} 8}$ & $65.5 \pm 0.1$ & $13.8 \pm 0.1$ & $19.6 \pm 0.1$ & $0.188 \pm 0.015$ & $0.96 \pm 0.02$ & -0.105 & 0.41 & 3.411 & 0.098 \\
\hline G9 & $66.0 \pm 0.8$ & $14.6 \pm 0.3$ & $16.2 \pm 0.4$ & $0.845 \pm 0.011$ & $2.99 \pm 0.12$ & -0.014 & 0.46 & 3.394 & 0.141 \\
\hline G10 & $68.3 \pm 0.5$ & $14.6 \pm 0.2$ & $14.1 \pm 0.2$ & $0.458 \pm 0.025$ & $2.97 \pm 0.08$ & -0.039 & 0.47 & 3.350 & 0.077 \\
\hline G11 & $67.7 \pm 1.3$ & $15.7 \pm 0.5$ & $13.6 \pm 0.8$ & $0.256 \pm 0.016$ & $3.00 \pm 0.15$ & -0.013 & 0.49 & 3.337 & 0.043 \\
\hline G12 & $67.8 \pm 1.0$ & $16.8 \pm 0.3$ & $12.3 \pm 0.6$ & $0.137 \pm 0.012$ & $2.93 \pm 0.16$ & 0.035 & 0.53 & 3.302 & 0.023 \\
\hline G13 & $67.4 \pm 0.4$ & $17.6 \pm 0.2$ & $11.9 \pm 0.2$ & $0.331 \pm 0.008$ & $2.98 \pm 0.05$ & 0.063 & 0.55 & 3.301 & 0.056 \\
\hline G14 & $68.2 \pm 0.1$ & $19.1 \pm 0.1$ & $9.75 \pm 0.1$ & $0.111 \pm 0.007$ & $2.92 \pm 0.04$ & 0.140 & 0.60 & 3.255 & 0.019 \\
\hline
\end{tabular}




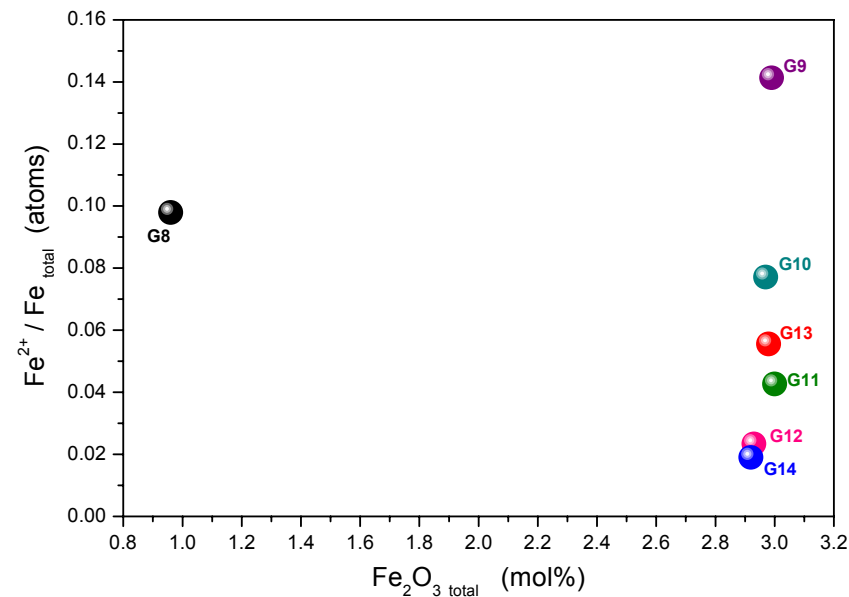

Fig. 50. Plot of the atomic ratio between the number of $\mathrm{Fe}^{2+}$ and $\mathrm{Fe}$ total as a function of $\mathrm{Fe}_{2} \mathrm{O}_{3 \text { total }}$ (in $\mathrm{mol} \%$ ).

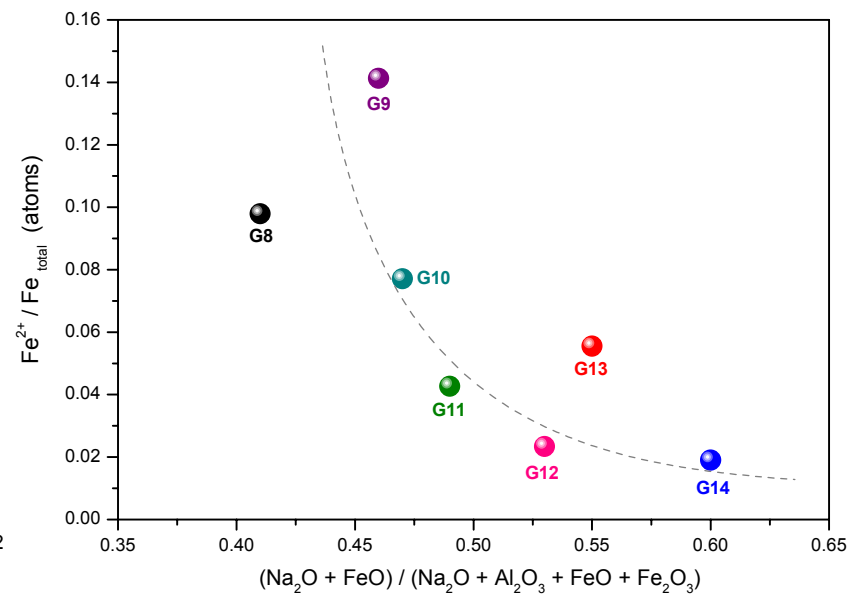

Fig. 51. Plot of the atomic ratio between the number of $\mathrm{Fe}^{2+}$ and $\mathrm{Fe}$ total as a function of the composition. The trend-line is a guide to the eye.

Peraluminous sample $\mathrm{G} 8$ has only $1 \mathrm{~mol} \%$ of $\mathrm{Fe}_{2} \mathrm{O}_{3}$, otherwise it will crystallize. From the reason of composition and $\mathrm{Fe}_{2} \mathrm{O}_{3}$ content, properties of these melts differ sometimes from the others and the points can lie out of the drawn trends (especially G8).

Figure 50 shows the atomic ratio between the number of $\mathrm{Fe}^{2+}$ and total amount of atomic $\mathrm{Fe}$ as a function of $\mathrm{Fe}_{2} \mathrm{O}_{3}$ total (in mol\%). Peralkaline $\mathrm{Fe}$-bearing samples have lower $\mathrm{Fe}^{2+} / \mathrm{Fe}$ total (atoms) ratio than peraluminous melts. $\mathrm{Fe}^{2+} / \mathrm{Fe}$ total (atoms) ratio does not depend on the $\mathrm{Fe}_{2} \mathrm{O}_{3}$ total (Fig. 50), but there is a relationship between this ratio and composition of the melt (Fig. 51). Webb (2005b) suggested that this behaviour is connected with the difference in charge balancing between peraluminous and peralkaline melts. In peraluminous melts, when there is not enough $\mathrm{Na}^{+}$to compensate negative charge of $\mathrm{Al}^{3+}$ - and $\mathrm{Fe}^{3+}$-tetrahedra, the role of charge balancer is played by $\mathrm{Fe}^{2+}$.

The very characteristic breaking point in all of the physical properties trends indicates minimal number of non-bridging oxygens occurring in the melt and that is in the moment, when peralkaline melt changes into peraluminous (see Table 9). This is shown in Figure 52 (glass transition temperature at $\log _{10} \eta=12$ as a function of NBO/T). As there is dependence between structure, composition and physical and thermodynamic properties of the melts, this behaviour will appear also in the visualisation of other data.

The maximum in $\mathrm{T}_{\mathrm{g}}{ }^{12}$ occurs slightly on the peraluminous side of the melt composition; and not exactly at $\mathrm{NBO} / \mathrm{T}=0$. In the investigated melts there is no sample which has NBO/T equals zero. The localization of the breaking point has been marked out between samples G3 and G4 in Fe-free melts and between samples G9 and G10 in Fe-bearing melts. 


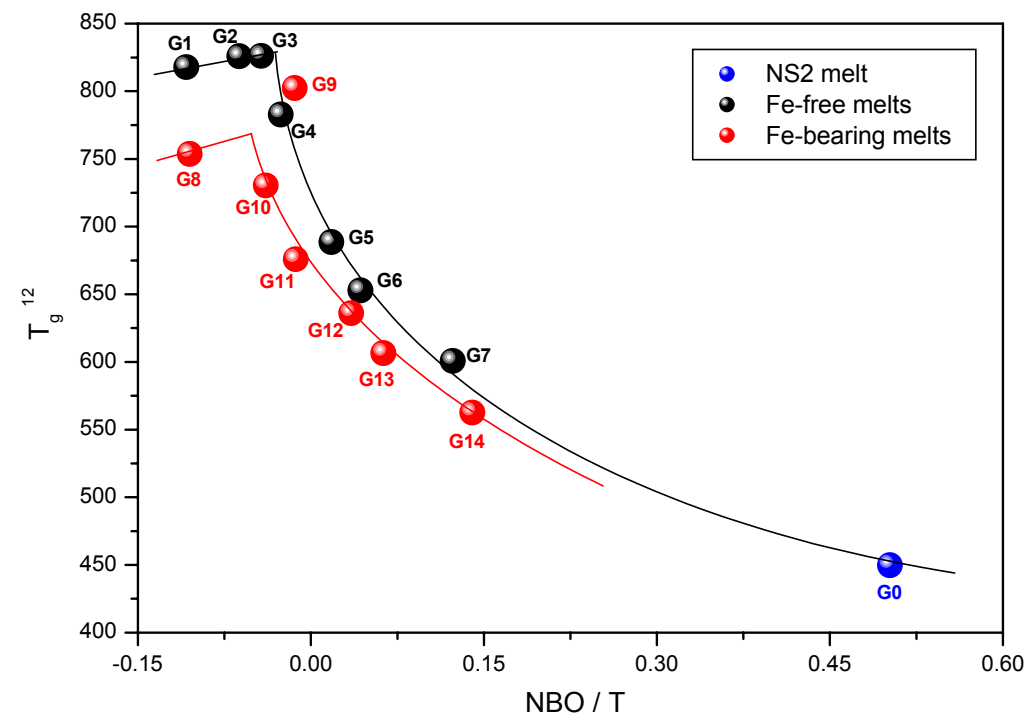

Fig. 52. Glass transition temperature at $\log _{10} \eta=12$ as a function of the parameter NBO/T.

Between NBO/T values and $\gamma$ for both the Fe-free and Fe-bearing melts there is a linear relationship described by an equation $N B O / T=-0.65+1.31 \gamma$ with $\mathrm{R}^{2}=0.976$ (Fig. 53). Sodium silicate sample G0 is not described by this correlation. To connect samples G1-G7 and G8-G14 with NS2 melt a curve needs to be drawn (Fig. 53).

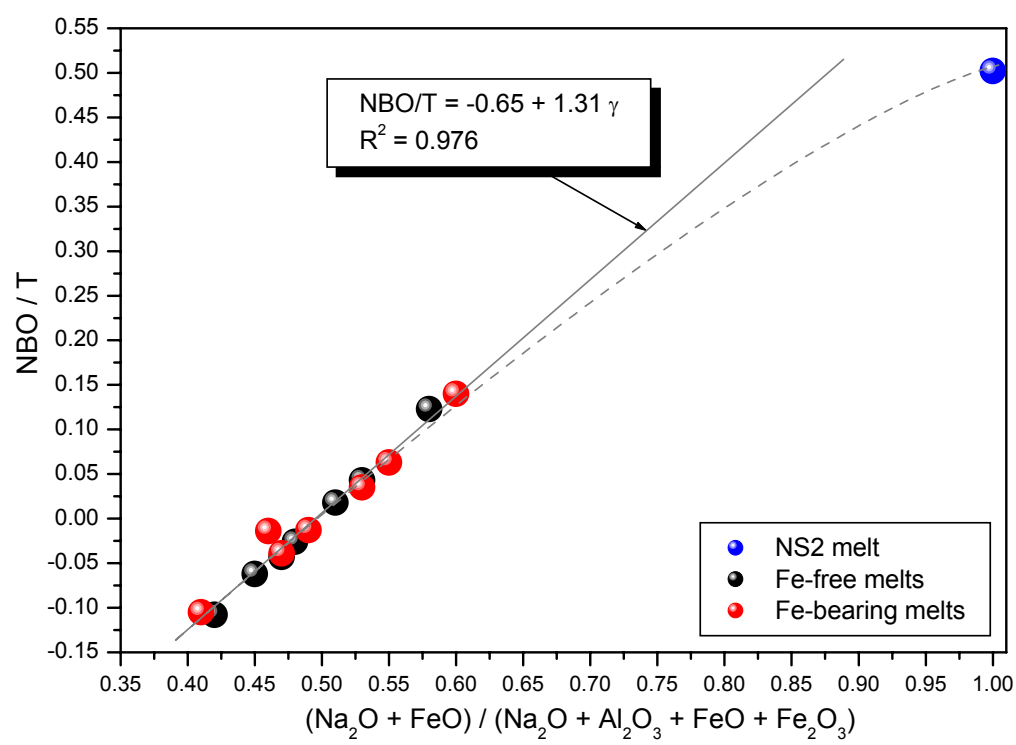

Fig. 53. Parameter $\mathrm{NBO} / \mathrm{T}$ for all of the samples as a function of their composition. The linear relationship between Fe-free (G1G7) and Fe-bearing samples (G8-G14) is indicated by a solid grey straight line. Dashed grey curve connects Fefree and Fe-bearing melts with NS2 melt. 
The oxidation state of Fe in melts G8-G14 is controlled by: (1) composition of the melt, (2) temperature and (3) oxygen fugacity (Jeoung et al., 2001; Berry et al., 2003) (the samples were made in air and measured in air-nitrogen atmosphere).

Neither microscopic observation nor microprobe analyses indicated the presence of crystals in Fe-free and Fe-bearing melts. The only one problem was with samples G8 and G9. During viscosity measurements the surface oxidized very fast, what required a polishing the surface before every new measurement.

\subsection{Density}

The densities of the glasses (see Table 10) as a function of composition together with their standard deviations from 10 measurements are shown in Figure 54. Density decreases as network-modifying $\mathrm{Na}^{+}$is removed from the peralkaline glass; and begins to increase as charge-balancing $\mathrm{Na}^{+}$is removed from the peraluminous glass. The same situation occurs in Fe-free as well in the Fe-bearing glasses. Fe-bearing glasses are denser and the trend-line is steeper. The characteristic minimum on the density plot is marked by samples G4 for Fe-free glasses and G11 for Fe-bearing glasses.

Figure 55 shows density data of the glasses from the system $\mathrm{Na}_{2} \mathrm{O}-\mathrm{Al}_{2} \mathrm{O}_{3}-\mathrm{SiO}_{2}$ containing $67 \mathrm{~mol} \% \mathrm{SiO}_{2}$ and $75 \mathrm{~mol} \% \mathrm{SiO}_{2}$. The presented data of Hunold \& Brückner (1980), Webb et al. (2007) and Fe-free samples from this study (G1-G7) create trends of the same shape. The dashed line drawn through the present data is simply a guide for the eye. A minimum in density is observed in all of these studies at $\gamma \sim 0.5$.

The data of Day \& Rindone (1962) show a similar minimum for $75 \mathrm{~mol}^{\circ} \mathrm{SiO}_{2}$ glasses. The $\pm 0.01 \mathrm{~g} \mathrm{~cm}^{-3}$ difference between the densities of the $67 \mathrm{~mol} \% \mathrm{SiO}_{2}$ glasses may be due to small differences in $\mathrm{SiO}_{2}$ content; or to differences is cooling rate. The present glasses were cooled down in three stages process:

- between $1650-700^{\circ} \mathrm{C}$ at $10^{\circ} \mathrm{C} \mathrm{min}^{-1}$;

- between $700-550^{\circ} \mathrm{C}$ at $1^{\circ} \mathrm{C} \min ^{-1}$;

- between $550-25^{\circ} \mathrm{C}$ at $10^{\circ} \mathrm{C} \mathrm{min}^{-1}$;

while those of Webb et al. (2007) were cooled at 5 and $10^{\circ} \mathrm{C} \mathrm{min}^{-1}$. Thus the glasses do not have the same fictive temperatures, but a change in density vs. composition trend is seen as the melt changes form peralkaline to peraluminous, and there is no longer enough $\mathrm{Na}^{+}$to charge-balance all of the $\mathrm{Al}^{3+}$ in tetrahedral coordination. 
Tab. 10. Densities, molar mass and molar volume of the glasses G0-G14 at room temperature. The standard deviations are calculated from 10 measurements.

\begin{tabular}{|c|c|c|c|}
\hline $\begin{array}{c}\text { melt } \\
\text { number }\end{array}$ & $\begin{array}{c}\text { density @25 } \\
\mathbf{~} \mathbf{~} \mathbf{~ c m}^{-3}\end{array}$ & $\begin{array}{c}\text { molar mass } \\
\mathbf{g ~ m o l}^{-1}\end{array}$ & $\begin{array}{c}\text { molar volume } \\
\mathbf{c m}^{\mathbf{3}} \mathbf{~ m o l}^{-1}\end{array}$ \\
\hline G0 & $2.485 \pm 0.005$ & $60.650 \pm 0.078$ & $24.407 \pm 0.051$ \\
\hline G1 & $2.419 \pm 0.006$ & $68.658 \pm 0.076$ & $28.383 \pm 0.051$ \\
G2 & $2.412 \pm 0.003$ & $67.524 \pm 0.129$ & $27.995 \pm 0.083$ \\
G3 & $2.414 \pm 0.001$ & $67.819 \pm 0.075$ & $28.094 \pm 0.048$ \\
G4 & $2.409 \pm 0.003$ & $67.774 \pm 0.113$ & $28.134 \pm 0.073$ \\
G5 & $2.415 \pm 0.001$ & $67.027 \pm 0.223$ & $27.755 \pm 0.144$ \\
G6 & $2.432 \pm 0.003$ & $67.048 \pm 0.175$ & $27.569 \pm 0.112$ \\
G7 & $2.444 \pm 0.003$ & $66.370 \pm 0.106$ & $27.156 \pm 0.068$ \\
\hline G8 & $2.460 \pm 0.004$ & $69.638 \pm 0.077$ & $28.301 \pm 0.050$ \\
G9 & $2.493 \pm 0.011$ & $70.701 \pm 0.410$ & $28.363 \pm 0.261$ \\
G10 & $2.485 \pm 0.010$ & $69.193 \pm 0.284$ & $27.839 \pm 0.181$ \\
G11 & $2.445 \pm 0.010$ & $69.146 \pm 0.707$ & $28.278 \pm 0.453$ \\
G12 & $2.485 \pm 0.008$ & $68.755 \pm 0.608$ & $27.665 \pm 0.386$ \\
G13 & $2.496 \pm 0.015$ & $68.438 \pm 0.220$ & $27.421 \pm 0.143$ \\
G14 & $2.512 \pm 0.003$ & $67.496 \pm 0.146$ & $26.874 \pm 0.092$ \\
\hline
\end{tabular}

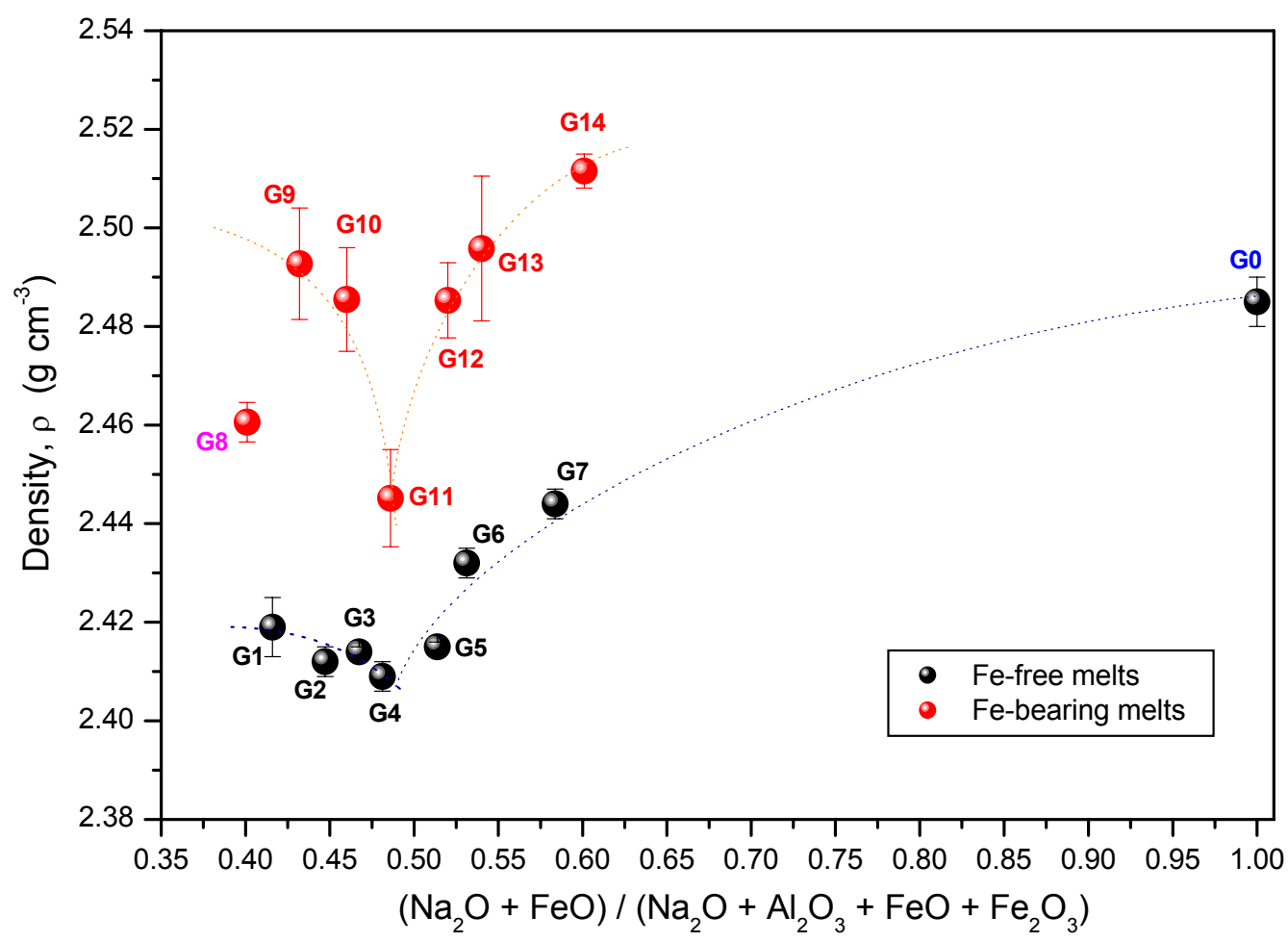

Fig. 54. Measured densities of the glasses as a function of their compositions. Sample G8 has less $\mathrm{Fe}^{3+}$ than other Fe-bearing samples. 


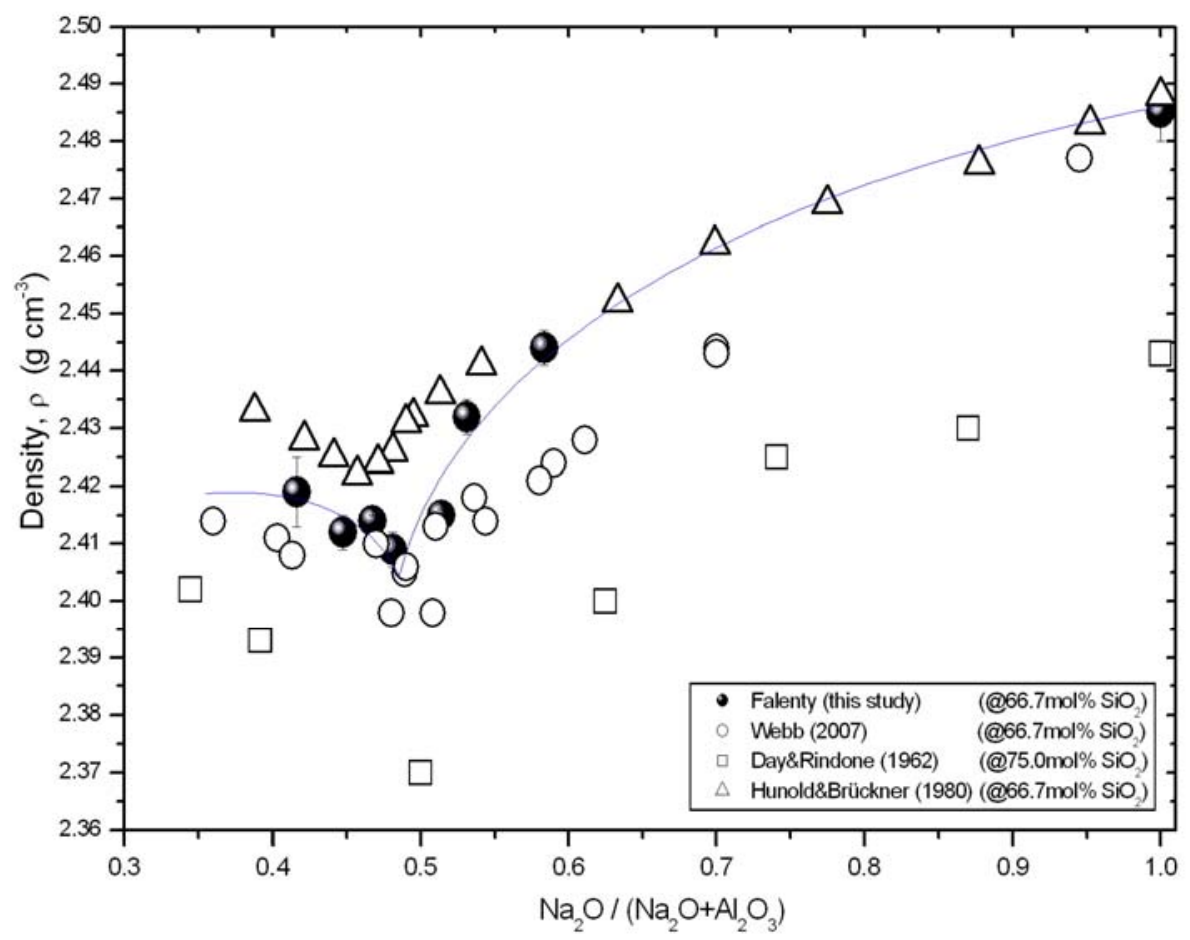

Fig. 55. Measured densities of the Fe-free sodium aluminosilicate glasses as a function of their compositions. Four sets of data have been used: black points - Falenty (samples G1-G7 - from this study); open circles - Webb (2007); open squares - Day \& Rindone (1962); open triangles Hunold \& Brückner (1980). The trend-line is a guide to the eye.

Figure 56 shows molar volume of the NS2 glass (blue point), Fe-free (black points) and Fe-bearing (red points) glasses as a function of composition. Here the effect of changing structure is not as clear as in the density plot. Data show that the structural units take up different volumes of space in peraluminous and peralkaline melts; the trend-line is similar to that in Figure 52.

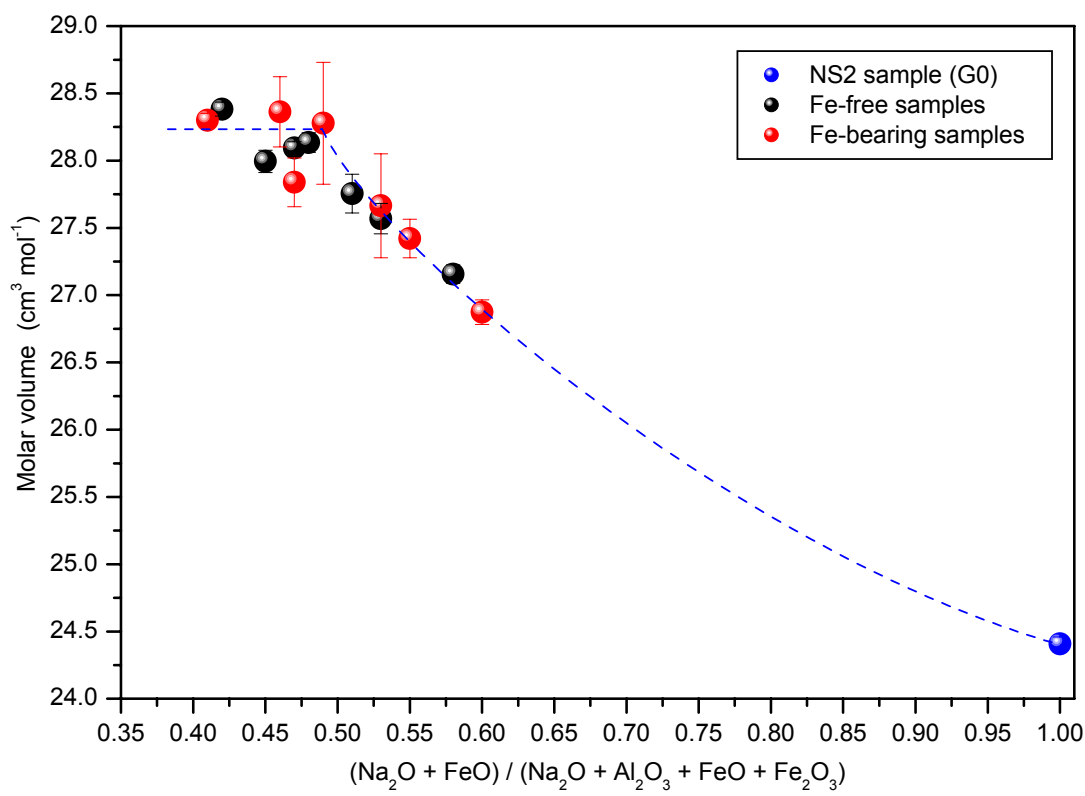

Fig. 56. Molar volume of the glasses as a function of composition. 


\subsection{Ultrasonic measurements}

Shear modulus $G$ and sound speed $V_{s}$ determined from the pulse echo overlap technique are presented in the Table 11. The shear moduli measured by ultrasonic methods at room temperature are $\sim 3.5 \mathrm{GPa}$ higher for Fe-free samples (Fig. 57a) and $\sim 1.8 \mathrm{GPa}$ higher for Fe-bearing samples (Fig. 57b) than the moduli determined by torsion methods. As there is, in general, a $500^{\circ} \mathrm{C}$ temperature difference between the ultrasonic and torsion data, an average temperature dependence of the shear modulus of $\sim-6.9 \mathrm{MPa}$ $\mathrm{K}^{-1}$ for Fe-free and $\sim-3.5 \mathrm{MPa} \mathrm{K}^{-1}$ for Fe-bearing glasses is calculated. The temperature dependence of the shear modulus of $\mathrm{Na}_{2} \mathrm{O}-\mathrm{Al}_{2} \mathrm{O}_{3}-\mathrm{SiO}_{2}$ glasses in the compilation by Ahrens (1995) is between -3 and $-10 \mathrm{MPa} \mathrm{K}^{-1}$.

Tab. 11. Shear modulus $G$ and sound speed $V_{s}$ determined from the pulse echo overlap technique for all of the samples investigated in this study.

\begin{tabular}{|c|c|c|}
\hline $\begin{array}{c}\text { melt } \\
\text { number }\end{array}$ & $\begin{array}{c}\text { Sound speed, } \mathbf{v} \text { s } \\
\mathbf{m ~ s}^{-1}\end{array}$ & $\begin{array}{c}\text { Shear modulus, G } \\
\mathbf{G P a}\end{array}$ \\
\hline G0 & $3471.60 \pm 20.51$ & $29.89 \pm 0.23$ \\
\hline G1 & $3918.85 \pm 75.84$ & $37.39 \pm 0.75$ \\
G2 & $3491.90 \pm 160.14$ & $37.84 \pm 1.09$ \\
G3 & $3953.50 \pm 26.28$ & $38.04 \pm 0.15$ \\
G4 & $3973.37 \pm 53.20$ & $38.07 \pm 0.62$ \\
G5 & $3919.16 \pm 19.18$ & $37.12 \pm 0.16$ \\
G6 & $3981.36 \pm 21.17$ & $36.65 \pm 0.19$ \\
G7 & $3754.51 \pm 22.73$ & $34.28 \pm 0.20$ \\
\hline \hline G8 & $3738.78 \pm 20.50$ & $34.23 \pm 0.29$ \\
G9 & $3682.87 \pm 31.03$ & $34.09 \pm 0.13$ \\
G10 & $3631.93 \pm 83.16$ & $33.56 \pm 0.51$ \\
G11 & $3714.70 \pm 35.61$ & $33.54 \pm 0.36$ \\
G12 & $3582.22 \pm 31.54$ & $32.23 \pm 0.27$ \\
G13 & $3524.93 \pm 24.36$ & $31.26 \pm 0.25$ \\
G14 & $3500.48 \pm 29.70$ & $30.94 \pm 0.19$ \\
\hline
\end{tabular}



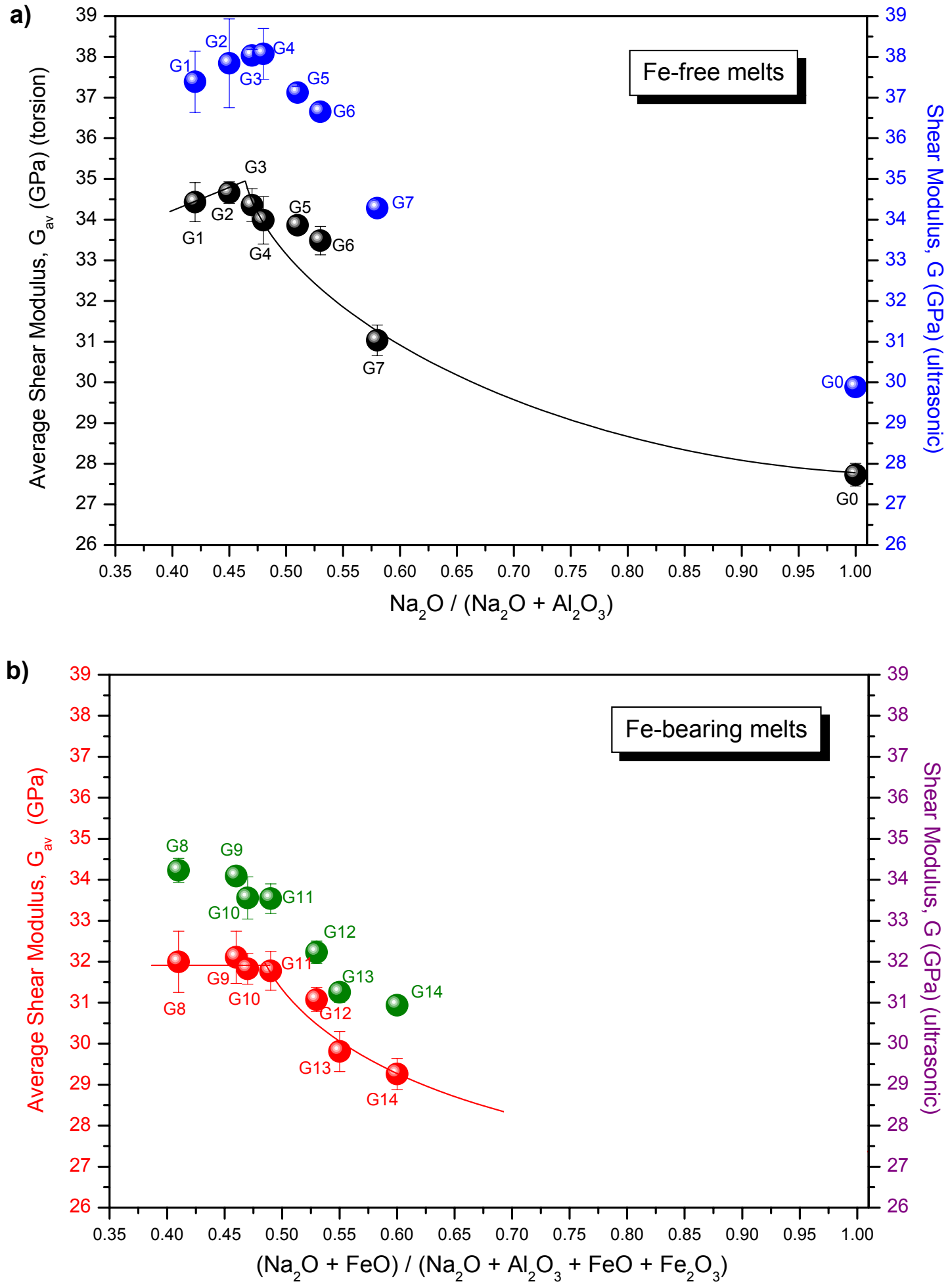

Fig. 57a, b. Shear modulus $G$ determined by pulse echo overlap technique (right axis) and average shear modulus $G_{\text {av }}$ from torsion apparatus (left axis) as a function of compositional parameter $\gamma=\left(\mathrm{Na}_{2} \mathrm{O}+\mathrm{FeO}\right) /\left(\mathrm{Na}_{2} \mathrm{O}+\mathrm{Al}_{2} \mathrm{O}_{3}+\mathrm{FeO}+\mathrm{Fe}_{2} \mathrm{O}_{3}\right)$. a) for NS2 (G0) and Fe-free samples (G1-G7); b) for Fe-bearing samples (G8-G14). The trend-lines are a guides to the eye. 
Figure 58 presents data of sound speed in the samples; determined by the ultrasonic method. Sound speed $V_{s}$ depends on the composition of the samples and is slower in Fe-bearing;

$$
V_{s}=\sqrt{\frac{G}{\rho}},
$$

where $G$ is shear modulus of the sample and $\rho$ is density.

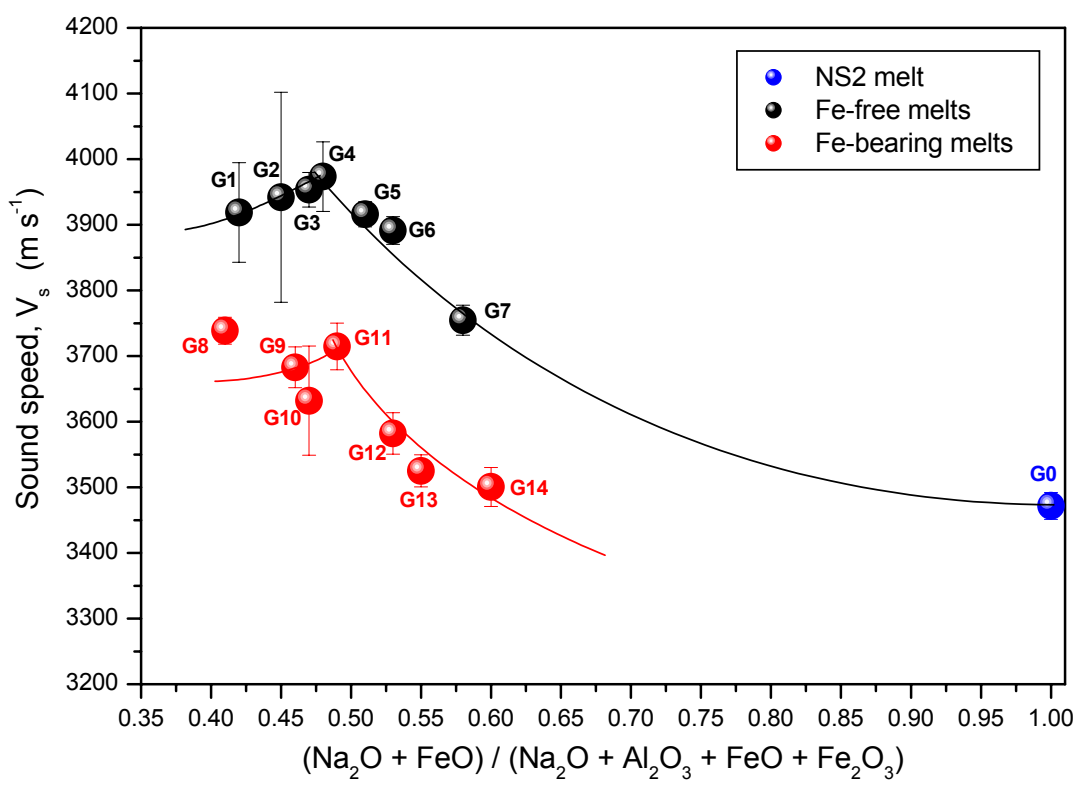

Fig. 58. Sound speed data for all of the samples as a function of compositional parameter $\gamma=\left(\mathrm{Na}_{2} \mathrm{O}+\mathrm{FeO}\right) /\left(\mathrm{Na}_{2} \mathrm{O}+\mathrm{Al}_{2} \mathrm{O}_{3}+\mathrm{FeO}+\mathrm{Fe}_{2} \mathrm{O}_{3}\right)$. Sample $\mathrm{G} 8$ has less $\mathrm{Fe}^{3+}$ than other samples. Trendlines are the guides to the eye.

\subsection{Viscosity}

\subsubsection{Viscosity as a function of temperature}

Viscosity as a function of temperature is presented in Figure 59a, b; and data are gathered in the Table 12. The viscosity data has been fit by an Arrhenian curve, and the A and $B$ parameters (with standard deviation) of the Arrhenian equation are given in the Table 13. The error of viscosity measurements is $\pm 0.06 \log _{10} \mathrm{~Pa} \mathrm{~s}$, and of temperature $\pm 0.5^{\circ} \mathrm{C}$. 
Tab. 12. Viscosity data for NS2 melt (G0), Fe-free melts (G1-G7) and Fe-bearing melts (G8-G14) as a function of temperature.

\begin{tabular}{|c|c|c|c|c|c|c|c|c|c|}
\hline \multicolumn{2}{|c|}{ G0 } & \multicolumn{2}{|c|}{ G1 } & \multicolumn{2}{|c|}{ G2 } & \multicolumn{2}{|c|}{ G3 } & \multicolumn{2}{|c|}{ G4 } \\
\hline$T$ & $\eta$ & T & $\eta$ & $\mathrm{T}$ & $\eta$ & $\mathrm{T}$ & $\eta$ & $\mathrm{T}$ & $\eta$ \\
\hline${ }^{\circ} \mathrm{C}$ & $\begin{array}{c}\log _{10} \\
\text { Pa s }\end{array}$ & ${ }^{\circ} \mathrm{C}$ & $\begin{array}{c}\log _{10} \\
\text { Pa s }\end{array}$ & ${ }^{\circ} \mathrm{C}$ & $\begin{array}{c}\log _{10} \\
\text { Pa s }\end{array}$ & ${ }^{\circ} \mathrm{C}$ & $\begin{array}{c}\log _{10} \\
\text { Pa s }\end{array}$ & ${ }^{\circ} \mathrm{C}$ & $\begin{array}{c}\log _{10} \\
\text { Pa s }\end{array}$ \\
\hline 521.79 & 8.76 & 938.54 & 9.26 & 948.71 & 9.34 & 806.16 & 9.10 & 928.37 & 9.62 \\
\hline 511.65 & 9.12 & 928.37 & 9.52 & 938.54 & 9.54 & 795.99 & 9.30 & 918.21 & 9.80 \\
\hline 501.50 & 9.53 & 908.04 & 9.87 & 928.37 & 9.69 & 775.66 & 9.70 & 908.04 & 10.08 \\
\hline 491.35 & 9.90 & 897.87 & 10.04 & 918.21 & 9.85 & 765.49 & 9.94 & 897.87 & 10.31 \\
\hline 481.20 & 10.37 & 887.70 & 10.29 & 908.04 & 10.12 & 755.32 & 10.16 & 887.70 & 10.51 \\
\hline 471.06 & 10.79 & 877.54 & 10.56 & 897.87 & 10.30 & 745.15 & 10.43 & 877.54 & 10.72 \\
\hline 460.91 & 11.41 & 867.37 & 10.75 & 887.70 & 10.56 & 734.98 & 10.73 & 867.37 & 10.97 \\
\hline 450.76 & 11.96 & 857.20 & 11.02 & 877.54 & 10.74 & 724.82 & 11.00 & 857.20 & 11.22 \\
\hline 440.61 & 12.62 & 847.03 & 11.29 & 867.37 & 11.01 & 714.65 & 11.20 & 847.03 & 11.50 \\
\hline & & 836.86 & 11.58 & 857.20 & 11.27 & 704.48 & 11.58 & & \\
\hline & & & & 847.03 & 11.48 & 694.31 & 11.95 & & \\
\hline & & & & 836.87 & 11.78 & & & & \\
\hline
\end{tabular}

\begin{tabular}{|c|c|c|c|c|c|c|c|c|c|}
\hline \multicolumn{2}{|c|}{ G5 } & \multicolumn{2}{|c|}{ G6 } & \multicolumn{2}{|c|}{ G7 } & \multicolumn{2}{|c|}{ G8 } & \multicolumn{2}{|c|}{ G9 } \\
\hline$T$ & $\eta$ & $\mathrm{T}$ & $\eta$ & $\mathrm{T}$ & $\eta$ & $\mathrm{T}$ & $\eta$ & $T$ & $\eta$ \\
\hline${ }^{\circ} \mathrm{C}$ & $\begin{array}{c}\log _{10} \\
\text { Pa s }\end{array}$ & ${ }^{\circ} \mathrm{C}$ & $\begin{array}{c}\log _{10} \\
\mathrm{~Pa} \mathrm{~S}\end{array}$ & ${ }^{\circ} \mathrm{C}$ & $\begin{array}{c}\log _{10} \\
\text { Pa s }\end{array}$ & ${ }^{\circ} \mathrm{C}$ & $\begin{array}{c}\log _{10} \\
\text { Pa s }\end{array}$ & ${ }^{\circ} \mathrm{C}$ & $\begin{array}{c}\log _{10} \\
\text { Pa s }\end{array}$ \\
\hline 918.21 & 9.04 & 765.49 & 9.10 & 704.48 & 9.01 & 887.14 & 9.10 & 876.99 & 10.22 \\
\hline 908.04 & 9.30 & 755.32 & 9.32 & 694.31 & 9.22 & 876.99 & 9.20 & 866.84 & 10.38 \\
\hline 897.87 & 9.50 & 745.15 & 9.60 & 684.15 & 9.48 & 866.84 & 9.44 & 856.69 & 10.61 \\
\hline 887.70 & 9.70 & 734.98 & 9.81 & 673.98 & 9.78 & 856.69 & 9.56 & 846.54 & 10.84 \\
\hline 877.54 & 9.85 & 724.82 & 10.02 & 663.81 & 10.05 & 846.54 & 9.91 & 836.39 & 11.02 \\
\hline 867.37 & 10.05 & 714.65 & 10.32 & 653.64 & 10.35 & 836.39 & 10.08 & 826.25 & 11.47 \\
\hline 857.20 & 10.30 & 704.48 & 10.60 & 643.48 & 10.64 & 826.25 & 10.22 & 816.10 & 11.55 \\
\hline 847.03 & 10.46 & 694.31 & 10.85 & 623.14 & 11.22 & 816.10 & 10.44 & 805.95 & 11.83 \\
\hline 836.86 & 10.71 & 684.15 & 11.11 & 612.97 & 11.60 & 805.95 & 10.84 & 795.80 & 12.28 \\
\hline 826.70 & 11.01 & 673.98 & 11.40 & 602.80 & 12.02 & 795.80 & 10.94 & & \\
\hline 816.53 & 11.28 & & & & & 785.65 & 11.27 & & \\
\hline
\end{tabular}

\begin{tabular}{|c|c|c|c|c|c|c|c|c|c|}
\hline \multicolumn{2}{|c|}{ G10 } & \multicolumn{2}{|c|}{ G11 } & \multicolumn{2}{|c|}{ G12 } & \multicolumn{2}{|c|}{ G13 } & \multicolumn{2}{|c|}{ G14 } \\
\hline $\mathrm{T}$ & $\eta$ & $\mathrm{T}$ & $\eta$ & $T$ & $\eta$ & $\mathrm{T}$ & $\eta$ & $\mathrm{T}$ & $\eta$ \\
\hline${ }^{\circ} \mathrm{C}$ & $\begin{array}{c}\log _{10} \\
\text { Pa s }\end{array}$ & ${ }^{\circ} \mathrm{C}$ & $\begin{array}{c}\log _{10} \\
\mathrm{~Pa} s\end{array}$ & ${ }^{\circ} \mathrm{C}$ & $\begin{array}{c}\log _{10} \\
\mathrm{~Pa} S\end{array}$ & ${ }^{\circ} \mathrm{C}$ & $\begin{array}{c}\log _{10} \\
\text { Pa s }\end{array}$ & ${ }^{\circ} \mathrm{C}$ & $\begin{array}{c}\log _{10} \\
\text { Pa s }\end{array}$ \\
\hline 847.03 & 9.30 & 816.53 & 8.87 & 735.19 & 9.26 & 694.52 & 9.37 & 664.01 & 8.83 \\
\hline 836.86 & 9.51 & 806.36 & 9.05 & 725.02 & 9.53 & 684.35 & 9.58 & 653.85 & 9.06 \\
\hline 826.70 & 9.75 & 796.19 & 9.26 & 714.85 & 9.81 & 674.18 & 9.80 & 643.68 & 9.31 \\
\hline 816.53 & 9.90 & 786.03 & 9.55 & 704.68 & 10.10 & 664.01 & 10.11 & 633.51 & 9.64 \\
\hline 806.36 & 10.10 & 775.86 & 9.65 & 694.52 & 10.38 & 653.85 & 10.39 & 623.34 & 9.89 \\
\hline 796.19 & 10.37 & 765.69 & 9.98 & 684.35 & 10.59 & 643.68 & 10.73 & 613.18 & 10.23 \\
\hline 786.03 & 10.54 & 755.52 & 10.02 & 664.01 & 11.24 & 633.51 & 11.10 & 603.01 & 10.56 \\
\hline 775.86 & 10.80 & 745.36 & 10.36 & 653.85 & 11.48 & 623.34 & 11.40 & 592.84 & 10.94 \\
\hline 765.69 & 11.06 & 735.19 & 10.45 & 643.68 & 11.70 & 613.18 & 11.79 & 582.67 & 11.34 \\
\hline 755.52 & 11.36 & 725.02 & 10.89 & & & 603.01 & 12.19 & 572.50 & 11.71 \\
\hline 745.36 & 1160 & 714.85 & 11.06 & & & & & & \\
\hline 735.19 & 11.97 & & & & & & & & \\
\hline
\end{tabular}


a)

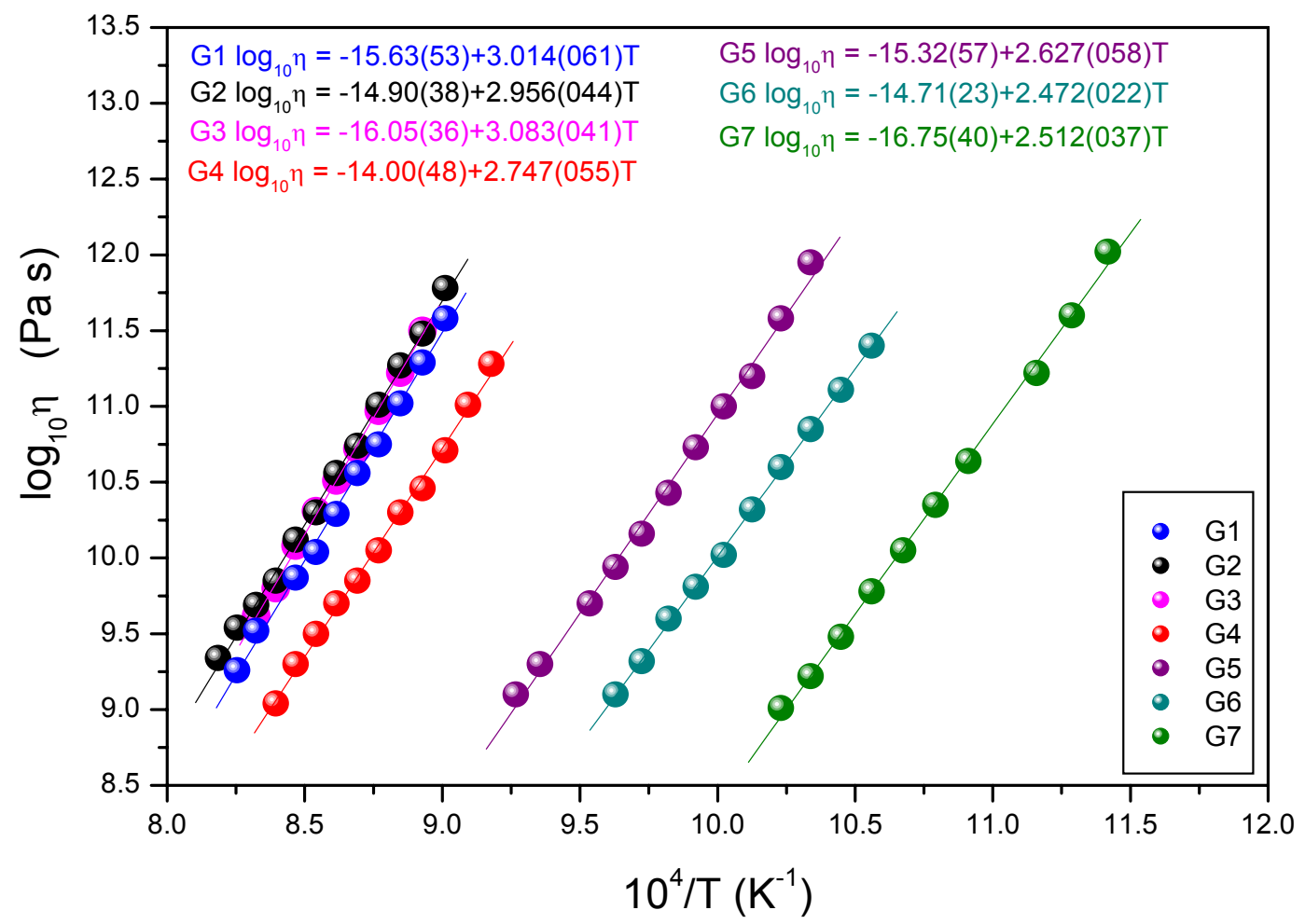

b)

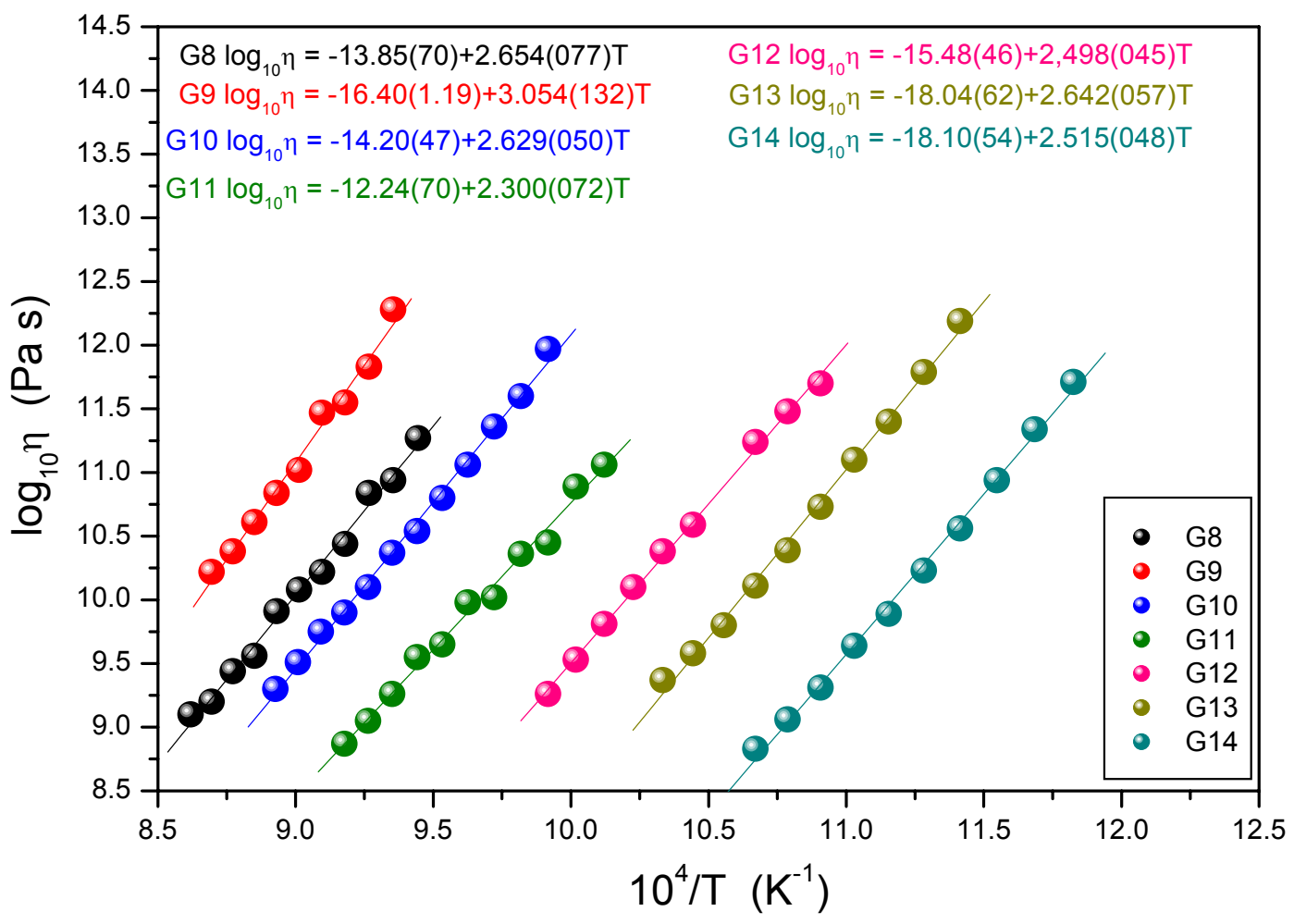

Fig. 59. Melt viscosity as a function of inverse temperature determined by the micropenetration method. The straight lines are the Arrhenian fits to the data (equations for Arrhenian fits above the data points). a) for Fe-free melts (G1-G7); b) for Fe-bearing melts (G8-G14). 
Table 13. Fits to the Arrhenian equation (Eq. 24) for the viscosity data of melts G0-G14. The activation energy $\mathrm{E}_{\mathrm{a}}$ calculated with using the Eq. $27 ; \gamma=\left(\mathrm{Na}_{2} \mathrm{O}+\mathrm{FeO}\right) /\left(\mathrm{Na}_{2} \mathrm{O}+\mathrm{Al}_{2} \mathrm{O}_{3}+\mathrm{FeO}+\mathrm{Fe}_{2} \mathrm{O}_{3}\right)$; $\log _{10}$ viscosity was determined for temperature $750^{\circ} \mathrm{C}$.

\begin{tabular}{|c|c|c|c|c|c|}
\hline Melt number & $\begin{array}{c}\mathbf{A} \\
\left(\log _{10} \mathrm{~Pa} \mathrm{~s}\right)\end{array}$ & $\begin{array}{c}\mathbf{B} \\
(\mathrm{K})\end{array}$ & $\begin{array}{c}\text { Activation Energy, } \mathbf{E}_{\mathbf{a}} \\
\left(\mathrm{kJ} \mathrm{mol}^{-1}\right)\end{array}$ & $\gamma$ & $\begin{array}{c}\log _{\mathbf{1 0}} \eta_{\mathbf{Q}}^{\mathbf{Q} 750^{\circ} \mathbf{C}} \\
(\mathrm{Pa} \mathbf{s})\end{array}$ \\
\hline \hline G0 & $-24.98 \pm 0.99$ & $2.673 \pm 0.074$ & $511.75 \pm 14.23$ & 1.00 & 1.145 \\
\hline G1 & $-15.63 \pm 0.53$ & $3.014 \pm 0.061$ & $577.10 \pm 11.68$ & 0.42 & 13.828 \\
G2 & $-14.90 \pm 0.38$ & $2.956 \pm 0.044$ & $565.99 \pm 8.42$ & 0.45 & 13.991 \\
G3 & $-16.05 \pm 0.36$ & $3.083 \pm 0.041$ & $590.31 \pm 7.85$ & 0.47 & 14.082 \\
G4 & $-14.00 \pm 0.48$ & $2.747 \pm 0.055$ & $525.97 \pm 10.53$ & 0.48 & 12.848 \\
G5 & $-15.32 \pm 0.57$ & $2.627 \pm 0.058$ & $503.00 \pm 11.11$ & 0.51 & 10.356 \\
G6 & $-14.71 \pm 0.23$ & $2.472 \pm 0.022$ & $473.32 \pm 4.21$ & 0.53 & 9.451 \\
G7 & $-16.75 \pm 0.40$ & $2.512 \pm 0.037$ & $480.98 \pm 7.08$ & 0.58 & 7.802 \\
\hline \hline G8 & $-13.85 \pm 0.70$ & $2.654 \pm 0.077$ & $508.16 \pm 14.74$ & 0.41 & 12.090 \\
G9 & $-16.40 \pm 1.19$ & $3.054 \pm 0.132$ & $584.75 \pm 25.27$ & 0.46 & 13.449 \\
G10 & $-14.20 \pm 0.47$ & $2.629 \pm 0.050$ & $503.38 \pm 9.57$ & 0.47 & 11.495 \\
G11 & $-12.24 \pm 0.70$ & $2.300 \pm 0.072$ & $440.38 \pm 13.79$ & 0.49 & 10.240 \\
G12 & $-15.48 \pm 0.46$ & $2.498 \pm 0.045$ & $478.34 \pm 8.62$ & 0.53 & 8.935 \\
G13 & $-18.04 \pm 0.62$ & $2.642 \pm 0.057$ & $505.87 \pm 10.91$ & 0.55 & 7.782 \\
G14 & $-18.10 \pm 0.54$ & $2.515 \pm 0.048$ & $481.63 \pm 9.19$ & 0.60 & 6.481 \\
\hline
\end{tabular}

Viscosity of peralkaline samples (with and without iron) increases together with increasing $\mathrm{Al}_{2} \mathrm{O}_{3}$ content. When the composition becomes peraluminous, viscosity does not change, just the opposite, stays constant.

The clearer change one observes in the plot of viscosity as a function of compositional parameter $\gamma$ at one chosen temperature. In Figure 60 the extrapolated $\log _{10}$ viscosity data are presented for $750^{\circ} \mathrm{C}$ (see also Tab. 13). Not all of the melts were analysed at $750^{\circ} \mathrm{C}$. Because of that the viscosity data for some melts need to be extrapolated beyond the investigated temperature range. Viscosity data as a function of $\left(\mathrm{Na}_{2} \mathrm{O}+\mathrm{FeO}\right) /\left(\mathrm{Na}_{2} \mathrm{O}+\mathrm{Al}_{2} \mathrm{O}_{3}+\mathrm{FeO}+\mathrm{Fe}_{2} \mathrm{O}_{3}\right)$ create a trend increasing from more to less peralkaline samples and then changes into a plateau in range of peraluminous composition. There can be also observed a weak maximum at 0.47 , i.e. in the peraluminous range.

The addition of iron to the sodium aluminosilicate melts decreases viscosity. As can be observed in Figure 60, the difference between viscosity of peralkaline Fe-free and Fe-bearing melts is smaller than the difference between viscosity of Fe-free and Febearing peraluminous melts. The same effect of the influence of $\mathrm{Fe}$ on the viscosity was confirmed by Cukierman \& Uhlmann, 1974; Mysen \& Virgo, 1985; Dingwell \& Virgo, 1988a,b or Webb, 2005b. 


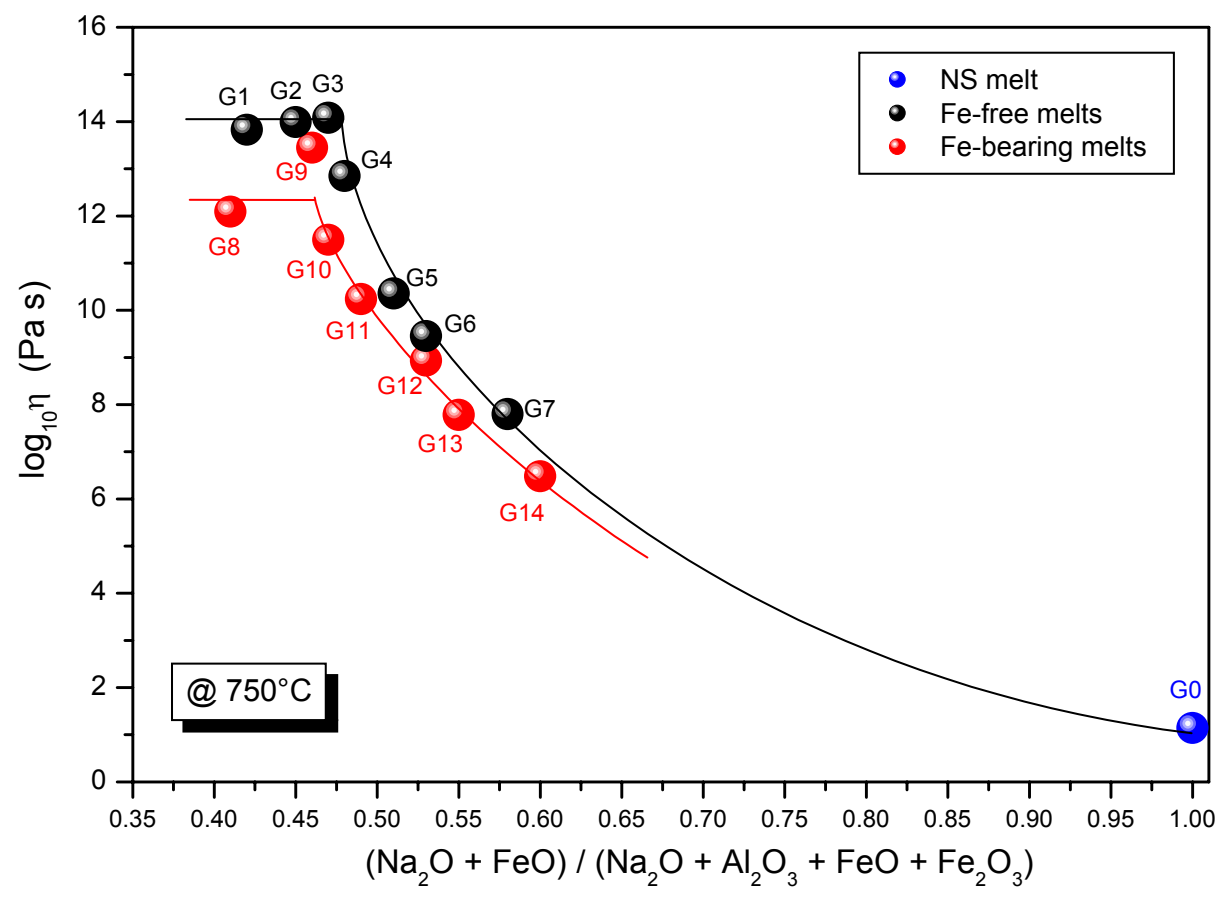

Fig. 60. Log $_{10}$ viscosity as a function of composition. Blue point is for NS2 melt (G0); black points are for Fe-free melts (G1-G7); red points are for Fe-bearing melts (G8-G14). The trend-lines are a guides to the eye.

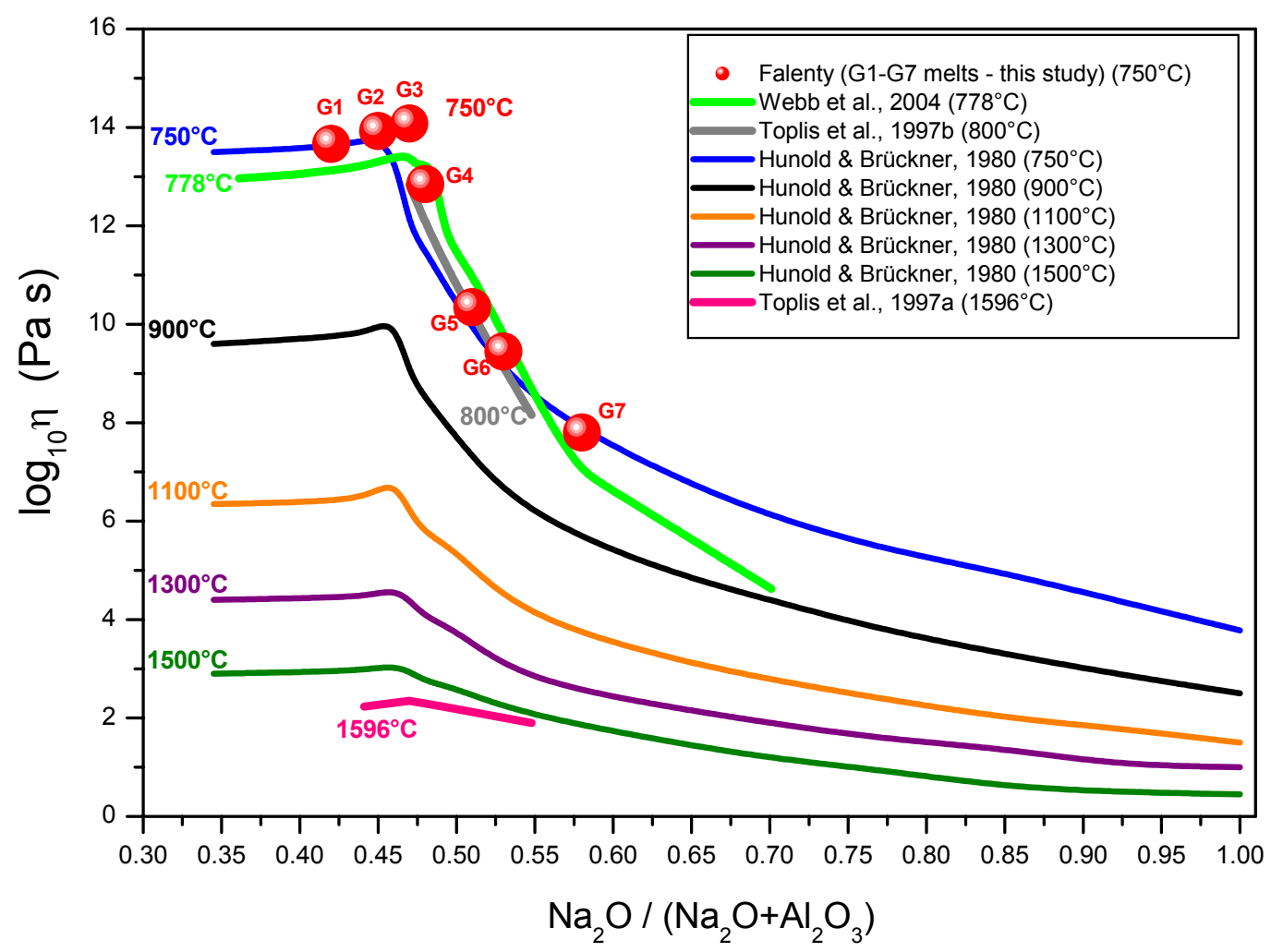

Fig. 61. $\log _{10}$ viscosity for sodium aluminosilicate melts at different temperatures as a function of composition. Coloured lines belong to the data of other authors (see legend); red points are for Fe-free melts from this study (G1-G7). 
As has been mentioned before, the viscosity depends on the temperature. Viscosity decreases with increasing temperature. Figure 61 shows viscosity data of Fefree melts (G1-G7) at $750^{\circ} \mathrm{C}$ compared to viscosity data of sodium aluminosilicate measured by Hunold \& Brückner (1980), Toplis et al. (1997a, b) and Webb et al. (2004) at different temperatures. There is small divergence between data of G1-G7 melts at $750^{\circ} \mathrm{C}$ and data of Hunold \& Brückner (1980) at the same temperature, however, Webb (2005b) assumed that the extrapolation of viscosity data beyond the investigation temperature range (using $A$ and $B$ parameters of the Arrhenian equation) can create an error in calculated viscosity up to $\sim 1 \log _{10} \mathrm{~Pa} \mathrm{~s}$.

\subsubsection{Activation energy for viscous flow}

Activation energy $E_{a}$ for NS2, Fe-free and Fe-bearing melts obtained from micropenetration measurements has been presented in Figure 62 as a function of compositional parameter $\gamma$ and in the Table 13. In Figure 62 data from this study are compared to other data of Fe-free melts (Webb et al., 2004; Toplis et al., 1997a, b) and Fe-bearing melts (Webb, 2005b). Because error bars are large, it is difficult to support a clear trend. Activation energy is the energy needed to trigger the flow mechanism in the melt. A related property is measured in heat capacity investigations, where heat needed to start flow is determined. From this reason the trend (being a guide to the eye in Figure 62) is similar to this one in Figure 76.

Activation energy of Fe-free peralkaline melts appears to be similar to the activation energy of Fe-bearing peralkaline melts. Starting from NS2 melt $E_{a}$ slowly decreases together with increasing the amount $\mathrm{Al}_{2} \mathrm{O}_{3}$ in the melts. The breaking point is close to $\gamma=0.53$. Fe-free and Fe-bearing peraluminous melts differ from each other. The activation of Fe-free peraluminous melts is higher than for Fe-bearing peraluminous melts and both of them are slightly higher than peralkaline melts. 


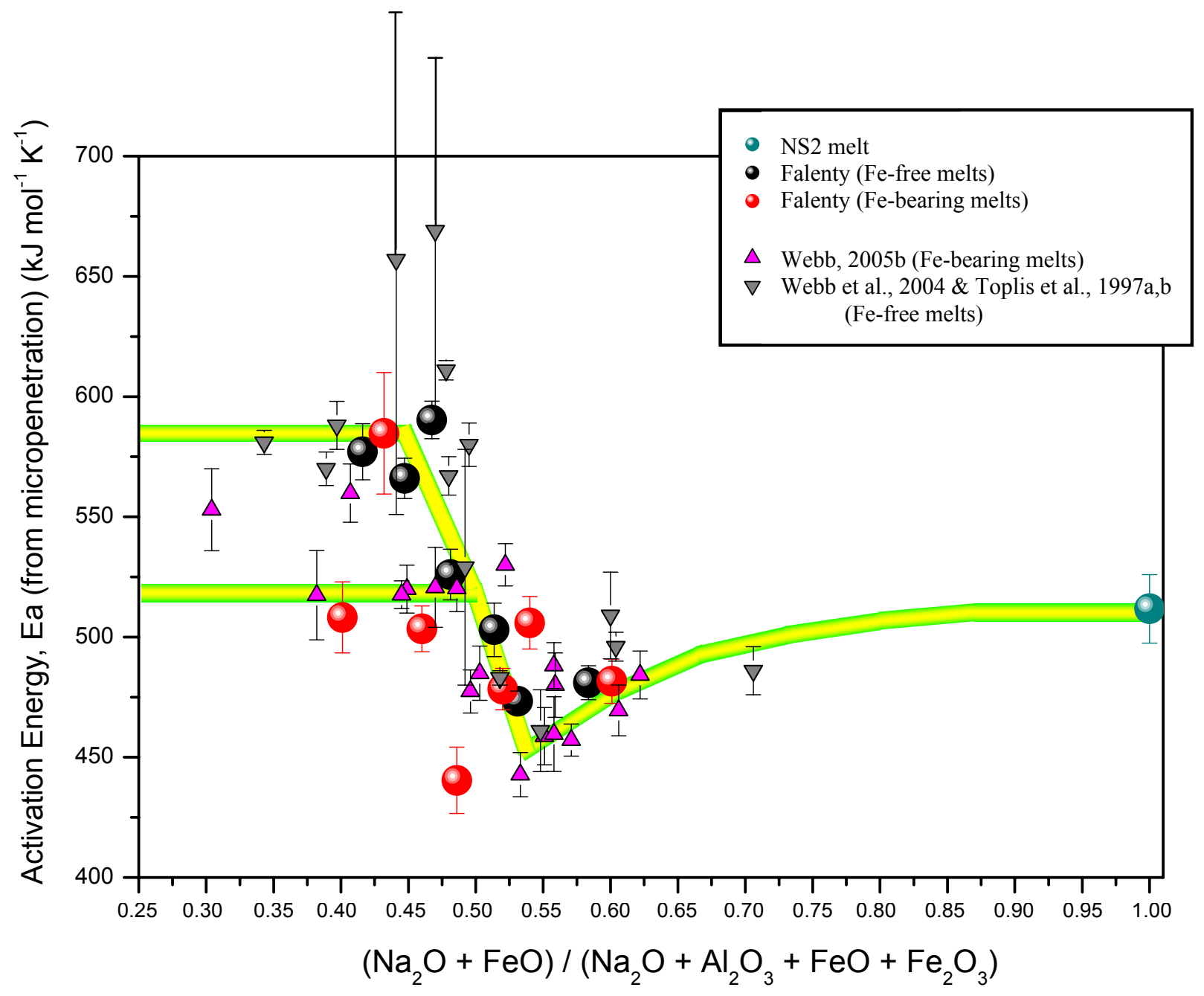

Fig. 62. Activation energy for NS2 (cyan point), Fe-free (black points) and Fe-bearing (red points) melts calculated from micropenetration measurements as a function of compositional parameter $\gamma$. Other plotted data come from Webb et al., 2004 and Toplis et al., 1997a, b (Fe-free melts - grey inverted triangles); and Webb, 2005b (Fe-bearing melts - pink triangles). 


\subsection{Heat capacity}

\subsubsection{Heat capacity data}

Typical results of heat capacity measurements (in $\mathrm{J} \mathrm{g}^{-1} \mathrm{~K}^{-1}$ ) for the glasses and liquids in the temperature range between 100 and $925^{\circ} \mathrm{C}$ with step of temperature of $5^{\circ} \mathrm{C}$ (heating/cooling rate: $20^{\circ} \mathrm{C} \mathrm{min}^{-1}$ ) for all of the samples investigated in this study are gathered in Appendix 1.

Heat capacity data are normally presented as a function of temperature (curves in Figure 66) or as a function of composition (Fig. 63) according to the models suggested by Stebbins et al. (1984), Richet (1984) and Richet (1987). The assumption of these models is that composition has no influence on the partial molar heat capacity; and the temperature dependence is expressed by Maier - Kelley equation (Eq. 28). However, in Figure 67 one observes a small compositional dependence for the peralkaline melts.

Heat capacity $c_{p}$ (in $\mathrm{J} \mathrm{g}^{-1} \mathrm{~K}^{-1}$ ) of the glass and of the liquid for all of the samples has been presented in Figure 63a and in the Table 14. Data come from measurements of heat capacity with the heating rate of $20^{\circ} \mathrm{C} \mathrm{min}{ }^{-1}$. Not all of the points in Figure 63a sit on the trend lines (samples G7, G8 and G14); as there is occasionally difficulty in obtaining a stable calibration in a non-temperature controlled laboratory and base line shift can occur. Absolute values can be slightly erroneous, but relative values (configurational heat capacities - see section 5.3.) are correct. However, it is easy to observe, that $c_{p}$ data of the liquid are higher than $c_{p}$ data of the glass and additionally within these two category (liquid and glass) Fe-free samples have higher heat capacity values than Fe-bearing samples. Additional remark is that $c_{p}$ of the peraluminous melts does not change and stays constant with composition. For peralkaline melts from composition where $\gamma \sim 0.48$ towards lower $\mathrm{Al}_{2} \mathrm{O}_{3}$ content the heat capacity slightly decreases.

Figure $63 \mathrm{~b}$ shows the same points of heat capacity but in $\mathrm{J} \mathrm{mol}^{-1} \mathrm{~K}^{-1}$. The models of Richet (1987) and Richet \& Bottinga (1985) have been used to fit the trends. These models have been created to calculate the heat capacity of the melts and glasses as a function of chemical composition and temperature. One observes that models work very well for heat capacity of the glasses and do not work for heat capacity of the liquids. 
a)

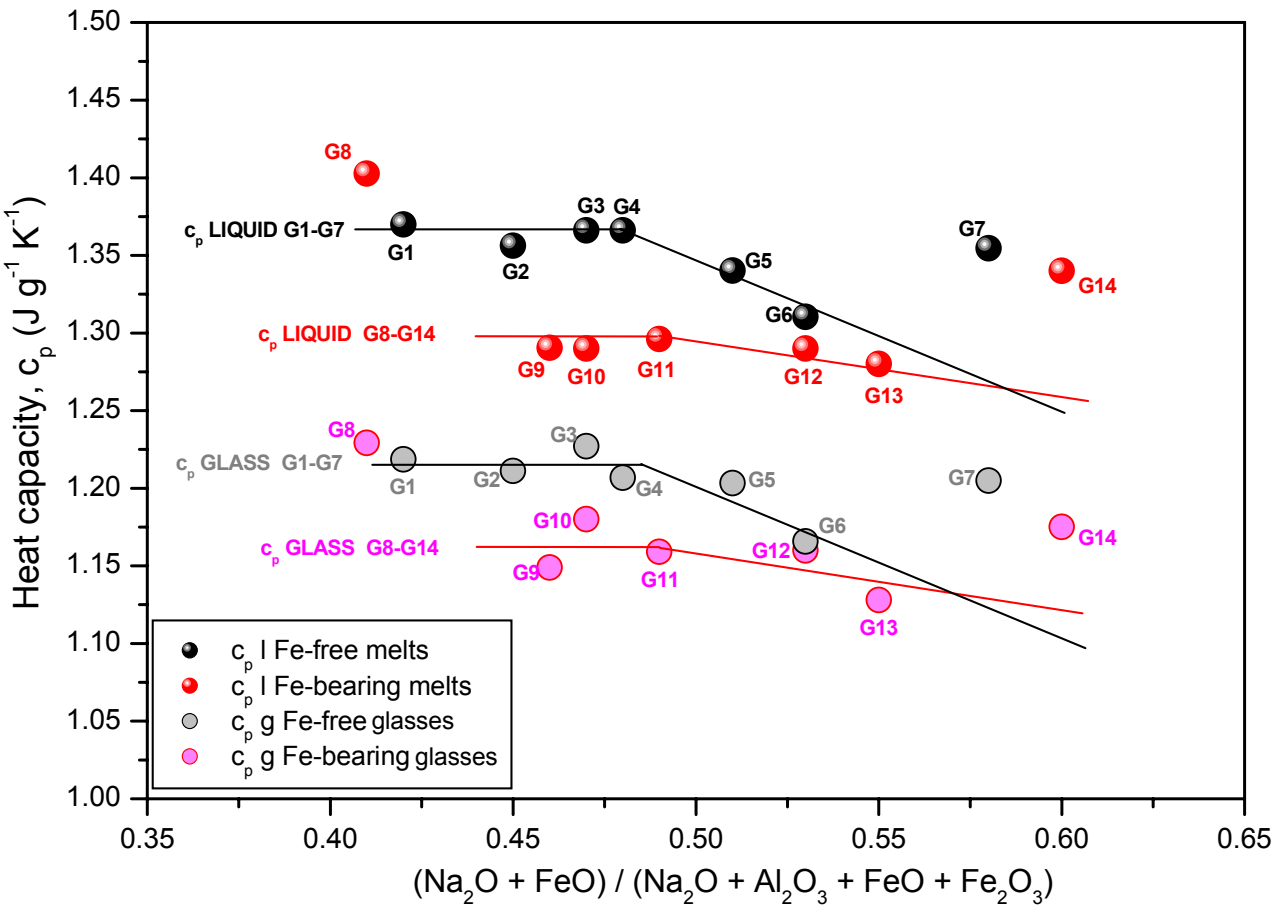

b)

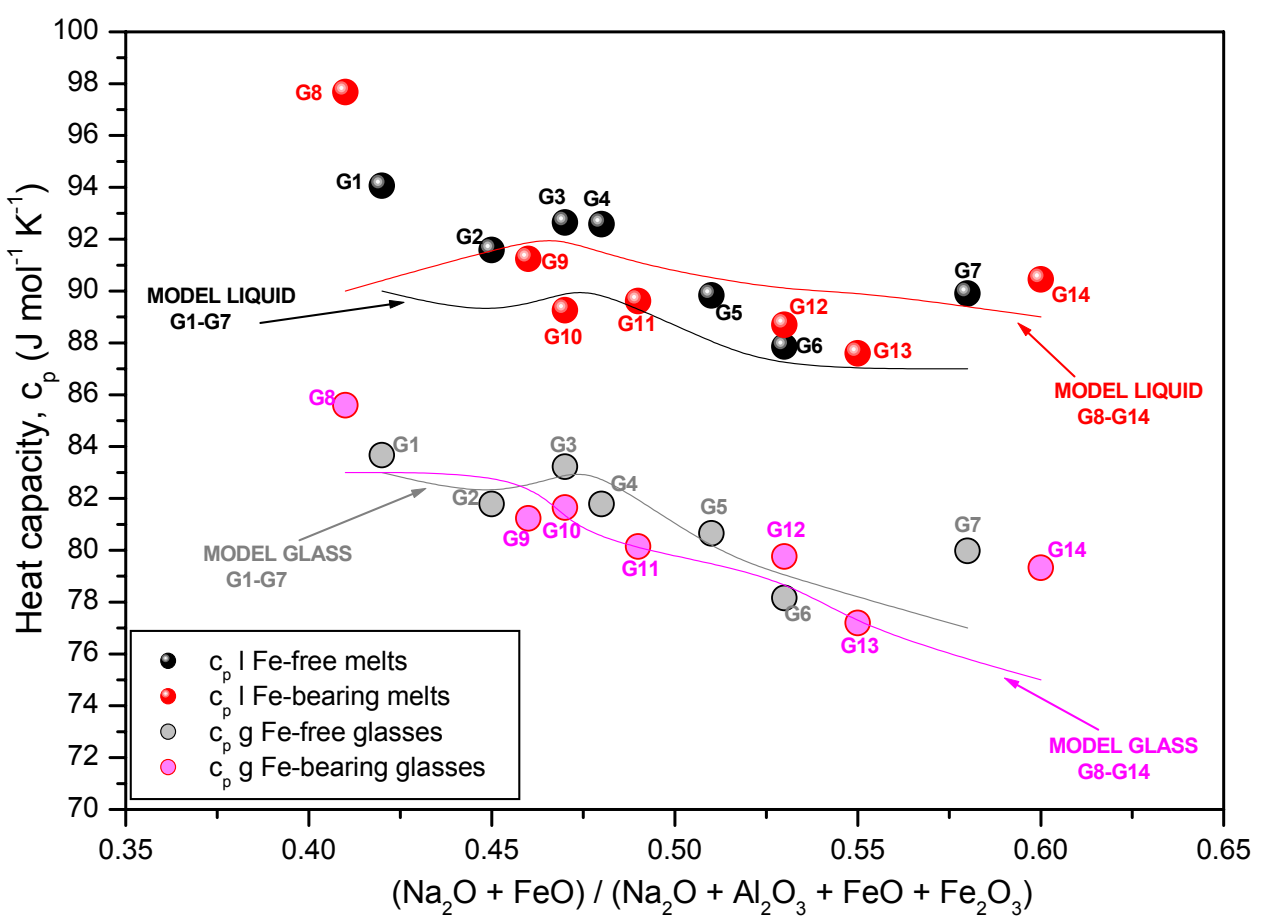

Fig. 63. Heat capacity of the glass $c_{p g}$ and heat capacity of the liquid $c_{p l}$ for peralkaline and peraluminous $\mathrm{Fe}$-free and $\mathrm{Fe}$-bearing samples as a function of compositional parameter $\gamma=\left(\mathrm{Na}_{2} \mathrm{O}+\mathrm{FeO}\right) /\left(\mathrm{Na}_{2} \mathrm{O}+\mathrm{Al}_{2} \mathrm{O}_{3}+\mathrm{FeO}+\mathrm{Fe}_{2} \mathrm{O}_{3}\right) ;$ a) in $\mathrm{J} \mathrm{g}^{-1} \mathrm{~K}^{-1}$; the trends are the guides to the eye; b) in $\mathrm{J} \mathrm{mol}^{-1} \mathrm{~K}^{-1}$; the lines are derived from the models of Richet (1987) and Richet \& Bottinga (1985). 


\begin{tabular}{|c|c|c|c|c|c|}
\hline $\begin{array}{c}\text { Melt } \\
\text { number }\end{array}$ & $\gamma$ & $\begin{array}{c}c_{p g} \\
\left(\mathrm{~J} \mathrm{~g}^{-1} \mathrm{~K}^{-1}\right)\end{array}$ & $\begin{array}{c}c_{p g} \\
\left(\mathrm{~J} \mathrm{~mol}^{-1} \mathrm{~K}^{-1}\right)\end{array}$ & $\begin{array}{c}c_{p l} \\
\left(\mathrm{~J} \mathrm{~g}^{-1} \mathrm{~K}^{-1}\right)\end{array}$ & $\begin{array}{c}c_{p l} \\
\left(\mathrm{~J} \mathrm{~mol}^{-1} \mathrm{~K}^{-1}\right)\end{array}$ \\
\hline $\mathbf{G 0}$ & 1.00 & 1.250 & 75.812 & 1.450 & 90.368 \\
\hline \hline $\mathbf{G 1}$ & 0.42 & 1.219 & 83.667 & 1.370 & 94.061 \\
$\mathbf{G 2}$ & 0.45 & 1.211 & 81.785 & 1.356 & 91.583 \\
$\mathbf{G 3}$ & 0.47 & 1.227 & 83.221 & 1.366 & 92.641 \\
$\mathbf{G 4}$ & 0.48 & 1.207 & 81.790 & 1.366 & 92.579 \\
$\mathbf{G 5}$ & 0.51 & 1.203 & 80.654 & 1.340 & 89.830 \\
$\mathbf{G 6}$ & 0.53 & 1.166 & 78.164 & 1.310 & 87.860 \\
$\mathbf{G 7}$ & 0.58 & 1.205 & 79.969 & 1.355 & 89.905 \\
\hline \hline $\mathbf{G 8}$ & 0.41 & 1.229 & 85.599 & 1.403 & 97.681 \\
$\mathbf{G 9}$ & 0.46 & 1.149 & 81.228 & 1.290 & 91.240 \\
$\mathbf{G 1 0}$ & 0.47 & 1.180 & 81.648 & 1.290 & 89.259 \\
$\mathbf{G 1 1}$ & 0.49 & 1.159 & 80.147 & 1.296 & 89.620 \\
$\mathbf{G 1 2}$ & 0.53 & 1.160 & 79.756 & 1.290 & 88.694 \\
$\mathbf{G 1 3}$ & 0.55 & 1.128 & 77.198 & 1.280 & 87.601 \\
$\mathbf{G 1 4}$ & 0.60 & 1.175 & 79.321 & 1.340 & 90.445 \\
\hline
\end{tabular}

Tab. 14. Table for all of the samples with compositional parameter $\gamma$; heat capacity of the glass $\mathrm{c}_{\mathrm{pg}}$ and liquid $\mathrm{c}_{\mathrm{pl}}$ (in $\mathrm{J} \mathrm{g}^{-1}$ $\mathrm{K}^{-1}$ and in $\mathrm{J} \mathrm{mol}^{-1} \mathrm{~K}^{-1}$ ).

\subsubsection{Glass transition temperature $T_{g}$ and fictive temperature $T_{f}$}

The glass transition temperatures (based on viscosity data) $\mathrm{T}_{\mathrm{g}}{ }^{12}$ (temperature at which $\eta=10^{12}$ Pas) are shown as a function of composition in Figure 64. The $\mathrm{T}_{\mathrm{g}}{ }^{12}$ values for Fe-free and Fe-bearing melts show a change in trends on the peraluminous side of the composition range $(\gamma \sim 0.475)$. This change in trend as a function of $\mathrm{Na} / \mathrm{Al}$ has been discussed previously by Toplis et al. (1997a, b) and Webb et al. (2004) in terms of the structural changes and the presence of triclusters in the melts with changing composition and also in terms of the changes in flow mechanism accompanying such changes in melt structure.

Because $\mathrm{Fe}$ ions have relatively low mobility than sodium, its high-field strength leads to much stronger bonds between $\mathrm{Fe}^{2+}$ as a network modifier and a surrounding structure. This influence of Fe on the structure can be observed in the glass transition $\left(T_{g}\right)$ plot. The similar situation exists in Ca-bearing melts. Increased field strength of Ca leads to disappearing the breaking point on the plot of $\mathrm{T}_{\mathrm{g}}$ (Shelby, 1985; Webb, 2007).

Fictive temperature $T_{f}$ is calculated from Eq. 30 for heating rate $20^{\circ} \mathrm{C} \mathrm{min}^{-1}$ and has been shown in the Table 15. It has been also observed that there is a linear relationship between fictive temperature $T_{f}$ measured for each sample for heating rate $20^{\circ} \mathrm{C} \mathrm{min}^{-1}$ and $\mathrm{T}_{\mathrm{g}}{ }^{12}$ (see Fig. 65). 


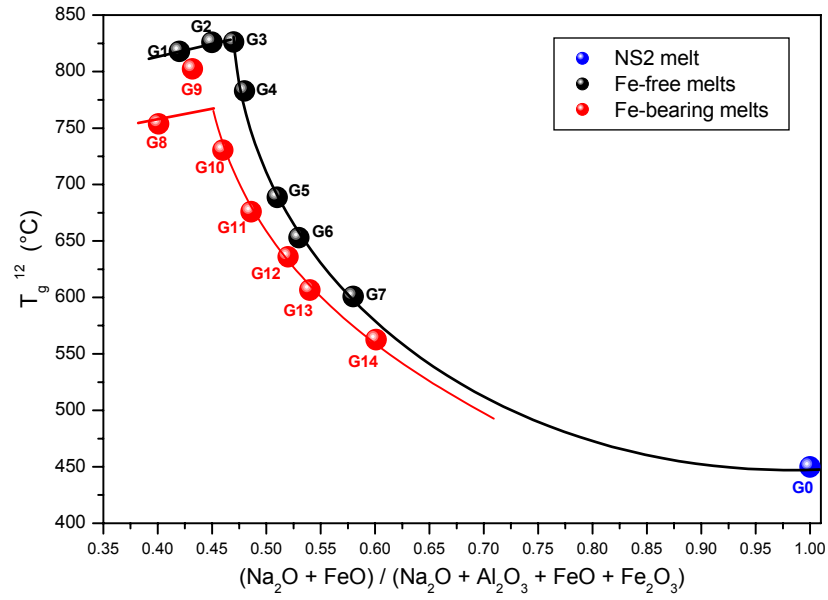

Fig. 64. A plot of $\mathrm{T}_{\mathrm{g}}{ }^{12}$ as a function of compositional parameter $\gamma$.

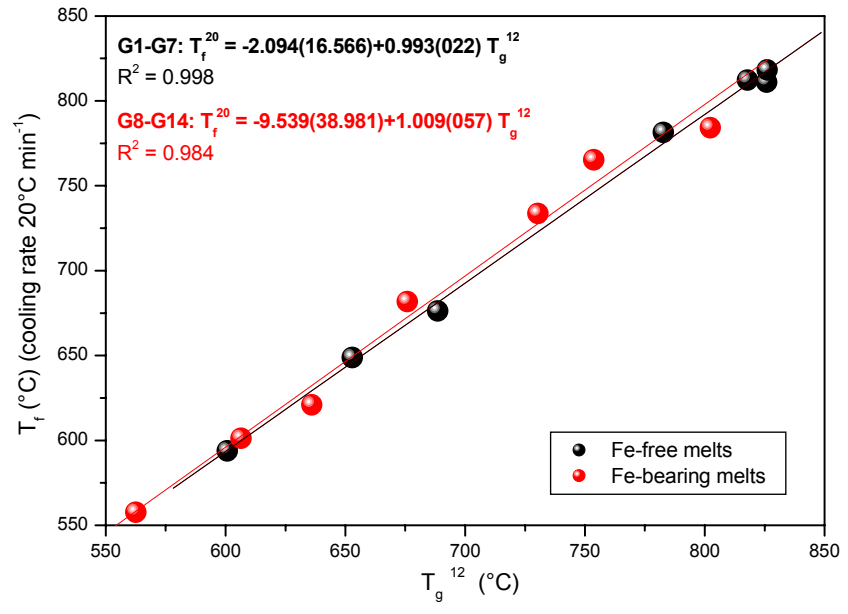

Fig. 65. A plot of $\mathrm{T}_{\mathrm{g}}{ }^{12}$ as a function of fictive temperature $\mathrm{T}_{\mathrm{f}}$ (for heating/cooling rate of $20^{\circ} \mathrm{C}$ $\min ^{-1}$ )

\begin{tabular}{|c|c|c|c|}
\hline $\begin{array}{c}\text { Melt } \\
\text { number }\end{array}$ & $\gamma$ & $T_{g}{ }^{12}\left({ }^{\circ} \mathrm{C}\right)$ & $T_{f}{ }^{20}\left({ }^{\circ} \mathrm{C}\right)$ \\
\hline $\mathbf{G 0}$ & 1.00 & 449.82 & 460.36 \\
\hline \hline $\mathbf{G 1}$ & 0.42 & 817.84 & 812.19 \\
$\mathbf{G 2}$ & 0.45 & 825.88 & 810.97 \\
$\mathbf{G 3}$ & 0.47 & 826.11 & 818.26 \\
$\mathbf{G}$ & 0.48 & 782.77 & 781.44 \\
$\mathbf{G 5}$ & 0.51 & 688.57 & 676.28 \\
$\mathbf{G 6}$ & 0.53 & 652.90 & 648.80 \\
$\mathbf{G 7}$ & 0.58 & 600.74 & 593.81 \\
\hline \hline $\mathbf{G 8}$ & 0.41 & 753.69 & 765.31 \\
$\mathbf{G 9}$ & 0.46 & 802.35 & 784.18 \\
$\mathbf{G 1 0}$ & 0.47 & 730.44 & 733.70 \\
$\mathbf{G 1 1}$ & 0.49 & 675.84 & 681.75 \\
$\mathbf{G 1 2}$ & 0.53 & 636.02 & 620.94 \\
$\mathbf{G 1 3}$ & 0.55 & 606.49 & 601.29 \\
$\mathbf{G 1 4}$ & 0.60 & 562.55 & 557.72 \\
\hline
\end{tabular}

Tab. 15. Glass transition temperature $T_{g}{ }^{12}$ at $\log _{10} \eta=12$; fictive temperature $T_{f}$ (for heating rate of $20^{\circ} \mathrm{C} \mathrm{min}^{-1}$ ).

\subsubsection{R parameter}

3R $\left(24.9 \mathrm{~J} \mathrm{~mol}^{-1} \mathrm{~K}^{-1}\right)$ is a classical limiting value of heat capacity determined empirically from the Dulong - Petit law of atomic heat. $3 R$ is valid only for small $b$ parameter of the Maier-Kelley equation (Eq. 28), that is for small atomic oscillation or for high temperatures. $3 R$ value, where $R$ is ideal gas constant $\left(8.314570(70) \mathrm{J} \mathrm{mol}^{-1} \mathrm{~K}^{-1}\right)$ and is equal:

$$
3 R=k_{B} \cdot N_{A}
$$

where $k_{B}$ is a Boltzmann's constant $\left(1.3806504 \cdot 10^{-23} \mathrm{~J} \mathrm{~K}^{-1}\right)$ and $N_{A}$ is Avogadro's number $\left(6.02214179 \cdot 10^{23} \mathrm{~mol}^{-1}\right)$. The Dulong - Petit limit of $3 \mathrm{R}$ is calculated per mole of atoms, not molecules. 

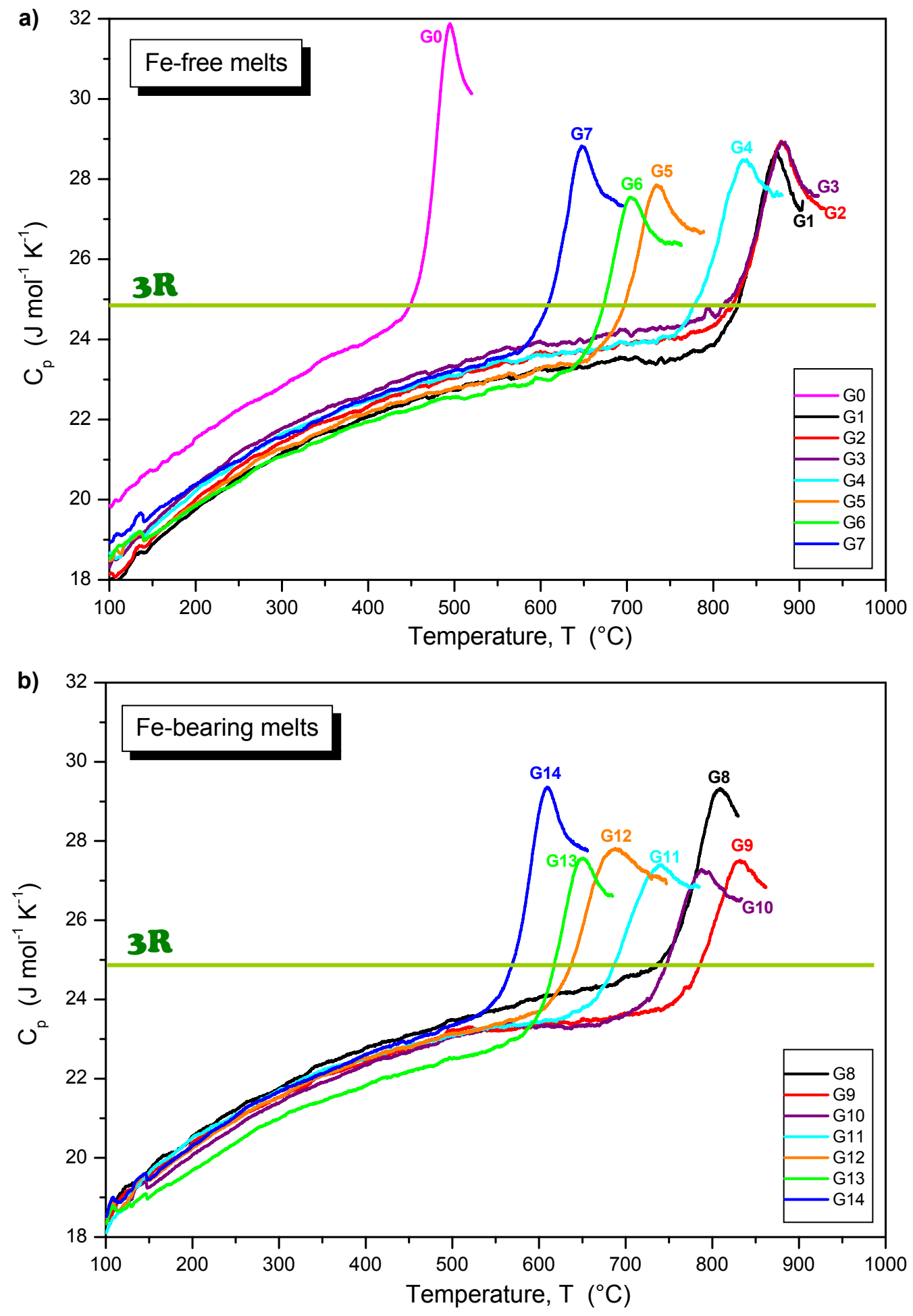

Fig. 66. Plots of classical limiting value $3 R$ for heat capacity calculated in mole per atoms. The horizontal line determine a 3R value for solids: $24.9 \mathrm{~J} \mathrm{~mol}^{-1} \mathrm{~K}^{-1}$. a) data for NS2 (G0) and Fe-free melts (G1-G7); b) data for Fe-bearing melts (G8-G14). 


\subsection{Torsion (forced oscillation)}

\subsubsection{Real and imaginary shear modulus}

The frequency dependent deformation data were obtained from $300-1000^{\circ} \mathrm{C}$. The maximum temperature is that at which it is no longer possible to measure the elastic (instantaneous) component of deformation; this occurs at viscosity of $\sim 10^{9} \mathrm{~Pa} \mathrm{~s}$. The minimum temperature is defined by the point at which the melt breaks away from the alumina torsion rod due to differences in thermal expansion of the two materials.

Figure 67 shows the variation in real and imaginary shear modulus as a function of angular frequency $(\omega=2 \pi f)$ times Maxwell relaxation time $\left(\tau_{M}\right)$. Data of real and imaginary shear modulus are presented in Appendix 2. The relaxation time is calculated from the Maxwell equation $\tau_{M}(T)=\eta_{N}(T) / G_{\infty}$ for Newtonian viscosity $\eta_{N}$ - determined from the micropenetration measurements, and elastic (infinite frequency) shear modulus $G_{\infty}$, which is taken to be the shear modulus at $1 \mathrm{~Hz}$ at the lowest temperature of measurement (see Tab. 16). The relaxation times for all of the melts are presented in Appendix 3. The studies of Rivers and Carmichael (1987), Webb (1992b) and Farnan and Stebbins (1994) (as well as many others) show that the Maxwell relationship successfully calculates the structural relaxation time of silicate melts. Plotting data from different temperatures on the same curve uses the principle of "thermorheological simplicity" which assumes that the structure and mechanism of flow in these melts does not change over the temperature interval of the measurements (e.g. Narayanaswamy, 1988).

Herzfeld \& Litovitz (1959) developed a general equation to describe the frequency dependence of the shear modulus of a material independent of its structure;

$$
G^{*}(\omega)=\frac{G_{\infty} \omega^{2} \tau^{2}}{1+\omega^{2} \tau^{2}}+i \frac{G_{\infty} \omega \tau}{1+\omega^{2} \tau^{2}}
$$

where $G_{\infty}$ is infinite frequency elastic shear modulus, $\tau$ is the structural relaxation time and $\omega$ is angular frequency (see also Nowick \& Berry, 1972; Webb, 1991). The dotted lines in Figure 67 are the real and imaginary shear modulus expected from the theory of Herzfeld \& Litovitz (1959). As can be seen in Figure 67, none of the present data is adequately described by this equation. The solid lines are fits to the data (see also Figures 68 and 69) based on the assumption that there is a distribution of relaxation times. Data of the lines are given in Appendix 4a,b (for samples G0 and G1-G7) and in Appendix 4c,d (for samples G8-G14). 
Fig. 67. Real and imaginary components of the shear modulus of the present melts as a function of $\log _{10} \omega \tau_{\mathrm{M}}$. Open circles are for real shear modulus; solid circles are for imaginary shear modulus; the dotted line are the model of Herzfeld \& Litovitz (1959) for a single structure melt; solid line of the fitting of the data points; grey line is fitting of the imaginary parameters to the real shear modulus of the sample. Not all the data has been shown; for some of the samples the plot has been cut at $\log _{10} \omega \tau_{\mathrm{M}}=10$.
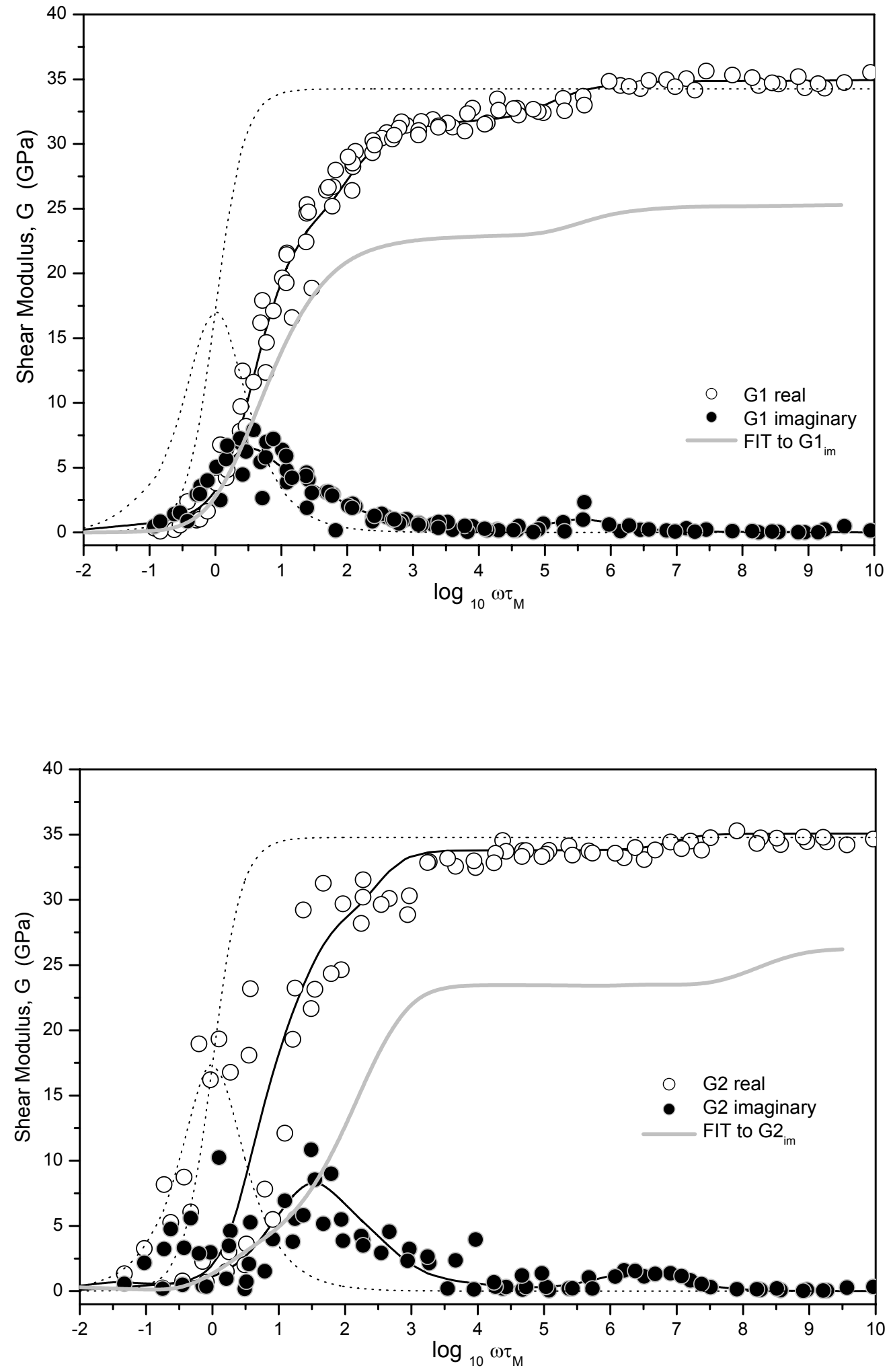
Fig. 67. continuation...
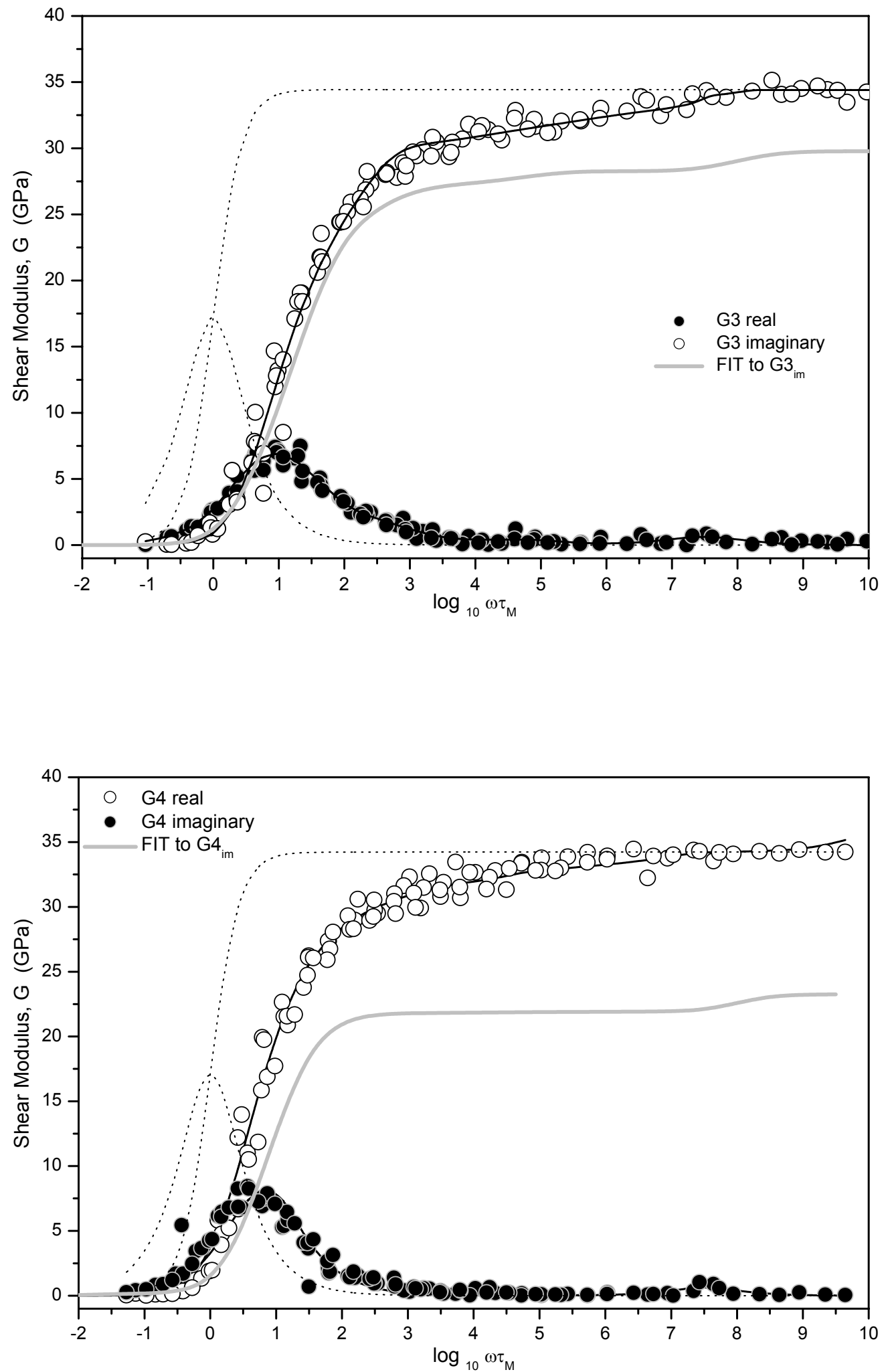
Fig. 67. continuation...
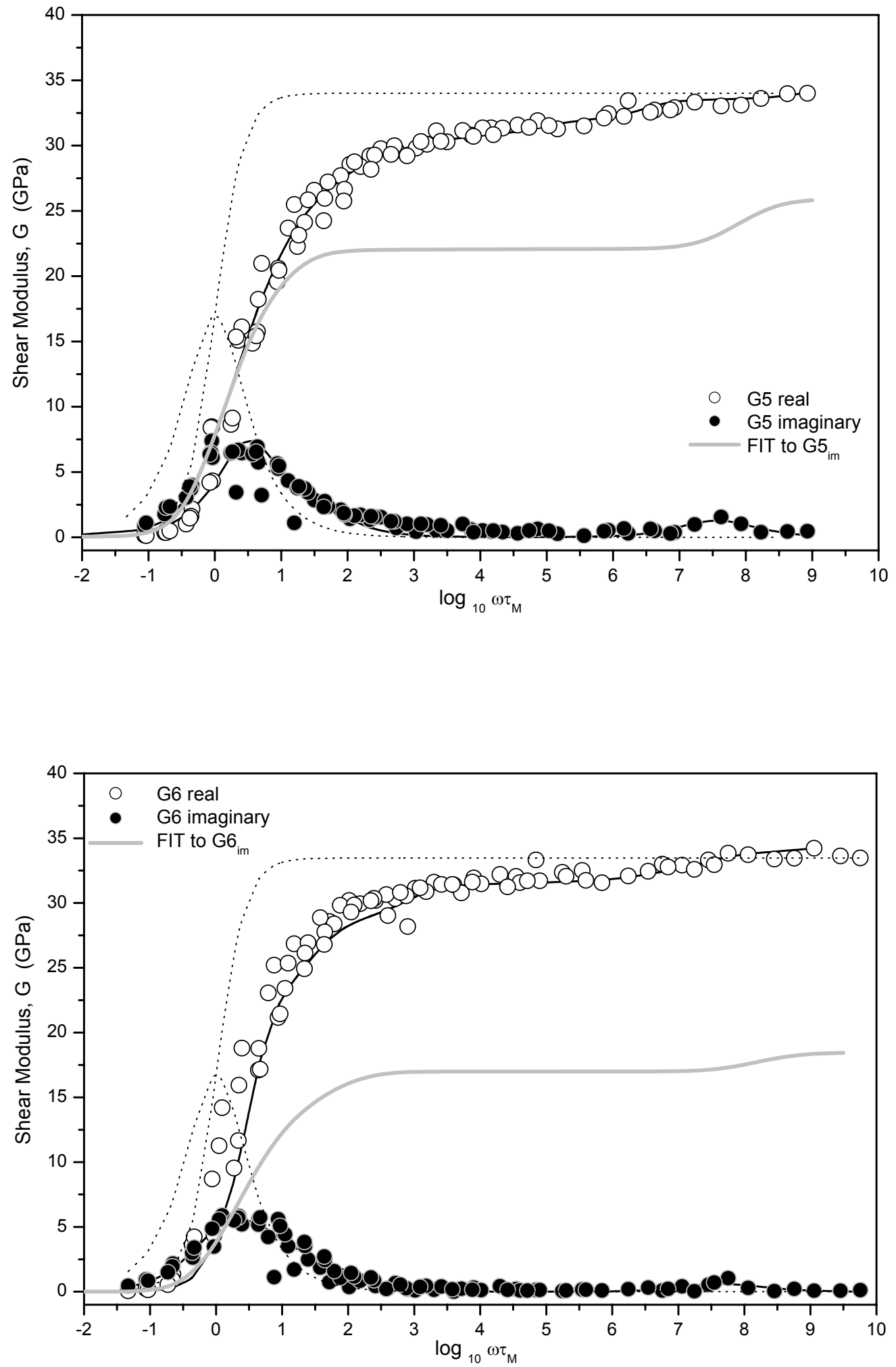
Fig. 67. continuation...
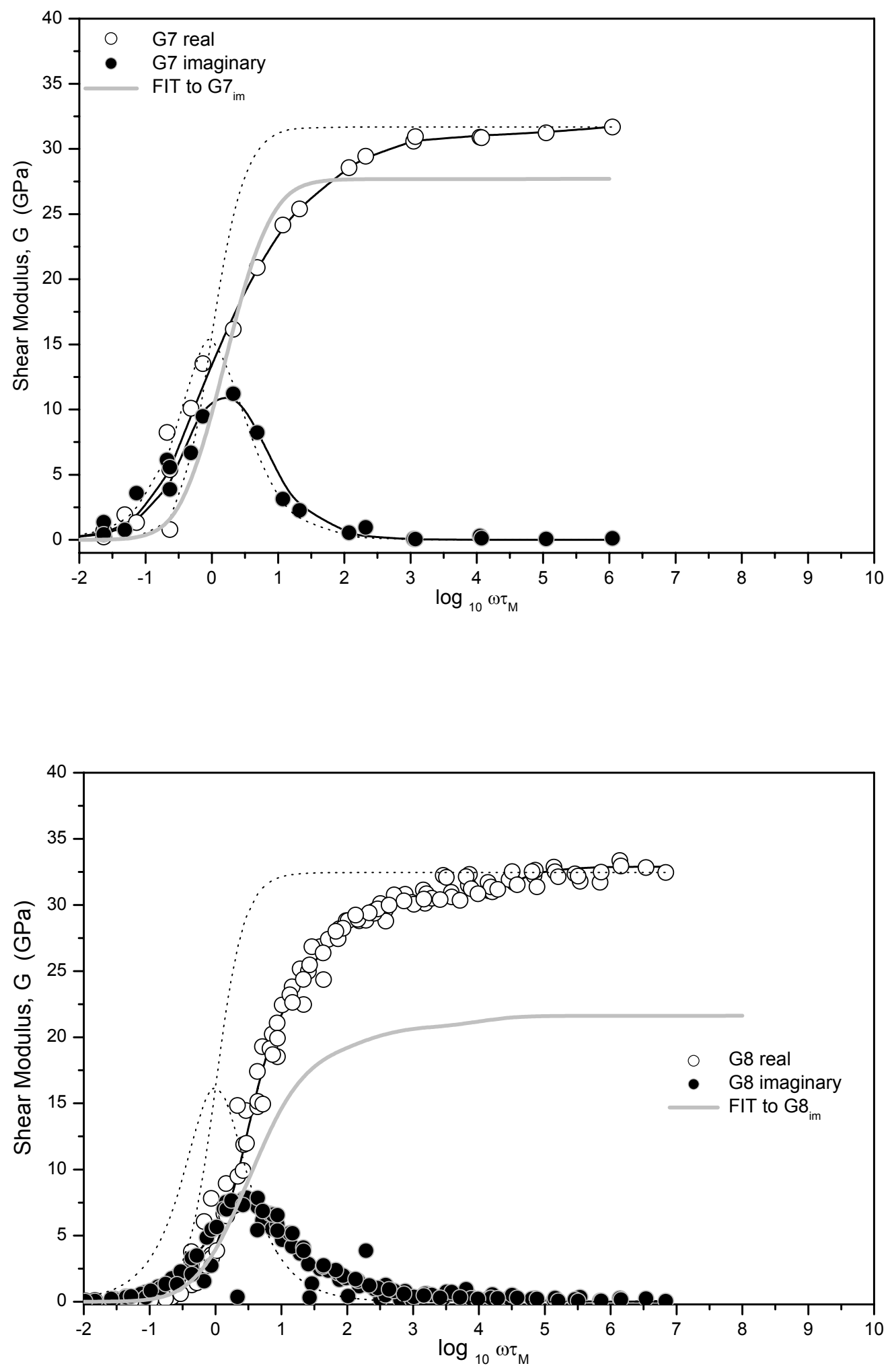
Fig. 67. continuation...
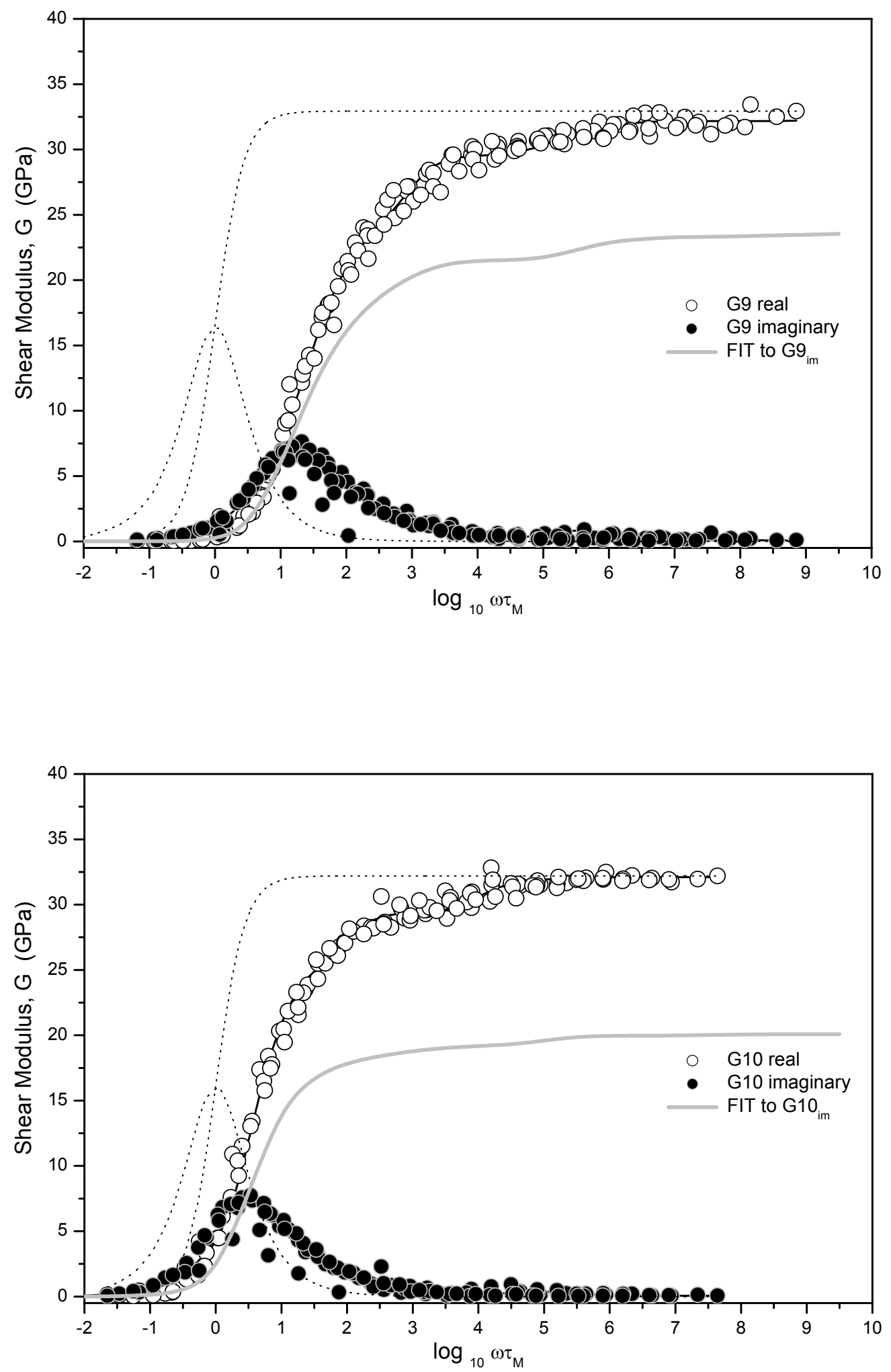
Fig. 67. continuation...
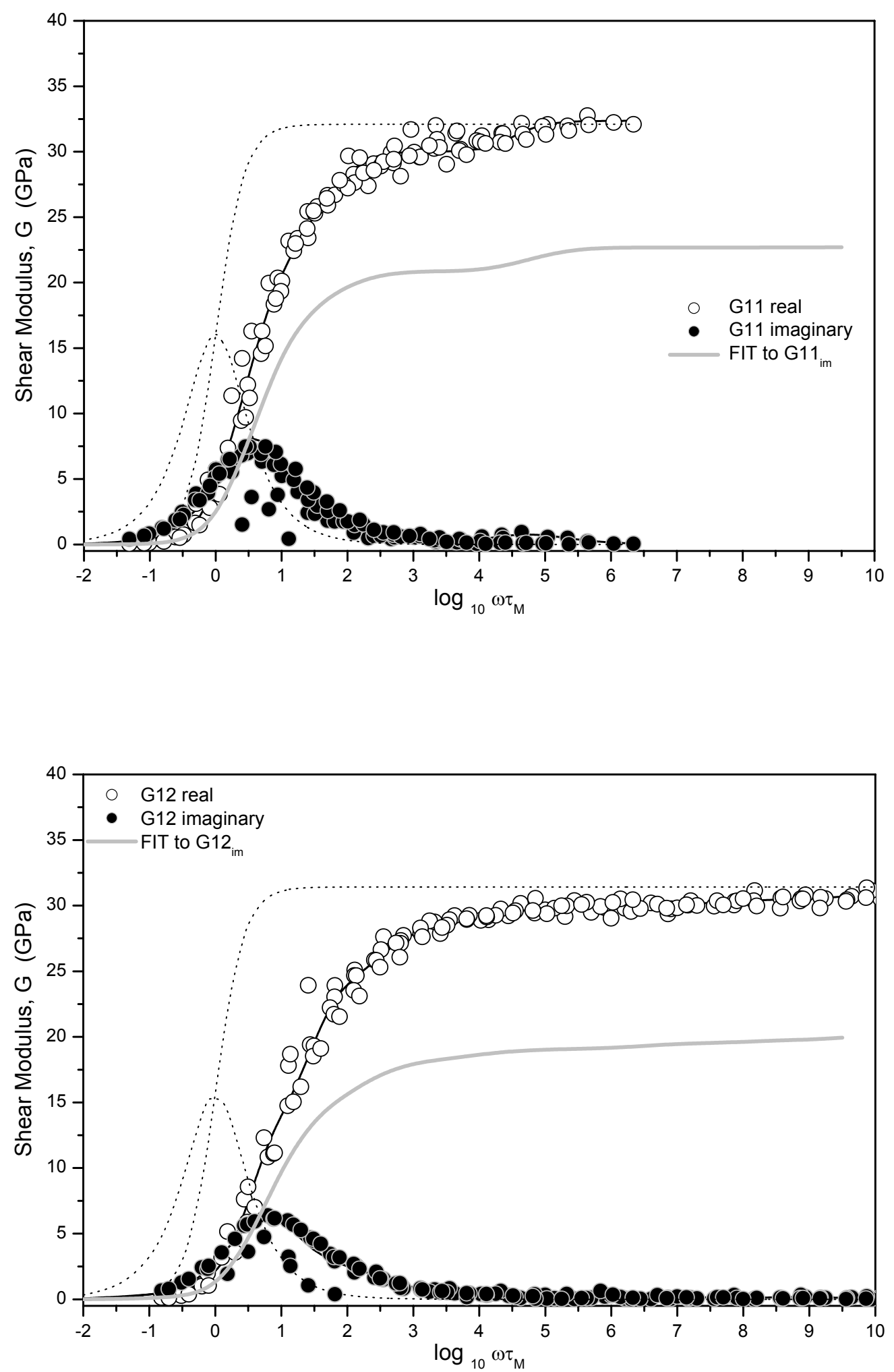
Fig. 67. continuation...
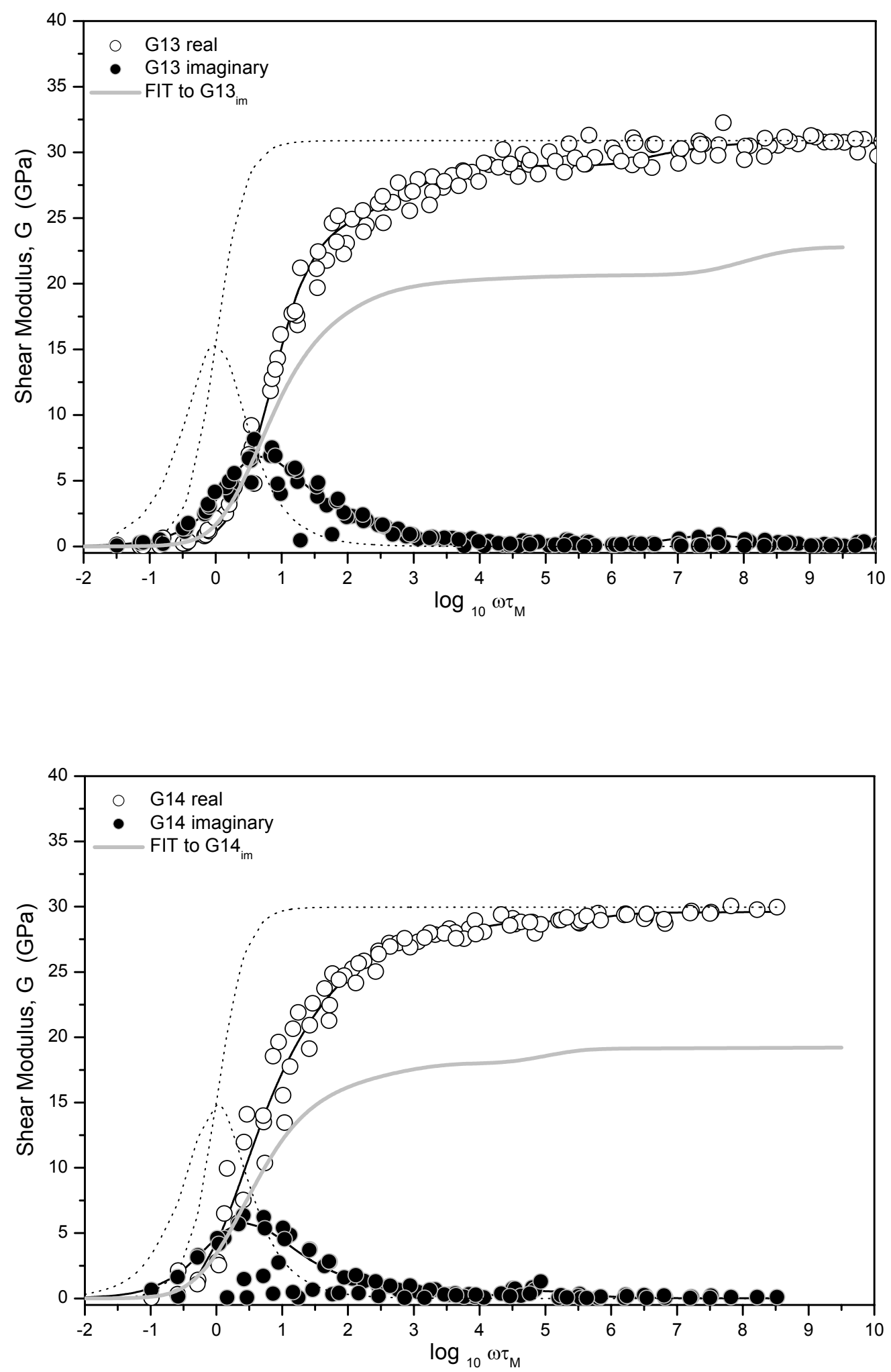

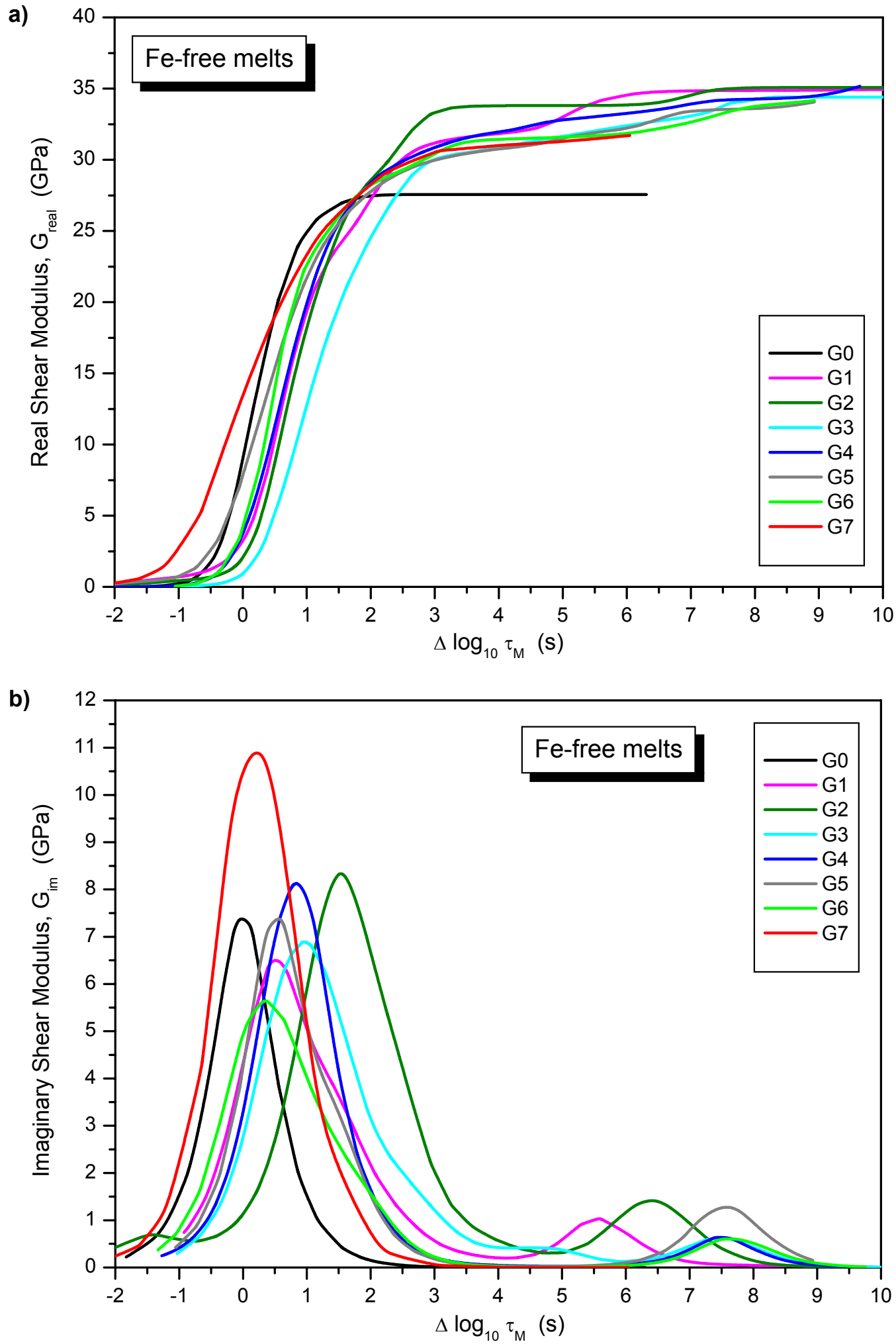

Fig. 68. The fits to the shear modulus data for NS2 (G0) and Fe-free melts (G1-G7), using Eq. 57. a) fitted lines for real shear modulus data; b) fitted lines for imaginary shear modulus data. See also Appendix $4 a, b$. 
a)

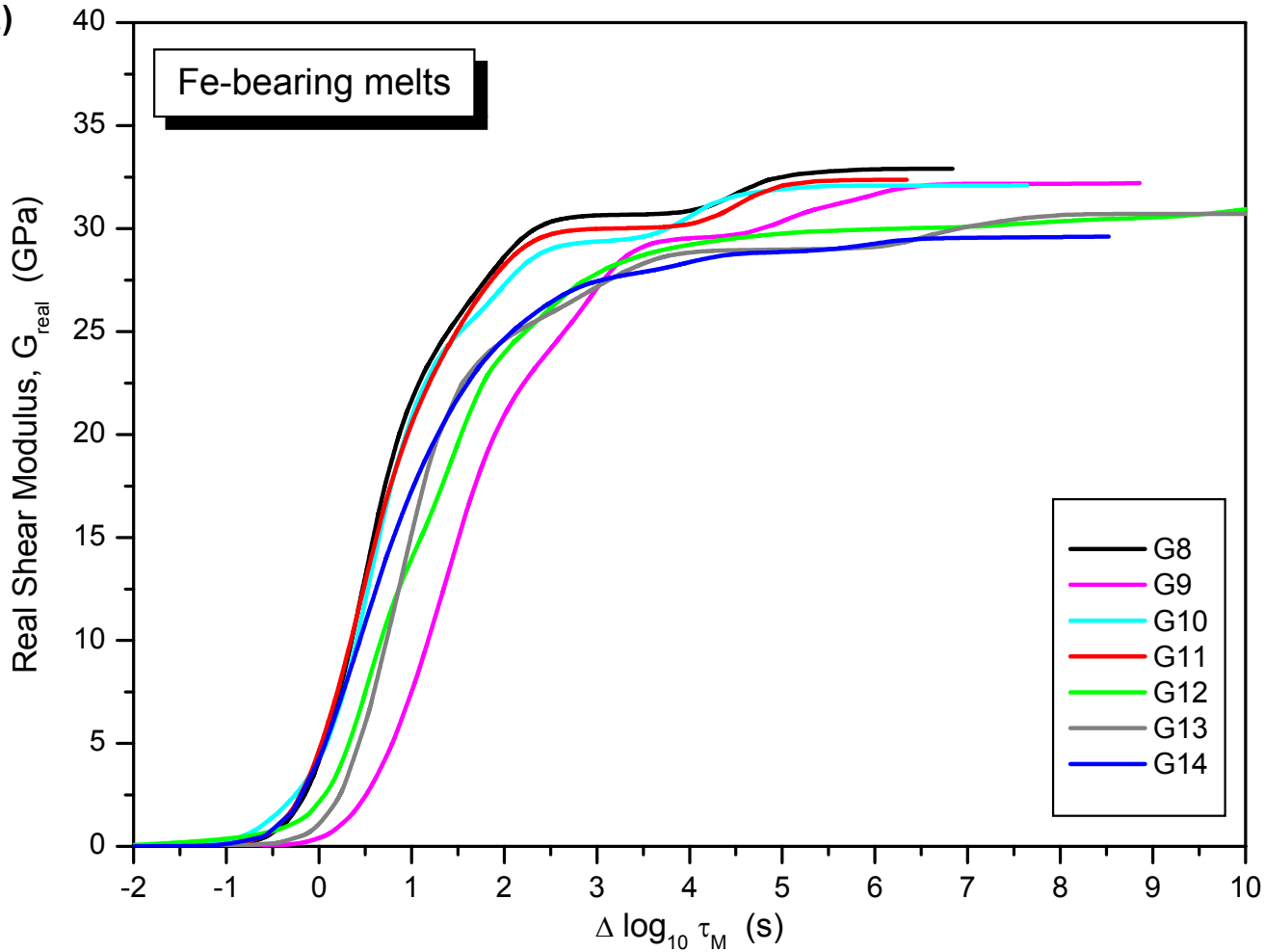

b)

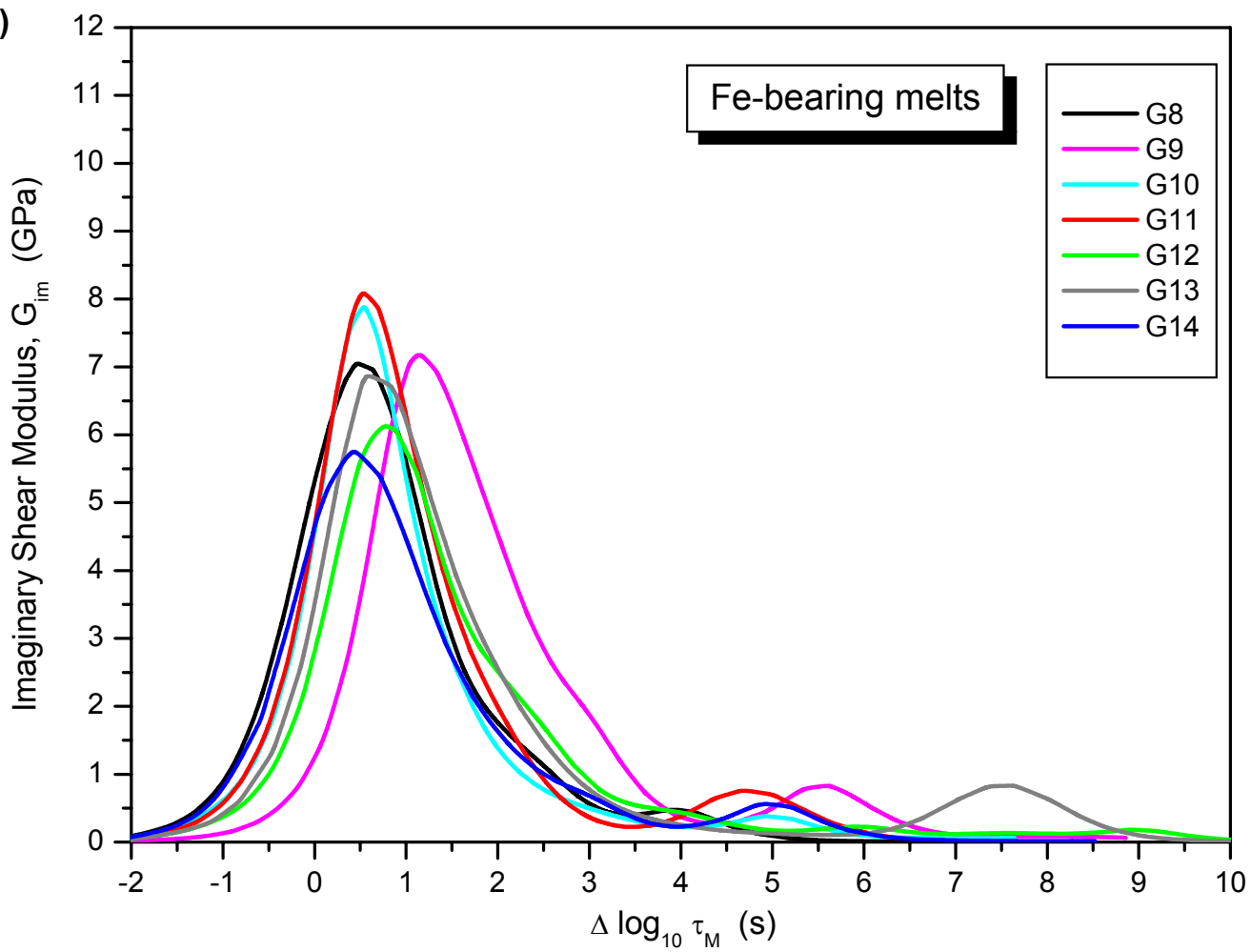

Fig. 69. The fits to the shear modulus data for Fe-bearing melts (G8-G14), using Eq. 57. a) fitted lines for real shear modulus data; b) fitted lines for imaginary shear modulus data. See also Appendix 4c,d. 
Instead of assuming the form of the distribution of relaxation time, the data have been fit by a summation of Eq. 57 :

$$
G^{*}(\omega \tau)=\sum_{x=-8.5}^{1.5} G_{x}^{*}\left(\omega \tau \cdot 10^{x}\right)
$$

The real and imaginary parts of the data have been fit separately. The resulting calculated distribution of relaxation time is shown in Figures 70 and 71 and the parameters of the fit are given in Appendix 5a,b.

Table 16 compares the $G_{\infty}$ values determined at $1 \mathrm{~Hz}$, with the average of the frequency independent $G_{a v}$ values determined from the forced oscillation measurements, and the room temperature elastic shear moduli determined on the glasses by ultrasonic methods - $G_{\text {ultra. }}$. The $G_{a v}$ moduli were determined over the temperature range between lowest measured temperatures up to $500-600^{\circ} \mathrm{C}$ (depending on the sample) and appear to be frequency independent and shows very little temperature dependence over the 50 $200^{\circ} \mathrm{C}$ (depending on the sample) range of calculation. The values of $G_{a v}$ and $G_{u l t r a}$ are shown in Figure $57 a, b$ as a function of the compositional parameter $\gamma$.

Tab. 16. Shear moduli $\mathrm{G}_{\mathrm{av}}$ and $\mathrm{G}_{\infty}$ from the torsion data together with that determined by ultrasonic techniques at room temperature $\mathrm{G}_{\text {ultra. }}$.

\begin{tabular}{|c|c|c|c|c|c|c|c|}
\hline \multirow[b]{3}{*}{ G0 } & \multicolumn{4}{|c|}{ Torsion Measurements } & \multirow{2}{*}{\multicolumn{3}{|c|}{$\begin{array}{c}\text { Pulse Echo Overlap Technique } \\
\text { Gultra }_{\text {(GPa) }} \\
\end{array}$}} \\
\hline & \multicolumn{3}{|c|}{$\mathrm{G}_{\mathrm{av}}(\mathrm{GPa})$} & \multirow{2}{*}{$\frac{\mathbf{G}_{\infty}(\mathbf{G P a})}{27.45}$} & & & \\
\hline & 27.730 & \pm & 0.278 & & 29.887 & \pm & 0.232 \\
\hline G1 & 34.431 & \pm & 0.479 & 34.26 & 37.389 & \pm & 0.754 \\
\hline G2 & 34.663 & \pm & 0.265 & 34.78 & 37.842 & \pm & 1.091 \\
\hline G3 & 34.358 & \pm & 0.402 & 34.43 & 38.036 & \pm & 0.147 \\
\hline G4 & 33.984 & \pm & 0.581 & 34.23 & 38.074 & \pm & 0.625 \\
\hline G5 & 33.863 & \pm & 0.219 & 34.01 & 37.122 & \pm & 0.155 \\
\hline G6 & 33.485 & \pm & 0.349 & 33.47 & 36.650 & \pm & 0.191 \\
\hline G7 & 31.034 & \pm & 0.376 & 31.68 & 34.284 & \pm & 0.198 \\
\hline G8 & 31.998 & \pm & 0.746 & 32.45 & 34.228 & \pm & 0.292 \\
\hline G9 & 32.108 & \pm & 0.636 & 32.94 & 34.090 & \pm & 0.129 \\
\hline G10 & 31.825 & \pm & 0.373 & 32.20 & 33.555 & \pm & 0.514 \\
\hline G11 & 31.775 & \pm & 0.472 & 32.09 & 33.540 & \pm & 0.362 \\
\hline G12 & 31.078 & \pm & 0.292 & 31.42 & 32.230 & \pm & 0.274 \\
\hline G13 & 29.810 & \pm & 0.489 & 30.89 & 31.256 & \pm & 0.247 \\
\hline G14 & 29.261 & \pm & 0.378 & 29.96 & 30.938 & \pm & 0.189 \\
\hline
\end{tabular}


a)

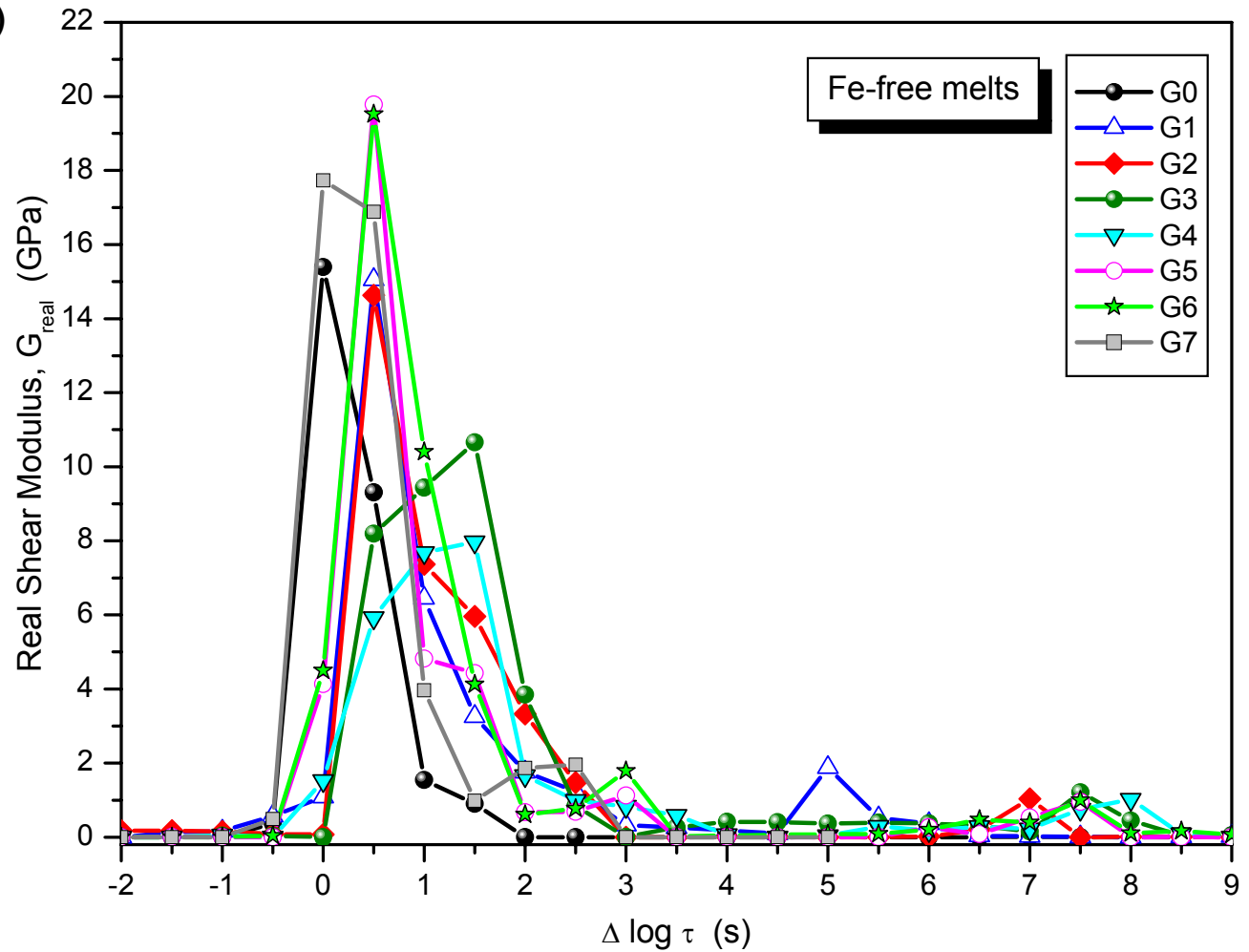

b)

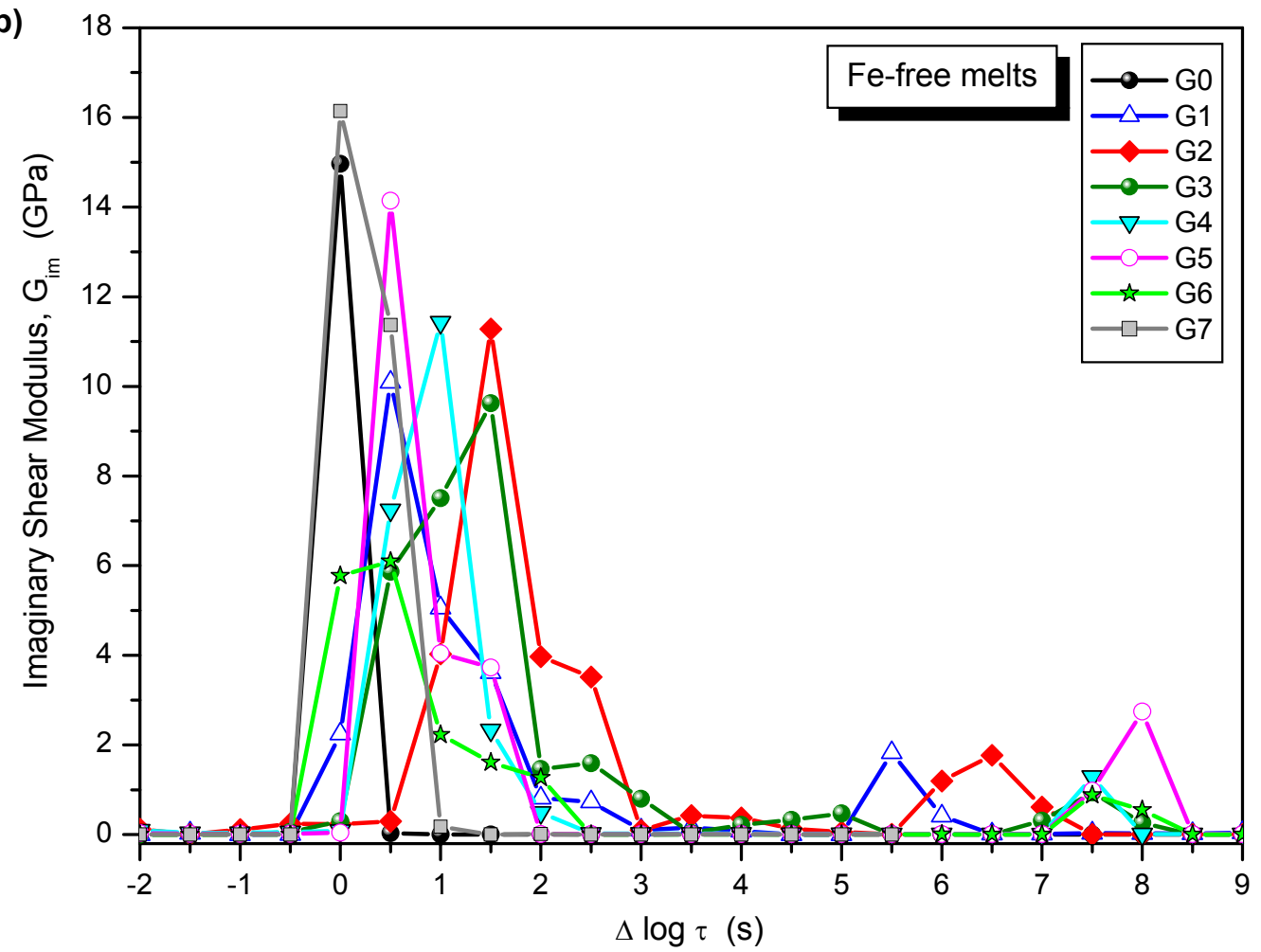

Fig. 70. The distribution of relaxation times for Fe-free samples (G0 and G1-G7) calculated from Eq. 58. The lines on the plot a) are calculated from the real component of the shear modulus, the lines from plot b) show the distributions calculated from the imaginary part of the modulus. The structural relaxation of $G 7$ and $G 0$ is centred on $\Delta \log _{10} \tau \sim 0$, while that of $G 2$ is centred on a timescale 1.5 order of magnitude faster than the Maxwell relaxation time. In all cases, a second relaxation is calculated for relaxation times between $\sim 5.5$ and $\sim 7.5$ orders of magnitude faster than the Maxwell relaxation time. See also Appendix 5a. 

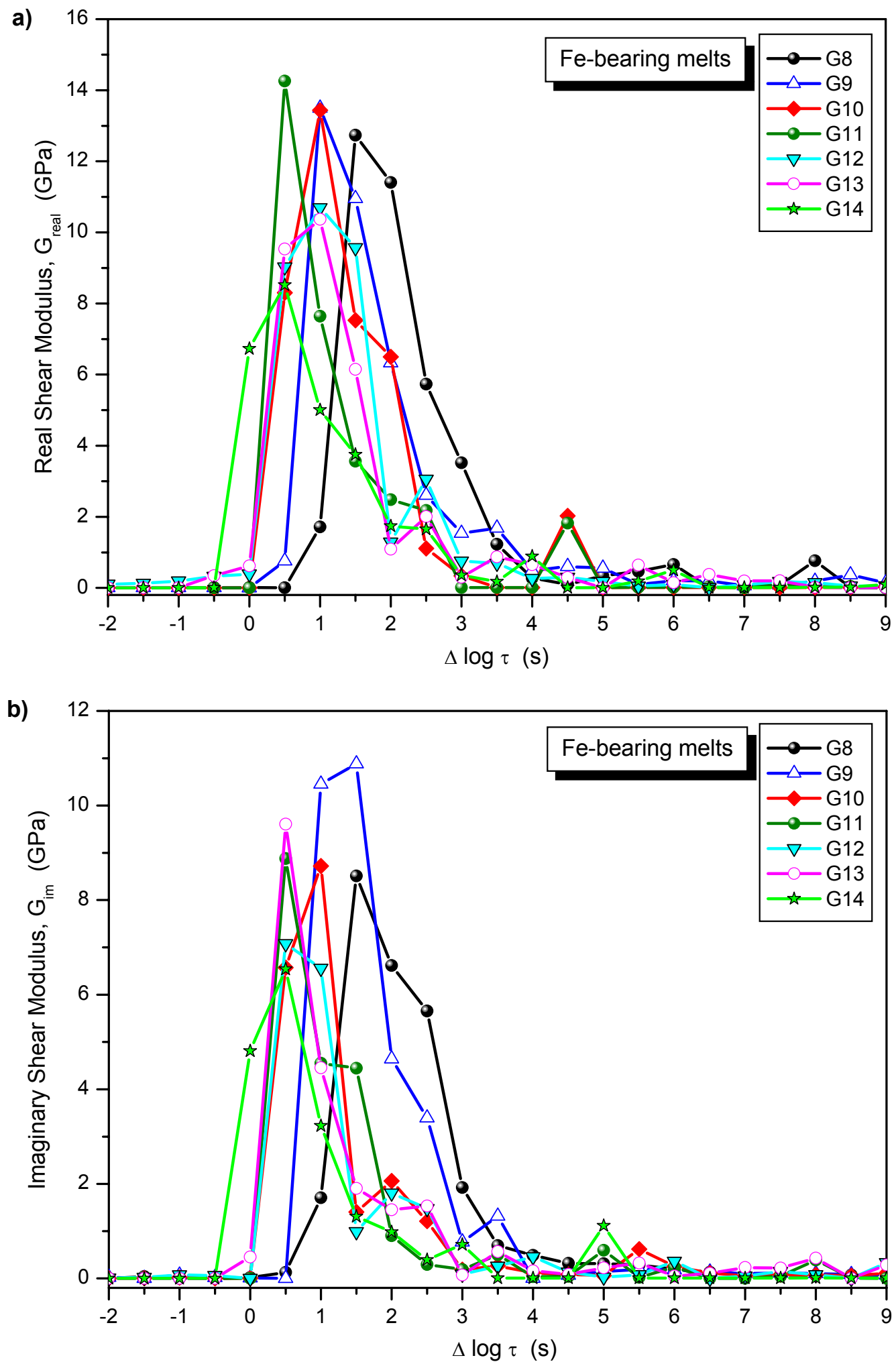

Fig. 71. The distribution of relaxation times for Fe-bearing samples (G8-G14) calculated from Eq. 58. The lines on the plot a) are calculated from the real component of the shear modulus, the lines from plot b) show the distributions calculated from the imaginary part of the modulus. There is no sample with structural relaxation centred on $\Delta \log _{10} \tau=0$. The other peaks are centred on a timescale 0.5-1.5 order of magnitude faster than the Maxwell relaxation time. Location of the second relaxation is not clear. See also Appendix $5 \mathrm{~b}$. 


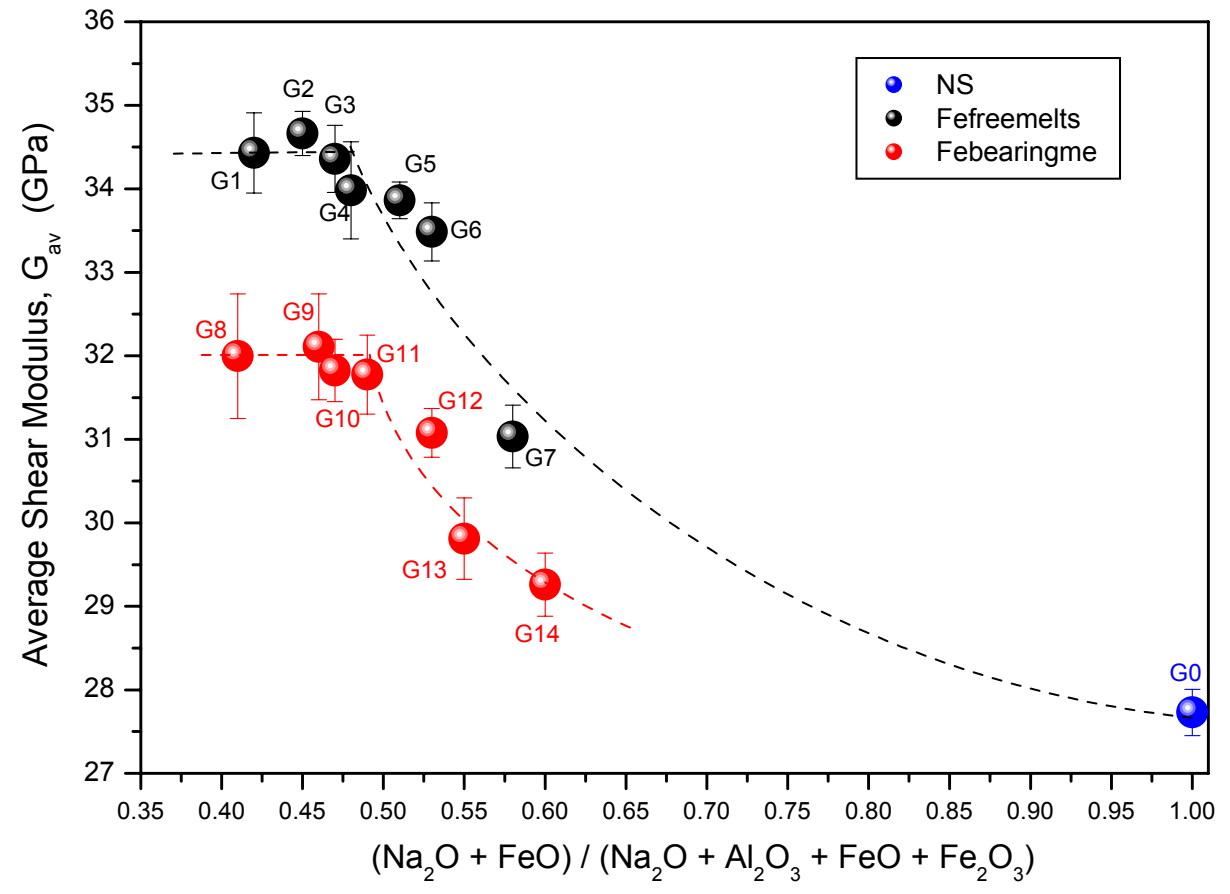

Fig. 72. The average elastic shear moduli as a function of composition - data obtained from torsion apparatus; blue point is for sodium silicate melt NS2 (G0), black points are for data of $\mathrm{G}_{\mathrm{av}}$ for Fefree samples (G1-G7), red points - for Fe-bearing melts (G8-G14). The dashed lines are the suggested trends in the data.

Two peaks are observed in the imaginary component of the shear modulus. Both peaks are accompanied by an increase in the real part of the shear modulus. Each peak in the imaginary modulus must be accompanied by an increase in shear modulus. The existence of this second fast relaxation peak is confirmed by the fitting of Eq. 58 independently to the real and imaginary modulus data.

The peak which occurs at the lowest frequencies is the $\alpha$-relaxation and therefore caused by the loss of energy to the motion of $\mathrm{Si}$ and $\mathrm{O}$ ions in the melt - the glass transition - which results in the change in shear modulus from 0 to $\sim 35 \mathrm{GPa}$. The second peak occurs $25.5-7.5$ orders of magnitude faster than the $\alpha$-peak (depending on the location of $\alpha$-peak) and is accompanied by a 2-3 GPa increase in the real part of the modulus. There are two energy loss processes (other than that associated with the lifetime of $\mathrm{Si}-\mathrm{O}$ bonds) expected in these melts - the motion of $\mathrm{Al}^{3+}$ ions and the motion of $\mathrm{Na}^{+}$ions - see Figure 73. 


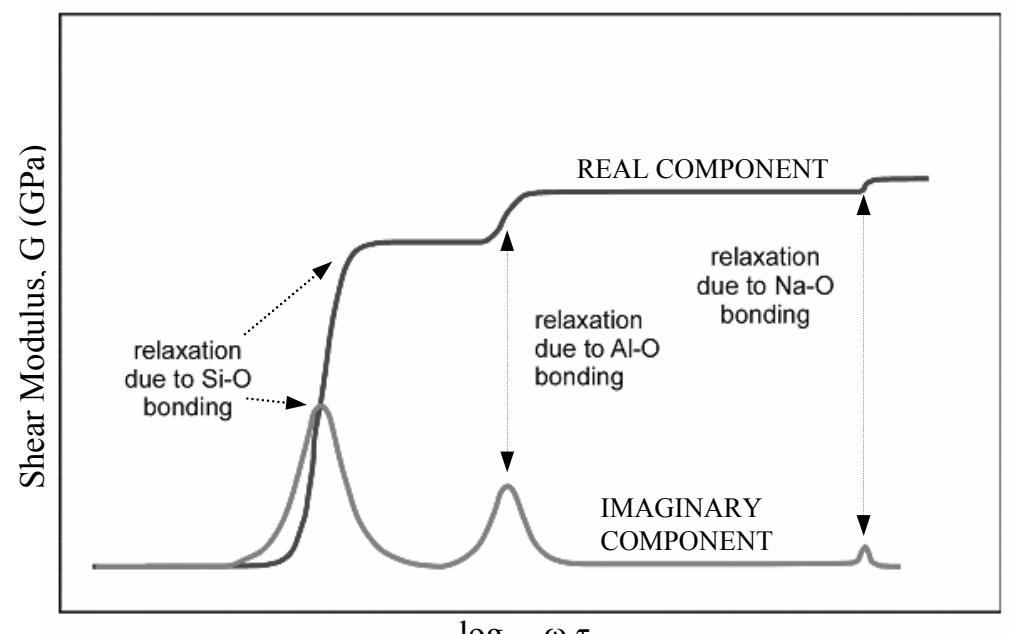

$\log _{10} \omega \tau_{M}$
Fig. 73. The known change in modulus with $\omega \tau_{\mathrm{M}}$ for the motion of $\mathrm{Si}$ and $\mathrm{O}$ atoms in silicate melts together with theoretically expected loss modulus associated with the motion of $\mathrm{Al}^{3+}$ and $\mathrm{Na}^{+}$atoms in the present melts.

The width of the $\alpha$-imaginary peak is the same for all the melt compositions with the FWHM (full width at half maximum) for NS2 melt equals 1.13 and the range 1.40-2.68 for G1-G14 (see Table 17) with no clear compositional dependence. However, the position of the peak moves from being centred on $\tau_{M}$ - the Na-rich compositions, to being centred on a timescale 1.5 order of magnitude smaller - the Al-rich compositions.

Such fast relaxation has never been seen before in a silicate melt. The data of Mills (1974), Bagdassarov et al. (1993) and Webb (1992a) all show that the $\alpha$-peak in silicate melts determined by mechanical spectroscopy is centred on $\log _{10} \omega \tau=0 \pm 0.5$.

\begin{tabular}{|c|c|}
\hline Melt number & $\begin{array}{c}\text { FWHM } \\
\log _{\mathbf{1 0}} \boldsymbol{\omega} \boldsymbol{\tau}_{\mathbf{M}}\end{array}$ \\
\hline \hline $\mathbf{G 0}$ & 1.13 \\
\hline $\mathbf{G 1}$ & 1.90 \\
$\mathbf{G 2}$ & 2.68 \\
$\mathbf{G 3}$ & 1.80 \\
$\mathbf{G 4}$ & 1.40 \\
$\mathbf{G 5}$ & 2.01 \\
$\mathbf{G 6}$ & 1.82 \\
$\mathbf{G 7}$ & 1.52 \\
\hline $\mathbf{G 8}$ & 1.83 \\
$\mathbf{G 9}$ & 1.57 \\
$\mathbf{G 1 0}$ & 1.67 \\
$\mathbf{G 1 1}$ & 1.81 \\
$\mathbf{G 1 2}$ & 1.67 \\
$\mathbf{G 1 3}$ & 1.69 \\
$\mathbf{G 1 4}$ & 1.80 \\
\hline
\end{tabular}

Tab. 17. FWHM (full width at half maximum) for $\alpha$-peak in investigated silicate melts: NS2 (G0), Fe-free (G1G7) and Fe-bearing (G8-G14) 


\subsubsection{Comparison of the shear moduli with literature data}

Shear modulus data determined in this study can be also compared with data of different silicates.

As seen in Figure 74, the maximum in the imaginary part of the shear modulus of G0 $\left(0.33 \mathrm{Na}_{2} \mathrm{O}-0.67 \mathrm{SiO}_{2}\right)$ is centred on $\log _{10} \omega \tau=0$. The data are compared to both the data of Mills (1974) on the same composition at the same deformation frequencies, as well as the theory of Herzfeld \& Litovitz (1959) for a melt with a single relaxation time and mechanism. Here, a $\mathrm{G}_{\infty}$ of $27.45 \mathrm{GPa}$ was determined.

Mills (1974) determined $\mathrm{G}_{\infty}=15 \mathrm{GPa}$ for the same melt composition (see Table 18). The room temperature shear modulus for this melt composition determined by ultrasonic methods is $29.89 \mathrm{GPa}$, with $24.1 \mathrm{GPa}$ presented in compilation of Ahrens (1995). There is good agreement between the present torsion data and that determination by ultrasonic techniques - for a dG/dT $=-6.9 \mathrm{MPa} \mathrm{K}^{-1}$. (see Table 11, Fig. 57a,b). A dG/dT of -12.0 MPa $\mathrm{K}^{-1}$ is needed to bring Mills' high temperature shear modulus into agreement with the present ultrasonic data. Both of these $d G / d T$ values are reasonable, with values of $-7.6 \mathrm{MPa} \mathrm{K}^{-1}$ and $-10.6 \mathrm{MPa} \mathrm{K}^{-1}$ being found in the literature for 30 and $35 \mathrm{~mol} \% \mathrm{Na}_{2} \mathrm{O}$ glasses (Bansal \& Doremus, 1986).

Tab. 18. Shear moduli of melts and glasses measured by different methods and authors.

\begin{tabular}{|c|c|c|c|c|c|}
\hline & $\begin{array}{l}\text { Torsion } \\
\text { (this study) }\end{array}$ & $\begin{array}{l}\text { Ultrasonic } \\
\text { value } \\
\text { (this study) }\end{array}$ & Mills (1974) & $\begin{array}{c}\text { Bansal \& } \\
\text { Doremus } \\
(1986)\end{array}$ & $\begin{array}{c}\text { Ahrens } \\
\text { (1995) }\end{array}$ \\
\hline & \multicolumn{5}{|c|}{ (GPa) } \\
\hline $\mathrm{SiO}_{2}$ & & & 29 & $\begin{array}{c}31.2 \\
\text { (room temp) }\end{array}$ & \\
\hline $\begin{array}{c}\text { Borosilicate } \\
\text { glass }\end{array}$ & $\begin{array}{l}26.04 \pm 1.64 \\
\text { (room temp) }\end{array}$ & $\begin{array}{l}26.37 \pm 0.25 \\
\text { (room temp) }\end{array}$ & & & \\
\hline $\begin{array}{c}33 \mathrm{Na}_{2} \mathrm{O}-67 \mathrm{SiO}_{2} \\
\text { (G0) }\end{array}$ & $\begin{array}{l}27.45 \pm 2.5 \\
\left(@ 400^{\circ} \mathrm{C}\right) \\
\end{array}$ & $\begin{array}{l}29.89 \pm 0.23 \\
\text { (room temp) }\end{array}$ & $\begin{array}{c}15 \\
\left(@ 414^{\circ} \mathrm{C}\right)\end{array}$ & & 24.1 \\
\hline $30 \mathrm{Na}_{2} \mathrm{O}-70 \mathrm{SiO}_{2}$ & & & & $\begin{array}{c}23.80 \\
\text { (room temp) }\end{array}$ & \\
\hline $35 \mathrm{Na}_{2} \mathrm{O}-65 \mathrm{SiO}_{2}$ & & & & $\begin{array}{c}23.02 \\
\text { (room temp) }\end{array}$ & \\
\hline $\mathrm{NaAISi}_{3} \mathrm{O}_{8}$ & & & & & 29.2 \\
\hline $\mathrm{Na}_{2} \mathrm{Al}_{2} \mathrm{Si}_{2} \mathrm{O}_{8}$ & & & & & 30.2 \\
\hline
\end{tabular}




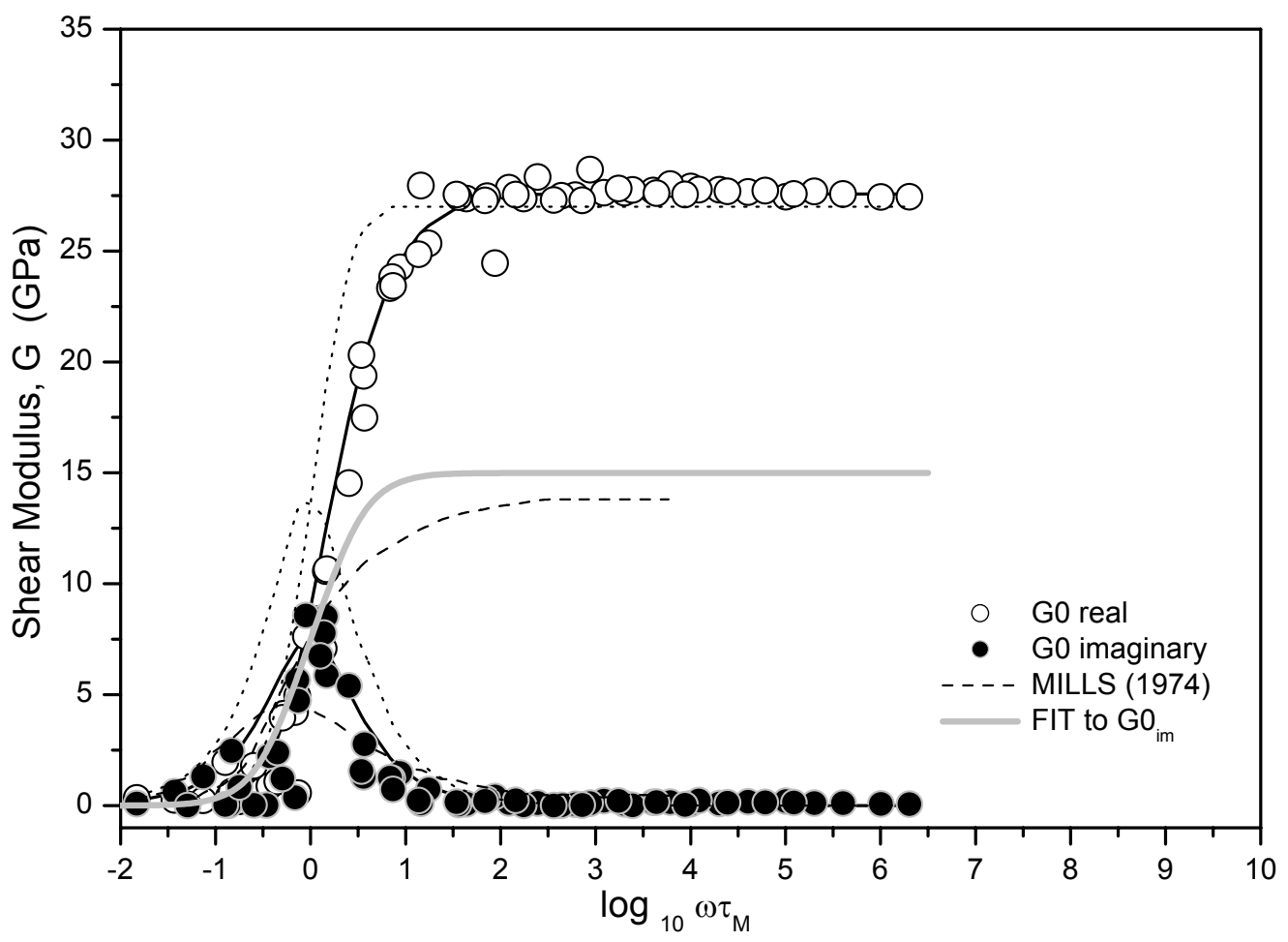

Fig. 74. Frequency dependent real and imaginary components of the shear modulus of $0.33 \mathrm{Na}_{2} \mathrm{O}-$ $0.67 \mathrm{SiO}_{2}$ (NS2, sample G0) The dashed line is the data of Mills (1974) on the same composition; the dotted line of the model of Herzfeld \& Litovitz (1959); solid line of the fitting; grey line is fitting to G0 imaginary parameters.

The greatest difficulty in designing a torsion machine is the rigidity of the connection between the torsion rods and the sample. If there is a slippage at this connection, low shear modulus values are measured. In the present work we do not melt the sample directly on the ceramic torsion rods; but use a rhyolite composition melt as glue. The high quality of the bonding between the torsion rods and the melts observed in scanning electron microscope (Fig. 45), together with the similarity between the elastic shear modulus determined by torsion and ultrasonic methods indicate that the low values of elastic shear modulus determined by Mills (1974) are due probably to slippage at the connections between sample and torsion rod. In his experiments, Mills (1974) used clamps between torsion rod and $50 \mathrm{~mm}$ long sample. These clamps sat inside his furnace. Mills determined the correct value of shear modulus for $\mathrm{SiO}_{2}$ melt because this melt was measured as a cylinder $50 \mathrm{~mm}$ long, which was held by clamps outside the furnace. The only time we get low $\mathrm{G}$ values similar to Mills is as the melt is in the process of detaching itself from the rod. 
The shear modulus of $\mathrm{SiO}_{2}$ glass at $27^{\circ} \mathrm{C}$ is $31.2 \mathrm{GPa}$ (Bansal \& Doremus, 1986) (Tab. 18). $\mathrm{G}$ at $700^{\circ} \mathrm{C}$ for a rhyolite composition has been found to be $30.5 \pm 2.5 \mathrm{GPa}$ via torsion measurements (Webb, 1992a). Bagdassarov et al. (1993) found G for haplogranitic glasses to vary from 23.90 to $29.61 \pm 0.05 \mathrm{GPa}$ at room temperature using ultrasonic techniques to $22.6-26.6 \pm 2.2 \mathrm{GPa}$ at temperatures $500-670^{\circ} \mathrm{C}$ in torsion. Bansal and Doremus (1986) report G for $30 \mathrm{Na}_{2} \mathrm{O}-70 \mathrm{SiO}_{2}$ at room temperature of $23.80 \mathrm{GPa}$ and $23.02 \mathrm{GPa}$ for $35 \mathrm{Na}_{2} \mathrm{O}-65 \mathrm{SiO}_{2}$. Our value of $\mathrm{G}_{\infty}$ for $\mathrm{NS} 2$ (sample $\mathrm{G} 0$ ) equals $27.45 \mathrm{GPa}$ (Tab. 18) is higher than the literature values of 23-24 GPa.

As seen in the Table 16, the $G$ for the present sodium aluminosilicate melts ranges from 31 to $35 \mathrm{GPa}$. Again these values are slightly higher than the 29-30 GPa in the literature. In addition, the variations in the radius of the ceramic rods may produce as much as $\sim 5 \%$ of error in shear modulus. 


\section{DISCUSSION}

\subsection{General}

Melts present great challenges due to their structural and thermodynamic complexity. With different experimental techniques and measurements it is possible to obtain various properties of glasses and melts which are controlled mostly by composition, but also by pressure, temperature and time. Changes in the physical and thermodynamic properties of melts are caused by rearrangement of the structure as a function of composition, pressure, temperature and time.

In this study melts of only four oxides have been used. The goal of the work was to obtain a good understanding of the relationship between structure and physical properties. There are a large number of studies of the physical properties and structure of silicate glass and melts in the literature. They simply present data without a controlled variation in composition. Here the effect of $\mathrm{Al}_{2} \mathrm{O}_{3}$ and $\mathrm{Fe}_{2} \mathrm{O}_{3}$ is investigated in a series of melts with constant $\mathrm{SiO}_{2}$ content and a controlled variation $\mathrm{Na}_{2} \mathrm{O} \cdot \mathrm{Al}_{2} \mathrm{O}_{3}\left(\mathrm{Fe}_{2} \mathrm{O}_{3}\right)$ ratio from peralkaline to peraluminous.

An increase of the complexity of the melt structure from only one oxide $\left(\mathrm{SiO}_{2}\right)$ towards multioxide compositions allows us to follow the role of each component, what is difficult in very complex systems. The $\mathrm{Na}_{2} \mathrm{O}-\mathrm{Al}_{2} \mathrm{O}_{3}-\mathrm{SiO}_{2}$ system is very common in both, industry and nature. These three oxides create many natural systems (Neuville \& Mysen, 1996) (i.e. albite, jadeite, nephelinite).

\subsection{Density and partial molar volume at room temperature}

The densities of the investigated samples (measured at room temperature) decrease in peralkaline melts with increasing $\mathrm{Al}_{2} \mathrm{O}_{3}$ content. The same trend is observed in Fe-free and Fe-bearing melts (Fig. 54). The breaking point and change of behaviour occurs at $\gamma \sim 0.5$, as the $\mathrm{Al}_{2} \mathrm{O}_{3}$ content increases in the peraluminous glasses.

In peralkaline melts, an addition of $\mathrm{Al}_{2} \mathrm{O}_{3}$ to the system $\mathrm{Na}_{2} \mathrm{O}-\mathrm{SiO}_{2}$ leads to changes the bond lengths and angles between tetrahedra. In this case not only the bond length between network formers and oxygen changes, but also an intertetrahedral angle network former $(T)$ - oxygen $(O)$ - network former $(T)$ (Taylor \& Brown, 1979b; Devine et al., 1987) and its energy (Meagher et al., 1980; Taylor et al., 1980). The Na diffusion channels which exist in sodium silicate melts disappear with increasing $\mathrm{Al}_{2} \mathrm{O}_{3}$ (Kargl \& Meyer, 2004); and $\mathrm{Na}$ is used to compensate the negative charge of Al-tetrahedra. Such 
rearrangement of the structure results in the decreasing density of the glasses, owing to the building the looser three-dimensional structure.

In peraluminous melt, there is not enough sodium to balance the negative charge of Al-tetrahedra and new structural units are formed, namely triclusters. That causes reduction of the network structure, which becomes denser, and distortions in the structure of tetrahedra. MacDowell \& Beall (1969) pointed out, that formation of the triclusters in the melt structure would increase density of the melt. Also Bottinga et al. (1982) indicated high compositional dependence of the density and also viscosity. The authors suggested that changes in the properties of the melt are connected with the changing coordination number of aluminium cation from tetrahedral to octahedral at $\gamma \sim 0.5$, but the percentage of aluminium with new coordination number is not sufficient to change the physical and thermodynamic properties. In this study, this reason was sufficient to assume that triclusters play more important role in the melt structure.

The partial molar volume of the oxides in these glasses has been calculated by a multi-linear regression between mole fraction of the oxide $x_{i}$ and molar volume of the oxide $V_{i}$ (see also Tab. 10):

$$
\mathrm{V}=\sum_{i} x_{i} V_{i}
$$

Fits to the molar volume data are presented in the Table 19. Because the very small amount of $\mathrm{FeO}$ introduces a large error in this calculation, $\mathrm{FeO}$ has been put together with $\mathrm{Fe}_{2} \mathrm{O}_{3}$ as $\mathrm{Fe}_{2} \mathrm{O}_{3}$ total. The calculation for each oxide in the glass structure has been separated into four parts:

(1) for all of the samples with fit $R^{2}=0.986$. Here one observes that the largest volume units in the melt structure are $\mathrm{Al}_{2} \mathrm{O}_{3}$ and $\mathrm{Fe}_{2} \mathrm{O}_{3}$ containing the cations with very high ionisation field strength (Tab. 1 ) and requiring a charge balancer. The fit parameter for $\mathrm{SiO}_{2}$ units is always constant and equals $\sim 26 \mathrm{~cm}^{3} \mathrm{~mol}^{-1}$;

(2) for peralkaline composition with fit $R^{2}=0.989$. The results do not vary significantly from first model;

(3) for peraluminous composition with fit $R^{2}=0.957$. Although (3) and (4) are not very different to (1) there is a large variation in $\mathrm{Na}_{2} \mathrm{O}$;

(4) for the model assuming the presence of $\mathrm{Al}_{2} \mathrm{O}_{3}$ in both Al-tetrahedra and triclusters in the peraluminous compositions of the glasses $\left(R^{2}=0.987\right)$. Here, there is no large increase in $\mathrm{Na}_{2} \mathrm{O}$ partial molar volume and the new structural units (triclusters) take less space in the glass structure than normal Al-tetrahedra. That would explain fast increase in the density with increasing peraluminosity of the glasses, what follows increasing number of triclusters. 
Tab. 19. Parameters of the fit to the molar volume data. Calculation has been separated into four models, in which (1) all of the samples have been used, (2) only peralkaline and (3) peraluminous samples and the (4) model including presence of $\mathrm{Al}_{2} \mathrm{O}_{3}$ in triclusters.

\begin{tabular}{|c|c|c|c|c|}
\cline { 2 - 5 } \multicolumn{1}{c|}{} & \multicolumn{4}{c|}{$\begin{array}{c}\text { Partial molar volume } \\
\left(\mathbf{c m}^{\mathbf{3}} \mathbf{m o l}^{-1}\right)\end{array}$} \\
\cline { 2 - 5 } & all & $\begin{array}{c}\text { peralkaline } \\
(1)\end{array}$ & $\begin{array}{c}\text { peraluminous } \\
(3)\end{array}$ & $\begin{array}{c}\text { + triclusters } \\
(4)\end{array}$ \\
\hline \hline $\mathbf{S i O}_{2}$ & $27.26 \pm 1.67$ & $26.42 \pm 5.03$ & $24.81 \pm 1.61$ & $26.85 \pm 1.71$ \\
$\mathbf{A l}_{2} \mathbf{O}_{3}$ & $39.01 \pm 3.31$ & $41.34 \pm 9.75$ & $39.19 \pm 2.43$ & $40.41 \pm 3.56$ \\
$\mathrm{Na}_{2} \mathbf{O}$ & $20.84 \pm 4.03$ & $20.11 \pm 10.05$ & $29.57 \pm 7.07$ & $19.41 \pm 3.47$ \\
$\mathrm{Fe}_{2} \mathbf{O}_{3}$ total & $35.34 \pm 5.25$ & $39.81 \pm 16.85$ & $32.49 \pm 3.28$ & $35.29 \pm 5.23$ \\
$\mathbf{A l}_{2} \mathbf{O}_{3}$ in triclusters & - & - & - & $37.08 \pm 3.78$ \\
\hline \hline $\mathbf{R}^{2}$ & 0.986 & 0.989 & 0.957 & 0.987 \\
\hline \hline
\end{tabular}

Comparison of density of the peralkaline melts from this study with other authors (Fig. 55) shows small disagreement, what can be explained by different cooling rates of the glasses. Density depends on a thermal history and especially on quenching rate (Angell, 1981; Angell, 1990). The higher density of the glasses is then caused by lower fictive temperature (Knoche et al., 1994).

\subsection{Heat capacity and configurational heat capacity}

The heat capacity of glasses is caused by the vibrations between atoms at temperature higher than room temperature (Tangeman \& Lange, 1998). With increasing temperature, there are more vibrational modes, what also causes higher heat capacity. The sudden increase in heat capacity just before reaching $T_{g}$ is explained as a need of additional energy to break the strongest bonds in the melt structure, to change the angles distances between atoms and coordination numbers (Richet \& Bottinga, 1995). Above $T_{g}$ the heat capacity of the liquid becomes constant over the $20^{\circ} \mathrm{C}$ range of temperature of our measurements.

The configurational state is due to the possibilities of the atoms arrangement over available potential energy minima (Richet \& Bottinga, 1995). Increasing temperature produces a heat, which is transformed into the potential energy and atoms are able to reach higher configurational states with higher energy (Fig. 75). 


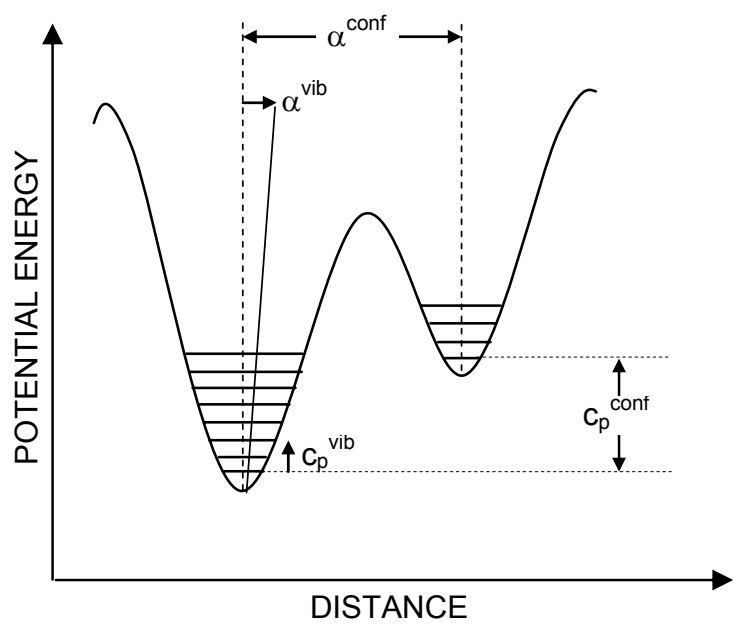

Fig. 75. Schema of the interatomic potentials. Heat capacity consists of vibrational and configurational part. Changes in the interatomic distances between atoms are connected with variations in vibrational and configurational thermal expansion coefficient of the material. Redrawn after Richet \& Bottinga, 1995.

Vibrational heat capacity is observed in glasses (below $T_{g}$ ) and glass transition occurs when Dulong - Petit harmonic limit of $3 R / g$ atom $/ K$ is reached. This kind of heat capacity does not depend strongly from composition (Haggerty et al., 1968; Richet \& Bottinga, 1986; Toplis et al., 2001, Roskosz et al., 2004). The compositional dependence is clear above $T_{g} .3 R / g$ atom/K (Fig. 66) introduced by Haggerty et al. (1968) expresses the moment of glass transition in the sample (confirmed by Richet \& Bottinga, 1986; Martens et al., 1987).

Configurational heat capacity $\mathrm{C}_{\mathrm{p}}$ conf is an energy required for structural changes which are strongly connected with increasing temperature (bond lengths and angles, coordination numbers, distances between atoms). The configurational heat capacity expresses the energy absorbed in reaction to changes of the potential energy, it means of the energy required for structure rearrangement (Simon, 1931; Bernal, 1936).

Configurational heat capacity $\mathrm{C}_{\mathrm{p}}{ }^{\text {conf }}$ (Tab. 20, Fig. 76) is a difference between heat capacity of the liquid $C_{p l}$ and heat capacity of the glass $C_{p g}$ at $T_{g}$ (Richet et al., 1986) and has been calculated by Eq. 29, reproduced here: $c_{p}^{\text {conf }}=c_{p l}-c_{p g}$. 
Tab. 20. Table for all of the samples with compositional parameter $\gamma$; heat capacity of the glass $\mathrm{c}_{\mathrm{pg}}$ and liquid $\mathrm{C}_{\mathrm{pl}}$ (in $\left.\mathrm{J} \mathrm{g}^{-1} \mathrm{~K}^{-1}\right)$; configurational heat capacity $\mathrm{C}_{\mathrm{p}}^{\text {conf }}\left(\mathrm{J} \mathrm{mol}^{-1} \mathrm{~K}^{-1}\right)$; parameter of MaierKelley equation $B_{e}\left(\mathrm{~kJ} \mathrm{~mol}^{-1}\right)$; configurational entropy $\mathrm{S}^{\mathrm{conf}}(\mathrm{T})\left(\mathrm{J} \mathrm{mol}^{-1} \mathrm{~K}^{-1}\right)$ and parameter $\mathrm{B}_{\mathrm{e}} / \mathrm{S}^{\mathrm{conf}}(\mathrm{T})$ $\left(10^{3} \mathrm{~K}^{-1}\right)$.

\begin{tabular}{|c|c|c|c|c|c|c|c|c|c|c|c|c|c|}
\hline \multirow{2}{*}{$\begin{array}{c}\begin{array}{c}\text { Melt } \\
\text { number }\end{array} \\
\text { G0 }\end{array}$} & \multirow{2}{*}{$\frac{\gamma}{1.00}$} & \multirow{2}{*}{$\begin{array}{c}c_{p g} \\
\left(\mathrm{~J} \mathrm{~g}^{-1} \mathrm{~K}^{-1}\right)\end{array}$} & \multirow{2}{*}{$\begin{array}{c}\begin{array}{c}C_{p l} \\
\left(\mathrm{~J} \mathrm{~g}^{-1} \mathrm{~K}^{-1}\right)\end{array} \\
1.450\end{array}$} & \multicolumn{3}{|c|}{$\begin{array}{c}C_{p}{ }^{\operatorname{conf}} \\
\left(\mathrm{J} \mathrm{mol}^{-1} \mathrm{~K}^{-1}\right)\end{array}$} & \multicolumn{3}{|c|}{$\begin{array}{c}\mathbf{B}_{\mathrm{e}} \\
\left(\mathrm{kJ} \mathrm{mol}{ }^{-1}\right)\end{array}$} & \multicolumn{3}{|c|}{$\begin{array}{c}\mathrm{S}^{\text {conf }}(\mathrm{T}) \\
\left(\mathrm{J} \mathrm{mol}^{-1} \mathrm{~K}^{-1}\right)\end{array}$} & $\begin{array}{c}\mathbf{B}_{\mathrm{e}} / \mathbf{S}^{\text {conf }}(\mathbf{T}) \\
\left(10^{3} \mathrm{~K}\right)\end{array}$ \\
\hline & & & & 14.28 & \pm & 0.42 & 189.01 & + & 3.93 & 7.759 & $t$ & 0.172 & $24.362 \pm 0.523$ \\
\hline N33 * & 1.00 & & & & & & \multicolumn{3}{|c|}{179.52} & \multicolumn{3}{|c|}{7.95} & 22.572 \\
\hline$\overline{\text { G1 }}$ & 0.42 & 1.219 & 1.370 & 10.60 & & 0.42 & 342.68 & & 9.84 & 9.273 & + & 0.292 & $36.955 \pm 1.112$ \\
\hline G2 & 0.45 & 1.211 & 56 & 9.98 & \pm & 0.45 & 371.60 & & 8.38 & 9.982 & & 0.239 & $37.227 \pm 0.866$ \\
\hline G3 & 0.47 & 1.227 & 6 & 9.66 & \pm & 0.47 & 331.38 & \pm & 9.02 & 8.928 & \pm & 0.258 & $37.117 \pm 1.041$ \\
\hline G4 & 0.48 & 1.207 & 1.366 & 10.92 & \pm & 0.48 & 472.82 & \pm & 15.77 & 13.221 & \pm & 0.462 & $35.763 \pm 1.221$ \\
\hline G5 & 0.51 & 1.203 & 1.340 & 9.79 & \pm & 0.51 & 304.22 & \pm & 9.69 & 9.368 & \pm & 0.316 & $32.474 \pm 1.065$ \\
\hline G6 & 0.53 & 1.166 & 1.310 & 9.87 & \pm & 0.53 & 306.25 & \pm & 6.43 & 9.785 & \pm & 0.220 & $31.299 \pm 0.680$ \\
\hline G7 & 0.58 & 1.205 & 1.355 & 10.22 & \pm & 0.58 & 260.59 & \pm & 4.88 & 8.893 & \pm & 0.176 & $29.302 \pm 0.565$ \\
\hline G8 & .41 & 1.229 & 1.403 & 12.04 & & 0.41 & 439.61 & & 25.71 & 12.661 & & 0.795 & $34.720 \pm 2.105$ \\
\hline G9 & .46 & 1.149 & 1.290 & 9.30 & $\perp$ & 0.46 & 323.27 & \pm & 26.03 & 8.932 & & 0.744 & $36.192 \pm 2.964$ \\
\hline G10 & 0.47 & 1.180 & 1.290 & 7.78 & \pm & 0.47 & 281.63 & \pm & 8.62 & 8.322 & $\mathbf{I}$ & 0.269 & $33.841 \pm 1.065$ \\
\hline G11 & 0.49 & 1.159 & 1.296 & 10.02 & \pm & 0.49 & 390.00 & \pm & 28.11 & 12.139 & \pm & 0.941 & $32.129 \pm 2.403$ \\
\hline G12 & 0.53 & 1.160 & 1.290 & 8.73 & \pm & 0.53 & 260.12 & \pm & 12.45 & 8.485 & \pm & 0.430 & $30.657 \pm 1.511$ \\
\hline G13 & 0.55 & 1.128 & 1.280 & 10.30 & \pm & 0.55 & 247.59 & \pm & 6.41 & 8.351 & \pm & 0.228 & $29.648 \pm 0.789$ \\
\hline G14 & 0.60 & 1.175 & 1.340 & 11.73 & \pm & 0.60 & 251.87 & \pm & 4.93 & 8.906 & \pm & 0.188 & $28.280 \pm 0.576$ \\
\hline
\end{tabular}

* sample N33 (compositional equivalent to the sample G0) has been placed in the table to confirm the correctness of the calculation. Data come from Richet (1984).

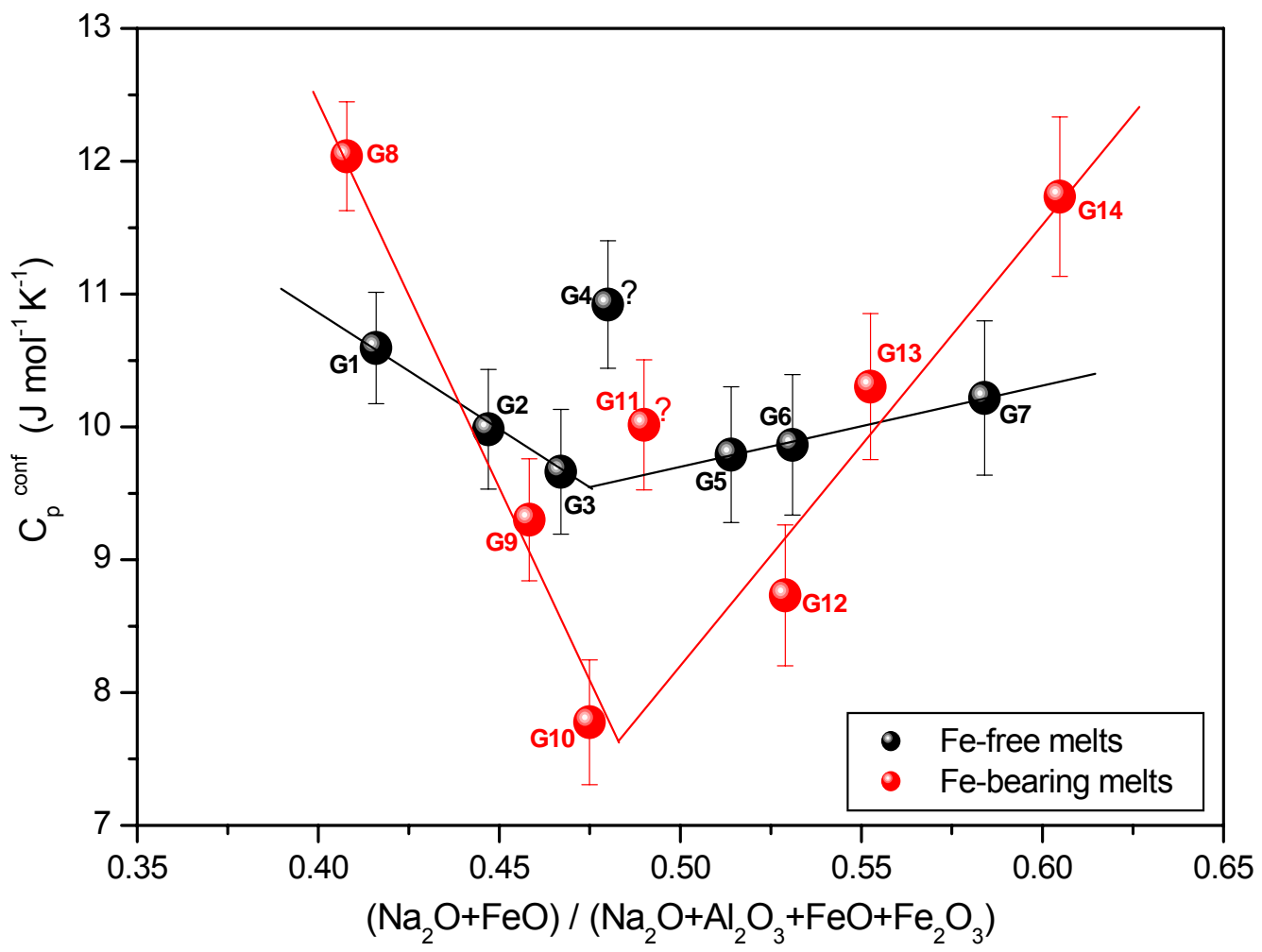

Fig. 76. Configurational heat capacity of the (in $\mathrm{J} \mathrm{mol}^{-1} \mathrm{~K}^{-1}$ ) for peralkaline and peraluminous $\mathrm{Fe}$ free (black points) and Fe-bearing (red points) samples as a function of compositional parameter $\gamma=\left(\mathrm{Na}_{2} \mathrm{O}+\mathrm{FeO}\right) /\left(\mathrm{Na}_{2} \mathrm{O}+\mathrm{Al}_{2} \mathrm{O}_{3}+\mathrm{FeO}+\mathrm{Fe}_{2} \mathrm{O}_{3}\right)$. The trends are the guides to the eye. 
Figure 76 presents the configurational heat capacity $\mathrm{C}_{\mathrm{p}}{ }^{\text {conf }}$ (in $\mathrm{J} \mathrm{mol}^{-1} \mathrm{~K}^{-1}$ ). The first observation is that trends connecting the data points have deep minimum at $\gamma \sim 0.48$. Addition $\mathrm{Al}_{2} \mathrm{O}_{3}$ to sodium silicate melts (sample $\mathrm{G}$ ) introduces a decrease in the configurational heat capacity because less energy is required for structural rearrangement as $\mathrm{Al}$ replaces network modifying $\mathrm{Na}$. At $\gamma \sim 0.48 \mathrm{C}_{\mathrm{p}}{ }^{\text {conf }}$ starts to increase and because of change of structure associated with the formation of triclusters, the more energy is needed for the flow in melt with triclusters. The presence of $\mathrm{Fe}^{3+}$ enhanced this effect.

In extreme peraluminous and peralkaline part of the plot the Fe-free melts (G1 and G7) have lower $C_{p}{ }^{\text {conf }}$ than Fe-bearing melts (respectively $G 8$ and G14). For the range $0.43>\gamma>0.56$ Fe-free melts have higher configurational heat capacity than Fe-bearing. Samples G4 and G11 are out of the trends. In spite of three repetitions of the measurements for each sample, their location can not be explained. To get satisfactory results one would need to prepare more samples with compositions filling the gap between the melts: G3-G4-G5 and G10-G11-G12.

As a first time the configurational heat capacity data are presented in a controlled series of compositions. In the literature, there exist data only for peralkaline melts (Toplis et al., 2001) and therefore, the breaking point at $\gamma \sim 0.5$ was not presented.

As has been mentioned before (see section 2.3.), in peralkaline range of melts $\mathrm{Al}$ is a tetrahedrally coordinated network former. Structure changes in peraluminous melt when $\mathrm{Al}^{3+}$ is in excess of $\mathrm{Na}^{+}$and there is no more cations to compensate a negative charge of Al-tetrahedra and triclusters are formed. That would explain a change close to the point when $\mathrm{Al} \sim \mathrm{Na}(\gamma \sim 0.5)$.

In the case of Fe-bearing peralkaline composition, as a first the complexes with sodium will be formed $\left(\mathrm{NaAl}^{4+}\right)$ and all $\mathrm{Fe}^{3+}$ stays in tetrahedral coordination. $\mathrm{Fe}^{3+}$ is then calculated follow the equation: $\mathrm{Fe}^{3+}=\left(\mathrm{Na}^{+}\right)-\left(\mathrm{Al}^{3+}\right)$. But in peraluminous melts, there is not enough $\mathrm{Na}$ to compensate the negative charge of tetrahedra. $\mathrm{Al}^{3+}$ and $\mathrm{Fe}^{3+}$ stay in tetrahedral coordination and they form triclusters. Weigel et al. (2006) measured that only $5 \%$ of $\mathrm{Fe}^{3+}$ changes the coordination number from tetrahedral to octahedral. 


\subsection{Configurational entropy $S^{\text {conf }}(T)$ and $B_{e}$ parameter}

Similar to configurational heat capacity $\mathrm{C}_{\mathrm{p}}{ }^{\text {conf }}$, the results of configurational entropy $S^{\text {conf }}$ and $B_{e}$ parameter have not been shown before in a controlled series of compositions.

Together with higher energy, the distribution of configurational states occurs, what is measured by configurational entropy $S^{\text {conf }}$ (Richet \& Bottinga, 1995). The configurational entropy of the melt can be determined e.g. using heat capacity measurements:

$$
S^{\text {conf }}(T)=S^{\text {conf }}\left(T_{g}\right)+\int_{T_{g}}^{T} \frac{C_{p}^{\text {conf }}}{T} d T,
$$

where $S^{\text {conf }}(T)$ is constant between $0 \mathrm{~K}$ and $T_{g}$ and $C_{p}^{\text {conf }}=$ constant. $S^{\text {conf }}\left(T_{g}\right)$ can be determined from viscosity measurements using the Adam-Gibbs relationship:

$$
\eta(T)=\eta_{0} \exp \left(\frac{B_{e}}{T S^{\text {conf }}(T)}\right),
$$

where $B_{e}$ is a constant (Richet \& Neuville, 1992; Toplis, 1998).

Changes in the viscosity and fragility in the investigated melts as a function of composition are connected with changes in configurational parameters. The link between configurational entropy and viscosity lies in Adam - Gibbs theory (Adam \& Gibbs, 1965; Richet, 1984; Bottinga \& Richet, 1996; Mysen, 1998):

$$
\ln \eta=A_{c}+\frac{B_{e}}{T S^{c o n f}(T)}
$$

(logarithmic form of Eq. 61), where $A_{c}$ and $B_{e}$ are constants, independent of temperature, but dependent on composition; and $S^{\text {conf }}(T)$ is configurational entropy. This configurational entropy model bonds viscous behaviour of aluminosilicates and their thermodynamic behaviour (Richet \& Neuville, 1992; Bottinga, 1994; Mysen, 1995b; Mysen, 1997). Adam Gibbs theory says that structural relaxation time is function of energy barrier needed to relocate a single silicate unit, and the number of units that must at the same time conquers their energetic barrier to reach a change in configuration (Toplis, 2001). 
The height of the peak in the $C_{p}$ curve (Fig. 66) upon heating can be described as a function of heating and cooling rate in terms of activation energy $\mathrm{H}$, which is found to be identical to that obtained from viscosity measurements (Scherer, 1984; Stevenson et al., 1996); with the structural relaxation time being given by Adam-Gibbs model (Crichton \& Moynihan, 1988):

$$
\tau_{p}=\tau_{0} \exp \left(\frac{B}{T S^{\text {conf }}(T)}\right)
$$

The Narayanaswamy model partitions the $B / S^{\text {conf }}(T)$ term into a structure dependent term $(1-x) H$ and a temperature dependent term $x H$ (Wilding et al., 1995; and references therein). Thus the determination of $C_{p}$ as a function of heating rate can be used to determine changes in melt structure with changing composition as well as the $B / S^{\text {conf }}(T)$ term.

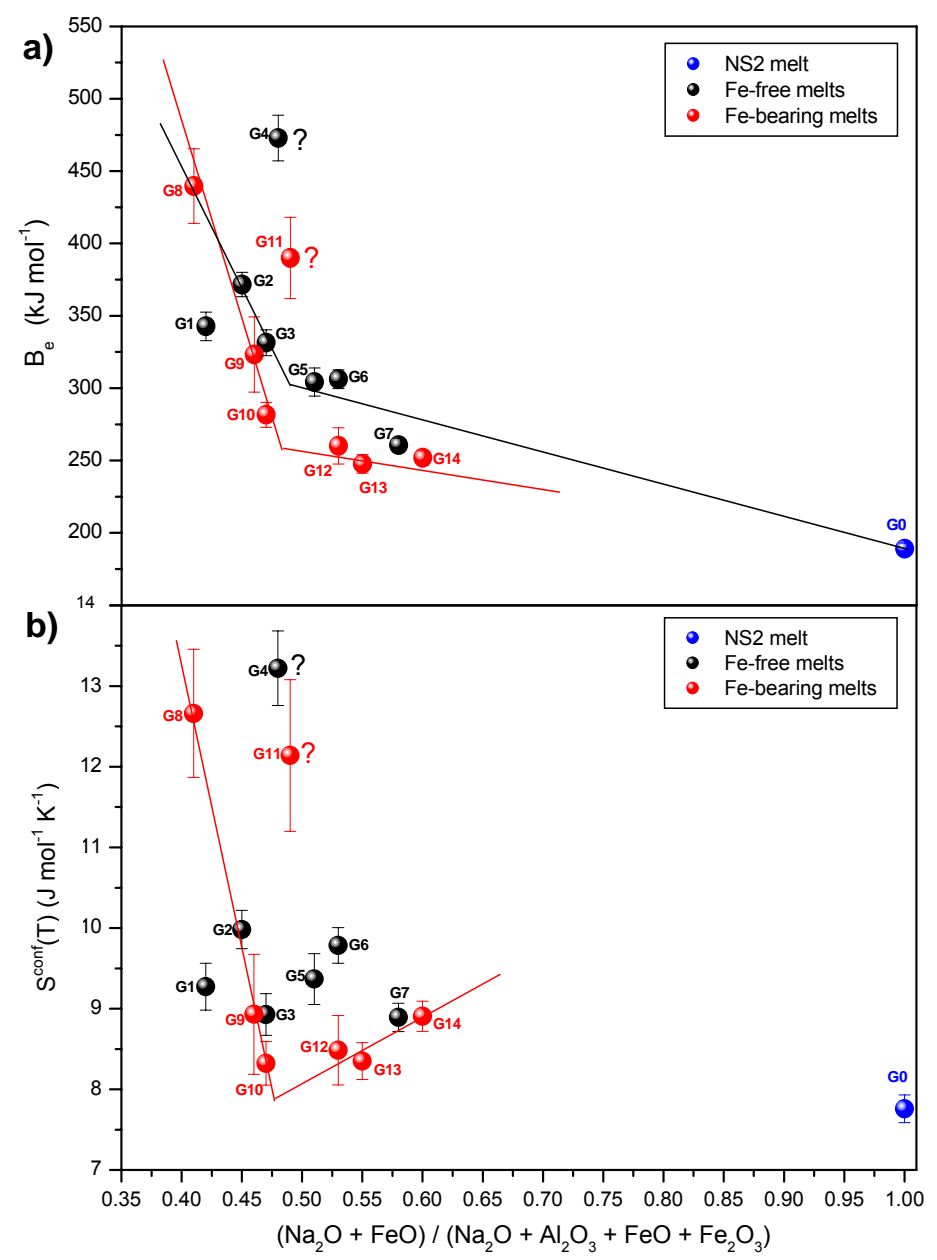

Fig. 77. a) $B_{e}$ parameter and b) configurational entropy $S^{\text {conf }}(T)$ for NS2 (blue point), Fe-free (black points) and Febearing (red points) melts as a function of compositional parameter $\gamma$. The trends are the guides to the eye. In the case of Fe-free melts in the plot $S^{\text {conf }}(T)$ vs. composition there is not enough data to support a breaking trend. 
How to connect calculated configurational entropy and the structure of the glasses?

The studies of Toplis (1998; 2001) and Russel et al. (2003) (provide an average value of $\eta_{0}$ for silicate melts which allows the calculation of the ratio $\mathrm{B}_{\mathrm{e}} / \mathrm{S}^{\text {conf }}(\mathrm{T})$ and ultimately a calculation of $S^{\text {conf }}\left(T_{g}\right)$. $S^{\text {conf }}\left(T_{g}\right)$ can be divided into two parts: (1) the topological contribution due to the different bonding within the glass; and (2) the chemical contribution - due to the mixing of different elements on the same structural site (Richet \& Neuville, 1992). Both contributions change and new entropies arise.

Similarly, $C_{p}^{\text {conf }}$ is made up of a chemical and topological contribution and can be used to support the interpretation of the variations in $S^{\text {conf }}(T)$ with composition. The calculation of $S^{\text {conf }}(T)$ as a function of composition will therefore allow discussion of the structure in terms of bonding and site preference of the $\mathrm{Al}^{3+}$ and $\mathrm{Fe}^{3+}$ atoms. $S^{\text {conf }}(T)$ is a measure of the range of structure.

In Figure 77 the $B_{e}$ parameter (Fig. 77a) and configurational entropy $S^{\text {conf }}(T)$ (Fig. 77b) for NS2, Fe-free and Fe-bearing melts as a function of compositional parameter $\gamma=\left(\mathrm{Na}_{2} \mathrm{O}+\mathrm{FeO}\right) /\left(\mathrm{Na}_{2} \mathrm{O}+\mathrm{Al}_{2} \mathrm{O}_{3}+\mathrm{FeO}+\mathrm{Fe}_{2} \mathrm{O}_{3}\right)$ has been presented. $\mathrm{B}_{\mathrm{e}}$ is:

$$
B_{e}=\frac{h f}{k_{B} T}
$$

where $h$ is Planck's constant $\left(6.626068 \cdot 10^{-34} \mathrm{~m}^{2} \mathrm{~kg} \mathrm{~s}^{-1}\right), f$ is frequency, $k_{B}$ is Boltzmann's constant and $T$ is temperature. $B_{e}$ was determined analytically from fit to the viscosity curve of the sample which heat capacity is measured (Richet, 1984; Bottinga \& Richet, 1996).

$B_{e}$ is a potential energy barrier to viscous flow and depends on the composition but is assumed to be temperature independent (Toplis, 1998). $B_{e}$ parameter has been then taken to determine configurational entropy $S^{\text {conf }}(T)$ using an Eq. 61 where $\eta(T)=\eta_{0} \exp \left(B_{e} / S^{\text {conf }}(T) T\right) \cdot \eta_{0}$ (marked also as $A_{0}$ or $A_{V F T}$ in Eq. 26) is the constant value of viscosity at infinite temperature. In this study this value has been fixed as -2.6 (Toplis, 1998). Choosing another value produces the same trend, just at slightly different absolute values.

The size of the region in the rearranging structure is expressed by the configurational entropy of this region (Adam \& Gibbs, 1965). $S^{\text {conf }}(T)$ is the sum of the configurational entropy of the smallest rearranging unit occurring in the structure - more precisely: the number of atoms taking part in the viscosity flow (Toplis, 1998; Adam \& Gibbs, 1965). 
$S^{\text {conf }}(T)$ reveals also a temperature dependence. The number of small subsystems increases and as, a consequence, $S^{\text {conf }}(T)$ also increases with temperature (Mysen, 1997; Toplis et al., 1997b; Toplis, 1998).

With change of the number of the smallest rearranging unit at glass transition $\left[z^{*}\left(T_{g}\right)\right]$ or height of the average potential energy barrier to viscous flow $(\Delta \mu)$, then the ratio $B_{e} / S^{\text {conf }}(T)$ also changes. $B_{e} / S^{\text {conf }}(T)$ relation shows a compositional dependence (Toplis, 1998).

In Figure 77 both $B_{e}$ and $S^{c o n f}(T)$ represent the breaking points at $\gamma \sim 0.5$, only in the case of Fe-free melts in the plot $S^{\text {conf }}(T)$ vs. composition there is not enough data to support a breaking trend. $\mathrm{Be}_{\mathrm{e}}$ increases with increasing $\mathrm{Al}_{2} \mathrm{O}_{3}$ content. In the peralkaline range of composition this increase is small, but in peraluminous melts $B_{e}$ parameter rises very fast. The breaking point indicates a change in a potential energy barrier to viscous flow, it means that with increasing $\mathrm{Al}_{2} \mathrm{O}_{3}$ content (close to $\gamma \sim 0.5$ ) new structure with higher potential energy arises and the flow mechanism is different too.

$S^{\text {conf }}(T)$ as a function of composition shows similar behaviour. Together with increasing $S^{\text {conf }}(T)$ decreases the size and increases the number of the units causing the rearrangement of the structure. Fe-bearing melts have slightly higher $S^{\text {conf }}(T)$ indicating smaller structural units in the network.

Because in the $B_{e}$ parameter and $S^{\text {conf }}(T)$ plots is observed large scatter to describe a structure of the melts is used a ratio $B_{e} / S^{\text {conf }}(T)$. $B_{e} / S^{\text {conf }}(T)$ ratio for the investigated samples as a function of their composition is shown in Figure 78. The trend is exactly the same like on viscosity or shear modulus plot and shows a structural change close to subaluminous point. 


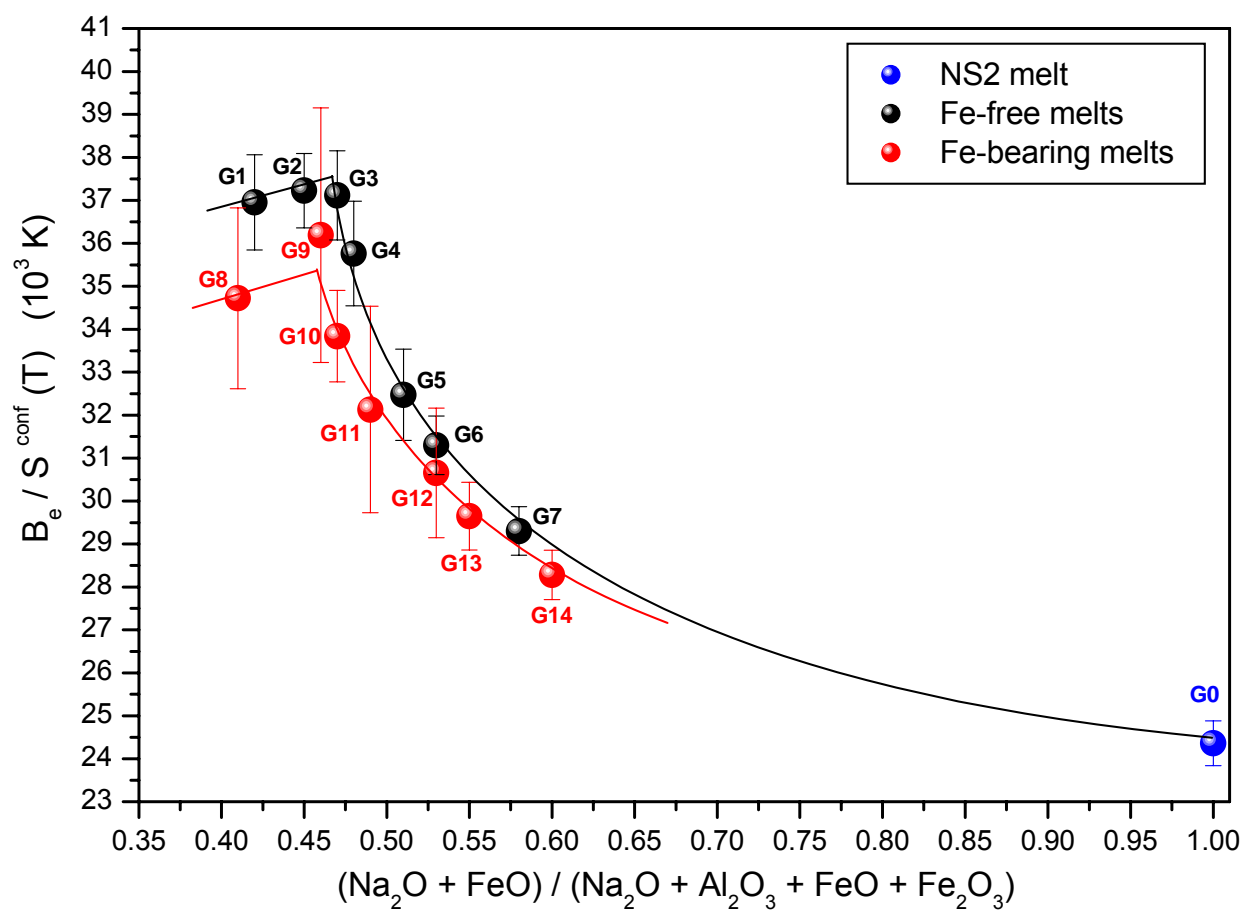

Fig. 78. $\mathrm{B}_{\mathrm{e}} / \mathrm{S}^{\mathrm{conf}}(\mathrm{T})$ as a function of compositional parameter $\gamma=\left(\mathrm{Na}_{2} \mathrm{O}+\mathrm{FeO}\right) /\left(\mathrm{Na}_{2} \mathrm{O}+\mathrm{Al}_{2} \mathrm{O}_{3}+\mathrm{FeO}+\right.$ $+\mathrm{Fe}_{2} \mathrm{O}_{3}$ ). Blue point is a NS2 melt (G0); black points are for Fe-free melts (G1-G7); red points are for Fe-bearing melts (G8-G14).

\subsection{Viscosity and fragility}

\subsubsection{Viscosity $\eta$}

Viscosity is temperature and composition dependent (Richet, 1984). The temperature dependence of the viscosity can be discussed using different theories, e.g. (1) Eyring equation (Glasstone et al., 1941), (2) the free volume theory (Cohen \& Grest, 1979), (3) Adam - Gibbs theory (Adam \& Gibbs, 1965) or (4) the mode coupling theory (Götze, 1991). Description of these theories with regard to the silicate melts viscosity has been done by Bottinga et al. (1995).

In the trend of $\log _{10}$ viscosity as a function of composition (Fig. 60) one observes a clear breaking point at $\gamma \sim 0.47$ for Fe-free melts (in agreement with the viscosity data of Toplis et al., 1997a,b and Webb et al., 2004); and at $\gamma \sim 0.46$ for Fe-bearing melts. For an exact position of the breaking points more compositions are required, especially in the case of Fe-bearing melts.

The viscosity increases from peralkaline composition toward peraluminous with increasing $\mathrm{Al}_{2} \mathrm{O}_{3}$ and simultaneously decreasing $\mathrm{Na}_{2} \mathrm{O}$. Close to the subaluminous point, 
but on the peraluminous side of the composition, the viscosity trend becomes independent on the compositional changes.

In the peralkaline melts amount of $\mathrm{Al}^{3+}$ and $\mathrm{Fe}^{3+}$ influences the viscosity through the degree of polymerization (Mysen et al., 1985b). The addition of these cations causes an increase of the melt polymerization and following higher viscosity of the peralkaline melts.

Toplis et al. (1997a,b) suggested that maximum in viscosity trends is shifted towards peraluminous composition because triclusters are created before they are necessary and thus some NBOs exist until $\gamma \sim 0.47$. This idea was confirmed with NMR measurements by Stebbins \& Xu (1997). It would be explained that not all $\mathrm{Na}$ plays a charge balancing role but is still connected with oxygen. Because in the peraluminous composition, there is less $\mathrm{Na}$ than $\mathrm{Al}$, then negative charge of $\mathrm{Al}$ - tetrahedra needs to be balanced in some different way, does not connected with sodium ions.

Stebbins \& Xu (1997) indicated a presence of $\sim 5 \%$ NBO on the peraluminous side of the melt composition in anorthite $\left(\mathrm{CaAl}_{2} \mathrm{Si}_{2} \mathrm{O}_{8}\right)$. That would mean that $\mathrm{Al}$ is not connected with charge balancing cations, but built own, fully compensated unit structure, e.g. triclusters.

With increasing amount of $\mathrm{Al}_{2} \mathrm{O}_{3}$ and $\mathrm{Fe}_{2} \mathrm{O}_{3}$ degree of polymerization should theoretically increases viscosity, what happens in the peralkaline range of the composition. But despite of higher number of triclusters, nothing like that occurs in the peraluminous melts. Higher peraluminosity does not affect the polymerization of the melt.

Change from tetrahedrally to octahedrally coordinated Al or Fe cations causes an increase the number of NBO and decrease in viscosity (Kozakevitsch, 1960; Riebling, 1966; Urbain et al., 1982). Toplis et al. (1997a) did not confirm this theory and says that viscosity of peraluminous melts stays constant. However, the numerous NMR measurements brought an answer, that there is not enough ${ }^{[\mathrm{VI}} \mathrm{Al}$ to influence the viscosity, so any other structural unit which can compensate negative charge of tetrahedra is triclusters. In this study the viscosity trend in peraluminous composition decreases insignificantly with increasing amount of $\mathrm{Al}_{2} \mathrm{O}_{3}$.

One observes a decrease in viscosity together with addition to the melt structure the cations with higher electronegativity, what evokes the weakening of network formeroxygen (T-O) bonds and the angle between T-O-T. The viscosity - temperature relationship of these melts increases in order borosilicate < ferrosilicate and galliosilicate < aluminosilicate (Dingwell \& Virgo, 1988a). This is consistant with that, what is observed in this study, the Fe-free melts have higher viscosity than Fe-bearing melts. 


\subsubsection{Frequency dependent shear viscosity $\eta^{*}(\omega)$ (Pa s) (from torsion)}

Viscosity can be also calculated from the torsion data:

$$
\eta^{*}(\omega)=\frac{G^{*}(\omega)}{i \omega}
$$

(Webb, 1992a). The real part of the viscosity of each melt is shown in Figure 79 as a function of frequency at each temperature and gathered in the Table 21. The Newtonian viscosity is given by the frequency independent part of the viscosity curve and is calculated at each temperature from the average of the frequency independent data; or where there are not enough data, the Newtonian viscosity is taken to be the lowest frequency datum.

Each of these viscosity data is plotted in Figure 80 as a function of inverse temperature, together with the viscosity data from the micropenetration measurements. The agreement between viscosities determined in the micropenetration and torsion experiments supports the robustness of the torsion $\mathrm{G}(\omega)$ data. This illustrates not only that viscosity can be determined from torsion data, but that the temperatures within the two furnaces (micropenetration and torsion) have been calibrated correctly. As the viscosities agree, the temperature calibrations of the two furnaces are correct, and there is no slippage at the torsion rod/melt interface - as also shown from the agreement between the torsion and the ultrasonic shear modulus values.

As the lowest frequency of the torsion measurements is $1 \mathrm{mHz}$, the highest viscosity expected to be accurately determined is $\sim 10^{13} \mathrm{~Pa} \mathrm{~s}$. Allowing for the distribution in relaxation times; all viscosities $>10^{15} \mathrm{~Pa} s$ are not relaxed and therefore slightly lower than the relaxed viscosity (see sample $\mathrm{G} 1$ in Figure $79 \mathrm{~b}$ ). 
Chapter 5 .
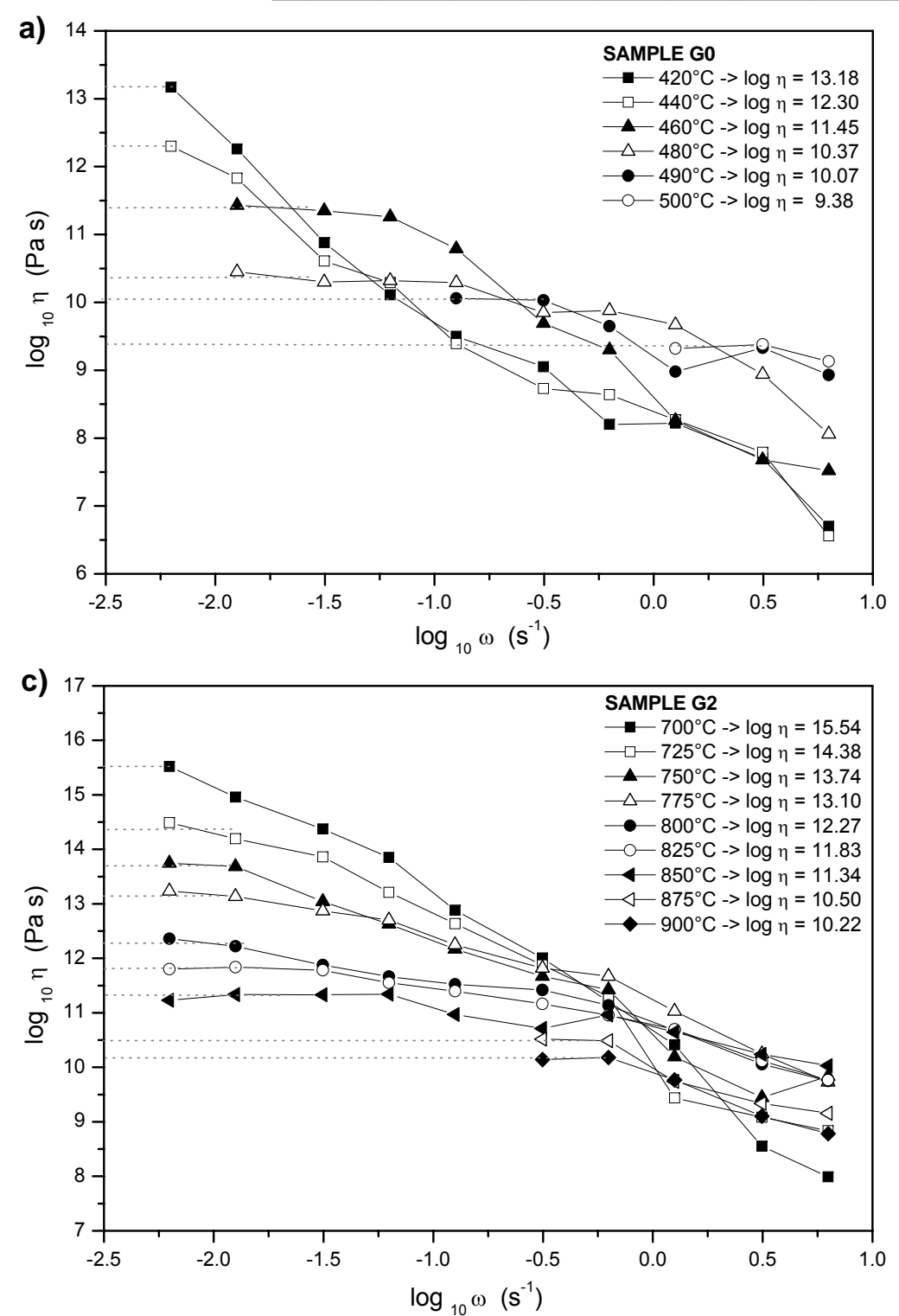

Discussion.
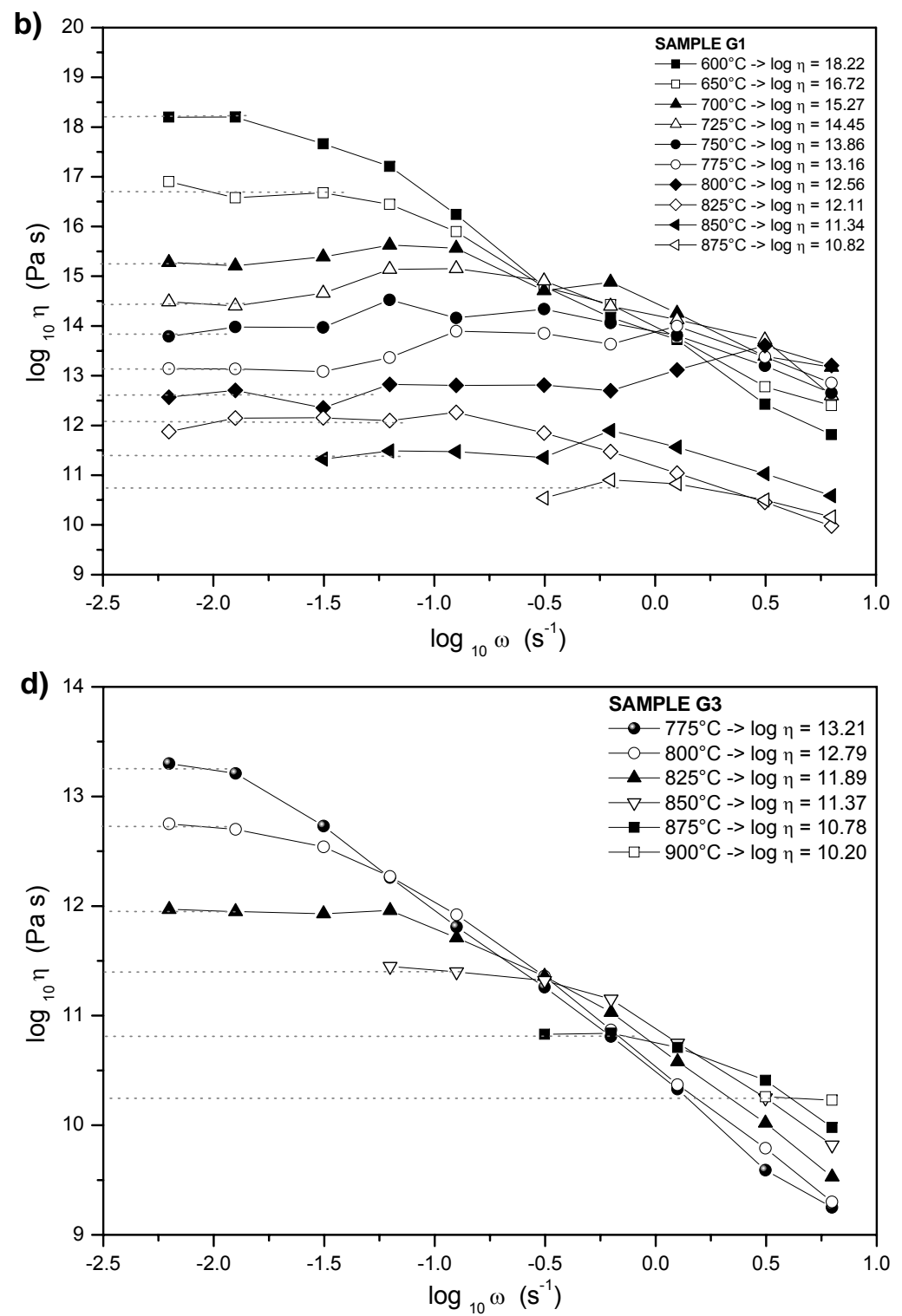

Fig. 79a, b, c, d. Frequency dependent viscosity of the present melts for a range of temperatures. a) sample G0; b) sample G1; c) sample G2; d) sample G3. 

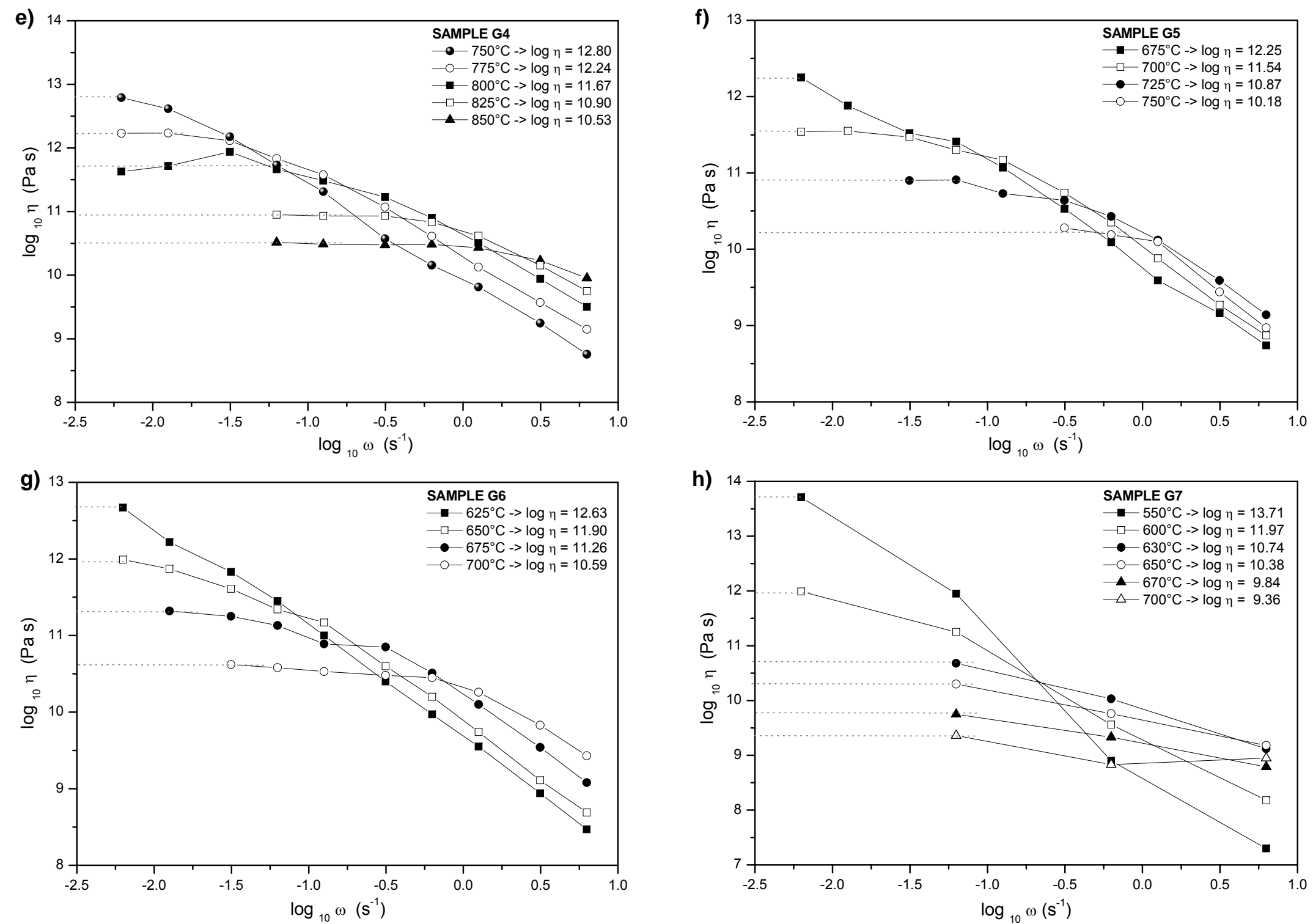

Fig. 79e, $\mathbf{f}, \mathbf{g}, \mathbf{h}$. Frequency dependent viscosity of the present melts for a range of temperatures. e) sample G4; f) sample G5; g) sample G6; $\mathbf{h}$ ) sample G7. 

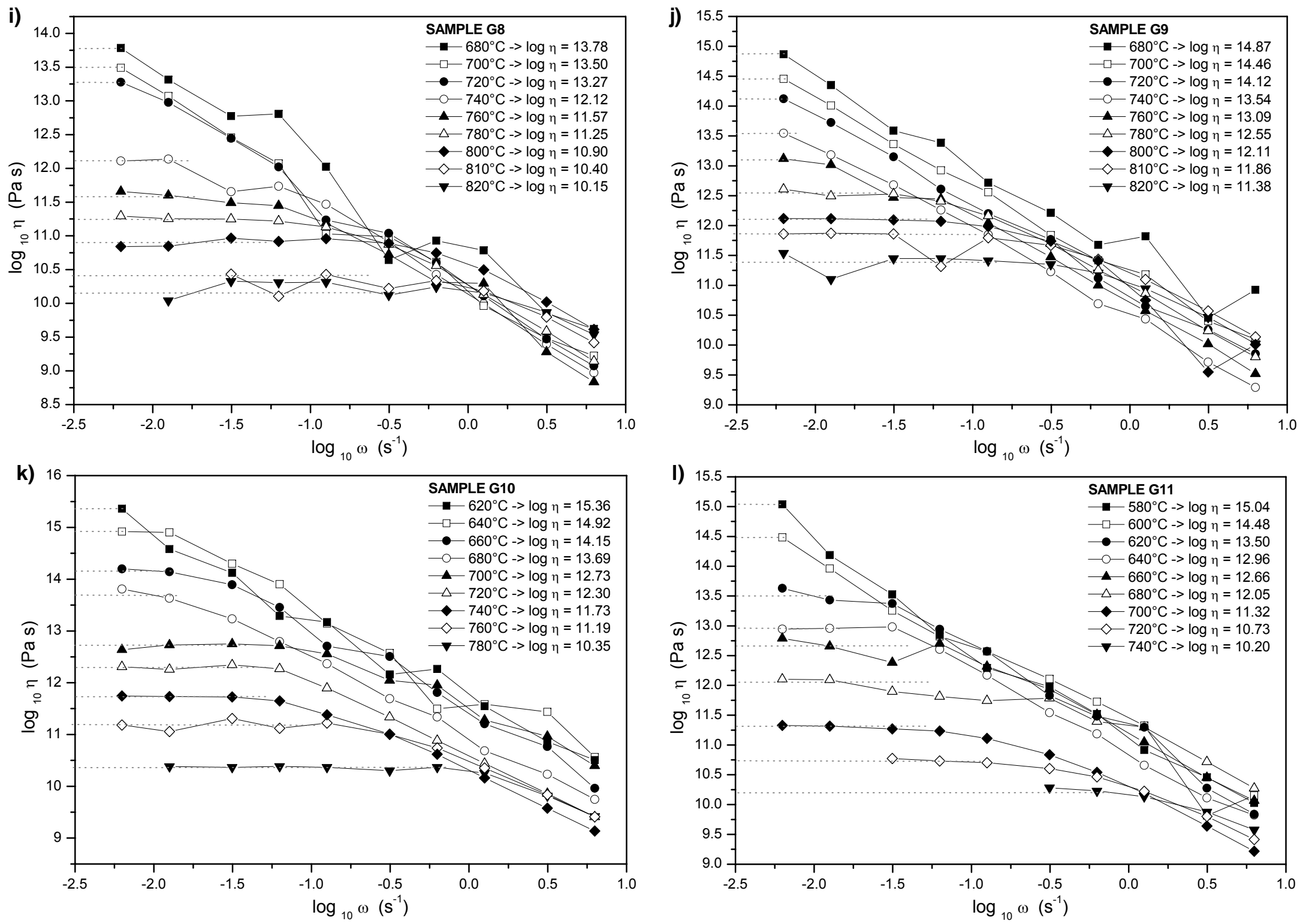

Fig. 79i, j, k, l. Frequency dependent viscosity of the present melts for a range of temperatures. i) sample G8; j) sample G9; k) sample G10; I) sample G11. 

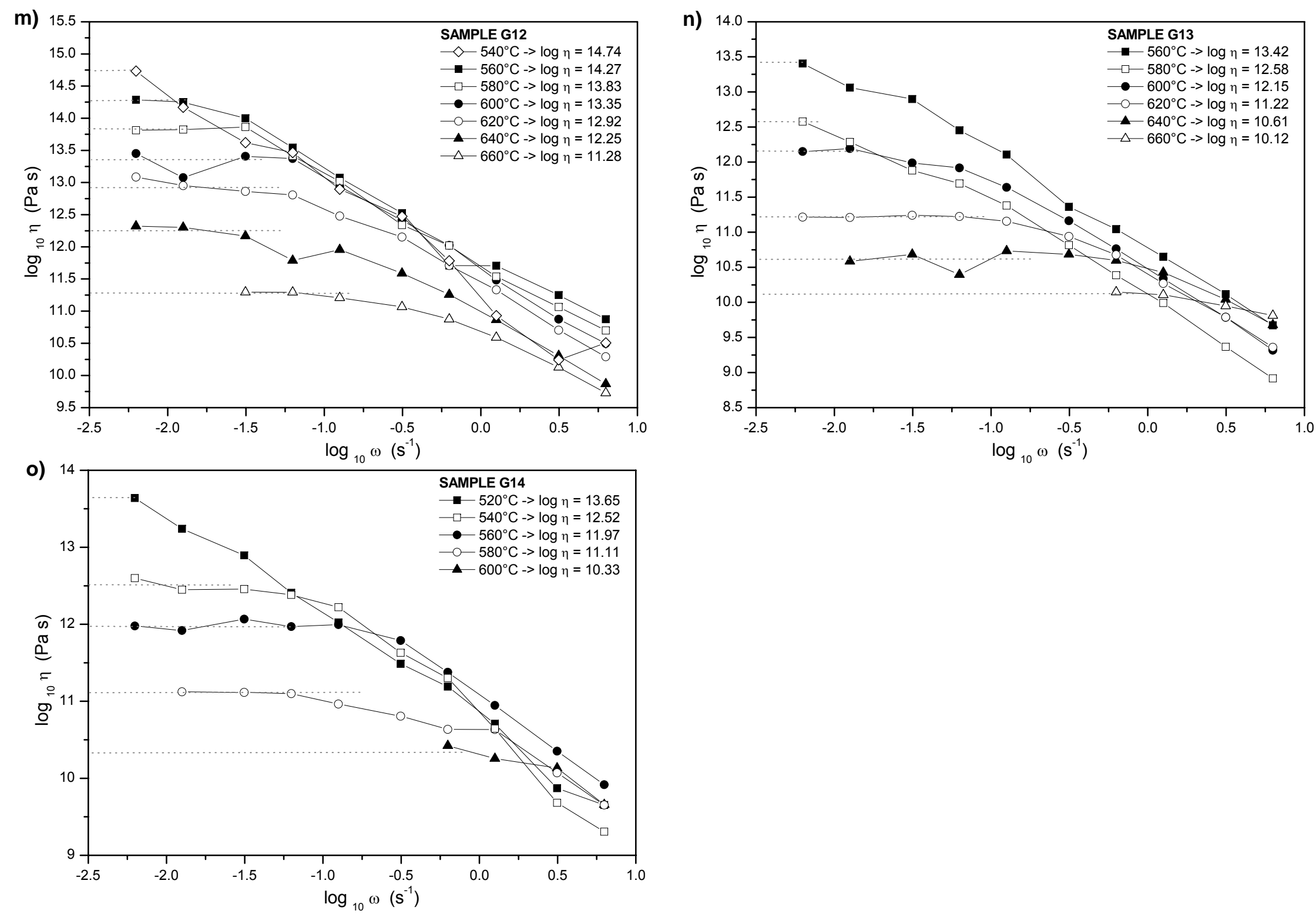

Fig. 79m, n, o. Frequency dependent viscosity of the present melts for a range of temperatures. $\mathbf{m}$ ) sample G12; $\mathbf{n}$ ) sample G13; o) sample G14. 


\section{Discussion.}
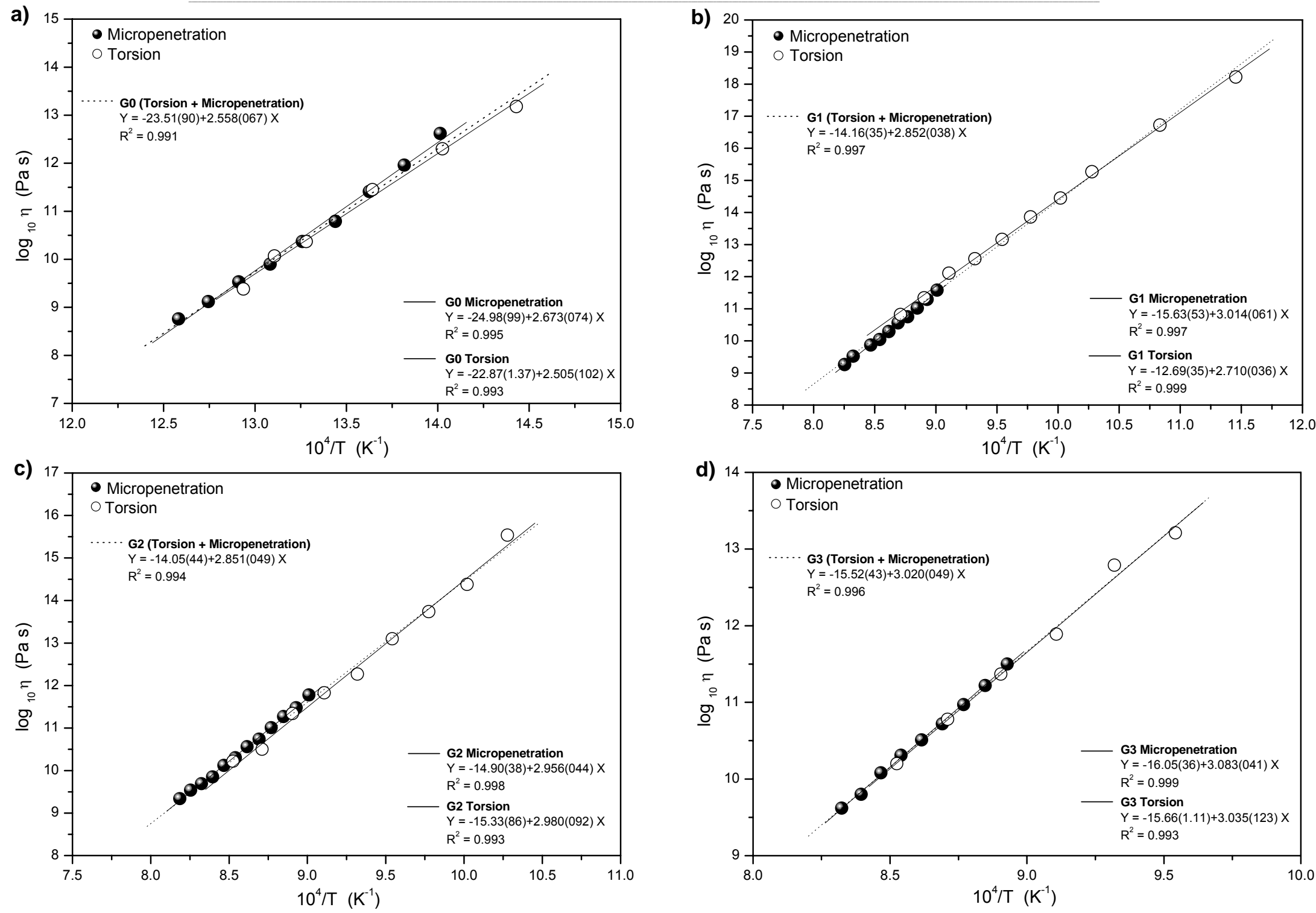

Fig. 80a, b, c, d. Viscosity determined by micropenetration and torsion as a function of inverse temperature. The two sets of viscosity data lie on the same Arrhenian line. a) sample G0; b) sample G1; c) sample G2; d) sample G3. 

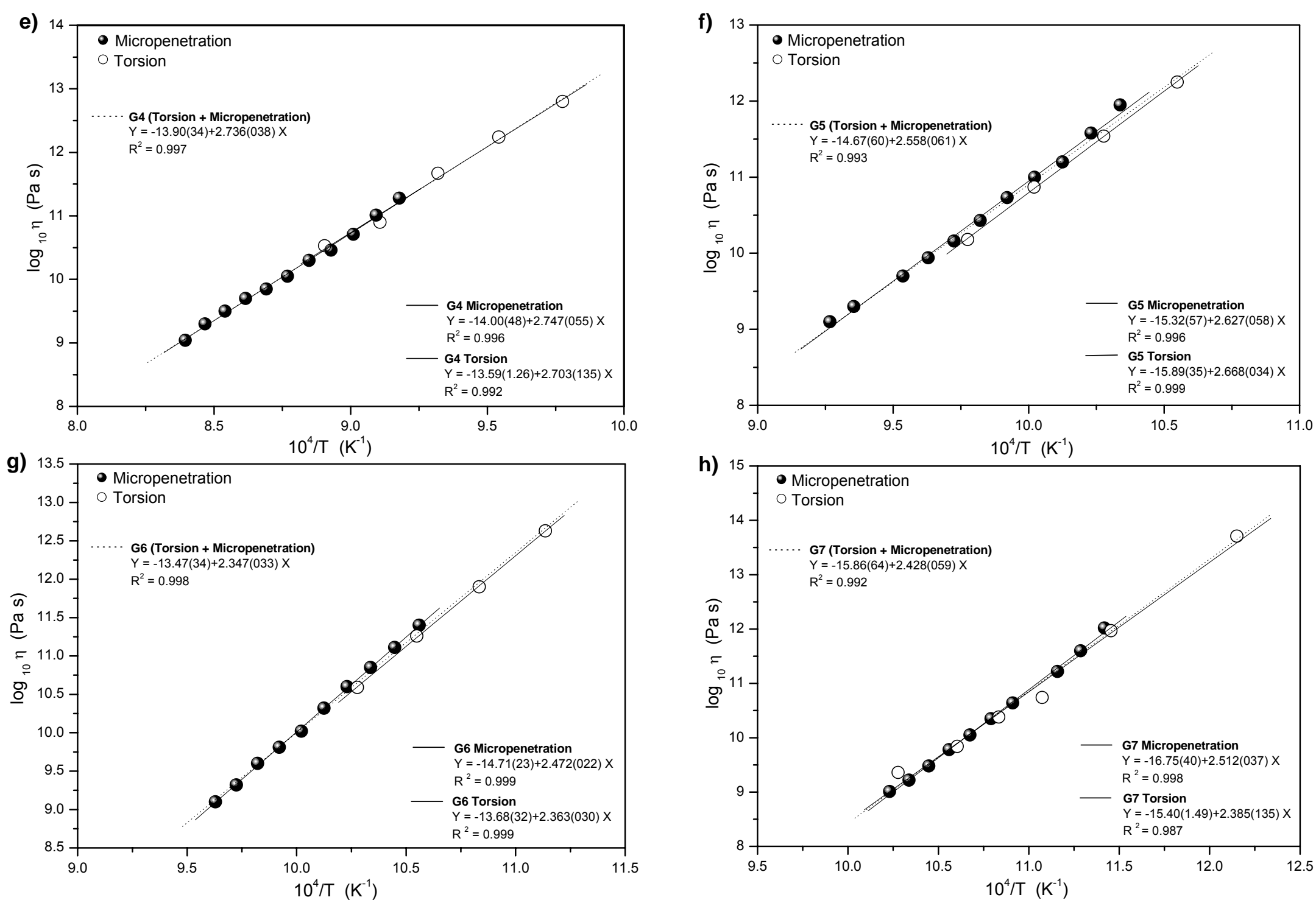

Fig. 80e, $\mathbf{f}, \mathbf{g}, \mathbf{h}$. Viscosity determined by micropenetration and torsion as a function of inverse temperature. The two sets of viscosity data lie on the same Arrhenian line. e) sample G4; f) sample G5; g) sample G6; h) sample G7. 

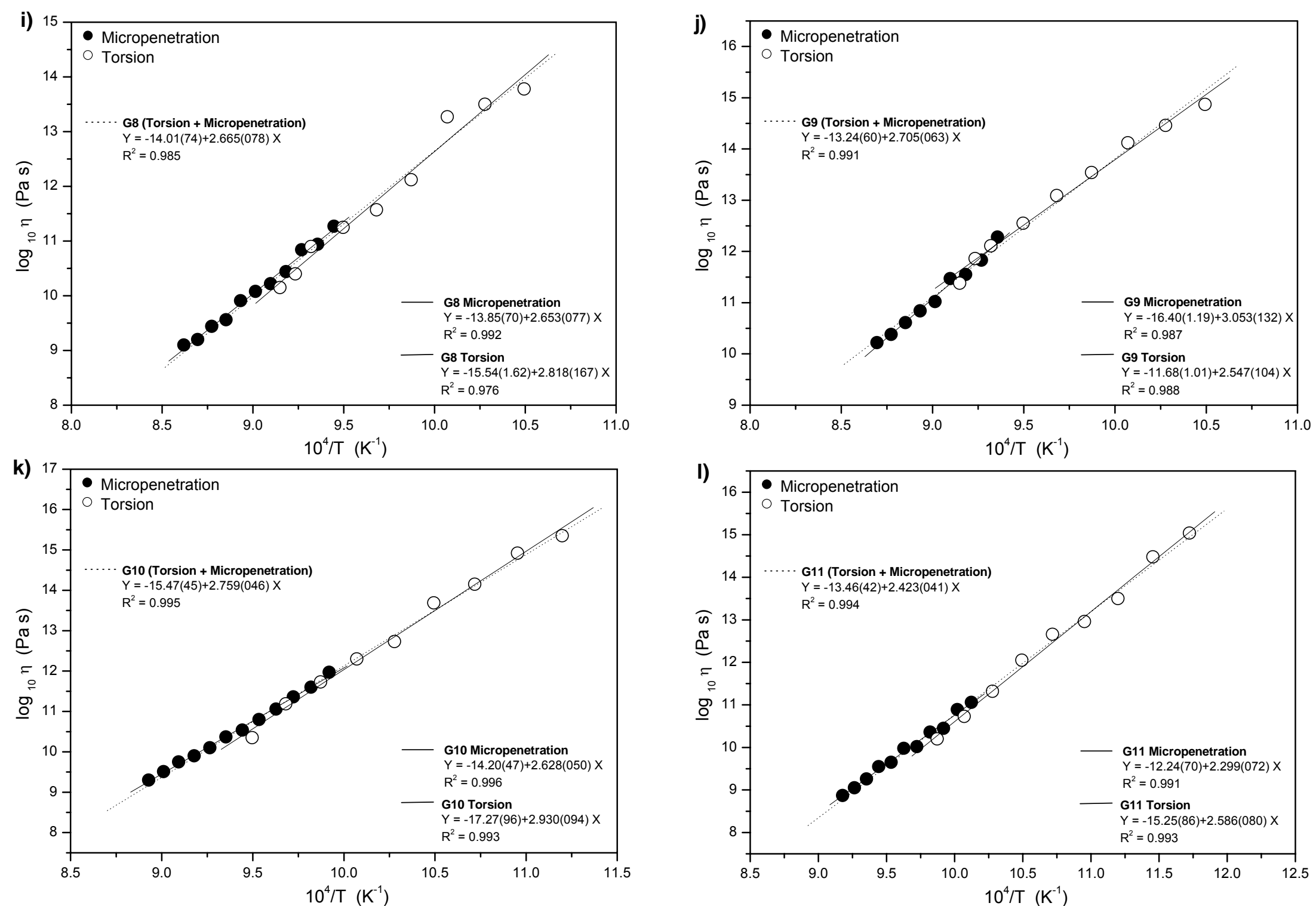

Fig. 80i, j, k, l. Viscosity determined by micropenetration and torsion as a function of inverse temperature. The two sets of viscosity data lie on the same Arrhenian line. i) sample G8; j) sample G9; k) sample G10; I) sample G11. 

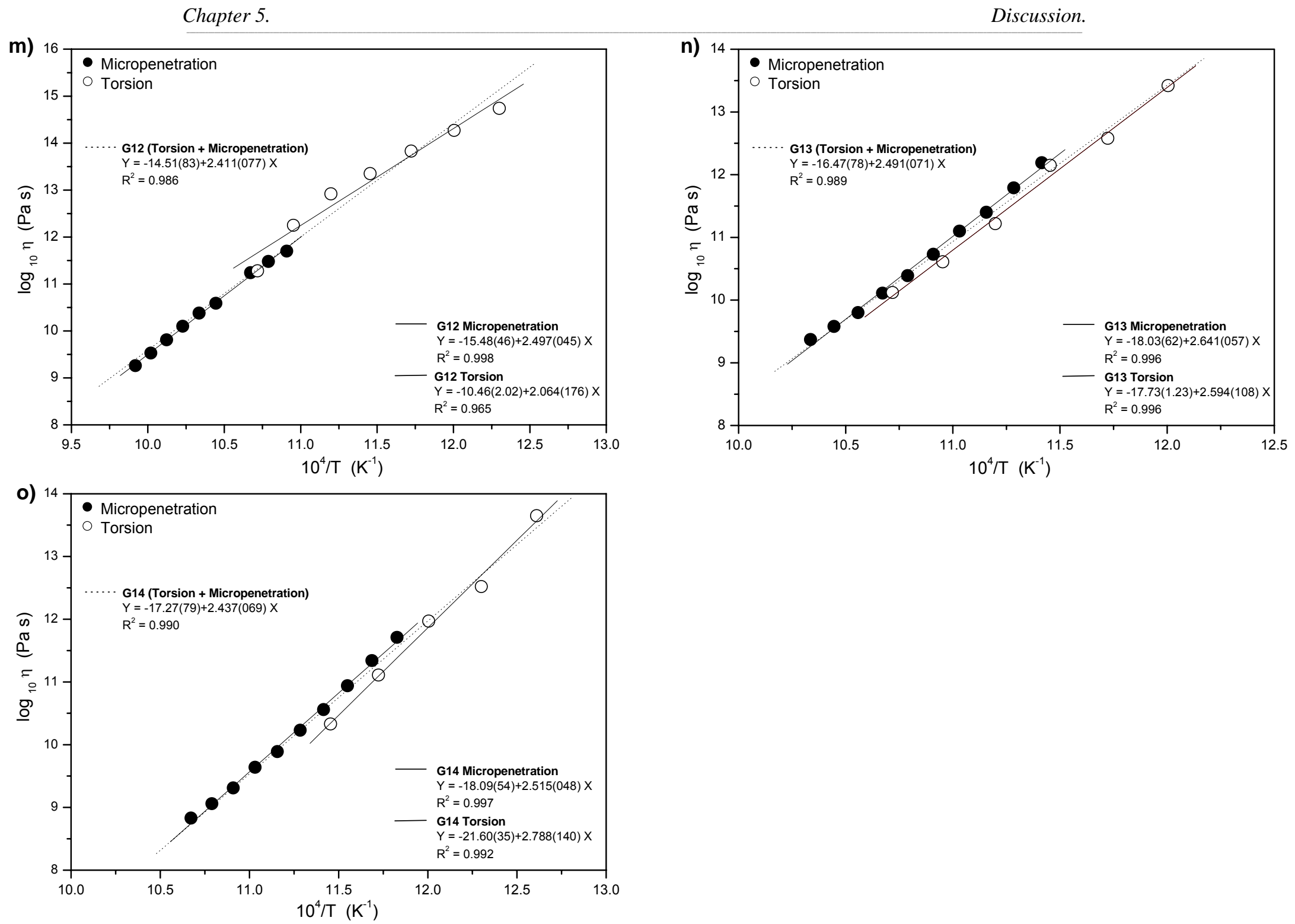

Fig. $\mathbf{8 0 m}, \mathbf{n}, \mathbf{o}$. Viscosity determined by micropenetration and torsion as a function of inverse temperature. The two sets of viscosity data lie on the same Arrhenian line. $\mathbf{m}$ ) sample G12; $\mathbf{n}$ ) sample G13; o) sample G14. 
Tab. 21. Shear viscosity data calculated from torsion data for NS2 melt (G0), Fe-free melts (G1-G7) and Fe-bearing melts (G8-G14) as a function of temperature (presented also in Figure 79).

\begin{tabular}{|c|c|c|c|c|c|c|c|c|c|}
\hline \multicolumn{2}{|c|}{ G0 } & \multicolumn{2}{c|}{ G1 } & \multicolumn{2}{c|}{ G2 } & \multicolumn{2}{c|}{ G3 } & \multicolumn{2}{c|}{ G4 } \\
\hline $\mathrm{T}$ & $\begin{array}{c}\eta \\
\log _{10}\end{array}$ & $\mathrm{~T}$ & $\begin{array}{c}\eta \\
{ }^{\circ} \mathrm{C}\end{array}$ & $\begin{array}{c}\mathrm{T} \\
\log _{10} \\
\mathrm{~Pa} \mathrm{~s}\end{array}$ & $\begin{array}{c}{ }^{\circ} \mathrm{C} \\
\mathrm{Pag}_{10}\end{array}$ & $\begin{array}{c}\mathrm{T} \\
\log _{10}\end{array}$ & $\begin{array}{c}{ }^{\circ} \mathrm{C} \\
\log _{10} \\
\mathrm{~Pa}\end{array}$ & $\begin{array}{c}\mathrm{T} \\
{ }^{\circ} \mathrm{C}\end{array}$ & $\begin{array}{c}\eta \\
\log _{10} \\
\mathrm{~Pa} \mathrm{~s}\end{array}$ \\
\hline 500 & 9.38 & 875 & 10.82 & 900 & 10.22 & 900 & 10.20 & 850 & 10.53 \\
490 & 10.07 & 850 & 11.34 & 875 & 10.50 & 875 & 10.78 & 825 & 10.90 \\
480 & 10.37 & 825 & 12.11 & 850 & 11.34 & 850 & 11.37 & 800 & 11.67 \\
460 & 11.45 & 800 & 12.56 & 825 & 11.83 & 825 & 11.89 & 775 & 12.24 \\
440 & 12.30 & 775 & 13.16 & 800 & 12.27 & 800 & 12.79 & 750 & 12.80 \\
420 & 13.18 & 750 & 13.86 & 775 & 13.10 & 775 & 13.21 & & \\
& & 725 & 14.45 & 750 & 13.74 & & & & \\
& & 700 & 15.27 & 725 & 14.38 & & & & \\
& & 650 & 16.72 & 700 & 15.54 & & & & \\
\hline
\end{tabular}

\begin{tabular}{|c|c|c|c|c|c|c|c|c|c|}
\hline \multicolumn{2}{|c|}{ G5 } & \multicolumn{2}{|c|}{ G6 } & \multicolumn{2}{|c|}{ G7 } & \multicolumn{2}{|c|}{ G8 } & \multicolumn{2}{|c|}{ G9 } \\
\hline $\begin{array}{c}\mathrm{T} \\
{ }^{\circ} \mathrm{C}\end{array}$ & $\begin{array}{c}\eta \\
\log _{10} \\
\text { Pa s }\end{array}$ & $\begin{array}{l}\mathrm{T} \\
{ }^{\circ} \mathrm{C}\end{array}$ & $\begin{array}{c}\eta \\
\log _{10} \\
\text { Pa s }\end{array}$ & $\begin{array}{c}\mathrm{T} \\
{ }^{\circ} \mathrm{C}\end{array}$ & $\begin{array}{c}\eta \\
\log _{10} \\
\text { Pa s }\end{array}$ & $\begin{array}{c}\mathrm{T} \\
{ }^{\circ} \mathrm{C}\end{array}$ & $\begin{array}{c}\eta \\
\log _{10} \\
\text { Pa s }\end{array}$ & $\begin{array}{c}\mathrm{T} \\
{ }^{\circ} \mathrm{C}\end{array}$ & $\begin{array}{c}\eta \\
\log _{10} \\
\text { Pa s }\end{array}$ \\
\hline 750 & 10.18 & 700 & 10.59 & 700 & 9.36 & 820 & 10.15 & 820 & 11.38 \\
\hline 725 & 10.87 & 675 & 11.26 & 670 & 9.84 & 810 & 10.40 & 810 & 11.86 \\
\hline 700 & 11.54 & 650 & 11.90 & 650 & 10.38 & 800 & 10.90 & 800 & 12.11 \\
\hline 675 & 12.25 & 625 & 12.63 & 630 & 10.74 & 780 & 11.25 & 780 & 12.55 \\
\hline & & & & 600 & 11.97 & 760 & 11.57 & 760 & 13.09 \\
\hline & & & & 550 & 13.71 & 740 & 12.12 & 740 & 13.54 \\
\hline & & & & & & 720 & 13.27 & 720 & 14.12 \\
\hline & & & & & & 700 & 13.50 & 700 & 14.46 \\
\hline & & & & & & 680 & 13.78 & 680 & 14.87 \\
\hline
\end{tabular}

\begin{tabular}{|c|c|c|c|c|c|c|c|c|c|}
\hline \multicolumn{2}{|c|}{ G10 } & \multicolumn{2}{c|}{ G11 } & \multicolumn{2}{c|}{ G12 } & \multicolumn{2}{c|}{ G13 } & \multicolumn{2}{c|}{ G14 } \\
\hline $\mathrm{T}$ & $\begin{array}{c}\eta \\
\log _{10}\end{array}$ & $\begin{array}{c}\mathrm{T} \\
{ }^{\circ} \mathrm{C}\end{array}$ & $\begin{array}{c}\eta \\
\log _{10} \\
{ }^{\circ} \mathrm{C}\end{array}$ & $\begin{array}{c}\mathrm{T} \\
{ }^{\circ} \mathrm{C}\end{array}$ & $\begin{array}{c}\eta \\
\log _{10} \\
\mathrm{~Pa} \mathrm{~s}\end{array}$ & $\begin{array}{c}\mathrm{T} \\
{ }^{\circ} \mathrm{C}\end{array}$ & $\begin{array}{c}\eta \\
\log _{10} \\
\mathrm{~Pa} \mathrm{~s}\end{array}$ & $\begin{array}{c}\mathrm{T} \\
{ }^{\circ} \mathrm{C}\end{array}$ & $\begin{array}{c}\eta \\
\log _{10} \\
\mathrm{~Pa} \mathrm{~s}\end{array}$ \\
\hline 780 & 10.35 & 740 & 10.20 & 660 & 11.28 & 660 & 10.12 & 600 & 10.33 \\
760 & 11.19 & 720 & 10.73 & 640 & 12.25 & 640 & 10.61 & 580 & 11.11 \\
740 & 11.73 & 700 & 11.32 & 620 & 12.92 & 620 & 11.22 & 560 & 11.97 \\
720 & 12.30 & 680 & 12.05 & 600 & 13.35 & 600 & 12.15 & 540 & 12.52 \\
700 & 12.73 & 660 & 12.66 & 580 & 13.83 & 580 & 12.58 & 520 & 13.65 \\
680 & 13.69 & 640 & 12.96 & 560 & 14.27 & 560 & 13.42 & & \\
660 & 14.15 & 620 & 13.50 & 540 & 14.74 & & & & \\
640 & 14.92 & 600 & 14.48 & & & & & & \\
620 & 15.36 & 580 & 15.04 & & & & & & \\
\hline
\end{tabular}

The agreement between viscosity determined using these two techniques also supports the shift in relaxation times to shorter values being real and not due to errors. The torsion data for stress, strain and strain-rate are correctly measured, as are the temperatures of the furnace. It also proved that there is no slippage on the surface between sample and alumina rod in torsion machine. 


\subsubsection{Fragility}

Fragility $(m)$ is the one parameter used to describe melts (Angell, 1988; Plazek \& Ngai, 1991; Böhmer \& Angell, 1992). It is not a structural parameter but describes the curvature of viscosity trends. Because the curvature of viscosity data as a function of inverse temperature can be different depending on the composition of the melt, the fragility value describes the shape of the $\log _{10}$ viscosity line as a function of inverse temperature. The widely accepted method to calculate fragility is the gradient of $\log _{10} \eta$ as a function of reduced temperature $T_{g} / T$ at $T_{g}$ (Toplis et al., 1997b; Casalini \& Roland, 2005; Webb, 2005):

$$
\text { fragility }=m=\left.\frac{d\left(\log _{10} \eta\right)}{d\left(T_{g} / T\right)}\right|_{T=T_{g}}
$$

where $T_{g}$ is the temperature at which $\eta=10^{12} \mathrm{~Pa} \mathrm{~s}$; and is the glass transition temperature. Value $m \sim 50$ indicates a fragile melt and shows the highest curvature of the viscosity plot (Fig. 81). Value $\mathrm{m} \sim 16$ is almost a straight line and is characteristic for strong melts $\left(\mathrm{SiO}_{2}\right.$-rich). Strong melts agree with Arrhenian equation (Eq. 24), whereas "fragile" have characteristic non-Arrhenian behaviour (Angell, 1988).

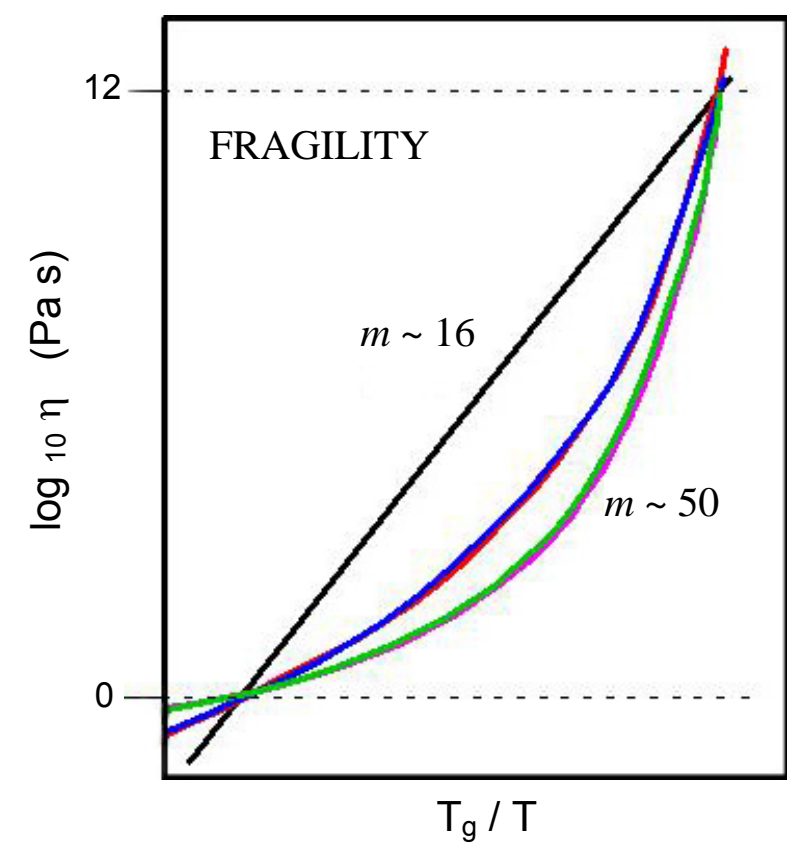

Fig. 81. Fragility of the melts characterised by the curvature of the viscosity plot as a function of reduced temperature. 
For the investigated melts fragility has been calculated from the gradient of the viscosity curve at the glass transition temperature using the equation:

$$
\text { fragility }=m=\frac{B \cdot 10^{4}}{T_{g}^{12}},
$$

where $B$ (in $K$ ) is the parameter of Arrhenian equation (see Eq. 24) and $\mathrm{T}_{\mathrm{g}}{ }^{12}$ (in $\mathrm{K}$ ) is the temperature, at which the $\log _{10}$ viscosity equals 12 (Toplis et al., 1997b). Data were presented in the Table 22 and showed in Figure 82. Fragility for sodium silicate melt (sample G0) is 36.98 and this is the most fragile sample of all of them. There is clear minimum in the fragility of the Fe-free and Fe-bearing melts at $\gamma \sim 0.49$ indicating some "stronger" than others behaviour of the samples.

Tab. 22. Fragility of the investigated samples calculated using Eq. 67.

\begin{tabular}{|c|c|}
\hline Melt number & FRAGILITY $\boldsymbol{~}$ \\
\hline \hline G0 & 36.98 \\
\hline G1 & 27.63 \\
G2 & 26.90 \\
G3 & 28.05 \\
G4 & 26.02 \\
G5 & 27.32 \\
G6 & 26.70 \\
G7 & 28.75 \\
\hline \hline G8 & 25.85 \\
G9 & 28.40 \\
G10 & 26.20 \\
G11 & 24.24 \\
G12 & 27.48 \\
G13 & 30.04 \\
G14 & 30.10 \\
\hline
\end{tabular}

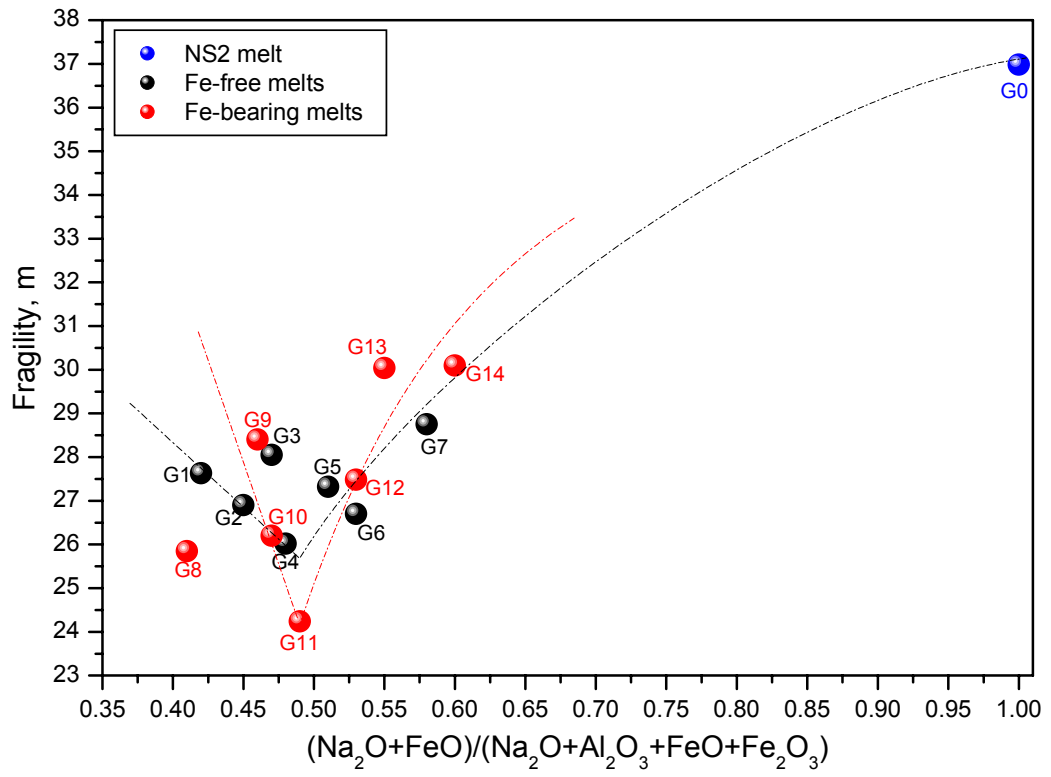

Fig. 82. Fragility of the investigated samples calculated from Eq. 67. The trends are the guides to the eye. Sample G8 lies outside the trend because contain less iron than the others. 


\subsection{Shear modulus}

\subsubsection{General}

The major observation from the relaxation times of the frequency dependent shear modulus data obtained in this study is that the most of the relaxation times of the samples deviate from Maxwell relaxation time and they are up to 1.3 orders of magnitude faster than Maxwell theory assumes (Fig. 83).

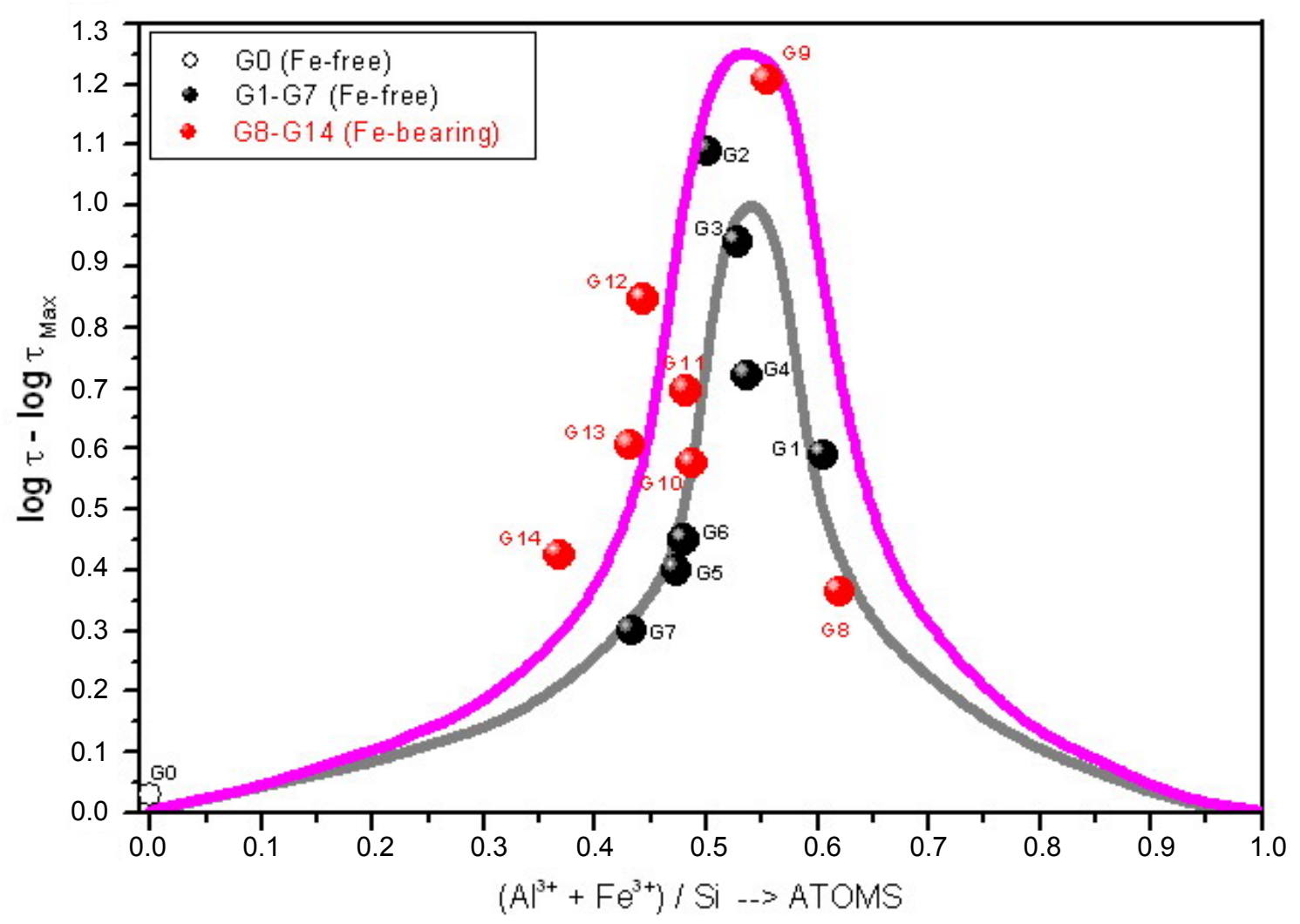

Fig. 83. Deviation of the relaxation times as a function of composition for all of the investigated samples. Sample $\mathrm{G} 8$ has only $1 \mathrm{~mol} \% \mathrm{Fe}_{2} \mathrm{O}_{3}$, otherwise it will crystallize.

The second major observation is the presence of a second energy loss peak occurring for different samples from $\sim 5.5$ to $\sim 7.5$ orders of magnitude faster than the slowest relaxation (Fig. 84). 


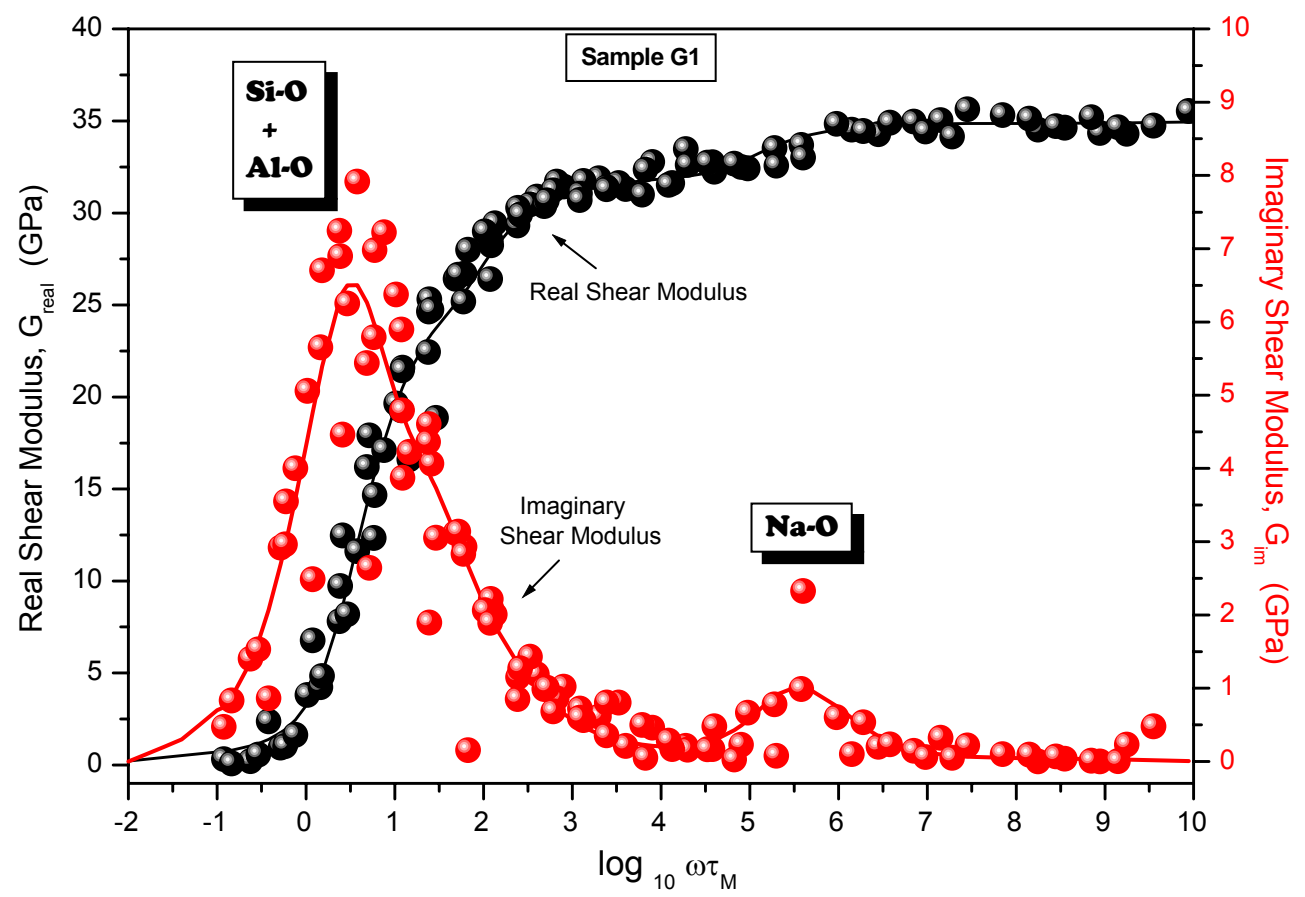

Fig. 84. The real and imaginary components of the shear modulus of $\mathrm{G} 1$ - the most peraluminous Fe-free melt composition - as a function of $\log _{10} \omega \tau$. Two peaks in the imaginary shear modulus are observable.

The first question is whether this relaxation faster than Maxwell relaxation time is real. To answer this, the temperature of the furnace was checked and controlled through whole time of the measurements. It needs to be mentioned than $\sim 30^{\circ} \mathrm{C}$ increase in temperature is equivalent to 1 order of magnitude decrease in viscosity - and therefore also in relaxation time (Webb, 1992b). The torsion furnace has been calibrated and there is a $\pm 3^{\circ} \mathrm{C}$ temperature variation across the $30 \mathrm{~mm}$ of the sample.

If there were a temperature variations during the measurement, the curves as a function of $\log _{10} \omega \tau$ would not be smooth. A further check was the successful comparison of the torsion viscosity with the micropenetration viscosity.

All of the frequency dependent data in Figures 68 and 69 show that a distribution in relaxation timescales is required to describe the observed frequency dependence of the shear modulus. The torsion measurements provide not only the shear modulus, but also the shear viscosity as a function of deformation frequency;

$$
\eta=\frac{\sigma}{\dot{\varepsilon}}=\frac{G^{*}(\omega)}{i \omega} \quad \text { as } \quad G=\frac{\sigma}{\dot{\varepsilon}}
$$

The shear viscosity determined from torsion data is presented in Figures 79 and 80. In Figure 79 , at constant temperature the real component of the shear viscosity increases together with decreasing frequency and becomes independent when it reaches 
a relaxed Newtonian viscosity. The value of relaxed Newtonian viscosity from torsion machine should agree with the viscosity obtained at the same temperature with micropenetration technique. As can be observed in Figure 80 , the results from both methods are identical. It confirms again that results of faster relaxation time from torsion machine are not due to the error during the measurements.

\subsubsection{Diffusion}

Of the three peaks expected in the imaginary part of the mechanical spectrum, only 2 are seen (Fig. 84). The second, fast structural relaxation is assumed to be associated with the diffusion of $\mathrm{Na}^{+}$in the melt. Given the relatively successful use of the Eyring relationship:

$$
D=\frac{k T}{\lambda \eta}
$$

for diffusivity - $D$ (as a transport property), Boltzmann's constant $-k$, temperature $T$, viscosity $\eta$, and jump distance $\lambda$, there is a relationship between the timescale of diffusion of Si and O and of viscous flow (e.g. Glasstone et al., 1941; Magaritz \& Hofmann, 1978; Watson, 1979; Dunn, 1982; Shimizu \& Kushiro, 1984; Mysen, 1995b; Reid et al., 2001).

In the calculation the relationship between diffusion, viscosity and relaxation time the other equation are also used. The Stokes equation connects diffusion coefficient $D$, rigid spheres of radius $a$ and the viscosity of the melt $\eta$ :

$$
D=\frac{k T}{6 \pi a \eta} .
$$

In the diffusion calculation this equation is combined very often with Einstein Smoluchowski equation (Farnan \& Stebbins, 1990b; Stebbins et al., 1995), which is the relationship between relaxation time $\tau$, diffusion coefficient $D$ and average jump distance $d$ (taken as $0.31 \mathrm{~nm}$ ):

$$
D=\frac{d^{2}}{6 \tau} .
$$




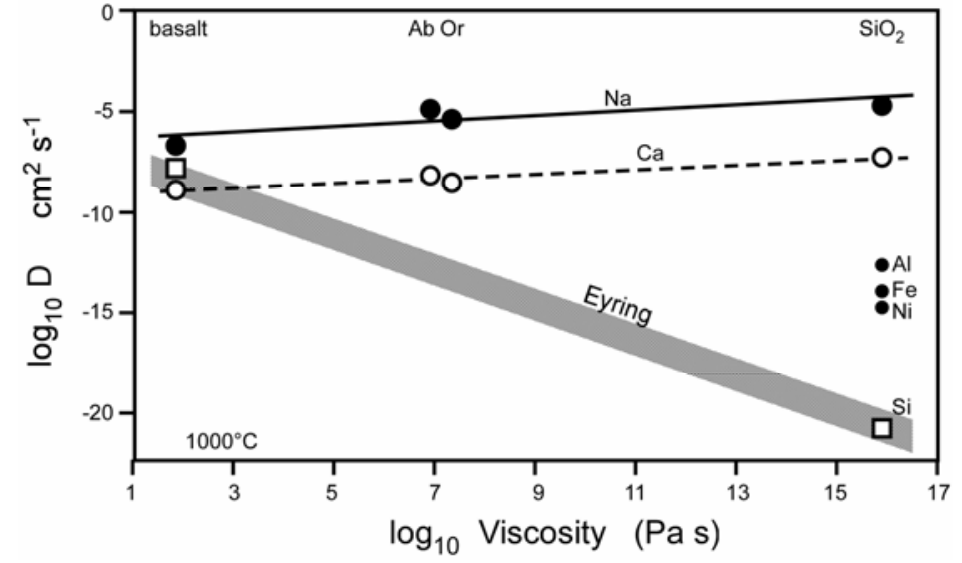

Fig. 85. $\mathrm{Na}, \mathrm{Ca}, \mathrm{Al}, \mathrm{Fe}, \mathrm{Ni}$ and $\mathrm{Si}$ tracer diffusivity data as a function of $\log _{10}$ viscosity ( $\mathrm{Pa}$ s) for basalt, Ca-Al-Si synthetic oxide melt, albite and orthoclase melts, and $\mathrm{SiO}_{2}$ melt at $1000^{\circ} \mathrm{C}$. Redrawn from Dingwell \& Webb, 1990.

The compilation of diffusivity data in Dingwell \& Webb (1990) (Fig. 85) illustrate the diffusion rate of $\mathrm{Na}^{+}$approaches that of $\mathrm{Si}$ and $\mathrm{O}$ in silicate melts at high temperature (low viscosities); but at viscosities of $\sim 10^{12} \mathrm{~Pa} \mathrm{~s}$, the diffusivity of $\mathrm{Na}^{+}$is 12 orders of magnitude faster than that of $\mathrm{Si}$ and $\mathrm{O}$ for a $\mathrm{Na}_{2} \mathrm{O}-\mathrm{Al}_{2} \mathrm{O}_{3}-\mathrm{SiO}_{2}$ and also for a $\mathrm{Na}_{2} \mathrm{O}-\mathrm{SiO}_{2}$ melt.

The diffusion data of Mungall et al. (1998) (Fig. 86) indicate a $\sim 5-7$ orders of magnitude difference between the lifetime of a $\mathrm{Na}-\mathrm{O}$ bond and a $\mathrm{Si}-\mathrm{O}$ bond. This indicates that the fast relaxation seen in our measurements is due to $\mathrm{Na}^{+}$diffusion (see also Day \& Rindone, 1962).

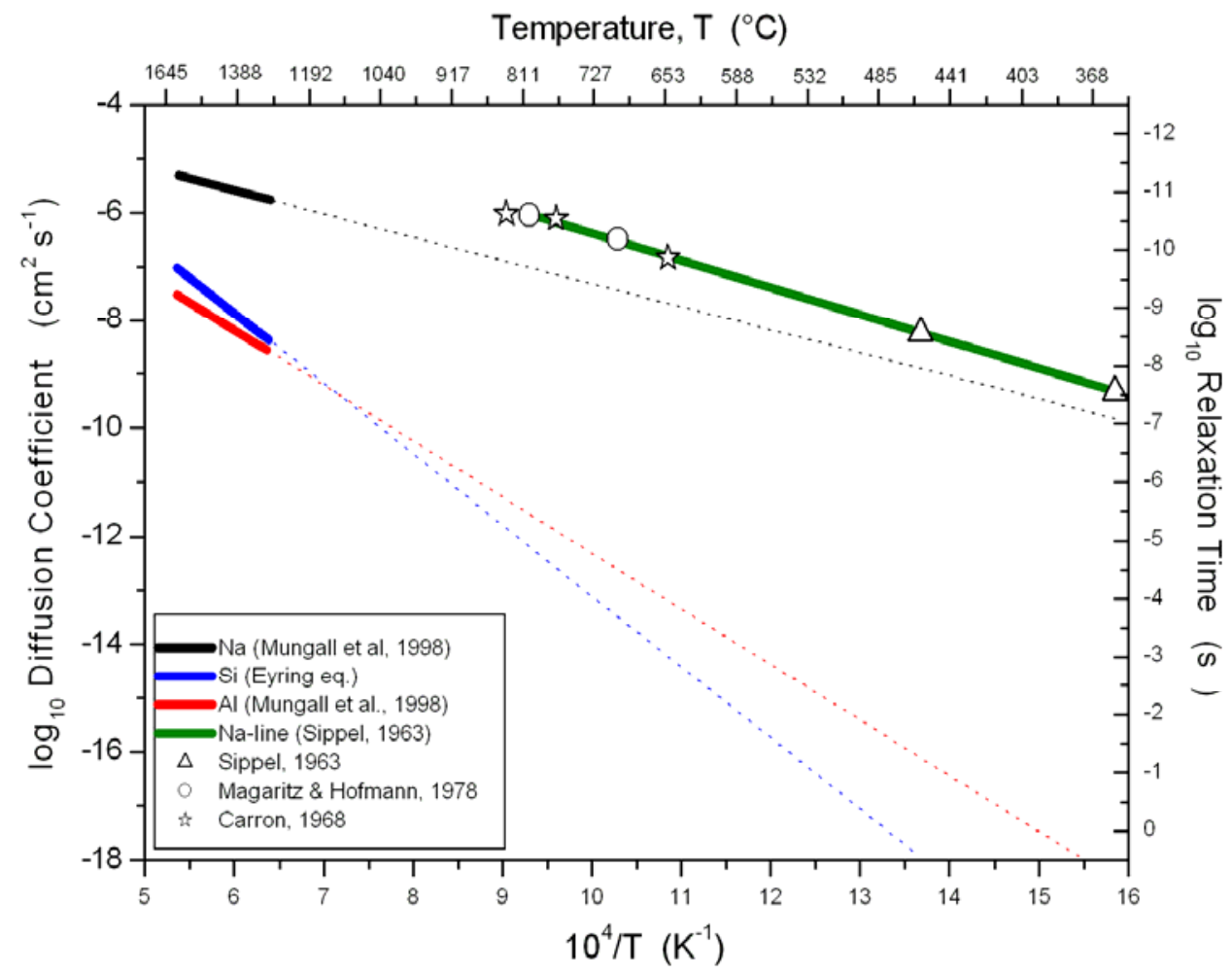

Fig. 86. Diffusivity data for $\mathrm{Al}, \mathrm{Si}$ and $\mathrm{Na}$ in a water-bearing haplogranitic melt (Mungall et al., 1998) - thick black, blue and red lines. These data are extrapolated in temperature to the conditions of the present torsion measurements in order to calculate the timescale of structural motion associated with the diffusion of each of these species. The thick green line shows Na diffusion data in obsidian. 
The bonds of the Na with BO are weaker than bonds Na-NBO (George \& Stebbins, 1996). From this reason $\mathrm{Na}$ can be more mobile and more ionic in the complex melts than in the binary silicates what was suggested by Hsieh et al. (1994) and Lam et al. (1980) with an X-ray photoelectron spectroscopy. Ionicity of sodium can also change the potential barriers to Na motion (Marchi et al., 1988). But the movement of this fast peak to slower times with increasing Al-content (see Fig. 87) agrees with the observations of Kargl \& Meyer (2004), who showed that an increase of the amount of Al in the melt composition causes a decrease in sodium mobility and relaxation time of the $\mathrm{Na}-\mathrm{O}$ bonds becomes slower (peak shifts to the left) (Fig. 87). Such observation is visible in the investigated melts. This further supports our identification of this peak as the $\mathrm{Na}$ peak. $\beta$-relaxation peak moves at about 2 orders of magnitude with increasing $\mathrm{Al}_{2} \mathrm{O}_{3}$ content from $15.9 \mathrm{~mol} \%$ (sample G6) to 19.9 mol\% (sample G1) in Fe-free melts. In Fe-bearing melt this correlation is difficult to do.

a)

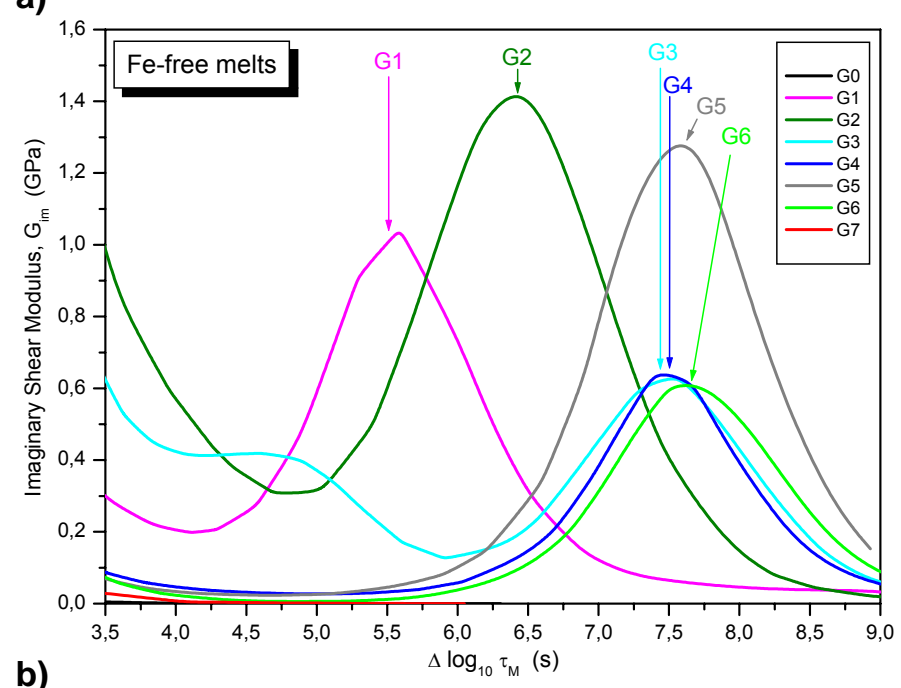

b)

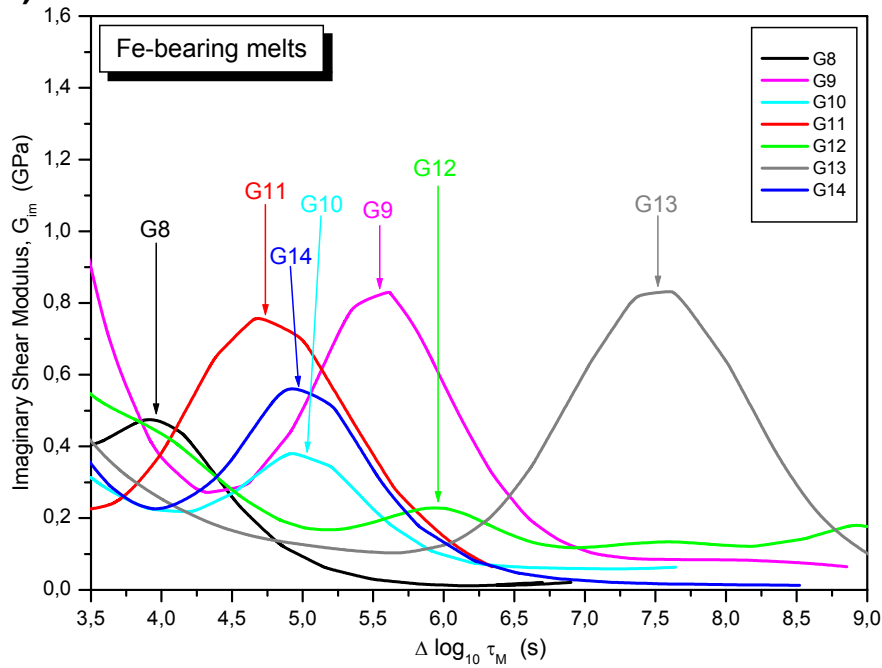

Fig. 87. Shift of the $\beta-$ relaxation toward slower relaxation time with increasing $\mathrm{Al}_{2} \mathrm{O}_{3}$ content. a) Fe-free melts; there is not enough data for sample $\mathrm{G} 7$ to indicate a $\beta$ relaxation peak; b) Fe-bearing melts. 
The expected $\mathrm{Al}^{3+}-\mathrm{O}$ relaxation peak cannot be resolved in our spectra. The data from Figure 85 as well as the extrapolated diffusion data from Mungall et al (1998) for $\mathrm{Al}^{3+}$ diffusion in $\mathrm{Na}_{2} \mathrm{O}-\mathrm{Al}_{2} \mathrm{O}_{3}-\mathrm{SiO}_{2}$ and $\mathrm{Na}_{2} \mathrm{O}-\mathrm{K}_{2} \mathrm{O}-\mathrm{Al}_{2} \mathrm{O}_{3}-\mathrm{SiO}_{2}-\mathrm{H}_{2} \mathrm{O}$ melts respectively (Fig. 86), suggest that the timescale of $\mathrm{Al}^{3+}$ diffusion is close to that of $\mathrm{Si}$ and $\mathrm{O}$ diffusion at the temperature and viscosity conditions of our measurements. Thus it appears that the $\mathrm{Al}^{3+}-\mathrm{O}$ peak is part of the Si-O peak (Fig. 86). These two peaks together appear as a broad peak in the imaginary data resulting in the calculation of a distribution of relaxation times. Although there is a large amount of scatter in the FWHM data for these imaginary peaks, the FWHM for $\mathrm{Na}_{2} \mathrm{O}-\mathrm{SiO}_{2}$ melt (1.13) is much less than that for the $\mathrm{Al}_{2} \mathrm{O}_{3}$-bearing melts (1.40-2.68). This supports the conclusion that the Si-O and Al-O peaks form one peak.

It can be seen from the frequency dependence of the shear modulus that the glass transition does not occur at the $\omega \tau_{\mathrm{M}}=1$ condition as expected from Maxwell theory and the model of Herzfeld \& Litovitz (1959). This is in contrast to all of the literature data which shows that the large peak in the imaginary part of the shear modulus is centred on $\omega \tau_{M} \sim 1$ (see Mills, 1974; Webb, 1991; Bagdassarov et al., 1993). The HPG8 melts of Bagdassarov et al. (1993) with $\mathrm{Al} / \mathrm{Si}^{\text {atom }}=0.20$ and $\gamma=0.53$ have structural relaxation times ranging from $3 \tau_{M}$ (for HPG8) to $\sim 0.8 \tau_{M}$ (for HPG8+fluorine). The natural obsidian of Webb (1992a) has a relaxation time $\sim \tau_{M}$ with $\mathrm{Al} / \mathrm{Si}^{\text {atom }}=0.20$ and $\gamma=0.54$. However, the data for the $\mathrm{Na}_{2} \mathrm{O}-\mathrm{SiO}_{2}$ melt do have the maximum in the imaginary part at $\omega \tau_{\mathrm{M}}=1$. The only difference between the $\mathrm{Na}_{2} \mathrm{O}-\mathrm{SiO}_{2}$ and the $\mathrm{Na}_{2} \mathrm{O}-\mathrm{Al}_{2} \mathrm{O}_{3}-\mathrm{SiO}_{2}$ melts is the presence of $\mathrm{Al}_{2} \mathrm{O}_{3}$. The majority of melts whose structural relaxation time has been compared to the Maxwell relationship have been $\mathrm{Al}_{2} \mathrm{O}_{3}$-free or -poor. The present melts have $\mathrm{Al} / \mathrm{Si}^{\text {atom }}=0.43$ for the peralkaline melts $(\gamma=0.58)$ to $\mathrm{Al} / \mathrm{Si}^{\text {atom }}=0.60$ for the peraluminous melts $(\gamma=0.42)$.

Thus, instead of the data expected (see Fig. 49) it appears that there is no separate energy loss peak for the lifetime of $\mathrm{Al}-\mathrm{O}$ bonds. Rather this peak is combined into the Si-O peak. Also FWHM of the slowest peak remains independent of composition.

It is assumed that the $\mathrm{Si}-\mathrm{O}$ is the longest lived bond in silicate melts, although this was determined on Al-free melts. The Al-O lifetime is therefore shorter than Si-O lifetime, but Mungall's plot (Fig. 86) begins to question this assumption. The relative lifetimes of Si-O and Al-O bonds will be further discussed in section 5.8 .

Thus one can begin to interpret the data in Figures 67, 68, 69, 70 and 71 . The first energy loss peak is assumed to be associated not only with the lifetime of Si-O bonds, but also of $\mathrm{Al}-\mathrm{O}$ bonds, with the slightly faster moving $\mathrm{Al}$; and thus creating a faster structural relaxation time than that calculated from the Maxwell equation which assumes a simple mono-structural melt. The second energy loss peak is then associated with the motion of $\mathrm{Na}$ in the melt. 
The data of Stebbins \& Sen (1998) (for borosilicate glass-forming liquid) show that the lifetime of $\mathrm{Si}-\mathrm{O}$ bonds is not always identified with viscosity. They found the long lived $\mathrm{B}-\mathrm{O}$ bonds to control flow and determine viscosity; with the shorter lived Si-O bonds forming the $\beta$-relaxation (Fig. 88).

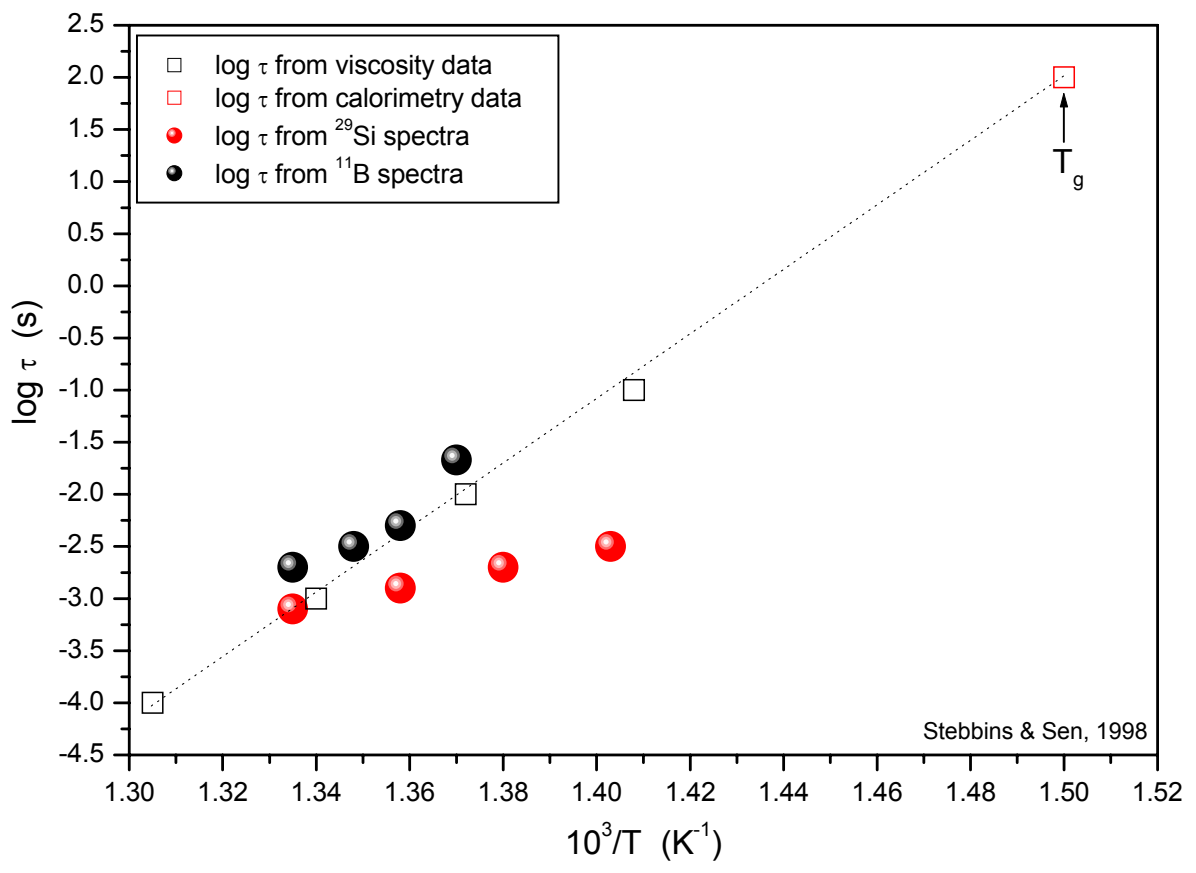

Fig. 88. Log $\tau$ derived from high temperature boron-11 (black solid circles) and silicon-29 (red solid circles) magic angle spinning nuclear magnetic resonance spectroscopy (MAS NMR) compared with shear relaxation times calculated from viscosity data (open black squares) and measured by differential scanning calorimetry (open red square). The dashed line is a fit to viscosity data as a guide to the eye. Redrawn after Stebbins \& Sen (1998).

\subsubsection{Attenuation}

The energy loss due to the movement of parts of the melt structure can also be analysed in terms of the attenuation $\mathrm{Q}^{-1}(\omega)$ (ratio between imaginary and real part of the shear modulus), where

$$
Q^{-1}(\omega)=\frac{J^{\prime \prime}(\omega)}{J^{\prime}(\omega)}
$$

for $J^{*}(\omega)=G^{*}(\omega)^{-1}$ (Webb \& Jackson, 2003; O'Connell \& Budiansky, 1978; Johnston \& Toksöz, 1981). Figure 90 shows $\log Q^{-1}$ for all of the melts as a function of inverse temperature. Two straight line trends can be seen in this plot - one associated with the 
slowest moving part of the melt structure (taken to me the lifetime of Si-O bonds); and one associated with the faster relaxation. The two regions have been fit separately to the equation:

$$
Q^{-1}(\omega)=Q_{0}^{-1} \omega^{\alpha} \exp \left(-\frac{\alpha E}{R T}\right)
$$

(Webb \& Jackson, 2003). As the low frequency peak indicates the energy lost in viscous flow its activation energy should be and is identical with the activation energy for viscous flow (see Tab. 23 and 24) in these melts. As there is no separate peak for Si-O and Al-O bonds, it is impossible to calculate the activation energy for both of them separately.

The result of average activation energy obtained in this study for bridging Si-O bonds in Fe-free melts is $543.67 \pm 52.98 \mathrm{~kJ} \mathrm{~mol}^{-1}$, what is identical with an activation energy of Si-O bond determined by Bockris \& Reddy (1970) $\left(\sim 540 \mathrm{~kJ} \mathrm{~mol}^{-1}\right)$, who worked with the silicate melts with binary metal oxide - silicate joins. For Fe-bearing melts the average activation energy determined by the attenuation calculation is $497.88 \pm 20.17 \mathrm{~kJ} \mathrm{~mol}^{-1}$.

The slope in the attenuation calculation in this study allows obtaining activation energy for the faster motion as $126.24 \pm 42.72 \mathrm{~kJ} \mathrm{~mol}^{-1}$. The activation energy for $\mathrm{Na}$ diffusion in silicate melts is $50-100 \mathrm{~kJ} \mathrm{~mol}^{-1}$ (Bansal \& Doremus, 1986), confirmed also by George \& Stebbins (1996) from slope of the relaxation curve as $70 \mathrm{~kJ} \mathrm{~mol}^{-1}$. This would further suggest that the second energy loss peak is for $\mathrm{Na}^{+}$diffusion. There was too much scatter in the plot of attenuation for the second peak in the Fe-bearing melts for it to be fit.

Change of the $\alpha$ parameter (from Eq. 73, see also Tab. 23 and 24) indicates a change in flow mechanism of the melt. Figure 89 presents $\alpha$ parameter as a function of $\gamma=\left(\mathrm{Na}_{2} \mathrm{O}+\mathrm{FeO}\right) /\left(\mathrm{Na}_{2} \mathrm{O}+\mathrm{Al}_{2} \mathrm{O}_{3}+\mathrm{FeO}+\mathrm{Fe}_{2} \mathrm{O}_{3}\right)$. Trend of Fe-free melts shows clear breaking point at $\gamma \sim 0.5$, what confirms all previous assumptions. $\alpha$ parameter of Fe-bearing melts seems to be constant, what cannot be explained considering small number of samples.

Tab. 23. Table of fit data $Q^{-1}$ for samples $G 0-G 7$ and activation energy for $\alpha$ - and $\beta$ - relaxation.

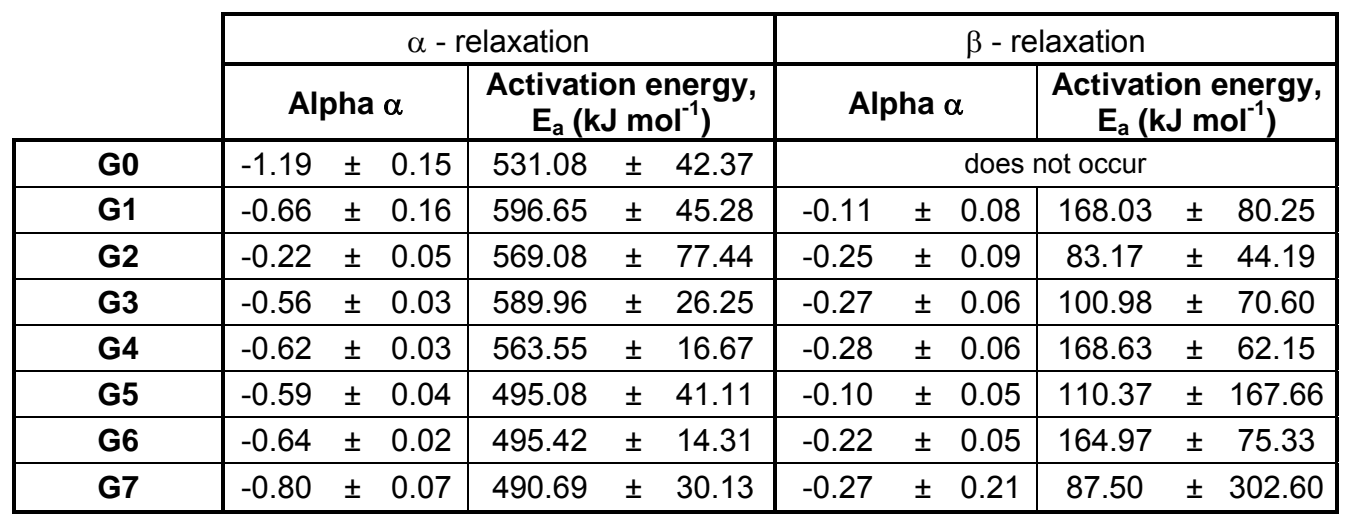


Tab. 24. Table of fit data $Q^{-1}$ for samples G0-G14 for $\alpha$ - relaxation.

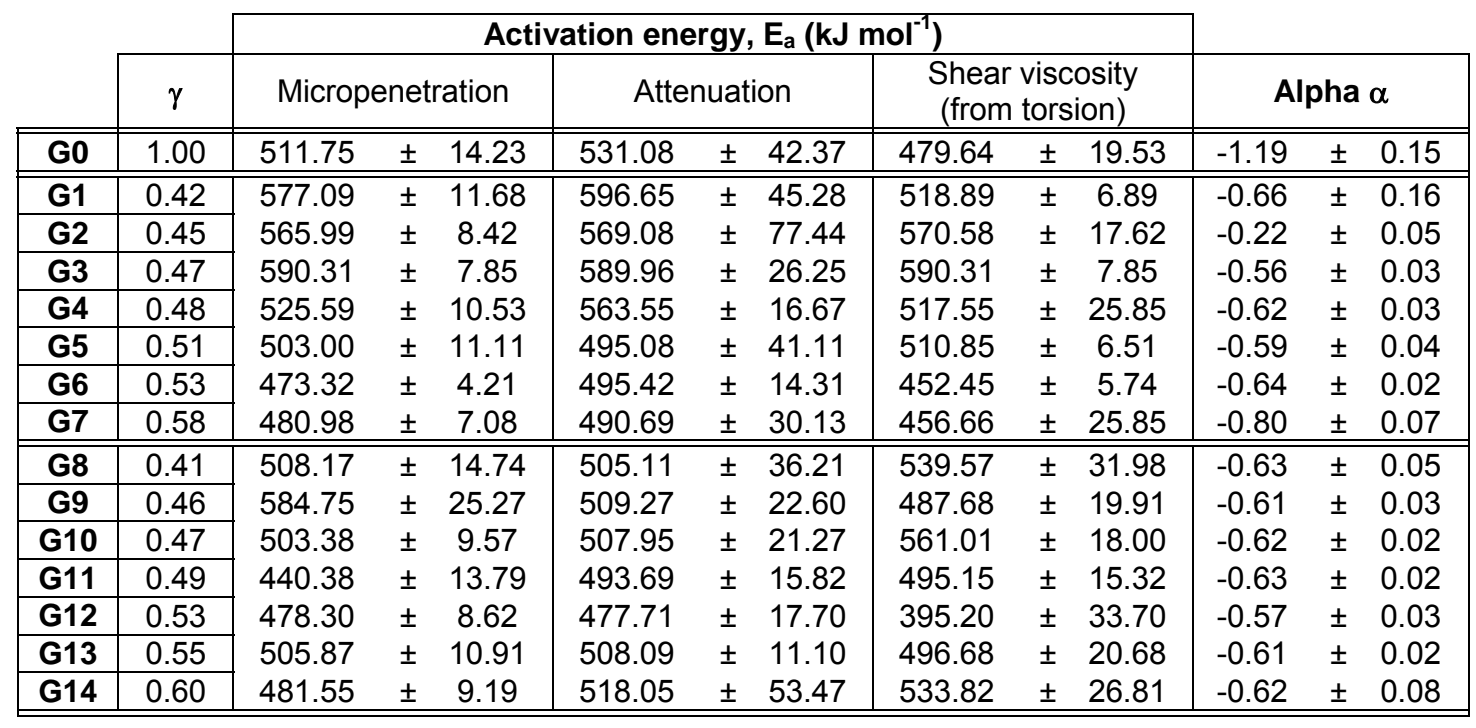

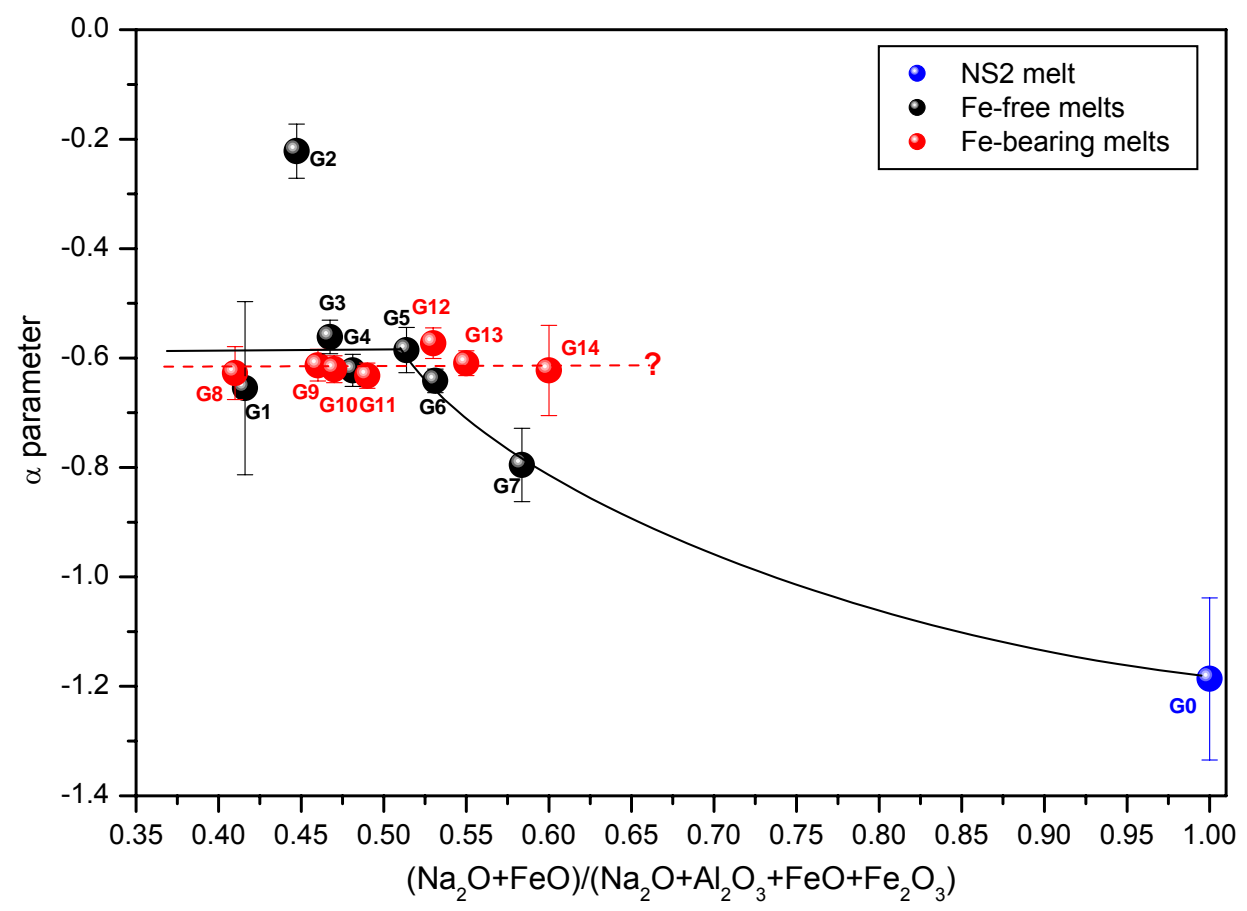

Fig. 89. $\alpha$ parameter as a function of $\gamma=\left(\mathrm{Na}_{2} \mathrm{O}+\mathrm{FeO}\right) /\left(\mathrm{Na}_{2} \mathrm{O}+\mathrm{Al}_{2} \mathrm{O}_{3}+\mathrm{FeO}+\mathrm{Fe}_{2} \mathrm{O}_{3}\right)$. 

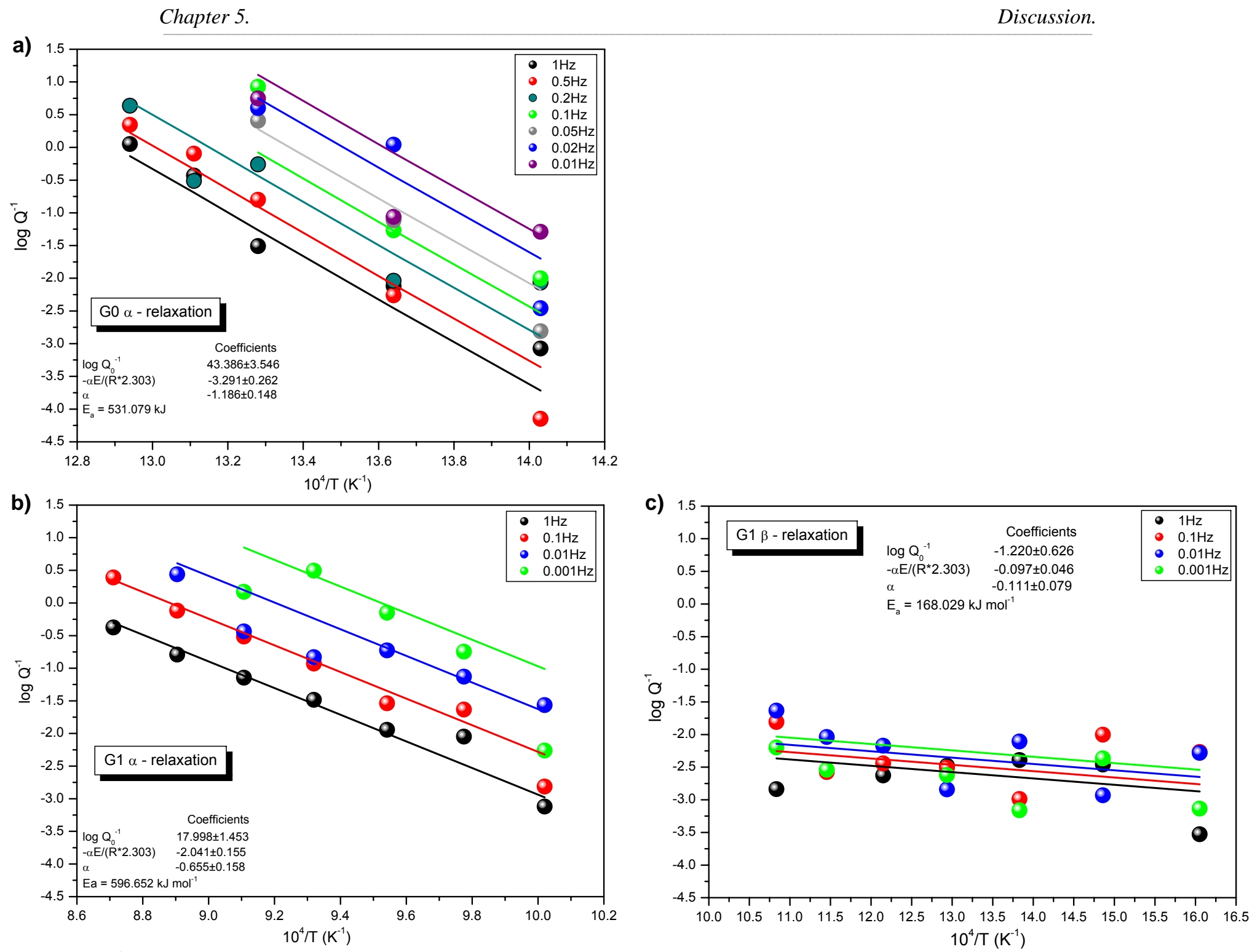

Fig. 90. Attenuation $\left(Q^{-1}\right)$ as a function of inverse temperature. The data are fit to Eq. (73). a) sample $G 0$ - $\alpha$-relaxation; b) sample $G 1-\alpha$-relaxation; $\left.\mathbf{c}\right)$ sample G1 - $\beta$-relaxation. 
d)
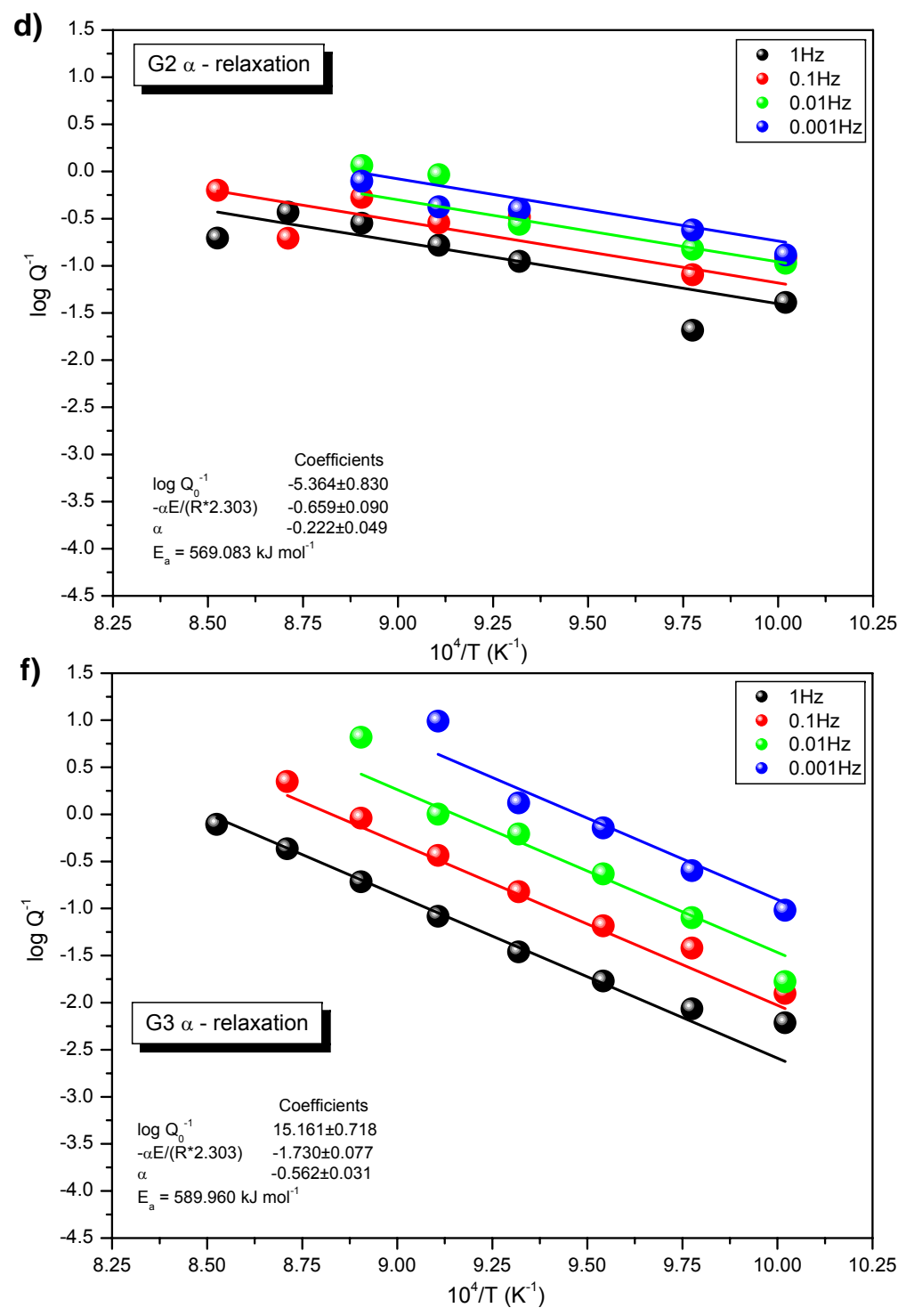

e)

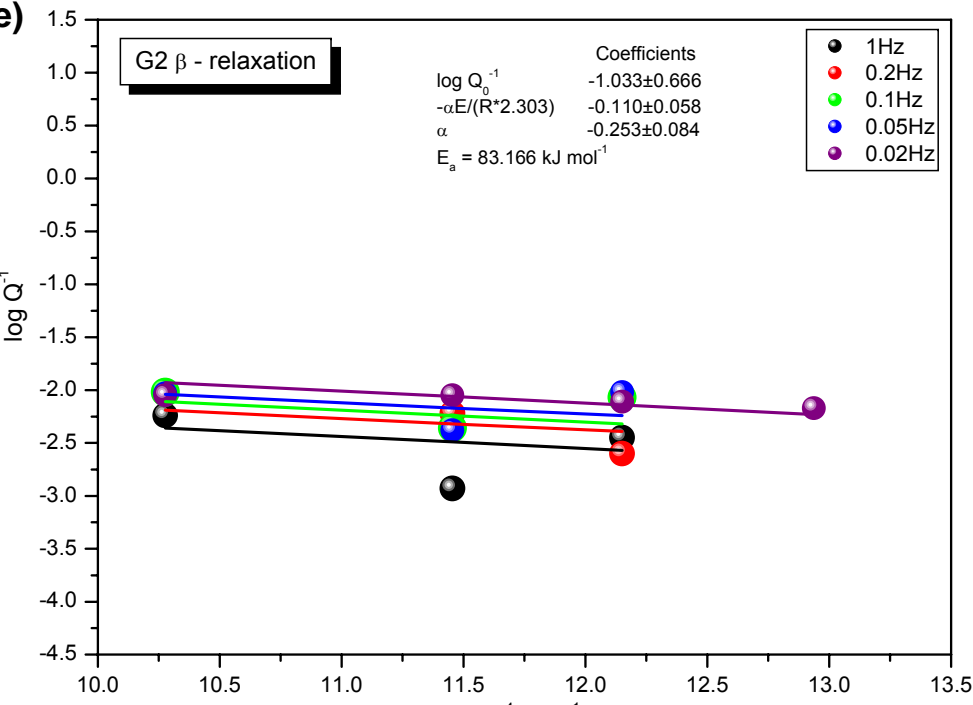

g) 1

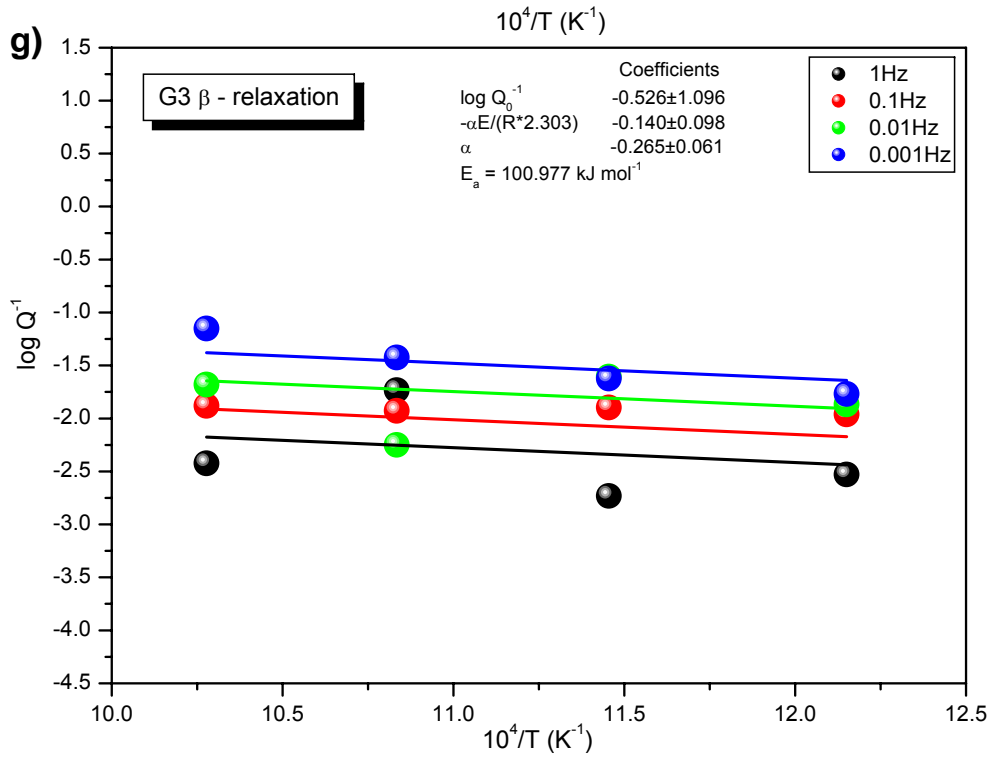

Fig. 90. continuation. Attenuation $\left(Q^{-1}\right)$ as a function of inverse temperature. The data are fit to Eq. (73). d) sample $G 2-\alpha$-relaxation; e) sample $G 2-\beta$ relaxation; f) sample $G 3$ - $\alpha$-relaxation; $\mathbf{g}$ ) sample $G 3$ - $\beta$-relaxation. 

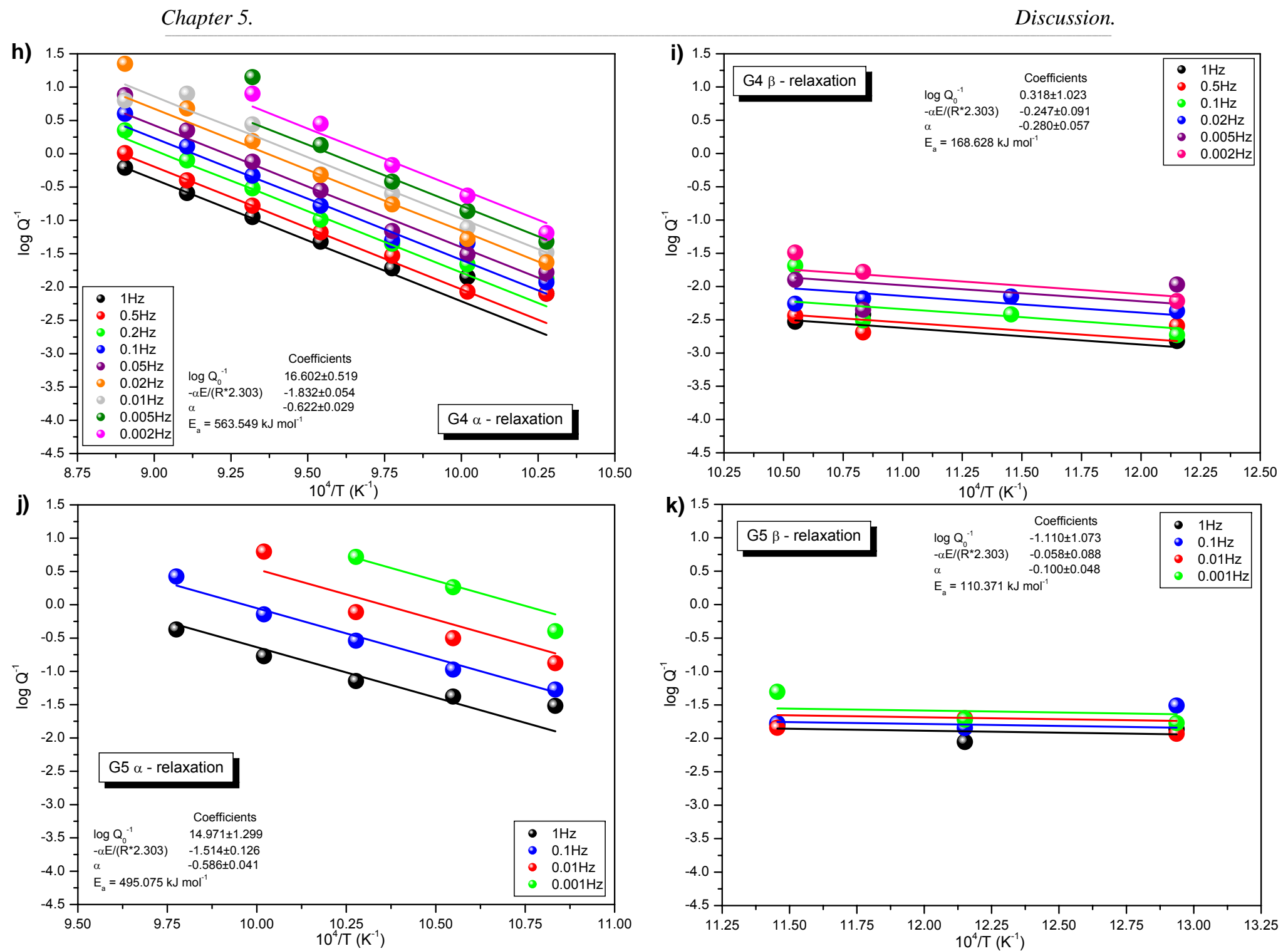

Fig. 90. continuation. Attenuation $\left(Q^{-1}\right)$ as a function of inverse temperature. The data are fit to Eq. (73). h) sample G4 - $\alpha$-relaxation; i) sample G4 - $\beta$ relaxation; j) sample $G 5$ - $\alpha$-relaxation; k) sample $G 5$ - $\beta$-relaxation. 

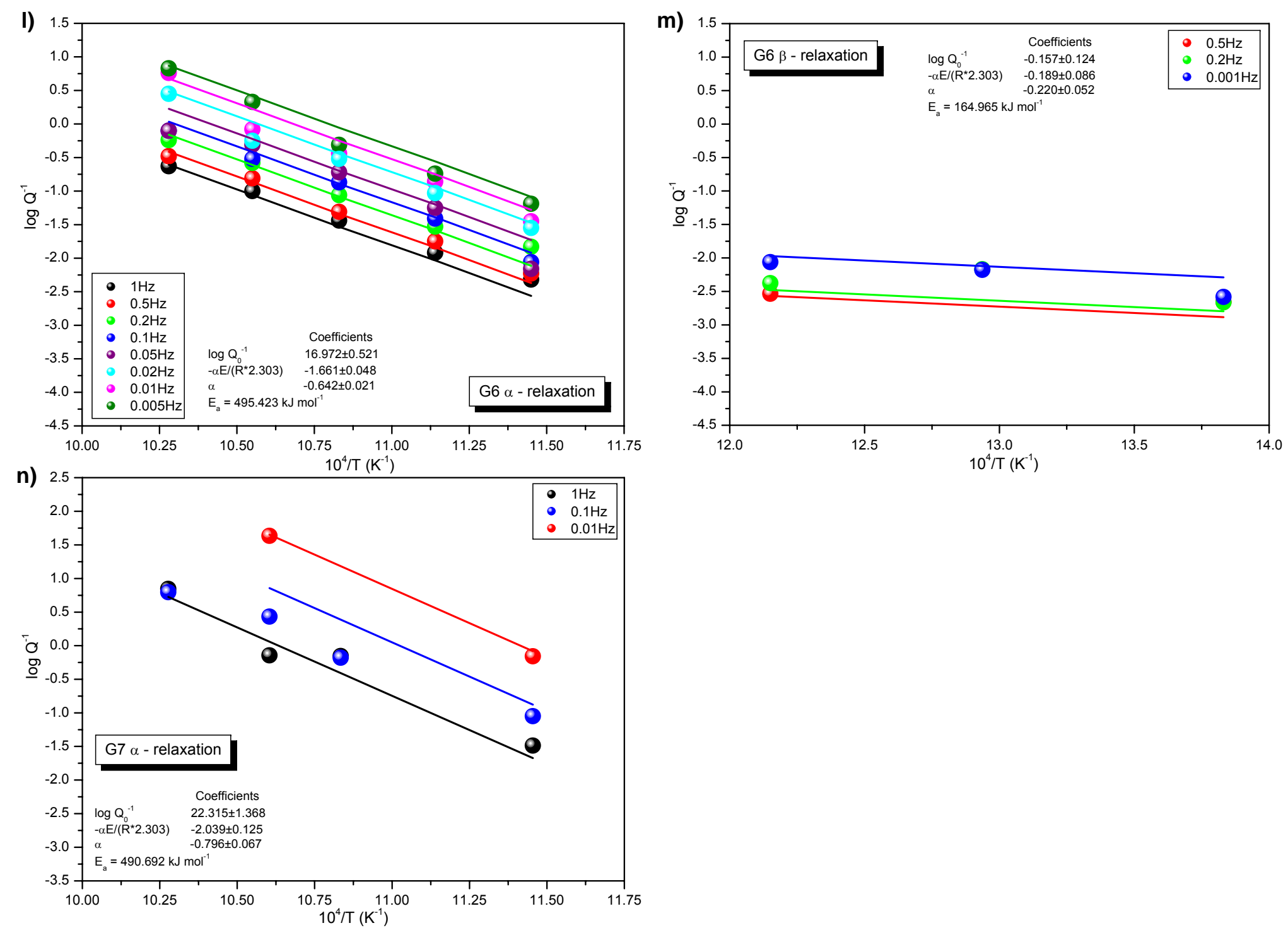

Fig. 90. continuation. Attenuation $\left(\mathrm{Q}^{-1}\right)$ as a function of inverse temperature. The data are fit to Eq. (73). I) sample $\mathrm{G} 6-\alpha$-relaxation; $\mathbf{m}$ ) sample $\mathrm{G} 6$ - $\beta$ relaxation; $\mathbf{n}$ ) sample $\mathrm{G} 7$ - $\alpha$-relaxation. 

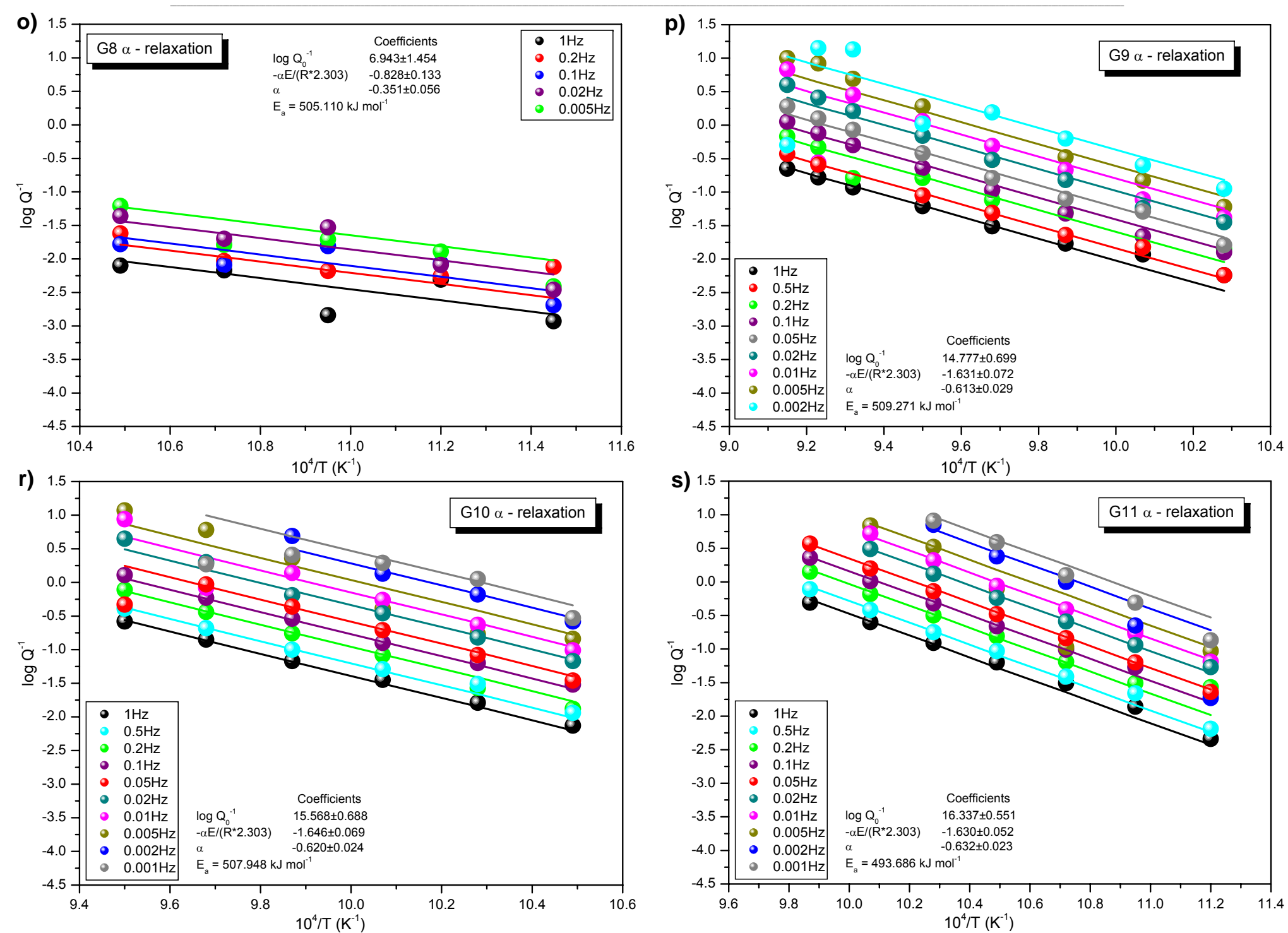

Fig. 90. continuation. Attenuation $\left(Q^{-1}\right)$ as a function of inverse temperature. The data are fit to Eq. (73). o) sample $G 8-\alpha$-relaxation; $\left.\mathbf{p}\right)$ sample $G 9-\alpha-$ relaxation; r) sample $\mathrm{G} 10$ - $\alpha$-relaxation; s) sample G11 - $\alpha$-relaxation. 

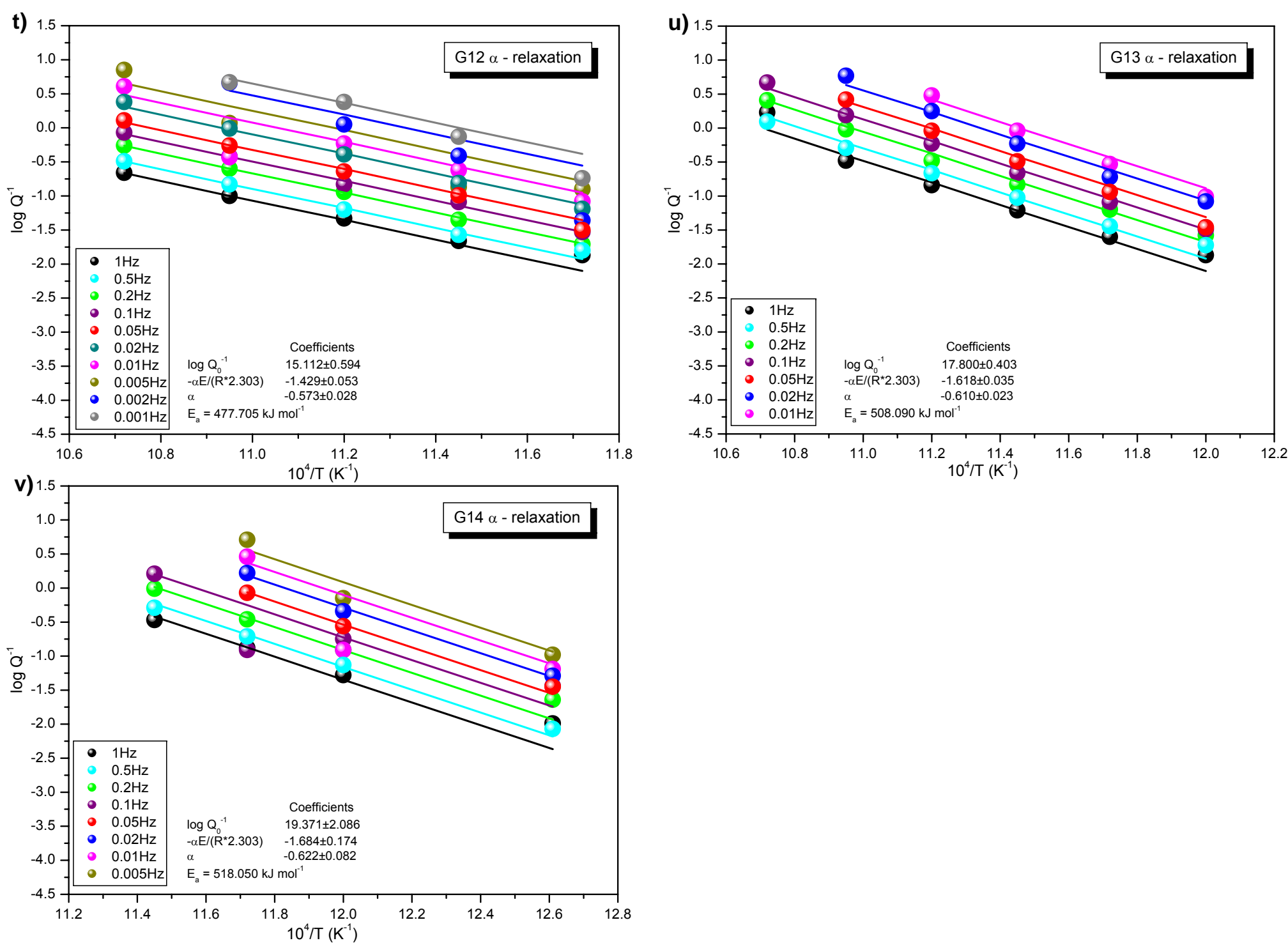

Fig. 90. continuation. Attenuation $\left(Q^{-1}\right)$ as a function of inverse temperature. The data are fit to Eq. (73). t) sample $\mathrm{G} 12-\alpha$-relaxation; $\left.\mathbf{u}\right)$ sample $\mathrm{G} 13-\alpha-$ relaxation; v) sample G14 - $\alpha$-relaxation. 


\subsection{Activation energy from all of the techniques}

Activation energy for viscous flow depends on the composition of the sample (Siewert \& Rosenhauer, 1997). The change in shape of the activation energy trend may be explained with bonding behaviour (MacKenzie, 1960; Bockris \& Reddy, 1970; Stein \& Spera, 1993).

Liquid $\mathrm{SiO}_{2}$ is a non-periodic arrangement of $\mathrm{SiO}_{4}$ tetrahedra connected by very strong covalent bonds at all corners (Bottinga \& Weill, 1972). To start the flow mechanism, these bonds need to be broken and because of their strength the activation energy must be very high (Hofmaier, 1968). The addition of some alkalies and alkali earths introduce new weaker bonds to the melt structure.

With increasing $\mathrm{Al}_{2} \mathrm{O}_{3}$ content in peralkaline sodium aluminosilicate melts occurs a weakening of the bonds between oxygen and silicate network former and increasing of Al-O bond lengths. As a result of that, activation energy $E_{a}$ decreases (Mysen et al., 1982; Seifert et al., 1982), what can be seen in Figure 62 in the $\gamma$ range between 1.00 and 0.53 . It is also observed, the activation energy between $1.0 \leq \gamma<0.53$ does not change significantly. That could be explained by a presence of structural units with similar $E_{a}$ (Seifert et al., 1982).

In the melts, where $\mathrm{Fe}^{2+}$ occurs as a network modifier, bonds between network formers and oxygen are longer and weaker what would explain lower activation energy in Fe-bearing melts. On the other hand, Seifert et al. (1982) suggested, that presence of two different structural units in the melt causes the creation of separate flow units with the weak bond between them.

Changing Na/Al ratio introduces a big spread in the activation energy results. Stein \& Spera (1993) shows that in the system $\mathrm{NaAISiO}_{4}$ activation energy changes between $515 \mathrm{~kJ} \mathrm{~mol}^{-1}$ and $340 \mathrm{~kJ} \mathrm{~mol}^{-1}$ with changing composition .

In this study, the results for samples with $0.42<\mathrm{Na} / \mathrm{Al}<0.58$ are: for Fe-free melts are between $473.3 \pm 4.2$ and $590.3 \pm 7.8 \mathrm{~kJ} \mathrm{~mol}^{-1}$, and for Fe-bearing melts: $440.4 \pm 13.8$ to $584.8 \pm 25.3 \mathrm{~kJ} \mathrm{~mol}^{-1}$.

Activation energies from torsion shear viscosity data and from micropenetration method are comparable, what was earlier shown with light spectroscopy by Lai et al. (1975), Bucaro \& Dardy (1977) and Siewert \& Rosenhauer (1997). Here, the activation energy using attenuation was also calculated (see Tab. 24). Figure 91 is a plot of activation energy determined from different techniques for all of the samples. So the viscosity should be the same from micropenetration, attenuation and torsion (shear viscosity), this data must plot on a straight line with slope $=1$. 


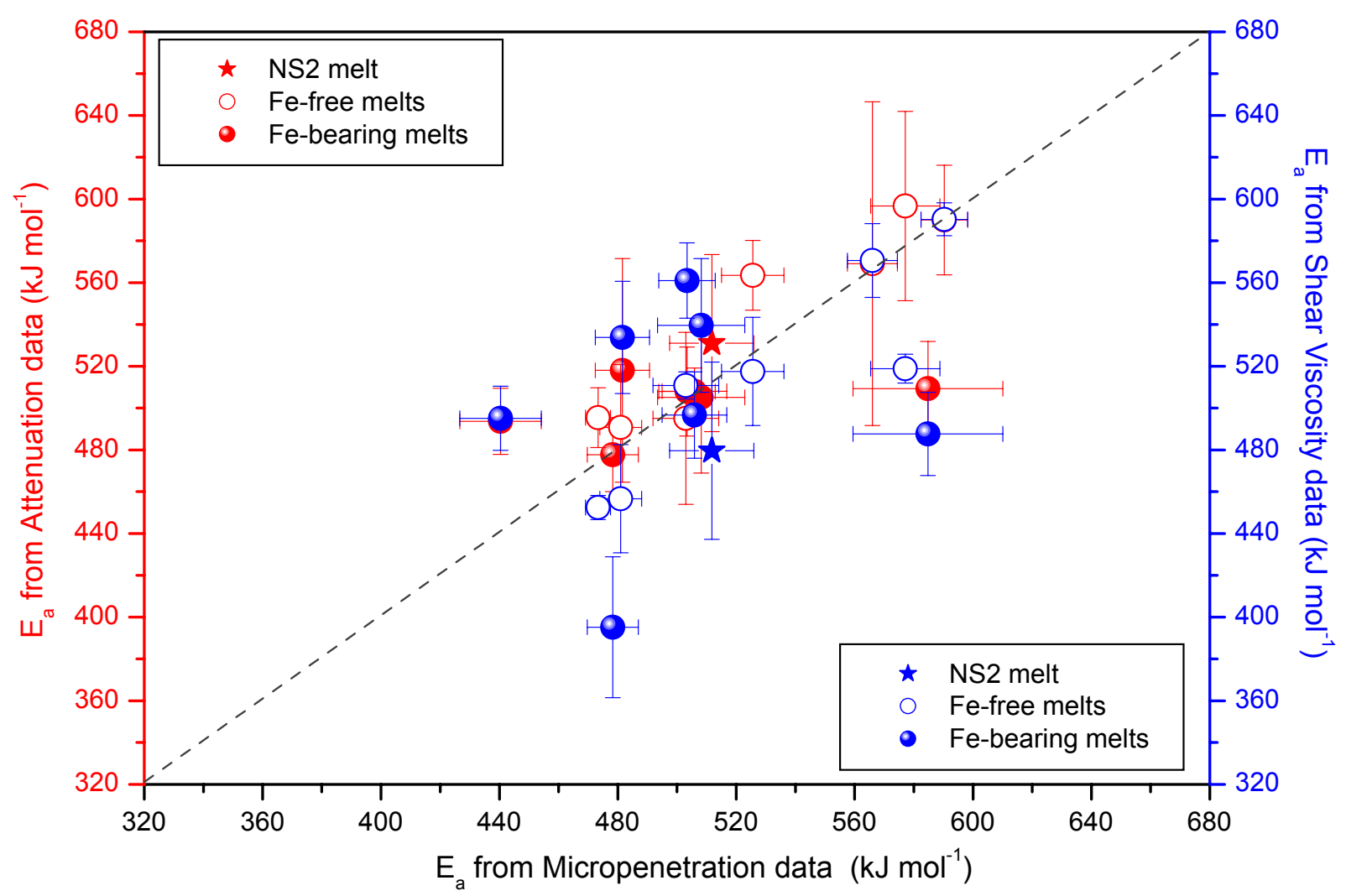

Fig. 91. Plot of the activation energy determined from the attenuation data and the viscosity against the activation energy from micropenetration data. Dashed line indicates slope $=1$.

\subsection{Fast relaxation time in aluminosilicate melts}

The rate of flow in the melts is controlled by the longest lived bonds, whereas there are structural relaxations for each of the bond types in the melt; and the slowest structural relaxation is identified with the rate of flow.

Thermodynamic and rheological properties of magma (viscosity, heat capacity, diffusivity, conductivity, expansivity, compressibility) are required to measure to better interpretation and understanding the flow mechanisms.

Stebbins \& Sen (1998) have investigated a microscopic dynamics and viscous flow in a borosilicate glass-forming liquid ( $44.5 \mathrm{~mol} \% \mathrm{Na}_{2} \mathrm{O}, 11.0 \mathrm{~mol}_{2} \mathrm{~B}_{2} \mathrm{O}_{5}$, and $44.5 \mathrm{~mol} \%$ $\mathrm{SiO}_{2}$ ). They have proved with NMR measurements that in multi - component oxide liquids some larger structural groups with different relaxation times are created. In borosilicate, the $\beta$ - relaxation (connected with breaking of $\mathrm{Si}-\mathrm{O}$ bonds) occurs at frequencies up to 50 times faster than Maxwell relaxation theory assumed; with the lifetime of B-O bonds being identical to $\tau_{M}$ (Fig. 88). The authors explain this fact with a creation of the polyhedra of 
$\mathrm{SiO}_{4}, \mathrm{BO}_{4}$ and $\mathrm{BO}_{3}$, which number depends on the temperature. These complex structural units influence the flow mechanism of the melt and lead to separation the measured relaxation time of Si-O bonds and that calculated from Maxwell equation (Eq. 3). Results obtained by Stebbins \& Sen (1998) confirm the results for aluminosilicates from this study that $\mathrm{Si}-\mathrm{O}$ bonds do not always control the flow in silicate melts.

In the case of aluminosilicate melts investigated in this study, it appears that, as suggested by Martens et al. (1987), the Si-rich units are "glued" together by Al-rich units.

The Si-rich units can be called "icebergs" and surrounding them Al-tetrahedra - as sea. The term "iceberg" was discussed by Bockris \& Kojonen (1960) for the structure of the alkali silicates and borates (see also references therein).

Stebbins (1995) suggested that $Q^{4}$ species can create the "icebergs" and $Q^{3}$ units - mica like "sheets". They move as relatively big parts of the structure and the lifetimes of the bonds creating the "icebergs" can be different than Maxwell theory assumes, because atoms in them are strongly connected. Through the flow the icebergs break the weakest bonds of network modifiers. The idea of such type of structure can explain our results.

Thus, the simple flow mechanism proposed by Farnan \& Stebbins (1994) and the relationship between viscosity and Si-O lifetime as determined by Liu et al. (1988) apply only to simple melt.

In the case where large structural units appear in the melt, a more complex relationship between flow mechanism, viscosity and bond lifetime occurs.

Figure 83 shows a difference between Maxwell relaxation time and relaxation times obtained in this study as a function of composition, presented here as $\left(\mathrm{Al}^{3+}+\mathrm{Fe}^{3+}\right) / \mathrm{Si}$ ratio (in atoms). Because in the melts occur also some local changes of structure, the relaxation times of aluminosilicate melts do not agree with Maxwell relaxation theory.

In Si-rich melts the flow mechanism is controlled by the lifetime of the $\mathrm{Si}-\mathrm{O}$ bonds. $\mathrm{Si}-\mathrm{O}$ bonds have the longest lifetime in the structure and because silicate melt is a simple melt, Maxwell relaxation works (Fig. 92 a).

With addition more and more $\mathrm{Al}_{2} \mathrm{O}_{3}$ the structure changes. New network former is introduced into the structure. As long as the ratio between $\mathrm{Al}$ and $\mathrm{Si}$ does not excess 1:3 (e.g. in albite) the Al-tetrahedra do not influence on the flow mechanism (Fig. 92b). As has been investigate (Dirken et al., 1997) in such melt there is no Al-O-Al bonds.

The increase in the number of Al-tetrahedra causes that these structural units begin to connect with each other, surrounding the separate silica rich clusters (icebergs) (Fig. 92c). The flow mechanism starts to be controlled by the shorter lifetime of Al-O bonds, in spite of presence longer lived $\mathrm{Si}-\mathrm{O}$ bonds. It means that the flow mechanism occurs faster than the structure is fully relaxed, what is observed as a peak in Figure 83. 
Figure 92d shows the stage when the melt is almost aluminate with some single Si-tetrahedra. The pure aluminate melt (Fig. 92e) is a simple melt again, where Maxwell relaxation time works.

Here, the original goal of the study - to determine the different behaviour of peralkaline and peraluminous melts - is decoupled from the observations of fast flow mechanism in these Al-rich melts. The existence of triclusters should have an effect on relaxation time and flow mechanism but this is overshadowed by the large effect of a large number of Al-tetrahedra surrounding icebergs of Si-tetrahedra. 

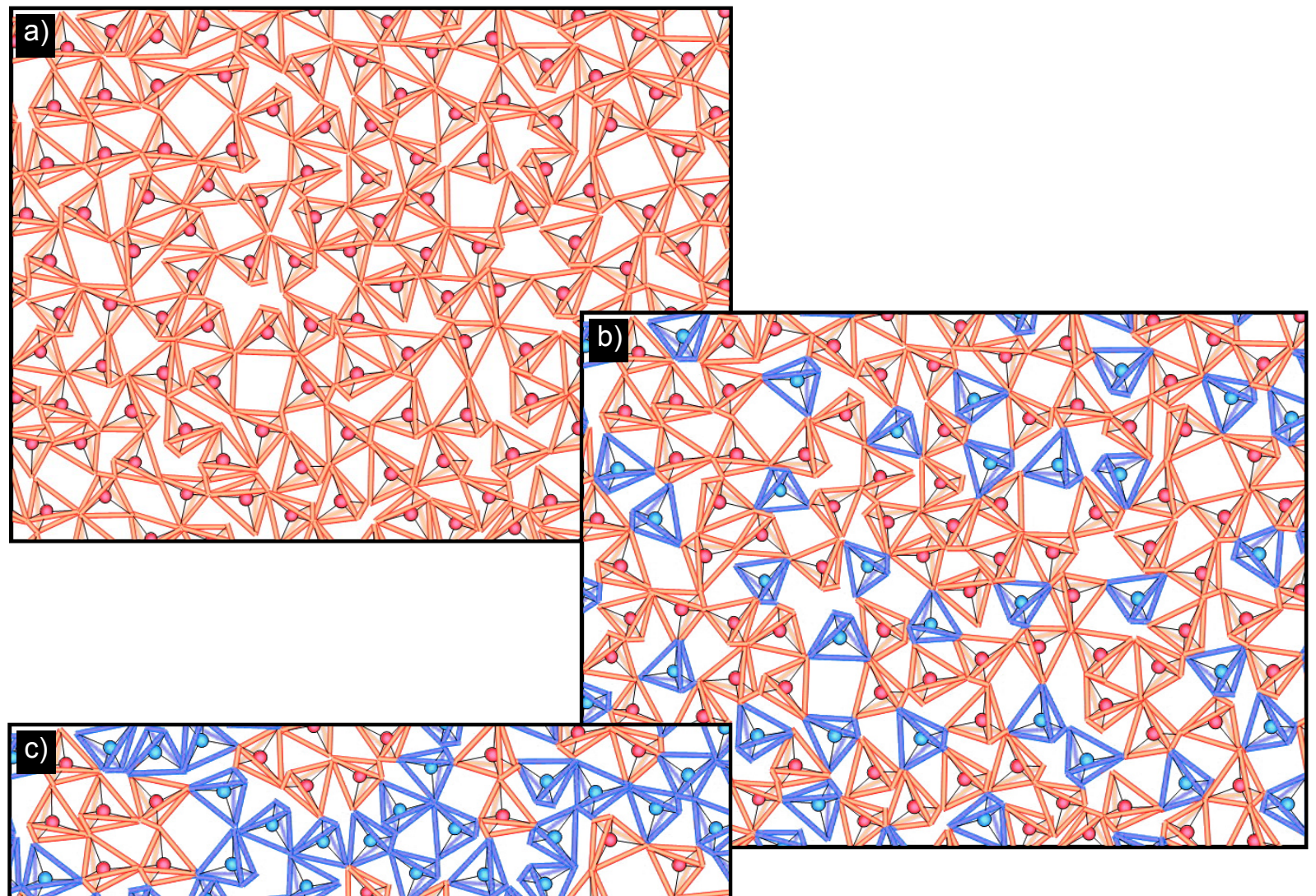

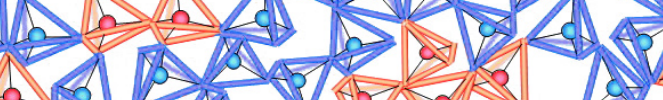

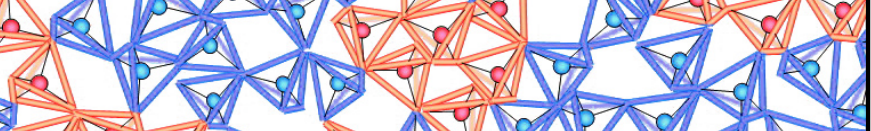

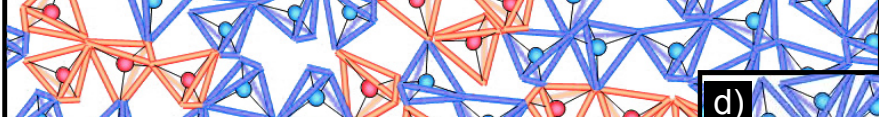

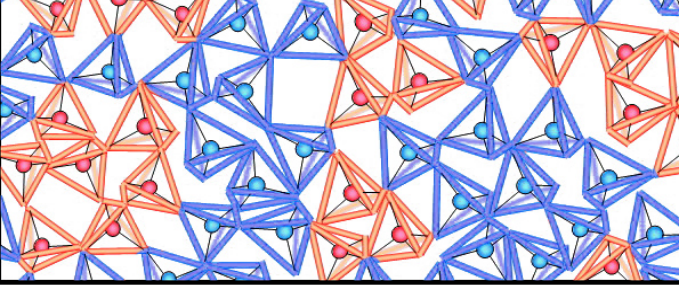

d)

d) 10010

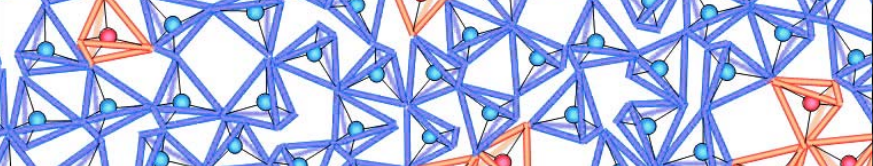

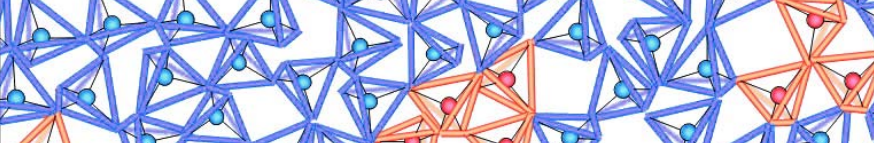

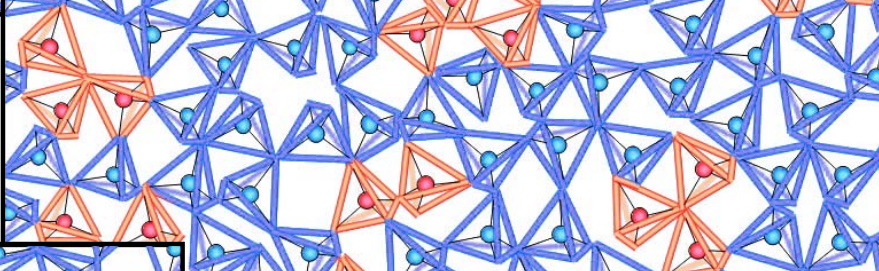

e) 10 (2)

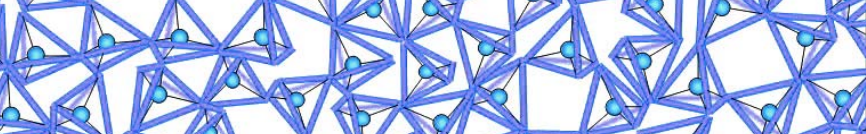

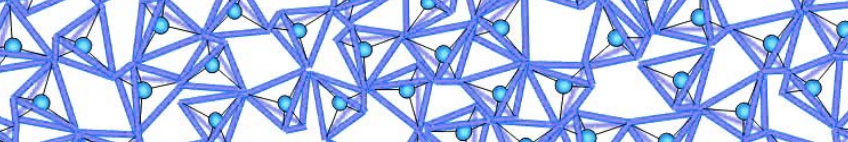

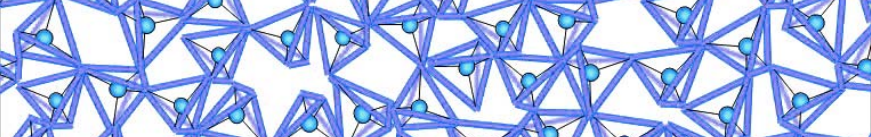

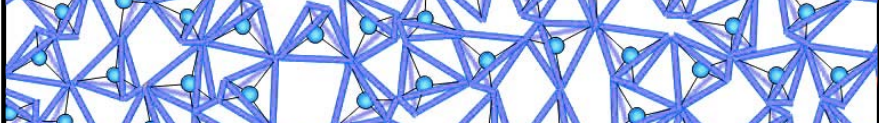

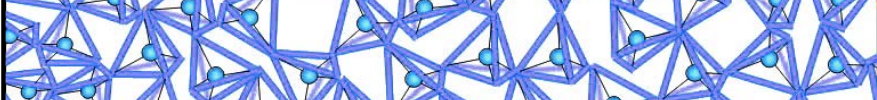

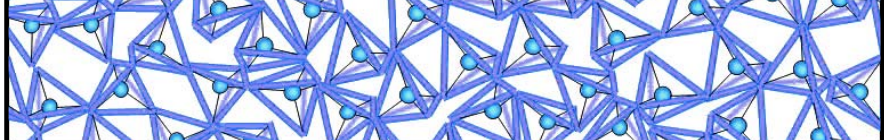

Fig. 92. Fast flow mechanism in aluminosilicate melts. Description in the text. Red: Si-tetrahedra; blue: Altetrahedra. 


\section{CONCLUSIONS}

The topology of our planet is dominated by the effect of silicate melts - in the form of mid-oceanic ridges, plumes, subduction zones, volcanoes and the differentiation into mantle and core at the creation of our planet. It therefore behoves us to understand the structure and physical and thermodynamic properties of silicate melts.

The structure of sodium aluminosilicate melts is taken to be built of network formers in tetrahedral coordination; and octahedrally coordinated network modifiers and charge balancers. In peralkaline structure, Si and Al form tetrahedra and $\mathrm{Na}$ is a network modifier and charge balancer. In peralkaline Fe-bearing melts $\mathrm{Fe}^{3+}$ is placed in tetrahedra and $\mathrm{Fe}^{2+}$ is octahedrally coordinated. The structural rearrangement occurs when composition changes into peraluminous $(\gamma \sim 0.5)$ - where there is no longer enough $\mathrm{Na}^{+}$to charge balance the $\mathrm{Al}^{3+}$ in tetrahedral coordination. There is no clear model of the peraluminous structure in aluminosilicate melts and for the purposes of this study here it is assumed that peraluminous structure consists $\mathrm{Si}, \mathrm{Al}$ (and $\mathrm{Fe}^{3+}$ in Fe-bearing melts) in tetrahedra which form triclusters, because of the lack in charge balancers $\left(\mathrm{Na}\right.$ and $\mathrm{Fe}^{2+}$ ). Negligible part of $\mathrm{Fe}^{3+}$ changes coordination from tetrahedral to octahedral, but this amount does not affect the change of structure.

Pure silicate or pure aluminate melt's and glass's networks show high similarity. However, when we start to change composition, one can observe strong variation in physical and thermodynamic properties. Into consideration should be taken not only the kind of the elements playing a network former and network modifiers role, but also the ratio between different ions.

The first observation is that viscosity, heat capacity and shear modulus data show a change in trend at $\gamma \sim 0.5$ indicating different structure in the melts as the composition changes from peralkaline to peraluminous. That is caused by the presence of triclusters. Here, the melt viscosity and glass density data confirm the information from previous studies, that there is a structural and flow mechanism change at $\gamma \sim 0.5$, as expected. The heat capacity data show simple trends as a function of composition.

For the first time the trends in configurational heat capacity as a function of composition indicate the presence of a change in structure. Literature data do not cover a controlled chemical composition range and thus have not observed this. With the addition of $\mathrm{Fe}_{2} \mathrm{O}_{3} / \mathrm{Al}_{2} \mathrm{O}_{3}$ this effect is additionally enlarged. The configurational entropy indicates that the range of structures available to the melt increases with increasing $\mathrm{Al}_{2} \mathrm{O}_{3}$ content.

The shear modulus and shear viscosity of $\mathrm{Na}_{2} \mathrm{O}-\mathrm{Al}_{2} \mathrm{O}_{3}-\mathrm{SiO}_{2}$ melts and the density of the glasses indicate that there is a change in melt structure at Al Na. Such a change in 
structure with composition also requires a change in flow mechanism with composition, and thus a change in the rates at which parts of the melt structure move. The rate of motion of structural units in silicate melts can be determined via forced oscillation methods.

A low-frequency forced oscillation technique has been used to measure the frequency and temperature dependence (to $1000^{\circ} \mathrm{C}$ ) of the shear modulus and viscosity of a range of $\mathrm{Na}_{2} \mathrm{O}-\mathrm{Al}_{2} \mathrm{O}_{3}-\mathrm{SiO}_{2}$ melts. The frequency range is between 1 and $0.001 \mathrm{~Hz}$, and thus the viscosity range is from $10^{8}-10^{15} \mathrm{~Pa} \mathrm{~s}$. The frequency-dependence of the shear modulus can be described by simple structural relaxation theory. The measured relaxation times $(\tau)$ for the simple peralkaline compositions agree with the Maxwell relaxation time $\left(\tau_{\mathrm{M}}\right)$. With increasing $\mathrm{Al}_{2} \mathrm{O}_{3}$ content, the structural relaxation time deviates from the calculated Maxwell relaxation time (gradually become shorter than $\tau_{\mathrm{M}}$ ) and after $\left(\mathrm{Al}^{3+}+\mathrm{Fe}^{3+}\right) / \mathrm{Si}^{\text {(atoms) }} \sim 0.55$ goes back and became longer again. The pure aluminate melts seem to be also simple and their relaxation times agree with Maxwell relaxation time $\left(\tau_{\mathrm{M}}\right)$. This is not effect of triclusters.

Such a decrease in $\tau / \tau_{\mathrm{M}}$ is observed for the first time. This shortening of relaxation times indicates that the large amount of $\mathrm{Al}^{3+}$ in these melts changes not only the structure, but also the flow mechanism of the melt. The shorter Al-O bond lifetimes appear to control the flow mechanism, in spite of the presence of the longer lived Si-O bonds. The structure flows faster than whole structure is relaxed. This has never been seen before. But also melts in this study have a much higher $\left(\mathrm{Al}^{3+}+\mathrm{Fe}^{3+}\right) / \mathrm{Si}^{\text {(atoms) }}$ ratio (0.62) than used in previous studies (0.2).

The structure of these melts is more complex than first thought. Not only is there the structural change with $\mathrm{Na} / \mathrm{Al}$ composition, but the large amount of $\mathrm{Al}^{3+}$ has created melts in which the motion of Al tetrahedra is as important as the motion of the $\mathrm{Si}$ tetrahedra. Thus, the theories created to describe the flow and structure of silicate melts need to be expanded to include the large effect due to the presence of almost as many Al tetrahedra as Si tetrahedra in the melt.

The torsion data show the lifetimes of motion of various structures in the melt. It was not possible to separate the lifetime of $\mathrm{Al}-\mathrm{O}$ bonds from $\mathrm{Si}-\mathrm{O}$ bonds, nor was the lifetime of $\mathrm{AISi}_{2} \mathrm{O}_{5}$ triclusters determined, but the lifetime of $\mathrm{Na}-\mathrm{O}$ bonds was measured. $\mathrm{A}$ second relaxation peak is seen at timescales $\sim 5.5-7.5$ orders of magnitude faster than the slowest relaxation time. This very fast relaxation is associated with the movement of $\mathrm{Na}^{+}$ in the melt.

What was determined here for the first time was the effect of a large number of fast $\mathrm{X}$-O lifetimes ( $\mathrm{X}=\mathrm{Al}$ in this case). The difference between $\mathrm{Si}-\mathrm{O}$ rich melts and shorter lived $\mathrm{Al}-\mathrm{O}$ rich melt means that a new structure controls the flow mechanism. This is not related 
to triclusters but simply to the lifetime of the different bonds. This effect is seen in the failure of the Maxwell relationship to calculate the structural relaxation times of melts with $\mathrm{Si}^{\text {atom }} \sim \mathrm{Al}^{\text {atom }}$.

Figure 93 shows predicted relaxation trend for aluminosilicate melts as a function of the Al/Si ratio. Relaxation times vary depending on the melt composition.

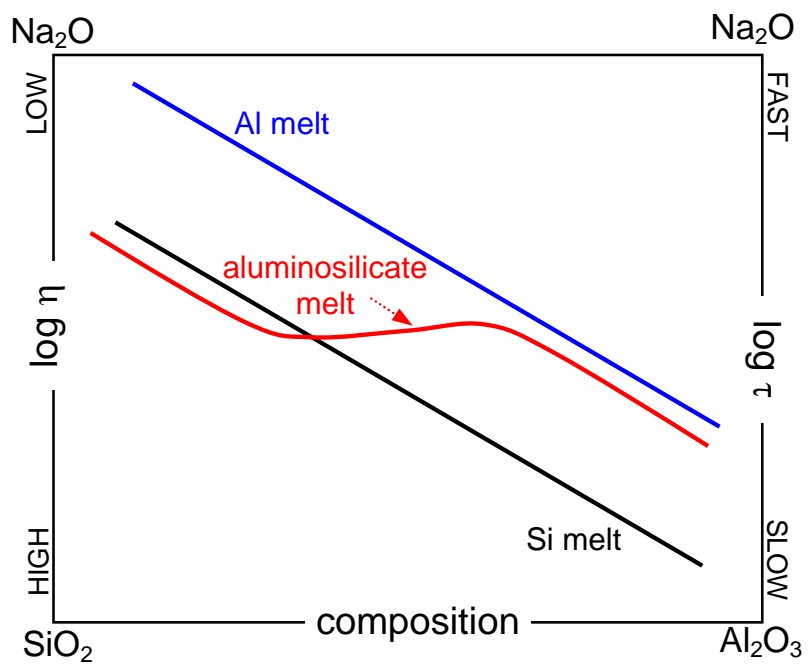

Fig. 93. Predicted relaxation trend for aluminosilicate melts as a function of composition.

Thus studies in which $\eta$ is related to $\tau_{\mathrm{M}}$ and to diffusion of Si or O will be in error in the composition range Si Al: this applies to phonolite melts, e.g. Laacher See (Eifel, Germany), Tenerife (Canary Islands, Spain), Sardinia (Italy), Dunedin Volcano (East Otago, New Zealand), Mont Dore (Auvergne, France), Bohemian Massif (Czech Republic) or Mount Saint-Hilaire (Québec, Canada).

The error could also occur in the modelling of the dynamic of the phonolitic volcano: wrong cooling rate gives wrong eruption temperature and viscosity, what influence on wrong calculation of the eruption rate and then on magma heat capacity, volume and temperature. 


\section{OUTLOOK}

The work described in this thesis concentrates on the structure of aluminosilicate melts as a function of composition and their physical and thermodynamic properties. In light of presented results the structural rearrangement has been shown to occur close to the subaluminous point. The failure of the Maxwell relaxation theory has been found and faster relaxation time of the complex melts has been measured.

Yet, more detailed studies are required in order to fully understand, what really occurs in these melts, e.g. NMR measurements of the bond lifetimes, diffusion measurements, and other torsion investigations.

To test of the present conclusions, the other series of the melts need to be investigated (Fig. 94), e.g. Ca-aluminosilicates, aluminosilicates with Al- and Si-end or K$\mathrm{Mg}$ or Na-K compositions. Choosing the end members of the series, the influence of triclusters can be omitted and then their effect on viscosity will not be observed, but the effect of changing bond lifetimes as a function of composition and structure will be revealed.

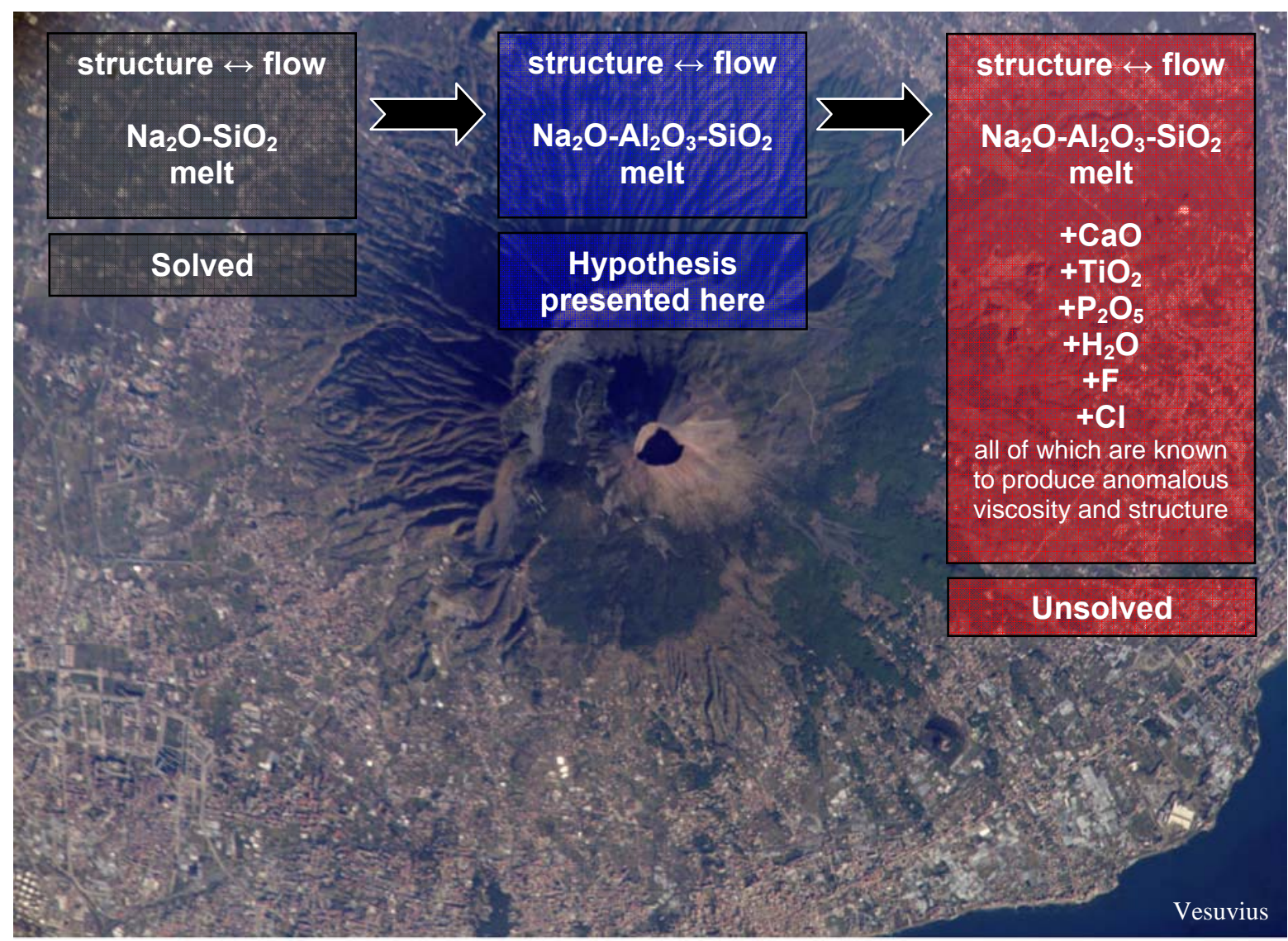

Fig. 94. Past, present and future of the studies of the relationship between structure and flow mechanism in silicate and aluminosilicate melts in the Earth. 


\section{LITERATURE}

Adam G \& JH Gibbs, On the temperature dependence of cooperative relaxation properties in glass - forming liquids. Journal of Chemical Physics, 43, 139-146, 1965.

Ahrens TJ, AGU Mineral Physics \& Crystallography. A Handbook of Physical Constants, 1995.

Allwardt JR, SK Lee \& JF Stebbins, Bonding preferences of non-bridging $O$ atoms: Evidence from ${ }^{17} \mathrm{O}$ MAS and 3 QMAS NMR on calcium aluminate and low-silica Ca-aluminosilicate glasses. American Mineralogist, 88, 949-954, 2003.

Angell CA, The glass transition: comparison of computer simulation and laboratory studies. Annals of the New York Academy of Sciences, 371, 136-150, 1981.

Angell CA, Structural instability and relaxation in liquid and glassy phases near the fragile liquid limit. Journal of Non-Crystalline Solids, 102, 205-221, 1988.

Angell CA, Dynamic processes in ionic glasses. Chemical Review, 90, 523-542, 1990.

Angell CA, Relaxation in liquids, polymers and plastic crystals - strong/fragile patterns and problems, Journal of Non-Crystalline Solids, 131-133, 13-31, 1991a.

Angell CA, Thermodynamic aspects of the glass transition in liquids and plastic crystals, Pure and Applied Chemistry, 63, 10, 1387-1392, 1991b.

Angell CA \& M Goldstein, eds., Dynamic aspects of structural change in liquids and glasses. Annals of the New York Academy of Sciences, 484, 1986.

Bagdassarov NS, DB Dingwell \& SL Webb, Effect of boron, phosphorus and fluorine on shear stress relaxation in haplogranitic melts, European Journal of Mineralogy, 5, 409-425, 1993.

Baker LL \& MJ Rutherford, The effect of dissolved water on the oxidation state of silicic melts. Geochimica et Cosmochimica Acta, 60, 2179-2187, 1996.

Bamford CR, A study of magnetic properties of iron in relation to its colouring action in glass. I. Iron in sodium borate glasses melted under oxidizing conditions. Physics and Chemistry of Glasses, 1, 159-164, 1960.

Bansal NP \& RH Doremus, Handbook of Glass Properties, 680pp, Academic Press, London, 1986.

Bell RJ \& P Dean. Discussions of the Faraday Society, 50, 55-61, 1970.

Beran A \& E Libowitzky, Spectroscopic methods in mineralogy, EMU Notes Mineral., 6, Budapest, Eötvös University Press, p. 661, 2004.

Bernal JD, An attempt at a molecular theory of liquid structure. Discussions of the Faraday Society, 336, 27-40, 1936.

Berry AJ, HSC O'Neill, KD Jayasuriya, SJ Campbell \& GJ Fornan, XANES calibrations for the oxidation state of iron in a silicate glass. American Mineralogist, 88, 967-977, 2003.

Bingham PA, JM Parker, TM Searle \& I Smith, Local structure and medium range ordering of tetrahedrally coordinated $\mathrm{Fe}^{3+}$ ions in alkali - alkaline earths - silica glasses. Journal of NonCrystalline Solids, 353, 24-25, 2479-2494, 2007.

Bockris J O'M \& E Kojonen, The compressibilities of certain molten alkali silicates and borates. Journal of the American Ceramic Society, 82, 4493-4497, 1960.

Bockris J O’M. \& AKN Reddy, Modern electrochemistry, 662, Plenum Press, New York, 1970.

Bockris J O'M., JD MacKenzie \& JA Kitchner, Viscous flow in silicate and binary liquid silicates. Transactions of the Faraday Society, 51, 1734-1748, 1955.

Böhmer R \& CA Angell, Correlation of the non-exponentiality and state dependence of mechanical relaxations with bond connectivity in Ge-As-Se supercooled liquids. Physical Review B, 45, 17, 10091-10094, 1992.

Botcharnikov R, J Köpke, F Holtz, C McCammon \& M Wilke, The effect of water activity on the oxidation and structural state of $\mathrm{Fe}$ in a ferro - basaltic melt. Geochimica et Cosmochimica Acta, 69, 5071-5085, 2005.

Bottinga $\mathrm{Y}$, Configurational entropy and non-Newtonian rheology of homogenous silicate liquids. Physical Review B, 49, 1, 1994. 
Bottinga Y \& DF Weill, The viscosity of magmatic silicate liquids: a model for calculation. American Journal of Science, 272, 438-475, 1972.

Bottinga $Y$ \& $P$ Richet, Silicate melt structural relaxation: rheology, kinetics, and Adam - Gibbs theory. Chemical Geology, 128, 129-141, 1996.

Bottinga Y, P Richet \& A Sipp, Viscosity regimes of homogenous silicate melts. American Mineralogist, 80, 305-318, 1995.

Bottinga $Y$, DF Weill \& $P$ Richet, Density calculations for silicate liquids. I. Revised method for aluminosilicate compositions. Geochimica et Cosmochimica Acta, 46, 909-919, 1982.

Brady JB, Chemical components and diffusion. American Journal of Scienc, 275, 1073-1088, 1975.

Brady JB, Diffusion data for silicate minerals, glasses, and liquids. In TJ Ahrens, Ed., Mineral physics and crystallography: A handbook of physical constants. American Geophysical Union, Washington, DC., 269-290, 1995.

Brawer SA, Theory of relaxation in viscous liquids and glasses. Journal of Chemical Physics, 81, 2, 954-975, 1984.

Brawer SA, Relaxation in viscous liquids and glasses. Journal of the American Ceramic Society, Columbus, OH, 1985.

Brown GE Jr., GA Waychunas, CW Ponader, WE Jackson \& DA McKeown, EXAFS and NEXAFS studies of cation environments in oxide glasses. Journal de Physique Colloque, Paris, 47, 661-668, 1986.

Brückner R \& $G$ Demharter, Systematische Untersuchungen über die Anwendbarkeit von Penetrationsviscosimetern. Glastechnische Berichte, 48, 1, 12-18, 1974.

Brückner $R$ \& $Y$ Yue, Non-Newtonian flow behaviour of glass melts as a consequence of viscoelasticity and anisotropic flow. Journal of Non-Crystalline Solids, 175, 118-128, 1994.

Bucaro JA \& HD Dardy, High - temperature strain relaxation in silica by optical correlation spectroscopy. Journal of Non-Crystalline Solids, 24, 121-129, 1977.

Bunker BC, RJ Kirkpatrick, RK Brow, GL Turner \& C Nelson, Local Structure of Alkaline-Earth Boroaluminate Crystals and Glasses: II, ${ }^{11} \mathrm{~B}$ and ${ }^{27} \mathrm{Al}$ MAS NMR Spectroscopy of AlkalineEarth Boroaluminate Glasses". Journal of the American Ceramic Society, 74, 6, 1430-38, 1991.

Burkhard DJM, Iron - bearing silicate glasses at ambient conditions. Journal of Non-Crystalline Solids, 275, 175-188, 2000.

Burnham CW, Thermodynamics of melting in experimental silicate - volatile systems. Geochimica et Cosmochimica Acta, 39, 1077-1084, 1975.

Bykov VN, VN Anfilogov \& SV Kuznetsov, Structure of Al-Si Melts from Raman Sp. Data, Geokhimija, 4, 331-337, 1996.

Bykov VN, AA Osipov \& VN Anfilogov, Structure of high-alkali aluminosilicate melts from the hightemperature Raman spectroscopic data, Glass Physics and Chemistry, Vol. 29, No. 2, 105107, 2003.

Calas G \& J Petiau, Coordination of iron in oxide glasses through high - resolution K-edge spectra: information from the pre-edge. Solid State Communications, 48, 625-629, 1983.

Carreño-Morelli E, SE Urreta \& R Schaller, Mechanical spectroscopy of thermal stress relaxation at metal-ceramic interfaces in aluminium-based composites. Acta Materialia, 48, 18-19, 47254733, 2000.

Carron JP, Autodiffusion du sodium et conductivité électrique dans les obsidiennes granitiques. Comptes Rendus Acad. Sci. Paris. Serie D, 266, 854-856, 1968.

Casalini R \& CM Roland, Why liquids are fragile. Physical Review E, 72, 031503/1-4, 2005.

Chakraborty S, Diffusion in silicate melts. Reviews in Mineralogy, 32, 411-504, 1995.

Cohen MH \& GS Grest, Liquid - glass transition, a free volume approach. Physical Review B, 20, 1077-1098, 1979.

Crichton SN \& CT Moynihan, Structural relaxation of lead silicate glass. Journal of Non-Crystalline Solids, 102, 222-227, 1988. 
Cruickshank-Miller C, The Stokes-Einstein Law for Diffusion in Solution Proceedings of the Royal Society of London. Series A, Containing Papers of a Mathematical and Physical Character, 106, 740, 724-749, 1924.

Cukierman M \& DR Uhlmann, Effects of iron oxidation state on viscosity, lunar composition 15555. Journal of Geophysical Research, 79, 1594-1598, 1974.

Day DE \& EG Rindone, Properties of soda aluminosilicate glasses: I, Refractive index, density, molar refractivity and infrared absorption spectra, Journal of the American Ceramic Society, 45, 579-581, 1962.

DeBolt MA, AJ Easteal, PB Macedo \& CT Moynihan, Analysis of structural relaxation in glass using rate heating data. Journal of the American Ceramic Society, 59, 16-21, 1976.

DeGrave $E$, D Chambaere, $P$ van Iseghem \& $R$ de Batist, Mössbauer spectroscopic study of some complex $\mathrm{M}_{2} \mathrm{O}-\mathrm{Mo}-\mathrm{M}_{2} \mathrm{O}_{3}-\mathrm{SiO}_{2}$ glasses. Journal de Physique Colloque, Paris, 41, 269-270, 1980.

Devine RAB, R Dupree, I Farnan \& JJ Capponi, Presure - induced bond - angle variation in amorphous $\mathrm{SiO}_{2}$. Physical Review B, 35, 2560-2562, 1987.

Dickenson MP \& PC Hess, Redox equilibria and the structural role of iron in aluminosilicate melts. Contributions to Mineralogy and Petrology, 78, 352-358, 1981.

Dickenson JE \& PC Hess, The structural role and homogenous redox equilibria of iron in peraluminous, metaluminous and peralkaline silicate melts. Contributions to Mineralogy and Petrology, 92, 207-217, 1985.

Dietzel AZ, Die Kationenfeldstirken und ihre Beziehungen zu Entglasungsvorgangen, zur Verbindungsbildung und zu den Schmelzpunkten von Silikaten. Z. Elektrochem., 48, 9-23, 1942.

Dingwell DB, Viscosity and Anelasticity of Melts. Mineral Physics and Crystallography, A Handbook of Physical Constants, AGU Reference Shelf 2, 209-217, 1995.

Dingwell DB, Volcanic dilemma - flow or blow? Science, 26, 1054-1056, 1996.

Dingwell DB, R Knoche \& SL Webb, The effect of $\mathrm{B}_{2} \mathrm{O}_{3}$ on the viscosity of haplogranitic liquids, American Mineralogist, 77, 457-461, 1992.

Dingwell DB \& D Virgo, Melt viscosities in the $\mathrm{Na}_{2} \mathrm{O}-\mathrm{FeO}-\mathrm{Fe}_{2} \mathrm{O}_{3}-\mathrm{SiO}_{2}$ system and factors controlling the relative viscosities of fully polymerized silicate melts, Geochimica Cosmochimica Acta, 52, 395-403, 1988a.

Dingwell DB \& D Virgo, Viscosity - oxidation state relationship for hedenbergitic melt. Carnegie Inst. Wash. Yearbook, Vol. 87, 48-53, 1988b.

Dingwell DB \& SL Webb, Structural relaxation in silicate melts and non-Newtonian melt rheology in geologic processes, Physics and Chemistry of Minerals, 16, 508-516, 1989.

Dingwell DB \& SL Webb, Relaxation in silicate melts, European Journal of Mineralogy, 2, 4, 427449, 1990.

Dirken PJ, SC Kohn, ME Smith \& ERH vanEck, Complete resolution of Si-O-Si and Si-O-Al arrangements in an aluminosilicate glass by 0-17 multiple quantum magic angle spinning NMR spectroscopy. Chemical Physics Lettters, 266, 568-574, 1997.

Douglas RW, WL Armstrong, JP Edward \& D Hall, A penetration viscometer. Glass Technology, 6 , 2, 52-55, 1965.

Dunn T, Oxygen diffusion in three silicate melts along the join diopside anorthite. Geochimica et Cosmochimica Acta, 47, 2292-2299, 1982.

Dyar MD, A review of Mössbauer data on inorganic glasses: the effects of composition on iron valency and coordination. American Mineralogist, 70, 304-316, 1985.

Farnan I \& JF Stebbins, High - temperature ${ }^{29}$ Si NMR investigation of solid and molten silicates. Journal of the American Ceramic Society, 112, 32-39, 1990a.

Farnan I \& JF Stebbins, Observation of slow atomic motions close to the glass transition using 2-D ${ }^{29}$ Si NMR. Journal of Non-Crystalline Solids, 124, 207-215, $1990 \mathrm{~b}$.

Farnan I \& JF Stebbins, The nature of the glass transition in a silica-rich oxide melt. Science, 265, 1206-1209, 1994. 
Fenstermacher JE, Optical absorption due to tetrahedral and octahedral ferric iron in silicate glasses. Journal of Non-Crystalline Solids, 38-39, 239-244, 1980.

Fox KE, T Furakawa \& WB White, Transition metal ions in silicate melts. Part 2: Iron in sodium silicate glasses. Physics and Chemistry of Glasses, 23, 169-178, 1982.

Freer R, Diffusion in silicate minerals and glasses: $A$ data digest and guide to the literature. Contributions to Mineralogy and Petrology, 76, 440-454, 1981.

Fulcher GS, Viscosity of simple soda - silicate $500^{\circ}$ to $1400^{\circ} \mathrm{C}$, Journal of the American Ceramic Society, 8, 6, 339, 1925.

Gaillard F, B Scaillet, M Pichavant \& JM Beny, The effect of water and $f \mathrm{O}_{2}$ on the ferric - ferrous ratio of silicic melts. Chemical Geology, 174, 255-273, 2001.

Gaskell PH, Vibrational spectra of simple silicate glasses. Discussions of the Faraday Society, 50, 82, 1970.

Gaskell PH \& AB Mistry, High - resolution transmission electron microscopy of small amorphous silica particles. Journal of Philosophy, 39, 245-257, 1979.

George AM \& JF Stebbins, Dynamics of $\mathrm{Na}$ in sodium aluminosilicate glasses and liquids. Physics and Chemistry of Minerals, 23, 526-534, 1996.

Giordano D, A Mangiacapra, M Potuzak, JK Russell, C Romano, DB Dingwell \& A Di Muro, An expanded non-Arrhenian model for silicate melt viscosity: A treatment for metaluminous, peraluminous and peralkaline liquids. Chemical Geology, 229, 42-56, 2006.

Glasstone S, KJ Laidler \& H Eyring, Theory of rate processes. McGraw-Hill, New York, 486, 1941.

Godovikov AA, Kchimicheskie osnovy sistematiki mineralov (Chemical Properties of Mineral Classification), Moskow: Nedra, 1979.

Goldman DS, Oxidation equilibrium of iron in borosilicate glas. Journal of the American Ceramic Society, 66, 205-209, 1983.

Goldschmidt VM, Skrifter norske Videnskaps. Akad. Oslo, I: Math.-naturvidensk, KI., 8, 7, 1926.

Goldstein M \& R Simha, eds. The glass transition and the nature of the glassy state. Annals of the New York Academy of Sciences, 279, 1976.

Goto T, S Yamamoto, I Ohno \& OL Anderson, Elastic constants of corundum up to $1825 \mathrm{~K}$, Journal of Geophysical Research, 94, 7588-7602, 1989.

Götze W, Aspects of structural glass transitions. In: Hansen JP, D Levsque \& J Zin-Justin, Eds., Liquids, freezing and glass transition. Part I, 292-503, Elsevier Science, Amsterdam, 1991.

Greaves GN, N Binsted \& CMB Henderson, The environments of modifiers in oxide glasses. In: Hodgson KO, B Hedman \& JE Penner-Hahn (eds): EXAFS and Near Edge Structure III. Springer - Verlag, Berlin, 297-301, 1984.

Gwinn R \& PC Hess, Iron and titanium solution properties in peraluminous and peralkaline rhyolitic liquids. Contributions to Mineralogy and Petrology, 101, 326-338, 1989.

Haggerty JS, AR Cooper \& JH Heasley, Heat capacity of three inorganic glasses and supercooled liquids. Physics and Chemistry of Glasses, 5, 130-136, 1968.

Hannoyer B, M Lenglet, J Dürr \& R Cortes, Spectroscopic evidence of octahedral iron (III) in soda lime silicate glasses. Journal of Non-Crystalline Solids, 151, 209-216, 1992.

Hawthorne FC, Spectroscopic methods in mineralogy and geology, Reviews in Mineralogy, 18, Washington (D.C.): Mineralogical Society of America, 1988.

Heinrichs H \& AG Herrmann, Praktikum der Analytischen Geochemie. Springer-Verlag, 420-425, 1998.

Hennet L, S Krishnan, A Bytchkov, T Key, D Thiaudière, P Melin, I Pozdnyakova, ML Saboungi \& DL Price, X-ray Diffraction on High-Temperature Liquids: Evolution Towards Time-Resolved Studies. International Journal of Thermophysics, 26, 4, 1127-1135, 2005.

Herrmann AG, Praktikum der Gesteinsanalyse: Chemisch-instrumentelle Methoden zur Bestimmung der Hauptkomponenten. Springer-Verlag, Berlin, 114-119, 1975.

Herzfeld KF \& TA Litovitz, Absorption and Dispersion of Ultrasonic Waves, 535, Academic Press, New York, 1959. 
Hess, KU and Dingwell DB, Viscosities of hydrous leucogranitic melts: A non-Arrhenian model. American Mineralogist, 81, 1297-1300, 1996.

Hess, PC \& MI Wood, Aluminium coordination in metaluminous and peralkaline silicate melts. Contributions to Mineralogy and Petrology, 81, 103-112, 1982.

Hirao K, N Soga \& M Kunugi, Mössbauer and ESR analysis of the distribution of $\mathrm{Fe}^{3+}$ in leucitetype iron silicate glasses and crystals. Journal of the American Ceramic Society, 62, 109110, 1979.

Hofmaier G, Viskosität und Struktur flüssiger Silikate: Berg und Hüttenin. Monatsh. Montan. Hochschule in Löben, 113, 270-281, 1968.

Hsieh $\mathrm{CH}, \mathrm{H}$ Jain, AC Miller \& El Kamitsos, X-ray photoelectron spectroscopy of Al- and Bsubstituted sodium trisilicate glasses. Journal of Non-Crystalline Solids, 168, 247-257, 1994.

Hummel W \& J Arndt, Variation of viscosity with temperature and composition in the plagioclase system. Contributions to Mineralogy and Petrology, 90, 83-92, 1985.

Hunold K \& R Brückner, Physikalische Eigenschaften und strukturelle Feinbau von NatriumAluminosilicatgläsern und -schmelzen. Glastechnische Berichte, 6S, 149-161, 1980.

Isard JO, Trans. Soc. Glass Technology, 43, 113, 1959.

luga D, C Morais, Z Gan, DR Neuville, L Cormier \& D Massiot, NMR Heteronuclear Correlation between Quadrupolar Nuclei in Solids. Journal of the American Ceramic Society, 127, 11540-11541, 2005.

Jaeger JC and NGW Cook. Fundamentals of rock mechanics ( $3^{\text {rd }}$ ed.), Chapman and Hall, New York , 593, 1979.

Jackson I, The laboratory study of seismic wave attenuation. In Mineral and Rock DeformationLaboratory Studies, Geophys. Monogr. Ser. 36:11-23, ed. B. E. Hobbs, H. C. Heard. Washington: American Geophysical Union, 1986.

Jeoung JS, WH Poisl \& MC Weinberg, Effect of oxidation state of iron on phase separation in sodium silicate glasses. Journal of the American Ceramic Society, 84, 8, 1859-1864, 2001.

Johnston WD, Oxidation - reduction equilibria in iron - containing glass. Journal of the American Ceramic Society, 47, 198-201, 1964.

Johnstone DH \& MN Toksöz, Definitions and terminology. Seismic wave attenuation. MN Toksöz and DH Johnstone, Society of Exploration Geophysics, 1-5, 1981.

Kargl F \& A Meyer, Inelastic neutron scattering on sodium aluminosilicate melts: sodium diffusion and intermediate range order, Chemical Geology, 213- 165-172, 2004.

Kilinc A, ISE Carmichael, ML Rivers \& RO Sack, The ferric - ferrous ratio of natural silicate liquids equilibrated in air. Contributions to Mineralogy and Petrology, 83, 136-140, 1983.

King IJ \& RWB Stephens, Experimental acoustics: Part I: Measurement of velocity of sound in terms of elastic modulus. Physics Education, 10, 6, 420-423, 1975.

Knoche R, DB Dingwell, FA Seifert \& SL Webb, Non-linear properties of supercooled liquids in the system $\mathrm{Na}_{2} \mathrm{O}-\mathrm{SiO}_{2}$. Chemical Geology, 116, 1-6, 1994.

Kress VC \& Carmichael ISE, The compressibility of silicate liquids containing $\mathrm{Fe}_{2} \mathrm{O}_{3}$ and the effect of composition, temperature, oxygen fugacity and pressure on their redox states. Contributions to Mineralogy and Petrology, 108, 82-92, 1991.

Kozakevitsch $\mathrm{P}$, Viscosité et elements structuraux des alumino-silicates fondues: laitiers $\mathrm{CaO}$ $\mathrm{Al}_{2} \mathrm{O}_{3}-\mathrm{SiO}_{2}$ entre 100 et $2000^{\circ} \mathrm{C}$. Review Métallurgie, 57, 149-160, 1960 .

Krupka KM, RA Robie \& BS Hemingway, High-temperature heat capacities of corundum, periclase, anorthite, $\mathrm{CaAl}_{2} \mathrm{Si}_{2} \mathrm{O}_{8}$ glass, moscovite, pyrophyllite, $\mathrm{KAISi}_{3} \mathrm{O}_{8}$ glass, grossular, and $\mathrm{NaAISi}_{3} \mathrm{O}_{8}$ glass. American Mineralogist, 64, 86-101, 1979.

Kubicki JD \& MJ Toplis, Molecular orbital calculations on aluminosilicate tricluster molecules: implications for the structure of aluminosilicate glasses. American Mineralogist, 87, 668-679, 2002.

Kurkjian CR \& EA Sigety, Coordination of the ferric ion in glass. Physics and Chemistry of Glasses, 9, 73-83, 1968. 
Kuryaeva RG, Degree of polymerization of aluminosilicate glasses and melts, Fiz. Khim. Stekla, 30, 2, 212-224, 2004 [Glass Phys. Chem. (Engl. transl.), 30, 2, 157-166, 2004].

Kushiro I, Changes in viscosity and structure of melt of $\mathrm{MaAlSi}_{2} \mathrm{O}_{6}$ composition at high pressures. Journal of Geophysical Research, 81, 6347-6350, 1976.

Lacy ED, Aluminum in glasses and melts. Physics and Chemistry of Glasses, 4, 234-238, 1963.

Lacy ED, Configuration change in silicates with particular reference to network structures. Acta Crystallographica, 18, 141, 1965.

Lai CC, PB Macedo \& CJ Montrose, Light-scattering measurements of structural relaxation in glass by digital correlation spectroscopy. Journal of the American Ceramic Society, 58, 3-4, 120 123, 1975.

Lam DJ, AP Paulikas \& BW Veal, X-ray photoemission spectroscopy studies of soda aluminosilicate glasses. Journal of Non-Crystalline Solids, 42, 41-47, 1980.

Lapp JC \& JE Shelby, Viscosity and thermal expansion of sodium and potassium galliosilicate glasses. Journal of the American Ceramic Society, 69, 2, 126-131, 1986.

Lee SK, GD Cody, Y Fei \& BO Mysen, The effect of $\mathrm{Na} / \mathrm{Si}$ on the structure of sodium silicate and aluminosilicate glasses quenched from melts at high pressure: A multi-nuclear (Al-27, Na-23, O-17) 1D and 2D solid - state NMR study. Chemical Geology, 229, 162-172, 2006.

Levy RA, CHP Lupis \& PA Flinn, Mössbauer analysis of the valence and coordination of iron cations in $\mathrm{SiO}_{2}-\mathrm{Na}_{2} \mathrm{O}-\mathrm{CaO}$ gasses. Physics and Chemistry of Glasses, 17, 94-103, 1976.

Litovitz TA \& P Macedo, Ultrasonic relaxation, viscosity and free volume in molten glasses. In: JA Prins, Ed., Physics of non-crystalline solids, Proceedings if the International Conference, North-Holland Publishing Co., Amsterdam, 220-230, 1965.

Liu SB, JF Stebbins, E Schneider \& A Pines, Diffusive motion in alkali-silicate melts: an NMR study at high temperature, Geochimica et Cosmochimica Acta, 52, 527- 538, 1988.

MacDowell JF \& GH Beall, Immiscibility and crystallization in $\mathrm{Al}_{2} \mathrm{O}_{3}-\mathrm{SiO}_{2}$ glasses. Journal of the American Ceramic Society, 52, 17-25, 1969.

MacFarlane RE \& JA Rayne, Ultrasonic attenuation of the noble metals. Physical Review, 162, 532-545, 1967.

MacKenzie JD, Structure of some inorganic glasses from high - temperature studies. In: MacKenzie JD, Modern Aspects of the Vitreous State, 188-218, Butterworths, Woburn, Mass., 1960.

Magaritz M \& AW Hofmann, Diffusion of $\mathrm{Sr}, \mathrm{Ba}$ and $\mathrm{Na}$ in obsidian. Geochimica et Cosmochimica Acta, 42, 595-605, 1978.

Magnien V, DR Neuville, L Cormier, BO Mysen, V Briois, S Belin, O Pinet \& P Richet, Kinetics of iron oxidation in silicate melts: a preliminary XANES study. Chemical Geology, 213, 253-263, 2004.

Maier CG \& ICK Kelly, An equation for the representation of high temperature heat content data. Journal of the American Chemical Society, 54, 3243-3246, 1932.

Marchi GD, P Mazzoldi \& A Miotello, Analysis of ionic conductivity in alkali and mixed - alkali aluminosilicate glasses. Journal of Non-Crystalline Solids, 105, 307-312, 1988.

Martens RM, M Rosenhauer, H Büttner \& K von Gehlen, Heat capacity and kinetic parameters in the glass transformation interval of diopside, anorthite and albite glass. Chemical Geology, 62, 49-70, 1987.

Matsumura T, D Okuyama, S Niioka, H Ishida, T Satoh, Y Murakami, H Toyosaki, Y Yamada, T Fukumura \& M Kawasaki, X-ray anomalous scattering of diluted magnetic oxide semiconductors: Possible evidence of lattice deformation for high temperature ferromagnetism. Physical Review B, 76, 115320-115325, 2007.

Maxwell JC, On the Dynamical Theory of Gases, Proceedings of the Royal Society of London 15, 167-171, (Also appeared in Philosophical Magazine, 32, 129-145), 1867.

May JE, Precise measurement of time delay. IRE International Convention Record, 6 (Pt. 2): 13442, 1958.

McMillan PF \& B Piriou, The structures and vibrational spectra of crystals and glasses in the silicaalumina system. Journal of Non-Crystalline Solids, 53, 279-298, 1982. 
McMillan PF, BT Poe, P Gillet \& B Reynard, A study of $\mathrm{SiO}_{2}$ glass and supercooled liquid to $1950 \mathrm{~K}$ via high temperature Raman spectroscopy, Geochimica et Cosmochimica Acta, 58, 36533664, 1994.

McSkimin HJ, Pulse superposition method for measuring ultrasonic wave velocities in solids. Journal of the Acoustical Society of America, 33, 12-16, 1961.

McSkimin HJ \& P Aadreatch, Analysis of pulse superposition method for measuring ultrasonic wave velocities as a function of temperature and pressure. Journal of the Acoustical Society of America, 34, 609-615, 1962.

Meade $\mathrm{C}$, RJ Hemley \& $\mathrm{HL}$ Mao, High-pressure x-ray diffraction of $\mathrm{SiO}_{2}$ glass. Physical Review Letters, 69, 9, 1387-1390, 1992.

Meagher EP, DK Swanson \& GV Gibbs, The calculation of tetrahedral Si-O and Al-O bridging bond lengths and angles (abstract). EOS, 61, 408, 1980.

Mills JJ, Low frequency storage and loss moduli of soda-silica glasses in the transformation range, Journal of Non-Crystalline Solids, 14, 255-268, 1974.

Moynihan CT, Structural relaxation and the glass transition. Reviews in Mineralogy and Geochemistry, 32, 1, 1-19, 1995.

Moynihan CT, Correlation between the width of the glass transition region and the temperature dependence of the viscosity of high- $\mathrm{T}_{\mathrm{g}}$ glasses. Journal of the American Ceramic Society, 76, 5, 1081-1087, 1993.

Moynihan CT, PB Macedo, CJ Montrose, CJ Montrose, PK Gupta, MA DeBolt, JF Dill, BE Dom, PW Drake, AJ Easteal, PB Elterman, RP Moeller, H Sasabe, JA Wilder, The glass transition and the nature of the glassy state. In: Structural relaxation in vitreous materials. Annals of the New York Academy of Sciences, 279, 1, 15-35, 1976.

Mungall JE, C Romano \& DB Dingwell, Multicomponent diffusion in the molten system $\mathrm{K}_{2} \mathrm{O}-\mathrm{Na}_{2} \mathrm{O}-$ $\mathrm{Al}_{2} \mathrm{O}_{3}-\mathrm{SiO}_{2}-\mathrm{H}_{2} \mathrm{O}$, American Mineralogist, 83, 685-699, 1998.

Mysen BO, Melting curves of rocks and viscosity of rock-forming melts. In: Touloukian YS, WR Judd \& RF Roy (eds), Physical Properties of Rocks and Minerals, of McGraw-Hill/CINDAS Data Series on Material Properties, Vol. II, McGraw-Hill, New York, 361-407, 1981.

Mysen BO, Magmatic silicate melts: Relations between bulk composition, structure and properties. In: Magmatic Processes: Physicochemical Principles (ed.: BO Mysen), 375-400. Geochemical Society, Special Publications, 1, 1987.

Mysen BO, Structure and properties of silicate melts, 354, Elsevier, Amsterdam, 1988.

Mysen $\mathrm{BO}$, Role of the $\mathrm{Al}$ in depolymerized, peralkaline aluminosilicate melts in the systems $\mathrm{LiO}_{2}$ $\mathrm{Al}_{2} \mathrm{O}_{3}-\mathrm{SiO}_{2}, \mathrm{Na}_{2} \mathrm{O}-\mathrm{Al}_{2} \mathrm{O}_{3}-\mathrm{SiO}_{2}$, and $\mathrm{K}_{2} \mathrm{O}-\mathrm{Al}_{2} \mathrm{O}_{3}-\mathrm{SiO}_{2}$. American Mineralogist, 75, 1-2, 120-134, 1990.

Mysen $\mathrm{BO}$, Structural behaviour of $\mathrm{Al}^{3+}$ in silicate melts: in-situ, high-temperature measurements as a function of bulk chemical composition. Geochimica et Cosmochimica Acta, 59, 455-474, 1995a.

Mysen BO, Experimental, in-situ, high-temperature studies of properties and structure of silicate melts relevant to magmatic temperatures. European Journal of Mineralogy, 7, 745-766, 1995b.

Mysen BO, Aluminosilicate melts: structure, composition and temperature. Contributions to Mineralogy and Petrology, 127, 104-118, 1997.

Mysen BO, Transport and configurational properties of silicate melts: relationship to melt structure at magmatic temperatures. Physics of the Earth and Planetary Interiors, 107, 23-32, 1998.

Mysen BO \& JD Frantz, Alkali silicate glass and melt structure in the temperature range $25-1651^{\circ} \mathrm{C}$ at atmospheric pressure and implications for mixing behaviour of structural units. Contributions to Mineralogy and Petrology, 117, 1-14, 1994a.

Mysen BO \& D Virgo, Influence of pressure, temperature, and bulk composition on melt structures in the system $\mathrm{NaAlSi}_{2} \mathrm{O}_{6}-\mathrm{NaFe}^{3+} \mathrm{Si}_{2} \mathrm{O}_{6}$. Journal of the American Ceramic Society, 278, 1307$1322,1978$.

Mysen BO \& D Virgo, Trace element partitioning and melt structure: an experimental study at 1 atm. pressure. Geochimica et Cosmochimica Acta, 44, 1917-1930, 1980. 
Mysen BO \& D Virgo, Iron bearing silicate melts - relations between pressure and redox equilibria. Physics and Chemistry of Minerals, 12, 4, 191-200, 1985.

Mysen BO, FA Seifert \& D Virgo, Structure and redox equilibria of iron - bearing silicate melts. American Mineralogist, 65, 867-884, 1980a.

Mysen BO, D Virgo \& \& FA Seifert, Relations between the anionic structure and viscosity of silicate melts - a Raman spectroscopic study. American Mineralogist, 65, 690-710, 1980b.

Mysen BO, D Virgo \& I Kushiro, The structural role of aluminium in silicate melts - a Raman spectroscopic study at 1 atmosphere. American Mineralogist, 66, 678-701, 1981a.

Mysen BO, D Virgo \& \& FA Seifert, Ferric iron as a network former and as a network modifier in melts relevant to petrological processes. Carnegie Institution of Washington Year Book, 80, 311-312, 1981b.

Mysen BO, D Virgo \& \& FA Seifert, The structure of silicate melts: implications for chemical and physical properties of natural magma, Reviews of Geophysics and Space Physics, 20, 3, 353-383, 1982.

Mysen BO, D Virgo \& \& FA Seifert, Redox equilibria of iron in alkaline earth silicate melts: relationships between melt structure, oxygen fugacity, temperature and properties of ironbearing silicate liquids. American Mineralogist, 69, 834-847, 1984.

Mysen BO, D Virgo, ER Neumann \& FA Seifert, Redox equilibria and the structural states of ferric and ferrous iron in the melts in the system $\mathrm{CaO}-\mathrm{MgO}-\mathrm{Al}_{2} \mathrm{O}_{3}-\mathrm{SiO}_{2}-\mathrm{Fe}-\mathrm{O}$ : relationships between redox equilibria, melt structure and liquidus phase equilibria. American Mineralogist, 70, 317-331, 1985a.

Mysen BO, D Virgo, CM Scarfe \& DJ Cronin, Viscosity and structure of iron- and aluminium bearing calcium silicate melts. American Mineralogist, 70, 487-498, $1985 \mathrm{~b}$.

Mysen $\mathrm{BO}, \mathrm{A}$ Lucier \& GD Cody, The structural behaviour of $\mathrm{Al}^{3+}$ in peralkaline melts and glasses in the system $\mathrm{Na}_{2} \mathrm{O}-\mathrm{Al}_{2} \mathrm{O}_{3}-\mathrm{SiO}_{2}$. American Mineralogist, 88, 1668-1678, 2003.

Nagel $R$ \& AG Balogh, Atomic transport in metal/ceramic interfaces under heavy ion irradiation, Nuclear Instruments and Methods, B156, 135-142, 1999.

Narayanaswamy OS, Model of structural relaxation in glass. Journal of the American Ceramic Society, 54, 491-498, 1971.

Narayanaswamy OS, Thermorheological simplicity in the glass transition, Journal of the American Ceramic Society, 71, 10, 900-904, 1988.

Neuville DR \& BO Mysen, Role of the aluminium in the silicate network: in-situ, high-temperature study of glasses and melts on the join $\mathrm{SiO}_{2}-\mathrm{NaAlO}_{2}$. Geochimica et Cosmochimica Acta, 60, 1727-1738, 1996.

Neuville DR, L Cormier, V Montouillout \& D Massiot, Local Al site distribution in aluminosilicate glasses by ${ }^{27} \mathrm{Al}$ MQMAS NMR. Journal of Non-Crystalline Solids, 353, 180-184, 2007.

Neuville DR, P Courtial, DB Dingwell \& P Richet, Thermodynamic and rheological properties of rhyolite and andesite melts. Contributions to Mineralogy and Petrology, 113, 572-581, 1993.

Nolet DA, RG Burns, SL Flamm \& JR Besancon, Spectra of Fe-Ti silicate glasses: implications to remote - sensing of planetary surfaces. Proceedings of the $10^{\text {th }}$ Lunar and Planetary Science Conference, 1775-1786, 1979.

Nowick AS \& BS Berry, Anelastic Relaxation in Solids. Academic Press, New York, U.S.A., 1972.

O'Connell RJ \& B Budiansky, Measures of dissipation in viscoelastic media, Geophysical Research Letters, 5, 5-8, 1978.

O'Reilley JM \& M Goldstein, eds., Structure and mobility in molecular and atomic glasses. Annals of the New York Academy of Sciences, 371, 1981.

Ohno I, S Yamamoto \& OL Anderson, Determination of elastic constants of trigonal crystals by the rectangular parallelepiped resonance method, Journal of Non-Crystalline Solids, 47, 1103$1108,1986$.

Okuno M, N Zotov, M Schmücker \& $\mathrm{H}$ Schneider, Structure of $\mathrm{SiO}_{2}-\mathrm{Al}_{2} \mathrm{O}_{3}$ glasses: Combined X-ray diffraction, IR and Raman studies. Journal of Non-Crystalline Solids, 351, 1032-1038, 2005.

Papadakis EP, Ultrasonic diffraction loss and phase change in anisotropic materials. Journal of the Acoustical Society of America, 40, 863-76, 1966. 
Papadakis EP, Ultrasonic velocity and attenuation: measurement methods with scientific and industrial applications. (Mason W P \& Thurston R N, eds.) Physical acoustics: principles and methods. New York: Academic Press, 12, 277-374, 1976.

Paul A, Oxidation - reduction equilibria in glass. Journal of Non-Crystalline Solids, 123, 354-362, 1990.

Piguet JL \& JE Shelby, Transformation-range behaviour of lithium galliosilicate glasses. Journal of the American Ceramic Society, 68, C232-C233, 1985.

Piguet JL, JC Lapp \& JE Shelby, Transformation range behavior of lithium galliosilicate glasses. Journal of the American Ceramic Society, 68, 6, 326-329, 1985.

Plazek DJ \& KL Ngai, Correlation of polymer segmental chain dynamics with temperature dependent time - scale shifts. Macromolecules, 24, 1222-1224, 1991.

Pocklington HC, Rough measurement of high viscosities. Proceedings of the Cambridge Philosophical Society, 36, 507-508, 1940.

Poe BT, PF McMillan, CA Angell \& RK Sato, $\mathrm{Al}$ and $\mathrm{Si}$ coordination in $\mathrm{SiO}_{2}-\mathrm{Al}_{2} \mathrm{O}_{3}$ glasses and liquids: a study by NMR and IR spectroscopy and MD simulations. Chemical Geology, 96, 333-349, 1992.

Poe BT, PF McMillan, B Coté, D Massiot \& JP Coutures, Structure and dynamics in calcium aluminate liquids: High temperature ${ }^{27} \mathrm{Al}$ NMR and Raman spectroscopy. Journal of the American Ceramic Society, 77, 1832-1838, 1994.

Poe B, F Seifert, TG Sharp \& Z Wu, ELNES spectroscopy of mixed Si coordination minerals. Physics and Chemistry of Minerals, 24, 477-487, 1997.

Reid JE, BT Poe, DC Rubie, N Zotov \& M Wiedenbeck, The self-diffusion of silicon and oxygen in diopside $\left(\mathrm{CaMgSi}_{2} \mathrm{O}_{6}\right)$ liquid up to $15 \mathrm{GPa}$. Chemical Geology, 174, 77-86, 2001.

Reid JE, A Suzuki, KI Funakoshi, H Terasaki, BT Poe, DC Rubie \& E Ohtani, The viscosity of $\mathrm{CaMgSi}_{2} \mathrm{O}_{6}$ liquid at pressures up to $13 \mathrm{GPa}$, Physics of the Earth and Planetary Interiors, 139, 45- 54, 2003.

Richet P, Viscosity and configurational entropy of silicate melts. Geochimica et Cosmochimica Acta, 48, 471-483, 1984.

Richet P, Heat capacity of silicate glasses. Chemical Geology, 62, 111-124, 1987.

Richet $P$, The physical basis of thermodynamics. With application to chemistry. Plenum Publishing, New York, 2001.

Richet $\mathrm{P}$, Enthalpy, volume and structural relaxation in glass - forming silicate melts. Journal of Thermal Analysis and Calorimetry, 69, 739-750, 2002.

Richet $P$ \& Y Bottinga, Heat capacity of aluminium - free liquid silicates. Geochimica et Cosmochimica Acta, 49, 471-486, 1985.

Richet P \& Y Bottinga, Thermochemical properties of silicate glasses and liquids. A review, Review Geophysics, 24, 1-25, 1986.

Richet $P$ \& Y Bottinga, Rheology and configurational entropy of silicate melts. In Stebbins JF, McMillan PF and Dingwell DB, Eds., Structure, Dynamics and Properties of Silicate Melts, 32, 67-93. Reviews in Mineralogy, Mineralogical Society of America, Washington, D.C., 1995.

Richet P \& DR Neuville, Thermodynamics of silicate melts: configurational properties. In: Saxena S., Ed., Thermodynamic data systematics and estimation, Advances in Physical Geochemistry, 132-160, Springer - Verlag, Berlin, 1992.

Richet P \& MJ Toplis, Thermodynamic aspects of the glass transition of silicates. Comptes Rendus de l'Académie des Sciences (Paris), série II. Sciences de la Terre et des planètes, 2, 191202, 2001

Richet P, RA Robie \& BS Hemingway, Low-temperature heat capacity of diopside glass $\left(\mathrm{CaMgSi}_{2} \mathrm{O}_{6}\right)$ : A calorimetric test of the configurational-entropy theory applied to the viscosity of liquid silicates. Geochimica et Cosmochimica Acta, 50, 7, 1521-1533, 1986.

Riebling EF, Structure of magnesium aluminosilicate liquids at $1700^{\circ} \mathrm{C}$. Canadian Journal of Chemistry, 42, 2811-2821, 1964. 
Riebling EF, Structure of aluminosilicate melts containing at least 50 mole $\% \mathrm{SiO}_{2}$ at $1500^{\circ} \mathrm{C}$. Journal of Chemical Physics, 44, 2857-2865, 1966.

Riebling EF, Structural similarities between a glass and its melt. Journal of the American Ceramic Society, 51, 143-149, 1968.

Riley WF, LD Sturges \& DH Morris, Mechanics of materials: Torsional loading of shafts, 272-344, John Wiley \& Sons, Inc., New York, 1999.

Risbud SH, RJ Kirkpatrick, AP Taglialavore \& B Montez, Solid-state NMR evidence of 4-, 5-, and 6fold aluminum sites in roller-quenched $\mathrm{SiO}_{2}-\mathrm{Al}_{2} \mathrm{O}_{3}$ glasses. Journal of the American Ceramic Society, 70, C10-C70, 1987.

Rivers ML \& LSE Carmichael, Ultrasonic studies of silicate melts. Journal of Geophysical Research, 92, 9247-9270, 1987.

Robie RA, BS Hemingway \& JR Fisher, Thermodynamic properties of minerals and related substances at $298.15 \mathrm{~K}$ and 1 bar (105 Pascals) pressure and at higher temperatures. United States Geological Survey Bulletin, 1452, 456, 1979.

Roselieb K, W Rammensee, H Büttner \& M Rosenhauer, Solubility and diffusion of noble gases in vitreous albite. Chemical Geology, 96, 241-266, 1992.

Roskosz M, MJ Toplis \& P Richet, The structural role of Ti in aluminosilicate liquids in the glass transition range: insights from heat capacity and shear viscosity measurements. Geochimica et Cosmochimica Acta, 68, 3, 591-606, 2004.

Russel C \& A Wiedenroth, The effect of glass composition on the thermodynamic of the $\mathrm{Fe}^{2+} / \mathrm{Fe}^{3+}$ equilibrium and the iron diffusivity in $\mathrm{Na}_{2} \mathrm{O} / \mathrm{MgO} / \mathrm{CaO} / \mathrm{Al}_{2} \mathrm{O}_{3} / \mathrm{SiO}_{2}$ melts. Chemical Geology, 213, 1-3, 125-135, 2004.

Russel JK, D Giordano \& DB Dingwell, High-temperature limits on viscosity of non-Arrhenian silicate melts, American Mineralogist, 88, 1390-1394, 2003.

Sack RO, ISE Carmichael, ML Rivers \& M Ghiorso, Ferric - ferrous equilibria in natural silicate liquids at 1 bar. Contributions to Mineralogy and Petrology, 75, 369-376, 1980.

Sartorius, Sartorius YDK 01, YDK 01-OD, YDK 01 LP. Density Determination Kit, User's manual. 2004

Sass F, Ch Bouché \& A Leitner, Dubbels Taschenbuch für den Machinenbau, Springer - Verlag, 382-384, 1966.

Sato RK, PF MacMillan, $\mathrm{P}$ Dennison \& $\mathrm{R}$ Dupree, High-resolution ${ }^{27} \mathrm{Al}$ and ${ }^{29} \mathrm{Si}$ MAS NMR investigation of $\mathrm{SiO}_{2}-\mathrm{Al}_{2} \mathrm{O}_{3}$ glasses. Journal of Physical Chemistry, 95, 11, 483-4489, $1991 \mathrm{a}$.

Sato RK, PF MacMillan, $\mathrm{P}$ Dennison \& R Dupree, A structural investigation of high alumina glasses in the $\mathrm{CaO}-\mathrm{Al}_{2} \mathrm{O}_{3}-\mathrm{SiO}_{2}$ system via Raman and magic angle spinning nuclear magnetic resonance spectroscopy. Physics and Chemistry of Glasses, 32, 149-154, 1991b.

Scherer GW, Use of the Adam - Gibbs equation in the analysis of structural relaxation. Journal of the American Ceramic Society, 67, 7, 504-511, 1984.

Scherer GW, Relaxation in glass and composites. 331, Wiley, New York, 1986.

Scherer GW, Theories of relaxation. Journal of Non-Crystalline Solids, 123, 75-89, 1990.

Schramm CM, BHWS DeJong \& VF Parziale, ${ }^{29}$ Si magic angle spinning NMR study of local silicon environments in-amorphous and crystalline lithium silicates. Journal of the American Ceramic Society, 106, 4396-4402, 1984.

Secco RA, MH Manghnani \& TC Liu, The bulk modulus - attenuation - viscosity systematics for diopside - anorthite melts. Geophysical Research Letters, 18, 1, 93-96, 1991.

Seifert FA \& M Olesch, Mössbauer spectroscopy of grandidierite, (Mg, $\mathrm{Fe}) \mathrm{Al}_{3} \mathrm{BSiO}_{9}$. American Mineralogist, 62, 547-553, 1977.

Seifert FA, BO Mysen \& D Virgo, Three - dimensional network structure of quenched melts (glass) in the system $\mathrm{SiO}_{2}-\mathrm{NaAlO}_{2}, \mathrm{SiO}_{2}-\mathrm{CaAl}_{2} \mathrm{O}_{4}$ and $\mathrm{SiO}_{2}-\mathrm{MgAl}_{2} \mathrm{O}_{4}$. American Mineralogist, 67, 696-717, 1982.

Seifert FA, D Virgo \& BO Mysen, Melt structures and redox equilibria in the system $\mathrm{Na}_{2} \mathrm{O}-\mathrm{FeO}-$ $\mathrm{Fe}_{2} \mathrm{O}_{3}-\mathrm{Al}_{2} \mathrm{O}_{3}-\mathrm{SiO}_{2}$. Carnegie Inst. Wach. Yearbook, 78, 511-519, 1979.

Shelby JE, Viscosity and thermal expansion of lithium aluminosilicate glasses. Journal of Applied Physics, 49, 12, 5885-5891, 1978. 
Shelby JE, Treatise on materials science and technology, 17, Glass II, Chapter 1, 1-40, 1979.

Shelby JE, Formation and properties of calcium aluminosilicate glasses. Journal of the American Ceramic Society, 68, 3, 155-158, 1985.

Shelby JE, Properties and structure of soda-lime aluminosilicate glasses. Journal of Applied Physics, 66, 5, 1947-1950, 1989.

Shelby JE, Introduction to glass science and technology. $2^{\text {nd }}$ ed. The Royal Society of Chemistry, Cambridge, 2005.

Shimizu N \& I Kushiro, Diffusivity of oxygen in jadeite and diopside melts at high pressures. Geochimica et Cosmochimica Acta, 48, 1295-1303, 1984.

Siewert R \& M Rosenhauer, Viscoelastic relaxation measurements in the system $\mathrm{SiO}_{2}-\mathrm{NaAlSiO}_{4}$ by photon correlation spectroscopy. American Mineralogist, 82, 1063-1072, 1997.

Simon F, Über der Zustand der unterkühlten Flüssigkeiten and Gläser. Zeitschrift für anorganische und allgemeine Chemie, 203, 219-227, 1931.

Sippel RF, Sodium self diffusion in natural minerals. Geochimica et Cosmochimica Acta, 27, 107120, 1963.

Spiering B \& FA Seifert, Iron in silicate glasses of granitic composition: a Mössbauer spectroscopic study. Contributions to Mineralogy and Petrology, 90, 63-73, 1985.

Stebbins JF, NMR evidence for five-coordinated silicon in a silicate glass at atmospheric pressure. Nature, 351, 638-639, 1991.

Stebbins JF, Dynamics and structure of silicate and oxide melts: nuclear magnetic resonance studies, In: Structure, Dynamics and Properties of Silicate Melts, Stebbins JF, PF McMillan \& DB Dingwell (eds), Reviews in Mineralogy, Mineralogical Society of America, Washington DC, 32, 191-246, 1995.

Stebbins JF \& I Farnan, The effects of temperature on silicate liquid structure: a multi-nuclear, high temperature NMR study. Science, 255, 586-589, 1992.

Stebbins JF \& PF McMillan, Compositional and temperature effects on five-coordinated silicon in ambient pressure silicate glasses, Journal of Non-Crystalline Solids, 160, 116-125, 1993.

Stebbins JF \& S Sen, Microscopic dynamics and viscous flow in a borosilicate glass - forming liquid. Journal of Non-Crystalline Solids, 224, 80-85, 1998.

Stebbins JF \& Z Xu, NMR evidence for excess non-bridging oxygen in an aluminosilicate glass, Nature, 390, 60-62, 1997.

Stebbins JF, ISE Carmichael \& LK Moret, Heat capacities and entropies of silicate liquids and glasses. Contributions to Mineralogy and Petrology, 86, 131-148, 1984.

Stebbins JF, JV Ogelsby \& SK Lee, Oxygen sites in silicate glasses; a new view from oxygen-17 NMR ; Chemical Geology, 174, 63-75, 2001.

Stebbins JF, S Sen \& I Farnan, Silicate species exchange, viscosity, and crystallization in a low silica melt: In situ high - temperature MAS NMR spectroscopy. American Mineralogist, 80, 861-864, 1995.

Steele FN \& RW Douglas, The absorption of iron in silicate and borate glasses. Physics and Chemistry of Glasses, 6, 246-252, 1965.

Stein DJ \& FJ Spera, Experimental rheometry of melts and supercooled liquids in the system $\mathrm{NaAlSiO}_{4}-\mathrm{SiO}_{2}$ : Implications for structure and dynamics. American Mineralogist, 78, 710-723, 1993.

Stevenson RJ, DB Dingwell, SL Webb \& NS Bagdassarov, The equivalence of enthalpy and shear stress relaxation in rhyolitic obsidian and quantification of the liquid-glass transition in volcanic processes, Journal of Volcanology and Geothermal Research, 68, 297-306, 1995.

Tammann G, Kristallisieren und Schmelzen. Johann Ambrosius Barth, Leipzig, 26-46, 1903.

Tammann G, Der Glaszustand. Leopold Voss, Leipzig, 123, 1933.

Tammann G, Aggregatzustände. Die Zustandsänderungen der Materie in Abhängigkeit von Druck und Temperatur. Leopold Voss, Leipzig, 2nd Edition, 1923. 
Tammann G \& W Hesse, Die Abhängigkeit der Viskosität von der Temperatur bei unterkühlten Flüssigkeiten, Zeitschrift für anorganische und allgemeine Chemie, 156, 1, 245-257, 1926.

Tangeman JA \& RA Lange, The effect of $\mathrm{Al}^{3+}, \mathrm{Fe}^{3+}$, and $\mathrm{Ti}^{4+}$ on the configurational heat capacities of sodium silicate liquids. Physics and Chemistry of Minerals, 26, 83-99, 1998.

Taylor MP \& GE Brown Jr., Structure of mineral glasses. I. The feldspar glasses $\mathrm{NaAISi}_{3} \mathrm{O}_{8}$, $\mathrm{KAISi}_{3} \mathrm{O}_{8}, \mathrm{CaAl}_{2} \mathrm{Si}_{2} \mathrm{O}_{8}$. Geochimica et Cosmochimica Acta, 43, 61-77, 1979a.

Taylor MP \& GE Brown Jr., Structure of mineral glasses. II. The $\mathrm{SiO}_{2}-\mathrm{NaAISiO}_{4}$ join. Geochimica et Cosmochimica Acta, 43, 1467-1473, $1979 \mathrm{~b}$.

Taylor TD \& GE Rindone, Properties of soda aluminosilicate glasses: V, low temperature viscosities. Journal of the American Ceramic Society, 53, 692-695, 1970.

Taylor MP, GE Brown Jr. \& PM Fenn, Structure of mineral glasses. III. $\mathrm{NaAISi}_{3} \mathrm{O}_{8}$ supercooled liquid at $850^{\circ} \mathrm{C}$ and the effects of thermal history. Geochimica et Cosmochimica Acta, 44, 109-117, 1980.

Terai R, Self-diffusion of sodium ions and electrical conductivity in sodium aluminosilicate glasses. Physics and Chemistry of Glasses, 10,146-152, 1969.

Toplis MJ, Energy barriers to viscous flow and the prediction of glass transition temperatures of molten silicates, American Mineralogist, 83, 480-490, 1998.

Toplis MJ, Quantitative links between microscopic properties and viscosity of liquids in the system $\mathrm{SiO}_{2}-\mathrm{Na}_{2} \mathrm{O}$. Chemical Geology, 174, 321-331, 2001.

Toplis M, DB Dingwell \& T Lenci, Peraluminous viscosity maxima in $\mathrm{Na}_{2} \mathrm{O}-\mathrm{Al}_{2} \mathrm{O}_{3}-\mathrm{SiO}_{2}$ liquids: The role of triclusters in tectosilicate melts. Geochimica et Cosmochimica Acta, 61, 2605-2612, 1997a.

Toplis M, DB Dingwell, K-U Hess \& T Lenci, Viscosity, fragility, and configurational entropy of melts along the join $\mathrm{SiO}_{2}-\mathrm{NaAlSiO}_{4}$. American Mineralogist, 82, 979-990, $1997 \mathrm{~b}$.

Toplis MJ, SC Kohn, ME Smith \& IJF Poplett, Fivefold - coordinated aluminium in tectosilicate glasses observed by triple quantum MAS NMAR. American Mineralogist, 85, 1556-1560, 2000.

Toplis MJ, J Gottsmann, R Knoche \& DB Dingwell, Heat capacities of haplogranitic glasses and liquids. Geochimica et Cosmochimica Acta, 65, 1985-1994, 2001.

Truell R, C Elbaum \& B Chick, Ultrasonic Methods in Solid State Physics, Academic Press, 1969.

Urbain G, Y Bottinga \& $P$ Richet, Viscosity of liquid silica, silicates and aluminosilicates. Geochimica et Cosmochimica Acta, 46, 1061-1072, 1982.

Virgo D \& BO Mysen, The structural state of iron in oxidized vs. reduced glasses at 1 atm.: a ${ }^{57} \mathrm{Fe}$ Mössbauer study. Physics and Chemistry of Minerals, 12, 65-76, 1985.

Virgo D, BO Mysen \& PA Danckwerth, Redox equilibria and the anionic structure of $\mathrm{Na}_{2} \mathrm{O} \cdot \mathrm{xSiO}_{2^{-}}$ Fe-O melts: effects of oxygen fugacity. Carnegie Institution of Washington Year Book, 82, 305-309, 1983.

Virgo D, BO Mysen \& FA Seifert, Relationship between the oxidation state of iron and the structure of silicate melts. Carnegie Institution of Washington Year Book, 80, 308-311, 1981.

Virgo D, BO Mysen, PA Danckwerth \& FA Seifert, The anionic structure of $1 \mathrm{~atm}$. melts in the system $\mathrm{SiO}_{2}-\mathrm{NaFeO}_{2}$. Carnegie Institution of Washington Year Book, 81, 347-349, 1982.

Virgo D, BO Mysen, PA Danckwerth \& FA Seifert, Speciation of $\mathrm{Fe}^{3+}$ in 1-atm $\mathrm{Na}_{2} \mathrm{O}-\mathrm{SiO}_{2}-\mathrm{Fe}-\mathrm{O}$ melts. Carnegie Institution of Washington Year Book, 81, 349-353, 1982a.

Vogel DRW, Structure and crystallization behaviour of glasses. Angewandte Chemie International Edition, 4, 2, 112-121, 1965.

Waff HS, The structural role of ferric iron in silicate melts. Canadian Mineralogist, 15, 198-199, 1977.

Wang CM \& H Chen, Mixed coordination of $\mathrm{Fe}^{3+}$ and its dependence on the iron content in sodium disilicate glasses. Physics and Chemistry of Glasses, 28, 39-47, 1987.

Wang Z, TF Cooney \& SK Sharma, High temperature structural investigations of $\mathrm{Na}_{2} \mathrm{O}-0.5 \mathrm{Fe}_{2} \mathrm{O}_{3}-$ $3 \mathrm{SiO}_{2}$ and $\mathrm{Na}_{2} \mathrm{O}-\mathrm{FeO}-3 \mathrm{SiO}_{2}$ melts and glasses. Contributions to Mineralogy and Petrology, 115, 112-122, 1993. 
Wang Z, TF Cooney \& SK Sharma, In situ structural investigations of iron - containing silicate liquids and glasses. Geochimica et Cosmochimica Acta, 59, 1571-1577, 1995.

Warren BE, X-ray diffraction in long chain liquids. Physical Review, 44, 969-973, 1933.

Watson EB, Calcium diffusion in a simple silicate melt to $30 \mathrm{kbar}$. Geochimica et Cosmochimica Acta, 43, 313-323, 1979.

Webb SL, Shear and volume relaxation in $\mathrm{Na}_{2} \mathrm{Si}_{2} \mathrm{O}_{5}$. American Mineralogist, 76, 1449-1454, 1991.

Webb SL, Low-frequency shear and structural relaxation in rhyolite melt. Physics and Chemistry of Minerals, 19, 240-245, 1992a.

Webb SL, Shear, volume, enthalpy and structural relaxation in silicate melts. Chemical Geology, 96, 449-457, 1992b.

Webb SL, Silicate melts at extreme conditions. In Miletich, R., Ed., Mineral Behaviour at Extreme Conditions, EMU Notes in Mineralogy, 7, Chapter 3, 65-94, 2005a.

Webb SL, Structure and rheology of $\mathrm{Fe}_{2} \mathrm{O}_{3}$-bearing $\mathrm{Na}_{2} \mathrm{O}-\mathrm{Al}_{2} \mathrm{O}_{3}-\mathrm{SiO}_{2}$ melts. European Journal of Mineralogy, 17, 223-232, 2005b.

Webb SL \& DB Dingwell, The onset of non-Newtonian rheology of silicate melts: A fiber elongation study. Physics and Chemistry of Minerals, 17, 125-132, 1990.

Webb SL \& DB Dingwell, Viscoelasticity. Reviews in Mineralogy and Geochemistry, 32, 1, 95-119, 1995.

Webb SL \& I Jackson, Anelasticity and microcreep in polycrystalline $\mathrm{MgO}$ at high temperature: an exploratory study. Physics and Chemistry of Minerals, 30, 157-166, 2003.

Webb SL, E Müller \& H Büttner, Anomalous rheology of peraluminous melts. American Mineralogist, 89, 812-818, 2004.

Webb SL, M Banaszak, U Köhler, S Rausch \& G Raschke, The viscosity of $\mathrm{Na}_{2} \mathrm{O}-\mathrm{CaO}-\mathrm{Al}_{2} \mathrm{O}_{3}-\mathrm{SiO}_{2}$ melts. European Journal of Mineralogy, submitted, 02/2007.

Weigel C, L Cormier, L Galoisy, G Calas, D Bowron \& B Beuneu, Determination of $\mathrm{Fe}^{3+}$ sites in a $\mathrm{NaFeSi}_{2} \mathrm{O}_{6}$ glass by neutron diffraction with isotopic substitution coupled with numerical simulation. Applied Physics Letters, 89, 14, 141911, 2006.

Wilding MC, SL Webb \& DB Dingwell, Evaluation of a relaxation geospeedometer for volcanic glasses. Chemical Geology, 125, 137-148, 1995.

Wilke M, H Behrens, DJM Burkhard \& S Rossano, The oxidation state of iron in silicic melt at 500 MPa water pressure. Chemical Geology, 189, 55-67, 2002.

Wilke M, C Schmidt, F Farges, V Malavergne, L Gautron, A Simionovici, M Hahn \& PE Petit, Structural environment of iron in hydrous aluminosilicate glass and melt - evidence from $X$ ray absorption spectroscopy. Chemical Geology, 229, 144-161, 2006.

Wispelaere SD, D Cabaret, C Levelut, S Rossano, AM Flank, P Parent \& F Farges, Na-, Al-, and Si K-edge XANES study of sodium silicate and sodium aluminosilicate glasses : influence of the glass surface. Chemical Geology, 213, 63-70, 2004.

Wood MI \& PC Hess, The structural role of $\mathrm{Al}_{2} \mathrm{O}_{3}$ and $\mathrm{TiO}_{2}$ in immiscible silicate liquids in the system $\mathrm{SiO}_{2}-\mathrm{MgO}-\mathrm{CaO}-\mathrm{FeO}-\mathrm{TiO}_{2}-\mathrm{Al}_{2} \mathrm{O}_{3}$. Contributions to Mineralogy and Petrology, 72, 319328, 1980.

Xue X \& M Kanzaki, NMR characteristics of possible oxygen sites in aluminosilicate glasses and melts: an ab initio study. Journal of Physical Chemistry B, 103, 10816-10830, 1999.

Yue YZ \& R Brückner, A new description and interpretation of the flow behaviour of glass forming melts. Journal of Non-Crystalline Solids, 180, 66-79, 1994.

Zachariasen WH,. Journal of the American Chemical Society, 54, 3841-3851 (1932).

Zdaniewski WA, GE Rindone \& DE Day, The internal friction of glasses. Journal of Materials Science, 14, 763-775, 1979.

Zotov N \& R Delaplane, Structure and crystallization kinetics of sodium tetrasilicate glass: a high temperature neutron diffraction study. Materials Science Forum, 321-324, 535-539, 2000. 



\section{APPENDICES}

Appendix 1

Heat capacity data (in $\mathrm{J} \mathrm{g}^{-1} \mathrm{~K}^{-1}$ ) for all of the samples with step of temperature of $5^{\circ} \mathrm{C}$ for

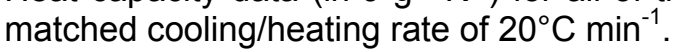

Appendix 2 .

Temperature $T, \log _{10} \eta$, frequency $f$, $\log _{10} \omega \tau_{\mathrm{M}}$, imaginary $\left(G_{\mathrm{im}}\right)$ and real ( $G$ real $)$ shear modulus and shear modulus of the melt $\left(G_{\text {melt }}\right)$ for the investigated samples.

Appendix 3.

Temperature $\mathrm{T}$, frequency $f, \mathrm{G}_{\infty}$, viscosity $\eta$, shear viscosity, relaxed Newtonian viscosity and relaxation times $\tau$ of the samples, determined by micropenetration and torsion measurements.

Appendix $4 a$.

Parameters of the lines fitted to the real shear modulus data for NS2 melt (G0) and Fe-free melts (G1-G7).

Appendix 4b.

Parameters of the lines fitted to the imaginary shear modulus data for NS2 melt (G0) and Fe-free melts (G1-G7).

Appendix 4c.

Parameters of the lines fitted to the real shear modulus data for Fe-bearing melts (G8-14).

Appendix 4d.

Parameters of the lines fitted to the imaginary shear modulus data for Fe-bearing melts (G8-G14).

Appendix 5a

Parameters of the fits to Eq. 58 for the NS (G0) and Fe-free melts (G1-G7).

Appendix 5b.

Parameters of the fits to Eq. 58 for the Fe-bearing melts (G8-G14). 

Appendix 1. Heat capacity data (in $\mathrm{J} \mathrm{g}^{-1} \mathrm{~K}^{-1}$ ) for all of the samples with step of temperature of $5^{\circ} \mathrm{C}$ for matched cooling/heating rate of $20^{\circ} \mathrm{C}$ min ${ }^{-1}$.

\begin{tabular}{|c|c|c|c|c|c|c|c|c|c|c|c|c|c|c|c|}
\hline \multirow[b]{2}{*}{$\mathrm{T}\left({ }^{\circ} \mathrm{C}\right)$} & G0 & G1 & G2 & G3 & G4 & G5 & G6 & G7 & G8 & G9 & G10 & G11 & G12 & G13 & G14 \\
\hline & \multicolumn{15}{|c|}{ Heat capacity $c_{p}\left(\mathrm{~J} \mathrm{~g}^{-1} \mathrm{~K}^{-1}\right)$} \\
\hline 100 & 0.981 & 0.906 & 0.898 & 0.906 & 0.923 & 0.917 & 0.918 & 0.936 & 0.911 & 0.882 & 0.887 & 0.874 & 0.888 & 0.885 & 0.893 \\
\hline 105 & 0.986 & 0.917 & 0.897 & 0.918 & 0.921 & 0.919 & 0.923 & 0.943 & 0.921 & 0.888 & 0.907 & 0.884 & 0.900 & 0.899 & 0.910 \\
\hline 110 & 0.987 & 0.919 & 0.898 & 0.918 & 0.918 & 0.924 & 0.936 & 0.948 & 0.926 & 0.899 & 0.911 & 0.893 & 0.902 & 0.904 & 0.914 \\
\hline 115 & 0.994 & 0.915 & 0.902 & 0.922 & 0.926 & 0.928 & 0.935 & 0.946 & 0.933 & 0.908 & 0.903 & 0.898 & 0.896 & 0.899 & 0.910 \\
\hline 120 & 1.001 & 0.918 & 0.908 & 0.928 & 0.935 & 0.938 & 0.939 & 0.951 & 0.940 & 0.915 & 0.909 & 0.903 & 0.902 & 0.903 & 0.913 \\
\hline 125 & 1.005 & 0.930 & 0.915 & 0.935 & 0.944 & 0.943 & 0.943 & 0.955 & 0.945 & 0.920 & 0.913 & 0.910 & 0.907 & 0.905 & 0.921 \\
\hline 130 & 1.008 & 0.940 & 0.927 & 0.941 & 0.948 & 0.947 & 0.947 & 0.965 & 0.947 & 0.921 & 0.918 & 0.923 & 0.915 & 0.906 & 0.926 \\
\hline 135 & 1.015 & 0.948 & 0.933 & 0.945 & 0.950 & 0.949 & 0.950 & 0.973 & 0.950 & 0.924 & 0.931 & 0.931 & 0.929 & 0.911 & 0.936 \\
\hline 140 & 1.019 & 0.953 & 0.933 & 0.951 & 0.946 & 0.948 & 0.940 & 0.963 & 0.956 & 0.930 & 0.936 & 0.934 & 0.937 & 0.916 & 0.942 \\
\hline 145 & 1.023 & 0.957 & 0.936 & 0.956 & 0.950 & 0.951 & 0.942 & 0.966 & 0.959 & 0.932 & 0.939 & 0.941 & 0.940 & 0.921 & 0.945 \\
\hline 150 & 1.024 & 0.952 & 0.943 & 0.961 & 0.955 & 0.955 & 0.946 & 0.972 & 0.964 & 0.937 & 0.933 & 0.947 & 0.934 & 0.916 & 0.939 \\
\hline 155 & 1.027 & 0.957 & 0.949 & 0.965 & 0.960 & 0.958 & 0.951 & 0.977 & 0.971 & 0.944 & 0.936 & 0.952 & 0.936 & 0.921 & 0.942 \\
\hline 160 & 1.034 & 0.960 & 0.953 & 0.971 & 0.965 & 0.963 & 0.955 & 0.981 & 0.977 & 0.949 & 0.939 & 0.958 & 0.940 & 0.925 & 0.947 \\
\hline 165 & 1.036 & 0.965 & 0.958 & 0.976 & 0.969 & 0.968 & 0.958 & 0.984 & 0.981 & 0.952 & 0.943 & 0.960 & 0.945 & 0.928 & 0.952 \\
\hline 170 & 1.041 & 0.970 & 0.963 & 0.981 & 0.974 & 0.972 & 0.960 & 0.987 & 0.984 & 0.956 & 0.948 & 0.964 & 0.949 & 0.932 & 0.956 \\
\hline 175 & 1.045 & 0.974 & 0.967 & 0.985 & 0.978 & 0.977 & 0.963 & 0.991 & 0.986 & 0.957 & 0.952 & 0.966 & 0.953 & 0.933 & 0.960 \\
\hline 180 & 1.047 & 0.979 & 0.972 & 0.991 & 0.983 & 0.982 & 0.968 & 0.994 & 0.987 & 0.961 & 0.957 & 0.971 & 0.957 & 0.935 & 0.964 \\
\hline 185 & 1.050 & 0.982 & 0.977 & 0.994 & 0.987 & 0.986 & 0.972 & 0.997 & 0.990 & 0.963 & 0.959 & 0.976 & 0.960 & 0.938 & 0.967 \\
\hline 190 & 1.054 & 0.986 & 0.980 & 0.998 & 0.990 & 0.989 & 0.976 & 1.001 & 0.994 & 0.966 & 0.963 & 0.980 & 0.964 & 0.942 & 0.971 \\
\hline 195 & 1.057 & 0.990 & 0.986 & 1.003 & 0.995 & 0.994 & 0.979 & 1.006 & 0.999 & 0.971 & 0.967 & 0.985 & 0.968 & 0.946 & 0.975 \\
\hline 200 & 1.066 & 0.995 & 0.991 & 1.009 & 1.000 & 0.999 & 0.982 & 1.008 & 1.006 & 0.979 & 0.972 & 0.990 & 0.971 & 0.949 & 0.979 \\
\hline 205 & 1.069 & 0.998 & 0.996 & 1.012 & 1.006 & 1.003 & 0.985 & 1.010 & 1.010 & 0.982 & 0.974 & 0.992 & 0.975 & 0.952 & 0.982 \\
\hline 210 & 1.072 & 1.003 & 1.000 & 1.017 & 1.009 & 1.007 & 0.989 & 1.015 & 1.014 & 0.985 & 0.979 & 0.995 & 0.979 & 0.955 & 0.986 \\
\hline 215 & 1.076 & 1.007 & 1.002 & 1.020 & 1.012 & 1.011 & 0.992 & 1.017 & 1.016 & 0.989 & 0.982 & 0.998 & 0.983 & 0.959 & 0.990 \\
\hline 220 & 1.079 & 1.010 & 1.006 & 1.022 & 1.016 & 1.013 & 0.996 & 1.020 & 1.019 & 0.991 & 0.985 & 1.001 & 0.986 & 0.962 & 0.995 \\
\hline 225 & 1.081 & 1.014 & 1.010 & 1.027 & 1.019 & 1.017 & 0.999 & 1.024 & 1.021 & 0.992 & 0.989 & 1.004 & 0.990 & 0.966 & 0.998 \\
\hline 230 & 1.086 & 1.017 & 1.013 & 1.031 & 1.021 & 1.020 & 1.003 & 1.028 & 1.027 & 0.996 & 0.993 & 1.006 & 0.994 & 0.969 & 1.001 \\
\hline 235 & 1.090 & 1.020 & 1.018 & 1.035 & 1.026 & 1.025 & 1.005 & 1.030 & 1.031 & 1.000 & 0.996 & 1.007 & 0.997 & 0.972 & 1.005 \\
\hline 240 & 1.094 & 1.023 & 1.023 & 1.040 & 1.030 & 1.029 & 1.008 & 1.033 & 1.035 & 1.003 & 0.999 & 1.011 & 1.000 & 0.976 & 1.008 \\
\hline 245 & 1.097 & 1.027 & 1.028 & 1.045 & 1.034 & 1.034 & 1.010 & 1.036 & 1.036 & 1.005 & 1.003 & 1.015 & 1.003 & 0.980 & 1.012 \\
\hline 250 & 1.100 & 1.030 & 1.031 & 1.048 & 1.038 & 1.038 & 1.013 & 1.038 & 1.039 & 1.007 & 1.006 & 1.018 & 1.005 & 0.982 & 1.015 \\
\hline 255 & 1.104 & 1.034 & 1.035 & 1.053 & 1.042 & 1.042 & 1.016 & 1.040 & 1.042 & 1.010 & 1.010 & 1.021 & 1.009 & 0.987 & 1.020 \\
\hline 260 & 1.108 & 1.039 & 1.037 & 1.054 & 1.044 & 1.044 & 1.021 & 1.044 & 1.047 & 1.014 & 1.013 & 1.023 & 1.012 & 0.989 & 1.023 \\
\hline 265 & 1.109 & 1.043 & 1.042 & 1.057 & 1.048 & 1.047 & 1.025 & 1.049 & 1.049 & 1.016 & 1.017 & 1.028 & 1.016 & 0.993 & 1.027 \\
\hline 270 & 1.113 & 1.045 & 1.044 & 1.059 & 1.051 & 1.050 & 1.030 & 1.053 & 1.052 & 1.018 & 1.020 & 1.030 & 1.020 & 0.998 & 1.031 \\
\hline 275 & 1.114 & 1.048 & 1.046 & 1.063 & 1.055 & 1.052 & 1.033 & 1.056 & 1.053 & 1.020 & 1.023 & 1.034 & 1.023 & 1.001 & 1.033 \\
\hline 280 & 1.117 & 1.050 & 1.049 & 1.065 & 1.057 & 1.054 & 1.034 & 1.058 & 1.055 & 1.023 & 1.024 & 1.037 & 1.025 & 1.003 & 1.034 \\
\hline 285 & 1.120 & 1.054 & 1.052 & 1.068 & 1.060 & 1.058 & 1.038 & 1.062 & 1.058 & 1.026 & 1.029 & 1.039 & 1.028 & 1.007 & 1.038 \\
\hline 290 & 1.122 & 1.056 & 1.055 & 1.072 & 1.064 & 1.061 & 1.040 & 1.064 & 1.059 & 1.029 & 1.031 & 1.043 & 1.029 & 1.007 & 1.040 \\
\hline
\end{tabular}


Appendix 1. Heat capacity data (in $\mathrm{J} \mathrm{g}^{-1} \mathrm{~K}^{-1}$ ) for all of the samples with step of temperature of $5^{\circ} \mathrm{C}$ for matched cooling/heating rate of $20^{\circ} \mathrm{C}$ min ${ }^{-1}$. Continuation...

\begin{tabular}{|c|c|c|c|c|c|c|c|c|c|c|c|c|c|c|c|}
\hline \multirow{2}{*}{$\mathrm{T}\left({ }^{\circ} \mathrm{C}\right)$} & G0 & G1 & G2 & G3 & G4 & G5 & G6 & G7 & G8 & G9 & G10 & G11 & G12 & G13 & G14 \\
\hline & \multicolumn{15}{|c|}{ Heat capacity $\mathrm{c}_{\mathrm{p}}\left(\mathrm{J} \mathrm{g}^{-1} \mathrm{~K}^{-1}\right)$} \\
\hline 295 & 1.125 & 1.059 & 1.059 & 1.076 & 1.069 & 1.065 & 1.044 & 1.066 & 1.063 & 1.031 & 1.033 & 1.045 & 1.030 & 1.010 & 1.041 \\
\hline 300 & 1.129 & 1.062 & 1.061 & 1.078 & 1.071 & 1.068 & 1.043 & 1.066 & 1.066 & 1.034 & 1.035 & 1.047 & 1.033 & 1.012 & 1.045 \\
\hline 305 & 1.132 & 1.066 & 1.063 & 1.080 & 1.072 & 1.070 & 1.045 & 1.070 & 1.068 & 1.035 & 1.039 & 1.050 & 1.036 & 1.016 & 1.047 \\
\hline 310 & 1.137 & 1.070 & 1.068 & 1.083 & 1.075 & 1.074 & 1.048 & 1.072 & 1.072 & 1.039 & 1.043 & 1.051 & 1.040 & 1.019 & 1.050 \\
\hline 315 & 1.140 & 1.073 & 1.069 & 1.085 & 1.077 & 1.076 & 1.051 & 1.074 & 1.076 & 1.042 & 1.044 & 1.053 & 1.043 & 1.021 & 1.052 \\
\hline 320 & 1.142 & 1.076 & 1.073 & 1.088 & 1.079 & 1.078 & 1.053 & 1.074 & 1.077 & 1.042 & 1.047 & 1.057 & 1.046 & 1.024 & 1.054 \\
\hline 325 & 1.144 & 1.078 & 1.075 & 1.090 & 1.081 & 1.081 & 1.055 & 1.077 & 1.079 & 1.044 & 1.049 & 1.060 & 1.048 & 1.024 & 1.056 \\
\hline 330 & 1.149 & 1.081 & 1.079 & 1.093 & 1.084 & 1.084 & 1.060 & 1.081 & 1.083 & 1.047 & 1.051 & 1.063 & 1.050 & 1.027 & 1.058 \\
\hline 335 & 1.152 & 1.084 & 1.081 & 1.096 & 1.086 & 1.087 & 1.063 & 1.084 & 1.086 & 1.050 & 1.053 & 1.064 & 1.055 & 1.030 & 1.060 \\
\hline 340 & 1.157 & 1.085 & 1.084 & 1.098 & 1.089 & 1.090 & 1.064 & 1.087 & 1.091 & 1.055 & 1.055 & 1.066 & 1.055 & 1.030 & 1.063 \\
\hline 345 & 1.161 & 1.087 & 1.084 & 1.099 & 1.089 & 1.091 & 1.066 & 1.089 & 1.093 & 1.059 & 1.058 & 1.070 & 1.057 & 1.032 & 1.065 \\
\hline 350 & 1.163 & 1.092 & 1.087 & 1.102 & 1.093 & 1.095 & 1.066 & 1.091 & 1.095 & 1.060 & 1.060 & 1.071 & 1.060 & 1.034 & 1.067 \\
\hline 355 & 1.167 & 1.095 & 1.089 & 1.106 & 1.094 & 1.098 & 1.068 & 1.093 & 1.098 & 1.062 & 1.061 & 1.073 & 1.062 & 1.036 & 1.069 \\
\hline 360 & 1.167 & 1.096 & 1.091 & 1.107 & 1.097 & 1.100 & 1.072 & 1.097 & 1.099 & 1.063 & 1.064 & 1.076 & 1.064 & 1.038 & 1.072 \\
\hline 365 & 1.171 & 1.098 & 1.092 & 1.109 & 1.099 & 1.101 & 1.076 & 1.100 & 1.101 & 1.064 & 1.065 & 1.077 & 1.065 & 1.039 & 1.073 \\
\hline 370 & 1.172 & 1.099 & 1.092 & 1.110 & 1.101 & 1.104 & 1.079 & 1.103 & 1.102 & 1.065 & 1.068 & 1.078 & 1.067 & 1.041 & 1.076 \\
\hline 375 & 1.174 & 1.102 & 1.096 & 1.113 & 1.104 & 1.107 & 1.081 & 1.106 & 1.105 & 1.067 & 1.071 & 1.078 & 1.071 & 1.044 & 1.079 \\
\hline 380 & 1.178 & 1.104 & 1.098 & 1.114 & 1.106 & 1.109 & 1.082 & 1.107 & 1.108 & 1.070 & 1.073 & 1.081 & 1.072 & 1.045 & 1.081 \\
\hline 385 & 1.179 & 1.104 & 1.100 & 1.115 & 1.107 & 1.110 & 1.081 & 1.109 & 1.109 & 1.071 & 1.075 & 1.082 & 1.073 & 1.046 & 1.083 \\
\hline 390 & 1.180 & 1.107 & 1.100 & 1.116 & 1.109 & 1.112 & 1.083 & 1.110 & 1.110 & 1.070 & 1.077 & 1.082 & 1.078 & 1.049 & 1.086 \\
\hline 395 & 1.183 & 1.110 & 1.102 & 1.118 & 1.110 & 1.112 & 1.085 & 1.112 & 1.112 & 1.072 & 1.080 & 1.085 & 1.080 & 1.050 & 1.087 \\
\hline 400 & 1.186 & 1.111 & 1.106 & 1.122 & 1.112 & 1.114 & 1.089 & 1.114 & 1.116 & 1.076 & 1.081 & 1.086 & 1.081 & 1.052 & 1.090 \\
\hline 405 & 1.189 & 1.114 & 1.109 & 1.122 & 1.113 & 1.116 & 1.091 & 1.117 & 1.118 & 1.079 & 1.083 & 1.087 & 1.081 & 1.055 & 1.092 \\
\hline 410 & 1.192 & 1.116 & 1.111 & 1.126 & 1.116 & 1.118 & 1.092 & 1.117 & 1.120 & 1.080 & 1.087 & 1.089 & 1.083 & 1.057 & 1.094 \\
\hline 415 & 1.196 & 1.119 & 1.114 & 1.127 & 1.118 & 1.119 & 1.094 & 1.119 & 1.121 & 1.081 & 1.088 & 1.090 & 1.085 & 1.060 & 1.096 \\
\hline 420 & 1.198 & 1.120 & 1.116 & 1.130 & 1.120 & 1.122 & 1.097 & 1.123 & 1.123 & 1.083 & 1.091 & 1.092 & 1.088 & 1.062 & 1.099 \\
\hline 425 & 1.202 & 1.121 & 1.118 & 1.134 & 1.123 & 1.124 & 1.098 & 1.124 & 1.124 & 1.084 & 1.090 & 1.094 & 1.089 & 1.063 & 1.099 \\
\hline 430 & 1.207 & 1.122 & 1.120 & 1.135 & 1.124 & 1.126 & 1.099 & 1.126 & 1.125 & 1.087 & 1.091 & 1.096 & 1.089 & 1.063 & 1.101 \\
\hline 435 & 1.210 & 1.124 & 1.120 & 1.137 & 1.125 & 1.126 & 1.099 & 1.126 & 1.125 & 1.089 & 1.093 & 1.097 & 1.091 & 1.065 & 1.102 \\
\hline 440 & 1.214 & 1.128 & 1.122 & 1.138 & 1.125 & 1.127 & 1.101 & 1.128 & 1.126 & 1.090 & 1.096 & 1.100 & 1.093 & 1.067 & 1.106 \\
\hline 445 & 1.222 & 1.128 & 1.124 & 1.140 & 1.126 & 1.130 & 1.102 & 1.130 & 1.129 & 1.091 & 1.096 & 1.101 & 1.094 & 1.068 & 1.105 \\
\hline 450 & 1.233 & 1.129 & 1.127 & 1.142 & 1.128 & 1.132 & 1.104 & 1.132 & 1.132 & 1.094 & 1.098 & 1.103 & 1.095 & 1.070 & 1.107 \\
\hline 455 & 1.247 & 1.130 & 1.130 & 1.146 & 1.131 & 1.135 & 1.106 & 1.135 & 1.134 & 1.095 & 1.101 & 1.103 & 1.096 & 1.072 & 1.109 \\
\hline 460 & 1.267 & 1.131 & 1.131 & 1.146 & 1.134 & 1.138 & 1.108 & 1.136 & 1.134 & 1.097 & 1.103 & 1.104 & 1.098 & 1.073 & 1.111 \\
\hline 465 & 1.294 & 1.131 & 1.131 & 1.148 & 1.136 & 1.138 & 1.111 & 1.139 & 1.135 & 1.099 & 1.102 & 1.106 & 1.099 & 1.074 & 1.111 \\
\hline 470 & 1.333 & 1.134 & 1.130 & 1.145 & 1.134 & 1.137 & 1.111 & 1.140 & 1.138 & 1.102 & 1.105 & 1.107 & 1.103 & 1.076 & 1.115 \\
\hline 475 & 1.381 & 1.134 & 1.133 & 1.148 & 1.136 & 1.139 & 1.112 & 1.141 & 1.137 & 1.103 & 1.106 & 1.109 & 1.103 & 1.077 & 1.115 \\
\hline 480 & 1.445 & 1.137 & 1.136 & 1.150 & 1.139 & 1.142 & 1.114 & 1.144 & 1.140 & 1.104 & 1.107 & 1.108 & 1.104 & 1.078 & 1.116 \\
\hline 485 & 1.507 & 1.140 & 1.137 & 1.151 & 1.140 & 1.144 & 1.116 & 1.145 & 1.142 & 1.106 & 1.111 & 1.110 & 1.107 & 1.081 & 1.120 \\
\hline
\end{tabular}


Appendix 1. Heat capacity data (in $\mathrm{J} \mathrm{g}^{-1} \mathrm{~K}^{-1}$ ) for all of the samples with step of temperature of $5^{\circ} \mathrm{C}$ for matched cooling/heating rate of $20^{\circ} \mathrm{C} \mathrm{min}^{-1}$. Continuation...

\begin{tabular}{|c|c|c|c|c|c|c|c|c|c|c|c|c|c|c|c|}
\hline \multirow[b]{2}{*}{$\mathrm{T}\left({ }^{\circ} \mathrm{C}\right)$} & G0 & G1 & G2 & G3 & G4 & G5 & G6 & G7 & G8 & G9 & G10 & G11 & G12 & G13 & G14 \\
\hline & \multicolumn{15}{|c|}{ Heat capacity $c_{p}\left(\mathrm{Jg}^{-1} K^{-1}\right)$} \\
\hline 490 & 1.555 & 1.143 & 1.140 & 1.153 & 1.142 & 1.147 & 1.116 & 1.146 & 1.144 & 1.107 & 1.114 & 1.112 & 1.109 & 1.084 & 1.122 \\
\hline 495 & 1.576 & 1.143 & 1.140 & 1.155 & 1.143 & 1.147 & 1.117 & 1.147 & 1.149 & 1.114 & 1.115 & 1.114 & 1.110 & 1.086 & 1.123 \\
\hline 500 & 1.559 & 1.147 & 1.140 & 1.155 & 1.142 & 1.146 & 1.120 & 1.150 & 1.150 & 1.115 & 1.117 & 1.115 & 1.111 & 1.086 & 1.127 \\
\hline 505 & 1.531 & 1.146 & 1.142 & 1.156 & 1.144 & 1.148 & 1.119 & 1.150 & 1.151 & 1.115 & 1.118 & 1.115 & 1.110 & 1.086 & 1.127 \\
\hline 510 & 1.510 & 1.148 & 1.146 & 1.160 & 1.147 & 1.152 & 1.119 & 1.149 & 1.151 & 1.116 & 1.120 & 1.116 & 1.113 & 1.088 & 1.131 \\
\hline 515 & 1.499 & 1.149 & 1.149 & 1.162 & 1.150 & 1.154 & 1.121 & 1.153 & 1.154 & 1.118 & 1.121 & 1.118 & 1.112 & 1.089 & 1.132 \\
\hline 520 & 1.490 & 1.150 & 1.149 & 1.163 & 1.152 & 1.155 & 1.123 & 1.155 & 1.153 & 1.118 & 1.119 & 1.119 & 1.113 & 1.090 & 1.134 \\
\hline 525 & & 1.151 & 1.150 & 1.165 & 1.154 & 1.154 & 1.124 & 1.155 & 1.155 & 1.117 & 1.123 & 1.121 & 1.115 & 1.091 & 1.135 \\
\hline 530 & & 1.152 & 1.150 & 1.164 & 1.153 & 1.154 & 1.125 & 1.156 & 1.155 & 1.115 & 1.123 & 1.123 & 1.116 & 1.092 & 1.138 \\
\hline 535 & & 1.153 & 1.153 & 1.168 & 1.156 & 1.158 & 1.127 & 1.157 & 1.158 & 1.115 & 1.125 & 1.122 & 1.117 & 1.093 & 1.142 \\
\hline 540 & & 1.155 & 1.155 & 1.171 & 1.157 & 1.158 & 1.130 & 1.161 & 1.159 & 1.113 & 1.127 & 1.124 & 1.119 & 1.095 & 1.146 \\
\hline 545 & & 1.156 & 1.156 & 1.173 & 1.159 & 1.160 & 1.131 & 1.164 & 1.161 & 1.113 & 1.128 & 1.124 & 1.121 & 1.097 & 1.151 \\
\hline 550 & & 1.157 & 1.155 & 1.174 & 1.160 & 1.159 & 1.128 & 1.164 & 1.164 & 1.115 & 1.130 & 1.126 & 1.123 & 1.099 & 1.156 \\
\hline 555 & & 1.157 & 1.160 & 1.176 & 1.163 & 1.163 & 1.130 & 1.166 & 1.163 & 1.114 & 1.131 & 1.124 & 1.123 & 1.100 & 1.163 \\
\hline 560 & & 1.160 & 1.163 & 1.179 & 1.163 & 1.165 & 1.132 & 1.166 & 1.165 & 1.114 & 1.131 & 1.124 & 1.125 & 1.102 & 1.174 \\
\hline 565 & & 1.163 & 1.159 & 1.177 & 1.159 & 1.159 & 1.133 & 1.168 & 1.166 & 1.115 & 1.133 & 1.127 & 1.127 & 1.107 & 1.188 \\
\hline 570 & & 1.164 & 1.160 & 1.176 & 1.159 & 1.160 & 1.134 & 1.171 & 1.168 & 1.116 & 1.131 & 1.128 & 1.127 & 1.109 & 1.203 \\
\hline 575 & & 1.165 & 1.159 & 1.176 & 1.161 & 1.163 & 1.133 & 1.171 & 1.168 & 1.116 & 1.130 & 1.128 & 1.128 & 1.111 & 1.223 \\
\hline 580 & & 1.166 & 1.161 & 1.179 & 1.163 & 1.165 & 1.134 & 1.177 & 1.172 & 1.119 & 1.130 & 1.127 & 1.132 & 1.113 & 1.248 \\
\hline 585 & & 1.168 & 1.164 & 1.182 & 1.165 & 1.167 & 1.134 & 1.183 & 1.175 & 1.120 & 1.130 & 1.127 & 1.133 & 1.119 & 1.279 \\
\hline 590 & & 1.167 & 1.169 & 1.184 & 1.169 & 1.169 & 1.137 & 1.191 & 1.177 & 1.117 & 1.128 & 1.130 & 1.133 & 1.125 & 1.314 \\
\hline 595 & & 1.168 & 1.173 & 1.185 & 1.169 & 1.172 & 1.142 & 1.199 & 1.177 & 1.120 & 1.127 & 1.132 & 1.137 & 1.132 & 1.353 \\
\hline 600 & & 1.168 & 1.173 & 1.185 & 1.168 & 1.172 & 1.141 & 1.210 & 1.179 & 1.123 & 1.129 & 1.131 & 1.138 & 1.141 & 1.385 \\
\hline 605 & & 1.169 & 1.172 & 1.183 & 1.169 & 1.171 & 1.142 & 1.221 & 1.180 & 1.122 & 1.129 & 1.133 & 1.141 & 1.152 & 1.407 \\
\hline 610 & & 1.171 & 1.170 & 1.182 & 1.167 & 1.170 & 1.142 & 1.234 & 1.180 & 1.122 & 1.129 & 1.132 & 1.146 & 1.169 & 1.416 \\
\hline 615 & & 1.172 & 1.170 & 1.182 & 1.169 & 1.170 & 1.144 & 1.253 & 1.181 & 1.123 & 1.129 & 1.133 & 1.153 & 1.188 & 1.405 \\
\hline 620 & & 1.173 & 1.172 & 1.183 & 1.171 & 1.173 & 1.147 & 1.276 & 1.181 & 1.123 & 1.129 & 1.131 & 1.160 & 1.211 & 1.388 \\
\hline 625 & & 1.172 & 1.172 & 1.184 & 1.172 & 1.177 & 1.149 & 1.307 & 1.183 & 1.123 & 1.129 & 1.135 & 1.168 & 1.234 & 1.373 \\
\hline 630 & & 1.173 & 1.173 & 1.184 & 1.171 & 1.177 & 1.152 & 1.333 & 1.183 & 1.124 & 1.127 & 1.137 & 1.177 & 1.261 & 1.360 \\
\hline 635 & & 1.175 & 1.172 & 1.185 & 1.171 & 1.176 & 1.158 & 1.371 & 1.184 & 1.122 & 1.127 & 1.140 & 1.189 & 1.287 & 1.352 \\
\hline 640 & & 1.178 & 1.172 & 1.183 & 1.171 & 1.178 & 1.162 & 1.402 & 1.183 & 1.124 & 1.130 & 1.144 & 1.204 & 1.309 & 1.347 \\
\hline 645 & & 1.177 & 1.175 & 1.189 & 1.176 & 1.182 & 1.166 & 1.421 & 1.185 & 1.125 & 1.129 & 1.143 & 1.220 & 1.324 & 1.343 \\
\hline 650 & & 1.178 & 1.174 & 1.184 & 1.173 & 1.181 & 1.173 & 1.425 & 1.189 & 1.127 & 1.131 & 1.149 & 1.239 & 1.329 & 1.341 \\
\hline 655 & & 1.181 & 1.172 & 1.186 & 1.172 & 1.184 & 1.181 & 1.414 & 1.189 & 1.128 & 1.132 & 1.151 & 1.260 & 1.327 & 1.341 \\
\hline 660 & & 1.179 & 1.173 & 1.188 & 1.175 & 1.190 & 1.195 & 1.397 & 1.187 & 1.125 & 1.131 & 1.155 & 1.280 & 1.317 & \\
\hline 665 & & 1.179 & 1.176 & 1.191 & 1.176 & 1.194 & 1.210 & 1.382 & 1.192 & 1.129 & 1.130 & 1.157 & 1.297 & 1.306 & \\
\hline 670 & & 1.179 & 1.176 & 1.192 & 1.176 & 1.197 & 1.225 & 1.370 & 1.191 & 1.127 & 1.131 & 1.167 & 1.311 & 1.296 & \\
\hline 675 & & 1.182 & 1.177 & 1.194 & 1.177 & 1.200 & 1.248 & 1.366 & 1.192 & 1.128 & 1.135 & 1.175 & 1.325 & 1.291 & \\
\hline 680 & & 1.182 & 1.177 & 1.193 & 1.178 & 1.211 & 1.274 & 1.363 & 1.193 & 1.129 & 1.134 & 1.184 & 1.328 & 1.284 & \\
\hline
\end{tabular}


Appendix 1. Heat capacity data (in $\mathrm{J} \mathrm{g}^{-1} \mathrm{~K}^{-1}$ ) for all of the samples with step of temperature of $5^{\circ} \mathrm{C}$ for matched cooling/heating rate of $20^{\circ} \mathrm{C}$ min ${ }^{-1}$. Continuation...

\begin{tabular}{|c|c|c|c|c|c|c|c|c|c|c|c|c|c|c|c|}
\hline \multirow{2}{*}{$\mathrm{T}\left({ }^{\circ} \mathrm{C}\right)$} & G0 & G1 & G2 & G3 & G4 & G5 & G6 & G7 & G8 & G9 & G10 & G11 & G12 & G13 & G14 \\
\hline & \multicolumn{15}{|c|}{ Heat capacity $c_{p}\left(\mathrm{~J} \mathrm{~g}^{-1} \mathrm{~K}^{-1}\right)$} \\
\hline 685 & & 1.185 & 1.179 & 1.194 & $\begin{array}{l}1.181 \\
\end{array}$ & 1.223 & 1.299 & $\begin{array}{l}1.359 \\
\end{array}$ & $\begin{array}{l}1.193 \\
\end{array}$ & 1.130 & 1.138 & 1.198 & 1.334 & $\begin{array}{l}1.283 \\
\end{array}$ & \\
\hline 690 & & 1.187 & 1.184 & 1.198 & 1.184 & 1.236 & 1.326 & 1.352 & 1.195 & 1.131 & 1.140 & 1.208 & 1.334 & & \\
\hline 695 & & 1.188 & 1.185 & 1.200 & 1.184 & 1.255 & 1.349 & 1.353 & 1.199 & 1.133 & 1.141 & 1.223 & 1.331 & & \\
\hline 700 & & 1.187 & 1.182 & 1.198 & 1.182 & 1.269 & 1.365 & & 1.203 & 1.134 & 1.142 & 1.238 & 1.326 & & \\
\hline 705 & & 1.188 & 1.185 & 1.192 & 1.182 & 1.291 & 1.368 & & 1.206 & 1.136 & 1.146 & 1.253 & 1.323 & & \\
\hline 710 & & 1.190 & 1.185 & 1.197 & 1.184 & 1.317 & 1.362 & & 1.204 & 1.134 & 1.149 & 1.268 & 1.318 & & \\
\hline 715 & & 1.192 & 1.185 & 1.200 & 1.182 & 1.341 & 1.350 & & 1.205 & 1.134 & 1.153 & 1.281 & 1.314 & & \\
\hline 720 & & 1.193 & 1.187 & 1.200 & 1.186 & 1.366 & 1.336 & & 1.210 & 1.136 & 1.156 & 1.294 & 1.309 & & \\
\hline 725 & & 1.189 & 1.187 & 1.201 & 1.184 & 1.383 & 1.326 & & 1.209 & 1.136 & 1.164 & 1.306 & 1.308 & & \\
\hline 730 & & 1.189 & 1.187 & 1.203 & 1.185 & 1.395 & 1.319 & & 1.215 & 1.139 & 1.166 & 1.312 & 1.300 & & \\
\hline 735 & & 1.192 & 1.185 & 1.199 & 1.185 & 1.393 & 1.315 & & 1.215 & 1.138 & 1.177 & 1.318 & 1.303 & & \\
\hline 740 & & 1.191 & 1.189 & 1.203 & 1.190 & 1.391 & 1.315 & & 1.223 & 1.139 & 1.187 & 1.320 & 1.298 & & \\
\hline 745 & & 1.193 & 1.192 & 1.202 & 1.191 & 1.377 & 1.310 & & 1.230 & 1.142 & 1.199 & 1.318 & 1.297 & & \\
\hline 750 & & 1.195 & 1.193 & 1.203 & 1.192 & 1.366 & 1.312 & & 1.237 & 1.147 & 1.216 & 1.315 & & & \\
\hline 755 & & 1.191 & 1.189 & 1.202 & 1.192 & 1.355 & & & 1.245 & 1.148 & 1.229 & 1.308 & & & \\
\hline 760 & & 1.191 & 1.191 & 1.204 & 1.199 & 1.351 & & & 1.257 & 1.154 & 1.248 & 1.304 & & & \\
\hline 765 & & 1.195 & 1.191 & 1.206 & 1.205 & 1.344 & & & 1.269 & 1.158 & 1.267 & 1.300 & & & \\
\hline 770 & & 1.200 & 1.193 & 1.207 & 1.211 & 1.344 & & & 1.289 & 1.167 & 1.285 & 1.299 & & & \\
\hline 775 & & 1.200 & 1.197 & 1.210 & 1.222 & 1.346 & & & 1.305 & 1.168 & 1.298 & 1.296 & & & \\
\hline 780 & & 1.204 & 1.197 & 1.211 & 1.233 & 1.344 & & & 1.327 & 1.181 & 1.312 & 1.297 & & & \\
\hline 785 & & 1.207 & 1.199 & 1.214 & 1.242 & 1.334 & & & 1.353 & 1.194 & 1.318 & 1.296 & & & \\
\hline 790 & & 1.205 & 1.200 & 1.218 & 1.257 & 1.341 & & & 1.379 & 1.207 & 1.317 & & & & \\
\hline 795 & & 1.209 & 1.202 & 1.225 & 1.272 & & & & 1.401 & 1.218 & 1.317 & & & & \\
\hline 800 & & 1.211 & 1.205 & 1.218 & 1.288 & & & & 1.421 & 1.238 & 1.311 & & & & \\
\hline 805 & & 1.219 & 1.209 & 1.219 & 1.306 & & & & 1.433 & 1.253 & 1.303 & & & & \\
\hline 810 & & 1.227 & 1.217 & 1.228 & 1.331 & & & & 1.436 & 1.268 & 1.300 & & & & \\
\hline 815 & & 1.227 & 1.224 & 1.236 & 1.355 & & & & 1.432 & 1.286 & 1.291 & & & & \\
\hline 820 & & 1.239 & 1.225 & 1.236 & 1.372 & & & & 1.422 & 1.301 & 1.286 & & & & \\
\hline 825 & & 1.250 & 1.236 & 1.245 & 1.386 & & & & 1.412 & 1.316 & 1.287 & & & & \\
\hline 830 & & 1.262 & 1.248 & 1.257 & 1.403 & & & & 1.403 & 1.319 & 1.283 & & & & \\
\hline 835 & & 1.279 & 1.261 & 1.267 & 1.408 & & & & & 1.319 & & & & & \\
\hline 840 & & 1.298 & 1.274 & 1.284 & 1.406 & & & & & 1.314 & & & & & \\
\hline 845 & & 1.322 & 1.292 & 1.303 & 1.400 & & & & & 1.309 & & & & & \\
\hline 850 & & 1.347 & 1.311 & 1.317 & 1.387 & & & & & 1.301 & & & & & \\
\hline 855 & & 1.375 & 1.341 & 1.339 & 1.382 & & & & & 1.295 & & & & & \\
\hline 860 & & 1.402 & 1.362 & 1.363 & 1.376 & & & & & 1.290 & & & & & \\
\hline 865 & & 1.425 & 1.388 & 1.386 & 1.374 & & & & & & & & & & \\
\hline 870 & & 1.450 & 1.408 & 1.404 & 1.366 & & & & & & & & & & \\
\hline 875 & & 1.459 & 1.425 & 1.424 & 1.367 & & & & & & & & & & \\
\hline
\end{tabular}


Appendix 1. Heat capacity data (in $\mathrm{J} \mathrm{g}^{-1} \mathrm{~K}^{-1}$ ) for all of the samples with step of temperature of $5^{\circ} \mathrm{C}$ for matched cooling/heating rate of $20^{\circ} \mathrm{C}$ min ${ }^{-1}$. Continuation...

\begin{tabular}{|c|c|c|c|c|c|c|c|c|c|c|c|c|c|c|c|}
\hline \multirow[b]{2}{*}{$\mathrm{T}\left({ }^{\circ} \mathrm{C}\right)$} & G0 & G1 & G2 & G3 & G4 & G5 & G6 & G7 & G8 & G9 & G10 & G11 & G12 & G13 & G14 \\
\hline & \multicolumn{15}{|c|}{ Heat capacity $c_{p}\left(\mathrm{Jg}^{-1} \mathrm{~K}^{-1}\right)$} \\
\hline $\begin{array}{l}880 \\
885 \\
890\end{array}$ & & $\begin{array}{l}1.451 \\
1.434 \\
1.417\end{array}$ & $\begin{array}{l}1.432 \\
1.424 \\
1.404\end{array}$ & $\begin{array}{l}1.431 \\
1.427 \\
1.410\end{array}$ & 1.366 & & & & & & & & & & \\
\hline 895 & & 1.404 & 1.395 & 1.400 & & & & & & & & & & & \\
\hline 900 & & 1.396 & 1.379 & 1.383 & & & & & & & & & & & \\
\hline 905 & & 1.392 & 1.373 & 1.377 & & & & & & & & & & & \\
\hline 910 & & 1.387 & 1.363 & 1.369 & & & & & & & & & & & \\
\hline 915 & & 1.382 & 1.359 & 1.366 & & & & & & & & & & & \\
\hline 920 & & 1.382 & 1.356 & 1.366 & & & & & & & & & & & \\
\hline 925 & & 1.378 & 1.350 & & & & & & & & & & & & \\
\hline
\end{tabular}


A-6 
Appendix 2. Temperature $T, \log _{10} \eta$, frequency $f, \log _{10} \omega \tau_{\mathrm{M}}$, imaginary $\left(G_{\mathrm{im}}\right)$ and real ( $(\mathrm{G}$ real) shear modulus and shear modulus of the melt ( $G$ melt) for the investigated samples.

\begin{tabular}{|c|c|c|c|c|c|c|}
\hline \multicolumn{7}{|c|}{ SAMPLE G0 } \\
\hline $\mathrm{T}\left({ }^{\circ} \mathrm{C}\right)$ & $\begin{array}{c}\log _{10} \\
\eta \\
\text { (Pa s) }\end{array}$ & $f(\mathrm{~Hz})$ & $\begin{array}{c}\log _{10} \\
\omega \tau_{M}\end{array}$ & $\begin{array}{c}\mathbf{G}_{\mathrm{im}} \\
(\mathrm{GPa})\end{array}$ & $\begin{array}{l}\mathbf{G}_{\text {real }} \\
(\mathrm{GPa})\end{array}$ & $\begin{array}{l}\mathbf{G}_{\text {melt }} \\
(\mathrm{GPa})\end{array}$ \\
\hline 380 & \multirow{10}{*}{$\begin{array}{l}\text { ने } \\
\text { فे }\end{array}$} & 1 & $\begin{array}{ll}6.305 \\
\end{array}$ & 0.065 & 27.446 & 27.446 \\
\hline 380 & & 0.5 & 6.004 & 0.067 & 27.436 & 27.436 \\
\hline 380 & & 0.2 & 5.606 & 0.107 & 27.565 & 27.565 \\
\hline 380 & & 0.1 & 5.305 & 0.107 & 27.687 & 27.687 \\
\hline 380 & & 0.05 & 5.004 & 0.201 & 27.449 & 27.450 \\
\hline 380 & & 0.02 & 4.606 & 0.170 & 27.657 & 27.658 \\
\hline 380 & & 0.01 & 4.305 & 0.092 & 27.763 & 27.763 \\
\hline 380 & & 0.005 & 4.004 & 0.079 & 27.906 & 27.907 \\
\hline 380 & & 0.002 & 3.606 & 0.146 & 27.725 & 27.726 \\
\hline 380 & & 0.001 & 3.305 & 0.076 & 27.632 & 27.632 \\
\hline 400 & \multirow{10}{*}{$\begin{array}{l}\stackrel{m}{\sim} \\
\underset{\dot{d}}{+}\end{array}$} & 1 & \begin{tabular}{c|}
5.088 \\
\end{tabular} & $\begin{array}{l}0.137 \\
\end{array}$ & 27.570 & 27.571 \\
\hline 400 & & 0.5 & 4.787 & 0.141 & 27.721 & 27.722 \\
\hline 400 & & 0.2 & 4.389 & 0.121 & 27.691 & 27.691 \\
\hline 400 & & 0.1 & 4.088 & 0.226 & 27.764 & 27.765 \\
\hline 400 & & 0.05 & 3.787 & 0.155 & 28.019 & 28.019 \\
\hline 400 & & 0.02 & 3.389 & 0.010 & 27.756 & 27.756 \\
\hline 400 & & 0.01 & 3.088 & 0.215 & 27.628 & 27.629 \\
\hline 400 & & 0.005 & 2.787 & 0.056 & 27.514 & 27.514 \\
\hline 400 & & 0.002 & 2.389 & 0.113 & 28.334 & 28.335 \\
\hline 400 & & 0.001 & 2.088 & 0.119 & 27.852 & 27.853 \\
\hline "420 & \multirow{10}{*}{ 尊 } & 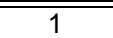 & $\begin{array}{l}3.942 \\
\end{array}$ & $\begin{array}{l}0.031 \\
\end{array}$ & 27.552 & 27.552 \\
\hline 420 & & 0.5 & 3.641 & 0.157 & 27.609 & 27.610 \\
\hline 420 & & 0.2 & 3.243 & 0.208 & 27.815 & 27.815 \\
\hline 420 & & 0.1 & 2.942 & 0.100 & 28.666 & 28.666 \\
\hline 420 & & 0.05 & 2.641 & 0.011 & 27.493 & 27.493 \\
\hline 420 & & 0.02 & 2.243 & 0.013 & 27.365 & 27.365 \\
\hline 420 & & 0.01 & 1.942 & 0.409 & 24.453 & 24.457 \\
\hline 420 & & 0.005 & 1.641 & 0.076 & 27.360 & 27.360 \\
\hline 420 & & 0.002 & 1.243 & 0.724 & 25.341 & 25.351 \\
\hline 420 & & 0.001 & 0.942 & 1.464 & 24.261 & 24.305 \\
\hline
\end{tabular}

\begin{tabular}{|c|c|c|c|c|c|c|}
\hline \multicolumn{7}{|c|}{ SAMPLE G0 } \\
\hline $\mathrm{T}\left({ }^{\circ} \mathrm{C}\right)$ & $\begin{array}{c}\log _{10} \\
\eta \\
\text { (Pa s) }\end{array}$ & $f(\mathrm{~Hz})$ & $\begin{array}{c}\log _{10} \\
\omega \tau_{M}\end{array}$ & $\begin{array}{c}\mathbf{G}_{\text {im }} \\
(\mathrm{GPa})\end{array}$ & $\begin{array}{l}\mathbf{G}_{\text {real }} \\
(\mathrm{GPa})\end{array}$ & $\begin{array}{l}\mathbf{G}_{\text {melt }} \\
(\mathrm{GPa})\end{array}$ \\
\hline 4440 & \multirow{10}{*}{$\stackrel{\circ}{\stackrel{\circ}{\sim}}$} & 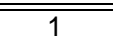 & 2.860 & 0.023 & 27.287 & 27.287 \\
\hline 440 & & 0.5 & 2.559 & 0.002 & 27.303 & 27.303 \\
\hline 440 & & 0.2 & 2.161 & 0.235 & 27.540 & 27.541 \\
\hline 440 & & 0.1 & 1.860 & 0.274 & 27.474 & 27.476 \\
\hline 440 & & 0.05 & 1.559 & 0.042 & 27.430 & 27.430 \\
\hline 440 & & 0.02 & 1.161 & 0.097 & 27.948 & 27.948 \\
\hline 440 & & 0.01 & 0.860 & 1.223 & 23.827 & 23.858 \\
\hline 440 & & 0.005 & 0.559 & 1.289 & 19.371 & 19.413 \\
\hline 440 & & 0.002 & 0.161 & 8.520 & 10.572 & 13.578 \\
\hline 440 & & 0.001 & -0.140 & 5.656 & 4.959 & 7.521 \\
\hline 2460 & \multirow{9}{*}{$\begin{array}{l}\stackrel{\infty}{+} \\
\underset{-}{-}\end{array}$} & 1 & 1.838 & 0.207 & 27.279 & 27.280 \\
\hline 460 & & 0.5 & 1.537 & 0.151 & 27.547 & 27.548 \\
\hline 460 & & 0.2 & 1.139 & 0.227 & 24.857 & 24.858 \\
\hline 460 & & 0.1 & 0.838 & 1.253 & 23.324 & 23.358 \\
\hline 460 & & 0.05 & 0.537 & 1.554 & 20.310 & 20.370 \\
\hline 460 & & 0.02 & 0.139 & 7.773 & 7.074 & 10.510 \\
\hline 460 & & 0.01 & -0.162 & 0.365 & 4.210 & 4.225 \\
\hline 460 & & 0.005 & -0.463 & 0.014 & 1.532 & 1.532 \\
\hline 460 & & 0.002 & -0.861 & 0.003 & $\begin{array}{l}1.032 \\
0.382\end{array}$ & $\begin{array}{l}1.502 \\
0.382\end{array}$ \\
\hline 480 & \multirow{9}{*}{ 로 } & 1 & 0.869 & 0.726 & 23.425 & 2023.437 \\
\hline 480 & & 0.5 & 0.568 & 2.767 & 17.474 & 17.692 \\
\hline 480 & & 0.2 & 0.170 & 5.869 & 10.652 & 12.162 \\
\hline 480 & & 0.1 & -0.131 & 4.727 & 0.559 & 4.760 \\
\hline 480 & & 0.05 & -0.432 & 2.231 & 0.871 & 2.395 \\
\hline 480 & & 0.02 & -0.830 & 2.467 & 0.619 & 2.543 \\
\hline 480 & & 0.01 & -1.131 & 1.312 & 0.235 & 1.332 \\
\hline 480 & & 0.005 & -1.432 & 0.626 & 0.255 & 0.676 \\
\hline 480 & & 0.002 & -1.830 & 0.088 & 0.343 & 0.354 \\
\hline
\end{tabular}


Appendix 2. Temperature $T, \log _{10} \eta$, frequency $f, \log _{10} \omega \tau_{\mathrm{M}}$, imaginary $\left(G_{\mathrm{im}}\right)$ and real ( $(\mathrm{G}$ real) shear modulus and shear modulus of the melt ( $G$ melt) for the investigated samples - continuation...

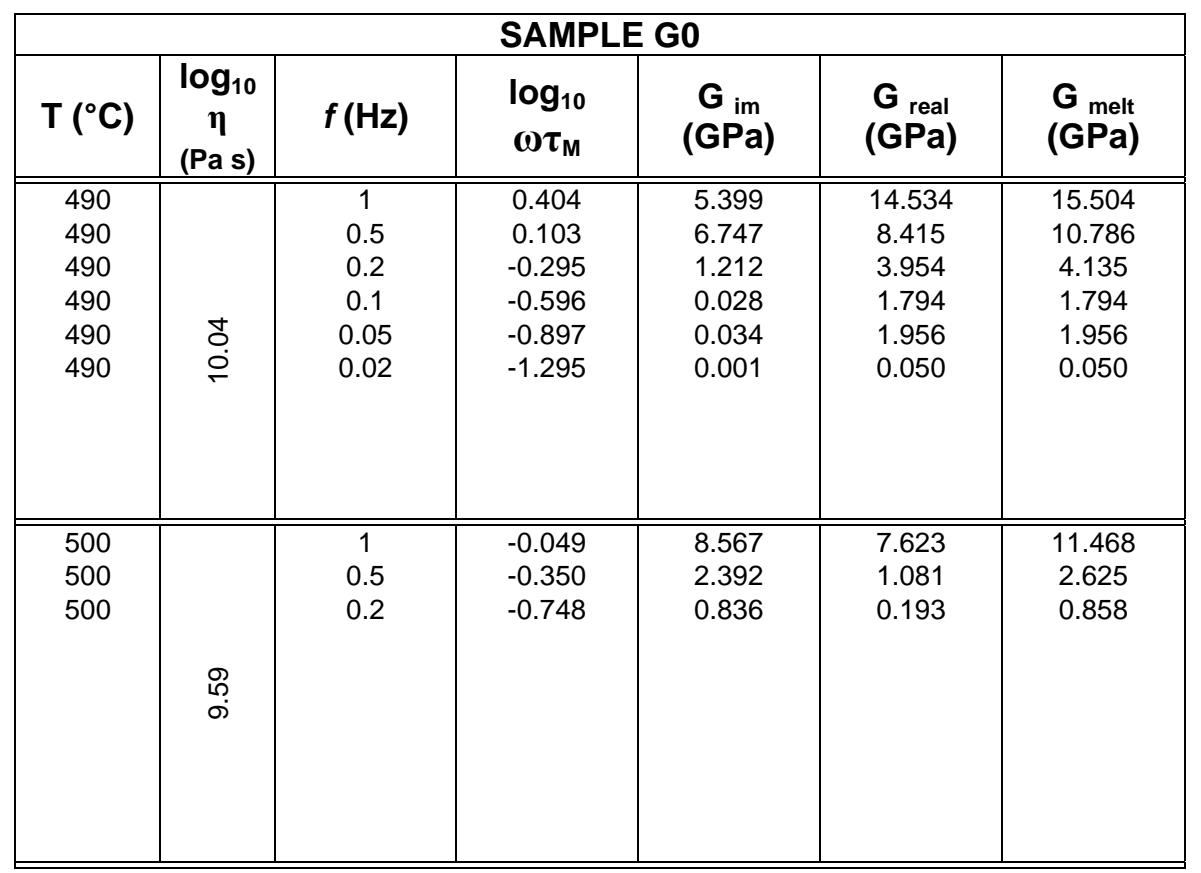

\begin{tabular}{|c|c|c|c|c|c|c|}
\hline \multicolumn{7}{|c|}{ SAMPLE G1 } \\
\hline $\mathrm{T}\left({ }^{\circ} \mathrm{C}\right)$ & $\begin{array}{c}\log _{10} \\
\eta \\
(\mathrm{Pa} \mathrm{s})\end{array}$ & $f(\mathrm{~Hz})$ & $\begin{array}{c}\log _{10} \\
\omega \tau_{M}\end{array}$ & $\begin{array}{c}\mathbf{G}_{\mathrm{im}} \\
(\mathrm{GPa})\end{array}$ & $\begin{array}{c}\mathbf{G}_{\text {real }} \\
(\mathrm{GPa})\end{array}$ & $\begin{array}{c}\mathbf{G}_{\text {melt }} \\
(\mathrm{GPa})\end{array}$ \\
\hline 350 & \multirow{10}{*}{ 离 } & 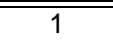 & 23.002 & 0.010 & 34.259 & 34.259 \\
\hline 350 & & 0.5 & 22.701 & 0.099 & 33.948 & 33.948 \\
\hline 350 & & 0.2 & 22.303 & 0.145 & 34.363 & 34.364 \\
\hline 350 & & 0.1 & 22.002 & 0.182 & 33.721 & 33.722 \\
\hline 350 & & 0.05 & 21.701 & 0.110 & 33.693 & 33.693 \\
\hline 350 & & 0.02 & 21.303 & 0.066 & 33.456 & 33.456 \\
\hline 350 & & 0.01 & 21.002 & 0.179 & 34.354 & 34.355 \\
\hline 350 & & 0.005 & 20.701 & 0.226 & 33.834 & 33.835 \\
\hline 350 & & 0.002 & 20.303 & 0.015 & 34.135 & 34.135 \\
\hline 350 & & 0.001 & 20.002 & 0.025 & 34.235 & 34.235 \\
\hline 400 & \multirow{10}{*}{$\begin{array}{l}\stackrel{+}{\sim} \\
\stackrel{\sim}{N}\end{array}$} & 1 & 19.408 & "0.119 & 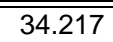 & 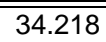 \\
\hline 400 & & 0.5 & 19.107 & 0.043 & 34.270 & 34.270 \\
\hline 400 & & 0.2 & 18.709 & 0.027 & 33.731 & 33.731 \\
\hline 400 & & 0.1 & 18.408 & 0.340 & 34.173 & 34.175 \\
\hline 400 & & 0.05 & 18.107 & 0.008 & 34.121 & 34.121 \\
\hline 400 & & 0.02 & 17.709 & 0.210 & 33.822 & 33.823 \\
\hline 400 & & 0.01 & 17.408 & 0.041 & 35.062 & 35.062 \\
\hline 400 & & 0.005 & 17.107 & 0.034 & 34.413 & 34.413 \\
\hline 400 & & 0.002 & 16.709 & 0.111 & 34.651 & 34.651 \\
\hline 400 & & 0.001 & 16.408 & 0.150 & 34.741 & 34.741 \\
\hline 450 & \multirow{10}{*}{$\begin{array}{l}\stackrel{2}{0} \\
\dot{0}\end{array}$} & 1 & 16.311 & 0.141 & 34.787 & 34.787 \\
\hline 450 & & 0.5 & 16.010 & 0.031 & 34.621 & 34.621 \\
\hline 450 & & 0.2 & 15.612 & 0.037 & 34.913 & 34.913 \\
\hline 450 & & 0.1 & 15.311 & 0.036 & 34.792 & 34.792 \\
\hline 450 & & 0.05 & 15.010 & 0.181 & 35.527 & 35.528 \\
\hline 450 & & 0.02 & 14.612 & 0.088 & 35.295 & 35.295 \\
\hline 450 & & 0.01 & 14.311 & 0.268 & 34.269 & 34.270 \\
\hline 450 & & 0.005 & 14.010 & 0.183 & 35.692 & 35.692 \\
\hline 450 & & 0.002 & 13.612 & 0.123 & 34.414 & 34.414 \\
\hline 450 & & 0.001 & 13.311 & 0.024 & 34.510 & 34.510 \\
\hline
\end{tabular}


Appendix 2. Temperature $T, \log _{10} \eta$, frequency $f, \log _{10} \omega \tau_{\mathrm{M}}$, imaginary $\left(G_{\mathrm{im}}\right)$ and real ( $(\mathrm{G}$ real) shear modulus and shear modulus of the melt ( $G$ melt) for the investigated samples - continuation..

\begin{tabular}{|c|c|c|c|c|c|c|}
\hline \multicolumn{7}{|c|}{ SAMPLE G1 } \\
\hline $\mathrm{T}\left({ }^{\circ} \mathrm{C}\right)$ & $\begin{array}{c}\log _{10} \\
\eta \\
\text { (Pa s) }\end{array}$ & $f(\mathrm{~Hz})$ & $\begin{array}{c}\log _{10} \\
\omega \tau_{M}\end{array}$ & $\begin{array}{c}\mathbf{G}_{\mathrm{im}} \\
(\mathrm{GPa})\end{array}$ & $\begin{array}{l}\mathbf{G}_{\text {real }} \\
(\mathrm{GPa})\end{array}$ & $\begin{array}{l}\mathbf{G}_{\text {melt }} \\
(\mathrm{GPa})\end{array}$ \\
\hline 500 & \multirow{10}{*}{ 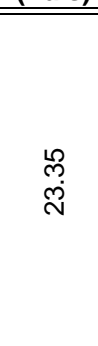 } & 1 & 13.615 & 0.113 & 34.257 & 34.258 \\
\hline 500 & & 0.5 & 13.314 & 0.069 & 34.596 & 34.596 \\
\hline 500 & & 0.2 & 12.916 & 0.117 & 34.408 & 34.408 \\
\hline 500 & & 0.1 & 12.615 & 0.107 & 34.703 & 34.704 \\
\hline 500 & & 0.05 & 12.314 & 0.024 & 34.692 & 34.692 \\
\hline 500 & & 0.02 & 11.916 & 0.132 & 34.584 & 34.584 \\
\hline 500 & & 0.01 & 11.615 & 0.049 & 34.218 & 34.218 \\
\hline 500 & & 0.005 & 11.314 & 0.177 & 34.921 & 34.922 \\
\hline 500 & & 0.002 & 10.916 & 0.341 & 33.956 & 33.958 \\
\hline 500 & & 0.001 & 10.615 & 0.082 & 34.319 & 34.319 \\
\hline 2550 & \multirow{10}{*}{ 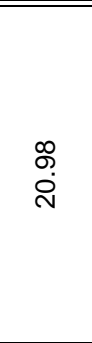 } & 1 & 11.247 & $\begin{array}{l}0.081 \\
\end{array}$ & 34.376 & 34.376 \\
\hline 550 & & 0.5 & 10.946 & 0.096 & 34.231 & 34.231 \\
\hline 550 & & 0.2 & 10.548 & 0.074 & 34.461 & 34.461 \\
\hline 550 & & 0.1 & 10.247 & 0.123 & 34.022 & 34.022 \\
\hline 550 & & 0.05 & 9.946 & 0.141 & 35.531 & 35.531 \\
\hline 550 & & 0.02 & 9.548 & 0.478 & 34.744 & 34.748 \\
\hline 550 & & 0.01 & 9.247 & 0.231 & 34.306 & 34.307 \\
\hline 550 & & 0.005 & 8.946 & 0.000 & 34.341 & 34.341 \\
\hline 550 & & 0.002 & 8.548 & 0.036 & 34.630 & 34.630 \\
\hline 550 & & 0.001 & 8.247 & 0.001 & 34.516 & 34.516 \\
\hline (2600 & \multirow{10}{*}{$\begin{array}{l}\infty \\
\infty \\
\infty \\
\infty \\
-1\end{array}$} & 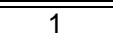 & $\begin{array}{l}9.149 \\
\end{array}$ & $\begin{array}{l}0.004 \\
\end{array}$ & "34.661 & 34.661 \\
\hline 600 & & 0.5 & 8.848 & 0.008 & 35.200 & 35.200 \\
\hline 600 & & 0.2 & 8.451 & 0.067 & 34.721 & 34.721 \\
\hline 600 & & 0.1 & 8.149 & 0.093 & 35.122 & 35.122 \\
\hline 600 & & 0.05 & 7.848 & 0.098 & 35.320 & 35.320 \\
\hline 600 & & 0.02 & 7.451 & 0.219 & 35.628 & 35.629 \\
\hline 600 & & 0.01 & 7.149 & 0.321 & 35.043 & 35.044 \\
\hline 600 & & 0.005 & 6.848 & 0.145 & 34.962 & 34.962 \\
\hline 600 & & 0.002 & 6.451 & 0.200 & 34.281 & 34.281 \\
\hline 600 & & 0.001 & 6.149 & 0.099 & 34.517 & 34.517 \\
\hline
\end{tabular}

\begin{tabular}{|c|c|c|c|c|c|c|}
\hline \multicolumn{7}{|c|}{ SAMPLE G1 } \\
\hline $\mathrm{T}\left({ }^{\circ} \mathrm{C}\right)$ & $\begin{array}{c}\log _{10} \\
\eta \\
\text { (Pa s) }\end{array}$ & $f(\mathrm{~Hz})$ & $\begin{array}{c}\log _{10} \\
\omega \tau_{M}\end{array}$ & $\begin{array}{c}\mathbf{G}_{\text {im }} \\
(\mathrm{GPa})\end{array}$ & $\begin{array}{l}\mathbf{G}_{\text {real }} \\
(\mathrm{GPa})\end{array}$ & $\begin{array}{l}\mathbf{G}_{\text {melt }} \\
(\mathrm{GPa})\end{array}$ \\
\hline 4650 & \multirow{10}{*}{$\begin{array}{l}\text { Tे } \\
\text { - } \\
\text { - }\end{array}$} & $\bar{~} 1$ & $\begin{array}{l}7.279 \\
\end{array}$ & $\begin{array}{l}0.050 \\
\end{array}$ & 34.157 & 34.157 \\
\hline 650 & & 0.5 & 6.978 & 0.060 & 34.418 & 34.418 \\
\hline 650 & & 0.2 & 6.581 & 0.232 & 34.898 & 34.899 \\
\hline 650 & & 0.1 & 6.279 & 0.535 & 34.446 & 34.450 \\
\hline 650 & & 0.05 & 5.978 & 0.605 & 34.838 & 34.843 \\
\hline 650 & & 0.02 & 5.581 & 0.989 & 33.686 & 33.700 \\
\hline 650 & & 0.01 & 5.279 & 0.780 & 33.520 & 33.529 \\
\hline 650 & & 0.005 & 4.978 & 0.667 & 32.420 & 34.427 \\
\hline 650 & & 0.002 & 4.581 & 0.169 & 32.755 & 33.756 \\
\hline 650 & & 0.001 & 4.279 & 0.212 & 33.477 & 33.478 \\
\hline "700 & \multirow{10}{*}{ ले } & $\bar{~} 1$ & $\begin{array}{c}5.602 \\
\end{array}$ & 2.329 & "33.004 & " 33.086 \\
\hline 700 & & 0.5 & 5.301 & 0.077 & 32.563 & 32.563 \\
\hline 700 & & 0.2 & 4.903 & 0.226 & 32.500 & 32.500 \\
\hline 700 & & 0.1 & 4.602 & 0.479 & 32.234 & 32.238 \\
\hline 700 & & 0.05 & 4.301 & 0.161 & 32.606 & 32.606 \\
\hline 700 & & 0.02 & 3.903 & 0.459 & 32.767 & 32.770 \\
\hline 700 & & 0.01 & 3.602 & 0.211 & 31.305 & 31.306 \\
\hline 700 & & 0.005 & 3.301 & 0.611 & 31.889 & 31.894 \\
\hline 700 & & 0.002 & 2.903 & 1.021 & 31.431 & 31.448 \\
\hline 700 & & 0.001 & 2.602 & 1.188 & 30.883 & 30.906 \\
\hline 725 & \multirow{10}{*}{$\begin{array}{l}\text { L } \\
\stackrel{\leftrightarrow}{+} \\
\text { J }\end{array}$} & 1 & 4.826 & 0.025 & 32.677 & 32.677 \\
\hline 725 & & 0.5 & 4.525 & 0.165 & 32.730 & 32.730 \\
\hline 725 & & 0.2 & 4.127 & 0.168 & 31.623 & 31.623 \\
\hline 725 & & 0.1 & 3.826 & 0.050 & 32.344 & 32.345 \\
\hline 725 & & 0.05 & 3.525 & 0.803 & 31.622 & 31.632 \\
\hline 725 & & 0.02 & 3.127 & 0.565 & 31.736 & 31.741 \\
\hline 725 & & 0.01 & 2.826 & 0.862 & 31.708 & 31.720 \\
\hline 725 & & 0.005 & 2.525 & 1.430 & 30.452 & 30.486 \\
\hline 725 & & 0.002 & 2.127 & 2.006 & 29.429 & 29.497 \\
\hline 725 & & 0.001 & 1.826 & 0.153 & 27.987 & 27.987 \\
\hline
\end{tabular}


Appendix 2. Temperature $T$, $\log _{10} \eta$, frequency $f$, $\log _{10} \omega \tau_{\mathrm{M}}$, imaginary $(G \mathrm{im})$ and real $\left(\mathrm{G}_{\text {real }}\right)$ shear modulus and shear modulus of the melt $(\mathrm{G}$ melt $)$ for the investigated samples - continuation..

\begin{tabular}{|c|c|c|c|c|c|c|}
\hline \multicolumn{7}{|c|}{ SAMPLE G1 } \\
\hline $\mathrm{T}\left({ }^{\circ} \mathrm{C}\right)$ & $\begin{array}{c}\log _{10} \\
\eta \\
\text { (Pa s) }\end{array}$ & $f(\mathrm{~Hz})$ & $\begin{array}{c}\log _{10} \\
\omega \tau_{M}\end{array}$ & $\begin{array}{c}\mathbf{G}_{\mathrm{im}} \\
(\mathrm{GPa})\end{array}$ & $\begin{array}{l}\mathbf{G}_{\text {real }} \\
(\mathrm{GPa})\end{array}$ & $\begin{array}{l}\mathbf{G}_{\text {melt }} \\
(\mathrm{GPa})\end{array}$ \\
\hline 750 & \multirow{10}{*}{ 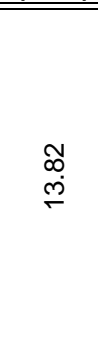 } & 1 & 4.088 & 0.282 & 31.523 & 31.524 \\
\hline 750 & & 0.5 & 3.787 & 0.497 & 30.993 & 30.997 \\
\hline 750 & & 0.2 & 3.389 & 0.803 & 31.401 & 31.411 \\
\hline 750 & & 0.1 & 3.088 & 0.720 & 31.044 & 31.052 \\
\hline 750 & & 0.05 & 2.787 & 0.685 & 31.232 & 31.240 \\
\hline 750 & & 0.02 & 2.389 & 1.150 & 30.269 & 30.291 \\
\hline 750 & & 0.01 & 2.088 & 2.094 & 28.235 & 28.313 \\
\hline 750 & & 0.005 & 1.787 & 2.930 & 26.700 & 26.860 \\
\hline 750 & & 0.002 & 1.389 & 1.896 & 25.313 & 25.384 \\
\hline 750 & & 0.001 & 1.088 & 3.872 & 21.589 & 21.933 \\
\hline 7775 & \multirow{10}{*}{$\begin{array}{l}\underset{\sim}{\mathcal{M}} \\
\stackrel{\rightarrow}{\rightarrow}\end{array}$} & 1 & 3.385 & $\begin{array}{l}0.356 \\
\end{array}$ & 31.285 & 31.287 \\
\hline 775 & & 0.5 & 3.084 & 0.594 & 30.698 & 30.704 \\
\hline 775 & & 0.2 & 2.686 & 0.989 & 30.377 & 30.393 \\
\hline 775 & & 0.1 & 2.385 & 0.851 & 29.313 & 29.326 \\
\hline 775 & & 0.05 & 2.084 & 2.217 & 28.516 & 28.602 \\
\hline 775 & & 0.02 & 1.686 & 3.119 & 26.414 & 26.597 \\
\hline 775 & & 0.01 & 1.385 & 4.606 & 24.633 & 25.060 \\
\hline 775 & & 0.005 & 1.084 & 4.792 & 21.454 & 21.982 \\
\hline 775 & & 0.002 & 0.686 & 5.438 & 16.193 & 17.082 \\
\hline 775 & & 0.001 & 0.385 & 6.898 & 9.730 & 11.927 \\
\hline 800 & \multirow{10}{*}{ 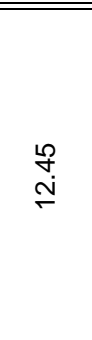 } & 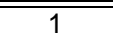 & 2.715 & $\begin{array}{ll}.006 \\
\end{array}$ & 30.672 & 30.688 \\
\hline 800 & & 0.5 & 2.414 & 1.268 & 29.907 & 29.934 \\
\hline 800 & & 0.2 & 2.016 & 2.063 & 28.996 & 29.069 \\
\hline 800 & & 0.1 & 1.715 & 3.135 & 26.642 & 26.826 \\
\hline 800 & & 0.05 & 1.414 & 4.066 & 24.749 & 25.081 \\
\hline 800 & & 0.02 & 1.016 & 6.374 & 19.652 & 20.660 \\
\hline 800 & & 0.01 & 0.715 & 2.643 & 17.912 & 18.106 \\
\hline 800 & & 0.005 & 0.414 & 4.460 & 12.468 & 13.242 \\
\hline 800 & & 0.002 & 0.016 & 5.057 & 3.808 & 6.331 \\
\hline 800 & & 0.001 & -0.285 & 2.918 & 0.935 & 3.064 \\
\hline
\end{tabular}

\begin{tabular}{|c|c|c|c|c|c|c|}
\hline \multicolumn{7}{|c|}{ SAMPLE G1 } \\
\hline $\mathrm{T}\left({ }^{\circ} \mathrm{C}\right)$ & $\begin{array}{c}\log _{10} \\
\eta \\
\text { (Pa s) }\end{array}$ & $f(\mathrm{~Hz})$ & $\begin{array}{c}\log _{10} \\
\omega \tau_{M}\end{array}$ & $\begin{array}{c}\mathbf{G}_{\text {im }} \\
(\mathrm{GPa})\end{array}$ & $\begin{array}{l}\mathbf{G}_{\text {real }} \\
(\mathrm{GPa})\end{array}$ & $\begin{array}{l}\mathbf{G}_{\text {melt }} \\
(\mathrm{GPa})\end{array}$ \\
\hline 825 & \multirow{10}{*}{$\begin{array}{l}-1 \\
-1 \\
-1\end{array}$} & $\bar{~} 1$ & $\begin{array}{l}2.076 \\
\end{array}$ & 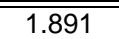 & 26.412 & 26.480 \\
\hline 825 & & 0.5 & 1.775 & 2.833 & 25.190 & 25.349 \\
\hline 825 & & 0.2 & 1.377 & 4.359 & 22.440 & 22.859 \\
\hline 825 & & 0.1 & 1.076 & 5.895 & 19.273 & 20.155 \\
\hline 825 & & 0.05 & 0.775 & 6.981 & 14.673 & 16.249 \\
\hline 825 & & 0.02 & 0.377 & 7.244 & 7.812 & 10.654 \\
\hline 825 & & 0.01 & 0.076 & 2.487 & 6.773 & 7.215 \\
\hline 825 & & 0.005 & -0.225 & 3.550 & 1.193 & 3.745 \\
\hline 825 & & 0.002 & -0.623 & 1.401 & 0.206 & 1.416 \\
\hline 825 & & 0.001 & -0.924 & 0.472 & 0.320 & 0.571 \\
\hline 850 & \multirow{8}{*}{$\begin{array}{l}\text { तิ } \\
\text { - }\end{array}$} & 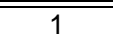 & 1.465 & 3.054 & 18.866 & 19.112 \\
\hline 850 & & 0.5 & 1.164 & 4.224 & 16.595 & 17.124 \\
\hline 850 & & 0.2 & 0.766 & 5.791 & 12.343 & 13.634 \\
\hline 850 & & 0.1 & 0.465 & 6.250 & 8.192 & 10.304 \\
\hline 850 & & 0.05 & 0.164 & 5.654 & 4.217 & 7.053 \\
\hline 850 & & 0.02 & -0.234 & 2.963 & 1.021 & 3.134 \\
\hline 850 & & 0.01 & -0.535 & 1.531 & 0.556 & 1.629 \\
\hline 850 & & 0.005 & -0.836 & 0.830 & 0.082 & 0.835 \\
\hline 875 & \multirow{8}{*}{ 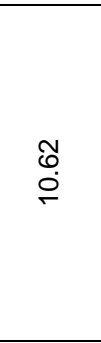 } & $\overline{11}$ & 0.880 & 7.222 & 17.109 & 18.571 \\
\hline 875 & & 0.5 & 0.579 & 7.916 & 11.614 & 14.055 \\
\hline 875 & & 0.2 & 0.181 & 6.704 & 4.831 & 8.263 \\
\hline 875 & & 0.1 & -0.120 & 4.000 & 1.630 & 4.320 \\
\hline 875 & & 0.05 & -0.421 & 0.862 & 2.373 & 2.524 \\
\hline & & & & & & \\
\hline & & & & & & \\
\hline & & & & & & \\
\hline
\end{tabular}


Appendix 2. Temperature $T, \log _{10} \eta$, frequency $f, \log _{10} \omega \tau_{\mathrm{M}}$, imaginary $\left(G_{\mathrm{im}}\right)$ and real ( $(\mathrm{G}$ real) shear modulus and shear modulus of the melt ( $G$ melt) for the investigated samples - continuation...

\begin{tabular}{|c|c|c|c|c|c|c|}
\hline \multicolumn{7}{|c|}{ SAMPLE G2 } \\
\hline $\mathrm{T}\left({ }^{\circ} \mathrm{C}\right)$ & $\begin{array}{c}\log _{10} \\
\eta \\
\text { (Pa s) }\end{array}$ & $f(\mathrm{~Hz})$ & $\begin{array}{c}\log _{10} \\
\omega \tau_{M}\end{array}$ & $\begin{array}{c}\mathbf{G}_{\mathrm{im}} \\
(\mathbf{G P a})\end{array}$ & $\begin{array}{l}\mathbf{G}_{\text {real }} \\
(\mathrm{GPa})\end{array}$ & $\begin{array}{l}\mathbf{G}_{\text {melt }} \\
(\mathrm{GPa})\end{array}$ \\
\hline 500 & \multirow{10}{*}{$\stackrel{m}{\underset{m}{\sim}}$} & $\overline{11}$ & 13.590 & 0.503 & 34.780 & 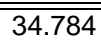 \\
\hline 500 & & 0.5 & 13.289 & 0.526 & 34.868 & 34.872 \\
\hline 500 & & 0.2 & 12.891 & 0.440 & 34.738 & 34.741 \\
\hline 500 & & 0.1 & 12.590 & 0.439 & 34.663 & 34.666 \\
\hline 500 & & 0.05 & 12.289 & 0.620 & 34.897 & 34.903 \\
\hline 500 & & 0.02 & 11.891 & 0.234 & 34.744 & 34.744 \\
\hline 500 & & 0.01 & 11.590 & 0.107 & 34.596 & 34.596 \\
\hline 500 & & 0.005 & 11.289 & 0.574 & 34.868 & 34.873 \\
\hline 500 & & 0.002 & 10.891 & 0.168 & 34.824 & 34.824 \\
\hline 500 & & 0.001 & 10.590 & 0.390 & 35.283 & 35.285 \\
\hline 2550 & \multirow{10}{*}{$\begin{array}{l}-1 \\
\text { - } \\
-1\end{array}$} & 1 & 11.267 & $\begin{array}{l}0.122 \\
\end{array}$ & 34.411 & 34.411 \\
\hline 550 & & 0.5 & 10.966 & 0.010 & 34.366 & 34.366 \\
\hline 550 & & 0.2 & 10.568 & 0.086 & 34.497 & 34.497 \\
\hline 550 & & 0.1 & 10.267 & 0.293 & 34.580 & 34.581 \\
\hline 550 & & 0.05 & 9.966 & 0.332 & 34.662 & 34.663 \\
\hline 550 & & 0.02 & 9.568 & 0.266 & 34.217 & 34.218 \\
\hline 550 & & 0.01 & 9.267 & 0.043 & 34.445 & 34.445 \\
\hline 550 & & 0.005 & 8.966 & 0.108 & 34.459 & 34.459 \\
\hline 550 & & 0.002 & 8.568 & 0.077 & 34.237 & 34.237 \\
\hline 550 & & 0.001 & 8.267 & 0.104 & 34.745 & 34.745 \\
\hline (2600 & \multirow{10}{*}{ 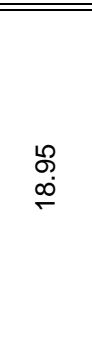 } & 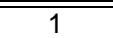 & $\begin{array}{l}9.210 \\
\end{array}$ & $\begin{array}{l}0.041 \\
\end{array}$ & 34.792 & 34.792 \\
\hline 600 & & 0.5 & 8.909 & 0.022 & 34.816 & 34.816 \\
\hline 600 & & 0.2 & 8.511 & 0.209 & 34.730 & 34.731 \\
\hline 600 & & 0.1 & 8.210 & 0.148 & 34.316 & 34.317 \\
\hline 600 & & 0.05 & 7.909 & 0.146 & 35.289 & 35.289 \\
\hline 600 & & 0.02 & 7.511 & 0.311 & 34.764 & 34.765 \\
\hline 600 & & 0.01 & 7.210 & 0.818 & 34.508 & 34.509 \\
\hline 600 & & 0.005 & 6.909 & 1.379 & 34.431 & 34.432 \\
\hline 600 & & 0.002 & 6.511 & 1.128 & 33.085 & 33.085 \\
\hline 600 & & 0.001 & 6.210 & 1.591 & 33.225 & 33.225 \\
\hline
\end{tabular}

\begin{tabular}{|c|c|c|c|c|c|c|}
\hline \multicolumn{7}{|c|}{ SAMPLE G2 } \\
\hline $\mathrm{T}\left({ }^{\circ} \mathrm{C}\right)$ & $\begin{array}{c}\log _{10} \\
\eta \\
\text { (Pa s) }\end{array}$ & $f(\mathrm{~Hz})$ & $\begin{array}{c}\log _{10} \\
\omega \tau_{M}\end{array}$ & $\begin{array}{l}\mathbf{G}_{\text {im }} \\
(\mathbf{G P a})\end{array}$ & $\begin{array}{l}\mathbf{G}_{\text {real }} \\
(\mathrm{GPa})\end{array}$ & $\begin{array}{l}G_{\text {melt }} \\
(\mathrm{GPa})\end{array}$ \\
\hline 650 & \multirow{10}{*}{$\begin{array}{l}\text { İ } \\
\underset{I}{-}\end{array}$} & 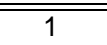 & 7.376 & 0.547 & 33.812 & 33.812 \\
\hline 650 & & 0.5 & 7.075 & 1.161 & 33.920 & 33.921 \\
\hline 650 & & 0.2 & 6.677 & 1.327 & 33.818 & 33.819 \\
\hline 650 & & 0.1 & 6.376 & 1.544 & 34.002 & 34.002 \\
\hline 650 & & 0.05 & 6.075 & 1.119 & 33.577 & 33.577 \\
\hline 650 & & 0.02 & 5.677 & 1.051 & 33.750 & 33.750 \\
\hline 650 & & 0.01 & 5.376 & 0.207 & 34.133 & 34.134 \\
\hline 650 & & 0.005 & 5.075 & 0.066 & 33.784 & 33.784 \\
\hline 650 & & 0.002 & 4.677 & 0.121 & 33.772 & 33.772 \\
\hline 650 & & 0.001 & 4.376 & 0.199 & 34.542 & 34.543 \\
\hline 700 & \multirow{5}{*}{$\begin{array}{l}\text { fे } \\
\text { ம் }\end{array}$} & $\bar{~} 1$ & 5.730 & 0.195 & 33.587 & 33.587 \\
\hline 700 & & 0.5 & 5.429 & 0.222 & 33.433 & 33.434 \\
\hline 700 & & 0.2 & 5.031 & 0.326 & 33.505 & 33.506 \\
\hline 700 & & 0.1 & 4.730 & 0.321 & 33.763 & 33.765 \\
\hline 700 & & 0.05 & 4.429 & 0.317 & 33.719 & 33.721 \\
\hline 725 & \multirow{10}{*}{ 离 } & 1 & 4.970 & 1.360 & 33.314 & 33.341 \\
\hline 725 & & 0.5 & 4.668 & 1.205 & 33.330 & 33.352 \\
\hline 725 & & 0.2 & 4.271 & 0.346 & 33.565 & 33.567 \\
\hline 725 & & 0.1 & 3.970 & 3.951 & 32.479 & 32.719 \\
\hline 725 & & 0.05 & 3.668 & 2.361 & 32.572 & 32.657 \\
\hline 725 & & 0.02 & 3.271 & 2.162 & 32.923 & 32.993 \\
\hline 725 & & 0.01 & 2.970 & 3.227 & 30.306 & 30.478 \\
\hline 725 & & 0.005 & 2.668 & 4.557 & 30.103 & 30.446 \\
\hline 725 & & 0.002 & 2.271 & 3.923 & 30.205 & 30.458 \\
\hline 725 & & 0.001 & 1.970 & 3.866 & 29.690 & 29.940 \\
\hline
\end{tabular}


Appendix 2. Temperature $T, \log _{10} \eta$, frequency $f, \log _{10} \omega \tau_{\mathrm{M}}$, imaginary $\left(G_{\mathrm{im}}\right)$ and real ( $(\mathrm{G}$ real) shear modulus and shear modulus of the melt ( $G$ melt) for the investigated samples - continuation...

\begin{tabular}{|c|c|c|c|c|c|c|}
\hline \multicolumn{7}{|c|}{ SAMPLE G2 } \\
\hline $\mathrm{T}\left({ }^{\circ} \mathrm{C}\right)$ & $\begin{array}{c}\log _{10} \\
\eta \\
\text { (Pa s) }\end{array}$ & $f(\mathrm{~Hz})$ & $\begin{array}{c}\log _{10} \\
\omega \tau_{M}\end{array}$ & $\begin{array}{c}\mathbf{G}_{\mathrm{im}} \\
(\mathrm{GPa})\end{array}$ & $\begin{array}{l}\mathbf{G}_{\text {real }} \\
(\mathrm{GPa})\end{array}$ & $\begin{array}{l}\mathbf{G}_{\text {melt }} \\
(\mathrm{GPa})\end{array}$ \\
\hline 750 & \multirow{10}{*}{ 蟌 } & 1 & 4.246 & 0.679 & 32.835 & 32.842 \\
\hline 750 & & 0.5 & 3.945 & 0.139 & 32.993 & 32.994 \\
\hline 750 & & 0.2 & 3.547 & 0.196 & 33.189 & 33.189 \\
\hline 750 & & 0.1 & 3.246 & 2.640 & 32.855 & 32.960 \\
\hline 750 & & 0.05 & 2.945 & 2.327 & 28.868 & 28.962 \\
\hline 750 & & 0.02 & 2.547 & 2.932 & 29.642 & 29.787 \\
\hline 750 & & 0.01 & 2.246 & 4.232 & 28.188 & 28.503 \\
\hline 750 & & 0.005 & 1.945 & 5.503 & 24.635 & 25.242 \\
\hline 750 & & 0.002 & 1.547 & 8.552 & 23.147 & 24.676 \\
\hline 750 & & 0.001 & 1.246 & 5.555 & 23.239 & 23.894 \\
\hline 800 & \multirow{10}{*}{ 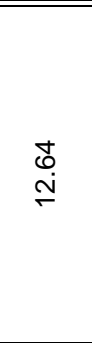 } & $\bar{~} 1$ & 2.900 & 3.508 & 31.538 & "31.733 \\
\hline 800 & & 0.5 & 2.598 & 8.998 & 30.799 & 32.086 \\
\hline 800 & & 0.2 & 2.201 & 6.051 & 30.106 & 30.708 \\
\hline 800 & & 0.1 & 1.900 & 8.572 & 26.067 & 27.440 \\
\hline 800 & & 0.05 & 1.598 & 8.240 & 23.249 & 24.666 \\
\hline 800 & & 0.02 & 1.201 & 5.271 & 23.179 & 23.771 \\
\hline 800 & & 0.01 & 0.900 & 4.612 & 16.775 & 17.398 \\
\hline 800 & & 0.005 & 0.598 & 2.967 & 16.206 & 16.475 \\
\hline 800 & & 0.002 & 0.201 & 3.323 & 8.745 & 9.355 \\
\hline 800 & & 0.001 & -0.100 & 3.223 & 8.169 & 8.782 \\
\hline 825 & \multirow{8}{*}{$\begin{array}{l}\text { Oे } \\
\text { తి }\end{array}$} & 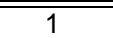 & \begin{tabular}{c|}
2.272 \\
\end{tabular} & $\begin{array}{l}5.162 \\
\end{array}$ & 31.273 & 31.696 \\
\hline 825 & & 0.5 & 1.971 & 5.814 & 29.223 & 29.796 \\
\hline & & & & & & \\
\hline 825 & & 0.02 & 0.573 & 4.446 & 7.664 & 8.860 \\
\hline 825 & & 0.01 & 0.272 & 5.595 & 6.067 & 8.253 \\
\hline 825 & & 0.005 & -0.029 & 4.765 & 5.268 & 7.104 \\
\hline 825 & & 0.002 & -0.427 & 2.175 & 3.264 & 3.922 \\
\hline 825 & & 0.001 & -0.728 & 0.562 & 1.336 & 1.449 \\
\hline
\end{tabular}

\begin{tabular}{|c|c|c|c|c|c|c|}
\hline \multicolumn{7}{|c|}{ SAMPLE G2 } \\
\hline $\mathrm{T}\left({ }^{\circ} \mathrm{C}\right)$ & $\begin{array}{c}\log _{10} \\
\eta \\
\text { (Pa s) }\end{array}$ & $f(\mathrm{~Hz})$ & $\begin{array}{c}\log _{10} \\
\omega \tau_{M}\end{array}$ & $\begin{array}{l}\mathbf{G}_{\text {im }} \\
(\mathbf{G P a})\end{array}$ & $\begin{array}{l}\mathbf{G}_{\text {real }} \\
(\mathrm{GPa})\end{array}$ & $\begin{array}{l}\mathbf{G}_{\text {melt }} \\
(\mathrm{GPa})\end{array}$ \\
\hline 850 & \multirow{10}{*}{$\begin{array}{l}\mathcal{F} \\
\text { - } \\
\text { - }\end{array}$} & $\overline{11}$ & $\begin{array}{ll}1.673 \\
\end{array}$ & 8.469 & 30.002 & 30.496 \\
\hline 850 & & 0.5 & 1.372 & 9.691 & 28.864 & 30.447 \\
\hline 850 & & 0.2 & 0.974 & 9.896 & 30.499 & 32.065 \\
\hline 850 & & 0.1 & 0.673 & 10.243 & 19.339 & 21.884 \\
\hline 850 & & 0.05 & 0.372 & 2.887 & 18.954 & 19.172 \\
\hline 850 & & 0.02 & -0.026 & 2.081 & 18.085 & 18.204 \\
\hline 850 & & 0.01 & -0.327 & 3.480 & 3.016 & 4.605 \\
\hline 850 & & 0.005 & -0.628 & 0.424 & 2.278 & 2.318 \\
\hline 850 & & 0.002 & -1.026 & 0.483 & 0.779 & 0.916 \\
\hline 850 & & 0.001 & -1.327 & 0.190 & 0.241 & 0.307 \\
\hline 875 & \multirow{5}{*}{\begin{tabular}{l}
\multirow{1}{0}{} \\
0 \\
0 \\
-1
\end{tabular}} & 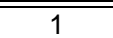 & $\begin{array}{ll}1.100 \\
\end{array}$ & 8.998 & 24.342 & 25.952 \\
\hline 875 & & 0.5 & 0.799 & 10.842 & 21.656 & 24.219 \\
\hline 875 & & 0.2 & 0.401 & 6.928 & 12.106 & 13.948 \\
\hline 875 & & 0.1 & 0.100 & 1.532 & 7.825 & 7.973 \\
\hline 875 & & 0.05 & -0.201 & 0.164 & 2.015 & 2.022 \\
\hline 900 & \multirow{8}{*}{$\begin{array}{l}\text { Q̦ } \\
\text { Oें }\end{array}$} & 1 & 0.551 & 3.793 & 19.308 & 19.678 \\
\hline 900 & & 0.5 & 0.250 & 3.984 & 5.503 & 6.794 \\
\hline 900 & & 0.2 & -0.148 & 0.734 & 3.618 & 3.692 \\
\hline 900 & & 0.1 & -0.449 & 0.950 & 1.506 & 1.781 \\
\hline 900 & & 0.05 & -0.750 & 0.347 & 0.687 & 0.770 \\
\hline & & & & & & \\
\hline & & & & & & \\
\hline & & & & & & \\
\hline
\end{tabular}


Appendix 2. Temperature $T$, $\log _{10} \eta$, frequency $f$, $\log _{10} \omega \tau_{\mathrm{M}}$, imaginary $(G \mathrm{im})$ and real $\left(\mathrm{G}_{\text {real }}\right)$ shear modulus and shear modulus of the melt $(\mathrm{G}$ melt $)$ for the investigated samples - continuation..

\begin{tabular}{|c|c|c|c|c|c|c|}
\hline \multicolumn{7}{|c|}{ SAMPLE G3 } \\
\hline $\mathrm{T}\left({ }^{\circ} \mathrm{C}\right)$ & $\begin{array}{c}\log _{10} \\
\eta \\
\text { (Pa s) }\end{array}$ & $f(\mathrm{~Hz})$ & $\begin{array}{c}\log _{10} \\
\omega \tau_{M}\end{array}$ & $\begin{array}{c}\mathbf{G}_{\mathrm{im}} \\
(\mathrm{GPa})\end{array}$ & $\begin{array}{l}\mathbf{G}_{\text {real }} \\
(\mathrm{GPa})\end{array}$ & $\begin{array}{l}\mathbf{G}_{\text {melt }} \\
(\mathrm{GPa})\end{array}$ \\
\hline 2550 & \multirow{10}{*}{$\begin{array}{l}\vec{y} \\
\stackrel{-}{N}\end{array}$} & 1 & 11.671 & $\begin{array}{l}0.102 \\
\end{array}$ & 34.434 & 34.434 \\
\hline 550 & & 0.5 & 11.370 & 0.037 & 34.650 & 34.650 \\
\hline 550 & & 0.2 & 10.972 & 0.081 & 34.381 & 34.381 \\
\hline 550 & & 0.1 & 10.671 & 0.379 & 34.737 & 34.739 \\
\hline 550 & & 0.05 & 10.370 & 0.319 & 33.786 & 33.788 \\
\hline 550 & & 0.02 & 9.972 & 0.290 & 34.250 & 34.251 \\
\hline 550 & & 0.01 & 9.671 & 0.455 & 33.474 & 33.477 \\
\hline 550 & & 0.005 & 9.370 & 0.217 & 34.424 & 34.425 \\
\hline 550 & & 0.002 & 8.972 & 0.329 & 34.511 & 34.512 \\
\hline 550 & & 0.001 & 8.671 & 0.579 & 34.068 & 34.073 \\
\hline "600 & \multirow{10}{*}{$\begin{array}{l}\stackrel{2}{N} \\
\stackrel{-}{-}\end{array}$} & 1 & \begin{tabular}{c|}
9.526 \\
\end{tabular} & $\begin{array}{l}0.064 \\
\end{array}$ & 34.375 & 34.375 \\
\hline 600 & & 0.5 & 9.225 & 0.276 & 34.705 & 34.706 \\
\hline 600 & & 0.2 & 8.827 & 0.033 & 34.097 & 34.097 \\
\hline 600 & & 0.1 & 8.526 & 0.446 & 35.138 & 35.141 \\
\hline 600 & & 0.05 & 8.225 & 0.076 & 34.313 & 34.313 \\
\hline 600 & & 0.02 & 7.827 & 0.236 & 33.850 & 33.851 \\
\hline 600 & & 0.01 & 7.526 & 0.853 & 34.361 & 34.361 \\
\hline 600 & & 0.005 & 7.225 & 0.008 & 32.915 & 32.915 \\
\hline 600 & & 0.002 & 6.827 & 0.117 & 32.467 & 32.468 \\
\hline 600 & & 0.001 & 6.526 & 0.807 & 33.896 & 33.906 \\
\hline "650 & \multirow{10}{*}{ 岇 } & 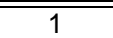 & $\begin{array}{ll}7.613 \\
\end{array}$ & $\begin{array}{l}0.626 \\
\end{array}$ & 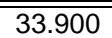 & 33.900 \\
\hline 650 & & 0.5 & 7.312 & 0.707 & 34.110 & 34.110 \\
\hline 650 & & 0.2 & 6.914 & 0.214 & 33.292 & 33.292 \\
\hline 650 & & 0.1 & 6.613 & 0.397 & 33.635 & 33.637 \\
\hline 650 & & 0.05 & 6.312 & 0.105 & 32.811 & 32.811 \\
\hline 650 & & 0.02 & 5.914 & 0.598 & 33.017 & 33.023 \\
\hline 650 & & 0.01 & 5.613 & 0.181 & 32.059 & 32.059 \\
\hline 650 & & 0.005 & 5.312 & 0.070 & 32.044 & 32.044 \\
\hline 650 & & 0.002 & 4.914 & 0.614 & 31.656 & 31.662 \\
\hline 650 & & 0.001 & 4.613 & 1.236 & 32.858 & 32.881 \\
\hline
\end{tabular}

\begin{tabular}{|c|c|c|c|c|c|c|}
\hline \multicolumn{7}{|c|}{ SAMPLE G3 } \\
\hline $\mathrm{T}\left({ }^{\circ} \mathrm{C}\right)$ & $\begin{array}{c}\log _{10} \\
\eta \\
\text { (Pa s) }\end{array}$ & $f(\mathrm{~Hz})$ & $\begin{array}{c}\log _{10} \\
\omega \tau_{M}\end{array}$ & $\begin{array}{l}\mathbf{G}_{\text {im }} \\
(\mathbf{G P a})\end{array}$ & $\begin{array}{l}\mathbf{G}_{\text {real }} \\
(\mathrm{GPa})\end{array}$ & $\begin{array}{l}G_{\text {melt }} \\
(\mathrm{GPa})\end{array}$ \\
\hline 700 & \multirow{10}{*}{$\begin{array}{l}\text { ठे } \\
\text { ம் }\end{array}$} & 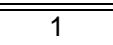 & 5.897 & 0.122 & 32.258 & 32.258 \\
\hline 700 & & 0.5 & 5.596 & 0.091 & 32.154 & 32.154 \\
\hline 700 & & 0.2 & 5.198 & 0.275 & 31.222 & 31.223 \\
\hline 700 & & 0.1 & 4.897 & 0.424 & 32.188 & 32.190 \\
\hline 700 & & 0.05 & 4.596 & 0.461 & 32.267 & 32.270 \\
\hline 700 & & 0.02 & 4.198 & 0.039 & 31.405 & 31.405 \\
\hline 700 & & 0.01 & 3.897 & 0.664 & 31.811 & 31.818 \\
\hline 700 & & 0.005 & 3.596 & 0.553 & 29.366 & 29.371 \\
\hline 700 & & 0.002 & 3.198 & 1.048 & 29.891 & 29.909 \\
\hline 700 & & 0.001 & 2.897 & 2.037 & 28.894 & 28.966 \\
\hline 7725 & \multirow{10}{*}{$\begin{array}{l}\stackrel{+}{\infty} \\
\dot{J}\end{array}$} & $\overline{11}$ & \begin{tabular}{c|}
5.103 \\
\end{tabular} & "0.191 & "31.182 & "31.182 \\
\hline 725 & & 0.5 & 4.802 & 0.191 & 31.453 & 31.454 \\
\hline 725 & & 0.2 & 4.404 & 0.120 & 30.616 & 30.616 \\
\hline 725 & & 0.1 & 4.103 & 0.397 & 31.709 & 31.711 \\
\hline 725 & & 0.05 & 3.802 & 0.077 & 30.695 & 30.695 \\
\hline 725 & & 0.02 & 3.404 & 0.343 & 30.484 & 30.486 \\
\hline 725 & & 0.01 & 3.103 & 0.491 & 29.424 & 29.428 \\
\hline 725 & & 0.005 & 2.802 & 1.517 & 27.796 & 27.838 \\
\hline 725 & & 0.002 & 2.404 & 2.484 & 27.307 & 27.420 \\
\hline 725 & & 0.001 & 2.103 & 2.478 & 25.918 & 25.922 \\
\hline 750 & \multirow{10}{*}{$\begin{array}{l}8 \\
\dot{+}\end{array}$} & $\overline{c 1}$ & 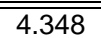 & 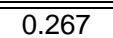 & 31.077 & 31.078 \\
\hline 750 & & 0.5 & 4.047 & 0.175 & 31.256 & 31.257 \\
\hline 750 & & 0.2 & 3.649 & 0.487 & 30.459 & 30.463 \\
\hline 750 & & 0.1 & 3.348 & 1.168 & 30.819 & 30.841 \\
\hline 750 & & 0.05 & 3.047 & 1.279 & 29.718 & 29.745 \\
\hline 750 & & 0.02 & 2.649 & 1.868 & 28.176 & 28.238 \\
\hline 750 & & 0.01 & 2.348 & 2.255 & 28.240 & 28.330 \\
\hline 750 & & 0.005 & 2.047 & 3.167 & 25.186 & 25.384 \\
\hline 750 & & 0.002 & 1.649 & 4.299 & 23.560 & 23.596 \\
\hline 750 & & 0.001 & 1.348 & 4.810 & 19.065 & 19.662 \\
\hline
\end{tabular}


Appendix 2. Temperature $T, \log _{10} \eta$, frequency $f, \log _{10} \omega \tau_{\mathrm{M}}$, imaginary $\left(G_{\mathrm{im}}\right)$ and real ( $(\mathrm{G}$ real) shear modulus and shear modulus of the melt ( $G$ melt) for the investigated samples - continuation..

\begin{tabular}{|c|c|c|c|c|c|c|}
\hline \multicolumn{7}{|c|}{ SAMPLE G3 } \\
\hline $\mathrm{T}\left({ }^{\circ} \mathrm{C}\right)$ & $\begin{array}{c}\log _{10} \\
\eta \\
\text { (Pa s) }\end{array}$ & $f(\mathrm{~Hz})$ & $\begin{array}{c}\log _{10} \\
\omega \tau_{M}\end{array}$ & $\begin{array}{c}\mathbf{G}_{\mathrm{im}} \\
(\mathrm{GPa})\end{array}$ & $\begin{array}{l}\mathbf{G}_{\text {real }} \\
(\mathrm{GPa})\end{array}$ & $\begin{array}{l}\mathbf{G}_{\text {melt }} \\
(\mathrm{GPa})\end{array}$ \\
\hline 7775 & \multirow{10}{*}{$\begin{array}{l}\hat{m} \\
\stackrel{g}{\rightarrow}\end{array}$} & 1 & 3.629 & $\begin{array}{l}0.503 \\
\end{array}$ & 29.680 & 29.684 \\
\hline 775 & & 0.5 & 3.328 & 0.548 & 29.408 & 29.413 \\
\hline 775 & & 0.2 & 2.930 & 1.194 & 27.877 & 27.902 \\
\hline 775 & & 0.1 & 2.629 & 1.832 & 28.013 & 28.073 \\
\hline 775 & & 0.05 & 2.328 & 2.571 & 26.855 & 26.978 \\
\hline 775 & & 0.02 & 1.930 & 3.609 & 24.408 & 24.674 \\
\hline 775 & & 0.01 & 1.629 & 5.078 & 21.782 & 22.367 \\
\hline 775 & & 0.005 & 1.328 & 7.497 & 19.060 & 20.482 \\
\hline 775 & & 0.002 & 0.930 & 7.162 & 14.676 & 15.013 \\
\hline 775 & & 0.001 & 0.629 & 5.632 & 7.836 & 9.650 \\
\hline 800 & \multirow{10}{*}{$\begin{array}{l}\stackrel{\infty}{\circ} \\
\stackrel{\leftrightarrow}{H}\end{array}$} & 1 & 2.944 & $\begin{array}{l}0.991 \\
\end{array}$ & 28.670 & 28.687 \\
\hline 800 & & 0.5 & 2.643 & 1.528 & 28.079 & 28.121 \\
\hline 800 & & 0.2 & 2.245 & 2.339 & 26.191 & 26.296 \\
\hline 800 & & 0.1 & 1.944 & 3.680 & 24.426 & 24.702 \\
\hline 800 & & 0.05 & 1.643 & 4.555 & 21.746 & 22.218 \\
\hline 800 & & 0.02 & 1.245 & 6.564 & 17.111 & 18.327 \\
\hline 800 & & 0.01 & 0.944 & 7.409 & 11.975 & 14.082 \\
\hline 800 & & 0.005 & 0.643 & 6.875 & 10.039 & 10.761 \\
\hline 800 & & 0.002 & 0.245 & 3.931 & 3.066 & 4.986 \\
\hline 800 & & 0.001 & -0.056 & 2.225 & 1.685 & 2.791 \\
\hline 825 & \multirow{10}{*}{$\begin{array}{l}\stackrel{m}{0} \\
\text { İ }\end{array}$} & 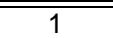 & \begin{tabular}{c|}
2.290 \\
\end{tabular} & 2.114 & 25.566 & 25.653 \\
\hline 825 & & 0.5 & 1.989 & 3.303 & 24.462 & 24.684 \\
\hline 825 & & 0.2 & 1.591 & 4.734 & 20.622 & 21.159 \\
\hline 825 & & 0.1 & 1.290 & 6.747 & 18.410 & 18.613 \\
\hline 825 & & 0.05 & 0.989 & 7.130 & 13.213 & 15.014 \\
\hline 825 & & 0.02 & 0.591 & 6.393 & 6.215 & 8.916 \\
\hline 825 & & 0.01 & 0.290 & 5.665 & 5.645 & 7.997 \\
\hline 825 & & 0.005 & -0.011 & 2.659 & 0.818 & 2.782 \\
\hline 825 & & 0.002 & -0.409 & 1.117 & 0.143 & 1.127 \\
\hline 825 & & 0.001 & -0.710 & 0.582 & 0.060 & 0.585 \\
\hline
\end{tabular}

\begin{tabular}{|c|c|c|c|c|c|c|}
\hline \multicolumn{7}{|c|}{ SAMPLE G3 } \\
\hline $\mathrm{T}\left({ }^{\circ} \mathrm{C}\right)$ & $\begin{array}{c}\log _{10} \\
\eta \\
\text { (Pa s) }\end{array}$ & $f(\mathrm{~Hz})$ & $\begin{array}{c}\log _{10} \\
\omega \tau_{M}\end{array}$ & $\begin{array}{l}\mathbf{G}_{\mathrm{im}} \\
(\mathrm{GPa})\end{array}$ & $\begin{array}{l}\mathbf{G}_{\text {real }} \\
(\mathrm{GPa})\end{array}$ & $\begin{array}{l}\mathbf{G}_{\text {melt }} \\
(\mathrm{GPa})\end{array}$ \\
\hline 850 & \multirow{9}{*}{$\begin{array}{l}\text { o } \\
\text { - } \\
\text { - }\end{array}$} & 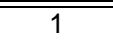 & 1.665 & 4.118 & 21.420 & 21.813 \\
\hline 850 & & 0.5 & 1.364 & 5.600 & 18.408 & 19.241 \\
\hline 850 & & 0.2 & 0.966 & 7.015 & 12.796 & 14.593 \\
\hline 850 & & 0.1 & 0.665 & 6.994 & 7.681 & 10.388 \\
\hline 850 & & 0.05 & 0.364 & 5.223 & 3.477 & 6.275 \\
\hline 850 & & 0.02 & -0.034 & 2.484 & 1.304 & 2.805 \\
\hline 850 & & 0.01 & -0.335 & 1.402 & 0.213 & 1.418 \\
\hline 850 & & 0.005 & -0.636 & 0.659 & 0.054 & 0.661 \\
\hline 850 & & 0.002 & -1.034 & 0.034 & 0.259 & 0.261 \\
\hline 875 & \multirow{5}{*}{$\begin{array}{l}-1 \\
0 \\
0 \\
-1\end{array}$} & 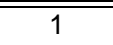 & 1.067 & 6.035 & 14.009 & 15.253 \\
\hline 875 & & 0.5 & 0.766 & 6.343 & 6.934 & 8.182 \\
\hline 875 & & 0.2 & 0.368 & 4.053 & 3.251 & 5.196 \\
\hline 875 & & 0.1 & 0.067 & 2.774 & 1.245 & 3.040 \\
\hline 875 & & 0.05 & -0.234 & 1.356 & 0.719 & 1.535 \\
\hline 900 & \multirow{5}{*}{ - } & 1 & 1.067 & 6.664 & 8.517 & 12.881 \\
\hline 900 & & 0.5 & 0.766 & 5.680 & 3.913 & 10.441 \\
\hline & & & & & & \\
\hline & & & & & & \\
\hline & & & & & & \\
\hline
\end{tabular}


Appendix 2. Temperature $T$, $\log _{10} \eta$, frequency $f$, $\log _{10} \omega \tau_{\mathrm{M}}$, imaginary $(G \mathrm{im})$ and real $\left(\mathrm{G}_{\text {real }}\right)$ shear modulus and shear modulus of the melt $(\mathrm{G}$ melt $)$ for the investigated samples - continuation..

\begin{tabular}{|c|c|c|c|c|c|c|}
\hline \multicolumn{7}{|c|}{ SAMPLE G4 } \\
\hline $\mathrm{T}\left({ }^{\circ} \mathrm{C}\right)$ & $\begin{array}{c}\log _{10} \\
\eta \\
\text { (Pa s) }\end{array}$ & $f(\mathrm{~Hz})$ & $\begin{array}{c}\log _{10} \\
\omega \tau_{M}\end{array}$ & $\begin{array}{c}\mathbf{G}_{\mathrm{im}} \\
(\mathrm{GPa})\end{array}$ & $\begin{array}{l}\mathbf{G}_{\text {real }} \\
(\mathrm{GPa})\end{array}$ & $\begin{array}{l}\mathbf{G}_{\text {melt }} \\
\text { (GPa) }\end{array}$ \\
\hline 550 & \multirow{10}{*}{$\begin{array}{l}\text { m } \\
\text { gे } \\
\text { जे }\end{array}$} & $\overline{11}$ & 9.643 & 0.052 & 34.233 & 34.233 \\
\hline 550 & & 0.5 & 9.342 & 0.088 & 34.215 & 34.215 \\
\hline 550 & & 0.2 & 8.944 & 0.256 & 34.421 & 34.422 \\
\hline 550 & & 0.1 & 8.643 & 0.063 & 34.122 & 34.122 \\
\hline 550 & & 0.05 & 8.342 & 0.117 & 34.290 & 34.290 \\
\hline 550 & & 0.02 & 7.944 & 0.146 & 34.081 & 34.081 \\
\hline 550 & & 0.01 & 7.643 & 0.908 & 33.542 & 33.548 \\
\hline 550 & & 0.005 & 7.342 & 0.368 & 34.386 & 34.388 \\
\hline 550 & & 0.002 & 6.944 & 0.203 & 33.757 & 33.757 \\
\hline 550 & & 0.001 & 6.643 & 0.083 & 32.231 & 32.231 \\
\hline 600 & \multirow{10}{*}{\begin{tabular}{l}
$\underset{f}{+}$ \\
\multirow{-}{*}{}
\end{tabular}} & 1 & 7.731 & 0.585 & 34.193 & 34.193 \\
\hline 600 & & 0.5 & 7.430 & 1.047 & 34.291 & 34.291 \\
\hline 600 & & 0.2 & 7.032 & 0.017 & 34.023 & 34.023 \\
\hline 600 & & 0.1 & 6.731 & 0.128 & 33.918 & 33.918 \\
\hline 600 & & 0.05 & 6.430 & 0.233 & 34.461 & 34.461 \\
\hline 600 & & 0.02 & 6.032 & 0.239 & 33.932 & 33.932 \\
\hline 600 & & 0.01 & 5.731 & 0.045 & 34.220 & 34.220 \\
\hline 600 & & 0.005 & 5.430 & 0.135 & 33.869 & 33.869 \\
\hline 600 & & 0.002 & 5.032 & 0.045 & 33.792 & 33.792 \\
\hline 600 & & 0.001 & 4.731 & 0.175 & 33.446 & 33.447 \\
\hline 650 & \multirow{10}{*}{ 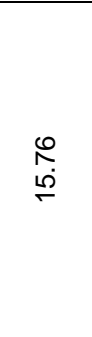 } & 1 & 6.026 & 0.129 & 33.678 & 33.678 \\
\hline 650 & & 0.5 & 5.725 & 0.069 & 33.438 & 33.438 \\
\hline 650 & & 0.2 & 5.327 & 0.063 & 33.008 & 33.008 \\
\hline 650 & & 0.1 & 5.026 & 0.101 & 32.839 & 32.839 \\
\hline 650 & & 0.05 & 4.725 & 0.019 & 33.370 & 33.370 \\
\hline 650 & & 0.02 & 4.327 & 0.219 & 32.803 & 32.804 \\
\hline 650 & & 0.01 & 4.026 & 0.578 & 32.667 & 32.672 \\
\hline 650 & & 0.005 & 3.725 & 0.149 & 33.461 & 33.462 \\
\hline 650 & & 0.002 & 3.327 & 0.545 & 32.556 & 32.560 \\
\hline 650 & & 0.001 & 3.026 & 0.313 & 32.318 & 32.319 \\
\hline
\end{tabular}

\begin{tabular}{|c|c|c|c|c|c|c|}
\hline \multicolumn{7}{|c|}{ SAMPLE G4 } \\
\hline $\mathrm{T}\left({ }^{\circ} \mathrm{C}\right)$ & $\begin{array}{c}\log _{10} \\
\eta \\
\text { (Pa s) }\end{array}$ & $f(\mathrm{~Hz})$ & $\begin{array}{c}\log _{10} \\
\omega \tau_{M}\end{array}$ & $\begin{array}{l}\mathbf{G}_{\text {im }} \\
(\mathbf{G P a})\end{array}$ & $\begin{array}{l}\mathbf{G}_{\text {real }} \\
(\mathrm{GPa})\end{array}$ & $\begin{array}{l}G_{\text {melt }} \\
(\mathrm{GPa})\end{array}$ \\
\hline 675 & \multirow{10}{*}{$\begin{array}{l}\stackrel{\circ}{\circ} \\
\dot{J}\end{array}$} & 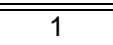 & 5.242 & 0.097 & 32.778 & 32.778 \\
\hline 675 & & 0.5 & 4.941 & 0.120 & 32.812 & 32.812 \\
\hline 675 & & 0.2 & 4.543 & 0.230 & 32.970 & 32.971 \\
\hline 675 & & 0.1 & 4.242 & 0.663 & 32.281 & 32.288 \\
\hline 675 & & 0.05 & 3.941 & 0.036 & 32.664 & 32.664 \\
\hline 675 & & 0.02 & 3.543 & 0.176 & 31.898 & 31.898 \\
\hline 675 & & 0.01 & 3.242 & 0.579 & 31.494 & 31.500 \\
\hline 675 & & 0.005 & 2.941 & 0.397 & 31.634 & 31.636 \\
\hline 675 & & 0.002 & 2.543 & 0.959 & 29.504 & 29.520 \\
\hline 675 & & 0.001 & 2.242 & 1.563 & 30.592 & 30.632 \\
\hline$\overline{7700}$ & \multirow{10}{*}{$\begin{array}{l}\underset{N}{+} \\
\underset{\sim}{+}\end{array}$} & $\overline{11}$ & 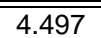 & "0.248 & "31.335 & "31.336 \\
\hline 700 & & 0.5 & 4.196 & 0.252 & 31.374 & 31.375 \\
\hline 700 & & 0.2 & 3.798 & 0.403 & 30.700 & 30.703 \\
\hline 700 & & 0.1 & 3.497 & 0.364 & 30.786 & 30.788 \\
\hline 700 & & 0.05 & 3.196 & 0.495 & 29.906 & 29.910 \\
\hline 700 & & 0.02 & 2.798 & 0.724 & 31.013 & 31.021 \\
\hline 700 & & 0.01 & 2.497 & 0.975 & 29.757 & 29.773 \\
\hline 700 & & 0.005 & 2.196 & 1.386 & 28.993 & 29.026 \\
\hline 700 & & 0.002 & 1.798 & 1.767 & 27.346 & 27.403 \\
\hline 700 & & 0.001 & 1.497 & 0.701 & 26.231 & 26.241 \\
\hline 725 & \multirow{10}{*}{$\begin{array}{l}\stackrel{M}{\Omega} \\
\stackrel{\leftrightarrow}{\rightarrow}\end{array}$} & $\overline{c 1}$ & $\begin{array}{l}3.790 \\
\end{array}$ & $\begin{array}{c}0.449 \\
\end{array}$ & 31.526 & 31.529 \\
\hline 725 & & 0.5 & 3.489 & 0.269 & 31.307 & 31.308 \\
\hline 725 & & 0.2 & 3.091 & 0.673 & 31.064 & 31.071 \\
\hline 725 & & 0.1 & 2.790 & 1.387 & 30.432 & 30.463 \\
\hline 725 & & 0.05 & 2.489 & 0.950 & 30.529 & 30.544 \\
\hline 725 & & 0.02 & 2.091 & 1.543 & 29.317 & 29.358 \\
\hline 725 & & 0.01 & 1.790 & 2.136 & 27.375 & 27.458 \\
\hline 725 & & 0.005 & 1.489 & 3.646 & 26.113 & 26.366 \\
\hline 725 & & 0.002 & 1.091 & 5.299 & 22.650 & 23.261 \\
\hline 725 & & 0.001 & 0.790 & 6.916 & 19.926 & 20.524 \\
\hline
\end{tabular}


Appendix 2. Temperature $T, \log _{10} \eta$, frequency $f, \log _{10} \omega \tau_{\mathrm{M}}$, imaginary $\left(G_{\mathrm{im}}\right)$ and real ( $(\mathrm{G}$ real) shear modulus and shear modulus of the melt ( $G$ melt) for the investigated samples - continuation...

\begin{tabular}{|c|c|c|c|c|c|c|}
\hline \multicolumn{7}{|c|}{ SAMPLE G4 } \\
\hline $\mathrm{T}\left({ }^{\circ} \mathrm{C}\right)$ & $\begin{array}{c}\log _{10} \\
\eta \\
\text { (Pa s) }\end{array}$ & $f(\mathrm{~Hz})$ & $\begin{array}{c}\log _{10} \\
\omega \tau_{M}\end{array}$ & $\begin{array}{c}\mathbf{G}_{\text {im }} \\
(\mathbf{G P a})\end{array}$ & $\begin{array}{l}\mathbf{G}_{\text {real }} \\
(\mathrm{GPa})\end{array}$ & $\begin{array}{l}\mathbf{G}_{\text {melt }} \\
\text { (GPa) }\end{array}$ \\
\hline 750 & \multirow{10}{*}{$\begin{array}{l}\stackrel{\llcorner}{\infty} \\
\stackrel{\leftrightarrow}{\sim}\end{array}$} & $\overline{11}$ & 3.117 & 0.565 & 29.964 & 29.969 \\
\hline 750 & & 0.5 & 2.816 & 0.878 & 29.474 & 29.487 \\
\hline 750 & & 0.2 & 2.418 & 1.298 & 28.959 & 28.988 \\
\hline 750 & & 0.1 & 2.117 & 1.430 & 28.265 & 28.301 \\
\hline 750 & & 0.05 & 1.816 & 1.860 & 26.752 & 26.816 \\
\hline 750 & & 0.02 & 1.418 & 4.101 & 23.780 & 24.131 \\
\hline 750 & & 0.01 & 1.117 & 5.374 & 21.531 & 22.192 \\
\hline 750 & & 0.005 & 0.816 & 7.497 & 19.753 & 21.127 \\
\hline 750 & & 0.002 & 0.418 & 8.260 & 12.200 & 13.712 \\
\hline 750 & & 0.001 & 0.117 & 6.165 & 5.819 & 8.478 \\
\hline "775 & \multirow{10}{*}{ 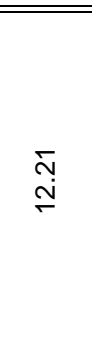 } & 1 & $\begin{array}{l}2.477 \\
\end{array}$ & $\bar{~} 1.404$ & 229.255 & $\bar{~} 29.289$ \\
\hline 775 & & 0.5 & 2.176 & 1.853 & 28.316 & 28.377 \\
\hline 775 & & 0.2 & 1.778 & 2.668 & 25.914 & 26.051 \\
\hline 775 & & 0.1 & 1.477 & 4.069 & 24.727 & 25.060 \\
\hline 775 & & 0.05 & 1.176 & 5.848 & 20.886 & 21.690 \\
\hline 775 & & 0.02 & 0.778 & 7.553 & 15.852 & 17.560 \\
\hline 775 & & 0.01 & 0.477 & 6.804 & 13.978 & 14.001 \\
\hline 775 & & 0.005 & 0.176 & 6.471 & 4.759 & 8.033 \\
\hline 775 & & 0.002 & -0.222 & 3.430 & 1.214 & 3.639 \\
\hline 775 & & 0.001 & -0.523 & 1.701 & 0.652 & 1.822 \\
\hline 800 & \multirow{10}{*}{$\begin{array}{l}8 \\
\stackrel{8}{-} \\
\stackrel{-}{1}\end{array}$} & 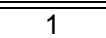 & $\begin{array}{ll}1.866 \\
\end{array}$ & $\begin{array}{l}3.143 \\
\end{array}$ & 28.058 & 28.233 \\
\hline 800 & & 0.5 & 1.565 & 4.341 & 26.065 & 26.424 \\
\hline 800 & & 0.2 & 1.167 & 6.459 & 21.566 & 22.512 \\
\hline 800 & & 0.1 & 0.866 & 7.890 & 16.895 & 18.646 \\
\hline 800 & & 0.05 & 0.565 & 8.447 & 11.020 & 13.885 \\
\hline 800 & & 0.02 & 0.167 & 6.095 & 3.927 & 7.251 \\
\hline 800 & & 0.01 & -0.134 & 3.668 & 1.336 & 3.903 \\
\hline 800 & & 0.005 & -0.435 & 5.452 & 0.382 & 5.466 \\
\hline 800 & & 0.002 & -0.833 & 0.825 & 0.105 & 0.831 \\
\hline 800 & & 0.001 & -1.134 & 0.424 & 0.177 & 0.459 \\
\hline
\end{tabular}

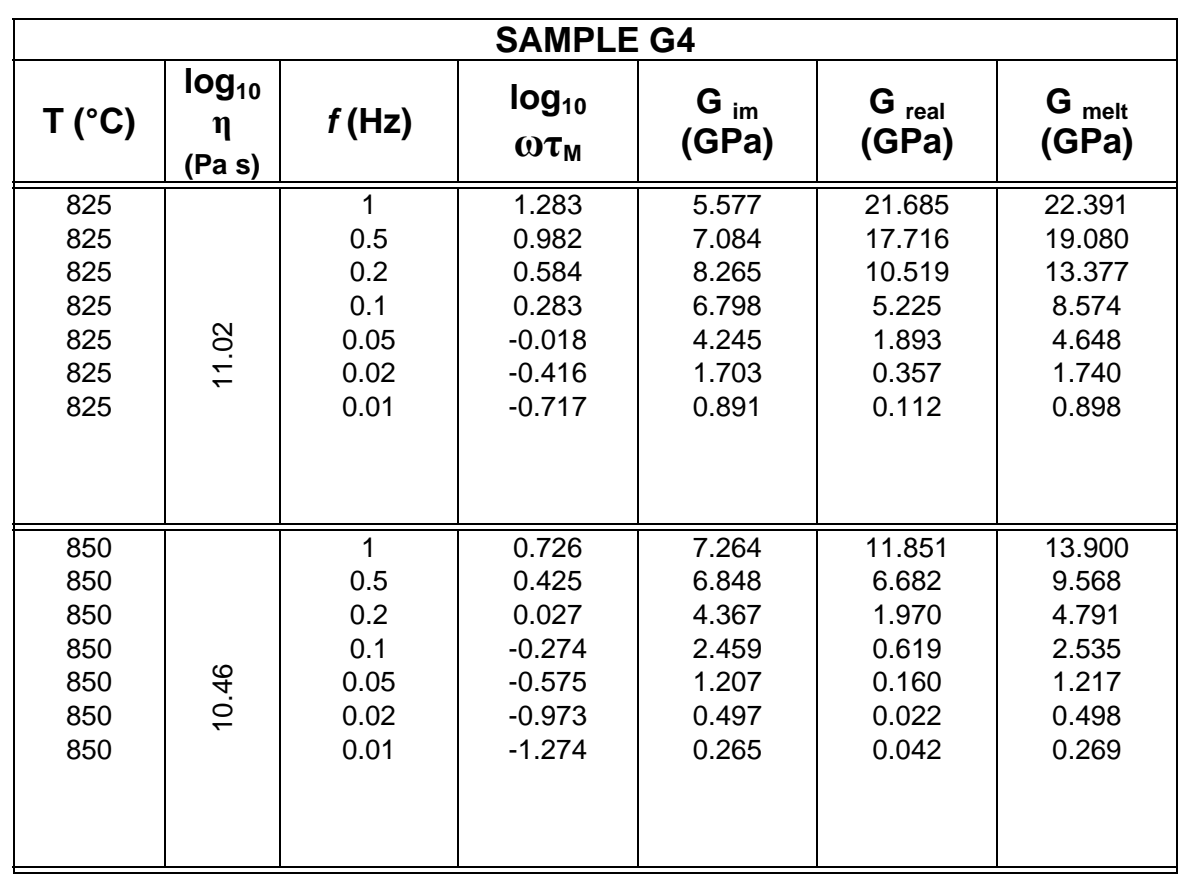


Appendix 2. Temperature $T$, $\log _{10} \eta$, frequency $f$, $\log _{10} \omega \tau_{\mathrm{M}}$, imaginary $(G \mathrm{im})$ and real $\left(\mathrm{G}_{\text {real }}\right)$ shear modulus and shear modulus of the melt $(\mathrm{G}$ melt $)$ for the investigated samples - continuation..

\begin{tabular}{|c|c|c|c|c|c|c|}
\hline \multicolumn{7}{|c|}{ SAMPLE G5 } \\
\hline $\mathrm{T}\left({ }^{\circ} \mathrm{C}\right)$ & $\begin{array}{c}\log _{10} \\
\eta \\
\text { (Pa s) }\end{array}$ & $f(\mathrm{~Hz})$ & $\begin{array}{c}\log _{10} \\
\omega \tau_{M}\end{array}$ & $\begin{array}{c}\mathbf{G}_{\mathrm{im}} \\
(\mathrm{GPa})\end{array}$ & $\begin{array}{l}\mathbf{G}_{\text {real }} \\
(\mathrm{GPa})\end{array}$ & $\begin{array}{c}\mathbf{G}_{\text {melt }} \\
(\mathrm{GPa})\end{array}$ \\
\hline 500 & \multirow{10}{*}{$\begin{array}{l}0 \\
\stackrel{0}{0} \\
\infty \\
-1\end{array}$} & 1 & 8.929 & 0.465 & 34.004 & 34.007 \\
\hline 500 & & 0.5 & 8.628 & 0.439 & 33.968 & 33.970 \\
\hline 500 & & 0.2 & 8.230 & 0.396 & 33.608 & 33.611 \\
\hline 500 & & 0.1 & 7.929 & 1.023 & 33.098 & 33.133 \\
\hline 500 & & 0.05 & 7.628 & 1.560 & 33.038 & 33.043 \\
\hline 500 & & 0.02 & 7.230 & 0.985 & 33.331 & 33.343 \\
\hline 500 & & 0.01 & 6.929 & 0.385 & 32.903 & 32.905 \\
\hline 500 & & 0.005 & 6.628 & 0.508 & 32.716 & 32.720 \\
\hline 500 & & 0.002 & 6.230 & 0.311 & 33.436 & 33.437 \\
\hline 500 & & 0.001 & 5.929 & 0.549 & 32.448 & 32.453 \\
\hline 2550 & \multirow{10}{*}{$\begin{array}{l}\stackrel{8}{0} \\
\dot{\leftrightarrow}\end{array}$} & 1 & 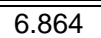 & $\begin{array}{l}0.289 \\
\end{array}$ & 32.735 & 32.736 \\
\hline 550 & & 0.5 & 6.563 & 0.634 & 32.548 & 32.554 \\
\hline 550 & & 0.2 & 6.165 & 0.667 & 32.238 & 32.245 \\
\hline 550 & & 0.1 & 5.864 & 0.459 & 32.105 & 32.108 \\
\hline 550 & & 0.05 & 5.563 & 0.130 & 31.503 & 31.504 \\
\hline 550 & & 0.02 & 5.165 & 0.272 & 31.283 & 31.284 \\
\hline 550 & & 0.01 & 4.864 & 0.639 & 31.909 & 31.915 \\
\hline 550 & & 0.005 & 4.563 & 0.306 & 31.566 & 31.568 \\
\hline 550 & & 0.002 & 4.165 & 0.449 & 31.352 & 31.355 \\
\hline 550 & & 0.001 & 3.864 & 0.587 & 30.799 & 30.804 \\
\hline (2600 & \multirow{10}{*}{$\begin{array}{l}\hat{N} \\
\stackrel{\dot{J}}{+}\end{array}$} & 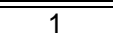 & 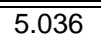 & $\begin{array}{l}0.502 \\
\end{array}$ & 31.524 & 31.528 \\
\hline 600 & & 0.5 & 4.735 & 0.522 & 31.396 & 31.400 \\
\hline 600 & & 0.2 & 4.337 & 0.401 & 31.339 & 31.342 \\
\hline 600 & & 0.1 & 4.036 & 0.529 & 31.350 & 31.354 \\
\hline 600 & & 0.05 & 3.735 & 1.021 & 31.146 & 31.162 \\
\hline 600 & & 0.02 & 3.337 & 0.540 & 31.131 & 31.135 \\
\hline 600 & & 0.01 & 3.036 & 0.429 & 29.807 & 29.810 \\
\hline 600 & & 0.005 & 2.735 & 0.722 & 29.647 & 29.656 \\
\hline 600 & & 0.002 & 2.337 & 1.348 & 29.209 & 29.240 \\
\hline 600 & & 0.001 & 2.036 & 1.419 & 28.554 & 28.589 \\
\hline
\end{tabular}

\begin{tabular}{|c|c|c|c|c|c|c|}
\hline \multicolumn{7}{|c|}{ SAMPLE G5 } \\
\hline $\mathrm{T}\left({ }^{\circ} \mathrm{C}\right)$ & $\begin{array}{c}\log _{10} \\
\eta \\
\text { (Pa s) }\end{array}$ & $f(\mathrm{~Hz})$ & $\begin{array}{c}\log _{10} \\
\omega \tau_{M}\end{array}$ & $\begin{array}{c}\mathbf{G}_{\text {im }} \\
(\mathrm{GPa})\end{array}$ & $\begin{array}{l}\mathbf{G}_{\text {real }} \\
(\mathrm{GPa})\end{array}$ & $\begin{array}{c}\mathbf{G}_{\text {melt }} \\
(\mathrm{GPa})\end{array}$ \\
\hline 625 & \multirow{10}{*}{ 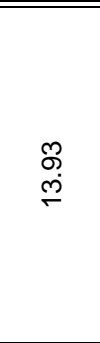 } & 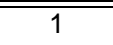 & 4.198 & 0.510 & 30.835 & 30.839 \\
\hline 625 & & 0.5 & 3.897 & 0.396 & 30.709 & 30.712 \\
\hline 625 & & 0.2 & 3.499 & 0.520 & 30.292 & 30.297 \\
\hline 625 & & 0.1 & 3.198 & 0.980 & 30.106 & 30.122 \\
\hline 625 & & 0.05 & 2.897 & 1.028 & 29.215 & 29.233 \\
\hline 625 & & 0.02 & 2.499 & 1.544 & 29.753 & 29.793 \\
\hline 625 & & 0.01 & 2.198 & 1.719 & 28.392 & 28.444 \\
\hline 625 & & 0.005 & 1.897 & 2.114 & 27.700 & 27.781 \\
\hline 625 & & 0.002 & 1.499 & 2.846 & 26.574 & 26.726 \\
\hline 625 & & 0.001 & 1.198 & 1.107 & 25.487 & 25.511 \\
\hline "650 & \multirow{10}{*}{$\stackrel{4}{4}$} & 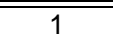 & $\begin{array}{l}3.406 \\
\end{array}$ & 0.920 & "30.333 & " 30.346 \\
\hline 650 & & 0.5 & 3.105 & 1.022 & 30.305 & 30.322 \\
\hline 650 & & 0.2 & 2.707 & 1.216 & 29.979 & 30.004 \\
\hline 650 & & 0.1 & 2.406 & 1.561 & 29.278 & 29.320 \\
\hline 650 & & 0.05 & 2.105 & 1.654 & 28.754 & 28.801 \\
\hline 650 & & 0.02 & 1.707 & 2.352 & 27.211 & 27.312 \\
\hline 650 & & 0.01 & 1.406 & 3.433 & 25.843 & 26.070 \\
\hline 650 & & 0.005 & 1.105 & 4.342 & 23.686 & 24.081 \\
\hline 650 & & 0.002 & 0.707 & 3.225 & 20.969 & 21.216 \\
\hline 650 & & 0.001 & 0.406 & 6.447 & 16.107 & 17.350 \\
\hline 675 & \multirow{10}{*}{$\begin{array}{l}\stackrel{\text { }}{\mathfrak{H}} \\
\text { }\end{array}$} & 1 & 2.655 & 1.226 & 29.327 & 29.352 \\
\hline 675 & & 0.5 & 2.354 & 1.605 & 28.183 & 28.228 \\
\hline 675 & & 0.2 & 1.956 & 1.746 & 26.652 & 26.709 \\
\hline 675 & & 0.1 & 1.655 & 2.762 & 25.955 & 26.101 \\
\hline 675 & & 0.05 & 1.354 & 3.735 & 24.125 & 24.412 \\
\hline 675 & & 0.02 & 0.956 & 5.252 & 20.607 & 21.266 \\
\hline 675 & & 0.01 & 0.655 & 5.740 & 18.224 & 19.107 \\
\hline 675 & & 0.005 & 0.354 & 6.685 & 15.089 & 15.532 \\
\hline 675 & & 0.002 & -0.044 & 7.378 & 8.480 & 9.128 \\
\hline 675 & & 0.001 & -0.345 & 3.988 & 2.175 & 4.543 \\
\hline
\end{tabular}


Appendix 2. Temperature $T, \log _{10} \eta$, frequency $f, \log _{10} \omega \tau_{\mathrm{M}}$, imaginary $\left(G_{\mathrm{im}}\right)$ and real ( $(\mathrm{G}$ real) shear modulus and shear modulus of the melt ( $G$ melt) for the investigated samples - continuation...

\begin{tabular}{|c|c|c|c|c|c|c|}
\hline \multicolumn{7}{|c|}{ SAMPLE G5 } \\
\hline $\mathrm{T}\left({ }^{\circ} \mathrm{C}\right)$ & $\begin{array}{c}\log _{10} \\
\eta \\
\text { (Pa s) }\end{array}$ & $f(\mathrm{~Hz})$ & $\begin{array}{c}\log _{10} \\
\omega \tau_{M}\end{array}$ & $\begin{array}{l}\mathbf{G}_{\text {im }} \\
(\mathbf{G P a})\end{array}$ & $\begin{array}{l}\mathbf{G}_{\text {real }} \\
(\mathrm{GPa})\end{array}$ & $\begin{array}{l}\mathbf{G}_{\text {melt }} \\
\text { (GPa) }\end{array}$ \\
\hline$\overline{700}$ & \multirow{10}{*}{ 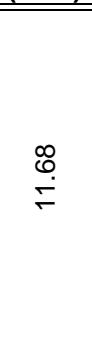 } & $\overline{11}$ & $\overline{|c| 943}$ & $\begin{array}{l}1.845 \\
\end{array}$ & 25.748 & 25.814 \\
\hline 700 & & 0.5 & 1.642 & 2.330 & 24.235 & 24.347 \\
\hline 700 & & 0.2 & 1.244 & 3.783 & 22.278 & 22.597 \\
\hline 700 & & 0.1 & 0.943 & 5.643 & 19.567 & 20.364 \\
\hline 700 & & 0.05 & 0.642 & 6.940 & 15.758 & 17.219 \\
\hline 700 & & 0.02 & 0.244 & 6.465 & 8.638 & 11.417 \\
\hline 700 & & 0.01 & -0.057 & 6.494 & 8.397 & 8.412 \\
\hline 700 & & 0.005 & -0.358 & 3.707 & 1.615 & 4.043 \\
\hline 700 & & 0.002 & -0.756 & 1.771 & 0.386 & 1.812 \\
\hline 700 & & 0.001 & -1.057 & 0.865 & 0.166 & 0.881 \\
\hline 725 & \multirow{9}{*}{$\begin{array}{l}8 \\
\text { - } \\
\text { ت }\end{array}$} & $\overline{11}$ & 1.267 & 3.904 & 23.141 & 23.468 \\
\hline 725 & & 0.5 & 0.966 & 5.464 & 20.439 & 21.157 \\
\hline 725 & & 0.2 & 0.568 & 6.374 & 14.856 & 16.585 \\
\hline 725 & & 0.1 & 0.267 & 6.553 & 9.131 & 11.850 \\
\hline 725 & & 0.05 & -0.034 & 6.104 & 4.324 & 7.481 \\
\hline 725 & & 0.02 & -0.432 & 3.040 & 1.014 & 3.205 \\
\hline 725 & & 0.01 & -0.733 & 2.261 & 0.361 & 2.290 \\
\hline 725 & & 0.005 & -1.034 & 1.107 & 0.131 & 1.115 \\
\hline \multicolumn{6}{|l|}{725} & \\
\hline 750 & \multirow{6}{*}{$\begin{array}{l}\stackrel{0}{0} \\
\text { @ं }\end{array}$} & $\overline{11}$ & 0.624 & 6.554 & 15.428 & 16.762 \\
\hline 750 & & 0.5 & 0.323 & 3.444 & 15.342 & 15.724 \\
\hline 750 & & 0.2 & -0.075 & 6.345 & 4.200 & 7.609 \\
\hline \multirow[t]{3}{*}{750} & & 0.1 & -0.376 & 3.892 & 1.465 & 4.158 \\
\hline & & 0.05 & -0.677 & 2.361 & 0.482 & 2.410 \\
\hline & & & & & & \\
\hline & & & & & & \\
\hline & & & & & & \\
\hline
\end{tabular}

\begin{tabular}{|c|c|c|c|c|c|c|}
\hline \multicolumn{7}{|c|}{ SAMPLE G6 } \\
\hline $\mathrm{T}\left({ }^{\circ} \mathrm{C}\right)$ & $\begin{array}{c}\log _{10} \\
\eta \\
\text { (Pa s) }\end{array}$ & $f(\mathrm{~Hz})$ & $\begin{array}{c}\log _{10} \\
\omega \tau_{M}\end{array}$ & $\begin{array}{c}\mathbf{G}_{\text {im }} \\
(\mathrm{GPa})\end{array}$ & $\begin{array}{c}\mathbf{G}_{\text {real }} \\
(\mathrm{GPa})\end{array}$ & $\begin{array}{l}\mathbf{G}_{\text {melt }} \\
(\mathrm{GPa})\end{array}$ \\
\hline "450 & \multirow{10}{*}{$\begin{array}{l}\stackrel{\infty}{+} \\
\stackrel{\rightarrow}{\rightarrow}\end{array}$} & $\bar{~} 1$ & $\begin{array}{l}9.757 \\
\end{array}$ & $\begin{array}{l}0.122 \\
\end{array}$ & $\begin{array}{c}33.471 \\
\end{array}$ & 33.471 \\
\hline 450 & & 0.5 & 9.456 & 0.074 & 33.626 & 33.626 \\
\hline 450 & & 0.2 & 9.058 & 0.074 & 34.231 & 34.231 \\
\hline 450 & & 0.1 & 8.757 & 0.222 & 33.451 & 33.162 \\
\hline 450 & & 0.05 & 8.456 & 0.047 & 33.393 & 33.293 \\
\hline 450 & & 0.02 & 8.058 & 0.304 & 33.727 & 33.428 \\
\hline 450 & & 0.01 & 7.757 & 1.051 & 33.840 & 33.540 \\
\hline 450 & & 0.005 & 7.456 & 0.536 & 33.330 & 33.130 \\
\hline 450 & & 0.002 & 7.058 & 0.409 & 32.915 & 32.915 \\
\hline 450 & & 0.001 & 6.757 & 0.086 & 33.001 & 33.001 \\
\hline "2500 & \multirow{10}{*}{ 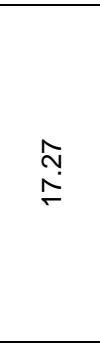 } & $\bar{~} 1$ & $=8.545$ & $\begin{array}{l}0.708 \\
\end{array}$ & "32.946 & " 32.946 \\
\hline 500 & & 0.5 & 7.244 & 0.031 & 32.593 & 32.593 \\
\hline 500 & & 0.2 & 6.846 & 0.222 & 32.771 & 32.771 \\
\hline 500 & & 0.1 & 6.545 & 0.324 & 32.451 & 32.451 \\
\hline 500 & & 0.05 & 6.244 & 0.197 & 32.079 & 32.079 \\
\hline 500 & & 0.02 & 5.846 & 0.100 & 31.556 & 31.556 \\
\hline 500 & & 0.01 & 5.545 & 0.164 & 32.509 & 32.509 \\
\hline 500 & & 0.005 & 5.244 & 0.059 & 32.360 & 32.360 \\
\hline 500 & & 0.002 & 4.846 & 0.133 & 33.327 & 33.327 \\
\hline 500 & & 0.001 & 4.545 & 0.212 & 32.051 & 32.052 \\
\hline 550 & \multirow{10}{*}{ 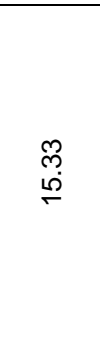 } & 1 & 5.602 & 0.155 & 31.754 & 31.754 \\
\hline 550 & & 0.5 & 5.300 & 0.094 & 32.058 & 32.058 \\
\hline 550 & & 0.2 & 4.903 & 0.133 & 31.710 & 31.710 \\
\hline 550 & & 0.1 & 4.602 & 0.101 & 31.574 & 31.574 \\
\hline 550 & & 0.05 & 4.300 & 0.425 & 32.198 & 32.201 \\
\hline 550 & & 0.02 & 3.903 & 0.322 & 31.934 & 31.935 \\
\hline 550 & & 0.01 & 3.602 & 0.004 & 31.402 & 31.402 \\
\hline 550 & & 0.005 & 3.300 & 0.149 & 31.609 & 31.609 \\
\hline 550 & & 0.002 & 2.903 & 0.206 & 28.179 & 28.179 \\
\hline 550 & & 0.001 & 2.602 & 0.251 & 29.024 & 29.025 \\
\hline
\end{tabular}


Appendix 2. Temperature $T$, $\log _{10} \eta$, frequency $f$, $\log _{10} \omega \tau_{\mathrm{M}}$, imaginary $(G \mathrm{im})$ and real $\left(\mathrm{G}_{\text {real }}\right)$ shear modulus and shear modulus of the melt $(\mathrm{G}$ melt $)$ for the investigated samples - continuation..

\begin{tabular}{|c|c|c|c|c|c|c|}
\hline \multicolumn{7}{|c|}{ SAMPLE G6 } \\
\hline $\mathrm{T}\left({ }^{\circ} \mathrm{C}\right)$ & $\begin{array}{c}\log _{10} \\
\eta \\
\text { (Pa s) }\end{array}$ & $f(\mathrm{~Hz})$ & $\begin{array}{c}\log _{10} \\
\omega \tau_{M}\end{array}$ & $\begin{array}{c}\mathbf{G}_{\mathrm{im}} \\
(\mathbf{G P a})\end{array}$ & $\begin{array}{l}\mathbf{G}_{\text {real }} \\
(\mathrm{GPa})\end{array}$ & $\begin{array}{l}\mathbf{G}_{\text {melt }} \\
(\mathrm{GPa})\end{array}$ \\
\hline 2575 & \multirow{10}{*}{$\begin{array}{l}\text { J } \\
\text { - }\end{array}$} & 1 & 4.716 & $\begin{array}{l}0.089 \\
\end{array}$ & 31.713 & 31.713 \\
\hline 575 & & 0.5 & 4.415 & 0.145 & 31.252 & 31.252 \\
\hline 575 & & 0.2 & 4.017 & 0.129 & 31.477 & 31.478 \\
\hline 575 & & 0.1 & 3.716 & 0.230 & 30.783 & 30.784 \\
\hline 575 & & 0.05 & 3.415 & 0.404 & 31.434 & 31.437 \\
\hline 575 & & 0.02 & 3.017 & 0.100 & 31.140 & 31.140 \\
\hline 575 & & 0.01 & 2.716 & 0.672 & 30.341 & 30.348 \\
\hline 575 & & 0.005 & 2.415 & 0.447 & 30.200 & 30.203 \\
\hline 575 & & 0.002 & 2.017 & 0.357 & 30.200 & 30.202 \\
\hline 575 & & 0.001 & 1.716 & 0.738 & 28.570 & 28.580 \\
\hline 600 & \multirow{10}{*}{$\begin{array}{l}-1 \\
\stackrel{9}{\oplus}\end{array}$} & $\bar{~} 1$ & "3.881 & 0.153 & 31.620 & $\overline{231.621}$ \\
\hline 600 & & 0.5 & 3.580 & 0.185 & 31.435 & 31.436 \\
\hline 600 & & 0.2 & 3.182 & 0.453 & 30.880 & 30.883 \\
\hline 600 & & 0.1 & 2.881 & 0.267 & 30.551 & 30.552 \\
\hline 600 & & 0.05 & 2.580 & 0.210 & 30.645 & 30.646 \\
\hline 600 & & 0.02 & 2.182 & 0.845 & 29.936 & 29.947 \\
\hline 600 & & 0.01 & 1.881 & 1.067 & 29.826 & 29.845 \\
\hline 600 & & 0.005 & 1.580 & 1.871 & 28.865 & 28.926 \\
\hline 600 & & 0.002 & 1.182 & 1.713 & 26.844 & 26.899 \\
\hline 600 & & 0.001 & 0.881 & 1.123 & 25.201 & 25.226 \\
\hline "625 & \multirow{10}{*}{$\begin{array}{l}\underset{\infty}{\infty} \\
\underset{H}{H}\end{array}$} & 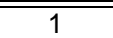 & $\begin{array}{l}3.092 \\
\end{array}$ & $\begin{array}{l}0.373 \\
\end{array}$ & 31.168 & 31.171 \\
\hline 625 & & 0.5 & 2.791 & 0.548 & 30.810 & 30.815 \\
\hline 625 & & 0.2 & 2.393 & 0.885 & 30.346 & 30.359 \\
\hline 625 & & 0.1 & 2.092 & 1.170 & 29.847 & 29.870 \\
\hline 625 & & 0.05 & 1.791 & 1.584 & 28.380 & 28.424 \\
\hline 625 & & 0.02 & 1.393 & 2.494 & 26.927 & 27.042 \\
\hline 625 & & 0.01 & 1.092 & 3.517 & 25.350 & 25.593 \\
\hline 625 & & 0.005 & 0.791 & 4.218 & 23.059 & 23.441 \\
\hline 625 & & 0.002 & 0.393 & 5.202 & 18.798 & 19.262 \\
\hline 625 & & 0.001 & 0.092 & 5.892 & 14.194 & 15.369 \\
\hline
\end{tabular}

\begin{tabular}{|c|c|c|c|c|c|c|}
\hline \multicolumn{7}{|c|}{ SAMPLE G6 } \\
\hline $\mathrm{T}\left({ }^{\circ} \mathrm{C}\right)$ & $\begin{array}{c}\log _{10} \\
\eta \\
\text { (Pa s) }\end{array}$ & $f(\mathrm{~Hz})$ & $\begin{array}{c}\log _{10} \\
\omega \tau_{M}\end{array}$ & $\begin{array}{c}\mathbf{G}_{\text {im }} \\
(\mathrm{GPa})\end{array}$ & $\begin{array}{l}\mathbf{G}_{\text {real }} \\
(\mathrm{GPa})\end{array}$ & $\begin{array}{c}\mathbf{G}_{\text {melt }} \\
(\mathrm{GPa})\end{array}$ \\
\hline "650 & & $\bar{~} 1$ & $\begin{array}{l}2.347 \\
\end{array}$ & $\begin{array}{l}1.099 \\
\end{array}$ & 30.177 & 30.197 \\
\hline 650 & & 0.5 & 2.046 & 1.450 & 29.310 & 29.346 \\
\hline 650 & & 0.2 & 1.648 & 2.439 & 27.769 & 27.876 \\
\hline 650 & & 0.1 & 1.347 & 3.519 & 26.137 & 26.373 \\
\hline 650 & ¿ & 0.05 & 1.046 & 4.425 & 23.413 & 23.828 \\
\hline 650 & $\stackrel{\sim}{\exists}$ & 0.02 & 0.648 & 5.613 & 18.769 & 19.900 \\
\hline 650 & & 0.01 & 0.347 & 5.823 & 15.940 & 16.653 \\
\hline 650 & & 0.005 & 0.046 & 5.566 & 11.260 & 12.150 \\
\hline 650 & & 0.002 & -0.352 & 2.637 & 2.979 & 3.979 \\
\hline 650 & & 0.001 & -0.653 & 2.169 & 1.346 & 2.553 \\
\hline "675 & & 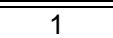 & $\begin{array}{ll}1.640 \\
\end{array}$ & 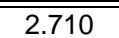 & 26.795 & 26.932 \\
\hline 675 & & 0.5 & 1.339 & 3.837 & 24.926 & 25.220 \\
\hline 675 & & 0.2 & 0.941 & 5.599 & 21.155 & 21.883 \\
\hline 675 & & 0.1 & 0.640 & 5.181 & 17.101 & 18.548 \\
\hline 675 & $\hat{m}$ & 0.05 & 0.339 & 5.710 & 11.673 & 14.101 \\
\hline 675 & تં & 0.02 & -0.059 & 4.862 & 8.698 & 9.157 \\
\hline 675 & & 0.01 & -0.360 & 3.001 & 3.626 & 4.707 \\
\hline 675 & & 0.005 & -0.661 & 1.960 & 0.917 & 2.164 \\
\hline 675 & & 0.002 & -1.059 & 0.930 & 0.230 & 0.958 \\
\hline 7700 & & $\overline{11}$ & $\begin{array}{c}0.970 \\
\end{array}$ & $\overline{5.074}$ & 21.440 & 22.257 \\
\hline 700 & & 0.5 & 0.669 & 5.732 & 17.167 & 18.746 \\
\hline 700 & & 0.2 & 0.271 & 5.498 & 9.540 & 12.513 \\
\hline 700 & & 0.1 & -0.030 & 3.482 & 4.367 & 7.651 \\
\hline 700 & ? & 0.05 & -0.331 & 3.396 & 4.228 & 4.555 \\
\hline 700 & 웜 & 0.02 & -0.729 & 1.512 & 0.542 & 1.606 \\
\hline 700 & & 0.01 & -1.030 & 0.849 & 0.146 & 0.861 \\
\hline 700 & & 0.005 & -1.331 & 0.460 & 0.068 & 0.465 \\
\hline
\end{tabular}


Appendix 2. Temperature $T, \log _{10} \eta$, frequency $f, \log _{10} \omega \tau_{\mathrm{M}}$, imaginary $\left(G_{\mathrm{im}}\right)$ and real ( $(\mathrm{G}$ real) shear modulus and shear modulus of the melt ( $G$ melt) for the investigated samples - continuation...

\begin{tabular}{|c|c|c|c|c|c|c|}
\hline \multicolumn{7}{|c|}{ SAMPLE G7 } \\
\hline $\mathrm{T}\left({ }^{\circ} \mathrm{C}\right)$ & $\begin{array}{c}\log _{10} \\
\eta \\
\text { (Pa s) }\end{array}$ & $f(\mathrm{~Hz})$ & $\begin{array}{c}\log _{10} \\
\omega \tau_{M}\end{array}$ & $\begin{array}{l}\mathbf{G}_{\mathrm{im}} \\
(\mathrm{GPa})\end{array}$ & $\begin{array}{l}\mathbf{G}_{\text {real }} \\
(\mathrm{GPa})\end{array}$ & $\begin{array}{l}\mathbf{G}_{\text {melt }} \\
\text { (GPa) }\end{array}$ \\
\hline 500 & & $\overline{11}$ & "6.047 & 0.111 & 31.678 & 31.678 \\
\hline 500 & & 0.1 & 5.047 & 0.062 & 31.228 & 31.228 \\
\hline 500 & & 0.01 & 4.047 & 0.286 & 30.903 & 30.904 \\
\hline 500 & & 0.001 & 3.047 & 0.071 & 30.588 & 30.588 \\
\hline 550 & & $\overline{11}$ & 4.073 & 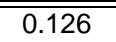 & 30.864 & 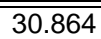 \\
\hline 550 & & 0.1 & 3.073 & 0.050 & 30.941 & 30.941 \\
\hline 550 & & 0.01 & 2.073 & 0.547 & 28.555 & 28.561 \\
\hline 550 & & 0.001 & 1.073 & 3.132 & 24.156 & 24.358 \\
\hline 类600 & & $\overline{11}$ & $\begin{array}{l}2.325 \\
\end{array}$ & 0.958 & 29.439 & 29.454 \\
\hline 600 & & 0.1 & 1.325 & 2.269 & 25.392 & 25.493 \\
\hline 600 & & 0.01 & 0.325 & 11.212 & 16.151 & 19.661 \\
\hline 600 & & 0.001 & -0.675 & 6.136 & 8.244 & 10.277 \\
\hline 630 & & 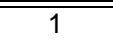 & 0.686 & 8.227 & 20.892 & 222.453 \\
\hline 630 & & 0.1 & -0.314 & 6.671 & 10.092 & 12.098 \\
\hline 630 & & 0.01 & -1.314 & 0.760 & 1.927 & 2.071 \\
\hline 650 & & 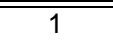 & "-0.135 & 9.480 & 13.520 & 1016.546 \\
\hline 650 & & 0.1 & -1.135 & 3.589 & 1.325 & 3.826 \\
\hline 650 & & 0.01 & -2.135 & 0.503 & 0.249 & 0.561 \\
\hline 670 & & 1 & -0.632 & 3.868 & "5.388 & 6.633 \\
\hline 670 & & 0.1 & -1.632 & 1.353 & 0.216 & 1.370 \\
\hline 670 & & 0.01 & -2.632 & 0.140 & 0.003 & 0.140 \\
\hline
\end{tabular}

\begin{tabular}{|c|c|c|c|c|c|c|}
\hline \multicolumn{7}{|c|}{ SAMPLE G7 } \\
\hline $\mathrm{T}\left({ }^{\circ} \mathrm{C}\right)$ & $\begin{array}{c}\log _{10} \\
\eta \\
(\mathrm{Pa} \mathrm{s})\end{array}$ & $f(\mathrm{~Hz})$ & $\begin{array}{c}\log _{10} \\
\omega \tau_{M}\end{array}$ & $\begin{array}{c}\mathbf{G}_{\mathrm{im}} \\
(\mathrm{GPa})\end{array}$ & $\begin{array}{l}\mathbf{G}_{\text {real }} \\
(\mathbf{G P a})\end{array}$ & $\begin{array}{l}G_{\text {melt }} \\
(\mathrm{GPa})\end{array}$ \\
\hline 700 & & 1 & -0.632 & 5.569 & 0.795 & 5.625 \\
\hline 700 & & 0.1 & -1.632 & 0.424 & 0.683 & 0.804 \\
\hline 700 & & 0.01 & -2.632 & 0.158 & 0.404 & 0.434 \\
\hline
\end{tabular}


Appendix 2. Temperature $T$, $\log _{10} \eta$, frequency $f$, $\log _{10} \omega \tau_{\mathrm{M}}$, imaginary $(G \mathrm{im})$ and real $\left(\mathrm{G}_{\text {real }}\right)$ shear modulus and shear modulus of the melt $(\mathrm{G}$ melt $)$ for the investigated samples - continuation..

\begin{tabular}{|c|c|c|c|c|c|c|}
\hline \multicolumn{7}{|c|}{ SAMPLE G8 } \\
\hline $\mathrm{T}\left({ }^{\circ} \mathrm{C}\right)$ & $\begin{array}{c}\log _{10} \\
\eta \\
\text { (Pa s) }\end{array}$ & $f(\mathrm{~Hz})$ & $\begin{array}{c}\log _{10} \\
\omega \tau_{M}\end{array}$ & $\begin{array}{c}\mathbf{G}_{\mathrm{im}} \\
(\mathrm{GPa})\end{array}$ & $\begin{array}{l}\mathbf{G}_{\text {real }} \\
(\mathrm{GPa})\end{array}$ & $\begin{array}{l}\mathbf{G}_{\text {melt }} \\
(\mathrm{GPa})\end{array}$ \\
\hline 600 & \multirow{10}{*}{ 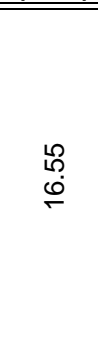 } & 1 & 6.838 & 0.039 & 32.451 & 32.451 \\
\hline 600 & & 0.5 & 6.537 & 0.233 & 32.827 & 32.828 \\
\hline 600 & & 0.2 & 6.139 & 0.250 & 33.358 & 33.359 \\
\hline 600 & & 0.1 & 5.838 & 0.064 & 31.715 & 31.715 \\
\hline 600 & & 0.05 & 5.537 & 0.340 & 31.789 & 31.791 \\
\hline 600 & & 0.02 & 5.139 & 0.115 & 32.863 & 32.863 \\
\hline 600 & & 0.01 & 4.838 & 0.232 & 32.225 & 32.225 \\
\hline 600 & & 0.005 & 4.537 & 0.123 & 31.683 & 31.683 \\
\hline 600 & & 0.002 & 4.139 & 0.214 & 31.715 & 31.716 \\
\hline 600 & & 0.001 & 3.838 & 0.540 & 31.493 & 31.498 \\
\hline 2620 & \multirow{10}{*}{ 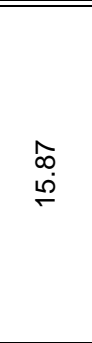 } & 1 & \begin{tabular}{c|}
6.157 \\
\end{tabular} & $\begin{array}{l}0.163 \\
\end{array}$ & 32.947 & 32.947 \\
\hline 620 & & 0.5 & 5.856 & 0.056 & 32.487 & 32.487 \\
\hline 620 & & 0.2 & 5.458 & 0.176 & 32.355 & 32.355 \\
\hline 620 & & 0.1 & 5.157 & 0.277 & 32.514 & 32.515 \\
\hline 620 & & 0.05 & 4.856 & 0.248 & 32.629 & 32.630 \\
\hline 620 & & 0.02 & 4.458 & 0.260 & 31.918 & 31.919 \\
\hline 620 & & 0.01 & 4.157 & 0.016 & 31.073 & 31.073 \\
\hline 620 & & 0.005 & 3.856 & 0.416 & 32.313 & 32.315 \\
\hline 620 & & 0.002 & 3.458 & 0.244 & 32.230 & 32.230 \\
\hline 620 & & 0.001 & 3.157 & 0.475 & 31.131 & 31.134 \\
\hline (2640 & \multirow{10}{*}{$\begin{array}{l}\text { N } \\
\stackrel{\leftrightarrow}{\leftrightarrow}\end{array}$} & 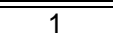 & 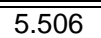 & $\begin{array}{l}0.047 \\
\end{array}$ & 32.180 & 32.180 \\
\hline 640 & & 0.5 & 5.205 & 0.056 & 32.132 & 32.132 \\
\hline 640 & & 0.2 & 4.807 & 0.217 & 32.526 & 32.527 \\
\hline 640 & & 0.1 & 4.506 & 0.505 & 32.523 & 32.526 \\
\hline 640 & & 0.05 & 4.205 & 0.538 & 30.993 & 30.997 \\
\hline 640 & & 0.02 & 3.807 & 0.943 & 32.140 & 32.154 \\
\hline 640 & & 0.01 & 3.506 & 0.746 & 32.078 & 32.086 \\
\hline 640 & & 0.005 & 3.205 & 0.598 & 30.846 & 30.852 \\
\hline 640 & & 0.002 & 2.807 & 0.153 & 30.659 & 30.659 \\
\hline 640 & & 0.001 & 2.506 & 0.190 & 30.101 & 30.102 \\
\hline
\end{tabular}

\begin{tabular}{|c|c|c|c|c|c|c|}
\hline \multicolumn{7}{|c|}{ SAMPLE G8 } \\
\hline $\mathrm{T}\left({ }^{\circ} \mathrm{C}\right)$ & $\begin{array}{c}\log _{10} \\
\eta \\
\text { (Pa s) }\end{array}$ & $f(\mathrm{~Hz})$ & $\begin{array}{c}\log _{10} \\
\omega \tau_{M}\end{array}$ & $\begin{array}{l}\mathbf{G}_{\text {im }} \\
(\mathbf{G P a})\end{array}$ & $\begin{array}{l}\mathbf{G}_{\text {real }} \\
(\mathrm{GPa})\end{array}$ & $\begin{array}{l}G_{\text {melt }} \\
(\mathrm{GPa})\end{array}$ \\
\hline 660 & \multirow{10}{*}{$\begin{array}{l}\stackrel{8}{0} \\
\dot{J}\end{array}$} & 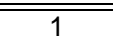 & 4.883 & 0.211 & 31.373 & 31.373 \\
\hline 660 & & 0.5 & 4.582 & 0.294 & 31.529 & 31.530 \\
\hline 660 & & 0.2 & 4.184 & 0.292 & 31.364 & 31.365 \\
\hline 660 & & 0.1 & 3.883 & 0.255 & 31.233 & 31.234 \\
\hline 660 & & 0.05 & 3.582 & 0.568 & 30.949 & 30.954 \\
\hline 660 & & 0.02 & 3.184 & 0.597 & 30.130 & 30.136 \\
\hline 660 & & 0.01 & 2.883 & 0.789 & 30.813 & 30.823 \\
\hline 660 & & 0.005 & 2.582 & 0.485 & 29.628 & 29.632 \\
\hline 660 & & 0.002 & 2.184 & 1.177 & 28.799 & 28.823 \\
\hline 660 & & 0.001 & 1.883 & 1.667 & 28.205 & 28.254 \\
\hline "680 & \multirow{10}{*}{$\begin{array}{l}8 \\
\dot{J}\end{array}$} & $\overline{11}$ & 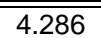 & "0.250 & "31.176 & 31.177 \\
\hline 680 & & 0.5 & 3.985 & 0.214 & 30.848 & 30.849 \\
\hline 680 & & 0.2 & 3.587 & 0.730 & 30.604 & 30.613 \\
\hline 680 & & 0.1 & 3.286 & 0.510 & 30.508 & 30.512 \\
\hline 680 & & 0.05 & 2.985 & 0.132 & 30.189 & 30.189 \\
\hline 680 & & 0.02 & 2.587 & 1.267 & 28.783 & 28.811 \\
\hline 680 & & 0.01 & 2.286 & 3.854 & 28.844 & 29.101 \\
\hline 680 & & 0.005 & 1.985 & 1.786 & 28.815 & 28.870 \\
\hline 680 & & 0.002 & 1.587 & 2.477 & 26.840 & 26.954 \\
\hline 680 & & 0.001 & 1.286 & 3.645 & 25.166 & 25.428 \\
\hline 7700 & \multirow{10}{*}{$\begin{array}{l}\stackrel{?}{+} \\
\stackrel{\leftrightarrow}{\rightarrow}\end{array}$} & $\overline{c 1}$ & $\begin{array}{l}3.713 \\
\end{array}$ & 0.332 & 30.346 & 30.348 \\
\hline 700 & & 0.5 & 3.412 & 0.300 & 30.411 & 30.413 \\
\hline 700 & & 0.2 & 3.014 & 0.365 & 30.056 & 30.058 \\
\hline 700 & & 0.1 & 2.713 & 0.816 & 30.769 & 30.779 \\
\hline 700 & & 0.05 & 2.412 & 0.967 & 29.375 & 29.391 \\
\hline 700 & & 0.02 & 2.014 & 0.423 & 28.814 & 28.817 \\
\hline 700 & & 0.01 & 1.713 & 2.349 & 27.404 & 27.505 \\
\hline 700 & & 0.005 & 1.412 & 2.843 & 24.986 & 25.147 \\
\hline 700 & & 0.002 & 1.014 & 4.692 & 22.443 & 22.928 \\
\hline 700 & & 0.001 & 0.713 & 6.148 & 19.292 & 20.248 \\
\hline
\end{tabular}


Appendix 2. Temperature $T$, $\log _{10} \eta$, frequency $f$, $\log _{10} \omega \tau_{\mathrm{M}}$, imaginary $(G \mathrm{im})$ and real $\left(\mathrm{G}_{\text {real }}\right)$ shear modulus and shear modulus of the melt $(\mathrm{G}$ melt $)$ for the investigated samples - continuation..

\begin{tabular}{|c|c|c|c|c|c|c|}
\hline \multicolumn{7}{|c|}{ SAMPLE G8 } \\
\hline $\mathrm{T}\left({ }^{\circ} \mathrm{C}\right)$ & $\begin{array}{c}\log _{10} \\
\eta \\
\text { (Pa s) }\end{array}$ & $f(\mathrm{~Hz})$ & $\begin{array}{c}\log _{10} \\
\omega \tau_{M}\end{array}$ & $\begin{array}{c}\mathbf{G}_{\mathrm{im}} \\
(\mathrm{GPa})\end{array}$ & $\begin{array}{l}\mathbf{G}_{\text {real }} \\
(\mathrm{GPa})\end{array}$ & $\begin{array}{l}\mathbf{G}_{\text {melt }} \\
(\mathrm{GPa})\end{array}$ \\
\hline 720 & \multirow{10}{*}{$\begin{array}{l}\infty \\
\stackrel{\infty}{\mathfrak{I}} \\
\stackrel{H}{ }\end{array}$} & 1 & 3.164 & 0.469 & 30.447 & 30.451 \\
\hline 720 & & 0.5 & 2.863 & 0.590 & 30.304 & 30.310 \\
\hline 720 & & 0.2 & 2.465 & 1.021 & 29.700 & 29.717 \\
\hline 720 & & 0.1 & 2.164 & 1.588 & 28.907 & 28.950 \\
\hline 720 & & 0.05 & 1.863 & 2.163 & 27.446 & 27.532 \\
\hline 720 & & 0.02 & 1.465 & 1.364 & 26.849 & 26.883 \\
\hline 720 & & 0.01 & 1.164 & 4.171 & 23.818 & 24.180 \\
\hline 720 & & 0.005 & 0.863 & 5.520 & 20.227 & 20.967 \\
\hline 720 & & 0.002 & 0.465 & 7.540 & 14.450 & 16.299 \\
\hline 720 & & 0.001 & 0.164 & 7.525 & 8.931 & 11.679 \\
\hline 740 & \multirow{10}{*}{ 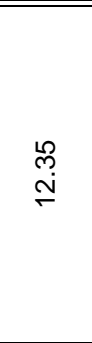 } & 1 & 2.636 & $\begin{array}{l}0.931 \\
\end{array}$ & 29.987 & 30.001 \\
\hline 740 & & 0.5 & 2.335 & 1.232 & 29.401 & 29.427 \\
\hline 740 & & 0.2 & 1.937 & 1.966 & 28.236 & 28.304 \\
\hline 740 & & 0.1 & 1.636 & 2.654 & 26.377 & 26.510 \\
\hline 740 & & 0.05 & 1.335 & 4.097 & 24.377 & 24.719 \\
\hline 740 & & 0.02 & 0.937 & 5.848 & 21.074 & 21.870 \\
\hline 740 & & 0.01 & 0.636 & 5.413 & 17.407 & 18.229 \\
\hline 740 & & 0.005 & 0.335 & 0.357 & 14.833 & 14.837 \\
\hline 740 & & 0.002 & -0.063 & 2.732 & 7.807 & 8.271 \\
\hline 740 & & 0.001 & -0.364 & 2.565 & 3.788 & 4.575 \\
\hline 760 & \multirow{10}{*}{$\begin{array}{l}\text { व } \\
\text { - } \\
-\end{array}$} & 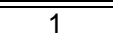 & 2.129 & $\begin{array}{l}1.711 \\
\end{array}$ & 29.235 & 29.286 \\
\hline 760 & & 0.5 & 1.828 & 2.385 & 28.008 & 28.109 \\
\hline 760 & & 0.2 & 1.430 & 0.312 & 25.470 & 25.471 \\
\hline 760 & & 0.1 & 1.129 & 5.181 & 23.207 & 23.778 \\
\hline 760 & & 0.05 & 0.828 & 6.643 & 19.138 & 20.258 \\
\hline 760 & & 0.02 & 0.430 & 7.765 & 11.883 & 14.195 \\
\hline 760 & & 0.01 & 0.129 & 7.015 & 6.649 & 9.665 \\
\hline 760 & & 0.005 & -0.172 & 1.542 & 6.066 & 6.259 \\
\hline 760 & & 0.002 & -0.570 & 2.000 & 0.618 & 2.094 \\
\hline 760 & & 0.001 & -0.871 & 1.137 & 0.165 & 1.149 \\
\hline
\end{tabular}

\begin{tabular}{|c|c|c|c|c|c|c|}
\hline \multicolumn{7}{|c|}{ SAMPLE G8 } \\
\hline $\mathrm{T}\left({ }^{\circ} \mathrm{C}\right)$ & $\begin{array}{c}\log _{10} \\
\eta \\
\text { (Pa s) }\end{array}$ & $f(\mathrm{~Hz})$ & $\begin{array}{c}\log _{10} \\
\omega \tau_{M}\end{array}$ & $\begin{array}{c}\mathbf{G}_{\text {im }} \\
(\mathrm{GPa})\end{array}$ & $\begin{array}{l}\mathbf{G}_{\text {real }} \\
(\mathrm{GPa})\end{array}$ & $\begin{array}{c}\mathbf{G}_{\text {melt }} \\
(\mathrm{GPa})\end{array}$ \\
\hline 780 & & 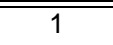 & 1.641 & 2.755 & 24.357 & 24.512 \\
\hline 780 & & 0.5 & 1.340 & 3.840 & 22.471 & 22.797 \\
\hline 780 & & 0.2 & 0.942 & 5.369 & 18.526 & 19.288 \\
\hline 780 & & 0.1 & 0.641 & 7.180 & 14.744 & 16.399 \\
\hline 780 & $\stackrel{m}{m}$ & 0.05 & 0.340 & 7.426 & 9.485 & 12.046 \\
\hline 780 & - & 0.02 & -0.058 & 5.392 & 3.551 & 6.456 \\
\hline 780 & & 0.01 & -0.359 & 3.292 & 1.263 & 3.526 \\
\hline 780 & & 0.005 & -0.660 & 1.760 & 0.351 & 1.794 \\
\hline 780 & & 0.002 & -1.058 & 0.713 & 0.081 & 0.717 \\
\hline 780 & & 0.001 & -1.359 & 0.391 & 0.033 & 0.393 \\
\hline 800 & & 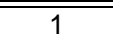 & $\begin{array}{ll}1.171 \\
\end{array}$ & "5.167 & 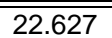 & 23.209 \\
\hline 800 & & 0.5 & 0.870 & 6.580 & 18.682 & 19.807 \\
\hline 800 & & 0.2 & 0.472 & 7.872 & 11.968 & 14.325 \\
\hline 800 & & 0.1 & 0.171 & 6.987 & 6.488 & 9.535 \\
\hline 800 & $\infty$ & 0.05 & -0.130 & 4.852 & 2.713 & 5.559 \\
\hline 800 & ○े & 0.02 & -0.528 & 2.279 & 0.601 & 2.357 \\
\hline 800 & & 0.01 & -0.829 & 1.036 & 0.659 & 1.228 \\
\hline 800 & & 0.005 & -1.130 & 0.580 & 0.058 & 0.582 \\
\hline 800 & & 0.002 & -1.528 & 0.176 & 0.178 & 0.251 \\
\hline 800 & & 0.001 & -1.829 & 0.055 & 0.097 & 0.112 \\
\hline 810 & & 1 & 0.943 & 26.543 & \begin{tabular}{|c|}
19.918 \\
\end{tabular} & 20.965 \\
\hline 810 & & 0.5 & 0.642 & 7.836 & 15.148 & 17.055 \\
\hline 810 & & 0.2 & 0.244 & 7.643 & 7.622 & 10.794 \\
\hline 810 & & 0.1 & -0.057 & 5.455 & 3.316 & 6.384 \\
\hline 810 & $\varnothing$ & 0.05 & -0.358 & 2.077 & 3.115 & 3.744 \\
\hline 810 & 웜 & 0.02 & -0.756 & 1.339 & 0.229 & 1.358 \\
\hline 810 & & 0.01 & -1.057 & 0.320 & 0.651 & 0.725 \\
\hline 810 & & 0.005 & -1.358 & 0.339 & 0.054 & 0.343 \\
\hline
\end{tabular}


Appendix 2. Temperature $T$, $\log _{10} \eta$, frequency $f$, $\log _{10} \omega \tau_{\mathrm{M}}$, imaginary $(G \mathrm{im})$ and real $\left(\mathrm{G}_{\text {real }}\right)$ shear modulus and shear modulus of the melt $(\mathrm{G}$ melt $)$ for the investigated samples - continuation..

\begin{tabular}{|c|c|c|c|c|c|c|}
\hline \multicolumn{7}{|c|}{ SAMPLE G8 } \\
\hline $\mathrm{T}\left({ }^{\circ} \mathrm{C}\right)$ & $\begin{array}{c}\log _{10} \\
\eta \\
\text { (Pa s) } \\
\end{array}$ & $f(\mathrm{~Hz})$ & $\begin{array}{c}\log _{10} \\
\omega \tau_{M}\end{array}$ & $\begin{array}{c}\mathbf{G}_{\text {im }} \\
(\mathrm{GPa})\end{array}$ & $\begin{array}{c}\mathbf{G}_{\text {real }} \\
(\mathrm{GPa})\end{array}$ & $\begin{array}{l}\text { G melt } \\
\text { (GPa) }\end{array}$ \\
\hline 820 & & 1 & 0.719 & 6.853 & 14.943 & 16.439 \\
\hline 820 & & 0.5 & 0.418 & 7.306 & 9.913 & 12.315 \\
\hline 820 & & 0.2 & 0.020 & 5.646 & 3.856 & 6.837 \\
\hline 820 & & 0.1 & -0.281 & 3.481 & 1.424 & 3.761 \\
\hline 820 & $\stackrel{?}{+}$ & 0.05 & -0.582 & 1.316 & 1.579 & 2.055 \\
\hline 820 & คे & 0.02 & -0.980 & 0.820 & 0.094 & 0.825 \\
\hline 820 & & 0.01 & -1.281 & 0.405 & 0.025 & 0.405 \\
\hline 820 & & 0.005 & -1.582 & 0.212 & 0.003 & 0.212 \\
\hline 820 & & 0.002 & -1.980 & 0.039 & 0.072 & 0.082 \\
\hline
\end{tabular}

\begin{tabular}{|c|c|c|c|c|c|c|}
\hline \multicolumn{7}{|c|}{ SAMPLE G9 } \\
\hline $\mathrm{T}\left({ }^{\circ} \mathrm{C}\right)$ & $\begin{array}{c}\log _{10} \\
\eta \\
\text { (Pa s) }\end{array}$ & $f(\mathrm{~Hz})$ & $\begin{array}{c}\log _{10} \\
\omega \tau_{M}\end{array}$ & $\begin{array}{l}\mathbf{G}_{\text {im }} \\
(\mathbf{G P a})\end{array}$ & $\begin{array}{l}\mathbf{G}_{\text {real }} \\
(\mathrm{GPa})\end{array}$ & $\begin{array}{c}\mathbf{G}_{\text {melt }} \\
(\mathrm{GPa})\end{array}$ \\
\hline 600 & \multirow{10}{*}{ 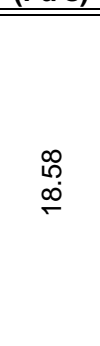 } & 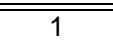 & 8.856 & 0.110 & 32.941 & 32.942 \\
\hline 600 & & 0.5 & 8.555 & 0.086 & 32.510 & 32.510 \\
\hline 600 & & 0.2 & 8.157 & 0.219 & 33.441 & 33.442 \\
\hline 600 & & 0.1 & 7.856 & 0.241 & 32.030 & 32.030 \\
\hline 600 & & 0.05 & 7.555 & 0.659 & 31.174 & 31.181 \\
\hline 600 & & 0.02 & 7.157 & 0.169 & 32.491 & 32.491 \\
\hline 600 & & 0.01 & 6.856 & 0.228 & 32.212 & 32.213 \\
\hline 600 & & 0.005 & 6.555 & 0.233 & 32.800 & 32.800 \\
\hline 600 & & 0.002 & 6.157 & 0.184 & 31.920 & 31.921 \\
\hline 600 & & 0.001 & 5.856 & 0.207 & 32.100 & 32.101 \\
\hline 620 & \multirow{10}{*}{$\begin{array}{l}\frac{9}{2} \\
\text { I }\end{array}$} & $\bar{~} 1$ & 8.073 & 0.114 & 31.710 & 31.710 \\
\hline 620 & & 0.5 & 7.772 & 0.058 & 31.840 & 31.840 \\
\hline 620 & & 0.2 & 7.374 & 0.157 & 32.101 & 32.102 \\
\hline 620 & & 0.1 & 7.073 & 0.124 & 31.876 & 31.876 \\
\hline 620 & & 0.05 & 6.772 & 0.339 & 32.837 & 32.838 \\
\hline 620 & & 0.02 & 6.374 & 0.064 & 32.583 & 32.583 \\
\hline 620 & & 0.01 & 6.073 & 0.588 & 31.924 & 31.929 \\
\hline 620 & & 0.005 & 5.772 & 0.261 & 31.413 & 31.414 \\
\hline 620 & & 0.002 & 5.374 & 0.130 & 31.175 & 31.175 \\
\hline 620 & & 0.001 & 5.073 & 0.272 & 31.066 & 31.068 \\
\hline 2640 & \multirow{10}{*}{$\begin{array}{l}\text { Dे } \\
\text { ने }\end{array}$} & $\bar{~} 1$ & $\begin{array}{ll}7.324 \\
\end{array}$ & $\overline{c 0.046}$ & 31.849 & 31.849 \\
\hline 640 & & 0.5 & 7.023 & 0.055 & 31.660 & 31.660 \\
\hline 640 & & 0.2 & 6.625 & 0.207 & 31.015 & 31.015 \\
\hline 640 & & 0.1 & 6.324 & 0.489 & 31.488 & 31.492 \\
\hline 640 & & 0.05 & 6.023 & 0.545 & 31.421 & 31.426 \\
\hline 640 & & 0.02 & 5.625 & 0.908 & 30.950 & 30.963 \\
\hline 640 & & 0.01 & 5.324 & 0.708 & 30.420 & 30.428 \\
\hline 640 & & 0.005 & 5.023 & 0.601 & 31.039 & 31.045 \\
\hline 640 & & 0.002 & 4.625 & 0.153 & 30.640 & 30.640 \\
\hline 640 & & 0.001 & 4.324 & 0.192 & 30.415 & 30.415 \\
\hline
\end{tabular}


Appendix 2. Temperature $T$, $\log _{10} \eta$, frequency $f$, $\log _{10} \omega \tau_{\mathrm{M}}$, imaginary $(G \mathrm{im})$ and real $\left(\mathrm{G}_{\text {real }}\right)$ shear modulus and shear modulus of the melt $(\mathrm{G}$ melt $)$ for the investigated samples - continuation..

\begin{tabular}{|c|c|c|c|c|c|c|}
\hline \multicolumn{7}{|c|}{ SAMPLE G9 } \\
\hline $\mathrm{T}\left({ }^{\circ} \mathrm{C}\right)$ & $\begin{array}{c}\log _{10} \\
\eta \\
\text { (Pa s) }\end{array}$ & $f(\mathrm{~Hz})$ & $\begin{array}{c}\log _{10} \\
\omega \tau_{M}\end{array}$ & $\begin{array}{c}\mathbf{G}_{\mathrm{im}} \\
(\mathrm{GPa})\end{array}$ & $\begin{array}{l}\mathbf{G}_{\text {real }} \\
(\mathrm{GPa})\end{array}$ & $\begin{array}{l}\mathbf{G}_{\text {melt }} \\
(\mathrm{GPa})\end{array}$ \\
\hline "660 & \multirow{10}{*}{ 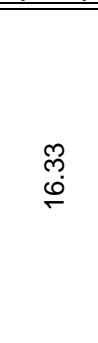 } & 1 & $\begin{array}{c}6.607 \\
\end{array}$ & 0.023 & 31.616 & 31.616 \\
\hline 660 & & 0.5 & 6.306 & 0.182 & 31.371 & 31.371 \\
\hline 660 & & 0.2 & 5.908 & 0.130 & 30.909 & 30.909 \\
\hline 660 & & 0.1 & 5.607 & 0.234 & 31.615 & 31.615 \\
\hline 660 & & 0.05 & 5.306 & 0.175 & 31.477 & 31.478 \\
\hline 660 & & 0.02 & 4.908 & 0.394 & 30.723 & 30.725 \\
\hline 660 & & 0.01 & 4.607 & 0.527 & 30.257 & 30.262 \\
\hline 660 & & 0.005 & 4.306 & 0.625 & 30.185 & 30.192 \\
\hline 660 & & 0.002 & 3.908 & 0.698 & 29.582 & 29.590 \\
\hline 660 & & 0.001 & 3.607 & 1.296 & 29.569 & 29.598 \\
\hline 2680 & \multirow{10}{*}{ 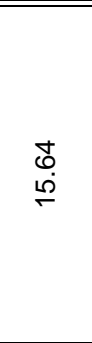 } & 1 & $\begin{array}{l}5.920 \\
\end{array}$ & $\begin{array}{l}0.263 \\
\end{array}$ & 30.827 & 30.828 \\
\hline 680 & & 0.5 & 5.619 & 0.045 & 30.956 & 30.956 \\
\hline 680 & & 0.2 & 5.221 & 0.417 & 30.587 & 30.589 \\
\hline 680 & & 0.1 & 4.920 & 0.150 & 30.809 & 30.809 \\
\hline 680 & & 0.05 & 4.619 & 0.257 & 30.127 & 30.128 \\
\hline 680 & & 0.02 & 4.221 & 0.328 & 30.628 & 30.630 \\
\hline 680 & & 0.01 & 3.920 & 0.764 & 30.232 & 30.242 \\
\hline 680 & & 0.005 & 3.619 & 0.611 & 29.277 & 29.283 \\
\hline 680 & & 0.002 & 3.221 & 1.413 & 28.123 & 28.158 \\
\hline 680 & & 0.001 & 2.920 & 2.320 & 26.826 & 26.926 \\
\hline 700 & \multirow{10}{*}{$\begin{array}{l}\infty \\
\stackrel{\circ}{+} \\
\dot{\sim}\end{array}$} & 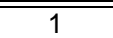 & \begin{tabular}{c|}
5.261 \\
\end{tabular} & $\begin{array}{l}0.176 \\
\end{array}$ & 30.600 & 30.601 \\
\hline 700 & & 0.5 & 4.960 & 0.177 & 30.486 & 30.486 \\
\hline 700 & & 0.2 & 4.562 & 0.426 & 29.893 & 29.896 \\
\hline 700 & & 0.1 & 4.261 & 0.368 & 29.240 & 29.242 \\
\hline 700 & & 0.05 & 3.960 & 0.486 & 30.029 & 30.033 \\
\hline 700 & & 0.02 & 3.562 & 1.018 & 28.910 & 28.928 \\
\hline 700 & & 0.01 & 3.261 & 1.182 & 28.438 & 28.463 \\
\hline 700 & & 0.005 & 2.960 & 1.629 & 27.175 & 27.224 \\
\hline 700 & & 0.002 & 2.562 & 2.875 & 25.430 & 25.592 \\
\hline 700 & & 0.001 & 2.261 & 4.031 & 24.026 & 24.362 \\
\hline
\end{tabular}

\begin{tabular}{|c|c|c|c|c|c|c|}
\hline \multicolumn{7}{|c|}{ SAMPLE G9 } \\
\hline $\mathrm{T}\left({ }^{\circ} \mathrm{C}\right)$ & $\begin{array}{c}\log _{10} \\
\eta \\
\text { (Pa s) }\end{array}$ & $f(\mathrm{~Hz})$ & $\begin{array}{c}\log _{10} \\
\omega \tau_{M}\end{array}$ & $\begin{array}{l}\mathbf{G}_{\mathrm{im}} \\
(\mathrm{GPa})\end{array}$ & $\begin{array}{l}\mathbf{G}_{\text {real }} \\
(\mathrm{GPa})\end{array}$ & $\begin{array}{c}\mathbf{G}_{\text {melt }} \\
(\mathrm{GPa})\end{array}$ \\
\hline 720 & \multirow{10}{*}{$\begin{array}{l}\stackrel{L}{m} \\
\stackrel{\sim}{+}\end{array}$} & 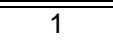 & 4.629 & 0.355 & 30.065 & 30.067 \\
\hline 720 & & 0.5 & 4.328 & 0.452 & 29.524 & 29.527 \\
\hline 720 & & 0.2 & 3.930 & 0.445 & 29.257 & 29.260 \\
\hline 720 & & 0.1 & 3.629 & 0.655 & 29.594 & 29.601 \\
\hline 720 & & 0.05 & 3.328 & 1.455 & 28.189 & 28.226 \\
\hline 720 & & 0.02 & 2.930 & 1.576 & 27.171 & 27.217 \\
\hline 720 & & 0.01 & 2.629 & 2.029 & 26.163 & 26.242 \\
\hline 720 & & 0.005 & 2.328 & 3.535 & 23.885 & 24.145 \\
\hline 720 & & 0.002 & 1.930 & 5.296 & 20.870 & 21.531 \\
\hline 720 & & 0.001 & 1.629 & 6.632 & 17.153 & 18.390 \\
\hline 7470 & \multirow{10}{*}{ 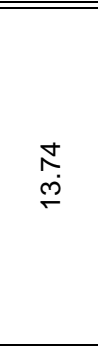 } & 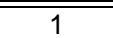 & $\begin{array}{c}4.022 \\
\end{array}$ & "0.488 & 28.423 & 28.428 \\
\hline 740 & & 0.5 & 3.721 & 0.647 & 28.331 & 28.339 \\
\hline 740 & & 0.2 & 3.323 & 1.365 & 27.184 & 27.218 \\
\hline 740 & & 0.1 & 3.022 & 1.232 & 26.015 & 26.044 \\
\hline 740 & & 0.05 & 2.721 & 2.115 & 26.878 & 26.961 \\
\hline 740 & & 0.02 & 2.323 & 3.517 & 23.410 & 23.673 \\
\hline 740 & & 0.01 & 2.022 & 4.559 & 21.450 & 21.929 \\
\hline 740 & & 0.005 & 1.721 & 5.996 & 18.060 & 19.029 \\
\hline 740 & & 0.002 & 1.323 & 7.642 & 12.155 & 14.358 \\
\hline 740 & & 0.001 & 1.022 & 6.960 & 6.959 & 9.842 \\
\hline 760 & \multirow{10}{*}{$\begin{array}{l}0 \\
\rightarrow \\
\stackrel{9}{\rightarrow}\end{array}$} & 1 & 3.438 & 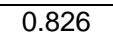 & 26.730 & 26.743 \\
\hline 760 & & 0.5 & 3.137 & 1.304 & 26.523 & 26.555 \\
\hline 760 & & 0.2 & 2.739 & 1.870 & 24.757 & 24.827 \\
\hline 760 & & 0.1 & 2.438 & 2.494 & 23.399 & 23.532 \\
\hline 760 & & 0.05 & 2.137 & 3.723 & 22.875 & 23.176 \\
\hline 760 & & 0.02 & 1.739 & 5.552 & 18.196 & 19.024 \\
\hline 760 & & 0.01 & 1.438 & 7.004 & 14.249 & 15.877 \\
\hline 760 & & 0.005 & 1.137 & 3.673 & 12.029 & 12.578 \\
\hline 760 & & 0.002 & 0.739 & 5.196 & 3.380 & 6.199 \\
\hline 760 & & 0.001 & 0.438 & 3.284 & 1.910 & 3.799 \\
\hline
\end{tabular}


Appendix 2. Temperature $T$, $\log _{10} \eta$, frequency $f$, $\log _{10} \omega \tau_{\mathrm{M}}$, imaginary $(G \mathrm{im})$ and real $\left(\mathrm{G}_{\text {real }}\right)$ shear modulus and shear modulus of the melt $(\mathrm{G}$ melt $)$ for the investigated samples - continuation..

\begin{tabular}{|c|c|c|c|c|c|c|}
\hline \multicolumn{7}{|c|}{ SAMPLE G9 } \\
\hline $\mathrm{T}\left({ }^{\circ} \mathrm{C}\right)$ & $\begin{array}{c}\log _{10} \\
\eta \\
\text { (Pa s) } \\
\end{array}$ & $f(\mathrm{~Hz})$ & $\begin{array}{c}\log _{10} \\
\omega \tau_{M}\end{array}$ & $\begin{array}{l}\mathbf{G}_{\mathrm{im}} \\
(\mathrm{GPa})\end{array}$ & $\begin{array}{l}\mathbf{G}_{\text {real }} \\
(\mathrm{GPa})\end{array}$ & $\begin{array}{l}\mathbf{G}_{\text {melt }} \\
(\mathrm{GPa})\end{array}$ \\
\hline 780 & \multirow{10}{*}{$\begin{array}{l}\stackrel{\leftrightarrow}{0} \\
\stackrel{\leftrightarrow}{H}\end{array}$} & 1 & 2.876 & 1.575 & 25.275 & 25.324 \\
\hline 780 & & 0.5 & 2.575 & 2.161 & 24.261 & 24.357 \\
\hline 780 & & 0.2 & 2.177 & 3.647 & 22.268 & 22.564 \\
\hline 780 & & 0.1 & 1.876 & 4.520 & 19.528 & 20.044 \\
\hline 780 & & 0.05 & 1.575 & 6.198 & 16.195 & 17.341 \\
\hline 780 & & 0.02 & 1.177 & 7.260 & 10.473 & 12.743 \\
\hline 780 & & 0.01 & 0.876 & 6.365 & 5.539 & 8.438 \\
\hline 780 & & 0.005 & 0.575 & 4.236 & 2.234 & 4.789 \\
\hline 780 & & 0.002 & 0.177 & 1.563 & 1.503 & 2.168 \\
\hline 780 & & 0.001 & -0.124 & 1.016 & 0.456 & 1.114 \\
\hline 800 & \multirow{10}{*}{ 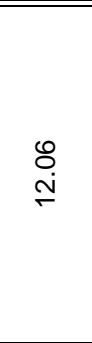 } & $\bar{~} 1$ & "2.336 & 2.553 & 21.642 & 21.792 \\
\hline 800 & & 0.5 & 2.035 & 0.443 & 20.748 & 20.753 \\
\hline 800 & & 0.2 & 1.637 & 2.811 & 17.498 & 17.723 \\
\hline 800 & & 0.1 & 1.336 & 6.445 & 12.774 & 14.308 \\
\hline 800 & & 0.05 & 1.035 & 6.865 & 8.150 & 10.656 \\
\hline 800 & & 0.02 & 0.637 & 4.817 & 2.946 & 5.646 \\
\hline 800 & & 0.01 & 0.336 & 2.938 & 1.048 & 3.119 \\
\hline 800 & & 0.005 & 0.035 & 1.556 & 0.321 & 1.589 \\
\hline 800 & & 0.002 & -0.363 & 0.653 & 0.049 & 0.655 \\
\hline 800 & & 0.001 & -0.664 & 0.327 & 0.024 & 0.328 \\
\hline 810 & \multirow{10}{*}{$\begin{array}{l}\stackrel{R}{T} \\
\stackrel{-}{-}\end{array}$} & 1 & 2.073 & 3.409 & 20.424 & 20.706 \\
\hline 810 & & 0.5 & 1.772 & 4.656 & 18.269 & 18.853 \\
\hline 810 & & 0.2 & 1.374 & 6.258 & 13.405 & 14.793 \\
\hline 810 & & 0.1 & 1.073 & 6.822 & 9.034 & 11.320 \\
\hline 810 & & 0.05 & 0.772 & 5.865 & 4.660 & 7.491 \\
\hline 810 & & 0.02 & 0.374 & 3.120 & 1.211 & 3.347 \\
\hline 810 & & 0.01 & 0.073 & 0.521 & 1.905 & 1.975 \\
\hline 810 & & 0.005 & -0.228 & 0.907 & 0.110 & 0.913 \\
\hline 810 & & 0.002 & -0.626 & 0.372 & 0.026 & 0.373 \\
\hline 810 & & 0.001 & -0.927 & 0.181 & 0.012 & 0.181 \\
\hline
\end{tabular}

\begin{tabular}{|c|c|c|c|c|c|c|}
\hline \multicolumn{7}{|c|}{ SAMPLE G9 } \\
\hline $\mathrm{T}\left({ }^{\circ} \mathrm{C}\right)$ & $\begin{array}{c}\log _{10} \\
\eta \\
\text { (Pa s) }\end{array}$ & $f(\mathrm{~Hz})$ & $\begin{array}{c}\log _{10} \\
\omega \tau_{M}\end{array}$ & $\begin{array}{c}\mathbf{G}_{\mathrm{im}} \\
(\mathrm{GPa})\end{array}$ & $\begin{array}{l}\mathbf{G}_{\text {real }} \\
(\mathrm{GPa})\end{array}$ & $\begin{array}{l}\mathbf{G}_{\text {melt }} \\
(\mathrm{GPa})\end{array}$ \\
\hline 820 & & 1 & 1.815 & 3.700 & 16.569 & 16.977 \\
\hline 820 & & 0.5 & 1.514 & 5.149 & 14.011 & 14.928 \\
\hline 820 & & 0.2 & 1.116 & 6.213 & 9.261 & 11.151 \\
\hline 820 & & 0.1 & 0.815 & 5.698 & 5.068 & 7.626 \\
\hline 820 & กొ? & 0.05 & 0.514 & 3.967 & 2.066 & 4.473 \\
\hline 820 & $ت$ & 0.02 & 0.116 & 1.831 & 0.461 & 1.888 \\
\hline 820 & & 0.01 & -0.185 & 0.992 & 0.146 & 1.003 \\
\hline 820 & & 0.005 & -0.486 & 0.497 & 0.049 & 0.499 \\
\hline 820 & & 0.002 & -0.884 & 0.089 & 0.178 & 0.199 \\
\hline 820 & & 0.001 & -1.185 & 0.122 & 0.047 & 0.130 \\
\hline
\end{tabular}


Appendix 2. Temperature $T$, $\log _{10} \eta$, frequency $f$, $\log _{10} \omega \tau_{\mathrm{M}}$, imaginary $(G \mathrm{im})$ and real $\left(\mathrm{G}_{\text {real }}\right)$ shear modulus and shear modulus of the melt $(\mathrm{G}$ melt $)$ for the investigated samples - continuation..

\begin{tabular}{|c|c|c|c|c|c|c|}
\hline \multicolumn{7}{|c|}{ SAMPLE G10 } \\
\hline $\mathrm{T}\left({ }^{\circ} \mathrm{C}\right)$ & $\begin{array}{c}\log _{10} \\
\eta \\
\text { (Pa s) }\end{array}$ & $f(\mathrm{~Hz})$ & $\begin{array}{c}\log _{10} \\
\omega \tau_{M}\end{array}$ & $\begin{array}{c}\mathbf{G}_{\mathrm{im}} \\
(\mathrm{GPa})\end{array}$ & $\begin{array}{l}\mathbf{G}_{\text {real }} \\
(\mathrm{GPa})\end{array}$ & $\begin{array}{l}\mathbf{G}_{\text {melt }} \\
(\mathrm{GPa})\end{array}$ \\
\hline 2560 & \multirow{10}{*}{ 帝 } & 1 & $\begin{array}{ll}7.642 \\
\end{array}$ & 0.062 & 32.199 & 32.199 \\
\hline 560 & & 0.5 & 7.341 & 0.135 & 31.958 & 31.958 \\
\hline 560 & & 0.2 & 6.943 & 0.051 & 31.723 & 31.723 \\
\hline 560 & & 0.1 & 6.642 & 0.076 & 32.019 & 32.020 \\
\hline 560 & & 0.05 & 6.341 & 0.207 & 32.229 & 32.230 \\
\hline 560 & & 0.02 & 5.943 & 0.132 & 32.489 & 32.489 \\
\hline 560 & & 0.01 & 5.642 & 0.212 & 32.040 & 32.040 \\
\hline 560 & & 0.005 & 5.341 & 0.241 & 31.658 & 31.658 \\
\hline 560 & & 0.002 & 4.943 & 0.116 & 31.503 & 31.503 \\
\hline 560 & & 0.001 & 4.642 & 0.383 & 31.462 & 31.464 \\
\hline 580 & \multirow{10}{*}{$\begin{array}{l}-1 \\
\dot{6} \\
\dot{\varphi}\end{array}$} & 1 & 6.902 & $\begin{array}{l}0.110 \\
\end{array}$ & 31.927 & 31.927 \\
\hline 580 & & 0.5 & 6.601 & 0.102 & 31.881 & 31.881 \\
\hline 580 & & 0.2 & 6.203 & 0.196 & 31.973 & 31.974 \\
\hline 580 & & 0.1 & 5.902 & 0.262 & 31.925 & 31.926 \\
\hline 580 & & 0.05 & 5.601 & 0.058 & 31.824 & 31.824 \\
\hline 580 & & 0.02 & 5.203 & 0.298 & 31.577 & 31.579 \\
\hline 580 & & 0.01 & 4.902 & 0.280 & 31.833 & 31.834 \\
\hline 580 & & 0.005 & 4.601 & 0.364 & 31.578 & 31.580 \\
\hline 580 & & 0.002 & 4.203 & 0.134 & 31.523 & 31.523 \\
\hline 580 & & 0.001 & 3.902 & 0.191 & 30.974 & 30.975 \\
\hline (2600 & \multirow{10}{*}{$\begin{array}{l}\text { ने } \\
\text { فे }\end{array}$} & 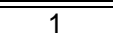 & $\begin{array}{ll}6.196 \\
\end{array}$ & $\begin{array}{l}0.046 \\
\end{array}$ & 31.825 & 31.825 \\
\hline 600 & & 0.5 & 5.895 & 0.056 & 32.028 & 32.028 \\
\hline 600 & & 0.2 & 5.497 & 0.212 & 31.906 & 31.907 \\
\hline 600 & & 0.1 & 5.196 & 0.486 & 31.281 & 31.285 \\
\hline 600 & & 0.05 & 4.895 & 0.546 & 31.454 & 31.459 \\
\hline 600 & & 0.02 & 4.497 & 0.928 & 31.619 & 31.633 \\
\hline 600 & & 0.01 & 4.196 & 0.763 & 32.821 & 32.830 \\
\hline 600 & & 0.005 & 3.895 & 0.577 & 29.762 & 29.768 \\
\hline 600 & & 0.002 & 3.497 & 0.155 & 31.057 & 31.057 \\
\hline 600 & & 0.001 & 3.196 & 0.185 & 29.281 & 29.282 \\
\hline
\end{tabular}

\begin{tabular}{|c|c|c|c|c|c|c|}
\hline \multicolumn{7}{|c|}{ SAMPLE G10 } \\
\hline $\mathrm{T}\left({ }^{\circ} \mathrm{C}\right)$ & $\begin{array}{c}\log _{10} \\
\eta \\
\text { (Pa s) }\end{array}$ & $f(\mathrm{~Hz})$ & $\begin{array}{c}\log _{10} \\
\omega \tau_{M}\end{array}$ & $\begin{array}{l}\mathbf{G}_{\text {im }} \\
(\mathbf{G P a})\end{array}$ & $\begin{array}{l}\mathbf{G}_{\text {real }} \\
(\mathrm{GPa})\end{array}$ & $\begin{array}{l}G_{\text {melt }} \\
(\mathrm{GPa})\end{array}$ \\
\hline 620 & \multirow{10}{*}{$\begin{array}{l}\text { N } \\
\text { ஸे }\end{array}$} & 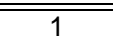 & 5.522 & 0.032 & 31.980 & 31.980 \\
\hline 620 & & 0.5 & 5.221 & 0.036 & 32.103 & 32.103 \\
\hline 620 & & 0.2 & 4.823 & 0.070 & 31.455 & 31.455 \\
\hline 620 & & 0.1 & 4.522 & 0.183 & 31.362 & 31.362 \\
\hline 620 & & 0.05 & 4.221 & 0.045 & 31.906 & 31.906 \\
\hline 620 & & 0.02 & 3.823 & 0.295 & 30.188 & 30.189 \\
\hline 620 & & 0.01 & 3.522 & 0.077 & 28.928 & 28.928 \\
\hline 620 & & 0.005 & 3.221 & 0.660 & 29.598 & 29.606 \\
\hline 620 & & 0.002 & 2.823 & 0.302 & 29.709 & 29.710 \\
\hline 620 & & 0.001 & 2.522 & 2.287 & 30.618 & 30.703 \\
\hline 2640 & \multirow{10}{*}{ 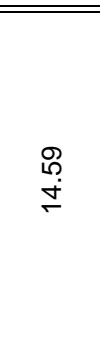 } & $\overline{11}$ & $\begin{array}{l}4.877 \\
\end{array}$ & "0.046 & " 31.334 & "31.334 \\
\hline 640 & & 0.5 & 4.576 & 0.171 & 30.488 & 30.488 \\
\hline 640 & & 0.2 & 4.178 & 0.096 & 30.242 & 30.242 \\
\hline 640 & & 0.1 & 3.877 & 0.039 & 30.857 & 30.857 \\
\hline 640 & & 0.05 & 3.576 & 0.234 & 30.548 & 30.549 \\
\hline 640 & & 0.02 & 3.178 & 0.350 & 29.566 & 29.568 \\
\hline 640 & & 0.01 & 2.877 & 0.632 & 28.953 & 28.960 \\
\hline 640 & & 0.005 & 2.576 & 0.785 & 28.665 & 28.676 \\
\hline 640 & & 0.002 & 2.178 & 1.421 & 27.977 & 28.013 \\
\hline 640 & & 0.001 & 1.877 & 0.329 & 26.758 & 26.760 \\
\hline 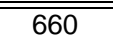 & \multirow{10}{*}{ बू } & $\overline{c 1}$ & $\begin{array}{l}4.260 \\
\end{array}$ & $\begin{array}{l}0.058 \\
\end{array}$ & 30.604 & 30.604 \\
\hline 660 & & 0.5 & 3.959 & 0.184 & 30.377 & 30.378 \\
\hline 660 & & 0.2 & 3.561 & 0.202 & 30.316 & 30.317 \\
\hline 660 & & 0.1 & 3.260 & 0.406 & 29.775 & 29.778 \\
\hline 660 & & 0.05 & 2.959 & 0.800 & 28.802 & 28.813 \\
\hline 660 & & 0.02 & 2.561 & 0.508 & 28.543 & 28.548 \\
\hline 660 & & 0.01 & 2.260 & 1.425 & 28.377 & 28.413 \\
\hline 660 & & 0.005 & 1.959 & 1.947 & 27.117 & 27.187 \\
\hline 660 & & 0.002 & 1.561 & 3.114 & 25.487 & 25.676 \\
\hline 660 & & 0.001 & 1.260 & 1.769 & 21.563 & 21.635 \\
\hline
\end{tabular}


Appendix 2. Temperature $T$, $\log _{10} \eta$, frequency $f$, $\log _{10} \omega \tau_{\mathrm{M}}$, imaginary $(G \mathrm{im})$ and real $\left(\mathrm{G}_{\text {real }}\right)$ shear modulus and shear modulus of the melt $(\mathrm{G}$ melt $)$ for the investigated samples - continuation..

\begin{tabular}{|c|c|c|c|c|c|c|}
\hline \multicolumn{7}{|c|}{ SAMPLE G10 } \\
\hline $\mathrm{T}\left({ }^{\circ} \mathrm{C}\right)$ & $\begin{array}{c}\log _{10} \\
\eta \\
\text { (Pa s) }\end{array}$ & $f(\mathrm{~Hz})$ & $\begin{array}{c}\log _{10} \\
\omega \tau_{M}\end{array}$ & $\begin{array}{c}\mathbf{G}_{\mathrm{im}} \\
(\mathrm{GPa})\end{array}$ & $\begin{array}{l}\mathbf{G}_{\text {real }} \\
(\mathrm{GPa})\end{array}$ & $\begin{array}{c}\mathbf{G}_{\text {melt }} \\
(\mathrm{GPa})\end{array}$ \\
\hline 680 & \multirow{10}{*}{ 孞 } & 1 & 3.669 & 0.221 & 29.710 & 29.711 \\
\hline 680 & & 0.5 & 3.368 & 0.338 & 29.522 & 29.524 \\
\hline 680 & & 0.2 & 2.970 & 0.384 & 29.138 & 29.141 \\
\hline 680 & & 0.1 & 2.669 & 0.863 & 28.250 & 28.263 \\
\hline 680 & & 0.05 & 2.368 & 0.977 & 28.262 & 28.279 \\
\hline 680 & & 0.02 & 1.970 & 1.847 & 27.060 & 27.123 \\
\hline 680 & & 0.01 & 1.669 & 2.470 & 25.502 & 25.621 \\
\hline 680 & & 0.005 & 1.368 & 3.390 & 23.515 & 23.758 \\
\hline 680 & & 0.002 & 0.970 & 5.377 & 20.318 & 21.017 \\
\hline 680 & & 0.001 & 0.669 & 5.093 & 17.371 & 18.103 \\
\hline 700 & \multirow{10}{*}{$\begin{array}{l}\text {-1 } \\
\stackrel{\text { Ij }}{H}\end{array}$} & 1 & $\begin{array}{l}3.102 \\
\end{array}$ & $\begin{array}{l}0.494 \\
\end{array}$ & 30.312 & 30.316 \\
\hline 700 & & 0.5 & 2.801 & 0.918 & 29.977 & 29.991 \\
\hline 700 & & 0.2 & 2.403 & 0.756 & 28.191 & 28.201 \\
\hline 700 & & 0.1 & 2.102 & 1.780 & 27.941 & 27.997 \\
\hline 700 & & 0.05 & 1.801 & 2.199 & 26.345 & 26.437 \\
\hline 700 & & 0.02 & 1.403 & 3.599 & 23.868 & 24.137 \\
\hline 700 & & 0.01 & 1.102 & 5.158 & 21.840 & 22.441 \\
\hline 700 & & 0.005 & 0.801 & 3.147 & 18.397 & 18.664 \\
\hline 700 & & 0.002 & 0.403 & 7.573 & 11.522 & 13.788 \\
\hline 700 & & 0.001 & 0.102 & 6.830 & 6.134 & 9.180 \\
\hline 720 & \multirow{10}{*}{\begin{tabular}{l}
$\underset{N}{N}$ \\
\multirow{H}{*}{}
\end{tabular}} & 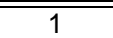 & \begin{tabular}{l|l}
2.558 \\
\end{tabular} & $\begin{array}{ll}1.020 \\
\end{array}$ & 28.492 & 28.510 \\
\hline 720 & & 0.5 & 2.257 & 1.438 & 27.750 & 27.788 \\
\hline 720 & & 0.2 & 1.859 & 2.185 & 26.087 & 26.178 \\
\hline 720 & & 0.1 & 1.558 & 3.040 & 24.297 & 24.486 \\
\hline 720 & & 0.05 & 1.257 & 4.304 & 22.137 & 22.552 \\
\hline 720 & & 0.02 & 0.859 & 6.218 & 17.770 & 18.827 \\
\hline 720 & & 0.01 & 0.558 & 7.354 & 13.420 & 15.303 \\
\hline 720 & & 0.005 & 0.257 & 4.391 & 10.896 & 11.748 \\
\hline 720 & & 0.002 & -0.141 & 4.537 & 3.344 & 5.636 \\
\hline 720 & & 0.001 & -0.442 & 2.554 & 1.319 & 2.874 \\
\hline
\end{tabular}

\begin{tabular}{|c|c|c|c|c|c|c|}
\hline \multicolumn{7}{|c|}{ SAMPLE G10 } \\
\hline $\mathrm{T}\left({ }^{\circ} \mathrm{C}\right)$ & $\begin{array}{c}\log _{10} \\
\eta \\
(\mathrm{Pa} \mathrm{s})\end{array}$ & $f(\mathrm{~Hz})$ & $\begin{array}{c}\log _{10} \\
\omega \tau_{M}\end{array}$ & $\begin{array}{c}\mathbf{G}_{\mathrm{im}} \\
(\mathrm{GPa})\end{array}$ & $\begin{array}{l}\mathbf{G}_{\text {real }} \\
(\mathrm{GPa})\end{array}$ & $\begin{array}{l}\mathbf{G}_{\text {melt }} \\
(\mathrm{GPa})\end{array}$ \\
\hline 740 & \multirow{10}{*}{$\begin{array}{l}\text { Tे } \\
\text { G }\end{array}$} & 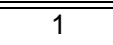 & 2.035 & 1.921 & 28.149 & 28.214 \\
\hline 740 & & 0.5 & 1.734 & 2.648 & 26.645 & 26.777 \\
\hline 740 & & 0.2 & 1.336 & 4.078 & 23.249 & 23.604 \\
\hline 740 & & 0.1 & 1.035 & 5.851 & 20.483 & 21.302 \\
\hline 740 & & 0.05 & 0.734 & 7.144 & 16.489 & 17.970 \\
\hline 740 & & 0.02 & 0.336 & 6.776 & 10.391 & 12.405 \\
\hline 740 & & 0.01 & 0.035 & 6.256 & 4.484 & 7.697 \\
\hline 740 & & 0.005 & -0.266 & 3.743 & 1.625 & 4.080 \\
\hline 740 & & 0.002 & -0.664 & 1.516 & 0.308 & 1.547 \\
\hline 740 & & 0.001 & -0.965 & 0.780 & 0.301 & 0.836 \\
\hline 760 & \multirow{10}{*}{$\begin{array}{l}\stackrel{d}{N} \\
\text { G }\end{array}$} & $\overline{11}$ & 1.533 & $\begin{array}{l}3.601 \\
\end{array}$ & 25.780 & 2026.030 \\
\hline 760 & & 0.5 & 1.232 & 4.829 & 23.290 & 23.786 \\
\hline 760 & & 0.2 & 0.834 & 6.340 & 17.470 & 18.585 \\
\hline 760 & & 0.1 & 0.533 & 7.735 & 13.038 & 15.159 \\
\hline 760 & & 0.05 & 0.232 & 7.074 & 7.603 & 10.384 \\
\hline 760 & & 0.02 & -0.166 & 4.678 & 2.327 & 5.225 \\
\hline 760 & & 0.01 & -0.467 & 1.851 & 2.238 & 2.904 \\
\hline 760 & & 0.005 & -0.768 & 1.435 & 0.236 & 1.454 \\
\hline 760 & & 0.002 & -1.166 & 0.320 & 0.443 & 0.546 \\
\hline 760 & & 0.001 & -1.467 & 0.214 & 0.116 & 0.244 \\
\hline 780 & \multirow{9}{*}{$\begin{array}{l}\stackrel{0}{2} \\
0 \\
0\end{array}$} & 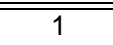 & 1.049 & $\bar{~} 5.172$ & 19.464 & 20.140 \\
\hline 780 & & 0.5 & 0.748 & 6.473 & 15.765 & 17.043 \\
\hline 780 & & 0.2 & 0.350 & 7.178 & 9.247 & 11.706 \\
\hline 780 & & 0.1 & 0.049 & 5.811 & 4.486 & 7.341 \\
\hline 780 & & 0.05 & -0.252 & 1.987 & 4.206 & 4.652 \\
\hline 780 & & 0.02 & -0.650 & 1.643 & 0.368 & 1.684 \\
\hline 780 & & 0.01 & -0.951 & 0.855 & 0.097 & 0.861 \\
\hline 780 & & 0.005 & -1.252 & 0.410 & 0.035 & 0.411 \\
\hline 780 & & 0.002 & -1.650 & 0.061 & 0.122 & 0.136 \\
\hline
\end{tabular}


Appendix 2. Temperature $T$, $\log _{10} \eta$, frequency $f$, $\log _{10} \omega \tau_{\mathrm{M}}$, imaginary $(G \mathrm{im})$ and real $\left(\mathrm{G}_{\text {real }}\right)$ shear modulus and shear modulus of the melt $(\mathrm{G}$ melt $)$ for the investigated samples - continuation..

\begin{tabular}{|c|c|c|c|c|c|c|}
\hline \multicolumn{7}{|c|}{ SAMPLE G11 } \\
\hline $\mathrm{T}\left({ }^{\circ} \mathrm{C}\right)$ & $\begin{array}{c}\log _{10} \\
\eta \\
\text { (Pa s) }\end{array}$ & $f(\mathrm{~Hz})$ & $\begin{array}{c}\log _{10} \\
\omega \tau_{M}\end{array}$ & $\begin{array}{c}\mathbf{G}_{\mathrm{im}} \\
(\mathrm{GPa})\end{array}$ & $\begin{array}{l}\mathbf{G}_{\text {real }} \\
(\mathrm{GPa})\end{array}$ & $\begin{array}{c}\mathbf{G}_{\text {melt }} \\
(\mathrm{GPa})\end{array}$ \\
\hline 540 & \multirow{10}{*}{$\begin{array}{l}\stackrel{2}{0} \\
\dot{\varphi}\end{array}$} & 1 & 6.343 & 0.047 & 32.094 & 32.094 \\
\hline 540 & & 0.5 & 6.042 & 0.056 & 32.240 & 32.240 \\
\hline 540 & & 0.2 & 5.644 & 0.218 & 32.761 & 32.762 \\
\hline 540 & & 0.1 & 5.343 & 0.496 & 31.973 & 31.977 \\
\hline 540 & & 0.05 & 5.042 & 0.557 & 32.085 & 32.090 \\
\hline 540 & & 0.02 & 4.644 & 0.944 & 32.166 & 32.179 \\
\hline 540 & & 0.01 & 4.343 & 0.730 & 31.402 & 31.411 \\
\hline 540 & & 0.005 & 4.042 & 0.605 & 31.223 & 31.229 \\
\hline 540 & & 0.002 & 3.644 & 0.157 & 31.408 & 31.408 \\
\hline 540 & & 0.001 & 3.343 & 0.202 & 32.001 & 32.002 \\
\hline 2560 & \multirow{10}{*}{$\begin{array}{l}\hat{m} \\
\text { فु }\end{array}$} & 1 & 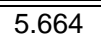 & $\begin{array}{l}0.151 \\
\end{array}$ & 32.055 & 32.055 \\
\hline 560 & & 0.5 & 5.362 & 0.006 & 31.614 & 31.614 \\
\hline 560 & & 0.2 & 4.965 & 0.070 & 31.939 & 31.939 \\
\hline 560 & & 0.1 & 4.664 & 0.077 & 31.354 & 31.354 \\
\hline 560 & & 0.05 & 4.362 & 0.487 & 31.385 & 31.389 \\
\hline 560 & & 0.02 & 3.965 & 0.046 & 30.852 & 30.852 \\
\hline 560 & & 0.01 & 3.664 & 0.160 & 31.584 & 31.585 \\
\hline 560 & & 0.005 & 3.362 & 0.314 & 30.941 & 30.942 \\
\hline 560 & & 0.002 & 2.965 & 0.612 & 31.704 & 31.710 \\
\hline 560 & & 0.001 & 2.664 & 0.421 & 29.909 & 29.912 \\
\hline 580 & \multirow{10}{*}{$\begin{array}{l}\underset{N}{\sim} \\
\underset{\sim}{+}\end{array}$} & 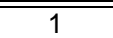 & 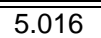 & $\begin{array}{l}0.084 \\
\end{array}$ & 31.325 & 31.325 \\
\hline 580 & & 0.5 & 4.715 & 0.113 & 30.928 & 30.928 \\
\hline 580 & & 0.2 & 4.317 & 0.131 & 30.725 & 30.725 \\
\hline 580 & & 0.1 & 4.016 & 0.262 & 30.761 & 30.762 \\
\hline 580 & & 0.05 & 3.715 & 0.376 & 30.195 & 30.198 \\
\hline 580 & & 0.02 & 3.317 & 0.311 & 30.239 & 30.240 \\
\hline 580 & & 0.01 & 3.016 & 0.553 & 29.968 & 29.974 \\
\hline 580 & & 0.005 & 2.715 & 0.528 & 30.440 & 30.444 \\
\hline 580 & & 0.002 & 2.317 & 0.485 & 27.398 & 27.403 \\
\hline 580 & & 0.001 & 2.016 & 1.722 & 29.673 & 29.723 \\
\hline
\end{tabular}

\begin{tabular}{|c|c|c|c|c|c|c|}
\hline \multicolumn{7}{|c|}{ SAMPLE G11 } \\
\hline $\mathrm{T}\left({ }^{\circ} \mathrm{C}\right)$ & $\begin{array}{c}\log _{10} \\
\eta \\
\text { (Pa s) }\end{array}$ & $f(\mathrm{~Hz})$ & $\begin{array}{c}\log _{10} \\
\omega \tau_{M}\end{array}$ & $\begin{array}{l}\mathbf{G}_{\mathrm{im}} \\
(\mathrm{GPa})\end{array}$ & $\begin{array}{l}\mathbf{G}_{\text {real }} \\
(\mathrm{GPa})\end{array}$ & $\begin{array}{c}\mathbf{G}_{\text {melt }} \\
(\mathrm{GPa})\end{array}$ \\
\hline 600 & \multirow{10}{*}{$\begin{array}{l}\vec{F} \\
\stackrel{+}{-}\end{array}$} & 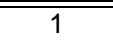 & 4.398 & 0.141 & 30.629 & 30.629 \\
\hline 600 & & 0.5 & 4.097 & 0.033 & 30.642 & 30.642 \\
\hline 600 & & 0.2 & 3.699 & 0.421 & 30.087 & 30.090 \\
\hline 600 & & 0.1 & 3.398 & 0.529 & 30.330 & 30.335 \\
\hline 600 & & 0.05 & 3.097 & 0.637 & 29.577 & 29.584 \\
\hline 600 & & 0.02 & 2.699 & 0.740 & 29.144 & 29.154 \\
\hline 600 & & 0.01 & 2.398 & 0.662 & 29.088 & 29.096 \\
\hline 600 & & 0.005 & 2.097 & 0.894 & 28.278 & 28.292 \\
\hline 600 & & 0.002 & 1.699 & 1.818 & 26.671 & 26.732 \\
\hline 600 & & 0.001 & 1.398 & 2.408 & 25.431 & 25.545 \\
\hline 620 & \multirow{10}{*}{ 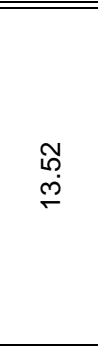 } & 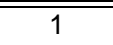 & 3.808 & "0.137 & 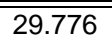 & 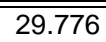 \\
\hline 620 & & 0.5 & 3.507 & 0.188 & 29.041 & 29.041 \\
\hline 620 & & 0.2 & 3.109 & 0.790 & 29.600 & 29.611 \\
\hline 620 & & 0.1 & 2.808 & 0.604 & 28.133 & 28.140 \\
\hline 620 & & 0.05 & 2.507 & 0.669 & 28.907 & 28.914 \\
\hline 620 & & 0.02 & 2.109 & 1.483 & 27.636 & 27.676 \\
\hline 620 & & 0.01 & 1.808 & 1.741 & 26.708 & 26.765 \\
\hline 620 & & 0.005 & 1.507 & 2.345 & 25.297 & 25.406 \\
\hline 620 & & 0.002 & 1.109 & 0.428 & 23.176 & 23.180 \\
\hline 620 & & 0.001 & 0.808 & 2.680 & 19.965 & 20.144 \\
\hline 640 & \multirow{10}{*}{ 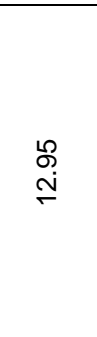 } & 1 & 3.244 & 0.419 & 30.480 & 30.482 \\
\hline 640 & & 0.5 & 2.943 & 0.646 & 29.684 & 29.691 \\
\hline 640 & & 0.2 & 2.545 & 0.912 & 29.235 & 29.249 \\
\hline 640 & & 0.1 & 2.244 & 1.531 & 28.373 & 28.414 \\
\hline 640 & & 0.05 & 1.943 & 1.745 & 27.515 & 27.570 \\
\hline 640 & & 0.02 & 1.545 & 2.960 & 25.819 & 25.988 \\
\hline 640 & & 0.01 & 1.244 & 4.014 & 23.389 & 23.731 \\
\hline 640 & & 0.005 & 0.943 & 3.791 & 20.361 & 20.711 \\
\hline 640 & & 0.002 & 0.545 & 3.615 & 16.288 & 16.684 \\
\hline 640 & & 0.001 & 0.244 & 5.529 & 11.363 & 12.637 \\
\hline
\end{tabular}


Appendix 2. Temperature $T, \log _{10} \eta$, frequency $f, \log _{10} \omega \tau_{\mathrm{M}}$, imaginary $\left(G_{\mathrm{im}}\right)$ and real ( $(\mathrm{G}$ real) shear modulus and shear modulus of the melt ( $G$ melt) for the investigated samples - continuation..

\begin{tabular}{|c|c|c|c|c|c|c|}
\hline \multicolumn{7}{|c|}{ SAMPLE G11 } \\
\hline $\mathrm{T}\left({ }^{\circ} \mathrm{C}\right)$ & $\begin{array}{c}\log _{10} \\
\eta \\
\text { (Pa s) }\end{array}$ & $f(\mathrm{~Hz})$ & $\begin{array}{c}\log _{10} \\
\omega \tau_{M}\end{array}$ & $\begin{array}{c}\mathbf{G}_{\text {im }} \\
(\mathbf{G P a})\end{array}$ & $\begin{array}{l}\mathbf{G}_{\text {real }} \\
(\mathrm{GPa})\end{array}$ & $\begin{array}{l}\mathbf{G}_{\text {melt }} \\
(\mathrm{GPa})\end{array}$ \\
\hline 2660 & \multirow{10}{*}{$\begin{array}{l}\underset{+}{+} \\
\underset{\sim}{+}\end{array}$} & $\overline{11}$ & $\begin{array}{l}2.704 \\
\end{array}$ & $\begin{array}{l}0.919 \\
\end{array}$ & 29.430 & $\bar{~} 29.445$ \\
\hline 660 & & 0.5 & 2.403 & 1.121 & 28.581 & 28.603 \\
\hline 660 & & 0.2 & 2.005 & 1.759 & 27.217 & 27.273 \\
\hline 660 & & 0.1 & 1.704 & 2.549 & 25.909 & 26.034 \\
\hline 660 & & 0.05 & 1.403 & 3.398 & 23.421 & 23.666 \\
\hline 660 & & 0.02 & 1.005 & 5.227 & 20.121 & 20.789 \\
\hline 660 & & 0.01 & 0.704 & 6.324 & 16.304 & 17.487 \\
\hline 660 & & 0.005 & 0.403 & 1.509 & 14.208 & 14.288 \\
\hline 660 & & 0.002 & 0.005 & 5.719 & 5.716 & 8.086 \\
\hline 660 & & 0.001 & -0.296 & 3.886 & 3.109 & 4.977 \\
\hline 680 & \multirow{10}{*}{$\begin{array}{l}\stackrel{8}{\infty} \\
\text { - }\end{array}$} & 1 & 2.187 & $\bar{~} 1.872$ & 29.551 & 29.610 \\
\hline 680 & & 0.5 & 1.886 & 2.596 & 27.816 & 27.937 \\
\hline 680 & & 0.2 & 1.488 & 3.969 & 25.480 & 25.788 \\
\hline 680 & & 0.1 & 1.187 & 4.924 & 22.429 & 22.963 \\
\hline 680 & & 0.05 & 0.886 & 6.073 & 18.334 & 19.313 \\
\hline 680 & & 0.02 & 0.488 & 6.971 & 12.199 & 14.051 \\
\hline 680 & & 0.01 & 0.187 & 6.481 & 7.370 & 9.814 \\
\hline 680 & & 0.005 & -0.114 & 3.922 & 4.931 & 6.301 \\
\hline 680 & & 0.002 & -0.512 & 2.472 & 1.034 & 2.680 \\
\hline 680 & & 0.001 & -0.813 & 1.266 & 0.328 & 1.308 \\
\hline 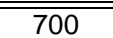 & \multirow{10}{*}{$\begin{array}{l}\stackrel{o}{+} \\
\text { ت }\end{array}$} & 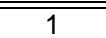 & $\begin{array}{ll}1.691 \\
\end{array}$ & $\begin{array}{l}3.267 \\
\end{array}$ & 26.436 & 26.637 \\
\hline 700 & & 0.5 & 1.390 & 4.334 & 24.124 & 24.510 \\
\hline 700 & & 0.2 & 0.992 & 6.142 & 19.337 & 20.289 \\
\hline 700 & & 0.1 & 0.691 & 6.911 & 14.572 & 16.128 \\
\hline 700 & & 0.05 & 0.390 & 6.798 & 9.463 & 11.651 \\
\hline 700 & & 0.02 & -0.008 & 5.124 & 3.893 & 6.435 \\
\hline 700 & & 0.01 & -0.309 & 3.398 & 1.635 & 3.771 \\
\hline 700 & & 0.005 & -0.610 & 1.854 & 0.564 & 1.938 \\
\hline 700 & & 0.002 & -1.008 & 0.822 & 0.115 & 0.830 \\
\hline 700 & & 0.001 & -1.309 & 0.424 & 0.052 & 0.427 \\
\hline
\end{tabular}

\begin{tabular}{|c|c|c|c|c|c|c|}
\hline \multicolumn{7}{|c|}{ SAMPLE G11 } \\
\hline $\mathrm{T}\left({ }^{\circ} \mathrm{C}\right)$ & $\begin{array}{c}\log _{10} \\
\eta \\
\text { (Pa s) }\end{array}$ & $f(\mathrm{~Hz})$ & $\begin{array}{c}\log _{10} \\
\omega \tau_{M}\end{array}$ & $\begin{array}{c}\mathbf{G}_{\mathrm{im}} \\
(\mathrm{GPa})\end{array}$ & $\begin{array}{l}\mathbf{G}_{\text {real }} \\
(\mathrm{GPa})\end{array}$ & $\begin{array}{l}\mathbf{G}_{\text {melt }} \\
(\mathrm{GPa})\end{array}$ \\
\hline 720 & \multirow{8}{*}{$\begin{array}{l}\tilde{\sigma} \\
\text { Oें } \\
\text { - }\end{array}$} & 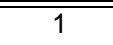 & 1.214 & 5.767 & 22.990 & 23.703 \\
\hline 720 & & 0.5 & 0.913 & 7.066 & 18.776 & 20.061 \\
\hline 720 & & 0.2 & 0.516 & 7.456 & 11.183 & 13.441 \\
\hline 720 & & 0.1 & 0.214 & 6.517 & 6.408 & 9.140 \\
\hline 720 & & 0.05 & -0.087 & 4.472 & 2.791 & 5.272 \\
\hline 720 & & 0.02 & -0.484 & 2.246 & 0.730 & 2.362 \\
\hline 720 & & 0.01 & -0.786 & 1.202 & 0.231 & 1.224 \\
\hline 720 & & 0.005 & -1.087 & 0.662 & 0.095 & 0.669 \\
\hline 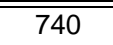 & \multirow{8}{*}{ ণ্ণ } & 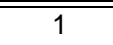 & 0.757 & 7.477 & 15.163 & 16.906 \\
\hline 740 & & 0.5 & 0.456 & 7.467 & 9.702 & 12.243 \\
\hline 740 & & 0.2 & 0.058 & 5.407 & 3.822 & 6.621 \\
\hline 740 & & 0.1 & -0.243 & 3.372 & 1.478 & 3.682 \\
\hline 740 & & 0.05 & -0.544 & 1.902 & 0.508 & 1.969 \\
\hline & & & & & & \\
\hline & & & & & & \\
\hline & & & & & & \\
\hline
\end{tabular}


Appendix 2. Temperature $T, \log _{10} \eta$, frequency $f, \log _{10} \omega \tau_{\mathrm{M}}$, imaginary $\left(G_{\mathrm{im}}\right)$ and real ( $(\mathrm{G}$ real) shear modulus and shear modulus of the melt ( $G$ melt) for the investigated samples - continuation..

\begin{tabular}{|c|c|c|c|c|c|c|}
\hline \multicolumn{7}{|c|}{ SAMPLE G12 } \\
\hline $\mathrm{T}\left({ }^{\circ} \mathrm{C}\right)$ & $\begin{array}{c}\log _{10} \\
\eta \\
\text { (Pa s) }\end{array}$ & $f(\mathrm{~Hz})$ & $\begin{array}{c}\log _{10} \\
\omega \tau_{M}\end{array}$ & $\begin{array}{c}\mathbf{G}_{\mathrm{im}} \\
(\mathrm{GPa})\end{array}$ & $\begin{array}{l}\mathbf{G}_{\text {real }} \\
(\mathrm{GPa})\end{array}$ & $\begin{array}{c}\mathbf{G}_{\text {melt }} \\
(\mathrm{GPa})\end{array}$ \\
\hline 340 & \multirow{10}{*}{ 穵 } & 1 & 15.576 & 0.022 & 31.420 & 31.420 \\
\hline 340 & & 0.5 & 15.275 & 0.037 & 31.316 & 31.316 \\
\hline 340 & & 0.2 & 14.877 & 0.028 & 31.543 & 31.543 \\
\hline 340 & & 0.1 & 14.576 & 0.044 & 30.880 & 30.880 \\
\hline 340 & & 0.05 & 14.275 & 0.222 & 31.072 & 31.073 \\
\hline 340 & & 0.02 & 13.877 & 0.018 & 31.320 & 31.320 \\
\hline 340 & & 0.01 & 13.576 & 0.057 & 31.180 & 31.180 \\
\hline 340 & & 0.005 & 13.275 & 0.251 & 30.946 & 30.947 \\
\hline 340 & & 0.002 & 12.877 & 0.107 & 31.006 & 31.006 \\
\hline 340 & & 0.001 & 12.576 & 0.034 & 31.092 & 31.092 \\
\hline 360 & \multirow{10}{*}{ 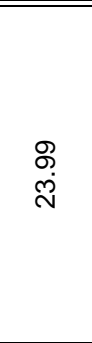 } & 1 & 214.289 & 0.014 & 31.494 & 31.494 \\
\hline 360 & & 0.5 & 13.988 & 0.018 & 31.404 & 31.404 \\
\hline 360 & & 0.2 & 13.590 & 0.017 & 31.006 & 31.006 \\
\hline 360 & & 0.1 & 13.289 & 0.206 & 30.914 & 30.915 \\
\hline 360 & & 0.05 & 12.988 & 0.278 & 30.938 & 30.939 \\
\hline 360 & & 0.02 & 12.590 & 0.267 & 31.384 & 31.385 \\
\hline 360 & & 0.01 & 12.289 & 0.095 & 31.217 & 31.217 \\
\hline 360 & & 0.005 & 11.988 & 0.117 & 30.869 & 30.869 \\
\hline 360 & & 0.002 & 11.590 & 0.050 & 30.643 & 30.643 \\
\hline 360 & & 0.001 & 11.289 & 0.185 & 30.700 & 30.701 \\
\hline 380 & \multirow{10}{*}{ 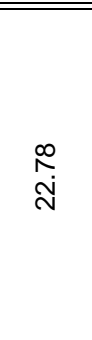 } & $\overline{11}$ & $\begin{array}{ll}13.080 \\
\end{array}$ & $\begin{array}{l}0.045 \\
\end{array}$ & 31.212 & 31.212 \\
\hline 380 & & 0.5 & 12.779 & 0.053 & 30.630 & 30.630 \\
\hline 380 & & 0.2 & 12.381 & 0.210 & 31.531 & 31.532 \\
\hline 380 & & 0.1 & 12.080 & 0.484 & 31.190 & 31.194 \\
\hline 380 & & 0.05 & 11.779 & 0.545 & 31.397 & 31.401 \\
\hline 380 & & 0.02 & 11.381 & 0.909 & 30.959 & 30.972 \\
\hline 380 & & 0.01 & 11.080 & 0.722 & 31.029 & 31.037 \\
\hline 380 & & 0.005 & 10.779 & 0.592 & 30.580 & 30.586 \\
\hline 380 & & 0.002 & 10.381 & 0.155 & 30.910 & 30.911 \\
\hline 380 & & 0.001 & 10.080 & 0.193 & 30.524 & 30.525 \\
\hline
\end{tabular}

\begin{tabular}{|c|c|c|c|c|c|c|}
\hline \multicolumn{7}{|c|}{ SAMPLE G12 } \\
\hline $\mathrm{T}\left({ }^{\circ} \mathrm{C}\right)$ & $\begin{array}{c}\log _{10} \\
\eta \\
\text { (Pa s) }\end{array}$ & $f(\mathrm{~Hz})$ & $\begin{array}{c}\log _{10} \\
\omega \tau_{M}\end{array}$ & $\begin{array}{l}\mathbf{G}_{\mathrm{im}} \\
(\mathrm{GPa})\end{array}$ & $\begin{array}{l}\mathbf{G}_{\text {real }} \\
(\mathrm{GPa})\end{array}$ & $\begin{array}{c}\mathbf{G}_{\text {melt }} \\
(\mathrm{GPa})\end{array}$ \\
\hline 400 & \multirow{10}{*}{\begin{tabular}{l}
\multirow{U}{0}{} \\
$\dot{N}$
\end{tabular}} & 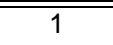 & 11.943 & 0.035 & 30.681 & 30.681 \\
\hline 400 & & 0.5 & 11.642 & 0.023 & 30.972 & 30.972 \\
\hline 400 & & 0.2 & 11.244 & 0.061 & 30.895 & 30.895 \\
\hline 400 & & 0.1 & 10.943 & 0.230 & 31.664 & 31.665 \\
\hline 400 & & 0.05 & 10.642 & 0.201 & 30.857 & 30.858 \\
\hline 400 & & 0.02 & 10.244 & 0.035 & 30.407 & 30.407 \\
\hline 400 & & 0.01 & 9.943 & 0.001 & 31.149 & 31.149 \\
\hline 400 & & 0.005 & 9.642 & 0.145 & 30.712 & 30.712 \\
\hline 400 & & 0.002 & 9.244 & 0.107 & 30.540 & 30.540 \\
\hline 400 & & 0.001 & 8.943 & 0.182 & 30.820 & 30.820 \\
\hline (420 & \multirow{10}{*}{ مُ } & 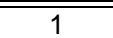 & $\begin{array}{l}10.871 \\
\end{array}$ & $\begin{array}{l}0.105 \\
\end{array}$ & "31.261 & "31.261 \\
\hline 420 & & 0.5 & 10.570 & 0.012 & 30.934 & 30.934 \\
\hline 420 & & 0.2 & 10.173 & 0.009 & 30.693 & 30.693 \\
\hline 420 & & 0.1 & 9.871 & 0.220 & 31.353 & 31.354 \\
\hline 420 & & 0.05 & 9.570 & 0.091 & 30.449 & 30.449 \\
\hline 420 & & 0.02 & 9.173 & 0.132 & 30.640 & 30.640 \\
\hline 420 & & 0.01 & 8.871 & 0.343 & 30.527 & 30.529 \\
\hline 420 & & 0.005 & 8.570 & 0.025 & 30.567 & 30.567 \\
\hline 420 & & 0.002 & 8.173 & 0.032 & 31.141 & 31.141 \\
\hline 420 & & 0.001 & 7.871 & 0.177 & 30.318 & 30.318 \\
\hline 440 & \multirow{10}{*}{ 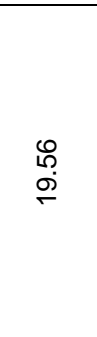 } & 1 & 9.860 & 0.046 & 30.605 & 30.605 \\
\hline 440 & & 0.5 & 9.559 & 0.011 & 30.328 & 30.328 \\
\hline 440 & & 0.2 & 9.161 & 0.067 & 29.815 & 29.815 \\
\hline 440 & & 0.1 & 8.860 & 0.088 & 30.353 & 30.353 \\
\hline 440 & & 0.05 & 8.559 & 0.123 & 29.808 & 29.809 \\
\hline 440 & & 0.02 & 8.161 & 0.010 & 30.372 & 30.372 \\
\hline 440 & & 0.01 & 7.860 & 0.330 & 30.045 & 30.046 \\
\hline 440 & & 0.005 & 7.559 & 0.039 & 29.963 & 29.963 \\
\hline 440 & & 0.002 & 7.161 & 0.038 & 30.033 & 30.033 \\
\hline 440 & & 0.001 & 6.860 & 0.125 & 29.364 & 29.364 \\
\hline
\end{tabular}


Appendix 2. Temperature $T$, $\log _{10} \eta$, frequency $f$, $\log _{10} \omega \tau_{\mathrm{M}}$, imaginary $(G \mathrm{im})$ and real $\left(\mathrm{G}_{\text {real }}\right)$ shear modulus and shear modulus of the melt $(\mathrm{G}$ melt $)$ for the investigated samples - continuation..

\begin{tabular}{|c|c|c|c|c|c|c|}
\hline \multicolumn{7}{|c|}{ SAMPLE G12 } \\
\hline $\mathrm{T}\left({ }^{\circ} \mathrm{C}\right)$ & $\begin{array}{c}\log _{10} \\
\eta \\
\text { (Pa s) }\end{array}$ & $f(\mathrm{~Hz})$ & $\begin{array}{c}\log _{10} \\
\omega \tau_{M}\end{array}$ & $\begin{array}{c}\mathbf{G}_{\mathrm{im}} \\
(\mathrm{GPa})\end{array}$ & $\begin{array}{l}\mathbf{G}_{\text {real }} \\
(\mathrm{GPa})\end{array}$ & $\begin{array}{l}\mathbf{G}_{\text {melt }} \\
(\mathrm{GPa})\end{array}$ \\
\hline 460 & \multirow{10}{*}{ 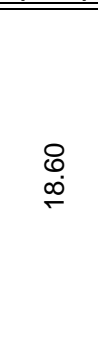 } & 1 & 8.904 & 0.083 & 30.516 & 30.516 \\
\hline 460 & & 0.5 & 8.603 & 0.066 & 30.663 & 30.663 \\
\hline 460 & & 0.2 & 8.205 & 0.101 & 29.968 & 29.969 \\
\hline 460 & & 0.1 & 7.904 & 0.112 & 30.367 & 30.367 \\
\hline 460 & & 0.05 & 7.603 & 0.157 & 30.376 & 30.376 \\
\hline 460 & & 0.02 & 7.205 & 0.097 & 30.374 & 30.375 \\
\hline 460 & & 0.01 & 6.904 & 0.036 & 29.757 & 29.757 \\
\hline 460 & & 0.005 & 6.603 & 0.011 & 30.204 & 30.204 \\
\hline 460 & & 0.002 & 6.205 & 0.053 & 29.795 & 29.795 \\
\hline 460 & & 0.001 & 5.904 & 0.193 & 29.842 & 29.843 \\
\hline 4880 & \multirow{10}{*}{$\begin{array}{l}\stackrel{5}{r} \\
\stackrel{H}{H}\end{array}$} & 1 & $\begin{array}{ll}7.999 \\
\end{array}$ & $\begin{array}{l}0.015 \\
\end{array}$ & 30.536 & 30.536 \\
\hline 480 & & 0.5 & 7.698 & 0.151 & 30.051 & 30.052 \\
\hline 480 & & 0.2 & 7.300 & 0.065 & 30.008 & 30.008 \\
\hline 480 & & 0.1 & 6.999 & 0.209 & 29.827 & 29.828 \\
\hline 480 & & 0.05 & 6.698 & 0.319 & 30.110 & 30.111 \\
\hline 480 & & 0.02 & 6.300 & 0.006 & 29.549 & 29.549 \\
\hline 480 & & 0.01 & 5.999 & 0.303 & 29.038 & 29.040 \\
\hline 480 & & 0.005 & 5.698 & 0.095 & 29.490 & 29.490 \\
\hline 480 & & 0.002 & 5.300 & 0.141 & 29.161 & 29.161 \\
\hline 480 & & 0.001 & 4.999 & 0.335 & 29.677 & 29.679 \\
\hline 2500 & \multirow{10}{*}{ 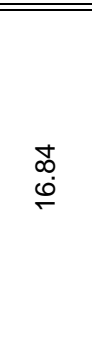 } & 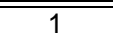 & $\begin{array}{ll}7.141 \\
\end{array}$ & $\begin{array}{l}0.151 \\
\end{array}$ & 30.061 & 30.061 \\
\hline 500 & & 0.5 & 6.840 & 0.072 & 29.813 & 29.813 \\
\hline 500 & & 0.2 & 6.442 & 0.040 & 29.775 & 29.775 \\
\hline 500 & & 0.1 & 6.141 & 0.162 & 30.520 & 30.520 \\
\hline 500 & & 0.05 & 5.840 & 0.633 & 29.927 & 29.934 \\
\hline 500 & & 0.02 & 5.442 & 0.012 & 30.383 & 30.383 \\
\hline 500 & & 0.01 & 5.141 & 0.072 & 29.809 & 29.809 \\
\hline 500 & & 0.005 & 4.840 & 0.383 & 29.433 & 29.435 \\
\hline 500 & & 0.002 & 4.442 & 0.271 & 29.223 & 29.224 \\
\hline 500 & & 0.001 & 4.141 & 0.349 & 28.923 & 28.925 \\
\hline
\end{tabular}

\begin{tabular}{|c|c|c|c|c|c|c|}
\hline \multicolumn{7}{|c|}{ SAMPLE G12 } \\
\hline $\mathrm{T}\left({ }^{\circ} \mathrm{C}\right)$ & $\begin{array}{c}\log _{10} \\
\eta \\
\text { (Pa s) }\end{array}$ & $f(\mathrm{~Hz})$ & $\begin{array}{c}\log _{10} \\
\omega \tau_{M}\end{array}$ & $\begin{array}{l}\mathbf{G}_{\text {im }} \\
(\mathbf{G P a})\end{array}$ & $\begin{array}{l}\mathbf{G}_{\text {real }} \\
(\mathrm{GPa})\end{array}$ & $\begin{array}{l}G_{\text {melt }} \\
(\mathrm{GPa})\end{array}$ \\
\hline 520 & \multirow{10}{*}{ 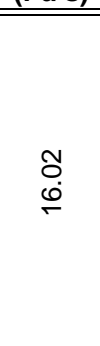 } & 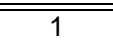 & 6.325 & 0.119 & 30.446 & 30.447 \\
\hline 520 & & 0.5 & 6.024 & 0.347 & 30.245 & 30.247 \\
\hline 520 & & 0.2 & 5.626 & 0.090 & 30.252 & 30.252 \\
\hline 520 & & 0.1 & 5.325 & 0.397 & 29.979 & 29.981 \\
\hline 520 & & 0.05 & 5.024 & 0.052 & 29.383 & 29.383 \\
\hline 520 & & 0.02 & 4.626 & 0.093 & 30.176 & 30.176 \\
\hline 520 & & 0.01 & 4.325 & 0.634 & 29.735 & 29.742 \\
\hline 520 & & 0.005 & 4.024 & 0.397 & 28.881 & 28.884 \\
\hline 520 & & 0.002 & 3.626 & 0.462 & 29.236 & 29.239 \\
\hline 520 & & 0.001 & 3.325 & 0.743 & 28.762 & 28.771 \\
\hline$\overline{540}$ & \multirow{10}{*}{$\begin{array}{l}\stackrel{\sim}{N} \\
\stackrel{\leftrightarrow}{\leftrightarrow}\end{array}$} & $\overline{11}$ & $\begin{array}{c}5.550 \\
\end{array}$ & "0.090 & " 30.084 & "30.084 \\
\hline 540 & & 0.5 & 5.249 & 0.024 & 29.987 & 29.987 \\
\hline 540 & & 0.2 & 4.851 & 0.048 & 30.561 & 30.561 \\
\hline 540 & & 0.1 & 4.550 & 0.170 & 29.603 & 29.603 \\
\hline 540 & & 0.05 & 4.249 & 0.417 & 29.259 & 29.262 \\
\hline 540 & & 0.02 & 3.851 & 0.442 & 29.260 & 29.263 \\
\hline 540 & & 0.01 & 3.550 & 0.820 & 28.929 & 28.941 \\
\hline 540 & & 0.005 & 3.249 & 0.586 & 28.836 & 28.842 \\
\hline 540 & & 0.002 & 2.851 & 0.827 & 27.731 & 27.744 \\
\hline 540 & & 0.001 & 2.550 & 1.519 & 27.628 & 27.670 \\
\hline$\overline{5600}$ & \multirow{10}{*}{ 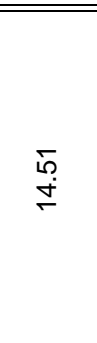 } & $\bar{~} 11$ & 4.813 & 0.236 & 29.632 & 29.633 \\
\hline 560 & & 0.5 & 4.512 & 0.280 & 29.437 & 29.438 \\
\hline 560 & & 0.2 & 4.114 & 0.320 & 29.130 & 29.132 \\
\hline 560 & & 0.1 & 3.813 & 0.162 & 28.939 & 28.940 \\
\hline 560 & & 0.05 & 3.512 & 0.526 & 28.508 & 28.513 \\
\hline 560 & & 0.02 & 3.114 & 0.747 & 27.897 & 27.907 \\
\hline 560 & & 0.01 & 2.813 & 1.098 & 27.343 & 27.365 \\
\hline 560 & & 0.005 & 2.512 & 1.566 & 26.644 & 26.690 \\
\hline 560 & & 0.002 & 2.114 & 2.240 & 25.083 & 25.183 \\
\hline 560 & & 0.001 & 1.813 & 0.384 & 23.918 & 23.921 \\
\hline
\end{tabular}


Appendix 2. Temperature $T$, $\log _{10} \eta$, frequency $f$, $\log _{10} \omega \tau_{\mathrm{M}}$, imaginary $(G \mathrm{im})$ and real $\left(\mathrm{G}_{\text {real }}\right)$ shear modulus and shear modulus of the melt $(\mathrm{G}$ melt $)$ for the investigated samples - continuation..

\begin{tabular}{|c|c|c|c|c|c|c|}
\hline \multicolumn{7}{|c|}{ SAMPLE G12 } \\
\hline $\mathrm{T}\left({ }^{\circ} \mathrm{C}\right)$ & $\begin{array}{c}\log _{10} \\
\eta \\
\text { (Pa s) }\end{array}$ & $f(\mathrm{~Hz})$ & $\begin{array}{c}\log _{10} \\
\omega \tau_{M}\end{array}$ & $\begin{array}{c}\mathbf{G}_{\text {im }} \\
(\mathbf{G P a})\end{array}$ & $\begin{array}{l}\mathbf{G}_{\text {real }} \\
(\mathrm{GPa})\end{array}$ & $\begin{array}{l}\mathbf{G}_{\text {melt }} \\
(\mathrm{GPa})\end{array}$ \\
\hline 580 & \multirow{10}{*}{ 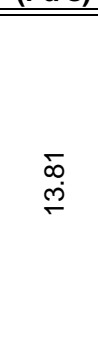 } & 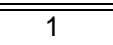 & 4.109 & 0.395 & 29.235 & 29.237 \\
\hline 580 & & 0.5 & 3.808 & 0.460 & 29.003 & 29.006 \\
\hline 580 & & 0.2 & 3.411 & 0.543 & 27.869 & 27.874 \\
\hline 580 & & 0.1 & 3.109 & 0.826 & 28.288 & 28.300 \\
\hline 580 & & 0.05 & 2.808 & 0.865 & 27.135 & 27.148 \\
\hline 580 & & 0.02 & 2.411 & 1.654 & 25.852 & 25.905 \\
\hline 580 & & 0.01 & 2.109 & 2.046 & 24.676 & 24.761 \\
\hline 580 & & 0.005 & 1.808 & 2.883 & 23.044 & 23.223 \\
\hline 580 & & 0.002 & 1.411 & 1.054 & 23.928 & 23.951 \\
\hline 580 & & 0.001 & 1.109 & 3.244 & 17.804 & 18.097 \\
\hline 年600 & \multirow{10}{*}{ 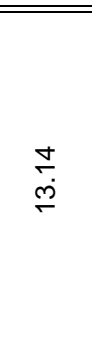 } & $\bar{~} 1$ & $\begin{array}{l}3.439 \\
\end{array}$ & $\bar{~} 0.622$ & 28.329 & 28.336 \\
\hline 600 & & 0.5 & 3.137 & 0.745 & 27.629 & 27.639 \\
\hline 600 & & 0.2 & 2.740 & 1.207 & 27.149 & 27.176 \\
\hline 600 & & 0.1 & 2.439 & 2.097 & 25.825 & 25.910 \\
\hline 600 & & 0.05 & 2.137 & 2.543 & 24.662 & 24.793 \\
\hline 600 & & 0.02 & 1.740 & 3.450 & 22.222 & 22.488 \\
\hline 600 & & 0.01 & 1.439 & 4.673 & 19.411 & 19.965 \\
\hline 600 & & 0.005 & 1.137 & 2.537 & 18.681 & 18.852 \\
\hline 600 & & 0.002 & 0.740 & 4.746 & 12.308 & 13.191 \\
\hline 600 & & 0.001 & 0.439 & 5.608 & 7.623 & 9.464 \\
\hline (620 & \multirow{10}{*}{$\begin{array}{l}\stackrel{\circ}{\leftrightarrow} \\
\text { ษิ }\end{array}$} & 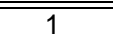 & $\begin{array}{l}2.798 \\
\end{array}$ & $\begin{array}{l}1.229 \\
\end{array}$ & 26.073 & 26.102 \\
\hline 620 & & 0.5 & 2.497 & 1.588 & 25.307 & 25.357 \\
\hline 620 & & 0.2 & 2.099 & 2.699 & 23.542 & 23.697 \\
\hline 620 & & 0.1 & 1.798 & 3.224 & 21.723 & 21.961 \\
\hline 620 & & 0.05 & 1.497 & 4.454 & 19.309 & 19.816 \\
\hline 620 & & 0.02 & 1.099 & 6.001 & 14.741 & 15.915 \\
\hline 620 & & 0.01 & 0.798 & 6.381 & 10.846 & 12.584 \\
\hline 620 & & 0.005 & 0.497 & 3.626 & 8.565 & 9.301 \\
\hline 620 & & 0.002 & 0.099 & 3.565 & 3.189 & 4.783 \\
\hline 620 & & 0.001 & -0.202 & 2.420 & 0.999 & 2.618 \\
\hline
\end{tabular}

\begin{tabular}{|c|c|c|c|c|c|c|}
\hline \multicolumn{7}{|c|}{ SAMPLE G12 } \\
\hline $\mathrm{T}\left({ }^{\circ} \mathrm{C}\right)$ & $\begin{array}{c}\log _{10} \\
\eta \\
\text { (Pa s) }\end{array}$ & $f(\mathrm{~Hz})$ & $\begin{array}{c}\log _{10} \\
\omega \tau_{M}\end{array}$ & $\begin{array}{l}\mathbf{G}_{\text {im }} \\
(\mathbf{G P a})\end{array}$ & $\begin{array}{l}\mathbf{G}_{\text {real }} \\
(\mathrm{GPa})\end{array}$ & $\begin{array}{c}\mathbf{G}_{\text {melt }} \\
(\mathrm{GPa})\end{array}$ \\
\hline 640 & & 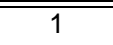 & 2.185 & 2.318 & 23.108 & 23.224 \\
\hline 640 & & 0.5 & 1.884 & 3.193 & 21.537 & 21.772 \\
\hline 640 & & 0.2 & 1.486 & 4.616 & 18.524 & 19.091 \\
\hline 640 & & 0.1 & 1.185 & 5.688 & 15.053 & 16.091 \\
\hline 640 & $\infty$ & 0.05 & 0.884 & 6.119 & 11.103 & 12.677 \\
\hline 640 & ت & 0.02 & 0.486 & 5.705 & 5.879 & 8.192 \\
\hline 640 & & 0.01 & 0.185 & 1.938 & 5.157 & 5.509 \\
\hline 640 & & 0.005 & -0.116 & 2.319 & 1.978 & 3.048 \\
\hline 640 & & 0.002 & -0.514 & 1.265 & 0.276 & 1.294 \\
\hline 640 & & 0.001 & -0.815 & 0.660 & 0.140 & 0.675 \\
\hline 660 & & 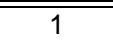 & 1.598 & "4.221 & 19.098 & 19.559 \\
\hline 660 & & 0.5 & 1.297 & 5.288 & 16.190 & 17.032 \\
\hline 660 & & 0.2 & 0.899 & 6.177 & 11.161 & 12.756 \\
\hline 660 & & 0.1 & 0.598 & 5.927 & 7.016 & 9.185 \\
\hline 660 & প্ল & 0.05 & 0.297 & 4.607 & 3.565 & 5.825 \\
\hline 660 & تં & 0.02 & -0.101 & 2.543 & 1.051 & 2.752 \\
\hline 660 & & 0.01 & -0.402 & 1.556 & 0.384 & 1.603 \\
\hline 660 & & 0.005 & -0.703 & 0.779 & 0.110 & 0.787 \\
\hline
\end{tabular}


Appendix 2. Temperature $T$, $\log _{10} \eta$, frequency $f$, $\log _{10} \omega \tau_{\mathrm{M}}$, imaginary $(G \mathrm{im})$ and real $\left(\mathrm{G}_{\text {real }}\right)$ shear modulus and shear modulus of the melt $(\mathrm{G}$ melt $)$ for the investigated samples - continuation..

\begin{tabular}{|c|c|c|c|c|c|c|}
\hline \multicolumn{7}{|c|}{ SAMPLE G13 } \\
\hline $\mathrm{T}\left({ }^{\circ} \mathrm{C}\right)$ & $\begin{array}{c}\log _{10} \\
\eta \\
\text { (Pa s) }\end{array}$ & $f(\mathrm{~Hz})$ & $\begin{array}{c}\log _{10} \\
\omega \tau_{M}\end{array}$ & $\begin{array}{c}\mathbf{G}_{\mathrm{im}} \\
(\mathrm{GPa})\end{array}$ & $\begin{array}{l}\mathbf{G}_{\text {real }} \\
(\mathrm{GPa})\end{array}$ & $\begin{array}{c}\mathbf{G}_{\text {melt }} \\
(\mathrm{GPa})\end{array}$ \\
\hline 320 & \multirow{10}{*}{ 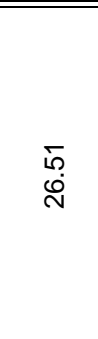 } & 1 & 16.820 & 0.088 & 30.887 & 30.887 \\
\hline 320 & & 0.5 & 16.519 & 0.067 & 30.945 & 30.945 \\
\hline 320 & & 0.2 & 16.121 & 0.185 & 31.151 & 31.151 \\
\hline 320 & & 0.1 & 15.820 & 0.121 & 30.608 & 30.609 \\
\hline 320 & & 0.05 & 15.519 & 0.415 & 30.654 & 30.657 \\
\hline 320 & & 0.02 & 15.121 & 0.259 & 30.719 & 30.720 \\
\hline 320 & & 0.01 & 14.820 & 0.093 & 30.988 & 30.988 \\
\hline 320 & & 0.005 & 14.519 & 0.251 & 30.609 & 30.610 \\
\hline 320 & & 0.002 & 14.121 & 0.027 & 29.761 & 29.761 \\
\hline 320 & & 0.001 & 13.820 & 0.317 & 32.020 & 32.021 \\
\hline 340 & \multirow{10}{*}{ 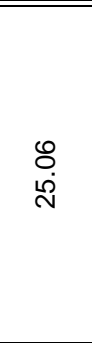 } & 1 & 215.367 & $\begin{array}{l}0.059 \\
\end{array}$ & 30.701 & 30.701 \\
\hline 340 & & 0.5 & 15.066 & 0.076 & 30.779 & 30.780 \\
\hline 340 & & 0.2 & 14.668 & 0.193 & 30.813 & 30.813 \\
\hline 340 & & 0.1 & 14.367 & 0.023 & 30.890 & 30.890 \\
\hline 340 & & 0.05 & 14.066 & 0.627 & 30.840 & 30.846 \\
\hline 340 & & 0.02 & 13.668 & 0.127 & 30.043 & 30.043 \\
\hline 340 & & 0.01 & 13.367 & 0.000 & 30.766 & 30.766 \\
\hline 340 & & 0.005 & 13.066 & & & \\
\hline 340 & & 0.002 & 12.668 & 0.048 & 29.934 & 29.934 \\
\hline 340 & & 0.001 & 12.367 & 0.919 & 30.672 & 30.686 \\
\hline 360 & \multirow{10}{*}{$\begin{array}{l}\stackrel{P}{\mathscr{N}} \\
\stackrel{N}{N}\end{array}$} & 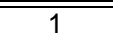 & 14.005 & $\begin{array}{l}0.058 \\
\end{array}$ & 30.838 & 30.838 \\
\hline 360 & & 0.5 & 13.704 & 0.052 & 30.627 & 30.627 \\
\hline 360 & & 0.2 & 13.306 & 0.221 & 30.701 & 30.702 \\
\hline 360 & & 0.1 & 13.005 & 0.152 & 30.487 & 30.488 \\
\hline 360 & & 0.05 & 12.704 & 0.213 & 30.307 & 30.308 \\
\hline 360 & & 0.02 & 12.306 & 0.082 & 30.785 & 30.785 \\
\hline 360 & & 0.01 & 12.005 & 0.003 & 30.323 & 30.323 \\
\hline 360 & & 0.005 & 11.704 & 0.252 & 30.560 & 30.561 \\
\hline 360 & & 0.002 & 11.306 & 0.249 & 30.166 & 30.167 \\
\hline 360 & & 0.001 & 11.005 & 0.384 & 28.952 & 28.954 \\
\hline
\end{tabular}

\begin{tabular}{|c|c|c|c|c|c|c|}
\hline \multicolumn{7}{|c|}{ SAMPLE G13 } \\
\hline $\mathrm{T}\left({ }^{\circ} \mathrm{C}\right)$ & $\begin{array}{c}\log _{10} \\
\eta \\
\text { (Pa s) }\end{array}$ & $f(\mathrm{~Hz})$ & $\begin{array}{c}\log _{10} \\
\omega \tau_{M}\end{array}$ & $\begin{array}{c}\mathbf{G}_{\text {im }} \\
(\mathrm{GPa})\end{array}$ & $\begin{array}{l}\mathbf{G}_{\text {real }} \\
(\mathrm{GPa})\end{array}$ & $\begin{array}{c}\mathbf{G}_{\text {melt }} \\
(\mathrm{GPa})\end{array}$ \\
\hline 380 & \multirow{10}{*}{$\begin{array}{l}\text { ปั } \\
\text { ป̃ }\end{array}$} & 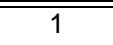 & 12.727 & 0.101 & 30.675 & 30.675 \\
\hline 380 & & 0.5 & 12.426 & 0.049 & 30.440 & 30.440 \\
\hline 380 & & 0.2 & 12.028 & 0.100 & 30.398 & 30.398 \\
\hline 380 & & 0.1 & 11.727 & 0.160 & 30.728 & 30.729 \\
\hline 380 & & 0.05 & 11.426 & 0.146 & 31.022 & 31.023 \\
\hline 380 & & 0.02 & 11.028 & 0.056 & 30.441 & 30.441 \\
\hline 380 & & 0.01 & 10.727 & 0.239 & 30.521 & 30.522 \\
\hline 380 & & 0.005 & 10.426 & 0.048 & 30.876 & 30.876 \\
\hline 380 & & 0.002 & 10.028 & 0.181 & 29.714 & 29.714 \\
\hline 380 & & 0.001 & 9.727 & 0.320 & 30.013 & 30.015 \\
\hline 400 & \multirow{10}{*}{$\begin{array}{l}\text { Nิ } \\
\text { ה }\end{array}$} & 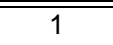 & 11.525 & 0.107 & 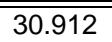 & 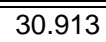 \\
\hline 400 & & 0.5 & 11.223 & 0.093 & 30.610 & 30.610 \\
\hline 400 & & 0.2 & 10.826 & 0.203 & 30.752 & 30.752 \\
\hline 400 & & 0.1 & 10.525 & 0.080 & 30.580 & 30.580 \\
\hline 400 & & 0.05 & 10.223 & 0.061 & 30.527 & 30.527 \\
\hline 400 & & 0.02 & 9.826 & 0.362 & 30.992 & 30.994 \\
\hline 400 & & 0.01 & 9.525 & 0.034 & 30.760 & 30.760 \\
\hline 400 & & 0.005 & 9.223 & 0.170 & 30.779 & 30.779 \\
\hline 400 & & 0.002 & 8.826 & 0.212 & 30.635 & 30.636 \\
\hline 400 & & 0.001 & 8.525 & 0.307 & 30.538 & 30.539 \\
\hline 420 & \multirow{10}{*}{$\begin{array}{l}\infty \\
\stackrel{\text { ¿ }}{\text { in }}\end{array}$} & 1 & 10.392 & 0.035 & 30.840 & 30.840 \\
\hline 420 & & 0.5 & 10.091 & 0.008 & 30.823 & 30.823 \\
\hline 420 & & 0.2 & 9.693 & 0.041 & 31.013 & 31.013 \\
\hline 420 & & 0.1 & 9.392 & 0.125 & 30.809 & 30.809 \\
\hline 420 & & 0.05 & 9.091 & 0.046 & 31.167 & 31.167 \\
\hline 420 & & 0.02 & 8.693 & 0.295 & 30.867 & 30.868 \\
\hline 420 & & 0.01 & 8.392 & 0.094 & 30.480 & 30.480 \\
\hline 420 & & 0.005 & 8.091 & 0.359 & 30.486 & 30.488 \\
\hline 420 & & 0.002 & 7.693 & 0.010 & 32.251 & 32.251 \\
\hline 420 & & 0.001 & 7.392 & 0.162 & 30.627 & 30.627 \\
\hline
\end{tabular}


Appendix 2. Temperature $T$, $\log _{10} \eta$, frequency $f$, $\log _{10} \omega \tau_{\mathrm{M}}$, imaginary $(G \mathrm{im})$ and real $\left(\mathrm{G}_{\text {real }}\right)$ shear modulus and shear modulus of the melt $(\mathrm{G}$ melt $)$ for the investigated samples - continuation..

\begin{tabular}{|c|c|c|c|c|c|c|}
\hline \multicolumn{7}{|c|}{ SAMPLE G13 } \\
\hline $\mathrm{T}\left({ }^{\circ} \mathrm{C}\right)$ & $\begin{array}{c}\log _{10} \\
\eta \\
\text { (Pa s) }\end{array}$ & $f(\mathrm{~Hz})$ & $\begin{array}{c}\log _{10} \\
\omega \tau_{M}\end{array}$ & $\begin{array}{c}\mathbf{G}_{\mathrm{im}} \\
(\mathrm{GPa})\end{array}$ & $\begin{array}{l}\mathbf{G}_{\text {real }} \\
(\mathrm{GPa})\end{array}$ & $\begin{array}{c}\mathbf{G}_{\text {melt }} \\
(\mathrm{GPa})\end{array}$ \\
\hline 440 & \multirow{10}{*}{ - } & 1 & 9.322 & 0.045 & 30.803 & 30.803 \\
\hline 440 & & 0.5 & 9.021 & 0.054 & 31.278 & 31.278 \\
\hline 440 & & 0.2 & 8.623 & 0.208 & 31.166 & 31.167 \\
\hline 440 & & 0.1 & 8.322 & 0.482 & 31.069 & 31.073 \\
\hline 440 & & 0.05 & 8.021 & 0.529 & 30.463 & 30.467 \\
\hline 440 & & 0.02 & 7.623 & 0.897 & 30.576 & 30.589 \\
\hline 440 & & 0.01 & 7.322 & 0.718 & 30.868 & 30.877 \\
\hline 440 & & 0.005 & 7.021 & 0.583 & 30.088 & 30.094 \\
\hline 440 & & 0.002 & 6.623 & 0.153 & 30.573 & 30.573 \\
\hline 440 & & 0.001 & 6.322 & 0.197 & 31.108 & 31.109 \\
\hline 4460 & \multirow{10}{*}{$\begin{array}{l}8 \\
\substack{0 \\
-1 \\
-1}\end{array}$} & 1 & $\begin{array}{l}8.311 \\
\end{array}$ & $\begin{array}{l}0.024 \\
\end{array}$ & 29.688 & 29.688 \\
\hline 460 & & 0.5 & 8.010 & 0.036 & 29.434 & 29.435 \\
\hline 460 & & 0.2 & 7.612 & 0.259 & 29.783 & 29.784 \\
\hline 460 & & 0.1 & 7.311 & 0.050 & 29.722 & 29.722 \\
\hline 460 & & 0.05 & 7.010 & 0.181 & 29.160 & 29.161 \\
\hline 460 & & 0.02 & 6.612 & 0.217 & 28.842 & 28.843 \\
\hline 460 & & 0.01 & 6.311 & 0.261 & 29.038 & 29.039 \\
\hline 460 & & 0.005 & 6.010 & 0.117 & 30.344 & 30.344 \\
\hline 460 & & 0.002 & 5.612 & 0.351 & 29.098 & 29.101 \\
\hline 460 & & 0.001 & 5.311 & 0.497 & 29.140 & 29.144 \\
\hline "480 & \multirow{10}{*}{ 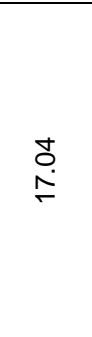 } & 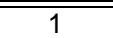 & $\begin{array}{ll}7.354 \\
\end{array}$ & $\begin{array}{l}0.074 \\
\end{array}$ & 30.621 & 30.621 \\
\hline 480 & & 0.5 & 7.053 & 0.043 & 30.282 & 30.282 \\
\hline 480 & & 0.2 & 6.655 & 0.166 & 30.628 & 30.629 \\
\hline 480 & & 0.1 & 6.354 & 0.159 & 30.732 & 30.733 \\
\hline 480 & & 0.05 & 6.053 & 0.112 & 29.934 & 29.934 \\
\hline 480 & & 0.02 & 5.655 & 0.334 & 31.310 & 31.312 \\
\hline 480 & & 0.01 & 5.354 & 0.448 & 30.644 & 30.647 \\
\hline 480 & & 0.005 & 5.053 & 0.053 & 30.051 & 30.051 \\
\hline 480 & & 0.002 & 4.655 & 0.441 & 29.850 & 29.853 \\
\hline 480 & & 0.001 & 4.354 & 0.136 & 30.212 & 30.213 \\
\hline
\end{tabular}

\begin{tabular}{|c|c|c|c|c|c|c|}
\hline \multicolumn{7}{|c|}{ SAMPLE G13 } \\
\hline $\mathrm{T}\left({ }^{\circ} \mathrm{C}\right)$ & $\begin{array}{c}\log _{10} \\
\eta \\
\text { (Pa s) }\end{array}$ & $f(\mathrm{~Hz})$ & $\begin{array}{c}\log _{10} \\
\omega \tau_{M}\end{array}$ & $\begin{array}{l}\mathbf{G}_{\text {im }} \\
(\mathbf{G P a})\end{array}$ & $\begin{array}{l}\mathbf{G}_{\text {real }} \\
(\mathrm{GPa})\end{array}$ & $\begin{array}{l}G_{\text {melt }} \\
(\mathrm{GPa})\end{array}$ \\
\hline 500 & \multirow{10}{*}{$\begin{array}{l}\underset{+}{+} \\
\stackrel{-}{-}\end{array}$} & 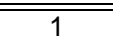 & 6.446 & 0.193 & 29.402 & 29.403 \\
\hline 500 & & 0.5 & 6.145 & 0.161 & 29.320 & 29.320 \\
\hline 500 & & 0.2 & 5.747 & 0.065 & 29.617 & 29.617 \\
\hline 500 & & 0.1 & 5.446 & 0.241 & 29.582 & 29.583 \\
\hline 500 & & 0.05 & 5.145 & 0.131 & 29.328 & 29.328 \\
\hline 500 & & 0.02 & 4.747 & 0.315 & 29.069 & 29.070 \\
\hline 500 & & 0.01 & 4.446 & 0.086 & 28.877 & 28.877 \\
\hline 500 & & 0.005 & 4.145 & 0.403 & 29.061 & 29.064 \\
\hline 500 & & 0.002 & 3.747 & 0.459 & 28.603 & 28.606 \\
\hline 500 & & 0.001 & 3.446 & 0.549 & 27.319 & 27.324 \\
\hline$\overline{520}$ & \multirow{10}{*}{ 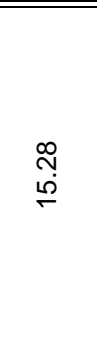 } & $\bar{~} 1$ & 5.584 & 0.010 & 29.091 & 29.091 \\
\hline 520 & & 0.5 & 5.283 & 0.069 & 28.480 & 28.480 \\
\hline 520 & & 0.2 & 4.885 & 0.346 & 28.351 & 28.353 \\
\hline 520 & & 0.1 & 4.584 & 0.024 & 28.160 & 28.160 \\
\hline 520 & & 0.05 & 4.283 & 0.233 & 28.858 & 28.859 \\
\hline 520 & & 0.02 & 3.885 & 0.611 & 28.489 & 28.496 \\
\hline 520 & & 0.01 & 3.584 & 0.633 & 28.239 & 28.246 \\
\hline 520 & & 0.005 & 3.283 & 0.587 & 28.155 & 28.161 \\
\hline 520 & & 0.002 & 2.885 & 0.773 & 26.869 & 26.880 \\
\hline 520 & & 0.001 & 2.584 & 1.465 & 26.190 & 26.231 \\
\hline 2540 & \multirow{10}{*}{$\begin{array}{l}\stackrel{0}{+} \\
\stackrel{+}{+}\end{array}$} & $\overline{c 1}$ & 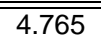 & 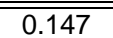 & 29.408 & 29.409 \\
\hline 540 & & 0.5 & 4.464 & 0.209 & 29.108 & 29.108 \\
\hline 540 & & 0.2 & 4.066 & 0.027 & 29.191 & 29.191 \\
\hline 540 & & 0.1 & 3.765 & 0.027 & 28.524 & 28.524 \\
\hline 540 & & 0.05 & 3.464 & 0.655 & 27.794 & 27.802 \\
\hline 540 & & 0.02 & 3.066 & 0.573 & 27.941 & 27.947 \\
\hline 540 & & 0.01 & 2.765 & 1.334 & 27.684 & 27.716 \\
\hline 540 & & 0.005 & 2.464 & 1.630 & 26.101 & 26.151 \\
\hline 540 & & 0.002 & 2.066 & 2.303 & 24.901 & 25.007 \\
\hline 540 & & 0.001 & 1.765 & 0.915 & 24.611 & 24.628 \\
\hline
\end{tabular}


Appendix 2. Temperature $T, \log _{10} \eta$, frequency $f, \log _{10} \omega \tau_{\mathrm{M}}$, imaginary $\left(G_{\mathrm{im}}\right)$ and real ( $(\mathrm{G}$ real) shear modulus and shear modulus of the melt ( $G$ melt) for the investigated samples - continuation...

\begin{tabular}{|c|c|c|c|c|c|c|}
\hline \multicolumn{7}{|c|}{ SAMPLE G13 } \\
\hline $\mathrm{T}\left({ }^{\circ} \mathrm{C}\right)$ & $\begin{array}{c}\log _{10} \\
\eta \\
\text { (Pa s) }\end{array}$ & $f(\mathrm{~Hz})$ & $\begin{array}{c}\log _{10} \\
\omega \tau_{M}\end{array}$ & $\begin{array}{c}\mathbf{G}_{\text {im }} \\
(\mathrm{GPa})\end{array}$ & $\begin{array}{l}\mathbf{G}_{\text {real }} \\
(\mathrm{GPa})\end{array}$ & $\begin{array}{l}\text { G melt } \\
\text { (GPa) }\end{array}$ \\
\hline 560 & \multirow{10}{*}{$\begin{array}{l}\stackrel{\infty}{\circ} \\
\stackrel{\leftrightarrow}{\rightarrow}\end{array}$} & 1 & 3.984 & 0.372 & 27.790 & 27.792 \\
\hline 560 & & 0.5 & 3.683 & 0.518 & 27.457 & 27.461 \\
\hline 560 & & 0.2 & 3.285 & 0.706 & 26.990 & 26.999 \\
\hline 560 & & 0.1 & 2.984 & 0.874 & 27.027 & 27.041 \\
\hline 560 & & 0.05 & 2.683 & 0.906 & 26.197 & 26.213 \\
\hline 560 & & 0.02 & 2.285 & 2.022 & 24.440 & 24.524 \\
\hline 560 & & 0.01 & 1.984 & 2.242 & 23.076 & 23.185 \\
\hline 560 & & 0.005 & 1.683 & 3.119 & 21.777 & 21.999 \\
\hline 560 & & 0.002 & 1.285 & 0.457 & 21.206 & 21.211 \\
\hline 560 & & 0.001 & 0.984 & 4.009 & 16.129 & 16.620 \\
\hline 580 & \multirow{10}{*}{\begin{tabular}{l} 
ֻ \\
\multirow{H}{*}{}
\end{tabular}} & 1 & $\begin{array}{l}3.241 \\
\end{array}$ & $\begin{array}{l}0.651 \\
\end{array}$ & 25.993 & 26.001 \\
\hline 580 & & 0.5 & 2.940 & 0.921 & 25.556 & 25.572 \\
\hline 580 & & 0.2 & 2.542 & 1.550 & 24.628 & 24.677 \\
\hline 580 & & 0.1 & 2.241 & 1.930 & 23.953 & 24.031 \\
\hline 580 & & 0.05 & 1.940 & 2.585 & 22.270 & 22.419 \\
\hline 580 & & 0.02 & 1.542 & 3.793 & 19.688 & 20.050 \\
\hline 580 & & 0.01 & 1.241 & 4.921 & 16.857 & 17.560 \\
\hline 580 & & 0.005 & 0.940 & 4.753 & 14.302 & 15.071 \\
\hline 580 & & 0.002 & 0.542 & 4.855 & 9.208 & 10.410 \\
\hline 580 & & 0.001 & 0.241 & 4.738 & 4.500 & 6.534 \\
\hline (2600 & \multirow{10}{*}{$\begin{array}{l}\underset{N}{N} \\
\underset{H}{N}\end{array}$} & $\bar{~} 1$ & $\begin{array}{l}2.531 \\
\end{array}$ & $\begin{array}{l}1.641 \\
\end{array}$ & 26.659 & 26.710 \\
\hline 600 & & 0.5 & 2.230 & 2.422 & 25.574 & 25.688 \\
\hline 600 & & 0.2 & 1.832 & 3.443 & 23.189 & 23.444 \\
\hline 600 & & 0.1 & 1.531 & 4.590 & 21.141 & 21.634 \\
\hline 600 & & 0.05 & 1.230 & 5.743 & 17.591 & 18.505 \\
\hline 600 & & 0.02 & 0.832 & 6.900 & 11.849 & 13.712 \\
\hline 600 & & 0.01 & 0.531 & 6.533 & 7.100 & 9.649 \\
\hline 600 & & 0.005 & 0.230 & 3.853 & 4.981 & 6.298 \\
\hline 600 & & 0.002 & -0.168 & 2.466 & 0.793 & 2.590 \\
\hline 600 & & 0.001 & -0.469 & 1.115 & 1.139 & 1.595 \\
\hline
\end{tabular}

\begin{tabular}{|c|c|c|c|c|c|c|}
\hline \multicolumn{7}{|c|}{ SAMPLE G13 } \\
\hline $\mathrm{T}\left({ }^{\circ} \mathrm{C}\right)$ & $\begin{array}{c}\log _{10} \\
\eta \\
(\mathrm{Pa} \mathrm{s})\end{array}$ & $f(\mathrm{~Hz})$ & $\begin{array}{c}\log _{10} \\
\omega \tau_{M}\end{array}$ & $\begin{array}{l}\mathbf{G}_{\mathrm{im}} \\
(\mathrm{GPa})\end{array}$ & $\begin{array}{l}\mathbf{G}_{\text {real }} \\
(\mathrm{GPa})\end{array}$ & $\begin{array}{l}\mathbf{G}_{\text {melt }} \\
(\mathrm{GPa})\end{array}$ \\
\hline 620 & & 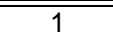 & 1.853 & 3.614 & 25.171 & 25.430 \\
\hline 620 & & 0.5 & 1.552 & 4.856 & 22.442 & 22.962 \\
\hline 620 & & 0.2 & 1.154 & 5.873 & 17.714 & 18.662 \\
\hline 620 & & 0.1 & 0.853 & 7.517 & 12.755 & 14.805 \\
\hline 620 & เి & 0.05 & 0.552 & 6.856 & 7.562 & 10.207 \\
\hline 620 & $ت$ & 0.02 & 0.154 & 4.512 & 2.514 & 5.165 \\
\hline 620 & & 0.01 & -0.147 & 2.643 & 0.875 & 2.784 \\
\hline 620 & & 0.005 & -0.448 & 1.375 & 0.234 & 1.395 \\
\hline 620 & & 0.002 & -0.846 & 0.512 & 0.045 & 0.514 \\
\hline 620 & & 0.001 & -1.147 & 0.259 & 0.018 & 0.259 \\
\hline 2640 & & 1 & $\begin{array}{ll}1.205 \\
\end{array}$ & 5.983 & $\begin{array}{ll}17.900 \\
\end{array}$ & 18.873 \\
\hline 640 & & 0.5 & 0.904 & 6.889 & 13.467 & 15.127 \\
\hline 640 & & 0.2 & 0.506 & 6.693 & 7.004 & 9.687 \\
\hline 640 & & 0.1 & 0.205 & 4.976 & 3.204 & 5.919 \\
\hline 640 & 8 & 0.05 & -0.096 & 3.026 & 1.159 & 3.241 \\
\hline 640 & - & 0.02 & -0.494 & 1.358 & 0.229 & 1.378 \\
\hline 640 & & 0.01 & -0.795 & 0.196 & 0.639 & 0.668 \\
\hline 640 & & 0.005 & -1.096 & 0.303 & 0.023 & 0.304 \\
\hline 640 & & 0.002 & -1.494 & 0.061 & 0.106 & 0.123 \\
\hline "660 & & $\bar{~} 1$ & "0.585 & 8.150 & "4.784 & 9.450 \\
\hline 660 & & 0.5 & 0.284 & 5.579 & 4.481 & 7.156 \\
\hline 660 & $\stackrel{\infty}{N}$ & 0.2 & -0.114 & 3.217 & 1.246 & 3.450 \\
\hline 660 & वे & 0.1 & -0.415 & 1.763 & 0.379 & 1.803 \\
\hline 6880 & $\begin{array}{l}\infty \\
\stackrel{0}{\circ} \\
\sigma^{\circ}\end{array}$ & $\overline{1}$ & -0.009 & 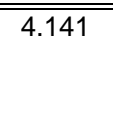 & 2.150 & 4.666 \\
\hline
\end{tabular}


Appendix 2. Temperature $T$, $\log _{10} \eta$, frequency $f, \log _{10} \omega \tau_{\mathrm{M}}$, imaginary $(G \mathrm{im})$ and real $\left(\mathrm{G}_{\text {real }}\right)$ shear modulus and shear modulus of the melt ( $G$ melt $)$ for the investigated samples - continuation..

\begin{tabular}{|c|c|c|c|c|c|c|}
\hline \multicolumn{7}{|c|}{ SAMPLE G14 } \\
\hline $\mathrm{T}\left({ }^{\circ} \mathrm{C}\right)$ & $\begin{array}{c}\log _{10} \\
\eta \\
\text { (Pa s) }\end{array}$ & $f(\mathrm{~Hz})$ & $\begin{array}{c}\log _{10} \\
\omega \tau_{M}\end{array}$ & $\begin{array}{c}\mathbf{G}_{\mathrm{im}} \\
(\mathrm{GPa})\end{array}$ & $\begin{array}{l}\mathbf{G}_{\text {real }} \\
(\mathrm{GPa})\end{array}$ & $\begin{array}{l}\mathbf{G}_{\text {melt }} \\
(\mathrm{GPa})\end{array}$ \\
\hline 420 & \multirow{10}{*}{ 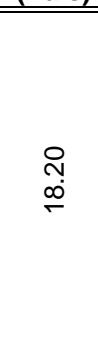 } & 1 & 8.521 & 0.094 & 29.965 & 29.965 \\
\hline 420 & & 0.5 & 8.220 & 0.098 & 29.764 & 29.764 \\
\hline 420 & & 0.2 & 7.822 & 0.097 & 30.055 & 30.055 \\
\hline 420 & & 0.1 & 7.521 & 0.209 & 29.573 & 29.574 \\
\hline 420 & & 0.05 & 7.220 & 0.112 & 29.665 & 29.665 \\
\hline 420 & & 0.02 & 6.822 & 0.059 & 28.705 & 28.705 \\
\hline 420 & & 0.01 & 6.521 & 0.187 & 29.158 & 29.159 \\
\hline 420 & & 0.005 & 6.220 & 0.266 & 29.470 & 29.471 \\
\hline 420 & & 0.002 & 5.822 & 0.036 & 29.067 & 29.067 \\
\hline 420 & & 0.001 & 5.521 & 0.339 & 28.728 & 28.730 \\
\hline 4440 & \multirow{10}{*}{ 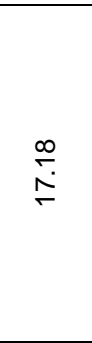 } & 1 & $7 \overline{7.503}$ & 0.034 & 29.471 & $\overline{229.471}$ \\
\hline 440 & & 0.5 & 7.202 & 0.034 & 29.503 & 29.503 \\
\hline 440 & & 0.2 & 6.804 & 0.219 & 29.034 & 29.035 \\
\hline 440 & & 0.1 & 6.503 & 0.249 & 29.072 & 29.073 \\
\hline 440 & & 0.05 & 6.202 & 0.035 & 29.362 & 29.362 \\
\hline 440 & & 0.02 & 5.804 & 0.035 & 29.505 & 29.505 \\
\hline 440 & & 0.01 & 5.503 & 0.181 & 28.797 & 28.798 \\
\hline 440 & & 0.005 & 5.202 & 0.042 & 28.956 & 28.956 \\
\hline 440 & & 0.002 & 4.804 & 0.824 & 28.819 & 28.831 \\
\hline 440 & & 0.001 & 4.503 & 0.749 & 29.109 & 29.119 \\
\hline 460 & \multirow{10}{*}{$\begin{array}{l}\underset{N}{\tilde{S}} \\
\stackrel{+}{-1}\end{array}$} & 1 & 6.541 & 0.027 & 29.456 & 29.456 \\
\hline 460 & & 0.5 & 6.240 & 0.182 & 29.391 & 29.391 \\
\hline 460 & & 0.2 & 5.842 & 0.138 & 28.959 & 28.960 \\
\hline 460 & & 0.1 & 5.541 & 0.015 & 28.942 & 28.942 \\
\hline 460 & & 0.05 & 5.240 & 0.234 & 28.979 & 28.980 \\
\hline 460 & & 0.02 & 4.842 & 0.665 & 27.961 & 27.969 \\
\hline 460 & & 0.01 & 4.541 & 0.748 & 28.671 & 28.681 \\
\hline 460 & & 0.005 & & & & \\
\hline 460 & & 0.002 & 3.842 & 0.315 & 28.303 & 28.304 \\
\hline 460 & & 0.001 & 3.541 & 0.288 & 28.312 & 28.314 \\
\hline
\end{tabular}

\begin{tabular}{|c|c|c|c|c|c|c|}
\hline \multicolumn{7}{|c|}{ SAMPLE G14 } \\
\hline $\mathrm{T}\left({ }^{\circ} \mathrm{C}\right)$ & $\begin{array}{c}\log _{10} \\
\eta \\
(\mathrm{Pa} \mathrm{s})\end{array}$ & $f(\mathrm{~Hz})$ & $\begin{array}{c}\log _{10} \\
\omega \tau_{M}\end{array}$ & $\begin{array}{c}\mathbf{G}_{\mathrm{im}} \\
(\mathrm{GPa})\end{array}$ & $\begin{array}{c}\mathbf{G}_{\text {real }} \\
(\mathrm{GPa})\end{array}$ & $\begin{array}{c}\mathbf{G}_{\text {melt }} \\
(\mathrm{GPa})\end{array}$ \\
\hline 480 & \multirow{10}{*}{$\begin{array}{l}\text { m. } \\
\text { فु }\end{array}$} & 1 & 5.629 & 0.030 & 29.281 & 29.281 \\
\hline 480 & & 0.5 & 5.328 & 0.070 & 29.173 & 29.173 \\
\hline 480 & & 0.2 & 4.930 & 1.290 & 28.647 & 28.676 \\
\hline 480 & & 0.1 & 4.629 & 0.200 & 28.830 & 28.831 \\
\hline 480 & & 0.05 & 4.328 & 0.367 & 29.391 & 29.393 \\
\hline 480 & & 0.02 & 3.930 & 0.130 & 28.943 & 28.943 \\
\hline 480 & & 0.01 & 3.629 & 0.384 & 28.028 & 28.030 \\
\hline 480 & & 0.005 & 3.328 & 0.687 & 27.860 & 27.868 \\
\hline 480 & & 0.002 & 2.930 & 0.640 & 27.131 & 27.139 \\
\hline 480 & & 0.001 & 2.629 & 0.947 & 27.207 & 27.223 \\
\hline$\overline{500}$ & \multirow{10}{*}{$\begin{array}{l}\stackrel{J}{+} \\
\text { - }\end{array}$} & 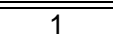 & $4 \quad 4.765$ & 0.361 & 28.803 & 28.806 \\
\hline 500 & & 0.5 & 4.464 & 0.171 & 28.566 & 28.567 \\
\hline 500 & & 0.2 & 4.066 & 0.069 & 28.070 & 28.070 \\
\hline 500 & & 0.1 & 3.765 & 0.123 & 27.541 & 27.541 \\
\hline 500 & & 0.05 & 3.464 & 0.300 & 27.963 & 27.964 \\
\hline 500 & & 0.02 & 3.066 & 0.491 & 27.308 & 27.312 \\
\hline 500 & & 0.01 & 2.765 & 0.648 & 27.224 & 27.232 \\
\hline 500 & & 0.005 & 2.464 & 0.937 & 26.610 & 26.627 \\
\hline 500 & & 0.002 & 2.066 & 1.525 & 25.254 & 25.300 \\
\hline 500 & & 0.001 & 1.765 & 0.317 & 24.875 & 24.878 \\
\hline 520 & \multirow{10}{*}{ 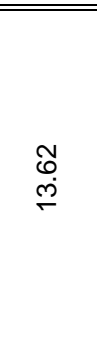 } & 1 & 3.944 & 0.282 & 27.902 & 27.904 \\
\hline 520 & & 0.5 & 3.643 & 0.233 & 27.579 & 27.580 \\
\hline 520 & & 0.2 & 3.245 & 0.641 & 27.997 & 28.004 \\
\hline 520 & & 0.1 & 2.944 & 0.976 & 26.902 & 26.919 \\
\hline 520 & & 0.05 & 2.643 & 0.965 & 26.953 & 26.970 \\
\hline 520 & & 0.02 & 2.245 & 1.328 & 25.848 & 25.882 \\
\hline 520 & & 0.01 & 1.944 & 1.607 & 24.717 & 24.770 \\
\hline 520 & & 0.005 & 1.643 & 2.469 & 23.733 & 23.861 \\
\hline 520 & & 0.002 & 1.245 & 0.069 & 21.908 & 21.908 \\
\hline 520 & & 0.001 & 0.944 & 2.743 & 19.629 & 19.819 \\
\hline
\end{tabular}


Appendix 2. Temperature $T$, $\log _{10} \eta$, frequency $f, \log _{10} \omega \tau_{\mathrm{M}}$, imaginary $(G \mathrm{im})$ and real $\left(\mathrm{G}_{\text {real }}\right)$ shear modulus and shear modulus of the melt ( $G$ melt $)$ for the investigated samples - continuation..

\begin{tabular}{|c|c|c|c|c|c|c|}
\hline \multicolumn{7}{|c|}{ SAMPLE G14 } \\
\hline $\mathrm{T}\left({ }^{\circ} \mathrm{C}\right)$ & $\begin{array}{c}\log _{10} \\
\eta \\
\text { (Pa s) }\end{array}$ & $f(\mathrm{~Hz})$ & $\begin{array}{c}\log _{10} \\
\omega \tau_{M}\end{array}$ & $\begin{array}{l}\mathbf{G}_{\mathrm{im}} \\
(\mathrm{GPa})\end{array}$ & $\begin{array}{l}\mathbf{G}_{\text {real }} \\
(\mathrm{GPa})\end{array}$ & $\begin{array}{l}\mathbf{G}_{\text {melt }} \\
(\mathrm{GPa})\end{array}$ \\
\hline 540 & \multirow{10}{*}{ 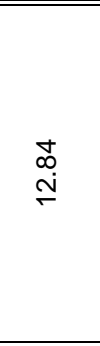 } & $\overline{c 1}$ & 3.164 & 0.040 & 27.629 & 27.629 \\
\hline 540 & & 0.5 & 2.863 & 0.048 & 27.570 & 27.570 \\
\hline 540 & & 0.2 & 2.465 & 0.176 & 26.358 & 26.359 \\
\hline 540 & & 0.1 & 2.164 & 0.398 & 25.660 & 25.663 \\
\hline 540 & & 0.05 & 1.863 & 0.424 & 24.410 & 24.414 \\
\hline 540 & & 0.02 & 1.465 & 0.663 & 22.597 & 22.607 \\
\hline 540 & & 0.01 & 1.164 & 0.480 & 20.647 & 20.653 \\
\hline 540 & & 0.005 & 0.863 & 0.359 & 18.550 & 18.553 \\
\hline 540 & & 0.002 & 0.465 & 0.070 & 14.095 & 14.096 \\
\hline 540 & & 0.001 & 0.164 & 0.063 & 9.949 & 9.949 \\
\hline 560 & \multirow{10}{*}{ 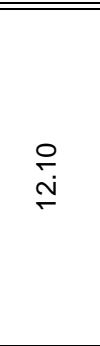 } & 1 & 2.421 & 1.304 & 25.025 & 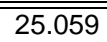 \\
\hline 560 & & 0.5 & 2.120 & 1.773 & 24.154 & 24.219 \\
\hline 560 & & 0.2 & 1.722 & 2.792 & 22.456 & 22.629 \\
\hline 560 & & 0.1 & 1.421 & 3.761 & 20.924 & 21.259 \\
\hline 560 & & 0.05 & 1.120 & 4.861 & 17.754 & 18.407 \\
\hline 560 & & 0.02 & 0.722 & 6.225 & 13.507 & 14.872 \\
\hline 560 & & 0.01 & 0.421 & 1.472 & 11.962 & 12.052 \\
\hline 560 & & 0.005 & 0.120 & 4.617 & 6.494 & 7.968 \\
\hline 560 & & 0.002 & -0.278 & 3.291 & 1.412 & 3.581 \\
\hline 560 & & 0.001 & -0.579 & 0.150 & 2.130 & 2.135 \\
\hline 580 & \multirow{9}{*}{ 羿 } & $\bar{~} 1$ & $\begin{array}{ll}1.713 \\
\end{array}$ & 2.829 & 21.262 & 21.450 \\
\hline 580 & & 0.5 & 1.412 & 3.705 & 19.125 & 19.480 \\
\hline 580 & & 0.2 & 1.014 & 5.400 & 15.555 & 16.465 \\
\hline 580 & & 0.1 & 0.713 & 1.717 & 13.993 & 14.098 \\
\hline 580 & & 0.05 & 0.412 & 6.365 & 7.534 & 9.862 \\
\hline 580 & & 0.02 & 0.014 & 4.619 & 2.797 & 5.399 \\
\hline 580 & & 0.01 & -0.287 & 3.143 & 1.089 & 3.327 \\
\hline 580 & & 0.005 & -0.588 & 1.632 & 0.317 & 1.663 \\
\hline 580 & & 0.002 & -0.986 & 0.665 & 0.052 & 0.667 \\
\hline
\end{tabular}

\begin{tabular}{|c|c|c|c|c|c|c|}
\hline \multicolumn{7}{|c|}{ SAMPLE G14 } \\
\hline $\mathrm{T}\left({ }^{\circ} \mathrm{C}\right)$ & $\begin{array}{c}\log _{10} \\
\eta \\
\text { (Pa s) }\end{array}$ & $f(\mathrm{~Hz})$ & $\begin{array}{c}\log _{10} \\
\omega \tau_{M}\end{array}$ & $\begin{array}{c}\mathbf{G}_{\mathrm{im}} \\
(\mathrm{GPa})\end{array}$ & $\begin{array}{l}\mathbf{G}_{\text {real }} \\
(\mathrm{GPa})\end{array}$ & $\begin{array}{l}\mathbf{G}_{\text {melt }} \\
(\mathrm{GPa})\end{array}$ \\
\hline 600 & \multirow{7}{*}{$\begin{array}{l}\stackrel{N}{N} \\
\stackrel{-}{0}\end{array}$} & 1 & 1.037 & 4.532 & 13.462 & 14.204 \\
\hline 600 & & 0.5 & 0.736 & 5.369 & 10.364 & 11.672 \\
\hline 600 & & 0.2 & 0.338 & 5.691 & 5.795 & 8.122 \\
\hline 600 & & 0.1 & 0.037 & 4.174 & 2.547 & 4.890 \\
\hline & & & & & & \\
\hline & & & & & & \\
\hline & & & & & & \\
\hline
\end{tabular}


A-38 
Appendix 3. Temperature $T$, frequency $f, \mathrm{G}_{\infty}$, viscosity, shear viscosity, relaxed Newtonian viscosity and relaxation times of the samples, determined by micropenetration and torsion measurements.

\begin{tabular}{|c|c|c|c|c|c|c|c|c|c|}
\hline \multirow[b]{2}{*}{$\mathbf{T}$} & \multirow[b]{2}{*}{ f } & \multirow[b]{2}{*}{$\mathbf{G}_{\infty}$} & \multicolumn{4}{|c|}{ MICROPENETRATION } & \multicolumn{3}{|c|}{ TORSION } \\
\hline & & & $\mathbf{A}$ & B & $\begin{array}{c}\log _{10} \\
\eta\end{array}$ & $\tau$ & $\begin{array}{l}\text { Shear } \\
\text { visc. } \\
\log _{10} \eta\end{array}$ & $\begin{array}{l}\text { Relaxed } \\
\text { Newt. visc. } \\
\log _{10} \eta\end{array}$ & $\tau$ \\
\hline${ }^{\circ} \mathrm{C}$ & $\mathrm{Hz}$ & GPa & $\log _{10} \mathrm{~Pa} \mathrm{~s}$ & $\mathrm{~K}$ & Pa s & $\mathbf{s}$ & $\mathrm{Pas}$ & Pas & $\mathbf{s}$ \\
\hline \multicolumn{10}{|c|}{ SAMPLE G0 } \\
\hline \multirow{3}{*}{ ৪ } & $\overline{11}$ & \multirow{3}{*}{27.45} & \multirow{3}{*}{$-24.98 \pm 0.99$} & \multirow{3}{*}{$\begin{array}{c}2.673 \\
\pm 0.074\end{array}$} & \multirow{3}{*}{9.59} & \multirow{3}{*}{0.142} & 9.13 & \multirow{3}{*}{9.38} & \multirow{3}{*}{0.087} \\
\hline & 0.5 & & & & & & 9.38 & & \\
\hline & 0.2 & & & & & & 9.32 & & \\
\hline \multirow{6}{*}{ ஓ } & 1 & \multirow{6}{*}{27.45} & \multirow{6}{*}{$-24.98 \pm 0.99$} & \multirow{6}{*}{$\begin{array}{c}2.673 \\
\pm 0.074\end{array}$} & & & 8.93 & & \\
\hline & 0.5 & & & & & & 9.33 & & \\
\hline & 0.2 & & & & & & 8.98 & 1007 & 0428 \\
\hline & 0.1 & & & & 10.04 & 0.403 & 9.65 & 10.07 & 0.428 \\
\hline & 0.05 & & & & & & 10.03 & & \\
\hline & 0.02 & & & & & & 10.06 & & \\
\hline & $\overline{c 1}$ & & & & & & 8.06 & & \\
\hline & 0.5 & & & & & & 8.94 & & \\
\hline & 0.2 & & & & & & 9.67 & & \\
\hline & 0.1 & & & & & & 9.88 & & \\
\hline$\stackrel{\infty}{\infty}$ & 0.05 & 27.45 & $-24.98 \pm 0.99$ & 2.673 & 10.51 & 1.177 & 9.85 & 10.37 & 0.854 \\
\hline & 0.02 & & & & & & 10.29 & & \\
\hline & 0.01 & & & & & & 10.32 & & \\
\hline & 0.005 & & & & & & 10.30 & & \\
\hline & 0.002 & & & & & & 10.45 & & \\
\hline & 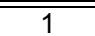 & & & & & & 7.52 & & \\
\hline & 0.5 & & & & & & 7.68 & & \\
\hline & 0.2 & & & & & & 8.26 & & \\
\hline & 0.1 & & & & & & 9.30 & & \\
\hline 8 & 0.05 & 27.45 & $-24.98 \pm 0.99$ & 2.673 & 11.48 & 10.948 & 9.69 & 11.45 & 10.269 \\
\hline & 0.02 & & & & & & 10.79 & & \\
\hline & 0.01 & & & & & & 11.26 & & \\
\hline & 0.005 & & & & & & 11.35 & & \\
\hline & 0.002 & & & & & & 11.43 & & \\
\hline & 1 & & & & & & 6.56 & & \\
\hline & 0.5 & & & & & & 7.79 & & \\
\hline & 0.2 & & & & & & 8.27 & & \\
\hline & 0.1 & & & & & & 8.64 & & \\
\hline 오 & 0.05 & & & 2.673 & & & 8.73 & & \\
\hline 寸 & 0.02 & 27.45 & $-24.98 \pm 0.99$ & \pm 0.074 & 12.50 & 115.386 & 9.39 & 12.30 & 72.698 \\
\hline & 0.01 & & & & & & 10.29 & & \\
\hline & 0.005 & & & & & & 10.61 & & \\
\hline & 0.002 & & & & & & 11.83 & & \\
\hline & 0.001 & & & & & & 12.30 & & \\
\hline & 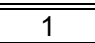 & & & & & & 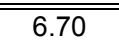 & & \\
\hline & 0.5 & & & & & & 7.70 & & \\
\hline & 0.2 & & & & & & 8.22 & & \\
\hline & 0.1 & & & & & & 8.20 & & \\
\hline 우 & 0.05 & & & 2.673 & & & 9.05 & & \\
\hline ₹ & 0.02 & 27.45 & $-24.98 \pm 0.99$ & \pm 0.074 & 13.58 & 1393.121 & 9.50 & 13.18 & 551.468 \\
\hline & 0.01 & & & & & & 10.11 & & \\
\hline & 0.005 & & & & & & 10.88 & & \\
\hline & 0.002 & & & & & & 12.26 & & \\
\hline & 0.001 & & & & & & 13.17 & & \\
\hline 400 & & 27.45 & $-24.98 \pm 0.99$ & $\begin{array}{c}2.673 \\
\pm 0.074\end{array}$ & 14.73 & 19503.938 & - & - & - \\
\hline 380 & & 27.45 & $-24.98 \pm 0.99$ & $\begin{array}{c}2.673 \\
\pm 0.074\end{array}$ & 15.94 & 320968.90 & - & - & - \\
\hline
\end{tabular}

\begin{tabular}{|c|c|c|c|c|c|c|c|c|c|}
\hline \multicolumn{10}{|c|}{ SAMPLE G1 } \\
\hline \multirow{5}{*}{$\underset{\infty}{\stackrel{1}{L}}$} & 1 & \multirow{5}{*}{34.26} & \multirow{5}{*}{$-15.63 \pm 0.53$} & \multirow{5}{*}{$\begin{array}{c}3.014 \\
\pm 0.061\end{array}$} & \multirow{5}{*}{10.62} & \multirow{5}{*}{1.208} & 10.16 & \multirow{5}{*}{10.82} & \multirow{5}{*}{1.928} \\
\hline & 0.5 & & & & & & 10.50 & & \\
\hline & 0.2 & & & & & & 10.83 & & \\
\hline & 0.1 & & & & & & 10.90 & & \\
\hline & 0.05 & & & & & & 10.54 & & \\
\hline \multirow{6}{*}{ ○ } & $\overline{11}$ & \multirow{6}{*}{34.26} & \multirow{6}{*}{$-15.63 \pm 0.53$} & \multirow{6}{*}{$\begin{array}{c}3.014 \\
\pm 0.061\end{array}$} & \multirow{6}{*}{11.20} & \multirow{6}{*}{4.640} & 10.59 & \multirow{6}{*}{11.34} & \multirow{6}{*}{6.386} \\
\hline & 0.5 & & & & & & 11.03 & & \\
\hline & 0.2 & & & & & & 11.56 & & \\
\hline & 0.1 & & & & & & 11.90 & & \\
\hline & 0.05 & & & & & & 11.36 & & \\
\hline & 0.02 & & & & & & 11.47 & & \\
\hline
\end{tabular}


Appendix 3. Temperature T, frequency $f, \mathrm{G}_{\infty}$, viscosity, shear viscosity, relaxed Newtonian viscosity and relaxation times of the samples, determined by micropenetration and torsion measurements.

Continuation...

\begin{tabular}{|c|c|c|c|c|c|c|c|c|c|}
\hline & & & \multicolumn{4}{|c|}{ MICROPENETRATION } & \multicolumn{3}{|c|}{ TORSION } \\
\hline $\mathbf{T}$ & f & $\mathbf{G}_{\infty}$ & A & B & $\begin{array}{c}\log _{10} \\
\eta\end{array}$ & $\tau$ & $\begin{array}{l}\text { Shear } \\
\text { visc. } \\
\log _{10} \eta\end{array}$ & $\begin{array}{c}\text { Relaxed } \\
\text { Newt. visc. } \\
\log _{10} \eta\end{array}$ & $\tau$ \\
\hline${ }^{\circ} \mathrm{C}$ & $\mathrm{Hz}$ & GPa & $\log _{10} \mathrm{~Pa} \mathrm{~S}$ & $\mathbf{K}$ & Pas & $\mathbf{s}$ & Pa s & $\mathrm{Pa} \mathrm{s}$ & s \\
\hline \multicolumn{10}{|c|}{ SAMPLE G1 } \\
\hline \multirow{2}{*}{850} & 0.01 & & \multirow{2}{*}{$-15.63 \pm 0.53$} & & \multirow{2}{*}{11.20} & \multirow{2}{*}{4.640} & 11.49 & \multirow{2}{*}{11.34} & \multirow{2}{*}{6.386} \\
\hline & 0.005 & & & & & & 11.32 & & \\
\hline \multirow{10}{*}{$\stackrel{\sim}{\infty}$} & 1 & & & & & & 9.98 & & \\
\hline & 0.5 & & & & & & 10.46 & & \\
\hline & 0.2 & & & & & & 11.04 & & \\
\hline & 0.1 & & & & & & 11.47 & & \\
\hline & 0.05 & 3426 & $-1563+0.53$ & 3.014 & 1181 & 18945 & 11.85 & 1211 & 37603 \\
\hline & 0.02 & 34.26 & $-15.03 \pm 0.53$ & \pm 0.061 & 11.81 & 18.945 & 12.26 & 12.11 & - 31.003 \\
\hline & 0.01 & & & & & & 12.10 & & \\
\hline & 0.005 & & & & & & 12.15 & & \\
\hline & 0.002 & & & & & & 12.15 & & \\
\hline & 0.001 & & & & & & 11.88 & & \\
\hline & 1 & & & & & & 13.20 & & \\
\hline & 0.5 & & & & & & 13.61 & & \\
\hline & 0.2 & & & & & & 13.12 & & \\
\hline & 0.1 & & & & & & 12.70 & & \\
\hline 8 & 0.05 & 3426 & $-1563+0.53$ & 3.014 & 1245 & 82598 & 12.81 & 1256 & 105981 \\
\hline$\infty$ & 0.02 & 34.20 & $-10.03 \pm 0.03$ & \pm 0.061 & & 82.598 & 12.80 & 12.56 & 105.901 \\
\hline & 0.01 & & & & & & 12.82 & & \\
\hline & 0.005 & & & & & & 12.35 & & \\
\hline & 0.002 & & & & & & 12.70 & & \\
\hline & 0.001 & & & & & & 12.57 & & \\
\hline & 1 & & & & & & 12.85 & & \\
\hline & 0.5 & & & & & & 13.38 & & \\
\hline & 0.2 & & & & & & 14.00 & & \\
\hline & 0.1 & & & & & & 13.63 & & \\
\hline$\stackrel{n}{\sim}$ & 0.05 & 3426 & $-1563+0.53$ & 3.014 & 1312 & 386.329 & 13.85 & 13,16 & 421917 \\
\hline N & 0.02 & 34.20 & & \pm 0.061 & & & 13.90 & & 421.911 \\
\hline & 0.01 & & & & & & 13.36 & & \\
\hline & 0.005 & & & & & & 13.08 & & \\
\hline & 0.002 & & & & & & 13.14 & & \\
\hline & 0.001 & & & & & & 13.14 & & \\
\hline & 1 & & & & & & 12.65 & & \\
\hline & 0.5 & & & & & & 13.20 & & \\
\hline & 0.2 & & & & & & 13.81 & & \\
\hline & 0.1 & & & & & & 14.06 & & \\
\hline 용 & 0.05 & 3426 & $-1563+0.53$ & 3.014 & 1382 & 1948454 & 14.34 & 13.86 & 2114.594 \\
\hline$\stackrel{2}{R}$ & 0.02 & 34.20 & & \pm 0.061 & & & 14.16 & 10.00 & 2114.094 \\
\hline & 0.01 & & & & & & 14.52 & & \\
\hline & 0.005 & & & & & & 13.97 & & \\
\hline & 0.002 & & & & & & 13.98 & & \\
\hline & 0.001 & & & & & & 13.79 & & \\
\hline & 1 & & & & & & 12.60 & & \\
\hline & 0.5 & & & & & & 13.72 & & \\
\hline & 0.2 & & & & & & 14.12 & & \\
\hline & 0.1 & & & & & & 14.40 & & \\
\hline$\stackrel{2}{\sim}$ & 0.05 & 3426 & $-1563+0.53$ & 3.014 & 1456 & 10656877 & 14.91 & 14.45 & 8226725 \\
\hline N & 0.02 & 34.26 & & \pm 0.061 & & & 15.15 & & \\
\hline & 0.01 & & & & & & 15.14 & & \\
\hline & 0.005 & & & & & & 14.66 & & \\
\hline & 0.002 & & & & & & 14.40 & & \\
\hline & 0.001 & & & & & & 14.49 & & \\
\hline & 1 & & & & & & 13.17 & & \\
\hline & 0.5 & & & & & & 13.39 & & \\
\hline & 0.2 & & & & & & 14.26 & & \\
\hline & 0.1 & & & & & & 14.88 & & \\
\hline 8 & 0.05 & 3426 & $-1563+0.53$ & 3.014 & 1534 & 63604893 & 14.71 & 15.27 & \\
\hline$r$ & 0.02 & $34 . \angle b$ & & \pm 0.061 & & & 15.56 & 15.27 & 54353.433 \\
\hline & 0.01 & & & & & & 15.63 & & \\
\hline & 0.005 & & & & & & 15.39 & & \\
\hline & 0.002 & & & & & & 15.21 & & \\
\hline & 0.001 & & & & & & 15.28 & & \\
\hline & 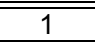 & & & & & & 12.40 & & \\
\hline 융 & 0.5 & 34.26 & $-15.63 \pm 0.53$ & $\begin{array}{c}3.014 \\
+0.061\end{array}$ & 17.02 & 3029002 & 12.78 & 16.72 & 1531887.9 \\
\hline & 0.2 & & & & & & 13.77 & & \\
\hline
\end{tabular}


Appendix 3. Temperature T, frequency $f, \mathrm{G}_{\infty}$, viscosity, shear viscosity, relaxed Newtonian viscosity and relaxation times of the samples, determined by micropenetration and torsion measurements. Continuation...

\begin{tabular}{|c|c|c|c|c|c|c|c|c|c|}
\hline & & & \multicolumn{4}{|c|}{ MICROPENETRATION } & \multicolumn{3}{|c|}{ TORSION } \\
\hline $\mathbf{T}$ & f & $\mathbf{G}_{\infty}$ & A & B & $\begin{array}{c}\log _{10} \\
\eta\end{array}$ & $\tau$ & $\begin{array}{l}\text { Shear } \\
\text { visc. } \\
\log _{10} \eta\end{array}$ & $\begin{array}{c}\text { Relaxed } \\
\text { Newt. visc. } \\
\log _{10} \eta\end{array}$ & $\tau$ \\
\hline${ }^{\circ} \mathrm{C}$ & $\mathrm{Hz}$ & GPa & $\log _{10} \mathrm{~Pa} \mathrm{~S}$ & $\mathbf{K}$ & Pa s & $\mathbf{s}$ & Pa s & Pa s & $\mathbf{s}$ \\
\hline \multicolumn{10}{|c|}{ SAMPLE G1 } \\
\hline \multirow{7}{*}{ ధ̊ } & 0.1 & \multirow{7}{*}{34.26} & \multirow{7}{*}{$-15.63 \pm 0.53$} & \multirow{7}{*}{$\begin{array}{c}3.014 \\
\pm 0.061\end{array}$} & \multirow{7}{*}{17.02} & \multirow{7}{*}{3029002} & 14.43 & \multirow{7}{*}{16.72} & \multirow{7}{*}{1531887.9} \\
\hline & 0.05 & & & & & & 14.78 & & \\
\hline & 0.02 & & & & & & 15.90 & & \\
\hline & 0.01 & & & & & & 16.44 & & \\
\hline & 0.005 & & & & & & 16.68 & & \\
\hline & 0.002 & & & & & & 16.58 & & \\
\hline & 0.001 & & & & & & 16.90 & & \\
\hline \multirow{10}{*}{8} & 1 & \multirow{10}{*}{34.26} & \multirow{10}{*}{$-15.63 \pm 0.53$} & \multirow{10}{*}{$\begin{array}{c}3.014 \\
\pm 0.061\end{array}$} & \multirow{10}{*}{18.89} & \multirow{10}{*}{224541877} & 11.82 & \multirow{10}{*}{18.22} & \multirow{10}{*}{48442548.4} \\
\hline & 0.5 & & & & & & 12.43 & & \\
\hline & 0.2 & & & & & & 13.72 & & \\
\hline & 0.1 & & & & & & 14.17 & & \\
\hline & 0.05 & & & & & & 14.79 & & \\
\hline & 0.02 & & & & & & 16.24 & & \\
\hline & 0.01 & & & & & & 17.21 & & \\
\hline & 0.005 & & & & & & 17.66 & & \\
\hline & 0.002 & & & & & & 18.20 & & \\
\hline & 0.001 & & & & & & 18.20 & & \\
\hline 550 & & 34.26 & $-15.63 \pm 0.53$ & $\begin{array}{c}3.014 \\
\pm 0.061\end{array}$ & 20.98 & $2.809 E+10$ & - & - & - \\
\hline 500 & & 34.26 & $-15.63 \pm 0.53$ & $\begin{array}{c}3.014 \\
\pm 0.061\end{array}$ & 23.35 & $6.562 \mathrm{E}+12$ & - & - & - \\
\hline 450 & & 34.26 & $-15.63 \pm 0.53$ & $\begin{array}{c}3.014 \\
\pm 0.061\end{array}$ & 26.05 & $3.260 E+15$ & - & - & - \\
\hline 400 & & 34.26 & $-15.63 \pm 0.53$ & $\begin{array}{c}3.014 \\
\pm 0.061\end{array}$ & 29.14 & $4.073 E+18$ & - & - & - \\
\hline 350 & & 34.26 & $-15.63 \pm 0.53$ & $\begin{array}{c}3.014 \\
\pm 0.061\end{array}$ & 32.74 & $1.598 \mathrm{E}+22$ & - & - & - \\
\hline
\end{tabular}

\begin{tabular}{|c|c|c|c|c|c|c|c|c|c|}
\hline \multicolumn{10}{|c|}{ SAMPLE G2 } \\
\hline \multirow{5}{*}{ ஓ } & 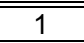 & \multirow{5}{*}{34.78} & \multirow{5}{*}{$-14.90 \pm 0.38$} & \multirow{5}{*}{$\begin{array}{c}2.956 \\
\pm 0.044\end{array}$} & \multirow{5}{*}{10.29} & \multirow{5}{*}{0.566} & 8.78 & \multirow{5}{*}{10.22} & \multirow{5}{*}{0.477} \\
\hline & 0.5 & & & & & & 9.10 & & \\
\hline & 0.2 & & & & & & 9.77 & & \\
\hline & 0.1 & & & & & & 10.18 & & \\
\hline & 0.05 & & & & & & 10.14 & & \\
\hline \multirow{5}{*}{$\underset{\infty}{\stackrel{1}{\infty}}$} & $\overline{1}$ & \multirow{5}{*}{34.78} & \multirow{5}{*}{$-14.90 \pm 0.38$} & \multirow{5}{*}{$\begin{array}{c}2.956 \\
\pm 0.044\end{array}$} & \multirow{5}{*}{10.84} & \multirow{5}{*}{2.004} & 9.16 & \multirow{5}{*}{10.50} & \multirow{5}{*}{0.909} \\
\hline & 0.5 & & & & & & 9.34 & & \\
\hline & 0.2 & & & & & & 9.74 & & \\
\hline & 0.1 & & & & & & 10.49 & & \\
\hline & 0.05 & & & & & & 10.52 & & \\
\hline \multirow{10}{*}{ 윯 } & 1 & \multirow{10}{*}{34.78} & \multirow{10}{*}{$-14.90 \pm 0.38$} & \multirow{10}{*}{$\begin{array}{c}2.956 \\
\pm 0.044\end{array}$} & \multirow{10}{*}{11.42} & \multirow{10}{*}{7.498} & 10.03 & \multirow{10}{*}{11.34} & \multirow{10}{*}{6.290} \\
\hline & 0.5 & & & & & & 10.24 & & \\
\hline & 0.2 & & & & & & 10.65 & & \\
\hline & 0.1 & & & & & & 10.96 & & \\
\hline & 0.05 & & & & & & 10.71 & & \\
\hline & 0.02 & & & & & & 10.97 & & \\
\hline & 0.01 & & & & & & 11.34 & & \\
\hline & 0.005 & & & & & & 11.33 & & \\
\hline & 0.002 & & & & & & 11.34 & & \\
\hline & 0.001 & & & & & & 11.23 & & \\
\hline \multirow{10}{*}{$\underset{\text { N }}{\stackrel{\infty}{n}}$} & 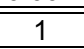 & \multirow{10}{*}{34.78} & & & & & 9.77 & & \\
\hline & 0.5 & & & & & & 10.12 & & \\
\hline & 0.2 & & & & & & 10.70 & & \\
\hline & 0.1 & & & & & & 10.96 & & \\
\hline & 0.05 & & & 2.956 & & & 11.16 & & \\
\hline & 0.02 & & $-14.90 \pm 0.38$ & \pm 0.044 & 12.02 & 29.191 & 11.40 & 11.83 & 19.431 \\
\hline & 0.01 & & & & & & 11.55 & & \\
\hline & 0.005 & & & & & & 11.78 & & \\
\hline & 0.002 & & & & & & 11.84 & & \\
\hline & 0.001 & & & & & & 11.80 & & \\
\hline & 1 & & & & & & 9.75 & & \\
\hline 용 & 0.5 & & & 2.956 & & & 10.06 & & 53533 \\
\hline$\infty$ & 0.2 & 34.78 & $-14.90 \pm 0.38$ & \pm 0.044 & 12.64 & 126.277 & 10.68 & 12.27 & 53.533 \\
\hline & 0.1 & & & & & & 11.14 & & \\
\hline
\end{tabular}


Appendix 3. Temperature $T$, frequency $f, \mathrm{G}_{\infty}$, viscosity, shear viscosity, relaxed Newtonian viscosity and relaxation times of the samples, determined by micropenetration and torsion measurements. Continuation...

\begin{tabular}{|c|c|c|c|c|c|c|c|c|c|}
\hline \multirow[b]{2}{*}{$\mathbf{T}$} & \multirow[b]{2}{*}{ f } & \multirow[b]{2}{*}{$\mathbf{G}_{\infty}$} & \multicolumn{4}{|c|}{ MICROPENETRATION } & \multicolumn{3}{|c|}{ TORSION } \\
\hline & & & A & B & $\begin{array}{c}\log _{10} \\
\eta\end{array}$ & $\tau$ & $\begin{array}{l}\text { Shear } \\
\text { visc. } \\
\log _{10} \eta\end{array}$ & $\begin{array}{c}\text { Relaxed } \\
\text { Newt. visc. } \\
\log _{10} \eta\end{array}$ & $\tau$ \\
\hline${ }^{\circ} \mathrm{C}$ & $\mathrm{Hz}$ & GPa & $\log _{10} \mathrm{~Pa} \mathrm{~S}$ & $\mathbf{K}$ & Pa S & $\mathbf{s}$ & $\mathrm{PaS}$ & Pas & $\mathbf{s}$ \\
\hline \multicolumn{10}{|c|}{ SAMPLE G2 } \\
\hline \multirow{6}{*}{ ○ } & 0.05 & \multirow{6}{*}{34.78} & \multirow{6}{*}{$-14.90 \pm 0.38$} & \multirow{6}{*}{$\begin{array}{c}2.956 \\
\pm 0.044\end{array}$} & \multirow{6}{*}{12.64} & \multirow{6}{*}{126.277} & 11.42 & \multirow{6}{*}{12.27} & \multirow{6}{*}{53.533} \\
\hline & 0.02 & & & & & & 11.52 & & \\
\hline & 0.01 & & & & & & 11.67 & & \\
\hline & 0.005 & & & & & & 11.88 & & \\
\hline & 0.002 & & & & & & 12.22 & & \\
\hline & 0.001 & & & & & & 12.36 & & \\
\hline \multirow{10}{*}{$\stackrel{n}{N}$} & $\overline{1}$ & \multirow{10}{*}{34.78} & \multirow{10}{*}{$-14.90 \pm 0.38$} & & & & 9.73 & & \\
\hline & 0.5 & & & & & & 10.25 & & \\
\hline & 0.2 & & & & & & 11.03 & & \\
\hline & 0.1 & & & & & & 11.67 & & \\
\hline & 0.05 & & & & & & 11.82 & & \\
\hline & 0.02 & & & \pm 0.044 & 13.30 & 573.329 & 12.25 & 13.10 & 361.927 \\
\hline & 0.01 & & & & & & 12.70 & & \\
\hline & 0.005 & & & & & & 12.87 & & \\
\hline & 0.002 & & & & & & 13.14 & & \\
\hline & 0.001 & & & & & & 13.24 & & \\
\hline & $\overline{1}$ & & & & & & 9.83 & & \\
\hline & 0.5 & & & & & & 9.45 & & \\
\hline & 0.2 & & & & & & 10.19 & & \\
\hline & 0.1 & & & & & & 11.42 & & \\
\hline 용 & 0.05 & & & 2.956 & & & 11.67 & & \\
\hline$\stackrel{n}{R}$ & 0.02 & 34.78 & $-14.90 \pm 0.38$ & \pm 0.044 & 13.99 & 2802.839 & 12.17 & 13.74 & 1579.870 \\
\hline & 0.01 & & & & & & 12.63 & & \\
\hline & 0.005 & & & & & & 13.04 & & \\
\hline & 0.002 & & & & & & 13.68 & & \\
\hline & 0.001 & & & & & & 13.75 & & \\
\hline & $\overline{1}$ & & & & & & 8.84 & & \\
\hline & 0.5 & & & & & & 9.08 & & \\
\hline & 0.2 & & & & & & 9.44 & & \\
\hline & 0.1 & & & & & & 11.30 & & \\
\hline$\stackrel{2}{2}$ & 0.05 & & & 2.956 & & & 11.88 & & \\
\hline $\mathfrak{N}$ & 0.02 & 34.78 & $-14.90 \pm 0.38$ & \pm 0.044 & 14.71 & 14836.133 & 12.64 & 14.38 & 6896.381 \\
\hline & 0.01 & & & & & & 13.21 & & \\
\hline & 0.005 & & & & & & 13.86 & & \\
\hline & 0.002 & & & & & & 14.19 & & \\
\hline & 0.001 & & & & & & 14.49 & & \\
\hline & $\overline{l 1}$ & & & & & & 7.99 & & \\
\hline & 0.5 & & & & & & 8.55 & & \\
\hline & 0.2 & & & & & & 10.41 & & \\
\hline & 0.1 & & & & & & 11.21 & & \\
\hline 요 & 0.05 & & & 2.956 & & & 12.00 & 1554 & 09683038 \\
\hline 운 & 0.02 & 34.78 & $-14.90 \pm 0.38$ & \pm 0.044 & 15.47 & 85552.678 & 12.88 & 15.54 & 99683.038 \\
\hline & 0.01 & & & & & & 13.85 & & \\
\hline & 0.005 & & & & & & 14.37 & & \\
\hline & 0.002 & & & & & & 14.96 & & \\
\hline & 0.001 & & & & & & 15.52 & & \\
\hline 650 & & 34.78 & $-14.90 \pm 0.38$ & $\begin{array}{c}2.956 \\
\pm 0.044\end{array}$ & 17.12 & 3781969.52 & - & - & - \\
\hline 600 & & 34.78 & $-14.90 \pm 0.38$ & $\begin{array}{c}2.956 \\
\pm 0.044\end{array}$ & 18.95 & 258040948 & - & - & - \\
\hline 550 & & 34.78 & $-14.90 \pm 0.38$ & $\begin{array}{c}2.956 \\
\pm 0.044 \\
\end{array}$ & 21.01 & $2.941 \mathrm{E}+10$ & - & - & - \\
\hline 500 & & 34.78 & $-14.90 \pm 0.38$ & $\begin{array}{c}2.956 \\
\pm 0.044\end{array}$ & 23.33 & $6.186 \mathrm{E}+12$ & - & - & - \\
\hline
\end{tabular}


Appendix 3. Temperature T, frequency $f, \mathrm{G}_{\infty}$, viscosity, shear viscosity, relaxed Newtonian viscosity and relaxation times of the samples, determined by micropenetration and torsion measurements.

Continuation...

\begin{tabular}{|c|c|c|c|c|c|c|c|c|c|}
\hline & & & \multicolumn{4}{|c|}{ MICROPENETRATION } & \multicolumn{3}{|c|}{ TORSION } \\
\hline $\mathbf{T}$ & $\mathbf{f}$ & $\mathbf{G}_{\infty}$ & A & B & $\begin{array}{c}\log _{10} \\
\eta\end{array}$ & $\tau$ & $\begin{array}{l}\text { Shear } \\
\text { visc. } \\
\log _{10} \eta\end{array}$ & $\begin{array}{c}\text { Relaxed } \\
\text { Newt. visc. } \\
\log _{10} \eta\end{array}$ & $\tau$ \\
\hline${ }^{\circ} \mathrm{C}$ & $\mathrm{Hz}$ & GPa & $\log _{10} \mathrm{Pas}$ & $\mathbf{K}$ & Pas & $\mathbf{s}$ & Pas & Pas & $\mathbf{s}$ \\
\hline \multicolumn{10}{|c|}{ SAMPLE G3 } \\
\hline \multirow{2}{*}{900} & 1 & \multirow{2}{*}{34.43} & \multirow{2}{*}{$-16.05 \pm 0.36$} & \multirow{2}{*}{$\begin{array}{c}3.083 \\
\pm 0.041 \\
\end{array}$} & \multirow{2}{*}{10.23} & \multirow{2}{*}{0.497} & 10.23 & \multirow{2}{*}{10.20} & \multirow{2}{*}{0.460} \\
\hline & 0.5 & & & & & & 10.26 & & \\
\hline \multirow{5}{*}{$\underset{\infty}{\stackrel{2}{\infty}}$} & 1 & \multirow{5}{*}{34.43} & \multirow{5}{*}{$-16.05 \pm 0.36$} & \multirow{5}{*}{$\begin{array}{c}3.083 \\
\pm 0.041\end{array}$} & \multirow{5}{*}{10.80} & & 9.98 & & \\
\hline & 0.5 & & & & & & 10.41 & & \\
\hline & 0.2 & & & & & 1.857 & 10.71 & 10.78 & 1.750 \\
\hline & 0.1 & & & & & & 10.84 & & \\
\hline & 0.05 & & & & & & 10.83 & & \\
\hline & 1 & & & & & & 9.82 & & \\
\hline & 0.5 & & & & & & 10.25 & & \\
\hline & 0.2 & & & & & & 10.75 & & \\
\hline & 0.1 & & & & & & 11.15 & & \\
\hline ధి & 0.05 & 34.43 & $-16.05 \pm 0.36$ & 3.083 & 11.40 & 7.356 & 11.32 & 11.37 & 6.808 \\
\hline & 0.02 & & & & & & 11.40 & & \\
\hline & 0.01 & & & & & & 11.45 & & \\
\hline & 0.005 & & & & & & - & & \\
\hline & 0.002 & & & & & & - & & \\
\hline & 1 & & & & & & 9.53 & & \\
\hline & 0.5 & & & & & & 10.02 & & \\
\hline & 0.2 & & & & & & 10.58 & & \\
\hline & 0.1 & & & & & & 11.03 & & \\
\hline\llcorner & 0.05 & & & 3.083 & & & 11.36 & & \\
\hline 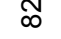 & 0.02 & 34.43 & $-16.05 \pm 0.36$ & \pm 0.041 & 12.03 & 31.022 & 11.71 & 11.89 & 22.543 \\
\hline & 0.01 & & & & & & 11.96 & & \\
\hline & 0.005 & & & & & & 11.93 & & \\
\hline & 0.002 & & & & & & 11.95 & & \\
\hline & 0.001 & & & & & & 11.97 & & \\
\hline & $\overline{c 1}$ & & & & & & 9.30 & & \\
\hline & 0.5 & & & & & & 9.79 & & \\
\hline & 0.2 & & & & & & 10.37 & & \\
\hline & 0.1 & & & & & & 10.87 & & \\
\hline & 0.05 & & & 3.083 & & & 11.36 & & \\
\hline$\infty$ & 0.02 & 34.43 & $-16.05 \pm 0.36$ & \pm 0.041 & 12.68 & 139.892 & 11.92 & 12.79 & $1 / 9.064$ \\
\hline & 0.01 & & & & & & 12.27 & & \\
\hline & 0.005 & & & & & & 12.54 & & \\
\hline & 0.002 & & & & & & 12.70 & & \\
\hline & 0.001 & & & & & & 12.75 & & \\
\hline & $\overline{11}$ & & & & & & 9.25 & & \\
\hline & 0.5 & & & & & & 9.59 & & \\
\hline & 0.2 & & & & & & 10.33 & & \\
\hline & 0.1 & & & & & & 10.81 & & \\
\hline 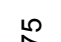 & 0.05 & & & 3.083 & & & 11.26 & & \\
\hline$\hat{\imath}$ & 0.02 & 34.43 & $-16.05 \pm 0.36$ & \pm 0.041 & 13.37 & 677.838 & 11.81 & 13.21 & 470.985 \\
\hline & 0.01 & & & & & & 12.26 & & \\
\hline & 0.005 & & & & & & 12.73 & & \\
\hline & 0.002 & & & & & & 13.21 & & \\
\hline & 0.001 & & & & & & 13.30 & & \\
\hline 750 & & 34.43 & $-16.05 \pm 0.36$ & $\begin{array}{c}3.083 \\
\pm 0.041 \\
\end{array}$ & 14.09 & 3547.749 & - & - & - \\
\hline 725 & & 34.43 & $-16.05 \pm 0.36$ & $\begin{array}{c}3.083 \\
\pm 0.041 \\
\end{array}$ & 14.84 & 20174.069 & - & - & - \\
\hline 700 & & 34.43 & $-16.05 \pm 0.36$ & $\begin{array}{c}3.083 \\
\pm 0.041 \\
\end{array}$ & 15.64 & 125436.353 & - & - & - \\
\hline 650 & & 34.43 & $-16.05 \pm 0.36$ & $\begin{array}{c}3.083 \\
\pm 0.041\end{array}$ & 17.35 & 6526177 & - & - & - \\
\hline 600 & & 34.43 & $-16.05 \pm 0.36$ & $\begin{array}{c}3.083 \\
\pm 0.041\end{array}$ & 19.26 & 533930613 & - & - & - \\
\hline 550 & & 34.43 & $-16.05 \pm 0.36$ & $\begin{array}{c}3.083 \\
\pm 0.041 \\
\end{array}$ & 21.41 & $7.460 \mathrm{E}+10$ & - & - & - \\
\hline
\end{tabular}


Appendix 3. Temperature T, frequency $f, \mathrm{G}_{\infty}$, viscosity, shear viscosity, relaxed Newtonian viscosity and relaxation times of the samples, determined by micropenetration and torsion measurements.

Continuation...

\begin{tabular}{|c|c|c|c|c|c|c|c|c|c|}
\hline & & & \multicolumn{4}{|c|}{ MICROPENETRATION } & \multicolumn{3}{|c|}{ TORSION } \\
\hline $\mathbf{T}$ & $\mathbf{f}$ & $\mathbf{G}_{\infty}$ & A & B & $\begin{array}{c}\log _{10} \\
\eta\end{array}$ & $\tau$ & $\begin{array}{c}\text { Shear } \\
\text { visc. } \\
\log _{10} \eta\end{array}$ & $\begin{array}{c}\text { Relaxed } \\
\text { Newt. visc. } \\
\log _{10} \eta\end{array}$ & $\tau$ \\
\hline${ }^{\circ} \mathrm{C}$ & $\mathrm{Hz}$ & GPa & $\log _{10} \mathrm{~Pa} \mathrm{~s}$ & $\mathbf{K}$ & Pa s & $\mathbf{s}$ & Pa s & $\mathrm{Pas}$ & $\mathbf{s}$ \\
\hline \multicolumn{10}{|c|}{ SAMPLE G4 } \\
\hline \multirow{7}{*}{ ధి } & 1 & \multirow{7}{*}{34.23} & \multirow{7}{*}{$-14.00 \pm 0.48$} & \multirow{7}{*}{$\begin{array}{c}2.747 \\
\pm 0.055\end{array}$} & \multirow{7}{*}{10.46} & \multirow{7}{*}{0.847} & 9.95 & \multirow{7}{*}{10.53} & \multirow{7}{*}{0.990} \\
\hline & 0.5 & & & & & & 10.23 & & \\
\hline & 0.2 & & & & & & 10.43 & & \\
\hline & 0.1 & & & & & & 10.48 & & \\
\hline & 0.05 & & & & & & 10.47 & & \\
\hline & 0.02 & & & & & & 10.49 & & \\
\hline & 0.01 & & & & & & 10.52 & & \\
\hline \multirow{7}{*}{$\underset{\infty}{\infty}$} & 1 & \multirow{7}{*}{34.23} & \multirow{7}{*}{$-14.00 \pm 0.48$} & \multirow{7}{*}{$\begin{array}{c}2.747 \\
\pm 0.055\end{array}$} & \multirow{7}{*}{11.02} & \multirow{7}{*}{3.055} & 9.75 & & \\
\hline & 0.5 & & & & & & 10.15 & & \\
\hline & 0.2 & & & & & & 10.62 & & \\
\hline & 0.1 & & & & & & 10.83 & 10.90 & 2.320 \\
\hline & 0.05 & & & & & & 10.93 & & \\
\hline & 0.02 & & & & & & 10.93 & & \\
\hline & 0.01 & & & & & & 10.95 & & \\
\hline & 1 & & & & & & 9.50 & & \\
\hline & 0.5 & & & & & & 9.94 & & \\
\hline & 0.2 & & & & & & 10.51 & & \\
\hline & 0.1 & & & & & & 10.90 & & \\
\hline ○ & 0.05 & & & 2.747 & & & 11.23 & & \\
\hline$\infty$ & 0.02 & 34.23 & $-14.00 \pm 0.48$ & \pm 0.055 & 11.60 & 11.692 & 11.49 & 11.67 & 13.663 \\
\hline & 0.01 & & & & & & 11.67 & & \\
\hline & 0.005 & & & & & & 11.94 & & \\
\hline & 0.002 & & & & & & 11.72 & & \\
\hline & 0.001 & & & & & & 11.63 & & \\
\hline & 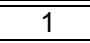 & & & & & & 9.15 & & \\
\hline & 0.5 & & & & & & 9.57 & & \\
\hline & 0.2 & & & & & & 10.13 & & \\
\hline & 0.1 & & & & & & 10.61 & & \\
\hline$\stackrel{2}{2}$ & 0.05 & & & 2.747 & & & 11.07 & 1224 & \\
\hline$\hat{\Lambda}$ & 0.02 & 34.23 & $-14.00 \pm 0.48$ & \pm 0.055 & 12.21 & 47.106 & 11.58 & 12.24 & 50.164 \\
\hline & 0.01 & & & & & & 11.83 & & \\
\hline & 0.005 & & & & & & 12.11 & & \\
\hline & 0.002 & & & & & & 12.24 & & \\
\hline & 0.001 & & & & & & 12.23 & & \\
\hline & 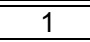 & & & & & & 8.75 & & \\
\hline & 0.5 & & & & & & 9.25 & & \\
\hline & 0.2 & & & & & & 9.81 & & \\
\hline & 0.1 & & & & & & 10.16 & & \\
\hline 용 & 0.05 & & & 2.747 & & & 10.57 & & \\
\hline$\stackrel{n}{n}$ & 0.02 & 34.23 & $-14.00 \pm 0.48$ & \pm 0.055 & 12.85 & 208.505 & 11.31 & 12.80 & 184.312 \\
\hline & 0.01 & & & & & & 11.73 & & \\
\hline & 0.005 & & & & & & 12.18 & & \\
\hline & 0.002 & & & & & & 12.62 & & \\
\hline & 0.001 & & & & & & 12.79 & & \\
\hline 725 & & 34.23 & $-14.00 \pm 0.48$ & $\begin{array}{c}2.747 \\
\pm 0.055 \\
\end{array}$ & 13.53 & 981.190 & - & - & - \\
\hline 700 & & 34.23 & $-14.00 \pm 0.48$ & $\begin{array}{c}2.747 \\
\pm 0.055 \\
\end{array}$ & 14.23 & 4999.810 & - & - & - \\
\hline 675 & & 34.23 & $-14.00 \pm 0.48$ & $\begin{array}{c}2.747 \\
\pm 0.055 \\
\end{array}$ & 14.98 & 27762.193 & - & - & - \\
\hline 650 & & 34.23 & $-14.00 \pm 0.48$ & $\begin{array}{c}2.747 \\
\pm 0.055\end{array}$ & 15.76 & 169154.854 & - & - & - \\
\hline 600 & & 34.23 & $-14.00 \pm 0.48$ & $\begin{array}{c}2.747 \\
\pm 0.055 \\
\end{array}$ & 17.47 & 8566363.46 & - & - & - \\
\hline 550 & & 34.23 & $-14.00 \pm 0.48$ & $\begin{array}{c}2.747 \\
\pm 0.055 \\
\end{array}$ & 19.38 & 698904974 & - & - & - \\
\hline
\end{tabular}


Appendix 3. Temperature T, frequency $f, \mathrm{G}_{\infty}$, viscosity, shear viscosity, relaxed Newtonian viscosity and relaxation times of the samples, determined by micropenetration and torsion measurements.

Continuation...

\begin{tabular}{|c|c|c|c|c|c|c|c|c|c|}
\hline & & & \multicolumn{4}{|c|}{ MICROPENETRATION } & \multicolumn{3}{|c|}{ TORSION } \\
\hline $\mathbf{T}$ & $\mathbf{f}$ & $\mathbf{G}_{\infty}$ & A & B & $\begin{array}{c}\log _{10} \\
\eta\end{array}$ & $\tau$ & $\begin{array}{l}\text { Shear } \\
\text { visc. } \\
\log _{10} \eta\end{array}$ & $\begin{array}{c}\text { Relaxed } \\
\text { Newt. visc. } \\
\log _{10} \eta\end{array}$ & $\tau$ \\
\hline${ }^{\circ} \mathrm{C}$ & $\mathrm{Hz}$ & GPa & $\log _{10} \mathrm{~Pa} \mathrm{~s}$ & $\mathbf{K}$ & Pas & $\mathbf{s}$ & Pa s & Pa s & $\mathbf{s}$ \\
\hline \multicolumn{10}{|c|}{ SAMPLE G5 } \\
\hline \multirow{5}{*}{$\stackrel{\circ}{\mathfrak{R}}$} & 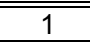 & \multirow{5}{*}{34.01} & \multirow{5}{*}{$-15.32 \pm 0.57$} & \multirow{5}{*}{$\begin{array}{c}2.627 \\
\pm 0.058\end{array}$} & \multirow{5}{*}{10.36} & \multirow{5}{*}{0.669} & 8.97 & \multirow{5}{*}{10.18} & \multirow{5}{*}{0.445} \\
\hline & 0.5 & & & & & & 9.44 & & \\
\hline & 0.2 & & & & & & 10.10 & & \\
\hline & 0.1 & & & & & & 10.19 & & \\
\hline & 0.05 & & & & & & 10.28 & & \\
\hline \multirow{8}{*}{$\stackrel{\mathscr{N}}{N}$} & 1 & \multirow{8}{*}{34.01} & \multirow{8}{*}{$-15.32 \pm 0.57$} & \multirow{8}{*}{$\begin{array}{c}2.627 \\
\pm 0.058\end{array}$} & \multirow{8}{*}{11.00} & \multirow{8}{*}{2.944} & 9.14 & \multirow{8}{*}{10.87} & \multirow{8}{*}{2.180} \\
\hline & 0.5 & & & & & & 9.59 & & \\
\hline & 0.2 & & & & & & 10.12 & & \\
\hline & 0.1 & & & & & & 10.43 & & \\
\hline & 0.05 & & & & & & 10.64 & & \\
\hline & 0.02 & & & & & & 10.73 & & \\
\hline & 0.01 & & & & & & 10.91 & & \\
\hline & 0.005 & & & & & & 10.90 & & \\
\hline \multirow{10}{*}{ ஓ } & 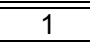 & \multirow{10}{*}{34.01} & \multirow{10}{*}{$-15.32 \pm 0.57$} & \multirow{10}{*}{$\begin{array}{c}2.627 \\
\pm 0.058\end{array}$} & & & 8.87 & & \\
\hline & 0.5 & & & & & & 9.27 & & \\
\hline & 0.2 & & & & & & 9.88 & & \\
\hline & 0.1 & & & & & & 10.35 & & \\
\hline & 0.05 & & & & & & 10.74 & & \\
\hline & 0.02 & & & & 11.68 & 13.970 & 11.17 & 11.54 & 10.195 \\
\hline & 0.01 & & & & & & 11.30 & & \\
\hline & 0.005 & & & & & & 11.47 & & \\
\hline & 0.002 & & & & & & 11.55 & & \\
\hline & 0.001 & & & & & & 11.54 & & \\
\hline & 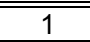 & & & & & & 8.74 & & \\
\hline & 0.5 & & & & & & 9.16 & & \\
\hline & 0.2 & & & & & & 9.59 & & \\
\hline & 0.1 & & & & & & 10.09 & & \\
\hline\llcorner & 0.05 & & & 2.627 & 1239 & & 10.53 & & \\
\hline$\hat{\theta}$ & 0.02 & 34.01 & $-15.32 \pm 0.57$ & \pm 0.058 & 12.39 & 71.972 & 11.07 & 12.25 & 52.287 \\
\hline & 0.01 & & & & & & 11.41 & & \\
\hline & 0.005 & & & & & & 11.52 & & \\
\hline & 0.002 & & & & & & 11.88 & & \\
\hline & 0.001 & & & & & & 12.25 & & \\
\hline 650 & & 34.01 & $-15.32 \pm 0.57$ & $\begin{array}{c}2.627 \\
\pm 0.058 \\
\end{array}$ & 13.14 & 405.235 & - & - & - \\
\hline 625 & & 34.01 & $-15.32 \pm 0.57$ & $\begin{array}{c}2.627 \\
\pm 0.058 \\
\end{array}$ & 13.93 & 2512.110 & - & - & - \\
\hline 600 & & 34.01 & $-15.32 \pm 0.57$ & $\begin{array}{c}2.627 \\
\pm 0.058 \\
\end{array}$ & 14.77 & 17288.228 & - & - & - \\
\hline 550 & & 34.01 & $-15.32 \pm 0.57$ & $\begin{array}{c}2.627 \\
\pm 0.058 \\
\end{array}$ & 16.60 & 1163738.49 & - & - & - \\
\hline 500 & & 34.01 & $-15.32 \pm 0.57$ & $\begin{array}{c}2.627 \\
\pm 0.058 \\
\end{array}$ & 18.66 & 135037734 & - & - & - \\
\hline
\end{tabular}

\begin{tabular}{|c|c|c|c|c|c|c|c|c|c|}
\hline \multicolumn{10}{|c|}{ SAMPLE G6 } \\
\hline \multirow{8}{*}{ 옥 } & 1 & \multirow{8}{*}{33.47} & \multirow{8}{*}{$-14.71 \pm 0.23$} & \multirow{8}{*}{$\begin{array}{c}2.472 \\
\pm 0.022\end{array}$} & \multirow{8}{*}{10.70} & \multirow{8}{*}{1.485} & 9.43 & \multirow{8}{*}{10.59} & \multirow{8}{*}{1.162} \\
\hline & 0.5 & & & & & & 9.83 & & \\
\hline & 0.2 & & & & & & 10.26 & & \\
\hline & 0.1 & & & & & & 10.45 & & \\
\hline & 0.05 & & & & & & 10.48 & & \\
\hline & 0.02 & & & & & & 10.53 & & \\
\hline & 0.01 & & & & & & 10.58 & & \\
\hline & 0.005 & & & & & & 10.62 & & \\
\hline \multirow{9}{*}{ 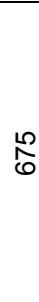 } & 1 & \multirow{9}{*}{33.47} & \multirow{9}{*}{$-14.71 \pm 0.23$} & \multirow{9}{*}{$\begin{array}{c}2.472 \\
\pm 0.022\end{array}$} & \multirow{9}{*}{11.37} & \multirow{9}{*}{6.950} & \begin{tabular}{c|}
9.08 \\
\end{tabular} & \multirow{9}{*}{11.26} & \multirow{9}{*}{5.437} \\
\hline & 0.5 & & & & & & 9.54 & & \\
\hline & 0.2 & & & & & & 10.10 & & \\
\hline & 0.1 & & & & & & 10.51 & & \\
\hline & 0.05 & & & & & & 10.85 & & \\
\hline & 0.02 & & & & & & 10.89 & & \\
\hline & 0.01 & & & & & & 11.13 & & \\
\hline & 0.005 & & & & & & 11.25 & & \\
\hline & 0.002 & & & & & & 11.32 & & \\
\hline
\end{tabular}


Appendix 3. Temperature $T$, frequency $f, \mathrm{G}_{\infty}$, viscosity, shear viscosity, relaxed Newtonian viscosity and relaxation times of the samples. determined by micropenetration and torsion measurements.

Continuation...

\begin{tabular}{|c|c|c|c|c|c|c|c|c|c|}
\hline & & & \multicolumn{4}{|c|}{ MICROPENETRATION } & \multicolumn{3}{|c|}{ TORSION } \\
\hline $\mathbf{T}$ & f & $\mathbf{G}_{\infty}$ & A & B & $\begin{array}{c}\log _{10} \\
\eta\end{array}$ & $\tau$ & $\begin{array}{c}\text { Shear } \\
\text { visc. } \\
\log _{10} \eta\end{array}$ & $\begin{array}{c}\text { Relaxed } \\
\text { Newt. visc. } \\
\log _{10} \eta\end{array}$ & $\tau$ \\
\hline${ }^{\circ} \mathrm{C}$ & $\mathrm{Hz}$ & GPa & $\log _{10} \mathrm{PaS}$ & $\mathbf{K}$ & Pas & $\mathbf{s}$ & Pa s & Pa s & $\mathbf{s}$ \\
\hline \multicolumn{10}{|c|}{ SAMPLE G6 } \\
\hline \multirow{10}{*}{ 융 } & 1 & \multirow{10}{*}{33.47} & \multirow{10}{*}{$-14.71 \pm 0.23$} & \multirow{10}{*}{$\begin{array}{c}2.472 \\
\pm 0.022\end{array}$} & \multirow{10}{*}{12.07} & \multirow{10}{*}{35.353} & 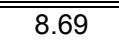 & \multirow{10}{*}{11.90} & \multirow{10}{*}{23.732} \\
\hline & 0.5 & & & & & & 9.11 & & \\
\hline & 0.2 & & & & & & 9.74 & & \\
\hline & 0.1 & & & & & & 10.20 & & \\
\hline & 0.05 & & & & & & 10.60 & & \\
\hline & 0.02 & & & & & & 11.17 & & \\
\hline & 0.01 & & & & & & 11.34 & & \\
\hline & 0.005 & & & & & & 11.61 & & \\
\hline & 0.002 & & & & & & 11.87 & & \\
\hline & 0.001 & & & & & & 11.99 & & \\
\hline \multirow{10}{*}{$\stackrel{\text { సै }}{6}$} & 1 & \multirow{10}{*}{33.47} & \multirow{10}{*}{$-14.71 \pm 0.23$} & \multirow{10}{*}{$\begin{array}{c}2.472 \\
\pm 0.022\end{array}$} & \multirow{10}{*}{12.82} & \multirow{10}{*}{196.877} & 8.47 & \multirow{10}{*}{12.63} & \multirow{10}{*}{127.451} \\
\hline & 0.5 & & & & & & 8.94 & & \\
\hline & 0.2 & & & & & & 9.55 & & \\
\hline & 0.1 & & & & & & 9.97 & & \\
\hline & 0.05 & & & & & & 10.40 & & \\
\hline & 0.02 & & & & & & 11.00 & & \\
\hline & 0.01 & & & & & & 11.45 & & \\
\hline & 0.005 & & & & & & 11.83 & & \\
\hline & 0.002 & & & & & & 12.22 & & \\
\hline & 0.001 & & & & & & 12.67 & & \\
\hline 600 & & 33.47 & $-14.71 \pm 0.23$ & $\begin{array}{c}2.472 \\
\pm 0.022 \\
\end{array}$ & 13.61 & 1209.697 & - & - & - \\
\hline 575 & & 33.47 & $-14.71 \pm 0.23$ & $\begin{array}{c}2.472 \\
\pm 0.022 \\
\end{array}$ & 14.44 & 8272.708 & - & - & - \\
\hline 550 & & 33.47 & $-14.71 \pm 0.23$ & $\begin{array}{c}2.472 \\
\pm 0.022\end{array}$ & 15.33 & 63583.751 & - & - & - \\
\hline 500 & & 33.47 & $-14.71 \pm 0.23$ & $\begin{array}{c}2.472 \\
\pm 0.022\end{array}$ & 17.27 & 5579716.25 & - & - & - \\
\hline 450 & & 33.47 & $-14.71 \pm 0.23$ & $\begin{array}{c}2.472 \\
\pm 0.022\end{array}$ & 19.48 & 909193294 & - & - & - \\
\hline
\end{tabular}

\begin{tabular}{|c|c|c|c|c|c|c|c|c|c|}
\hline \multicolumn{10}{|c|}{ SAMPLE G7 } \\
\hline \multirow{3}{*}{ 웃 } & 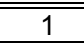 & \multirow{3}{*}{31.68} & \multirow{3}{*}{$-16.75 \pm 0.40$} & \multirow{3}{*}{$\begin{array}{c}2.512 \\
\pm 0.037\end{array}$} & \multirow{3}{*}{9.07} & \multirow{3}{*}{0.037} & 8.95 & \multirow{3}{*}{9.36} & \multirow{3}{*}{0.072} \\
\hline & 0.1 & & & & & & 8.83 & & \\
\hline & 0.01 & & & & & & 9.36 & & \\
\hline \multirow{3}{*}{$\frac{0}{0}$} & 1 & \multirow{3}{*}{31.68} & \multirow{3}{*}{$-16.75 \pm 0.40$} & \multirow{3}{*}{$\begin{array}{c}2.512 \\
\pm 0.037\end{array}$} & \multirow{3}{*}{9.89} & \multirow{3}{*}{0.246} & 8.79 & \multirow{3}{*}{9.84} & \multirow{3}{*}{0.218} \\
\hline & 0.1 & & & & & & 9.33 & & \\
\hline & 0.01 & & & & & & 9.75 & & \\
\hline \multirow{3}{*}{ 유 } & 1 & \multirow{3}{*}{31.68} & \multirow{3}{*}{$-16.75 \pm 0.40$} & \multirow{3}{*}{$\begin{array}{c}2.512 \\
\pm 0.037\end{array}$} & \multirow{3}{*}{10.47} & \multirow{3}{*}{0.929} & 9.18 & \multirow{3}{*}{10.38} & \multirow{3}{*}{0.757} \\
\hline & 0.1 & & & & & & 9.76 & & \\
\hline & 0.01 & & & & & & 10.30 & & \\
\hline \multirow{3}{*}{ ஜ్లి } & 1 & \multirow{3}{*}{31.68} & \multirow{3}{*}{$-16.75 \pm 0.40$} & \multirow{3}{*}{$\begin{array}{c}2.512 \\
\pm 0.037\end{array}$} & \multirow{3}{*}{11.07} & \multirow{3}{*}{3.723} & 9.12 & \multirow{3}{*}{10.74} & \multirow{3}{*}{1.735} \\
\hline & 0.1 & & & & & & 10.03 & & \\
\hline & 0.01 & & & & & & 10.68 & & \\
\hline & 1 & & & & & & 8.18 & & \\
\hline ○ & 0.1 & 2160 & $1675+010$ & 2.512 & 1202 & 22010 & 9.56 & 1107 & 20161 \\
\hline 8 & 0.01 & 31.68 & $-10.15 \pm 0.40$ & \pm 0.037 & 12.03 & 33.040 & 11.25 & 11.91 & 29.461 \\
\hline & 0.001 & & & & & & 11.99 & & \\
\hline & 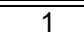 & & & & & & 7.30 & & \\
\hline 0 & 0.1 & 2100 & $1075+010$ & 2.512 & 1270 & & 8.90 & & \\
\hline 员 & 0.01 & 31.68 & $-16.15 \pm 0.40$ & \pm 0.037 & 13.78 & 1883.505 & 11.95 & 13.71 & 1618.984 \\
\hline & 0.001 & & & & & & 13.71 & & \\
\hline 500 & & 31.68 & $-16.75 \pm 0.40$ & $\begin{array}{c}2.512 \\
\pm 0.037\end{array}$ & 15.75 & 177513.061 & - & - & - \\
\hline
\end{tabular}


Appendix 3. Temperature T, frequency $f, \mathrm{G}_{\infty}$, viscosity, shear viscosity, relaxed Newtonian viscosity and relaxation times of the samples. determined by micropenetration and torsion measurements. Continuation...

\begin{tabular}{|c|c|c|c|c|c|c|c|c|c|}
\hline & & & \multicolumn{4}{|c|}{ MICROPENETRATION } & \multicolumn{3}{|c|}{ TORSION } \\
\hline $\mathbf{T}$ & f & $\mathbf{G}_{\infty}$ & A & B & $\begin{array}{c}\log _{10} \\
\eta\end{array}$ & $\tau$ & $\begin{array}{l}\text { Shear } \\
\text { visc. } \\
\log _{10} \eta\end{array}$ & $\begin{array}{c}\text { Relaxed } \\
\text { Newt. visc. } \\
\log _{10} \eta \\
\end{array}$ & $\tau$ \\
\hline${ }^{\circ} \mathrm{C}$ & $\mathrm{Hz}$ & GPa & $\log _{10} \mathrm{~Pa} \mathrm{~s}$ & $\mathbf{K}$ & Pa s & $\mathbf{s}$ & Pas & Pa s & $\mathbf{s}$ \\
\hline \multicolumn{10}{|c|}{ SAMPLE G8 } \\
\hline \multirow{9}{*}{ ণ্ণি } & 1 & \multirow{9}{*}{32.45} & \multirow{9}{*}{$-13.85 \pm 0.70$} & \multirow{9}{*}{$\begin{array}{c}2.654 \\
\pm 0.077\end{array}$} & \multirow{9}{*}{10.43} & \multirow{9}{*}{0.833} & 9.54 & \multirow{9}{*}{10.15} & \\
\hline & 0.5 & & & & & & 9.87 & & \\
\hline & 0.2 & & & & & & 10.15 & & \\
\hline & 0.1 & & & & & & 10.24 & & \\
\hline & 0.05 & & & & & & 10.12 & & 0.435 \\
\hline & 0.02 & & & & & & 10.31 & & \\
\hline & 0.01 & & & & & & 10.31 & & \\
\hline & 0.005 & & & & & & 10.33 & & \\
\hline & 0.002 & & & & & & 10.04 & & \\
\hline & 1 & & & & & & 9.42 & & \\
\hline & 0.5 & & & & & & 9.80 & & \\
\hline & 0.2 & & & & & & 10.18 & & \\
\hline 으 & 0.1 & 3245 & & 2.654 & & 1396 & 10.34 & 10.40 & 0.774 \\
\hline $\bar{\infty}$ & 0.05 & 32.45 & $-13.85 \pm 0.10$ & \pm 0.077 & 10.66 & 1.396 & 10.22 & 10.40 & $0.7 / 4$ \\
\hline & 0.02 & & & & & & 10.43 & & \\
\hline & 0.01 & & & & & & 10.11 & & \\
\hline & 0.005 & & & & & & 10.43 & & \\
\hline & 1 & & & & & & 9.62 & & \\
\hline & 0.5 & & & & & & 10.02 & & \\
\hline & 0.2 & & & & & & 10.50 & & \\
\hline & 0.1 & & & & & & 10.75 & & \\
\hline ○ & 0.05 & & & 2.654 & & & 10.89 & & \\
\hline$\infty$ & 0.02 & 32.45 & $-13.85 \pm 0.10$ & \pm 0.077 & 10.88 & 2.361 & 10.96 & 10.90 & 2.448 \\
\hline & 0.01 & & & & & & 10.92 & & \\
\hline & 0.005 & & & & & & 10.97 & & \\
\hline & 0.002 & & & & & & 10.85 & & \\
\hline & 0.001 & & & & & & 10.84 & & \\
\hline & $\overline{1}$ & & & & & & 9.14 & & \\
\hline & 0.5 & & & & & & 9.59 & & \\
\hline & 0.2 & & & & & & 10.13 & & \\
\hline & 0.1 & & & & & & 10.56 & & \\
\hline ৪ & 0.05 & & & 2.654 & & & 10.87 & & \\
\hline$\stackrel{\infty}{\wedge}$ & 0.02 & 32.45 & $-13.85 \pm 0.10$ & \pm 0.077 & 11.35 & 6.965 & 11.13 & 11.25 & 5.480 \\
\hline & 0.01 & & & & & & 11.22 & & \\
\hline & 0.005 & & & & & & 11.25 & & \\
\hline & 0.002 & & & & & & 11.25 & & \\
\hline & 0.001 & & & & & & 11.29 & & \\
\hline & 1 & & & & & & 8.84 & & \\
\hline & 0.5 & & & & & & 9.28 & & \\
\hline & 0.2 & & & & & & 10.29 & & \\
\hline & 0.1 & & & & & & 10.32 & & \\
\hline 8 & 0.05 & & & 2.654 & & & 10.73 & & \\
\hline$\stackrel{0}{1}$ & 0.02 & 32.45 & $-13.85 \pm 0.10$ & \pm 0.077 & 11.84 & 21.425 & 11.19 & $11.5 /$ & 11.449 \\
\hline & 0.01 & & & & & & 11.45 & & \\
\hline & 0.005 & & & & & & 11.49 & & \\
\hline & 0.002 & & & & & & 11.60 & & \\
\hline & 0.001 & & & & & & 11.66 & & \\
\hline & 1 & & & & & & 8.97 & & \\
\hline & 0.5 & & & & & & 9.39 & & \\
\hline & 0.2 & & & & & & 9.99 & & \\
\hline & 0.1 & & & & & & 10.43 & & \\
\hline 우 & 0.05 & 3245 & $1385+070$ & 2.654 & 1235 & & 10.92 & 1212 & 40623 \\
\hline N & 0.02 & 32.45 & $-13.85 \pm 0.10$ & \pm 0.077 & 12.35 & 68.893 & 11.47 & 12.12. & 40.623 \\
\hline & 0.01 & & & & & & 11.74 & & \\
\hline & 0.005 & & & & & & 11.66 & & \\
\hline & 0.002 & & & & & & 12.14 & & \\
\hline & 0.001 & & & & & & 12.11 & & \\
\hline & 1 & & & & & & 9.07 & & \\
\hline & 0.5 & & & & & & 9.47 & & \\
\hline & 0.2 & & & & & & 10.11 & & \\
\hline O & 0.1 & & & 2.654 & & & 10.60 & & \\
\hline $\mathfrak{N}$ & 0.05 & 32.45 & $-13.85 \pm 0.10$ & \pm 0.077 & 12.88 & 232.198 & 11.04 & 13.21 & $5 / 3.810$ \\
\hline & 0.02 & & & & & & 11.24 & & \\
\hline & 0.01 & & & & & & 12.02 & & \\
\hline & 0.005 & & & & & & 12.44 & & \\
\hline
\end{tabular}


Appendix 3. Temperature T, frequency $f, \mathrm{G}_{\infty}$, viscosity, shear viscosity, relaxed Newtonian viscosity and relaxation times of the samples. determined by micropenetration and torsion measurements.

Continuation...

\begin{tabular}{|c|c|c|c|c|c|c|c|c|c|}
\hline & & & \multicolumn{4}{|c|}{ MICROPENETRATION } & \multicolumn{3}{|c|}{ TORSION } \\
\hline $\mathbf{T}$ & f & $\mathbf{G}_{\infty}$ & A & B & $\begin{array}{c}\log _{10} \\
\eta\end{array}$ & $\tau$ & $\begin{array}{l}\text { Shear } \\
\text { visc. } \\
\log _{10} \eta\end{array}$ & $\begin{array}{c}\text { Relaxed } \\
\text { Newt. visc. } \\
\log _{10} \eta\end{array}$ & $\tau$ \\
\hline${ }^{\circ} \mathrm{C}$ & $\mathrm{Hz}$ & GPa & $\log _{10} \mathrm{~Pa} \mathrm{~S}$ & $\mathbf{K}$ & Pa s & $\mathbf{s}$ & Pa s & $\mathrm{Pas}$ & $\mathbf{s}$ \\
\hline \multicolumn{10}{|c|}{ SAMPLE G8 } \\
\hline \multirow{2}{*}{720} & 0.002 & \multirow{2}{*}{32.45} & \multirow{2}{*}{$-13.85 \pm 0.70$} & \multirow{2}{*}{$\begin{array}{c}2.654 \\
\pm 0.077 \\
\end{array}$} & \multirow{2}{*}{12.88} & \multirow{2}{*}{232.198} & 12.98 & \multirow{2}{*}{13.27} & \multirow{2}{*}{573.810} \\
\hline & 0.001 & & & & & & 13.28 & & \\
\hline \multirow{10}{*}{ 요 } & 1 & \multirow{10}{*}{32.45} & \multirow{10}{*}{$-13.85 \pm 0.70$} & \multirow{10}{*}{$\begin{array}{c}2.654 \\
\pm 0.077\end{array}$} & \multirow{10}{*}{13.43} & \multirow{10}{*}{822.684} & 9.22 & \multirow{10}{*}{13.50} & \multirow{10}{*}{974.470} \\
\hline & 0.5 & & & & & & 9.48 & & \\
\hline & 0.2 & & & & & & 9.96 & & \\
\hline & 0.1 & & & & & & 10.61 & & \\
\hline & 0.05 & & & & & & 10.99 & & \\
\hline & 0.02 & & & & & & 11.03 & & \\
\hline & 0.01 & & & & & & 12.07 & & \\
\hline & 0.005 & & & & & & 12.46 & & \\
\hline & 0.002 & & & & & & 13.07 & & \\
\hline & 0.001 & & & & & & 13.49 & & \\
\hline \multirow{10}{*}{$\begin{array}{l}8 \\
\& \\
0\end{array}$} & 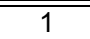 & \multirow{10}{*}{32.45} & \multirow{10}{*}{$-13.85 \pm 0.70$} & \multirow{10}{*}{$\begin{array}{c}2.654 \\
\pm 0.077\end{array}$} & \multirow{10}{*}{14.00} & \multirow{10}{*}{3073.737} & 9.62 & \multirow{10}{*}{13.78} & \multirow{10}{*}{1856.814} \\
\hline & 0.5 & & & & & & 9.85 & & \\
\hline & 0.2 & & & & & & 10.78 & & \\
\hline & 0.1 & & & & & & 10.93 & & \\
\hline & 0.05 & & & & & & 10.64 & & \\
\hline & 0.02 & & & & & & 12.02 & & \\
\hline & 0.01 & & & & & & 12.81 & & \\
\hline & 0.005 & & & & & & 12.77 & & \\
\hline & 0.002 & & & & & & 13.31 & & \\
\hline & 0.001 & & & & & & 13.78 & & \\
\hline 660 & & 32.45 & $-13.85 \pm 0.70$ & $\begin{array}{c}2.654 \\
\pm 0.077\end{array}$ & 14.60 & 12151.838 & - & - & - \\
\hline 640 & & 32.45 & $-13.85 \pm 0.70$ & $\begin{array}{c}2.654 \\
\pm 0.077\end{array}$ & 15.22 & 51023.674 & - & - & - \\
\hline 620 & & 32.45 & $-13.85 \pm 0.70$ & $\begin{array}{c}2.654 \\
\pm 0.077\end{array}$ & 15.87 & 228461.612 & - & - & - \\
\hline 600 & & 32.45 & $-13.85 \pm 0.70$ & $\begin{array}{c}2.654 \\
\pm 0.077\end{array}$ & 16.55 & 1095682.75 & - & - & - \\
\hline
\end{tabular}

\begin{tabular}{|c|c|c|c|c|c|c|c|c|c|}
\hline \multicolumn{10}{|c|}{ SAMPLE G9 } \\
\hline \multirow{10}{*}{ స్ర } & 1 & \multirow{10}{*}{32.94} & \multirow{10}{*}{$-16.40 \pm 1.19$} & \multirow{10}{*}{$\begin{array}{c}3.054 \\
\pm 0.132\end{array}$} & \multirow{10}{*}{11.53} & \multirow{10}{*}{10.396} & 10.02 & \multirow{10}{*}{11.38} & \multirow{10}{*}{7.282} \\
\hline & 0.5 & & & & & & 10.46 & & \\
\hline & 0.2 & & & & & & 10.94 & & \\
\hline & 0.1 & & & & & & 11.21 & & \\
\hline & 0.05 & & & & & & 11.35 & & \\
\hline & 0.02 & & & & & & 11.41 & & \\
\hline & 0.01 & & & & & & 11.45 & & \\
\hline & 0.005 & & & & & & 11.45 & & \\
\hline & 0.002 & & & & & & 11.10 & & \\
\hline & 0.001 & & & & & & 11.54 & & \\
\hline \multirow{10}{*}{$\frac{0}{\infty}$} & 1 & \multirow{10}{*}{32.94} & \multirow{10}{*}{$-16.40 \pm 1.19$} & \multirow{10}{*}{$\begin{array}{c}3.054 \\
\pm 0.132\end{array}$} & \multirow{10}{*}{11.79} & \multirow{10}{*}{18.831} & 10.13 & \multirow{10}{*}{11.86} & \multirow{10}{*}{21.992} \\
\hline & 0.5 & & & & & & 10.57 & & \\
\hline & 0.2 & & & & & & 11.10 & & \\
\hline & 0.1 & & & & & & 11.44 & & \\
\hline & 0.05 & & & & & & 11.67 & & \\
\hline & 0.02 & & & & & & 11.80 & & \\
\hline & 0.01 & & & & & & 11.32 & & \\
\hline & 0.005 & & & & & & 11.86 & & \\
\hline & 0.002 & & & & & & 11.87 & & \\
\hline & 0.001 & & & & & & 11.86 & & \\
\hline \multirow{10}{*}{ ৪ి } & 1 & \multirow{10}{*}{32.94} & \multirow{10}{*}{$-16.40 \pm 1.19$} & \multirow{10}{*}{$\begin{array}{c}3.054 \\
\pm 0.132\end{array}$} & \multirow{10}{*}{12.06} & \multirow{10}{*}{34.489} & 10.01 & \multirow{10}{*}{12.11} & \multirow{10}{*}{39.107} \\
\hline & 0.5 & & & & & & 9.55 & & \\
\hline & 0.2 & & & & & & 10.75 & & \\
\hline & 0.1 & & & & & & 11.41 & & \\
\hline & 0.05 & & & & & & 11.74 & & \\
\hline & 0.02 & & & & & & 11.98 & & \\
\hline & 0.01 & & & & & & 12.07 & & \\
\hline & 0.005 & & & & & & 12.09 & & \\
\hline & 0.002 & & & & & & 12.12 & & \\
\hline & 0.001 & & & & & & 12.12 & & \\
\hline
\end{tabular}


Appendix 3. Temperature T, frequency $f, \mathrm{G}_{\infty}$, viscosity, shear viscosity, relaxed Newtonian viscosity and relaxation times of the samples. determined by micropenetration and torsion measurements. Continuation...

\begin{tabular}{|c|c|c|c|c|c|c|c|c|c|}
\hline & & & \multicolumn{4}{|c|}{ MICROPENETRATION } & \multicolumn{3}{|c|}{ TORSION } \\
\hline $\mathbf{T}$ & f & $\mathbf{G}_{\infty}$ & A & B & $\begin{array}{c}\log _{10} \\
\eta\end{array}$ & $\tau$ & $\begin{array}{c}\text { Shear } \\
\text { visc. } \\
\log _{10} \eta\end{array}$ & $\begin{array}{c}\text { Relaxed } \\
\text { Newt. visc. } \\
\log _{10} \eta\end{array}$ & $\tau$ \\
\hline${ }^{\circ} \mathrm{C}$ & $\mathrm{Hz}$ & GPa & $\log _{10} \mathrm{~Pa} \mathrm{~S}$ & $\mathbf{K}$ & Pas & $\mathbf{s}$ & Pa s & Pa s & $\mathbf{s}$ \\
\hline \multicolumn{10}{|c|}{ SAMPLE G9 } \\
\hline \multirow{10}{*}{$\stackrel{\infty}{\infty}$} & 1 & \multirow{10}{*}{32.94} & \multirow{10}{*}{$-16.40 \pm 1.19$} & \multirow{10}{*}{$\begin{array}{c}3.054 \\
\pm 0.132\end{array}$} & \multirow{10}{*}{12.60} & \multirow{10}{*}{119.746} & 9.80 & \multirow{10}{*}{12.55} & \multirow{10}{*}{107.710} \\
\hline & 0.5 & & & & & & 10.24 & & \\
\hline & 0.2 & & & & & & 10.86 & & \\
\hline & 0.1 & & & & & & 11.26 & & \\
\hline & 0.05 & & & & & & 11.70 & & \\
\hline & 0.02 & & & & & & 12.16 & & \\
\hline & 0.01 & & & & & & 12.41 & & \\
\hline & 0.005 & & & & & & 12.53 & & \\
\hline & 0.002 & & & & & & 12.49 & & \\
\hline & 0.001 & & & & & & 12.61 & & \\
\hline & 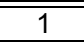 & & & & & & 9.52 & & \\
\hline & 0.5 & & & & & & 10.02 & & \\
\hline & 0.2 & & & & & & 10.57 & & \\
\hline & 0.1 & & & & & & 11.00 & & \\
\hline 8 & 0.05 & & & 3.054 & & & 11.47 & & \\
\hline$\stackrel{0}{r}$ & 0.02 & 32.94 & $-16.40 \pm 1.19$ & \pm 0.132 & 13.16 & 436.286 & 12.05 & 13.09 & $3 / 3.470$ \\
\hline & 0.01 & & & & & & 12.45 & & \\
\hline & 0.005 & & & & & & 12.47 & & \\
\hline & 0.002 & & & & & & 13.02 & & \\
\hline & 0.001 & & & & & & 13.12 & & \\
\hline & 1 & & & & & & 9.29 & & \\
\hline & 0.5 & & & & & & 9.71 & & \\
\hline & 0.2 & & & & & & 10.44 & & \\
\hline & 0.1 & & & & & & 10.69 & & \\
\hline 웅 & 0.05 & & & 3.054 & & & 11.23 & & \\
\hline 寺 & 0.02 & 32.94 & $-16.40 \pm 1.19$ & \pm 0.132 & $13 . / 4$ & $16 / 2.833$ & 11.85 & 13.54 & 1052.582 \\
\hline & 0.01 & & & & & & 12.26 & & \\
\hline & 0.005 & & & & & & 12.68 & & \\
\hline & 0.002 & & & & & & 13.18 & & \\
\hline & 0.001 & & & & & & 13.54 & & \\
\hline & 1 & & & & & & 9.85 & & \\
\hline & 0.5 & & & & & & 10.26 & & \\
\hline & 0.2 & & & & & & 10.65 & & \\
\hline & 0.1 & & & & & & 11.12 & & \\
\hline ○ิ & 0.05 & & & 3.054 & & & 11.77 & 1412 & \\
\hline $\mathfrak{N}$ & 0.02 & 32.94 & $-16.40 \pm 1.19$ & \pm 0.132 & 14.35 & $67 / 0.904$ & 12.20 & 14.12 & 4001.806 \\
\hline & 0.01 & & & & & & 12.61 & & \\
\hline & 0.005 & & & & & & 13.15 & & \\
\hline & 0.002 & & & & & & 13.72 & & \\
\hline & 0.001 & & & & & & 14.12 & & \\
\hline & 1 & & & & & & 10.10 & & \\
\hline & 0.5 & & & & & & 10.40 & & \\
\hline & 0.2 & & & & & & 11.18 & & \\
\hline & 0.1 & & & & & & 11.42 & & \\
\hline 요 1 & 0.05 & 3294 & & 3.054 & 1498 & & 11.84 & 1446 & 8754.997 \\
\hline 온 & 0.02 & 32.94 & $-16.40 \pm 1.19$ & \pm 0.132 & 14.98 & 29027.024 & 12.56 & 14.46 & $8 / 54.991$ \\
\hline & 0.01 & & & & & & 12.92 & & \\
\hline & 0.005 & & & & & & 13.36 & & \\
\hline & 0.002 & & & & & & 14.01 & & \\
\hline & 0.001 & & & & & & 14.46 & & \\
\hline & 1 & & & & & & 10.92 & & \\
\hline & 0.5 & & & & & & 10.46 & & \\
\hline & 0.2 & & & & & & 11.82 & & \\
\hline & 0.1 & & & & & & 11.68 & & \\
\hline$\infty$ & 0.05 & 3294 & & 3.054 & 1564 & & 12.21 & 1487 & \\
\hline ఝొ & 0.02 & 32.94 & $-16.40 \pm 1.19$ & \pm 0.132 & 15.64 & 132219.22 & 12.72 & $14.8 /$ & 22503.808 \\
\hline & 0.01 & & & & & & 13.39 & & \\
\hline & 0.005 & & & & & & 13.59 & & \\
\hline & 0.002 & & & & & & 14.35 & & \\
\hline & 0.001 & & & & & & 14.87 & & \\
\hline 660 & & 32.94 & $-16.40 \pm 1.19$ & $\begin{array}{c}3.054 \\
\pm 0.132 \\
\end{array}$ & 16.33 & 643310.088 & - & - & - \\
\hline 640 & & 32.94 & $-16.40 \pm 1.19$ & $\begin{array}{c}3.054 \\
\pm 0.132 \\
\end{array}$ & 17.04 & 3353084.93 & - & - & - \\
\hline
\end{tabular}


Appendix 3. Temperature T, frequency $f, \mathrm{G}_{\infty}$, viscosity, shear viscosity, relaxed Newtonian viscosity and relaxation times of the samples. determined by micropenetration and torsion measurements. Continuation...

\begin{tabular}{|c|c|c|c|c|c|c|c|c|c|}
\hline & & & \multicolumn{4}{|c|}{ MICROPENETRATION } & \multicolumn{3}{|c|}{ TORSION } \\
\hline $\mathbf{T}$ & f & $\mathbf{G}_{\infty}$ & A & B & $\begin{array}{c}\log _{10} \\
\eta\end{array}$ & $\tau$ & $\begin{array}{c}\text { Shear } \\
\text { visc. } \\
\log _{10} \eta\end{array}$ & $\begin{array}{c}\text { Relaxed } \\
\text { Newt. visc. } \\
\log _{10} \eta\end{array}$ & $\tau$ \\
\hline${ }^{\circ} \mathrm{C}$ & $\mathrm{Hz}$ & GPa & $\log _{10} \mathrm{~Pa} \mathrm{~S}$ & $\mathbf{K}$ & Pa s & $\mathbf{s}$ & Pas & Pa s & $\mathbf{s}$ \\
\hline \multicolumn{10}{|c|}{ SAMPLE G9 } \\
\hline 620 & & 32.94 & $-16.40 \pm 1.19$ & $\begin{array}{c}3.054 \\
\pm 0.132 \\
\end{array}$ & 17.79 & 18818555.7 & - & - & - \\
\hline 600 & & 32.94 & $-16.40 \pm 1.19$ & $\begin{array}{c}3.054 \\
\pm 0.132 \\
\end{array}$ & 18.58 & 114301772 & - & - & - \\
\hline
\end{tabular}

\begin{tabular}{|c|c|c|c|c|c|c|c|c|c|}
\hline \multicolumn{10}{|c|}{ SAMPLE G10 } \\
\hline \multirow{9}{*}{$\stackrel{\circ}{\infty}$} & 1 & \multirow{9}{*}{32.20} & \multirow{9}{*}{$-14.20 \pm 0.47$} & \multirow{9}{*}{$\begin{array}{c}2.629 \\
\pm 0.050\end{array}$} & \multirow{9}{*}{10.76} & \multirow{9}{*}{1.782} & 9.42 & \multirow{9}{*}{10.35} & \multirow{9}{*}{0.695} \\
\hline & 0.5 & & & & & & 9.81 & & \\
\hline & 0.2 & & & & & & 10.26 & & \\
\hline & 0.1 & & & & & & 10.37 & & \\
\hline & 0.05 & & & & & & 10.30 & & \\
\hline & 0.02 & & & & & & 10.37 & & \\
\hline & 0.01 & & & & & & 10.38 & & \\
\hline & 0.005 & & & & & & 10.37 & & \\
\hline & 0.002 & & & & & & 10.38 & & \\
\hline \multirow{10}{*}{$\stackrel{\circ}{\circ}$} & 1 & \multirow{10}{*}{32.20} & \multirow{10}{*}{$-14.20 \pm 0.47$} & \multirow{10}{*}{$\begin{array}{c}2.629 \\
\pm 0.050\end{array}$} & \multirow{10}{*}{11.24} & & 9.41 & & \\
\hline & 0.5 & & & & & & 9.84 & & \\
\hline & 0.2 & & & & & & 10.35 & & \\
\hline & 0.1 & & & & & & 10.74 & & \\
\hline & 0.05 & & & & & & 11.00 & & \\
\hline & 0.02 & & & & & 5.424 & 11.22 & 11.19 & 4.810 \\
\hline & 0.01 & & & & & & 11.12 & & \\
\hline & 0.005 & & & & & & 11.31 & & \\
\hline & 0.002 & & & & & & 11.06 & & \\
\hline & 0.001 & & & & & & 11.18 & & \\
\hline & 1 & & & & & & 9.14 & & \\
\hline & 0.5 & & & & & & 9.58 & & \\
\hline & 0.2 & & & & & & 10.16 & & \\
\hline & 0.1 & & & & & & 10.62 & & \\
\hline 오 & 0.05 & 3220 & $1420+017$ & 2.629 & 1174 & & 11.01 & & \\
\hline$\frac{1}{4}$ & 0.02 & 32.20 & $-14 . \angle U \pm 0.4 I$ & \pm 0.050 & $11 . / 4$ & 17.249 & 11.38 & $11 . / 3$ & $16.6 / 8$ \\
\hline & 0.01 & & & & & & 11.65 & & \\
\hline & 0.005 & & & & & & 11.73 & & \\
\hline & 0.002 & & & & & & 11.73 & & \\
\hline & 0.001 & & & & & & 11.74 & & \\
\hline & $\overline{1} 1$ & & & & & & 9.41 & & \\
\hline & 0.5 & & & & & & 9.86 & & \\
\hline & 0.2 & & & & & & 10.44 & & \\
\hline & 0.1 & & & & & & 10.88 & & \\
\hline 요 & 0.05 & 3220 & $1420+0.4$ & 2.629 & & 57465 & 11.34 & & \\
\hline $\mathbb{N}$ & 0.02 & 32.20 & $-14 . \angle U \pm 0.4 I$ & \pm 0.050 & 12.21 & 51.460 & 11.89 & 12.30 & 61.966 \\
\hline & 0.01 & & & & & & 12.27 & & \\
\hline & 0.005 & & & & & & 12.35 & & \\
\hline & 0.002 & & & & & & 12.26 & & \\
\hline & 0.001 & & & & & & 12.31 & & \\
\hline & $\overline{11}$ & & & & & & 10.40 & & \\
\hline & 0.5 & & & & & & 10.97 & & \\
\hline & 0.2 & & & & & & 11.28 & & \\
\hline & 0.1 & & & & & & 11.95 & & \\
\hline 용 & 0.05 & 3220 & & 2.629 & & & 12.05 & & \\
\hline 인 & 0.02 & 32.20 & $-14.20 \pm 0.47$ & \pm 0.050 & 12.81 & 201.158 & 12.56 & 12.13 & 166.184 \\
\hline & 0.01 & & & & & & 12.71 & & \\
\hline & 0.005 & & & & & & 12.75 & & \\
\hline & 0.002 & & & & & & 12.73 & & \\
\hline & 0.001 & & & & & & 12.64 & & \\
\hline & 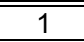 & & & & & & 9.75 & & \\
\hline & 0.5 & & & & & & 10.23 & & \\
\hline & 0.2 & & & & & & 10.69 & & \\
\hline & 0.1 & & & & & & 11.34 & & \\
\hline ᄋ & 0.05 & 3220 & $1420+0.4$ & 2.629 & 1338 & 742172 & 11.69 & 1369 & 1521086 \\
\hline 0 & 0.02 & 32.20 & $--14 . \angle U \pm U .4 I$ & \pm 0.050 & 13.38 & 142.112 & 12.37 & 13.69 & 1521.080 \\
\hline & 0.01 & & & & & & 12.79 & & \\
\hline & 0.005 & & & & & & 13.23 & & \\
\hline & 0.002 & & & & & & 13.63 & & \\
\hline & 0.001 & & & & & & 13.81 & & \\
\hline
\end{tabular}


Appendix 3. Temperature T, frequency $f, \mathrm{G}_{\infty}$, viscosity, shear viscosity, relaxed Newtonian viscosity and relaxation times of the samples. determined by micropenetration and torsion measurements. Continuation...

\begin{tabular}{|c|c|c|c|c|c|c|c|c|c|}
\hline & & & \multicolumn{4}{|c|}{ MICROPENETRATION } & \multicolumn{3}{|c|}{ TORSION } \\
\hline $\mathbf{T}$ & f & $\mathbf{G}_{\infty}$ & A & B & $\begin{array}{c}\log _{10} \\
\eta\end{array}$ & $\tau$ & $\begin{array}{l}\text { Shear } \\
\text { visc. } \\
\log _{10} \eta\end{array}$ & $\begin{array}{c}\text { Relaxed } \\
\text { Newt. visc. } \\
\log _{10} \eta\end{array}$ & $\tau$ \\
\hline${ }^{\circ} \mathrm{C}$ & $\mathrm{Hz}$ & GPa & $\log _{10} \mathrm{~Pa} \mathrm{~s}$ & $\mathbf{K}$ & Pa S & $\mathbf{s}$ & Pa S & Pas & $\mathbf{s}$ \\
\hline \multicolumn{10}{|c|}{ SAMPLE G10 } \\
\hline \multirow{10}{*}{$\stackrel{8}{8}$} & $\overline{11}$ & \multirow{10}{*}{32.20} & \multirow{10}{*}{$-14.20 \pm 0.47$} & \multirow{10}{*}{$\begin{array}{c}2.629 \\
\pm 0.050\end{array}$} & \multirow{10}{*}{13.97} & \multirow{10}{*}{2895.878} & 9.96 & \multirow{10}{*}{14.15} & \multirow{10}{*}{4386.860} \\
\hline & 0.5 & & & & & & 10.77 & & \\
\hline & 0.2 & & & & & & 11.21 & & \\
\hline & 0.1 & & & & & & 11.81 & & \\
\hline & 0.05 & & & & & & 12.51 & & \\
\hline & 0.02 & & & & & & 12.71 & & \\
\hline & 0.01 & & & & & & 13.46 & & \\
\hline & 0.005 & & & & & & 13.89 & & \\
\hline & 0.002 & & & & & & 14.14 & & \\
\hline & 0.001 & & & & & & 14.20 & & \\
\hline \multirow{10}{*}{ 옹 } & 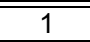 & \multirow{10}{*}{32.20} & \multirow{10}{*}{$-14.20 \pm 0.47$} & \multirow{10}{*}{$\begin{array}{c}2.629 \\
\pm 0.050\end{array}$} & \multirow{10}{*}{14.59} & \multirow{10}{*}{11993.902} & 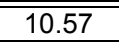 & \multirow{10}{*}{14.92} & \multirow{10}{*}{25831.746} \\
\hline & 0.5 & & & & & & 11.44 & & \\
\hline & 0.2 & & & & & & 11.58 & & \\
\hline & 0.1 & & & & & & 11.50 & & \\
\hline & 0.05 & & & & & & 12.57 & & \\
\hline & 0.02 & & & & & & 13.14 & & \\
\hline & 0.01 & & & & & & 13.90 & & \\
\hline & 0.005 & & & & & & 14.30 & & \\
\hline & 0.002 & & & & & & 14.90 & & \\
\hline & 0.001 & & & & & & 14.92 & & \\
\hline \multirow{10}{*}{ ণัญे } & 1 & \multirow{10}{*}{32.20} & \multirow{10}{*}{$-14.20 \pm 0.47$} & \multirow{10}{*}{$\begin{array}{c}2.629 \\
\pm 0.050\end{array}$} & \multirow{10}{*}{15.23} & \multirow{10}{*}{52940.246} & 10.51 & \multirow{10}{*}{15.36} & \\
\hline & 0.5 & & & & & & 10.86 & & \\
\hline & 0.2 & & & & & & 11.54 & & \\
\hline & 0.1 & & & & & & 12.27 & & \\
\hline & 0.05 & & & & & & 12.16 & & \\
\hline & 0.02 & & & & & & 13.17 & & 71146.536 \\
\hline & 0.01 & & & & & & 13.29 & & \\
\hline & 0.005 & & & & & & 14.12 & & \\
\hline & 0.002 & & & & & & 14.58 & & \\
\hline & 0.001 & & & & & & 15.36 & & \\
\hline 600 & & 32.20 & $-14.20 \pm 0.47$ & $\begin{array}{c}2.629 \\
\pm 0.050\end{array}$ & 15.91 & 250124.756 & - & - & - \\
\hline 580 & & 32.20 & $-14.20 \pm 0.47$ & $\begin{array}{c}2.629 \\
\pm 0.050\end{array}$ & 16.61 & 1271015.61 & - & - & - \\
\hline 560 & & 32.20 & $-14.20 \pm 0.47$ & $\begin{array}{c}2.629 \\
\pm 0.050 \\
\end{array}$ & 17.35 & 6983069.65 & - & - & - \\
\hline
\end{tabular}

\begin{tabular}{|c|c|c|c|c|c|c|c|c|c|}
\hline \multicolumn{10}{|c|}{ SAMPLE G11 } \\
\hline \multirow{5}{*}{ 乐 } & 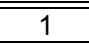 & \multirow{5}{*}{32.09} & \multirow{5}{*}{$-12.24 \pm 0.70$} & \multirow{5}{*}{$\begin{array}{c}2.300 \\
\pm 0.072\end{array}$} & \multirow{5}{*}{10.46} & \multirow{5}{*}{0.910} & 9.58 & \multirow{5}{*}{10.20} & \multirow{5}{*}{0.494} \\
\hline & 0.5 & & & & & & 9.88 & & \\
\hline & 0.2 & & & & & & 10.13 & & \\
\hline & 0.1 & & & & & & 10.23 & & \\
\hline & 0.05 & & & & & & 10.28 & & \\
\hline \multirow{8}{*}{$\stackrel{N}{N}$} & 1 & \multirow{8}{*}{32.09} & \multirow{8}{*}{$-12.24 \pm 0.70$} & \multirow{8}{*}{$\begin{array}{c}2.300 \\
\pm 0.072\end{array}$} & \multirow{8}{*}{10.92} & \multirow{8}{*}{2.608} & 9.41 & \multirow{8}{*}{10.73} & \multirow{8}{*}{1.673} \\
\hline & 0.5 & & & & & & 9.80 & & \\
\hline & 0.2 & & & & & & 10.22 & & \\
\hline & 0.1 & & & & & & 10.47 & & \\
\hline & 0.05 & & & & & & 10.60 & & \\
\hline & 0.02 & & & & & & 10.70 & & \\
\hline & 0.01 & & & & & & 10.73 & & \\
\hline & 0.005 & & & & & & 10.77 & & \\
\hline \multirow{9}{*}{ ஓ } & $\overline{11}$ & \multirow{9}{*}{32.09} & \multirow{9}{*}{$-12.24 \pm 0.70$} & \multirow{9}{*}{$\begin{array}{c}2.300 \\
\pm 0.072\end{array}$} & \multirow{9}{*}{11.40} & \multirow{9}{*}{7.806} & 9.22 & \multirow{9}{*}{11.32} & \multirow{9}{*}{6.510} \\
\hline & 0.5 & & & & & & $\begin{array}{c}9.64 \\
10.10\end{array}$ & & \\
\hline & $\begin{array}{l}0.2 \\
0.1\end{array}$ & & & & & & $\begin{array}{l}10.19 \\
10.54\end{array}$ & & \\
\hline & 0.05 & & & & & & 10.84 & & \\
\hline & 0.02 & & & & & & 11.11 & & \\
\hline & 0.01 & & & & & & 11.23 & & \\
\hline & 0.005 & & & & & & 11.27 & & \\
\hline & 0.002 & & & & & & 11.32 & & \\
\hline & 0.001 & & & & & & 11.33 & & \\
\hline \multirow{3}{*}{ 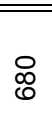 } & $\overline{11}$ & \multirow{3}{*}{32.09} & \multirow{3}{*}{$-12.24 \pm 0.70$} & \multirow{3}{*}{$\begin{array}{c}2.300 \\
\pm 0.072\end{array}$} & \multirow{3}{*}{11.89} & \multirow{3}{*}{24.462} & 10.27 & & \\
\hline & 0.5 & & & & & & 10.72 & 12.05 & 34.960 \\
\hline & $\begin{array}{l}0.2 \\
0.1\end{array}$ & & & & & & $\begin{array}{l}11.30 \\
11.39\end{array}$ & & \\
\hline
\end{tabular}


Appendix 3. Temperature T, frequency $f, \mathrm{G}_{\infty}$, viscosity, shear viscosity, relaxed Newtonian viscosity and relaxation times of the samples. determined by micropenetration and torsion measurements. Continuation...

\begin{tabular}{|c|c|c|c|c|c|c|c|c|c|}
\hline & & & \multicolumn{4}{|c|}{ MICROPENETRATION } & \multicolumn{3}{|c|}{ TORSION } \\
\hline $\mathbf{T}$ & $\mathbf{f}$ & $\mathbf{G}_{\infty}$ & A & B & $\begin{array}{c}\log _{10} \\
\eta\end{array}$ & $\tau$ & $\begin{array}{c}\text { Shear } \\
\text { visc. } \\
\log _{10} \eta\end{array}$ & $\begin{array}{c}\text { Relaxed } \\
\text { Newt. visc. } \\
\log _{10} \eta\end{array}$ & $\tau$ \\
\hline${ }^{\circ} \mathrm{C}$ & $\mathrm{Hz}$ & GPa & $\log _{10} \mathrm{~Pa} \mathrm{~s}$ & $\mathbf{K}$ & Pas & $\mathbf{s}$ & Pa s & Pa s & $\mathbf{s}$ \\
\hline \multicolumn{10}{|c|}{ SAMPLE G11 } \\
\hline \multirow{6}{*}{$\begin{array}{l}\circ \\
\stackrel{0}{0}\end{array}$} & 0.05 & \multirow{6}{*}{32.09} & \multirow{6}{*}{$-12.24 \pm 0.70$} & \multirow{6}{*}{$\begin{array}{c}2.300 \\
\pm 0.072\end{array}$} & \multirow{6}{*}{11.89} & \multirow{6}{*}{24.462} & 11.79 & \multirow{6}{*}{12.05} & \multirow{6}{*}{34.960} \\
\hline & 0.02 & & & & & & 11.74 & & \\
\hline & 0.01 & & & & & & 11.81 & & \\
\hline & 0.005 & & & & & & 11.90 & & \\
\hline & 0.002 & & & & & & 12.09 & & \\
\hline & 0.001 & & & & & & 12.10 & & \\
\hline \multirow{10}{*}{$\stackrel{8}{0}$} & $\overline{11}$ & \multirow{10}{*}{32.09} & & & & & 10.07 & & \\
\hline & 0.5 & & & & & & 10.45 & & \\
\hline & 0.2 & & & & & & 11.05 & & \\
\hline & 0.1 & & & & & & 11.51 & & \\
\hline & 0.05 & & & 2.300 & & & 11.93 & & \\
\hline & 0.02 & & $-12.24 \pm 0.70$ & \pm 0.072 & 12.41 & 80.513 & 12.32 & 12.66 & 142.421 \\
\hline & 0.01 & & & & & & 12.70 & & \\
\hline & 0.005 & & & & & & 12.38 & & \\
\hline & 0.002 & & & & & & 12.66 & & \\
\hline & 0.001 & & & & & & 12.79 & & \\
\hline & 1 & & & & & & 9.82 & & \\
\hline & 0.5 & & & & & & 10.11 & & \\
\hline & 0.2 & & & & & & 10.66 & & \\
\hline & 0.1 & & & & & & 11.19 & & \\
\hline 오 & 0.05 & 3200 & $1221+070$ & 2.300 & 1205 & 270187 & 11.54 & 1206 & 284167 \\
\hline కె & 0.02 & 32.09 & $-12.24 \pm 0.10$ & \pm 0.072 & 12.90 & 279.101 & 12.17 & 12.90 & 204.101 \\
\hline & 0.01 & & & & & & 12.61 & & \\
\hline & 0.005 & & & & & & 12.98 & & \\
\hline & 0.002 & & & & & & 12.96 & & \\
\hline & 0.001 & & & & & & 12.94 & & \\
\hline & 1 & & & & & & 9.84 & & \\
\hline & 0.5 & & & & & & 10.28 & & \\
\hline & 0.2 & & & & & & 11.30 & & \\
\hline & 0.1 & & & & & & 11.48 & & \\
\hline ○ & 0.05 & 2200 & $1221+070$ & 2.300 & 1252 & 1023567 & 11.83 & 1250 & 085311 \\
\hline 0 & 0.02 & 32.09 & $-12.24 \pm 0.10$ & \pm 0.072 & 10.02 & $10<3.001$ & 12.57 & 10.00 & 900.311 \\
\hline & 0.01 & & & & & & 12.94 & & \\
\hline & 0.005 & & & & & & 13.37 & & \\
\hline & 0.002 & & & & & & 13.43 & & \\
\hline & 0.001 & & & & & & 13.63 & & \\
\hline & 1 & & & & & & 10.15 & & \\
\hline & 0.5 & & & & & & 9.82 & & \\
\hline & 0.2 & & & & & & 11.33 & & \\
\hline & 0.1 & & & & & & 11.73 & & \\
\hline ○ & 0.05 & 2200 & & 2.300 & & & 12.11 & & \\
\hline 8 & 0.02 & 32.09 & $-12.24 \pm 0.70$ & \pm 0.072 & 14.11 & 3982.802 & 12.57 & 14.48 & 9409.647 \\
\hline & 0.01 & & & & & & 12.82 & & \\
\hline & 0.005 & & & & & & 13.25 & & \\
\hline & 0.002 & & & & & & 13.96 & & \\
\hline & 0.001 & & & & & & 14.48 & & \\
\hline & 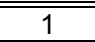 & & & & & & 10.03 & & \\
\hline & 0.5 & & & & & & 10.46 & & \\
\hline & 0.2 & & & & & & 10.92 & & \\
\hline & 0.1 & & & & & & 11.52 & & \\
\hline & 0.05 & & & 2.300 & & & 11.98 & & \\
\hline i & 0.02 & 32.09 & $-12.24 \pm 0.70$ & \pm 0.072 & 14.72 & 16517.018 & 12.29 & 15.04 & 34164.364 \\
\hline & 0.01 & & & & & & 12.84 & & \\
\hline & 0.005 & & & & & & 13.53 & & \\
\hline & 0.002 & & & & & & 14.19 & & \\
\hline & 0.001 & & & & & & 15.04 & & \\
\hline 560 & & 32.09 & $-12.24 \pm 0.70$ & $\begin{array}{c}2.300 \\
\pm 0.072\end{array}$ & 15.37 & 73339.525 & - & - & - \\
\hline 540 & & 32.09 & $-12.24 \pm 0.70$ & $\begin{array}{c}2.300 \\
\pm 0.072\end{array}$ & 16.05 & 350426.752 & - & - & - \\
\hline
\end{tabular}


Appendix 3. Temperature T, frequency $f, \mathrm{G}_{\infty}$, viscosity, shear viscosity, relaxed Newtonian viscosity and relaxation times of the samples. determined by micropenetration and torsion measurements. Continuation...

\begin{tabular}{|c|c|c|c|c|c|c|c|c|c|}
\hline & & & \multicolumn{4}{|c|}{ MICROPENETRATION } & \multicolumn{3}{|c|}{ TORSION } \\
\hline $\mathbf{T}$ & f & $\mathbf{G}_{\infty}$ & A & B & $\begin{array}{c}\log _{10} \\
\eta\end{array}$ & $\tau$ & $\begin{array}{l}\text { Shear } \\
\text { visc. } \\
\log _{10} \eta\end{array}$ & $\begin{array}{c}\text { Relaxed } \\
\text { Newt. visc. } \\
\log _{10} \eta\end{array}$ & $\tau$ \\
\hline${ }^{\circ} \mathrm{C}$ & $\mathrm{Hz}$ & GPa & $\log _{10} \mathrm{~Pa} \mathrm{~s}$ & $\mathbf{K}$ & Pa s & $\mathbf{s}$ & Pas & $\mathrm{Pa} \mathrm{s}$ & $\mathbf{s}$ \\
\hline \multicolumn{10}{|c|}{ SAMPLE G12 } \\
\hline \multirow{8}{*}{ 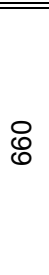 } & 1 & \multirow{8}{*}{31.42} & \multirow{8}{*}{$-15.48 \pm 0.46$} & \multirow{8}{*}{$\begin{array}{c}2.498 \\
\pm 0.045\end{array}$} & \multirow{8}{*}{11.30} & \multirow{8}{*}{6.310} & 9.73 & \multirow{8}{*}{11.28} & \\
\hline & 0.5 & & & & & & 10.13 & & \\
\hline & 0.2 & & & & & & 10.59 & & \\
\hline & 0.1 & & & & & & 10.87 & & 6061 \\
\hline & 0.05 & & & & & & 11.07 & & 0.004 \\
\hline & 0.02 & & & & & & 11.21 & & \\
\hline & 0.01 & & & & & & 11.29 & & \\
\hline & 0.005 & & & & & & 11.29 & & \\
\hline & $\overline{1}$ & & & & & & 9.87 & & \\
\hline & 0.5 & & & & & & 10.31 & & \\
\hline & 0.2 & & & & & & 10.87 & & \\
\hline & 0.1 & & & & & & 11.26 & & \\
\hline 우 & 0.05 & 3142 & $-1548+046$ & 2.498 & 1188 & 24354 & 11.59 & 1225 & 56.598 \\
\hline छे & 0.02 & 31.42 & $-15.48 \pm 0.46$ & \pm 0.045 & 11.88 & 24.354 & 11.96 & 12.25 & 56.598 \\
\hline & 0.01 & & & & & & 11.79 & & \\
\hline & 0.005 & & & & & & 12.17 & & \\
\hline & 0.002 & & & & & & 12.30 & & \\
\hline & 0.001 & & & & & & 12.32 & & \\
\hline & 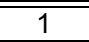 & & & & & & 10.29 & & \\
\hline & 0.5 & & & & & & 10.70 & & \\
\hline & 0.2 & & & & & & 11.33 & & \\
\hline & 0.1 & & & & & & 11.71 & & \\
\hline 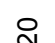 & 0.05 & 3142 & $-1548+046$ & 2.498 & 1250 & 99865 & 12.15 & 1292 & 264728 \\
\hline तु & 0.02 & 31.42 & $-15.48 \pm 0.46$ & \pm 0.045 & 12.50 & 99.865 & 12.48 & 12.92 & $264.7 \angle 8$ \\
\hline & 0.01 & & & & & & 12.81 & & \\
\hline & 0.005 & & & & & & 12.86 & & \\
\hline & 0.002 & & & & & & 12.95 & & \\
\hline & 0.001 & & & & & & 13.09 & & \\
\hline & 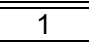 & & & & & & 10.50 & & \\
\hline & 0.5 & & & & & & 10.88 & & \\
\hline & 0.2 & & & & & & 11.48 & & \\
\hline & 0.1 & & & & & & 12.02 & & \\
\hline ㅇ & 0.05 & 3142 & $-1548+046$ & 2.498 & 1314 & 436850 & 12.41 & 1335 & 712524 \\
\hline 8 & 0.02 & 31.42 & $-15.48 \pm U .46$ & \pm 0.045 & 13.14 & 436.850 & 12.94 & 13.35 & 112.524 \\
\hline & 0.01 & & & & & & 13.37 & & \\
\hline & 0.005 & & & & & & 13.41 & & \\
\hline & 0.002 & & & & & & 13.08 & & \\
\hline & 0.001 & & & & & & 13.45 & & \\
\hline & $\overline{1} 1$ & & & & & & 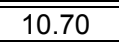 & & \\
\hline & 0.5 & & & & & & 11.07 & & \\
\hline & 0.2 & & & & & & 11.54 & & \\
\hline & 0.1 & & & & & & 12.02 & & \\
\hline$\infty$ & 0.05 & 3142 & $-1548+046$ & 2.498 & 1381 & 2047879 & 12.34 & 1383 & 2151788 \\
\hline in & 0.02 & 31.42 & $-15.48 \pm 0.46$ & \pm 0.045 & & 2041.819 & 13.02 & & 2151.188 \\
\hline & 0.01 & & & & & & 13.41 & & \\
\hline & 0.005 & & & & & & 13.86 & & \\
\hline & 0.002 & & & & & & 13.82 & & \\
\hline & 0.001 & & & & & & 13.81 & & \\
\hline & 1 & & & & & & 10.87 & & \\
\hline & 0.5 & & & & & & 11.25 & & \\
\hline & 0.2 & & & & & & 11.71 & & \\
\hline & 0.1 & & & & & & 11.71 & & \\
\hline 8 & 0.05 & 3142 & $-1548+046$ & 2.498 & 1451 & 10339412 & 12.52 & 1427 & 5926515 \\
\hline in & 0.02 & 31.42 & $-15.48 \pm 0.46$ & \pm 0.045 & 14.51 & 10339.412 & 13.07 & 14.27 & 5926.515 \\
\hline & 0.01 & & & & & & 13.54 & & \\
\hline & 0.005 & & & & & & 14.00 & & \\
\hline & 0.002 & & & & & & 14.25 & & \\
\hline & 0.001 & & & & & & 14.29 & & \\
\hline & 1 & & & & & & $\overline{10.51}$ & & \\
\hline & 0.5 & & & & & & 10.24 & & \\
\hline & 0.2 & & & & & & 10.93 & & \\
\hline 운 & 0.1 & 31.42 & $-15.48 \pm 0.46$ & $\begin{array}{r}2.498 \\
+0.045\end{array}$ & 15.25 & 56530.742 & 11.78 & 14.74 & 17490.386 \\
\hline & 0.05 & & & & & & 12.47 & & \\
\hline & 0.02 & & & & & & 12.90 & & \\
\hline & 0.01 & & & & & & 13.47 & & \\
\hline
\end{tabular}


Appendix 3. Temperature T, frequency $f, \mathrm{G}_{\infty}$, viscosity, shear viscosity, relaxed Newtonian viscosity and relaxation times of the samples. determined by micropenetration and torsion measurements. Continuation...

\begin{tabular}{|c|c|c|c|c|c|c|c|c|c|}
\hline & & & \multicolumn{4}{|c|}{ MICROPENETRATION } & \multicolumn{3}{|c|}{ TORSION } \\
\hline $\mathbf{T}$ & f & $\mathbf{G}_{\infty}$ & A & B & $\begin{array}{c}\log _{10} \\
\eta\end{array}$ & $\tau$ & $\begin{array}{l}\text { Shear } \\
\text { visc. } \\
\log _{10} \eta\end{array}$ & $\begin{array}{c}\text { Relaxed } \\
\text { Newt. visc. } \\
\log _{10} \eta\end{array}$ & $\tau$ \\
\hline${ }^{\circ} \mathrm{C}$ & $\mathrm{Hz}$ & GPa & $\log _{10} \mathrm{PaS}$ & $\mathbf{K}$ & Pa s & $\mathbf{s}$ & Pa s & Pa s & $\mathbf{s}$ \\
\hline \multicolumn{10}{|c|}{ SAMPLE G12 } \\
\hline \multirow{3}{*}{ 운 } & 0.005 & \multirow{3}{*}{31.42} & \multirow{3}{*}{$-15.48 \pm 0.46$} & \multirow{3}{*}{$\begin{array}{c}2.498 \\
\pm 0.045\end{array}$} & \multirow{3}{*}{15.25} & \multirow{3}{*}{56530.742} & 13.62 & \multirow{3}{*}{14.74} & \multirow{3}{*}{17490.386} \\
\hline & 0.002 & & & & & & 14.17 & & \\
\hline & 0.001 & & & & & & 14.73 & & \\
\hline 520 & & 31.42 & $-15.48 \pm 0.46$ & $\begin{array}{c}2.498 \\
\pm 0.045\end{array}$ & 16.02 & 336735.264 & - & - & - \\
\hline 500 & & 31.42 & $-15.48 \pm 0.46$ & $\begin{array}{c}2.498 \\
\pm 0.045 \\
\end{array}$ & 16.84 & 2199865.92 & - & - & - \\
\hline 480 & & 31.42 & $-15.48 \pm 0.46$ & $\begin{array}{c}2.498 \\
\pm 0.045\end{array}$ & 17.70 & 15878264.5 & - & - & - \\
\hline 460 & & 31.42 & $-15.48 \pm 0.46$ & $\begin{array}{c}2.498 \\
\pm 0.045\end{array}$ & 18.60 & 127659557 & - & - & - \\
\hline 440 & & 31.42 & $-15.48 \pm 0.46$ & $\begin{array}{c}2.498 \\
\pm 0.045\end{array}$ & 19.56 & 1153689848 & - & - & - \\
\hline 420 & & 31.42 & $-15.48 \pm 0.46$ & $\begin{array}{c}2.498 \\
\pm 0.045\end{array}$ & 20.57 & 1183878995 & - & - & - \\
\hline 400 & & 31.42 & $-15.48 \pm 0.46$ & $\begin{array}{c}2.498 \\
\pm 0.045\end{array}$ & 21.64 & $1.395 \mathrm{E}+11$ & - & - & - \\
\hline 380 & & 31.42 & $-15.48 \pm 0.46$ & $\begin{array}{c}2.498 \\
\pm 0.045\end{array}$ & 22.78 & $1.912 \mathrm{E}+12$ & - & - & - \\
\hline 360 & & 31.42 & $-15.48 \pm 0.46$ & $\begin{array}{c}2.498 \\
\pm 0.045 \\
\end{array}$ & 23.99 & $3.093 E+13$ & - & - & - \\
\hline 340 & & 31.42 & $-15.48 \pm 0.46$ & $\begin{array}{c}2.498 \\
\pm 0.045 \\
\end{array}$ & 25.28 & $6.000 \mathrm{E}+14$ & - & - & - \\
\hline
\end{tabular}

\begin{tabular}{|c|c|c|c|c|c|c|c|c|c|}
\hline \multicolumn{10}{|c|}{ SAMPLE G13 } \\
\hline \multirow{4}{*}{$\stackrel{8}{0}$} & 1 & \multirow{4}{*}{30.89} & \multirow{4}{*}{$-18.04 \pm 0.62$} & \multirow{4}{*}{$\begin{array}{c}2.642 \\
\pm 0.057\end{array}$} & \multirow{4}{*}{10.28} & \multirow{4}{*}{0.612} & 9.81 & \multirow{4}{*}{10.12} & \multirow{4}{*}{0.427} \\
\hline & 0.5 & & & & & & 9.95 & & \\
\hline & 0.2 & & & & & & 10.11 & & \\
\hline & 0.1 & & & & & & 10.15 & & \\
\hline \multirow{9}{*}{ 웅 } & $\overline{c 1}$ & \multirow{9}{*}{30.89} & \multirow{9}{*}{$-18.04 \pm 0.62$} & \multirow{9}{*}{$\begin{array}{c}2.642 \\
\pm 0.057\end{array}$} & \multirow{9}{*}{10.90} & \multirow{9}{*}{2.554} & \begin{tabular}{c|}
9.68 \\
\end{tabular} & \multirow{9}{*}{10.61} & \multirow{9}{*}{1.319} \\
\hline & 0.5 & & & & & & 10.04 & & \\
\hline & 0.2 & & & & & & 10.43 & & \\
\hline & 0.1 & & & & & & 10.60 & & \\
\hline & 0.05 & & & & & & 10.68 & & \\
\hline & 0.02 & & & & & & 10.73 & & \\
\hline & 0.01 & & & & & & 10.39 & & \\
\hline & 0.005 & & & & & & 10.68 & & \\
\hline & 0.002 & & & & & & 10.59 & & \\
\hline \multirow{10}{*}{ స్తి } & 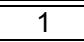 & \multirow{10}{*}{30.89} & \multirow{10}{*}{$-18.04 \pm 0.62$} & \multirow{10}{*}{$\begin{array}{c}2.642 \\
\pm 0.057\end{array}$} & \multirow{10}{*}{11.54} & \multirow{10}{*}{11.357} & 9.36 & \multirow{10}{*}{11.22} & \\
\hline & 0.5 & & & & & & 9.79 & & \\
\hline & 0.2 & & & & & & 10.27 & & \\
\hline & 0.1 & & & & & & 10.68 & & \\
\hline & 0.05 & & & & & & 10.94 & & \\
\hline & 0.02 & & & & & & 11.16 & & $5.3 / 3$ \\
\hline & 0.01 & & & & & & 11.22 & & \\
\hline & 0.005 & & & & & & 11.24 & & \\
\hline & 0.002 & & & & & & 11.21 & & \\
\hline & 0.001 & & & & & & 11.21 & & \\
\hline & 1 & & & & & & 9.32 & & \\
\hline & 0.5 & & & & & & 9.79 & & \\
\hline & 0.2 & & & & & & 10.34 & & \\
\hline & 0.1 & & & & & & 10.76 & & \\
\hline 용 & 0.05 & & & 2.642 & & & 11.16 & & \\
\hline 8 & 0.02 & 30.89 & $-18.04 \pm 0.62$ & \pm 0.057 & 12.22 & 54.080 & 11.64 & 12.15 & 45.133 \\
\hline & 0.01 & & & & & & 11.92 & & \\
\hline & 0.005 & & & & & & 11.99 & & \\
\hline & 0.002 & & & & & & 12.19 & & \\
\hline & 0.001 & & & & & & 12.15 & & \\
\hline & 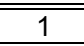 & & & & & & 8.92 & & \\
\hline & 0.5 & & & & & & 9.37 & & \\
\hline 요 & 0.2 & 3089 & $1804+062$ & & 1293 & 277070 & 9.99 & 1258 & 123007 \\
\hline$\infty$ & 0.1 & 30.89 & $-18.04 \pm 0.62$ & \pm 0.057 & 12.93 & 271.010 & 10.39 & 12.08 & 123.092 \\
\hline & 0.05 & & & & & & 10.82 & & \\
\hline & 0.02 & & & & & & 11.38 & & \\
\hline
\end{tabular}


Appendix 3. Temperature $T$, frequency $f, \mathrm{G}_{\infty}$, viscosity, shear viscosity, relaxed Newtonian viscosity and relaxation times of the samples. determined by micropenetration and torsion measurements. Continuation...

\begin{tabular}{|c|c|c|c|c|c|c|c|c|c|}
\hline & & & \multicolumn{4}{|c|}{ MICROPENETRATION } & \multicolumn{3}{|c|}{ TORSION } \\
\hline $\mathbf{T}$ & f & $\mathbf{G}_{\infty}$ & A & B & $\begin{array}{c}\log _{10} \\
\eta\end{array}$ & $\tau$ & $\begin{array}{l}\text { Shear } \\
\text { visc. } \\
\log _{10} \eta\end{array}$ & $\begin{array}{c}\text { Relaxed } \\
\text { Newt. visc. } \\
\log _{10} \eta\end{array}$ & $\tau$ \\
\hline${ }^{\circ} \mathrm{C}$ & $\mathrm{Hz}$ & GPa & $\log _{10} \mathrm{~Pa} \mathrm{~s}$ & $\mathbf{K}$ & Pa s & $\mathbf{s}$ & Pas & Pa s & $\mathbf{s}$ \\
\hline \multicolumn{10}{|c|}{ SAMPLE G13 } \\
\hline \multirow{4}{*}{ 吕 } & 0.01 & \multirow{4}{*}{30.89} & \multirow{4}{*}{$-18.04 \pm 0.62$} & \multirow{4}{*}{$\begin{array}{c}2.642 \\
\pm 0.057\end{array}$} & \multirow{4}{*}{12.93} & \multirow{4}{*}{277.070} & 11.69 & \multirow{4}{*}{12.58} & \multirow{4}{*}{123.092} \\
\hline & 0.005 & & & & & & 11.88 & & \\
\hline & 0.002 & & & & & & 12.29 & & \\
\hline & 0.001 & & & & & & 12.58 & & \\
\hline \multirow{10}{*}{$\stackrel{\circ}{\circ}$} & 1 & \multirow{10}{*}{30.89} & \multirow{10}{*}{$-18.04 \pm 0.62$} & \multirow{10}{*}{$\begin{array}{c}2.642 \\
\pm 0.057\end{array}$} & \multirow{10}{*}{13.68} & \multirow{10}{*}{1535.378} & 9.67 & \multirow{10}{*}{13.42} & \multirow{10}{*}{851.587} \\
\hline & 0.5 & & & & & & 10.12 & & \\
\hline & 0.2 & & & & & & 10.65 & & \\
\hline & 0.1 & & & & & & 11.04 & & \\
\hline & 0.05 & & & & & & 11.36 & & \\
\hline & 0.02 & & & & & & 12.11 & & \\
\hline & 0.01 & & & & & & 12.45 & & \\
\hline & 0.005 & & & & & & 12.90 & & \\
\hline & 0.002 & & & & & & 13.06 & & \\
\hline & 0.001 & & & & & & 13.40 & & \\
\hline 540 & & 30.89 & $-18.04 \pm 0.62$ & $\begin{array}{c}2.642 \\
\pm 0.057 \\
\end{array}$ & 14.46 & 9256.079 & - & - & - \\
\hline 520 & & 30.89 & $-18.04 \pm 0.62$ & $\begin{array}{c}2.642 \\
\pm 0.057 \\
\end{array}$ & 15.28 & 61093.320 & - & - & - \\
\hline 500 & & 30.89 & $-18.04 \pm 0.62$ & $\begin{array}{c}2.642 \\
\pm 0.057 \\
\end{array}$ & 16.14 & 444600.602 & - & - & - \\
\hline 480 & & 30.89 & $-18.04 \pm 0.62$ & $\begin{array}{c}2.642 \\
\pm 0.057 \\
\end{array}$ & 17.04 & 3595301.17 & - & - & - \\
\hline 460 & & 30.89 & $-18.04 \pm 0.62$ & $\begin{array}{c}2.642 \\
\pm 0.057 \\
\end{array}$ & 18.00 & 32586482.1 & - & - & - \\
\hline 440 & & 30.89 & $-18.04 \pm 0.62$ & $\begin{array}{c}2.642 \\
\pm 0.057 \\
\end{array}$ & 19.01 & 334229901 & - & - & - \\
\hline 420 & & 30.89 & $-18.04 \pm 0.62$ & $\begin{array}{c}2.642 \\
\pm 0.057 \\
\end{array}$ & 20.08 & 3921105590 & - & - & - \\
\hline 400 & & 30.89 & $-18.04 \pm 0.62$ & $\begin{array}{c}2.642 \\
\pm 0.057 \\
\end{array}$ & 21.22 & $5.325 \mathrm{E}+10$ & - & - & - \\
\hline 380 & & 30.89 & $-18.04 \pm 0.62$ & $\begin{array}{c}2.642 \\
\pm 0.057 \\
\end{array}$ & 22.42 & $8.485 E+11$ & - & - & - \\
\hline 360 & & 30.89 & $-18.04 \pm 0.62$ & $\begin{array}{c}2.642 \\
\pm 0.057\end{array}$ & 23.70 & $1.610 \mathrm{E}+13$ & - & - & - \\
\hline 340 & & 30.89 & $-18.04 \pm 0.62$ & $\begin{array}{c}2.642 \\
\pm 0.057\end{array}$ & 25.06 & $3.704 \mathrm{E}+14$ & - & - & - \\
\hline 320 & & 30.89 & $-18.04 \pm 0.62$ & $\begin{array}{c}2.642 \\
\pm 0.057 \\
\end{array}$ & 26.51 & $1.052 \mathrm{E}+16$ & - & - & - \\
\hline
\end{tabular}

\begin{tabular}{|c|c|c|c|c|c|c|c|c|c|}
\hline \multicolumn{10}{|c|}{ SAMPLE G14 } \\
\hline \multirow{4}{*}{8} & 1 & \multirow{4}{*}{29.96} & \multirow{4}{*}{$-18.10 \pm 0.54$} & \multirow{4}{*}{$\begin{array}{c}2.515 \\
\pm 0.048\end{array}$} & \multirow{4}{*}{10.72} & \multirow{4}{*}{1.734} & 99.66 & \multirow{4}{*}{10.33} & \multirow{4}{*}{0.713} \\
\hline & 0.5 & & & & & & 10.13 & & \\
\hline & 0.2 & & & & & & 10.26 & & \\
\hline & 0.1 & & & & & & 10.42 & & \\
\hline \multirow{9}{*}{ 串 } & $\overline{11}$ & \multirow{9}{*}{29.96} & \multirow{9}{*}{$-18.10 \pm 0.54$} & \multirow{9}{*}{$\begin{array}{c}2.515 \\
\pm 0.048\end{array}$} & \multirow{9}{*}{11.39} & \multirow{9}{*}{8.217} & 9.65 & \multirow{9}{*}{11.11} & \multirow{9}{*}{4.299} \\
\hline & 0.5 & & & & & & 10.07 & & \\
\hline & 0.2 & & & & & & 10.63 & & \\
\hline & 0.1 & & & & & & 10.64 & & \\
\hline & 0.05 & & & & & & 10.81 & & \\
\hline & 0.02 & & & & & & 10.97 & & \\
\hline & 0.01 & & & & & & 11.10 & & \\
\hline & 0.005 & & & & & & 11.12 & & \\
\hline & 0.002 & & & & & & 11.12 & & \\
\hline \multirow{10}{*}{ \&్రి } & $\overline{1}$ & \multirow{10}{*}{29.96} & \multirow{10}{*}{$-18.10 \pm 0.54$} & \multirow{10}{*}{$\begin{array}{c}2.515 \\
\pm 0.048\end{array}$} & \multirow{10}{*}{12.10} & \multirow{10}{*}{41.950} & 9.92 & \multirow{10}{*}{11.97} & \multirow{10}{*}{31.145} \\
\hline & 0.5 & & & & & & 10.35 & & \\
\hline & 0.2 & & & & & & 10.95 & & \\
\hline & 0.1 & & & & & & 11.38 & & \\
\hline & 0.05 & & & & & & 11.79 & & \\
\hline & 0.02 & & & & & & 12.00 & & \\
\hline & 0.01 & & & & & & 11.97 & & \\
\hline & 0.005 & & & & & & 12.07 & & \\
\hline & 0.002 & & & & & & 11.92 & & \\
\hline & 0.001 & & & & & & 11.98 & & \\
\hline
\end{tabular}


Appendix 3. Temperature $T$, frequency $f, G_{\infty}$, viscosity, shear viscosity, relaxed Newtonian viscosity and relaxation times of the samples. determined by micropenetration and torsion measurements. Continuation...

\begin{tabular}{|c|c|c|c|c|c|c|c|c|c|}
\hline & & & \multicolumn{4}{|c|}{ MICROPENETRATION } & \multicolumn{3}{|c|}{ TORSION } \\
\hline $\mathbf{T}$ & $\mathbf{f}$ & $\mathbf{G}_{\infty}$ & A & B & $\begin{array}{c}\log _{10} \\
\eta\end{array}$ & $\tau$ & $\begin{array}{c}\text { Shear } \\
\text { visc. } \\
\log _{10} \eta\end{array}$ & $\begin{array}{c}\text { Relaxed } \\
\text { Newt. visc. } \\
\log _{10} \eta\end{array}$ & $\tau$ \\
\hline${ }^{\circ} \mathrm{C}$ & $\mathrm{Hz}$ & GPa & $\log _{10} \mathrm{~Pa} \mathrm{~s}$ & $\mathbf{K}$ & Pas & $\mathbf{s}$ & Pa s & Pa s & $\mathbf{s}$ \\
\hline \multicolumn{10}{|c|}{ SAMPLE G14 } \\
\hline \multirow{10}{*}{ 号 } & 1 & \multirow{10}{*}{29.96} & \multirow{10}{*}{$-18.10 \pm 0.54$} & \multirow{10}{*}{$\begin{array}{c}2.515 \\
\pm 0.048\end{array}$} & \multirow{10}{*}{12.84} & \multirow{10}{*}{232.052} & 9.31 & \multirow{10}{*}{12.52} & \multirow{10}{*}{110.506} \\
\hline & 0.5 & & & & & & 9.68 & & \\
\hline & 0.2 & & & & & & 10.65 & & \\
\hline & 0.1 & & & & & & 11.30 & & \\
\hline & 0.05 & & & & & & 11.63 & & \\
\hline & 0.02 & & & & & & 12.22 & & \\
\hline & 0.01 & & & & & & 12.38 & & \\
\hline & 0.005 & & & & & & 12.46 & & \\
\hline & 0.002 & & & & & & 12.45 & & \\
\hline & 0.001 & & & & & & 12.60 & & \\
\hline \multirow{10}{*}{ ్ి } & 1 & \multirow{10}{*}{29.96} & \multirow{10}{*}{$-18.10 \pm 0.54$} & \multirow{10}{*}{$\begin{array}{c}2.515 \\
\pm 0.048\end{array}$} & \multirow{10}{*}{13.62} & \multirow{10}{*}{1399.289} & 9.65 & \multirow{10}{*}{13.65} & \multirow{10}{*}{1490.688} \\
\hline & 0.5 & & & & & & 9.87 & & \\
\hline & 0.2 & & & & & & 10.71 & & \\
\hline & 0.1 & & & & & & 11.19 & & \\
\hline & 0.05 & & & & & & 11.49 & & \\
\hline & 0.02 & & & & & & 12.02 & & \\
\hline & 0.01 & & & & & & 12.41 & & \\
\hline & 0.005 & & & & & & 12.90 & & \\
\hline & 0.002 & & & & & & 13.24 & & \\
\hline & 0.001 & & & & & & 13.64 & & \\
\hline 500 & & 29.96 & $-18.10 \pm 0.54$ & $\begin{array}{c}2.515 \\
\pm 0.048 \\
\end{array}$ & 14.44 & 9259.919 & - & - & - \\
\hline 480 & & 29.96 & $-18.10 \pm 0.54$ & $\begin{array}{c}2.515 \\
\pm 0.048 \\
\end{array}$ & 15.31 & 67749.071 & - & - & - \\
\hline 460 & & 29.96 & $-18.10 \pm 0.54$ & $\begin{array}{c}2.515 \\
\pm 0.048 \\
\end{array}$ & 16.22 & 552540.818 & - & - & - \\
\hline 440 & & 29.96 & $-18.10 \pm 0.54$ & $\begin{array}{c}2.515 \\
\pm 0.048 \\
\end{array}$ & 17.18 & 5069431.36 & - & - & - \\
\hline 420 & & 29.96 & $-18.10 \pm 0.54$ & $\begin{array}{c}2.515 \\
\pm 0.048 \\
\end{array}$ & 18.20 & 52858539.6 & - & - & - \\
\hline
\end{tabular}


Appendix 4a. Parameters of the lines fitted to the real shear modulus data for NS2 melt (G0) and Fe-free melts (G1-G7). See also Fig. 68a.

\begin{tabular}{|c|c|c|c|c|c|c|c|c|c|c|c|c|c|c|c|}
\hline \multicolumn{16}{|c|}{ FITS TO THE REAL SHEAR MODULUS DATA } \\
\hline \multicolumn{2}{|c|}{ G0 } & \multicolumn{2}{|c|}{ G1 } & \multicolumn{2}{|c|}{ G2 } & \multicolumn{2}{|c|}{ G3 } & \multicolumn{2}{|c|}{ G4 } & \multicolumn{2}{|c|}{ G5 } & \multicolumn{2}{|c|}{ G6 } & \multicolumn{2}{|c|}{ G7 } \\
\hline $\begin{array}{l}\Delta \log _{10} \\
\tau_{\mathrm{M}}(\mathrm{s}) \\
\end{array}$ & $\begin{array}{c}G_{\text {real }} \\
(\mathrm{GPa}) \\
\end{array}$ & $\begin{array}{l}\Delta \log _{10} \\
\tau_{\mathrm{M}}(\mathrm{s}) \\
\end{array}$ & $\begin{array}{l}G_{\text {real }} \\
(\mathrm{GPa}) \\
\end{array}$ & $\begin{array}{l}\Delta \log _{10} \\
\tau_{\mathrm{M}}(\mathrm{s}) \\
\end{array}$ & $\begin{array}{c}G_{\text {real }} \\
(\mathrm{GPa}) \\
\end{array}$ & $\begin{array}{l}\Delta \log _{10} \\
\tau_{\mathrm{M}}(\mathrm{s}) \\
\end{array}$ & $\begin{array}{l}G_{\text {real }} \\
(G P a)\end{array}$ & $\begin{array}{l}\Delta \log _{10} \\
\tau_{\mathrm{M}}(\mathrm{s})\end{array}$ & $\begin{array}{l}G_{\text {real }} \\
(\mathrm{GPa})\end{array}$ & $\begin{array}{l}\Delta \log _{10} \\
\tau_{\mathrm{M}}(\mathrm{s}) \\
\end{array}$ & $\begin{array}{l}G_{\text {real }} \\
(\mathrm{GPa})\end{array}$ & $\begin{array}{l}\Delta \log _{10} \\
\tau_{M}(\mathrm{~s}) \\
\end{array}$ & $\begin{array}{l}G_{\text {real }} \\
(\mathrm{GPa})\end{array}$ & $\begin{array}{l}\Delta \log _{10} \\
\tau_{M}(\mathrm{~s}) \\
\end{array}$ & $\begin{array}{c}G_{\text {real }} \\
(\mathrm{GPa}) \\
\end{array}$ \\
\hline $\begin{array}{l}-1.830 \\
\end{array}$ & 0.008 & $\begin{array}{l}-2.000 \\
\end{array}$ & 0.000 & -2.000 & 0.000 & $\begin{array}{l}-2.000 \\
\end{array}$ & 0.001 & -2.000 & 0.002 & -2.000 & 0.207 & -1.057 & 0.052 & -2.632 & 0.020 \\
\hline-1.432 & 0.040 & -1.500 & 0.000 & -1.600 & 0.000 & -1.600 & 0.003 & -1.800 & 0.005 & -1.800 & 0.299 & -1.034 & 0.058 & -2.632 & 0.020 \\
\hline-1.295 & 0.067 & -1.000 & 0.001 & -1.300 & 0.000 & -1.300 & 0.011 & -1.600 & 0.011 & -1.600 & 0.372 & -0.756 & 0.203 & -2.135 & 0.148 \\
\hline-1.131 & 0.127 & -0.500 & 0.009 & -1.000 & 0.000 & -1.034 & 0.029 & -1.400 & 0.024 & -1.400 & 0.436 & -0.733 & 0.225 & -1.632 & 0.561 \\
\hline-0.897 & 0.330 & 0.000 & 0.081 & -0.605 & 0.002 & -0.710 & 0.076 & -1.274 & 0.038 & -1.200 & 0.528 & -0.677 & 0.288 & -1.632 & 0.561 \\
\hline-0.861 & 0.383 & 0.385 & 0.357 & -0.304 & 0.007 & -0.636 & 0.094 & -1.134 & 0.063 & -1.057 & 0.650 & -0.432 & 0.826 & -1.314 & 1.147 \\
\hline-0.830 & 0.437 & 0.414 & 0.393 & -0.088 & 0.019 & -0.409 & 0.196 & -0.973 & 0.111 & -1.034 & 0.676 & -0.376 & 1.039 & -1.135 & 1.736 \\
\hline-0.748 & 0.616 & 0.686 & 0.849 & 0.029 & 0.032 & -0.335 & 0.254 & -0.833 & 0.183 & -0.756 & 1.268 & -0.358 & 1.120 & -0.675 & 4.980 \\
\hline-0.596 & 1.155 & 0.715 & 0.913 & 0.095 & 0.044 & -0.234 & 0.369 & -0.717 & 0.281 & -0.733 & 1.346 & -0.345 & 1.180 & -0.632 & 5.448 \\
\hline-0.464 & 1.960 & 0.775 & 1.057 & 0.213 & 0.075 & -0.056 & 0.735 & -0.575 & 0.482 & -0.677 & 1.562 & -0.075 & 3.188 & -0.632 & 5.448 \\
\hline-0.432 & 2.214 & 1.016 & 1.888 & 0.330 & 0.128 & -0.034 & 0.801 & -0.523 & 0.589 & -0.432 & 2.983 & -0.057 & 3.389 & -0.314 & 9.493 \\
\hline-0.350 & 3.009 & 1.076 & 2.178 & 0.396 & 0.174 & -0.011 & 0.877 & -0.435 & 0.829 & -0.376 & 3.426 & -0.044 & 3.536 & -0.135 & 11.826 \\
\hline-0.295 & 3.670 & 1.084 & 2.223 & 0.490 & 0.267 & 0.067 & 1.190 & -0.416 & 0.892 & -0.358 & 3.584 & -0.034 & 3.648 & 0.325 & 17.284 \\
\hline-0.163 & 5.698 & 1.088 & 2.243 & 0.514 & 0.298 & 0.245 & 2.310 & -0.274 & 1.522 & -0.345 & 3.698 & 0.244 & 8.044 & 0.686 & 20.849 \\
\hline-0.140 & 6.107 & 1.377 & 4.456 & 0.692 & 0.667 & 0.290 & 2.698 & -0.222 & 1.830 & -0.075 & 6.727 & 0.267 & 8.507 & 1.073 & 23.961 \\
\hline-0.131 & 6.268 & 1.385 & 4.542 & 0.697 & 0.682 & 0.364 & 3.438 & -0.134 & 2.471 & -0.057 & 6.979 & 0.323 & 9.709 & 1.325 & 25.537 \\
\hline-0.049 & 7.875 & 1.389 & 4.581 & 0.727 & 0.783 & 0.368 & 3.484 & -0.018 & 3.538 & -0.044 & 7.158 & 0.354 & 10.425 & 2.073 & 28.615 \\
\hline 0.103 & 11.168 & 1.414 & 4.847 & 0.791 & 1.039 & 0.591 & 6.349 & 0.027 & 4.022 & -0.034 & 7.292 & 0.406 & 11.640 & 2.325 & 29.357 \\
\hline 0.139 & 11.956 & 1.686 & 8.308 & 0.912 & 1.764 & 0.629 & 6.909 & 0.117 & 5.097 & 0.244 & 11.439 & 0.568 & 15.568 & 3.047 & 30.616 \\
\hline 0.161 & 12.456 & 1.715 & 8.729 & 0.993 & 2.493 & 0.643 & 7.111 & 0.167 & 5.756 & 0.267 & 11.788 & 0.624 & 16.844 & 3.073 & 30.637 \\
\hline 0.170 & 12.647 & 1.775 & 9.621 & 1.029 & 2.893 & 0.665 & 7.439 & 0.176 & 5.875 & 0.323 & 12.645 & 0.642 & 17.249 & 4.047 & 31.021 \\
\hline 0.404 & 17.445 & 1.787 & 9.808 & 1.092 & 3.744 & 0.766 & 8.994 & 0.283 & 7.470 & 0.354 & 13.130 & 0.655 & 17.526 & 4.073 & 31.030 \\
\hline 0.537 & 19.802 & 1.826 & 10.416 & 1.095 & 3.783 & 0.766 & 8.994 & 0.418 & 9.761 & 0.406 & 13.923 & 0.707 & 18.571 & 5.047 & 31.273 \\
\hline 0.559 & 20.172 & 2.016 & 13.525 & 1.213 & 5.945 & 0.930 & 11.629 & 0.425 & 9.884 & 0.568 & 16.363 & 0.943 & 22.077 & 6.047 & 31.702 \\
\hline 0.568 & 20.311 & 2.076 & 14.503 & 1.330 & 8.861 & 0.944 & 11.850 & 0.477 & 10.824 & 0.624 & 17.161 & 0.956 & 22.214 & & \\
\hline 0.838 & 23.695 & 2.084 & 14.641 & 1.388 & 10.570 & 0.966 & 12.206 & 0.565 & 12.467 & 0.642 & 17.419 & 0.966 & 22.314 & & \\
\hline 0.860 & 23.903 & 2.088 & 14.702 & 1.391 & 10.664 & 0.989 & 12.577 & 0.584 & 12.825 & 0.655 & 17.599 & 1.105 & 23.511 & & \\
\hline 0.869 & 23.980 & 2.127 & 15.329 & 1.396 & 10.810 & 1.067 & 13.829 & 0.726 & 15.421 & 0.707 & 18.294 & 1.198 & 24.160 & & \\
\hline 0.942 & 24.566 & 2.385 & 19.110 & 1.490 & 13.829 & 1.067 & 13.829 & 0.778 & 16.323 & 0.943 & 21.119 & 1.244 & 24.459 & & \\
\hline 1.139 & 25.718 & 2.389 & 19.160 & 1.689 & 20.010 & 1.245 & 16.488 & 0.790 & 16.533 & 0.956 & 21.258 & 1.267 & 24.602 & & \\
\hline 1.161 & 25.820 & 2.414 & 19.491 & 1.692 & 20.094 & 1.290 & 17.106 & 0.816 & 16.981 & 0.966 & 21.361 & 1.354 & 25.145 & & \\
\hline 1.243 & 26.147 & 2.525 & 20.869 & 1.697 & 20.224 & 1.328 & 17.617 & 0.866 & 17.811 & 1.105 & 22.724 & 1.406 & 25.462 & & \\
\hline 1.537 & 26.942 & 2.602 & 21.753 & 1.727 & 21.041 & 1.348 & 17.876 & 0.982 & 19.644 & 1.198 & 23.524 & 1.499 & 26.028 & & \\
\hline 1.559 & 26.986 & 2.686 & 22.654 & 1.791 & 22.554 & 1.364 & 18.077 & 1.091 & 21.212 & 1.244 & 23.886 & 1.642 & 26.834 & & \\
\hline 1.641 & 27.125 & 2.715 & 22.948 & 1.993 & 25.952 & 1.591 & 20.736 & 1.117 & 21.565 & 1.267 & 24.056 & 1.655 & 26.901 & & \\
\hline 1.838 & 27.357 & 2.787 & 23.640 & 2.029 & 26.357 & 1.629 & 21.141 & 1.167 & 22.205 & 1.354 & 24.666 & 1.707 & 27.156 & & \\
\hline 1.860 & 27.375 & 2.826 & 23.998 & 2.087 & 26.921 & 1.643 & 21.282 & 1.176 & 22.312 & 1.406 & 24.997 & 1.897 & 27.920 & & \\
\hline
\end{tabular}


Appendix 4a. Parameters of the lines fitted to the real shear modulus data for NS2 melt (G0) and Fe-free melts (G1-G7) - continuation...

\begin{tabular}{|c|c|c|c|c|c|c|c|c|c|c|c|c|c|c|c|}
\hline \multicolumn{16}{|c|}{ FITS TO THE REAL SHEAR MODULUS DATA } \\
\hline \multicolumn{2}{|c|}{ G0 } & \multicolumn{2}{|c|}{ G1 } & \multicolumn{2}{|c|}{$\mathbf{G 2}$} & \multicolumn{2}{|c|}{ G3 } & \multicolumn{2}{|c|}{ G4 } & \multicolumn{2}{|c|}{ G5 } & \multicolumn{2}{|c|}{ G6 } & \multicolumn{2}{|c|}{ G7 } \\
\hline $\begin{array}{l}\Delta \log _{10} \\
\tau_{\mathrm{M}}(\mathrm{s})\end{array}$ & $\begin{array}{l}G_{\text {real }} \\
(G P a)\end{array}$ & $\begin{array}{l}\Delta \log _{10} \\
\tau_{M}(\mathrm{~s})\end{array}$ & $\begin{array}{c}G_{\text {real }} \\
(\mathrm{GPa})\end{array}$ & $\begin{array}{l}\Delta \log _{10} \\
\tau_{M} \text { (s) }\end{array}$ & $\begin{array}{l}G_{\text {real }} \\
(\mathrm{GPa})\end{array}$ & $\begin{array}{l}\Delta \log _{10} \\
\tau_{M} \text { (s) }\end{array}$ & $\begin{array}{l}G_{\text {real }} \\
(\mathrm{GPa})\end{array}$ & $\begin{array}{l}\Delta \log _{10} \\
\tau_{M}(s)\end{array}$ & $\begin{array}{l}G_{\text {real }} \\
(\mathrm{GPa})\end{array}$ & $\begin{array}{l}\Delta \log _{10} \\
\tau_{M}(s)\end{array}$ & $\begin{array}{c}G_{\text {real }} \\
(\mathrm{GPa})\end{array}$ & $\begin{array}{l}\Delta \log _{10} \\
\tau_{M}(s)\end{array}$ & $\begin{array}{l}G_{\text {real }} \\
(\mathrm{GPa})\end{array}$ & $\begin{array}{l}\Delta \log _{10} \\
\tau_{M}(\mathrm{~s})\end{array}$ & $\begin{array}{c}G_{\text {real }} \\
(\mathrm{GPa})\end{array}$ \\
\hline 1.942 & 27.431 & 2.903 & 24.672 & 2.095 & 26.989 & 1.649 & 21.345 & 1.283 & 23.547 & 1.499 & 25.548 & 1.943 & 28.067 & & \\
\hline 2.088 & 27.495 & 3.084 & 26.103 & 2.119 & 27.187 & 1.665 & 21.505 & 1.418 & 24.867 & 1.642 & 26.290 & 1.956 & 28.106 & & \\
\hline 2.161 & 27.516 & 3.088 & 26.130 & 2.330 & 28.376 & 1.930 & 23.952 & 1.477 & 25.368 & 1.655 & 26.352 & 2.036 & 28.326 & & \\
\hline 2.243 & 27.532 & 3.127 & 26.408 & 2.388 & 28.595 & 1.944 & 24.068 & 1.489 & 25.467 & 1.707 & 26.591 & 2.105 & 28.494 & & \\
\hline 2.389 & 27.550 & 3.301 & 27.519 & 2.391 & 28.606 & 1.989 & 24.444 & 1.497 & 25.533 & 1.897 & 27.384 & 2.198 & 28.698 & & \\
\hline 2.559 & 27.561 & 3.385 & 27.993 & 2.396 & 28.623 & 2.047 & 24.920 & 1.565 & 26.052 & 1.943 & 27.559 & 2.337 & 28.974 & & \\
\hline 2.641 & 27.563 & 3.389 & 28.013 & 2.420 & 28.705 & 2.103 & 25.363 & 1.778 & 27.385 & 1.956 & 27.608 & 2.354 & 29.008 & & \\
\hline 2.787 & 27.566 & 3.419 & 28.169 & 2.689 & 29.582 & 2.245 & 26.429 & 1.790 & 27.450 & 2.036 & 27.894 & 2.406 & 29.108 & & \\
\hline 2.860 & 27.567 & 3.525 & 28.694 & 2.692 & 29.594 & 2.290 & 26.748 & 1.798 & 27.493 & 2.105 & 28.127 & 2.499 & 29.291 & & \\
\hline 2.942 & 27.568 & 3.602 & 29.037 & 2.727 & 29.727 & 2.328 & 27.014 & 1.816 & 27.585 & 2.198 & 28.417 & 2.655 & 29.615 & & \\
\hline 3.088 & 27.569 & 3.720 & 29.501 & 2.818 & 30.097 & 2.348 & 27.150 & 1.866 & 27.829 & 2.337 & 28.799 & 2.707 & 29.729 & & \\
\hline 3.243 & 27.569 & 3.787 & 29.734 & 2.887 & 30.414 & 2.404 & 27.522 & 2.091 & 28.753 & 2.354 & 28.843 & 2.735 & 29.792 & & \\
\hline 3.305 & 27.569 & 3.826 & 29.858 & 2.993 & 30.924 & 2.629 & 28.836 & 2.117 & 28.845 & 2.406 & 28.968 & 2.897 & 30.178 & & \\
\hline 3.389 & 27.569 & 3.903 & 30.084 & 3.029 & 31.098 & 2.643 & 28.903 & 2.176 & 29.039 & 2.499 & 29.179 & 3.036 & 30.516 & & \\
\hline 3.606 & 27.569 & 4.088 & 30.534 & 3.087 & 31.376 & 2.649 & 28.933 & 2.196 & 29.103 & 2.655 & 29.484 & 3.105 & 30.673 & & \\
\hline 3.641 & 27.569 & 4.117 & 30.594 & 3.119 & 31.523 & 2.802 & 29.544 & 2.242 & 29.241 & 2.707 & 29.572 & 3.198 & 30.862 & & \\
\hline 3.787 & 27.569 & 4.127 & 30.612 & 3.188 & 31.820 & 2.897 & 29.812 & 2.418 & 29.715 & 2.735 & 29.617 & 3.337 & 31.082 & & \\
\hline 3.942 & 27.569 & 4.280 & 30.872 & 3.388 & 32.454 & 2.930 & 29.889 & 2.477 & 29.854 & 2.897 & 29.849 & 3.406 & 31.163 & & \\
\hline 4.004 & 27.569 & 4.301 & 30.902 & 3.391 & 32.462 & 2.944 & 29.918 & 2.489 & 29.883 & 3.036 & 30.015 & 3.499 & 31.248 & & \\
\hline 4.088 & 27.569 & 4.419 & 31.052 & 3.420 & 32.526 & 3.047 & 30.100 & 2.497 & 29.902 & 3.105 & 30.089 & 3.735 & 31.373 & & \\
\hline 4.305 & 27.569 & 4.525 & 31.165 & 3.586 & 32.790 & 3.103 & 30.176 & 2.543 & 30.004 & 3.198 & 30.182 & 3.864 & 31.410 & & \\
\hline 4.389 & 27.569 & 4.581 & 31.217 & 3.689 & 32.884 & 3.198 & 30.279 & 2.790 & 30.499 & 3.337 & 30.308 & 3.897 & 31.418 & & \\
\hline 4.606 & 27.569 & 4.602 & 31.236 & 3.692 & 32.886 & 3.328 & 30.389 & 2.798 & 30.514 & 3.406 & 30.366 & 4.036 & 31.444 & & \\
\hline 4.787 & 27.569 & 4.720 & 31.335 & 3.818 & 32.957 & 3.348 & 30.404 & 2.816 & 30.547 & 3.499 & 30.442 & 4.165 & 31.464 & & \\
\hline 5.004 & 27.569 & 4.826 & 31.418 & 3.887 & 32.982 & 3.404 & 30.444 & 2.941 & 30.765 & 3.735 & 30.608 & 4.198 & 31.469 & & \\
\hline 5.088 & 27.569 & 4.903 & 31.477 & 4.087 & 33.023 & 3.596 & 30.573 & 3.026 & 30.906 & 3.864 & 30.684 & 4.337 & 31.488 & & \\
\hline 5.305 & 27.569 & 4.979 & 31.536 & 4.119 & 33.027 & 3.629 & 30.595 & 3.091 & 31.008 & 3.897 & 30.702 & 4.563 & 31.519 & & \\
\hline 5.606 & 27.569 & 5.117 & 31.647 & 4.188 & 33.033 & 3.649 & 30.608 & 3.117 & 31.048 & 4.036 & 30.775 & 4.735 & 31.544 & & \\
\hline 6.004 & 27.569 & 5.280 & 31.785 & 4.388 & 33.044 & 3.802 & 30.713 & 3.196 & 31.164 & 4.165 & 30.839 & 4.864 & 31.563 & & \\
\hline 6.305 & 27.569 & 5.301 & 31.804 & 4.420 & 33.045 & 3.897 & 30.781 & 3.242 & 31.227 & 4.198 & 30.855 & 5.036 & 31.591 & & \\
\hline & & 5.419 & 31.915 & 4.586 & 33.048 & 4.047 & 30.896 & 3.327 & 31.341 & 4.337 & 30.927 & 5.165 & 31.613 & & \\
\hline & & 5.581 & 32.072 & 4.727 & 33.049 & 4.103 & 30.940 & 3.489 & 31.533 & 4.563 & 31.069 & 5.563 & 31.696 & & \\
\hline & & 5.602 & 32.093 & 4.818 & 33.050 & 4.198 & 31.014 & 3.497 & 31.542 & 4.735 & 31.212 & 5.864 & 31.792 & & \\
\hline & & 5.720 & 32.203 & 4.887 & 33.050 & 4.348 & 31.134 & 3.543 & 31.590 & 4.864 & 31.344 & 5.929 & 31.819 & & \\
\hline & & 5.979 & 32.422 & 5.028 & 33.050 & 4.404 & 31.178 & 3.725 & 31.759 & 5.036 & 31.538 & 6.165 & 31.939 & & \\
\hline & & 6.117 & 32.520 & 5.119 & 33.051 & 4.596 & 31.331 & 3.790 & 31.809 & 5.165 & 31.675 & 6.230 & 31.979 & & \\
\hline & & 6.150 & 32.540 & 5.188 & 33.051 & 4.613 & 31.345 & 3.798 & 31.815 & 5.563 & 31.952 & 6.563 & 32.233 & & \\
\hline
\end{tabular}


Appendix 4a. Parameters of the lines fitted to the real shear modulus data for NS2 melt (G0) and Fe-free melts (G1-G7) - continuation...

\begin{tabular}{|c|c|c|c|c|c|c|c|c|c|c|c|c|c|c|c|}
\hline \multicolumn{16}{|c|}{ FITS TO THE REAL SHEAR MODULUS DATA } \\
\hline \multicolumn{2}{|c|}{ G0 } & \multicolumn{2}{|c|}{ G1 } & \multicolumn{2}{|c|}{ G2 } & \multicolumn{2}{|c|}{ G3 } & \multicolumn{2}{|c|}{ G4 } & \multicolumn{2}{|c|}{ G5 } & \multicolumn{2}{|c|}{ G6 } & \multicolumn{2}{|c|}{ G7 } \\
\hline $\begin{array}{l}\Delta \log _{10} \\
\tau_{M}(s)\end{array}$ & $\begin{array}{l}G_{\text {real }} \\
(\mathrm{GPa})\end{array}$ & $\begin{array}{l}\Delta \log _{10} \\
\tau_{M} \text { (s) }\end{array}$ & $\begin{array}{l}\mathbf{G}_{\text {real }} \\
(\mathrm{GPa})\end{array}$ & $\begin{array}{l}\Delta \log _{10} \\
\tau_{M}(s)\end{array}$ & $\begin{array}{c}G_{\text {real }} \\
(\mathrm{GPa})\end{array}$ & $\begin{array}{l}\Delta \log _{10} \\
\tau_{\mathrm{M}}(\mathrm{s})\end{array}$ & $\begin{array}{l}G_{\text {real }} \\
(\mathrm{GPa})\end{array}$ & $\begin{array}{l}\Delta \log _{10} \\
\tau_{M}(\mathrm{~s})\end{array}$ & $\begin{array}{l}\mathbf{G}_{\text {real }} \\
(\mathrm{GPa})\end{array}$ & $\begin{array}{l}\Delta \log _{10} \\
\tau_{M}(\mathrm{~s})\end{array}$ & $\begin{array}{l}G_{\text {real }} \\
(\mathrm{GPa})\end{array}$ & $\begin{array}{l}\Delta \log _{10} \\
\tau_{M}(s)\end{array}$ & $\begin{array}{l}\mathbf{G}_{\text {real }} \\
(\mathrm{GPa})\end{array}$ & $\begin{array}{l}\Delta \log _{10} \\
\tau_{M}(s)\end{array}$ & $\begin{array}{l}\mathbf{G}_{\text {real }} \\
(\mathrm{GPa})\end{array}$ \\
\hline & & 6.280 & 32.612 & 5.426 & 33.051 & 4.802 & 31.492 & 3.941 & 31.912 & 5.864 & 32.114 & 6.628 & 32.289 & & \\
\hline & & 6.419 & 32.676 & 5.452 & 33.051 & 4.897 & 31.565 & 4.026 & 31.969 & 5.929 & 32.159 & 6.864 & 32.504 & & \\
\hline & & 6.451 & 32.689 & 5.586 & 33.051 & 4.914 & 31.578 & 4.196 & 32.093 & 6.165 & 32.380 & 6.929 & 32.567 & & \\
\hline & & 6.581 & 32.743 & 5.727 & 33.051 & 5.103 & 31.722 & 4.242 & 32.131 & 6.230 & 32.459 & 7.230 & 32.899 & & \\
\hline & & 6.849 & 32.889 & 5.753 & 33.051 & 5.198 & 31.794 & 4.327 & 32.211 & 6.563 & 32.960 & 7.628 & 33.430 & & \\
\hline & & 6.979 & 32.997 & 5.887 & 33.052 & 5.312 & 31.881 & 4.497 & 32.389 & 6.628 & 33.056 & 7.929 & 33.720 & & \\
\hline & & 7.150 & 33.197 & 6.028 & 33.052 & 5.596 & 32.099 & 4.543 & 32.439 & 6.864 & 33.324 & 8.230 & 33.878 & & \\
\hline & & 7.280 & 33.402 & 6.151 & 33.053 & 5.613 & 32.112 & 4.725 & 32.619 & 6.929 & 33.372 & 8.628 & 34.029 & & \\
\hline & & 7.451 & 33.735 & 6.426 & 33.059 & 5.897 & 32.325 & 4.731 & 32.624 & 7.230 & 33.495 & 8.929 & 34.162 & & \\
\hline & & 7.849 & 34.462 & 6.452 & 33.061 & 5.914 & 32.337 & 4.941 & 32.769 & 7.628 & 33.546 & & & & \\
\hline & & 8.150 & 34.741 & 6.727 & 33.085 & 6.312 & 32.617 & 5.026 & 32.813 & 7.929 & 33.580 & & & & \\
\hline & & 8.247 & 34.794 & 6.753 & 33.089 & 6.526 & 32.755 & 5.032 & 32.816 & 8.230 & 33.643 & & & & \\
\hline & & 8.451 & 34.872 & 7.028 & 33.177 & 6.613 & 32.809 & 5.242 & 32.907 & 8.628 & 33.822 & & & & \\
\hline & & 8.548 & 34.899 & 7.151 & 33.257 & 6.827 & 32.940 & 5.327 & 32.943 & 8.929 & 34.063 & & & & \\
\hline & & 8.849 & 34.973 & 7.415 & 33.555 & 6.914 & 32.998 & 5.430 & 32.989 & & & & & & \\
\hline & & 8.946 & 34.997 & 7.426 & 33.570 & 7.225 & 33.282 & 5.725 & 33.128 & & & & & & \\
\hline & & 9.150 & 35.043 & 7.452 & 33.608 & 7.312 & 33.395 & 5.731 & 33.130 & & & & & & \\
\hline & & 9.247 & 35.060 & 7.716 & 33.994 & 7.526 & 33.820 & 6.026 & 33.267 & & & & & & \\
\hline & & 9.548 & 35.091 & 7.727 & 34.008 & 7.613 & 34.000 & 6.032 & 33.270 & & & & & & \\
\hline & & 9.946 & 35.102 & 7.753 & 34.042 & 7.827 & 34.100 & 6.430 & 33.482 & & & & & & \\
\hline & & 10.247 & 35.104 & 8.114 & 34.396 & 7.952 & 34.200 & 6.643 & 33.626 & & & & & & \\
\hline & & 10.548 & 35.104 & 8.151 & 34.422 & 8.102 & 34.300 & 6.731 & 33.693 & & & & & & \\
\hline & & 10.615 & 35.104 & 8.415 & 34.576 & 8.253 & 34.400 & 6.944 & 33.869 & & & & & & \\
\hline & & 10.916 & 35.104 & 8.452 & 34.594 & 8.403 & 34.400 & 7.032 & 33.943 & & & & & & \\
\hline & & 10.946 & 35.104 & 8.716 & 34.696 & 8.554 & 34.400 & 7.342 & 34.140 & & & & & & \\
\hline & & 11.247 & 35.104 & 9.114 & 34.761 & 8.704 & 34.400 & 7.430 & 34.174 & & & & & & \\
\hline & & 11.314 & 35.104 & 9.415 & 34.773 & 8.855 & 34.400 & 7.643 & 34.223 & & & & & & \\
\hline & & 11.615 & 35.104 & 9.625 & 34.775 & 9.006 & 34.400 & 7.731 & 34.235 & & & & & & \\
\hline & & 11.916 & 35.104 & 9.716 & 34.776 & 9.156 & 34.400 & 7.944 & 34.256 & & & & & & \\
\hline & & 12.314 & 35.104 & 9.926 & 34.776 & 9.307 & 34.400 & 8.342 & 34.302 & & & & & & \\
\hline & & 12.615 & 35.104 & 10.114 & 34.777 & 9.457 & 34.400 & 8.643 & 34.360 & & & & & & \\
\hline & & 12.916 & 35.104 & 10.324 & 34.777 & 9.608 & 34.400 & 8.944 & 34.451 & & & & & & \\
\hline & & 13.311 & 35.104 & 10.415 & 34.777 & 9.800 & 34.400 & 9.342 & 34.764 & & & & & & \\
\hline & & 13.314 & 35.104 & 10.625 & 34.777 & 10.000 & 34.400 & 9.643 & 35.162 & & & & & & \\
\hline & & 13.612 & 35.104 & 10.926 & 34.777 & 10.200 & 34.400 & & & & & & & & \\
\hline & & 13.615 & 35.104 & 11.324 & 34.777 & 10.400 & 34.400 & & & & & & & & \\
\hline & & 14.010 & 35.104 & 11.625 & 34.777 & 10.600 & 34.400 & & & & & & & & \\
\hline
\end{tabular}


Appendix 4a. Parameters of the lines fitted to the real shear modulus data for NS2 melt (G0) and Fe-free melts (G1-G7) - continuation...

\begin{tabular}{|c|c|c|c|c|c|c|c|c|c|c|c|c|c|c|c|}
\hline \multicolumn{16}{|c|}{ FITS TO THE REAL SHEAR MODULUS DATA } \\
\hline \multicolumn{2}{|c|}{ G0 } & \multicolumn{2}{|c|}{ G1 } & \multicolumn{2}{|c|}{ G2 } & \multicolumn{2}{|c|}{ G3 } & \multicolumn{2}{|c|}{ G4 } & \multicolumn{2}{|c|}{ G5 } & \multicolumn{2}{|c|}{ G6 } & \multicolumn{2}{|c|}{ G7 } \\
\hline $\begin{array}{l}\Delta \log _{10} \\
\tau_{M}(s)\end{array}$ & $\begin{array}{c}G_{\text {real }} \\
(\mathrm{GPa})\end{array}$ & $\begin{array}{l}\Delta \log _{10} \\
\tau_{M}(\mathrm{~s})\end{array}$ & $\begin{array}{l}G_{\text {real }} \\
(\mathrm{GPa})\end{array}$ & $\begin{array}{l}\Delta \log _{10} \\
\tau_{M}(\mathrm{~s})\end{array}$ & $\begin{array}{c}G_{\text {real }} \\
(\mathrm{GPa})\end{array}$ & $\begin{array}{l}\Delta \log _{10} \\
\tau_{M}(s)\end{array}$ & $\begin{array}{l}G_{\text {real }} \\
(\mathrm{GPa})\end{array}$ & $\begin{array}{l}\Delta \log _{10} \\
\tau_{M}(s)\end{array}$ & $\begin{array}{l}\mathbf{G}_{\text {real }} \\
(\mathrm{GPa})\end{array}$ & $\begin{array}{l}\Delta \log _{10} \\
\tau_{M}(\mathrm{~s})\end{array}$ & $\begin{array}{c}G_{\text {real }} \\
(\mathrm{GPa})\end{array}$ & $\begin{array}{l}\Delta \log _{10} \\
\tau_{M}(s)\end{array}$ & $\begin{array}{l}\mathbf{G}_{\text {real }} \\
(\mathrm{GPa})\end{array}$ & $\begin{array}{l}\Delta \log _{10} \\
\tau_{M}(s)\end{array}$ & $\begin{array}{c}G_{\text {real }} \\
(\mathrm{GPa})\end{array}$ \\
\hline & & $\begin{array}{l}14.311 \\
14.612 \\
15.010 \\
15.311 \\
15.612\end{array}$ & $\begin{array}{l}35.104 \\
35.104 \\
35.104 \\
35.104 \\
35.104\end{array}$ & $\begin{array}{l}11.926 \\
12.133 \\
12.324 \\
12.434 \\
12.625 \\
12.832 \\
13.133 \\
13.434 \\
13.832 \\
14.133 \\
14.434 \\
14.832 \\
15.133\end{array}$ & $\begin{array}{l}34.777 \\
34.777 \\
34.777 \\
34.777 \\
34.777 \\
34.777 \\
34.777 \\
34.777 \\
34.777 \\
34.777 \\
34.777 \\
34.777 \\
34.777\end{array}$ & $\begin{array}{l}10.800 \\
11.000 \\
11.200 \\
11.400 \\
11.600 \\
11.800 \\
12.000 \\
12.200 \\
12.400 \\
12.600 \\
12.800\end{array}$ & $\begin{array}{l}34.400 \\
34.400 \\
34.400 \\
34.400 \\
34.400 \\
34.400 \\
34.400 \\
34.400 \\
34.400 \\
34.400 \\
34.400\end{array}$ & & & & & & & & \\
\hline
\end{tabular}


Appendix 4b. Parameters of the lines fitted to the imaginary shear modulus data for NS2 melt (G0) and Fe-free melts (G1-G7). See also Fig. 68b.

\begin{tabular}{|c|c|c|c|c|c|c|c|c|c|c|c|c|c|c|c|}
\hline \multicolumn{16}{|c|}{ FITS TO THE IMAGINARY SHEAR MODULUS DATA } \\
\hline \multicolumn{2}{|c|}{ G0 } & \multicolumn{2}{|c|}{ G1 } & \multicolumn{2}{|c|}{$\mathbf{G 2}$} & \multicolumn{2}{|c|}{ G3 } & \multicolumn{2}{|c|}{ G4 } & \multicolumn{2}{|c|}{ G5 } & \multicolumn{2}{|c|}{ G6 } & \multicolumn{2}{|c|}{ G7 } \\
\hline $\begin{array}{l}\Delta \log _{10} \\
\tau_{\mathrm{M}}(\mathrm{s})\end{array}$ & $\begin{array}{c}\mathbf{G}_{\mathrm{im}} \\
(\mathrm{GPa})\end{array}$ & $\begin{array}{l}\Delta \log _{10} \\
\tau_{M}(\mathrm{~s})\end{array}$ & $\begin{array}{c}\mathbf{G}_{\mathrm{im}} \\
(\mathrm{GPa})\end{array}$ & $\begin{array}{l}\Delta \log _{10} \\
\tau_{M}(\mathrm{~s})\end{array}$ & $\begin{array}{c}\mathbf{G}_{\text {im }} \\
(\mathrm{GPa})\end{array}$ & $\begin{array}{l}\Delta \log _{10} \\
\tau_{\mathrm{M}}(\mathrm{s})\end{array}$ & $\begin{array}{c}\mathbf{G}_{\text {im }} \\
(\mathrm{GPa})\end{array}$ & $\begin{array}{l}\Delta \log _{10} \\
\tau_{\mathrm{M}} \text { (s) }\end{array}$ & $\begin{array}{c}\mathbf{G}_{\mathrm{im}} \\
(\mathrm{GPa})\end{array}$ & $\begin{array}{l}\Delta \log _{10} \\
\tau_{M}(\mathrm{~s}) \\
\end{array}$ & $\begin{array}{c}\mathbf{G}_{\mathrm{im}} \\
(\mathrm{GPa})\end{array}$ & $\begin{array}{l}\Delta \log _{10} \\
\tau_{M}(\mathrm{~s})\end{array}$ & $\begin{array}{c}\mathbf{G}_{\mathrm{im}} \\
(\mathrm{GPa})\end{array}$ & $\begin{array}{l}\Delta \log _{10} \\
\tau_{M}(\mathrm{~s}) \\
\end{array}$ & $\begin{array}{c}\mathbf{G}_{\mathrm{im}} \\
(\mathrm{GPa})\end{array}$ \\
\hline-1.830 & 0.222 & -1.000 & 0.032 & -0.605 & 0.042 & \begin{tabular}{c|}
-1.034 \\
\end{tabular} & 0.297 & -1.274 & 0.247 & -1.057 & 0.426 & $\begin{array}{c}-1.331 \\
\end{array}$ & 0.372 & $\begin{array}{l}-2.632 \\
\end{array}$ & 0.046 \\
\hline-1.432 & 0.553 & -0.500 & 0.102 & -0.304 & 0.085 & -0.710 & 0.617 & -1.134 & 0.316 & -1.034 & 0.448 & -1.059 & 0.694 & -2.632 & 0.046 \\
\hline-1.295 & 0.757 & 0.385 & 0.777 & -0.088 & 0.139 & -0.636 & 0.727 & -0.973 & 0.430 & -0.756 & 0.828 & -1.030 & 0.741 & -2.135 & 0.145 \\
\hline-1.131 & 1.102 & 0.414 & 0.829 & 0.029 & 0.182 & -0.409 & 1.192 & -0.833 & 0.569 & -0.733 & 0.871 & -0.729 & 1.454 & -1.632 & 0.461 \\
\hline-0.897 & 1.868 & 0.686 & 1.505 & 0.095 & 0.212 & -0.335 & 1.396 & -0.717 & 0.724 & -0.677 & 0.986 & -0.661 & 1.686 & -1.632 & 0.461 \\
\hline-0.861 & 2.022 & 0.715 & 1.601 & 0.213 & 0.278 & -0.234 & 1.726 & -0.575 & 0.979 & -0.432 & 1.703 & -0.653 & 1.713 & -1.314 & 0.957 \\
\hline-0.830 & 2.168 & 0.775 & 1.812 & 0.330 & 0.364 & -0.056 & 2.469 & -0.523 & 1.092 & -0.376 & 1.925 & -0.360 & 3.070 & -1.135 & 1.440 \\
\hline-0.748 & 2.591 & 1.016 & 2.898 & 0.396 & 0.423 & -0.034 & 2.575 & -0.435 & 1.320 & -0.358 & 2.005 & -0.352 & 3.111 & -0.675 & 4.026 \\
\hline-0.596 & 3.566 & 1.076 & 3.219 & 0.490 & 0.526 & -0.011 & 2.691 & -0.416 & 1.376 & -0.345 & 2.063 & -0.331 & 3.228 & -0.632 & 4.409 \\
\hline-0.464 & 4.606 & 1.084 & 3.266 & 0.514 & 0.555 & 0.067 & 3.110 & -0.274 & 1.864 & -0.075 & 3.641 & -0.059 & 4.676 & -0.632 & 4.409 \\
\hline-0.432 & 4.873 & 1.088 & 3.288 & 0.692 & 0.836 & 0.245 & 4.178 & -0.222 & 2.080 & -0.057 & 3.777 & -0.030 & 4.803 & -0.314 & 8.054 \\
\hline-0.350 & 5.574 & 1.377 & 4.964 & 0.697 & 0.845 & 0.290 & 4.458 & -0.134 & 2.503 & -0.044 & 3.874 & 0.046 & 5.094 & -0.135 & 10.206 \\
\hline-0.295 & 6.040 & 1.385 & 5.009 & 0.727 & 0.907 & 0.364 & 4.911 & -0.018 & 3.168 & -0.034 & 3.947 & 0.092 & 5.243 & 0.325 & 11.528 \\
\hline-0.163 & 6.994 & 1.389 & 5.029 & 0.791 & 1.049 & 0.368 & 4.937 & 0.027 & 3.461 & 0.244 & 6.231 & 0.271 & 5.595 & 0.686 & 8.459 \\
\hline-0.140 & 7.118 & 1.414 & 5.161 & 0.912 & 1.382 & 0.591 & 6.078 & 0.117 & 4.097 & 0.267 & 6.403 & 0.339 & 5.641 & 1.073 & 4.276 \\
\hline-0.131 & 7.162 & 1.686 & 6.163 & 0.993 & 1.662 & 0.629 & 6.229 & 0.167 & 4.473 & 0.323 & 6.788 & 0.347 & 5.643 & 1.325 & 2.495 \\
\hline-0.049 & 7.443 & 1.715 & 6.222 & 1.029 & 1.802 & 0.643 & 6.279 & 0.176 & 4.540 & 0.354 & 6.976 & 0.393 & 5.643 & 2.073 & 0.459 \\
\hline 0.103 & 7.288 & 1.775 & 6.312 & 1.092 & 2.078 & 0.665 & 6.356 & 0.283 & 5.385 & 0.406 & 7.225 & 0.640 & 5.244 & 2.325 & 0.258 \\
\hline 0.139 & 7.129 & 1.787 & 6.326 & 1.095 & 2.090 & 0.766 & 6.649 & 0.418 & 6.411 & 0.568 & 7.429 & 0.648 & 5.223 & 3.047 & 0.049 \\
\hline 0.161 & 7.006 & 1.826 & 6.358 & 1.213 & 2.718 & 0.766 & 6.649 & 0.425 & 6.460 & 0.624 & 7.293 & 0.669 & 5.161 & 3.073 & 0.046 \\
\hline 0.170 & 6.955 & 2.016 & 6.246 & 1.330 & 3.503 & 0.930 & 6.886 & 0.477 & 6.808 & 0.642 & 7.227 & 0.791 & 4.760 & 4.047 & 0.005 \\
\hline 0.404 & 5.124 & 2.076 & 6.117 & 1.388 & 3.961 & 0.944 & 6.891 & 0.565 & 7.327 & 0.655 & 7.176 & 0.881 & 4.444 & 4.073 & 0.005 \\
\hline 0.537 & 4.027 & 2.084 & 6.096 & 1.391 & 3.986 & 0.966 & 6.893 & 0.584 & 7.425 & 0.707 & 6.934 & 0.941 & 4.231 & 5.047 & 0.001 \\
\hline 0.559 & 3.852 & 2.088 & 6.086 & 1.396 & 4.026 & 0.989 & 6.888 & 0.726 & 7.969 & 0.943 & 5.478 & 0.970 & 4.130 & 6.047 & 0.000 \\
\hline 0.568 & 3.786 & 2.127 & 5.975 & 1.490 & 4.873 & 1.067 & 6.816 & 0.778 & 8.078 & 0.956 & 5.399 & 1.046 & 3.872 & & \\
\hline 0.838 & 2.142 & 2.385 & 5.022 & 1.689 & 6.955 & 1.067 & 6.816 & 0.790 & 8.096 & 0.966 & 5.341 & 1.092 & 3.716 & & \\
\hline 0.860 & 2.037 & 2.389 & 5.007 & 1.692 & 6.988 & 1.245 & 6.388 & 0.816 & 8.125 & 1.105 & 4.606 & 1.182 & 3.433 & & \\
\hline 0.869 & 1.998 & 2.414 & 4.908 & 1.697 & 7.040 & 1.290 & 6.236 & 0.866 & 8.136 & 1.198 & 4.219 & 1.339 & 2.989 & & \\
\hline 0.942 & 1.698 & 2.525 & 4.489 & 1.727 & 7.375 & 1.328 & 6.096 & 0.982 & 7.923 & 1.244 & 4.055 & 1.347 & 2.969 & & \\
\hline 1.139 & 1.089 & 2.602 & 4.215 & 1.791 & 8.042 & 1.348 & 6.021 & 1.091 & 7.405 & 1.267 & 3.979 & 1.393 & 2.850 & & \\
\hline 1.161 & 1.034 & 2.686 & 3.936 & 1.993 & 9.656 & 1.364 & 5.960 & 1.117 & 7.239 & 1.354 & 3.709 & 1.580 & 2.416 & & \\
\hline 1.243 & 0.858 & 2.715 & 3.847 & 2.029 & 9.819 & 1.591 & 4.956 & 1.167 & 6.887 & 1.406 & 3.556 & 1.640 & 2.285 & & \\
\hline 1.537 & 0.438 & 2.787 & 3.637 & 2.087 & 9.994 & 1.629 & 4.771 & 1.176 & 6.822 & 1.499 & 3.273 & 1.648 & 2.269 & & \\
\hline 1.559 & 0.415 & 2.826 & 3.529 & 2.095 & 10.009 & 1.643 & 4.705 & 1.283 & 5.945 & 1.642 & 2.789 & 1.716 & 2.127 & & \\
\hline 1.641 & 0.344 & 2.903 & 3.330 & 2.119 & 10.043 & 1.649 & 4.675 & 1.418 & 4.798 & 1.655 & 2.742 & 1.791 & 1.974 & & \\
\hline 1.838 & 0.219 & 3.084 & 2.901 & 2.330 & 9.651 & 1.665 & 4.599 & 1.477 & 4.330 & 1.707 & 2.553 & 1.881 & 1.798 & & \\
\hline 1.860 & 0.208 & 3.088 & 2.893 & 2.388 & 9.360 & 1.930 & 3.411 & 1.489 & 4.236 & 1.897 & 1.863 & 2.017 & 1.534 & & \\
\hline
\end{tabular}


Appendix 4b. Parameters of the lines fitted to the imaginary shear modulus data for NS2 melt (G0) and Fe-free melts (G1-G7) - continuation...

\begin{tabular}{|c|c|c|c|c|c|c|c|c|c|c|c|c|c|c|c|}
\hline \multicolumn{16}{|c|}{ FITS TO THE IMAGINARY SHEAR MODULUS DATA } \\
\hline \multicolumn{2}{|c|}{ G0 } & \multicolumn{2}{|c|}{ G1 } & \multicolumn{2}{|c|}{ G2 } & \multicolumn{2}{|c|}{ G3 } & \multicolumn{2}{|c|}{ G4 } & \multicolumn{2}{|c|}{ G5 } & \multicolumn{2}{|c|}{ G6 } & \multicolumn{2}{|c|}{ G7 } \\
\hline $\begin{array}{l}\Delta \log _{10} \\
\tau_{M}(s)\end{array}$ & $\begin{array}{c}\mathbf{G}_{\text {im }} \\
(\mathrm{GPa})\end{array}$ & $\begin{array}{l}\Delta \log _{10} \\
\tau_{M}(s)\end{array}$ & $\begin{array}{l}\mathbf{G}_{\text {im }} \\
(\mathrm{GPa})\end{array}$ & $\begin{array}{l}\Delta \log _{10} \\
\tau_{M}(s) \\
\end{array}$ & $\begin{array}{c}\mathbf{G}_{\text {im }} \\
(\mathrm{GPa})\end{array}$ & $\begin{array}{l}\Delta \log _{10} \\
\tau_{M}(s)\end{array}$ & $\begin{array}{c}\mathbf{G}_{\text {im }} \\
(\mathbf{G P a})\end{array}$ & $\begin{array}{l}\Delta \log _{10} \\
\tau_{M} \text { (s) }\end{array}$ & $\begin{array}{c}\mathbf{G}_{\text {im }} \\
(\mathrm{GPa})\end{array}$ & $\begin{array}{l}\Delta \log _{10} \\
\tau_{M}(s)\end{array}$ & $\begin{array}{c}\mathbf{G}_{\mathrm{im}} \\
(\mathrm{GPa})\end{array}$ & $\begin{array}{l}\Delta \log _{10} \\
\tau_{\mathrm{M}}(\mathrm{s})\end{array}$ & $\begin{array}{c}\mathbf{G}_{\text {im }} \\
(\mathrm{GPa})\end{array}$ & $\begin{array}{l}\Delta \log _{10} \\
\tau_{M}(s)\end{array}$ & $\begin{array}{c}\mathbf{G}_{\mathrm{im}} \\
(\mathrm{GPa})\end{array}$ \\
\hline 1.942 & 0.172 & 3.127 & 2.805 & 2.391 & 9.342 & 1.944 & 3.359 & 1.497 & 4.174 & 1.943 & 1.708 & 2.046 & 1.478 & & \\
\hline 2.088 & 0.123 & 3.301 & 2.424 & 2.396 & 9.315 & 1.989 & 3.195 & 1.565 & 3.683 & 1.956 & 1.666 & 2.092 & 1.387 & & \\
\hline 2.161 & 0.104 & 3.385 & 2.241 & 2.420 & 9.171 & 2.047 & 3.000 & 1.778 & 2.462 & 2.036 & 1.423 & 2.182 & 1.213 & & \\
\hline 2.243 & 0.086 & 3.389 & 2.233 & 2.689 & 6.926 & 2.103 & 2.831 & 1.790 & 2.406 & 2.105 & 1.235 & 2.347 & 0.912 & & \\
\hline 2.389 & 0.062 & 3.419 & 2.168 & 2.692 & 6.896 & 2.245 & 2.477 & 1.798 & 2.369 & 2.198 & 1.014 & 2.393 & 0.834 & & \\
\hline 2.559 & 0.042 & 3.525 & 1.929 & 2.727 & 6.544 & 2.290 & 2.383 & 1.816 & 2.289 & 2.337 & 0.751 & 2.415 & 0.800 & & \\
\hline 2.641 & 0.034 & 3.602 & 1.751 & 2.818 & 5.655 & 2.328 & 2.309 & 1.866 & 2.083 & 2.354 & 0.723 & 2.580 & 0.569 & & \\
\hline 2.787 & 0.025 & 3.720 & 1.474 & 2.887 & 4.992 & 2.348 & 2.272 & 2.091 & 1.361 & 2.406 & 0.645 & 2.602 & 0.543 & & \\
\hline 2.860 & 0.021 & 3.787 & 1.319 & 2.993 & 4.071 & 2.404 & 2.172 & 2.117 & 1.293 & 2.499 & 0.526 & 2.716 & 0.424 & & \\
\hline 2.942 & 0.017 & 3.826 & 1.233 & 3.029 & 3.787 & 2.629 & 1.799 & 2.176 & 1.154 & 2.655 & 0.373 & 2.791 & 0.358 & & \\
\hline 3.088 & 0.012 & 3.903 & 1.072 & 3.087 & 3.356 & 2.643 & 1.776 & 2.196 & 1.109 & 2.707 & 0.334 & 2.881 & 0.293 & & \\
\hline 3.243 & 0.009 & 4.088 & 0.749 & 3.119 & 3.137 & 2.649 & 1.766 & 2.242 & 1.013 & 2.735 & 0.314 & 2.903 & 0.279 & & \\
\hline 3.305 & 0.008 & 4.117 & 0.707 & 3.188 & 2.700 & 2.802 & 1.521 & 2.418 & 0.707 & 2.897 & 0.221 & 3.017 & 0.215 & & \\
\hline 3.389 & 0.006 & 4.127 & 0.694 & 3.388 & 1.736 & 2.897 & 1.373 & 2.477 & 0.626 & 3.036 & 0.166 & 3.092 & 0.181 & & \\
\hline 3.606 & 0.004 & 4.280 & 0.516 & 3.391 & 1.724 & 2.930 & 1.322 & 2.489 & 0.610 & 3.105 & 0.144 & 3.182 & 0.148 & & \\
\hline 3.641 & 0.003 & 4.301 & 0.496 & 3.420 & 1.615 & 2.944 & 1.301 & 2.497 & 0.600 & 3.198 & 0.119 & 3.301 & 0.113 & & \\
\hline 3.787 & 0.003 & 4.419 & 0.403 & 3.586 & 1.107 & 3.047 & 1.149 & 2.543 & 0.545 & 3.337 & 0.091 & 3.415 & 0.087 & & \\
\hline 3.942 & 0.002 & 4.525 & 0.344 & 3.689 & 0.876 & 3.103 & 1.069 & 2.790 & 0.325 & 3.406 & 0.080 & 3.580 & 0.059 & & \\
\hline 4.004 & 0.002 & 4.581 & 0.320 & 3.692 & 0.870 & 3.198 & 0.939 & 2.798 & 0.320 & 3.499 & 0.068 & 3.602 & 0.056 & & \\
\hline 4.088 & 0.001 & 4.602 & 0.313 & 3.818 & 0.652 & 3.328 & 0.780 & 2.816 & 0.308 & 3.735 & 0.047 & 3.716 & 0.043 & & \\
\hline 4.305 & 0.001 & 4.720 & 0.283 & 3.887 & 0.556 & 3.348 & 0.758 & 2.941 & 0.239 & 3.864 & 0.039 & 3.881 & 0.030 & & \\
\hline 4.389 & 0.001 & 4.826 & 0.273 & 4.087 & 0.352 & 3.404 & 0.700 & 3.027 & 0.202 & 3.897 & 0.037 & 3.903 & 0.028 & & \\
\hline 4.606 & 0.000 & 4.903 & 0.274 & 4.119 & 0.327 & 3.596 & 0.548 & 3.091 & 0.178 & 4.036 & 0.032 & 4.017 & 0.022 & & \\
\hline 4.787 & 0.000 & 4.979 & 0.282 & 4.188 & 0.279 & 3.629 & 0.529 & 3.117 & 0.169 & 4.165 & 0.028 & 4.301 & 0.012 & & \\
\hline 5.004 & 0.000 & 5.117 & 0.309 & 4.388 & 0.177 & 3.649 & 0.518 & 3.196 & 0.146 & 4.198 & 0.028 & 4.415 & 0.010 & & \\
\hline 5.088 & 0.000 & 5.280 & 0.350 & 4.420 & 0.165 & 3.802 & 0.458 & 3.242 & 0.134 & 4.337 & 0.025 & 4.545 & 0.008 & & \\
\hline 5.305 & 0.000 & 5.301 & 0.355 & 4.586 & 0.114 & 3.897 & 0.436 & 3.328 & 0.116 & 4.563 & 0.023 & 4.602 & 0.007 & & \\
\hline 5.606 & 0.000 & 5.419 & 0.377 & 4.727 & 0.084 & 4.047 & 0.418 & 3.489 & 0.089 & 4.735 & 0.023 & 4.716 & 0.006 & & \\
\hline 6.004 & 0.000 & 5.581 & 0.382 & 4.818 & 0.069 & 4.103 & 0.414 & 3.497 & 0.087 & 4.864 & 0.024 & 4.846 & 0.006 & & \\
\hline 6.305 & 0.000 & 5.602 & 0.380 & 4.887 & 0.060 & 4.198 & 0.412 & 3.543 & 0.082 & 5.036 & 0.026 & 4.903 & 0.006 & & \\
\hline & & 5.720 & 0.363 & 5.028 & 0.047 & 4.348 & 0.415 & 3.725 & 0.063 & 5.165 & 0.029 & 5.244 & 0.007 & & \\
\hline & & 5.979 & 0.315 & 5.119 & 0.041 & 4.404 & 0.416 & 3.790 & 0.058 & 5.563 & 0.046 & 5.301 & 0.008 & & \\
\hline & & 6.117 & 0.306 & 5.188 & 0.037 & 4.596 & 0.419 & 3.798 & 0.058 & 5.864 & 0.076 & 5.545 & 0.012 & & \\
\hline & & 6.150 & 0.306 & 5.426 & 0.033 & 4.613 & 0.419 & 3.941 & 0.049 & 5.929 & 0.086 & 5.602 & 0.014 & & \\
\hline & & 6.280 & 0.319 & 5.452 & 0.033 & 4.802 & 0.409 & 4.027 & 0.045 & 6.165 & 0.137 & 5.846 & 0.024 & & \\
\hline & & 6.419 & 0.353 & 5.586 & 0.035 & 4.897 & 0.396 & 4.196 & 0.039 & 6.230 & 0.156 & 6.244 & 0.058 & & \\
\hline & & 6.451 & 0.364 & 5.727 & 0.041 & 4.914 & 0.393 & 4.242 & 0.037 & 6.563 & 0.316 & 6.545 & 0.116 & & \\
\hline
\end{tabular}


Appendix 4b. Parameters of the lines fitted to the imaginary shear modulus data for NS2 melt (G0) and Fe-free melts (G1-G7) - continuation...

\begin{tabular}{|c|c|c|c|c|c|c|c|c|c|c|c|c|c|c|c|}
\hline \multicolumn{16}{|c|}{ FITS TO THE IMAGINARY SHEAR MODULUS DATA } \\
\hline \multicolumn{2}{|c|}{ G0 } & \multicolumn{2}{|c|}{ G1 } & \multicolumn{2}{|c|}{ G2 } & \multicolumn{2}{|c|}{ G3 } & \multicolumn{2}{|c|}{ G4 } & \multicolumn{2}{|c|}{ G5 } & \multicolumn{2}{|c|}{ G6 } & \multicolumn{2}{|c|}{ G7 } \\
\hline $\begin{array}{l}\Delta \log _{10} \\
\tau_{M}(\mathrm{~s})\end{array}$ & $\begin{array}{c}\mathbf{G}_{\text {im }} \\
(\mathrm{GPa})\end{array}$ & $\begin{array}{l}\log _{10} \\
\tau_{M}(\mathbf{s})\end{array}$ & $\begin{array}{c}\mathrm{G}_{\mathrm{im}} \\
(\mathrm{GPa})\end{array}$ & $\begin{array}{l}\log _{10} \\
\tau_{\mathrm{M}} \text { (s) }\end{array}$ & $\begin{array}{c}\mathbf{G}_{i m} \\
(\mathbf{G P a})\end{array}$ & $\begin{array}{l}\log _{10} \\
\tau_{M}(\mathbf{s})\end{array}$ & $\begin{array}{c}G_{i m} \\
\text { (GPa) }\end{array}$ & $\begin{array}{l}\Delta \log _{10} \\
\tau_{M} \text { (s) }\end{array}$ & $\begin{array}{c}\mathbf{G}_{\mathrm{im}} \\
(\mathrm{GPa})\end{array}$ & $\begin{array}{l}\log _{10} \\
\tau_{M}(\mathrm{~s})\end{array}$ & $\begin{array}{c}\mathbf{G}_{i m} \\
\text { (GPa) }\end{array}$ & $\begin{array}{l}\Delta \log _{10} \\
\tau_{M}(\mathrm{~s})\end{array}$ & $\begin{array}{c}\mathbf{G}_{i m} \\
(\mathrm{GPa})\end{array}$ & $\begin{array}{l}\Delta \log _{10} \\
\tau_{M}(\mathrm{~s})\end{array}$ & $\begin{array}{c}\mathbf{G}_{\text {im }} \\
\text { (GPa) }\end{array}$ \\
\hline & & 6.581 & 0.422 & 5.753 & 0.043 & 20.103 & 0.345 & 4.328 & 0.035 & 6.628 & 0.363 & 6.757 & 0.185 & & \\
\hline & & 6.849 & 0.624 & 5.887 & 0.054 & 5.198 & 0.311 & 4.497 & 0.032 & 6.864 & 0.596 & 6.846 & 0.225 & & \\
\hline & & 6.979 & 0.771 & 6.028 & 0.070 & 5.312 & 0.267 & 4.543 & 0.031 & 6.929 & 0.678 & 7.058 & 0.344 & & \\
\hline & & 7.150 & 1.002 & 6.151 & 0.091 & 5.596 & 0.173 & 4.725 & 0.028 & 7.230 & 1.129 & 7.244 & 0.467 & & \\
\hline & & 7.280 & 1.172 & $\begin{array}{l}6.426 \\
6.426\end{array}$ & 0.166 & 5.613 & 0.169 & 4.731 & 0.028 & 7.628 & 1.368 & $\begin{array}{l}7.244 \\
7.456\end{array}$ & 0.583 & & \\
\hline & & $\begin{array}{l}7.451 \\
7.451\end{array}$ & 1.305 & $\begin{array}{l}0.420 \\
6.452\end{array}$ & 0.176 & $\begin{array}{l}5.010 \\
5.897\end{array}$ & 0.127 & $\begin{array}{l}4.941 \\
4.941\end{array}$ & 0.027 & $\begin{array}{l}7.020 \\
7.929\end{array}$ & $\begin{array}{l}1.300 \\
1.043\end{array}$ & $\begin{array}{l}7.4500 \\
7.545\end{array}$ & 0.610 & & \\
\hline & & $\begin{array}{l}7.451 \\
7.849\end{array}$ & 0.973 & $\begin{array}{l}0.452 \\
6.727\end{array}$ & 0.325 & $\begin{array}{l}5.891 \\
5.914\end{array}$ & 0.126 & $\begin{array}{l}4.941 \\
5.027\end{array}$ & 0.027 & $\begin{array}{l}7.929 \\
8.230\end{array}$ & $\begin{array}{l}1.043 \\
0.649\end{array}$ & $\begin{array}{l}7.545 \\
7.757\end{array}$ & $\begin{array}{l}0.010 \\
0.610\end{array}$ & & \\
\hline & & 8.150 & 0.556 & 6.753 & 0.344 & 6.312 & 0.157 & 5.032 & 0.027 & 8.628 & 0.292 & 8.058 & 0.499 & & \\
\hline & & $\begin{array}{l}8.150 \\
8.247\end{array}$ & $\begin{array}{l}0.556 \\
0.452\end{array}$ & $\begin{array}{l}6.153 \\
7.028\end{array}$ & $\begin{array}{l}0.344 \\
0.608\end{array}$ & $\begin{array}{l}6.312 \\
6.526\end{array}$ & 0.218 & $\begin{array}{l}5.032 \\
5.242\end{array}$ & $\begin{array}{l}.027 \\
0.028\end{array}$ & $\begin{array}{l}8.628 \\
8.929\end{array}$ & $\begin{array}{l}0.292 \\
0.153\end{array}$ & $\begin{array}{l}8.058 \\
8.456\end{array}$ & $\begin{array}{l}.499 \\
0.268\end{array}$ & & \\
\hline & & 8.451 & 0.288 & 7.151 & 0.761 & 6.613 & 0.254 & 5.328 & 0.029 & & & 8.757 & 0.142 & & \\
\hline & & 8.548 & 0.232 & 7.415 & 1.083 & 6.827 & 0.360 & 5.430 & 0.030 & & & 9.058 & 0.072 & & \\
\hline & & 8.849 & 0.117 & $\begin{array}{l}7.410 \\
7.426\end{array}$ & 1.094 & $\begin{array}{l}6.914 \\
6.027\end{array}$ & 0.408 & $\begin{array}{l}5.725 \\
5.725\end{array}$ & 0.039 & & & 9.456 & 0.029 & & \\
\hline & & 8.946 & 0.093 & 7.452 & 1.118 & 7.225 & 0.570 & 5.731 & 0.039 & & & 9.757 & 0.015 & & \\
\hline & & 9.150 & 0.058 & 7.716 & 1.236 & 7.312 & 0.604 & 6.027 & 0.059 & & & & & & \\
\hline & & 9.247 & 0.047 & 7.727 & 1.236 & 7.526 & 0.633 & 6.032 & 0.060 & & & & & & \\
\hline & & 9.548 & 0.023 & 7.753 & 1.235 & 7.613 & 0.617 & 6.430 & 0.123 & & & & & & \\
\hline & & 9.946 & 0.009 & 8.114 & 1.008 & 7.827 & 0.527 & 6.643 & 0.189 & & & & & & \\
\hline & & 10.247 & 0.005 & 8.151 & 0.965 & 8.225 & 0.303 & 6.731 & 0.226 & & & & & & \\
\hline & & 10.548 & 0.002 & $\begin{array}{l}8.415 \\
8.151\end{array}$ & 0.638 & $\begin{array}{l}8.526 \\
8.020\end{array}$ & 0.168 & 6.944 & 0.344 & & & & & & \\
\hline & & 10.615 & 0.002 & $\begin{array}{l}0.415 \\
8.452\end{array}$ & $\begin{array}{l}0.0305 \\
0.595\end{array}$ & $\begin{array}{l}0.620 \\
8.671\end{array}$ & $\begin{array}{l}0.120 \\
0.122\end{array}$ & $\begin{array}{l}0.944 \\
7.032\end{array}$ & 0.405 & & & & & & \\
\hline & & 10.916 & 0.001 & 8.716 & 0.344 & 8.827 & 0.086 & 7.342 & 0.614 & & & & & & \\
\hline & & 10.946 & 0.001 & 9.114 & 0.141 & 8.972 & 0.062 & 7.430 & 0.646 & & & & & & \\
\hline & & 11.247 & 0.001 & 9.415 & 0.071 & 9.225 & 0.035 & 7.643 & 0.622 & & & & & & \\
\hline & & 11.314 & 0.000 & 9.625 & 0.044 & 9.370 & 0.025 & 7.731 & 0.574 & & & & & & \\
\hline & & 11.615 & 0.000 & 9.716 & 0.035 & 9.526 & 0.018 & $\begin{array}{l}7.944 \\
7.944\end{array}$ & 0.422 & & & & & & \\
\hline & & 11.916 & 0.000 & $\begin{array}{l}9.926 \\
9.920\end{array}$ & 0.022 & $\begin{array}{l}9.671 \\
9.670\end{array}$ & 0.013 & $\begin{array}{l}8.342 \\
8.342\end{array}$ & 0.194 & & & & & & \\
\hline & & 12.314 & 0.000 & 10.114 & 0.014 & $\begin{array}{l}5.011 \\
9.972\end{array}$ & 0.006 & $\begin{array}{l}0.644 \\
8.643\end{array}$ & 0.104 & & & & & & \\
\hline & & 12.615 & 0.000 & 10.324 & 0.009 & 10.370 & 0.003 & 8.944 & 0.057 & & & & & & \\
\hline & & 12.916 & 0.000 & 10.415 & 0.007 & 10.671 & 0.001 & 9.342 & 0.025 & & & & & & \\
\hline & & 13.311 & 0.000 & 10.625 & 0.004 & 10.972 & 0.001 & 9.643 & 0.013 & & & & & & \\
\hline & & 13.314 & 0.000 & 10.926 & 0.002 & 11.370 & 0.000 & & & & & & & & \\
\hline & & 13.612 & 0.000 & 11.324 & 0.001 & 11.671 & 0.000 & & & & & & & & \\
\hline & & 13.615 & 0.000 & $\begin{array}{l}11.625 \\
11.625\end{array}$ & 0.000 & & & & & & & & & & \\
\hline & & 14.010 & 0.000 & $\begin{array}{l}11.926 \\
11.926\end{array}$ & 0.000 & & & & & & & & & & \\
\hline & & 14.311 & 0.000 & 12133 & 0.000 & & & & & & & & & & \\
\hline & & 14.612 & 0.000 & 12.324 & 0.000 & & & & & & & & & & \\
\hline & & 15.010 & 0.000 & 12.434 & 0.000 & & & & & & & & & & \\
\hline
\end{tabular}


Appendix 4b. Parameters of the lines fitted to the imaginary shear modulus data for NS2 melt (G0) and Fe-free melts (G1-G7) - continuation..

\begin{tabular}{|c|c|c|c|c|c|c|c|c|c|c|c|c|c|c|c|}
\hline \multicolumn{16}{|c|}{ FITS TO THE IMAGINARY SHEAR MODULUS DATA } \\
\hline \multicolumn{2}{|c|}{ G0 } & \multicolumn{2}{|c|}{ G1 } & \multicolumn{2}{|c|}{ G2 } & \multicolumn{2}{|c|}{ G3 } & \multicolumn{2}{|c|}{ G4 } & \multicolumn{2}{|c|}{ G5 } & \multicolumn{2}{|c|}{ G6 } & \multicolumn{2}{|c|}{ G7 } \\
\hline $\begin{array}{l}\Delta \log _{10} \\
\tau_{M}(s)\end{array}$ & $\begin{array}{l}\mathbf{G}_{\text {im }} \\
(\mathrm{GPa})\end{array}$ & $\begin{array}{l}\Delta \log _{10} \\
\tau_{\mathrm{M}}(\mathrm{s})\end{array}$ & $\begin{array}{l}\mathbf{G}_{\text {im }} \\
(\mathrm{GPa})\end{array}$ & $\begin{array}{l}\Delta \log _{10} \\
\tau_{M} \text { (s) }\end{array}$ & $\begin{array}{l}\mathbf{G}_{\text {im }} \\
(\mathrm{GPa})\end{array}$ & $\begin{array}{l}\Delta \log _{10} \\
\tau_{M}(\mathrm{~s})\end{array}$ & $\begin{array}{l}\mathbf{G}_{\mathrm{im}} \\
(\mathrm{GPa})\end{array}$ & $\begin{array}{l}\Delta \log _{10} \\
\tau_{M}(s)\end{array}$ & $\begin{array}{l}\mathbf{G}_{\mathrm{im}} \\
(\mathrm{GPa})\end{array}$ & $\begin{array}{l}\Delta \log _{10} \\
\tau_{M}(s)\end{array}$ & $\begin{array}{c}\mathbf{G}_{\text {im }} \\
(\mathrm{GPa})\end{array}$ & $\begin{array}{l}\Delta \log _{10} \\
\tau_{M}(s)\end{array}$ & $\begin{array}{l}\mathbf{G}_{\mathrm{im}} \\
(\mathrm{GPa})\end{array}$ & $\begin{array}{l}\Delta \log _{10} \\
\tau_{\mathrm{M}}(\mathrm{s})\end{array}$ & $\begin{array}{l}\mathbf{G}_{\mathrm{im}} \\
(\mathrm{GPa})\end{array}$ \\
\hline & & $\begin{array}{l}15.311 \\
15.612 \\
16.010 \\
16.311 \\
16.408 \\
16.709 \\
17.107 \\
17.408 \\
17.709 \\
18.107 \\
18.408 \\
18.709 \\
19.107 \\
19.408 \\
20.002 \\
20.303 \\
20.701 \\
21.002 \\
21.303 \\
21.701 \\
22.002 \\
22.303 \\
22.701 \\
23.002 \\
24.223 \\
24.524 \\
24.922 \\
25.223 \\
25.524 \\
25.922 \\
26.223 \\
26.524 \\
26.922 \\
27.223\end{array}$ & $\begin{array}{l}0.000 \\
0.000 \\
0.000 \\
0.000 \\
0.000 \\
0.000 \\
0.000 \\
0.000 \\
0.000 \\
0.000 \\
0.000 \\
0.000 \\
0.000 \\
0.000 \\
0.000 \\
0.000 \\
0.000 \\
0.000 \\
0.000 \\
0.000 \\
0.000 \\
0.000 \\
0.000 \\
0.000 \\
0.000 \\
0.000 \\
0.000 \\
0.000 \\
0.000 \\
0.000 \\
0.000 \\
0.000 \\
0.000 \\
0.000\end{array}$ & $\begin{array}{l}12.625 \\
12.832 \\
13.133 \\
13.434 \\
13.832 \\
14.133 \\
14.434 \\
14.832 \\
15.133\end{array}$ & $\begin{array}{l}0.000 \\
0.000 \\
0.000 \\
0.000 \\
0.000 \\
0.000 \\
0.000 \\
0.000 \\
0.000\end{array}$ & & & & & & & & & & \\
\hline
\end{tabular}


Appendix 4c. Parameters of the lines fitted to the real shear modulus data for Fe-bearing melts (G8-G14). See also Fig. 69a.

\begin{tabular}{|c|c|c|c|c|c|c|c|c|c|c|c|c|c|}
\hline \multicolumn{14}{|c|}{ FITS TO THE REAL SHEAR MODULUS DATA } \\
\hline \multicolumn{2}{|c|}{ G8 } & \multicolumn{2}{|c|}{ G9 } & \multicolumn{2}{|c|}{ G10 } & \multicolumn{2}{|c|}{ G11 } & \multicolumn{2}{|c|}{ G12 } & \multicolumn{2}{|c|}{ G13 } & \multicolumn{2}{|c|}{ G14 } \\
\hline $\begin{array}{l}\Delta \log _{10} \\
\tau_{M}(\mathrm{~s}) \\
\end{array}$ & $\begin{array}{c}\mathbf{G}_{\text {real }} \\
(\mathrm{GPa})\end{array}$ & $\begin{array}{l}\Delta \log _{10} \\
\tau_{\mathrm{M}}(\mathrm{s}) \\
\end{array}$ & $\begin{array}{c}G_{\text {real }} \\
(\mathrm{GPa})\end{array}$ & $\begin{array}{l}\Delta \log _{10} \\
\tau_{M}(\mathbf{s}) \\
\end{array}$ & $\begin{array}{c}G_{\text {real }} \\
(\mathrm{GPa}) \\
\end{array}$ & $\begin{array}{l}\Delta \log _{10} \\
\tau_{\mathrm{M}}(\mathrm{s}) \\
\end{array}$ & $\begin{array}{c}\mathbf{G}_{\text {real }} \\
(\mathrm{GPa}) \\
\end{array}$ & $\begin{array}{l}\Delta \log _{10} \\
\tau_{M}(\mathrm{~s}) \\
\end{array}$ & $\begin{array}{l}G_{\text {real }} \\
(\mathrm{GPa}) \\
\end{array}$ & $\begin{array}{l}\Delta \log _{10} \\
\tau_{M}(\mathrm{~s}) \\
\end{array}$ & $\begin{array}{c}G_{\text {real }} \\
(\mathrm{GPa}) \\
\end{array}$ & $\begin{array}{l}\Delta \log _{10} \\
\tau_{\mathrm{M}}(\mathrm{s}) \\
\end{array}$ & $\begin{array}{r}\mathbf{G}_{\text {real }} \\
(\mathrm{GPa}) \\
\end{array}$ \\
\hline-2.000 & 0.006 & -2.000 & 0.003 & -2.000 & 0.001 & -2.000 & 0.000 & -2.000 & 0.068 & -2.000 & 0.004 & -2.000 & 0.002 \\
\hline-1.600 & 0.013 & -1.600 & 0.007 & -1.600 & 0.002 & -1.600 & 0.001 & -1.500 & 0.185 & -1.750 & 0.007 & -1.600 & 0.007 \\
\hline-1.300 & 0.018 & -1.300 & 0.011 & -1.289 & 0.004 & -1.300 & 0.004 & -1.100 & 0.322 & -1.494 & 0.013 & -1.300 & 0.022 \\
\hline-1.000 & 0.024 & -1.185 & 0.012 & -1.093 & 0.008 & -1.064 & 0.011 & -0.815 & 0.467 & -1.147 & 0.037 & -0.986 & 0.085 \\
\hline-0.796 & 0.029 & -0.927 & 0.017 & -0.891 & 0.018 & -0.851 & 0.030 & -0.703 & 0.543 & -1.096 & 0.045 & -0.588 & 0.487 \\
\hline-0.595 & 0.034 & -0.884 & 0.018 & -0.791 & 0.028 & -0.763 & 0.045 & -0.514 & 0.717 & -0.846 & 0.110 & -0.579 & 0.506 \\
\hline-0.398 & 0.040 & -0.664 & 0.026 & -0.590 & 0.066 & -0.557 & 0.115 & -0.402 & 0.863 & -0.795 & 0.133 & -0.287 & 1.660 \\
\hline-0.294 & 0.045 & -0.626 & 0.029 & -0.575 & 0.071 & -0.550 & 0.119 & -0.202 & 1.270 & -0.494 & 0.381 & -0.278 & 1.717 \\
\hline-0.149 & 0.055 & -0.486 & 0.041 & -0.394 & 0.158 & -0.365 & 0.276 & -0.116 & 1.543 & -0.469 & 0.413 & 0.014 & 4.360 \\
\hline-0.097 & 0.060 & -0.363 & 0.059 & -0.289 & 0.252 & -0.317 & 0.342 & -0.101 & 1.601 & -0.448 & 0.443 & 0.037 & 4.630 \\
\hline-0.073 & 0.063 & -0.228 & 0.094 & -0.274 & 0.270 & -0.256 & 0.449 & 0.099 & 2.685 & -0.415 & 0.492 & 0.120 & 5.634 \\
\hline 0.104 & 0.093 & -0.185 & 0.110 & -0.093 & 0.600 & -0.249 & 0.465 & 0.185 & 3.405 & -0.168 & 1.076 & 0.164 & 6.193 \\
\hline 0.152 & 0.106 & -0.124 & 0.140 & -0.036 & 0.764 & -0.064 & 1.048 & 0.297 & 4.622 & -0.147 & 1.151 & 0.338 & 8.559 \\
\hline 0.204 & 0.123 & 0.035 & 0.263 & 0.109 & 1.400 & -0.029 & 1.217 & 0.439 & 6.559 & -0.114 & 1.277 & 0.412 & 9.608 \\
\hline 0.228 & 0.132 & 0.073 & 0.307 & 0.124 & 1.487 & -0.016 & 1.283 & 0.486 & 7.274 & -0.096 & 1.353 & 0.421 & 9.739 \\
\hline 0.405 & 0.239 & 0.116 & 0.366 & 0.209 & 2.067 & 0.142 & 2.447 & 0.497 & 7.439 & -0.009 & 1.785 & 0.465 & 10.373 \\
\hline 0.453 & 0.285 & 0.177 & 0.470 & 0.265 & 2.544 & 0.149 & 2.520 & 0.598 & 8.996 & 0.154 & 2.999 & 0.713 & 13.849 \\
\hline 0.470 & 0.303 & 0.336 & 0.889 & 0.410 & 4.144 & 0.237 & 3.505 & 0.740 & 11.011 & 0.205 & 3.507 & 0.722 & 13.968 \\
\hline 0.602 & 0.500 & 0.374 & 1.033 & 0.425 & 4.338 & 0.272 & 3.970 & 0.798 & 11.751 & 0.230 & 3.780 & 0.736 & 14.155 \\
\hline 0.626 & 0.547 & 0.438 & 1.321 & 0.525 & 5.753 & 0.285 & 4.144 & 0.884 & 12.758 & 0.241 & 3.901 & 0.863 & 15.724 \\
\hline 0.706 & 0.743 & 0.514 & 1.757 & 0.606 & 7.050 & 0.443 & 6.766 & 0.899 & 12.929 & 0.284 & 4.427 & 0.944 & 16.665 \\
\hline 0.771 & 0.948 & 0.575 & 2.196 & 0.663 & 8.000 & 0.450 & 6.906 & 1.099 & 14.988 & 0.506 & 7.756 & 1.014 & 17.433 \\
\hline 0.851 & 1.270 & 0.637 & 2.725 & 0.711 & 8.853 & 0.523 & 8.314 & 1.109 & 15.099 & 0.531 & 8.163 & 1.037 & 17.681 \\
\hline 0.903 & 1.532 & 0.739 & 3.827 & 0.726 & 9.123 & 0.635 & 10.546 & 1.137 & 15.385 & 0.542 & 8.337 & 1.120 & 18.523 \\
\hline 0.927 & 1.664 & 0.772 & 4.246 & 0.826 & 10.981 & 0.670 & 11.241 & 1.185 & 15.879 & 0.552 & 8.511 & 1.164 & 18.949 \\
\hline 1.036 & 2.399 & 0.815 & 4.836 & 0.907 & 12.556 & 0.683 & 11.484 & 1.297 & 17.122 & 0.585 & 9.044 & 1.245 & 19.698 \\
\hline 1.104 & 2.986 & 0.876 & 5.759 & 0.964 & 13.641 & 0.744 & 12.665 & 1.411 & 18.482 & 0.832 & 12.667 & 1.412 & 21.098 \\
\hline 1.152 & 3.459 & 1.022 & 8.200 & 1.109 & 16.309 & 0.751 & 12.807 & 1.439 & 18.829 & 0.853 & 12.934 & 1.421 & 21.169 \\
\hline 1.169 & 3.642 & 1.035 & 8.430 & 1.110 & 16.323 & 0.824 & 14.157 & 1.486 & 19.416 & 0.904 & 13.551 & 1.465 & 21.508 \\
\hline 1.204 & 4.045 & 1.073 & 9.097 & 1.124 & 16.562 & 0.936 & 16.099 & 1.497 & 19.549 & 0.940 & 13.963 & 1.643 & 22.760 \\
\hline 1.228 & 4.331 & 1.116 & 9.838 & 1.209 & 17.885 & 0.971 & 16.673 & 1.598 & 20.767 & 0.984 & 14.465 & 1.713 & 23.190 \\
\hline 1.337 & 5.833 & 1.137 & 10.190 & 1.224 & 18.102 & 0.984 & 16.872 & 1.740 & 22.231 & 1.154 & 16.300 & 1.722 & 23.244 \\
\hline 1.405 & 6.922 & 1.177 & 10.861 & 1.265 & 18.655 & 1.100 & 18.626 & 1.798 & 22.726 & 1.205 & 16.853 & 1.765 & 23.488 \\
\hline 1.453 & 7.729 & 1.323 & 13.053 & 1.410 & 20.262 & 1.142 & 19.201 & 1.808 & 22.812 & 1.230 & 17.127 & 1.863 & 24.005 \\
\hline 1.470 & 8.028 & 1.336 & 13.235 & 1.411 & 20.270 & 1.149 & 19.301 & 1.813 & 22.844 & 1.241 & 17.244 & 1.944 & 24.394 \\
\hline 1.602 & 10.384 & 1.374 & 13.745 & 1.425 & 20.401 & 1.222 & 20.231 & 1.884 & 23.347 & 1.285 & 17.748 & 2.066 & 24.921 \\
\hline 1.625 & 10.791 & 1.438 & 14.549 & 1.525 & 21.236 & 1.237 & 20.413 & 2.099 & 24.461 & 1.531 & 20.645 & 2.120 & 25.137 \\
\hline
\end{tabular}


Appendix 4c. Parameters of the lines fitted to the real shear modulus data for Fe-bearing melts (G8-G14) - continuation...

\begin{tabular}{|c|c|c|c|c|c|c|c|c|c|c|c|c|c|}
\hline \multicolumn{14}{|c|}{ FITS TO THE REAL SHEAR MODULUS DATA } \\
\hline \multicolumn{2}{|c|}{ G8 } & \multicolumn{2}{|c|}{ G9 } & \multicolumn{2}{|c|}{ G10 } & \multicolumn{2}{|c|}{ G11 } & \multicolumn{2}{|c|}{ G12 } & \multicolumn{2}{|c|}{ G13 } & \multicolumn{2}{|c|}{ G14 } \\
\hline $\begin{array}{l}\Delta \log _{10} \\
\tau_{M}(s)\end{array}$ & $\begin{array}{c}\mathbf{G}_{\text {real }} \\
(\mathrm{GPa})\end{array}$ & $\begin{array}{l}\Delta \log _{10} \\
\tau_{M}(s)\end{array}$ & $\begin{array}{l}G_{\text {real }} \\
(\mathrm{GPa})\end{array}$ & $\begin{array}{l}\Delta \log _{10} \\
\tau_{M}(\mathrm{~s})\end{array}$ & $\begin{array}{c}G_{\text {real }} \\
(\mathrm{GPa})\end{array}$ & $\begin{array}{l}\Delta \log _{10} \\
\tau_{M}(\mathrm{~s})\end{array}$ & $\begin{array}{c}G_{\text {real }} \\
(\mathrm{GPa})\end{array}$ & $\begin{array}{l}\Delta \log _{10} \\
\tau_{M}(\mathrm{~s})\end{array}$ & $\begin{array}{l}G_{\text {real }} \\
(\mathrm{GPa})\end{array}$ & $\begin{array}{l}\Delta \log _{10} \\
\tau_{M}(s)\end{array}$ & $\begin{array}{c}G_{\text {real }} \\
(\mathrm{GPa})\end{array}$ & $\begin{array}{l}\Delta \log _{10} \\
\tau_{M}(\mathrm{~s})\end{array}$ & $\begin{array}{l}G_{\text {real }} \\
(\mathrm{GPa})\end{array}$ \\
\hline 1.626 & 10.804 & 1.514 & 15.442 & 1.606 & 21.847 & 1.272 & 20.822 & 2.109 & 24.508 & 1.542 & 20.767 & 2.164 & 25.305 \\
\hline 1.706 & 12.198 & 1.575 & 16.123 & 1.663 & 22.255 & 1.401 & 22.182 & 2.114 & 24.525 & 1.552 & 20.888 & 2.245 & 25.600 \\
\hline 1.735 & 12.676 & 1.629 & 16.694 & 1.720 & 22.679 & 1.443 & 22.577 & 2.137 & 24.625 & 1.683 & 22.264 & 2.421 & 26.182 \\
\hline 1.771 & 13.276 & 1.637 & 16.779 & 1.726 & 22.721 & 1.450 & 22.647 & 2.185 & 24.818 & 1.765 & 22.973 & 2.464 & 26.313 \\
\hline 1.851 & 14.532 & 1.721 & 17.651 & 1.809 & 23.357 & 1.523 & 23.291 & 2.411 & 25.749 & 1.832 & 23.470 & 2.465 & 26.316 \\
\hline 1.903 & 15.319 & 1.739 & 17.840 & 1.826 & 23.492 & 1.635 & 24.183 & 2.439 & 25.868 & 1.853 & 23.609 & 2.629 & 26.769 \\
\hline 1.926 & 15.652 & 1.772 & 18.181 & 1.907 & 24.166 & 1.670 & 24.440 & 2.497 & 26.118 & 1.940 & 24.099 & 2.643 & 26.804 \\
\hline 1.927 & 15.663 & 1.815 & 18.625 & 1.964 & 24.644 & 1.704 & 24.678 & 2.512 & 26.183 & 1.984 & 24.312 & 2.765 & 27.072 \\
\hline 2.036 & 17.166 & 1.876 & 19.261 & 2.021 & 25.139 & 1.744 & 24.950 & 2.550 & 26.348 & 2.066 & 24.644 & 2.863 & 27.248 \\
\hline 2.104 & 18.053 & 1.930 & 19.815 & 2.110 & 25.876 & 1.799 & 25.310 & 2.740 & 27.085 & 2.230 & 25.188 & 2.930 & 27.351 \\
\hline 2.152 & 18.645 & 2.022 & 20.758 & 2.124 & 25.990 & 1.824 & 25.468 & 2.798 & 27.280 & 2.241 & 25.220 & 2.944 & 27.370 \\
\hline 2.169 & 18.853 & 2.035 & 20.891 & 2.224 & 26.736 & 1.936 & 26.134 & 2.808 & 27.314 & 2.285 & 25.354 & 3.066 & 27.521 \\
\hline 2.228 & 19.551 & 2.073 & 21.272 & 2.265 & 27.011 & 1.971 & 26.331 & 2.813 & 27.328 & 2.464 & 25.880 & 3.164 & 27.620 \\
\hline 2.240 & 19.693 & 2.137 & 21.888 & 2.358 & 27.566 & 2.005 & 26.516 & 2.851 & 27.446 & 2.531 & 26.076 & 3.245 & 27.692 \\
\hline 2.324 & 20.633 & 2.177 & 22.262 & 2.411 & 27.835 & 2.100 & 27.017 & 3.109 & 28.077 & 2.542 & 26.106 & 3.328 & 27.758 \\
\hline 2.337 & 20.769 & 2.261 & 22.987 & 2.419 & 27.876 & 2.142 & 27.225 & 3.114 & 28.085 & 2.584 & 26.223 & 3.464 & 27.860 \\
\hline 2.405 & 21.497 & 2.323 & 23.481 & 2.425 & 27.902 & 2.222 & 27.609 & 3.137 & 28.132 & 2.683 & 26.480 & 3.541 & 27.919 \\
\hline 2.470 & 22.156 & 2.328 & 23.520 & 2.525 & 28.320 & 2.272 & 27.837 & 3.249 & 28.334 & 2.765 & 26.667 & 3.629 & 27.991 \\
\hline 2.541 & 22.838 & 2.336 & 23.582 & 2.659 & 28.749 & 2.336 & 28.115 & 3.325 & 28.458 & 2.885 & 26.903 & 3.643 & 28.003 \\
\hline 2.625 & 23.569 & 2.438 & 24.314 & 2.663 & 28.758 & 2.401 & 28.386 & 3.411 & 28.586 & 2.940 & 26.995 & 3.765 & 28.116 \\
\hline 2.626 & 23.575 & 2.562 & 25.094 & 2.720 & 28.901 & 2.403 & 28.392 & 3.439 & 28.626 & 2.984 & 27.066 & 3.842 & 28.195 \\
\hline 2.735 & 24.388 & 2.575 & 25.171 & 2.809 & 29.084 & 2.443 & 28.551 & 3.512 & 28.727 & 3.066 & 27.187 & 3.930 & 28.292 \\
\hline 2.771 & 24.627 & 2.629 & 25.468 & 2.826 & 29.114 & 2.523 & 28.851 & 3.550 & 28.777 & 3.241 & 27.428 & 3.944 & 28.308 \\
\hline 2.883 & 25.263 & 2.721 & 25.934 & 2.964 & 29.319 & 2.637 & 29.222 & 3.627 & 28.871 & 3.283 & 27.487 & 4.066 & 28.443 \\
\hline 2.926 & 25.479 & 2.739 & 26.021 & 3.021 & 29.385 & 2.670 & 29.316 & 3.808 & 29.061 & 3.285 & 27.490 & 4.328 & 28.674 \\
\hline 2.927 & 25.482 & 2.876 & 26.624 & 3.025 & 29.389 & 2.704 & 29.404 & 3.813 & 29.064 & 3.446 & 27.719 & 4.464 & 28.746 \\
\hline 2.939 & 25.541 & 2.920 & 26.799 & 3.057 & 29.422 & 2.799 & 29.618 & 3.851 & 29.099 & 3.464 & 27.745 & 4.503 & 28.762 \\
\hline 3.036 & 25.976 & 2.930 & 26.839 & 3.110 & 29.470 & 2.824 & 29.665 & 4.024 & 29.237 & 3.584 & 27.923 & 4.541 & 28.775 \\
\hline 3.169 & 26.558 & 2.960 & 26.956 & 3.224 & 29.553 & 2.971 & 29.876 & 4.109 & 29.296 & 3.683 & 28.068 & 4.629 & 28.800 \\
\hline 3.184 & 26.625 & 3.022 & 27.188 & 3.326 & 29.608 & 2.999 & 29.905 & 4.114 & 29.298 & 3.747 & 28.158 & 4.765 & 28.827 \\
\hline 3.240 & 26.888 & 3.137 & 27.596 & 3.358 & 29.622 & 3.005 & 29.910 & 4.141 & 29.316 & 3.765 & 28.183 & 4.804 & 28.833 \\
\hline 3.324 & 27.305 & 3.221 & 27.877 & 3.411 & 29.643 & 3.035 & 29.938 & 4.249 & 29.385 & 3.885 & 28.346 & 4.842 & 28.838 \\
\hline 3.337 & 27.369 & 3.261 & 28.008 & 3.419 & 29.646 & 3.100 & 29.988 & 4.325 & 29.430 & 3.984 & 28.473 & 4.930 & 28.851 \\
\hline 3.470 & 28.093 & 3.323 & 28.204 & 3.525 & 29.682 & 3.222 & 30.053 & 4.442 & 29.498 & 4.066 & 28.571 & 5.202 & 28.895 \\
\hline 3.541 & 28.484 & 3.328 & 28.220 & 3.659 & 29.722 & 3.300 & 30.082 & 4.512 & 29.537 & 4.145 & 28.659 & 5.240 & 28.904 \\
\hline 3.554 & 28.553 & 3.438 & 28.559 & 3.720 & 29.742 & 3.336 & 30.093 & 4.550 & 29.558 & 4.283 & 28.795 & 5.328 & 28.928 \\
\hline 3.582 & 28.698 & 3.562 & 28.916 & 3.723 & 29.743 & 3.401 & 30.111 & 4.627 & 29.598 & 4.354 & 28.855 & 5.503 & 28.990 \\
\hline
\end{tabular}


Appendix 4c. Parameters of the lines fitted to the real shear modulus data for Fe-bearing melts (G8-G14) - continuation...

\begin{tabular}{|c|c|c|c|c|c|c|c|c|c|c|c|c|c|}
\hline \multicolumn{14}{|c|}{ FITS TO THE REAL SHEAR MODULUS DATA } \\
\hline \multicolumn{2}{|c|}{ G8 } & \multicolumn{2}{|c|}{ G9 } & \multicolumn{2}{|c|}{ G10 } & \multicolumn{2}{|c|}{ G11 } & \multicolumn{2}{|c|}{ G12 } & \multicolumn{2}{|c|}{ G13 } & \multicolumn{2}{|c|}{ G14 } \\
\hline $\begin{array}{l}\Delta \log _{10} \\
\tau_{M}(\mathrm{~s})\end{array}$ & $\begin{array}{c}G_{\text {real }} \\
(\mathrm{GPa})\end{array}$ & $\begin{array}{l}\Delta \log _{10} \\
\tau_{M}(\mathrm{~s})\end{array}$ & $\begin{array}{c}G_{\text {real }} \\
\text { (GPa) }\end{array}$ & $\begin{array}{l}\Delta \log _{10} \\
\tau_{\mathrm{M}}(\mathrm{s})\end{array}$ & $\begin{array}{c}G_{\text {real }} \\
(\mathrm{GPa})\end{array}$ & $\begin{array}{l}\Delta \log _{10} \\
\tau_{M}(s)\end{array}$ & $\begin{array}{l}G_{\text {real }} \\
\text { (GPa) }\end{array}$ & $\begin{array}{l}\Delta \log _{10} \\
\tau_{\mathrm{M}}(\mathrm{s})\end{array}$ & $\begin{array}{l}G_{\text {real }} \\
(\mathrm{GPa})\end{array}$ & $\begin{array}{l}\Delta \log _{10} \\
\tau_{M}(\mathrm{~s})\end{array}$ & $\begin{array}{l}G_{\text {real }} \\
\text { (GPa) }\end{array}$ & $\begin{array}{l}\Delta \log _{10} \\
\tau_{M}(\mathrm{~s})\end{array}$ & $\begin{array}{l}G_{\text {real }} \\
\text { (GPa) }\end{array}$ \\
\hline 3.625 & 28.919 & 3.607 & 29.035 & 3.724 & 29.743 & 3.403 & 30.111 & 4.813 & 29.686 & 4.446 & 28.925 & 5.521 & 28.998 \\
\hline 3.735 & 29.412 & 3.619 & 29.067 & 3.809 & 29.776 & 3.523 & 30.138 & 4.840 & 29.697 & 4.464 & 28.938 & 5.541 & 29.006 \\
\hline 3.855 & 29.830 & 3.629 & 29.093 & 4.021 & 29.908 & 3.637 & 30.163 & 4.851 & 29.702 & 4.584 & 29.014 & 5.629 & 29.048 \\
\hline 3.883 & 29.906 & 3.721 & 29.314 & 4.024 & 29.910 & 3.695 & 30.177 & 4.999 & 29.760 & 4.655 & 29.053 & 5.804 & 29.147 \\
\hline 3.926 & 30.014 & 3.908 & 29.685 & 4.025 & 29.911 & 3.698 & 30.178 & 5.024 & 29.769 & 4.747 & 29.097 & 5.822 & 29.158 \\
\hline 3.939 & 30.043 & 3.920 & 29.705 & 4.057 & 29.941 & 3.704 & 30.179 & 5.141 & 29.807 & 4.765 & 29.105 & 5.842 & 29.170 \\
\hline 4.036 & 30.224 & 3.930 & 29.723 & 4.110 & 29.998 & 3.799 & 30.208 & 5.249 & 29.836 & 4.885 & 29.153 & 6.202 & 29.394 \\
\hline 4.184 & 30.405 & 3.960 & 29.772 & 4.326 & 30.342 & 3.996 & 30.309 & 5.300 & 29.849 & 5.053 & 29.220 & 6.220 & 29.404 \\
\hline 4.240 & 30.453 & 4.022 & 29.868 & 4.358 & 30.408 & 3.999 & 30.311 & 5.325 & 29.854 & 5.145 & 29.262 & 6.240 & 29.413 \\
\hline 4.253 & 30.463 & 4.221 & 30.140 & 4.419 & 30.543 & 4.005 & 30.315 & 5.442 & 29.878 & 5.283 & 29.342 & 6.503 & 29.500 \\
\hline 4.257 & 30.466 & 4.261 & 30.190 & 4.422 & 30.548 & 4.035 & 30.339 & 5.550 & 29.897 & 5.311 & 29.360 & 6.521 & 29.504 \\
\hline 4.324 & 30.512 & 4.306 & 30.245 & 4.454 & 30.622 & 4.100 & 30.398 & 5.627 & 29.910 & 5.354 & 29.391 & 6.541 & 29.508 \\
\hline 4.541 & 30.623 & 4.324 & 30.267 & 4.659 & 31.088 & 4.300 & 30.671 & 5.698 & 29.921 & 5.446 & 29.462 & 6.804 & 29.540 \\
\hline 4.554 & 30.629 & 4.328 & 30.272 & 4.720 & 31.207 & 4.336 & 30.735 & 5.840 & 29.942 & 5.584 & 29.576 & 6.822 & 29.542 \\
\hline 4.558 & 30.631 & 4.562 & 30.551 & 4.723 & 31.211 & 4.394 & 30.846 & 5.904 & 29.952 & 5.612 & 29.599 & 7.202 & 29.559 \\
\hline 4.582 & 30.642 & 4.607 & 30.602 & 4.724 & 31.213 & 4.403 & 30.863 & 5.999 & 29.966 & 5.655 & 29.634 & 7.220 & 29.560 \\
\hline 4.625 & 30.662 & 4.619 & 30.616 & 4.755 & 31.268 & 4.637 & 31.344 & 6.024 & 29.970 & 5.747 & 29.704 & 7.503 & 29.568 \\
\hline 4.855 & 30.778 & 4.625 & 30.623 & 5.024 & 31.584 & 4.695 & 31.451 & 6.141 & 29.986 & 6.010 & 29.871 & 7.521 & 29.569 \\
\hline 4.883 & 30.794 & 4.629 & 30.628 & 5.025 & 31.585 & 4.698 & 31.456 & 6.205 & 29.994 & 6.053 & 29.894 & 7.822 & 29.578 \\
\hline 4.939 & 30.828 & 4.908 & 30.927 & 5.057 & 31.607 & 4.704 & 31.465 & 6.300 & 30.004 & 6.145 & 29.945 & 8.220 & 29.592 \\
\hline 4.956 & 30.839 & 4.920 & 30.939 & 5.153 & 31.658 & 4.996 & 31.813 & 6.325 & 30.007 & 6.311 & 30.038 & 8.521 & 29.607 \\
\hline 4.993 & 30.863 & 4.960 & 30.979 & 5.221 & 31.684 & 4.999 & 31.815 & 6.442 & 30.017 & 6.322 & 30.044 & & \\
\hline 5.184 & 30.997 & 5.023 & 31.039 & 5.326 & 31.712 & 5.035 & 31.839 & 6.603 & 30.032 & 6.354 & 30.062 & & \\
\hline 5.240 & 31.041 & 5.073 & 31.084 & 5.358 & 31.719 & 5.300 & 31.943 & 6.698 & 30.042 & 6.446 & 30.117 & & \\
\hline 5.253 & 31.051 & 5.221 & 31.201 & 5.422 & 31.730 & 5.336 & 31.950 & 6.840 & 30.060 & 6.612 & 30.217 & & \\
\hline 5.257 & 31.054 & 5.261 & 31.229 & 5.454 & 31.734 & 5.394 & 31.960 & 6.860 & 30.063 & 6.623 & 30.223 & & \\
\hline 5.294 & 31.084 & 5.306 & 31.257 & 5.522 & 31.742 & 5.695 & 31.990 & 6.904 & 30.069 & 6.655 & 30.242 & & \\
\hline 5.554 & 31.314 & 5.324 & 31.268 & 5.723 & 31.757 & 5.698 & 31.990 & 6.999 & 30.086 & 7.010 & 30.424 & & \\
\hline 5.558 & 31.318 & 5.374 & 31.296 & 5.724 & 31.757 & 5.996 & 32.010 & 7.141 & 30.114 & 7.021 & 30.429 & & \\
\hline 5.582 & 31.340 & 5.607 & 31.401 & 5.755 & 31.759 & 5.999 & 32.010 & 7.161 & 30.119 & 7.053 & 30.443 & & \\
\hline 5.692 & 31.447 & 5.619 & 31.405 & 5.920 & 31.765 & 6.394 & 32.078 & 7.205 & 30.129 & 7.311 & 30.548 & & \\
\hline 5.765 & 31.520 & 5.625 & 31.408 & 6.024 & 31.769 & 6.695 & 32.094 & 7.300 & 30.152 & 7.322 & 30.553 & & \\
\hline 5.855 & 31.611 & 5.772 & 31.462 & 6.025 & 31.769 & & & 7.559 & 30.227 & 7.354 & 30.564 & & \\
\hline 5.883 & 31.639 & 5.856 & 31.493 & 6.153 & 31.772 & & & 7.603 & 30.240 & 7.392 & 30.578 & & \\
\hline 5.956 & 31.712 & 5.908 & 31.512 & 6.221 & 31.774 & & & 7.698 & 30.269 & 7.612 & 30.651 & & \\
\hline 5.993 & 31.748 & 5.920 & 31.517 & 6.422 & 31.778 & & & 7.860 & 30.317 & 7.623 & 30.654 & & \\
\hline 6.066 & 31.816 & 6.023 & 31.555 & 6.454 & 31.779 & & & 7.871 & 30.320 & 7.693 & 30.673 & & \\
\hline
\end{tabular}


Appendix 4c. Parameters of the lines fitted to the real shear modulus data for Fe-bearing melts (G8-G14) - continuation...

\begin{tabular}{|c|c|c|c|c|c|c|c|c|c|c|c|c|c|}
\hline \multicolumn{14}{|c|}{ FITS TO THE REAL SHEAR MODULUS DATA } \\
\hline \multicolumn{2}{|c|}{ G8 } & \multicolumn{2}{|c|}{ G9 } & \multicolumn{2}{|c|}{ G10 } & \multicolumn{2}{|c|}{ G11 } & \multicolumn{2}{|c|}{ G12 } & \multicolumn{2}{|c|}{ G13 } & \multicolumn{2}{|c|}{ G14 } \\
\hline $\begin{array}{l}\Delta \log _{10} \\
\tau_{M} \text { (s) }\end{array}$ & $\begin{array}{c}G_{\text {real }} \\
(\mathrm{GPa})\end{array}$ & $\begin{array}{l}\Delta \log _{10} \\
\tau_{\mathrm{M}} \text { (s) }\end{array}$ & $\begin{array}{c}G_{\text {real }} \\
\text { (GPa) }\end{array}$ & $\begin{array}{l}\Delta \log _{10} \\
\tau_{\mathrm{M}}(\mathrm{s})\end{array}$ & $\begin{array}{c}G_{\text {real }} \\
(\mathrm{GPa})\end{array}$ & $\begin{array}{l}\Delta \log _{10} \\
\tau_{\mathrm{M}} \text { (s) }\end{array}$ & $\begin{array}{c}\mathbf{G}_{\text {real }} \\
\text { (GPa) }\end{array}$ & $\begin{array}{l}\Delta \log _{10} \\
\tau_{\mathrm{M}}(\mathrm{s})\end{array}$ & $\begin{array}{c}G_{\text {real }} \\
(\mathrm{GPa})\end{array}$ & $\begin{array}{l}\Delta \log _{10} \\
\tau_{\mathrm{M}}(\mathrm{s})\end{array}$ & $\begin{array}{c}G_{\text {real }} \\
\text { (GPa) }\end{array}$ & $\begin{array}{l}\Delta \log _{10} \\
\tau_{\mathrm{M}}(\mathrm{s})\end{array}$ & $\begin{array}{c}G_{\text {real }} \\
\text { (GPa) }\end{array}$ \\
\hline 6.253 & 31.962 & (6.073 & 31.574 & 6.522 & 31.780 & & & 7.904 & 30.329 & 8.010 & 30.728 & & \\
\hline 6.257 & 31.965 & 6.157 & 31.606 & 6.723 & 31.785 & & & 7.999 & 30.355 & 8.021 & 30.729 & & \\
\hline 6.294 & 31.987 & 6.306 & 31.661 & 6.755 & 31.785 & & & 8.161 & 30.396 & 8.091 & 30.736 & & \\
\hline 6.464 & 32.065 & 6.324 & 31.667 & 6.920 & 31.789 & & & 8.173 & 30.398 & 8.311 & 30.750 & & \\
\hline 6.554 & 32.093 & 6.374 & 31.685 & 7.153 & 31.794 & & & 8.205 & 30.406 & 8.322 & 30.750 & & \\
\hline 6.558 & 32.094 & 6.555 & 31.748 & 7.221 & 31.795 & & & 8.559 & 30.470 & 8.392 & 30.753 & & \\
\hline 6.692 & 32.124 & 6.607 & 31.764 & 7.454 & 31.800 & & & 8.570 & 30.472 & 8.525 & 30.758 & & \\
\hline 6.765 & 32.137 & 6.625 & 31.770 & 7.522 & 31.801 & & & 8.603 & 30.477 & 8.623 & 30.761 & & \\
\hline 6.956 & 32.167 & 6.772 & 31.811 & 7.920 & 31.810 & & & 8.860 & 30.516 & 8.693 & 30.763 & & \\
\hline 6.993 & 32.173 & 6.856 & 31.831 & 8.221 & 31.817 & & & 8.871 & 30.517 & 8.826 & 30.766 & & \\
\hline 7.066 & 32.185 & 7.023 & 31.865 & & & & & 8.904 & 30.523 & 9.021 & 30.770 & & \\
\hline 7.257 & 32.221 & 7.073 & 31.874 & & & & & 8.943 & 30.529 & 9.091 & 30.772 & & \\
\hline 7.294 & 32.230 & 7.157 & 31.889 & & & & & 9.161 & 30.574 & 9.223 & 30.774 & & \\
\hline 7.464 & 32.281 & 7.324 & 31.920 & & & & & 9.173 & 30.577 & 9.322 & 30.776 & & \\
\hline 7.692 & 32.393 & 7.374 & 31.929 & & & & & 9.244 & 30.596 & 9.392 & 30.777 & & \\
\hline 7.765 & 32.444 & 7.555 & 31.968 & & & & & 9.559 & 30.708 & 9.525 & 30.780 & & \\
\hline 7.993 & 32.641 & 7.772 & 32.029 & & & & & 9.570 & 30.713 & 9.693 & 30.782 & & \\
\hline 8.066 & 32.709 & 7.856 & 32.059 & & & & & 9.642 & 30.746 & 9.727 & 30.782 & & \\
\hline 8.464 & 32.998 & 8.073 & 32.158 & & & & & 9.860 & 30.857 & 9.826 & 30.783 & & \\
\hline 8.765 & 33.160 & 8.073 & 32.158 & & & & & 9.871 & 30.864 & 10.028 & 30.784 & & \\
\hline & & 8.157 & 32.207 & & & & & 9.943 & 30.903 & 10.091 & 30.784 & & \\
\hline & & 8.157 & 32.207 & & & & & 10.080 & 30.976 & 10.223 & 30.785 & & \\
\hline & & 8.555 & 32.282 & & & & & 10.173 & 31.019 & 10.392 & 30.785 & & \\
\hline & & 8.555 & 32.282 & & & & & 10.244 & 31.048 & 10.426 & 30.785 & & \\
\hline & & 8.856 & 32.125 & & & & & 10.381 & 31.090 & 10.525 & 30.785 & & \\
\hline & & 8.856 & 32.125 & & & & & 10.570 & 31.124 & 10.727 & 30.785 & & \\
\hline & & & & & & & & 10.642 & 31.132 & 10.826 & 30.785 & & \\
\hline & & & & & & & & 10.779 & 31.141 & 11.005 & 30.785 & & \\
\hline & & & & & & & & 10.871 & 31.145 & 11.028 & 30.785 & & \\
\hline & & & & & & & & 10.943 & 31.147 & 11.223 & 30.785 & & \\
\hline & & & & & & & & 11.080 & 31.150 & 11.306 & 30.785 & & \\
\hline & & & & & & & & 11.244 & 31.151 & 11.426 & 30.785 & & \\
\hline & & & & & & & & 11.289 & 31.151 & 11.525 & 30.785 & & \\
\hline & & & & & & & & 11.381 & 31.152 & 11.704 & 30.785 & & \\
\hline & & & & & & & & 11.590 & 31.152 & 11.727 & 30.785 & & \\
\hline & & & & & & & & 11.642 & 31.152 & 12.005 & 30.785 & & \\
\hline & & & & & & & & 11.779 & 31.152 & 12.028 & 30.785 & & \\
\hline
\end{tabular}


Appendix 4c. Parameters of the lines fitted to the real shear modulus data for Fe-bearing melts (G8-G14) - continuation...

\begin{tabular}{|c|c|c|c|c|c|c|c|c|c|c|c|c|c|}
\hline \multicolumn{14}{|c|}{ FITS TO THE REAL SHEAR MODULUS DATA } \\
\hline \multicolumn{2}{|c|}{ G8 } & \multicolumn{2}{|c|}{ G9 } & \multicolumn{2}{|c|}{ G10 } & \multicolumn{2}{|c|}{ G11 } & \multicolumn{2}{|c|}{ G12 } & \multicolumn{2}{|c|}{ G13 } & \multicolumn{2}{|c|}{ G14 } \\
\hline $\begin{array}{l}\Delta \log _{10} \\
\tau_{M}(\mathrm{~s})\end{array}$ & $\begin{array}{c}\mathbf{G}_{\text {real }} \\
(\mathrm{GPa})\end{array}$ & $\begin{array}{l}\Delta \log _{10} \\
\tau_{M}(s)\end{array}$ & $\begin{array}{c}\mathbf{G}_{\text {real }} \\
(\mathrm{GPa})\end{array}$ & $\begin{array}{l}\Delta \log _{10} \\
\tau_{M} \text { (s) }\end{array}$ & $\begin{array}{c}G_{\text {real }} \\
(\mathrm{GPa})\end{array}$ & $\begin{array}{l}\Delta \log _{10} \\
\tau_{M}(s)\end{array}$ & $\begin{array}{c}G_{\text {real }} \\
(\mathrm{GPa})\end{array}$ & $\begin{array}{l}\Delta \log _{10} \\
\tau_{M}(s)\end{array}$ & $\begin{array}{c}G_{\text {real }} \\
(\mathrm{GPa})\end{array}$ & $\begin{array}{l}\Delta \log _{10} \\
\tau_{M}(s)\end{array}$ & $\begin{array}{c}G_{\text {real }} \\
(\mathrm{GPa})\end{array}$ & $\begin{array}{l}\Delta \log _{10} \\
\tau_{M}(s)\end{array}$ & $\begin{array}{c}G_{\text {real }} \\
(\mathrm{GPa})\end{array}$ \\
\hline & & & & & & & & $\begin{array}{l}11.943 \\
11.988 \\
12.080 \\
12.289 \\
12.381 \\
12.576 \\
12.590 \\
12.779 \\
12.877 \\
12.988 \\
13.080 \\
13.275 \\
13.289 \\
13.576 \\
13.590 \\
13.877 \\
13.988 \\
14.275 \\
14.289 \\
14.576 \\
14.877 \\
15.275 \\
15.576\end{array}$ & $\begin{array}{l}31.152 \\
31.152 \\
31.152 \\
31.153 \\
31.153 \\
31.153 \\
31.153 \\
31.153 \\
31.153 \\
31.153 \\
31.153 \\
31.153 \\
31.153 \\
31.153 \\
31.153 \\
31.153 \\
31.153 \\
31.153 \\
31.153 \\
31.153 \\
31.153 \\
31.153 \\
31.153\end{array}$ & $\begin{array}{l}12.306 \\
12.367 \\
12.426 \\
12.668 \\
12.704 \\
12.727 \\
13.005 \\
13.066 \\
13.306 \\
13.367 \\
13.668 \\
13.704 \\
13.820 \\
14.005 \\
14.066 \\
14.121 \\
14.367 \\
14.519 \\
14.668 \\
14.820 \\
15.066 \\
15.121 \\
15.367 \\
15.519 \\
15.820 \\
16.121 \\
16.519 \\
16.820\end{array}$ & $\begin{array}{l}30.785 \\
30.785 \\
30.785 \\
30.785 \\
30.785 \\
30.785 \\
30.785 \\
30.785 \\
30.785 \\
30.785 \\
30.785 \\
30.785 \\
30.785 \\
30.785 \\
30.785 \\
30.785 \\
30.785 \\
30.785 \\
30.785 \\
30.785 \\
30.785 \\
30.785 \\
30.785 \\
30.785 \\
30.785 \\
30.785 \\
30.785 \\
30.785\end{array}$ & & \\
\hline
\end{tabular}


Appendix 4d. Parameters of the lines fitted to the imaginary shear modulus data for Fe-bearing melts (G8-G14). See also Fig. 69b.

\begin{tabular}{|c|c|c|c|c|c|c|c|c|c|c|c|c|c|}
\hline \multicolumn{14}{|c|}{ FITS TO THE IMAGINARY SHEAR MODULUS DATA } \\
\hline \multicolumn{2}{|c|}{ G8 } & \multicolumn{2}{|c|}{ G9 } & \multicolumn{2}{|c|}{ G10 } & \multicolumn{2}{|c|}{ G11 } & \multicolumn{2}{|c|}{ G12 } & \multicolumn{2}{|c|}{ G13 } & \multicolumn{2}{|c|}{ G14 } \\
\hline $\begin{array}{l}\Delta \log _{10} \\
\tau_{M}(s)\end{array}$ & $\begin{array}{c}\mathbf{G}_{\text {im }} \\
(\mathrm{GPa})\end{array}$ & $\begin{array}{l}\Delta \log _{10} \\
\tau_{M}(s)\end{array}$ & $\begin{array}{c}\mathbf{G}_{\text {im }} \\
(\mathrm{GPa})\end{array}$ & $\begin{array}{l}\Delta \log _{10} \\
\tau_{M}(s)\end{array}$ & $\begin{array}{c}\mathbf{G}_{\text {im }} \\
(\mathrm{GPa})\end{array}$ & $\begin{array}{l}\Delta \log _{10} \\
\tau_{M}(\mathrm{~s})\end{array}$ & $\begin{array}{c}\mathbf{G}_{\mathrm{im}} \\
(\mathrm{GPa})\end{array}$ & $\begin{array}{l}\Delta \log _{10} \\
\tau_{M}(\mathrm{~s})\end{array}$ & $\begin{array}{c}\mathbf{G}_{\mathrm{im}} \\
(\mathrm{GPa})\end{array}$ & $\begin{array}{l}\Delta \log _{10} \\
\tau_{M}(s)\end{array}$ & $\begin{array}{c}\mathbf{G}_{\mathrm{im}} \\
(\mathrm{GPa})\end{array}$ & $\begin{array}{l}\Delta \log _{10} \\
\tau_{M}(\mathrm{~s})\end{array}$ & $\begin{array}{c}\mathbf{G}_{\mathrm{im}} \\
(\mathrm{GPa})\end{array}$ \\
\hline 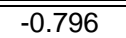 & 0.140 & $2-2.000$ & 0.033 & $2-2.000$ & 0.033 & $2-2.000$ & 0.060 & -2.000 & 0.050 & $2-2.000$ & 0.040 & -2.000 & 0.073 \\
\hline-0.595 & 0.176 & -1.600 & 0.059 & -1.600 & 0.083 & -1.600 & 0.147 & -1.600 & 0.112 & -1.750 & 0.072 & -1.600 & 0.182 \\
\hline-0.398 & 0.247 & -1.300 & 0.100 & -1.289 & 0.165 & -1.300 & 0.286 & -1.300 & 0.201 & -1.494 & 0.129 & -1.300 & 0.363 \\
\hline-0.294 & 0.303 & -1.185 & 0.123 & -1.093 & 0.255 & -1.064 & 0.479 & -1.000 & 0.359 & -1.147 & 0.286 & -0.986 & 0.744 \\
\hline-0.149 & 0.409 & -0.927 & 0.192 & -0.891 & 0.398 & -0.851 & 0.748 & -0.815 & 0.516 & -1.096 & 0.322 & -0.588 & 1.792 \\
\hline-0.097 & 0.457 & -0.884 & 0.207 & -0.791 & 0.497 & -0.763 & 0.890 & -0.703 & 0.646 & -0.846 & 0.571 & -0.579 & 1.826 \\
\hline-0.073 & 0.481 & -0.664 & 0.308 & -0.590 & 0.781 & -0.557 & 1.295 & -0.514 & 0.953 & -0.795 & 0.642 & -0.287 & 3.195 \\
\hline 0.104 & 0.704 & -0.626 & 0.331 & -0.575 & 0.807 & -0.550 & 1.312 & -0.402 & 1.206 & -0.494 & 1.266 & -0.278 & 3.243 \\
\hline 0.152 & 0.781 & -0.486 & 0.436 & -0.394 & 1.213 & -0.365 & 1.793 & -0.202 & 1.834 & -0.469 & 1.337 & 0.014 & 4.718 \\
\hline 0.204 & 0.874 & -0.363 & 0.563 & -0.289 & 1.529 & -0.317 & 1.941 & -0.116 & 2.192 & -0.448 & 1.401 & 0.037 & 4.816 \\
\hline 0.228 & 0.919 & -0.228 & 0.751 & -0.274 & 1.581 & -0.256 & 2.153 & -0.101 & 2.263 & -0.415 & 1.506 & 0.120 & 5.127 \\
\hline 0.405 & 1.338 & -0.185 & 0.824 & -0.093 & 2.338 & -0.249 & 2.181 & 0.099 & 3.327 & -0.168 & 2.540 & 0.164 & 5.269 \\
\hline 0.453 & 1.477 & -0.124 & 0.942 & -0.036 & 2.626 & -0.064 & 3.007 & 0.185 & 3.857 & -0.147 & 2.651 & 0.338 & 5.666 \\
\hline 0.470 & 1.530 & 0.035 & 1.335 & 0.109 & 3.487 & -0.029 & 3.197 & 0.297 & 4.558 & -0.114 & 2.828 & 0.412 & 5.742 \\
\hline 0.602 & 1.998 & 0.073 & 1.452 & 0.124 & 3.585 & -0.016 & 3.266 & 0.439 & 5.337 & -0.096 & 2.931 & 0.421 & 5.747 \\
\hline 0.626 & 2.093 & 0.116 & 1.596 & 0.209 & 4.152 & 0.142 & 4.247 & 0.486 & 5.547 & -0.009 & 3.452 & 0.465 & 5.757 \\
\hline 0.706 & 2.441 & 0.177 & 1.826 & 0.265 & 4.538 & 0.149 & 4.297 & 0.497 & 5.591 & 0.154 & 4.553 & 0.713 & 5.396 \\
\hline 0.771 & 2.752 & 0.336 & 2.567 & 0.410 & 5.498 & 0.237 & 4.885 & 0.598 & 5.918 & 0.205 & 4.906 & 0.722 & 5.372 \\
\hline 0.851 & 3.169 & 0.374 & 2.780 & 0.425 & 5.590 & 0.272 & 5.115 & 0.740 & 6.125 & 0.230 & 5.075 & 0.736 & 5.333 \\
\hline 0.903 & 3.462 & 0.438 & 3.169 & 0.525 & 6.137 & 0.285 & 5.194 & 0.798 & 6.134 & 0.241 & 5.147 & 0.863 & 4.944 \\
\hline 0.927 & 3.598 & 0.514 & 3.680 & 0.606 & 6.497 & 0.443 & 6.069 & 0.884 & 6.068 & 0.284 & 5.431 & 0.944 & 4.665 \\
\hline 1.036 & 4.253 & 0.575 & 4.126 & 0.663 & 6.694 & 0.450 & 6.102 & 0.899 & 6.046 & 0.506 & 6.483 & 1.014 & 4.413 \\
\hline 1.104 & 4.684 & 0.637 & 4.593 & 0.711 & 6.830 & 0.523 & 6.366 & 1.099 & 5.499 & 0.531 & 6.541 & 1.037 & 4.327 \\
\hline 1.152 & 4.988 & 0.739 & 5.379 & 0.726 & 6.865 & 0.635 & 6.582 & 1.109 & 5.456 & 0.542 & 6.562 & 1.120 & 4.020 \\
\hline 1.169 & 5.097 & 0.772 & 5.625 & 0.826 & 7.020 & 0.670 & 6.607 & 1.137 & 5.343 & 0.552 & 6.580 & 1.164 & 3.856 \\
\hline 1.204 & 5.320 & 0.815 & 5.930 & 0.907 & 7.030 & 0.683 & 6.612 & 1.185 & 5.140 & 0.585 & 6.621 & 1.245 & 3.558 \\
\hline 1.228 & 5.467 & 0.876 & 6.323 & 0.964 & 6.970 & 0.744 & 6.606 & 1.297 & 4.625 & 0.832 & 6.337 & 1.412 & 2.994 \\
\hline 1.337 & 6.104 & 1.022 & 6.954 & 1.109 & 6.556 & 0.751 & 6.603 & 1.411 & 4.117 & 0.853 & 6.277 & 1.421 & 2.965 \\
\hline 1.405 & 6.447 & 1.035 & 6.986 & 1.110 & 6.553 & 0.824 & 6.539 & 1.439 & 3.998 & 0.904 & 6.120 & 1.465 & 2.831 \\
\hline 1.453 & 6.649 & 1.073 & 7.058 & 1.124 & 6.495 & 0.936 & 6.364 & 1.486 & 3.808 & 0.940 & 5.999 & 1.643 & 2.345 \\
\hline 1.470 & 6.714 & 1.116 & 7.097 & 1.209 & 6.102 & 0.971 & 6.294 & 1.497 & 3.767 & 0.984 & 5.837 & 1.713 & 2.180 \\
\hline 1.602 & 7.051 & 1.137 & 7.101 & 1.224 & 6.025 & 0.984 & 6.268 & 1.598 & 3.408 & 1.154 & 5.145 & 1.722 & 2.159 \\
\hline 1.625 & 7.082 & 1.177 & 7.084 & 1.265 & 5.811 & 1.100 & 5.989 & 1.740 & 3.013 & 1.205 & 4.929 & 1.765 & 2.066 \\
\hline 1.626 & 7.083 & 1.323 & 6.788 & 1.410 & 5.035 & 1.142 & 5.878 & 1.798 & 2.881 & 1.230 & 4.824 & 1.863 & 1.870 \\
\hline 1.706 & 7.137 & 1.336 & 6.747 & 1.411 & 5.030 & 1.149 & 5.857 & 1.808 & 2.858 & 1.241 & 4.779 & 1.944 & 1.723 \\
\hline 1.735 & 7.139 & 1.374 & 6.616 & 1.425 & 4.957 & 1.222 & 5.649 & 1.813 & 2.850 & 1.285 & 4.593 & 2.066 & 1.524 \\
\hline 1.771 & 7.130 & 1.438 & 6.364 & 1.525 & 4.467 & 1.237 & 5.605 & 1.884 & 2.710 & 1.531 & 3.671 & 2.120 & 1.442 \\
\hline
\end{tabular}


Appendix 4d. Parameters of the lines fitted to the imaginary shear modulus data for Fe-bearing melts (G8-G14) - continuation...

\begin{tabular}{|c|c|c|c|c|c|c|c|c|c|c|c|c|c|}
\hline \multicolumn{14}{|c|}{ FITS TO THE IMAGINARY SHEAR MODULUS DATA } \\
\hline \multicolumn{2}{|c|}{ G8 } & \multicolumn{2}{|c|}{ G9 } & \multicolumn{2}{|c|}{ G10 } & \multicolumn{2}{|c|}{ G11 } & \multicolumn{2}{|c|}{ G12 } & \multicolumn{2}{|c|}{ G13 } & \multicolumn{2}{|c|}{ G14 } \\
\hline $\begin{array}{l}\Delta \log _{10} \\
\tau_{\mathrm{M}}(\mathrm{s}) \\
\end{array}$ & $\begin{array}{c}\mathbf{G}_{\text {im }} \\
(\mathbf{G P a}) \\
\end{array}$ & $\begin{array}{l}\Delta \log _{10} \\
\tau_{M}(\mathrm{~s}) \\
\end{array}$ & $\begin{array}{c}\mathbf{G}_{\text {im }} \\
(\mathrm{GPa})\end{array}$ & $\begin{array}{l}\Delta \log _{10} \\
\tau_{\mathrm{M}}(\mathrm{s}) \\
\end{array}$ & $\begin{array}{c}\mathbf{G}_{\text {im }} \\
(\mathbf{G P a}) \\
\end{array}$ & $\begin{array}{l}\Delta \log _{10} \\
\tau_{\mathrm{M}}(\mathrm{s}) \\
\end{array}$ & $\begin{array}{c}\mathbf{G}_{\text {im }} \\
(\mathrm{GPa})\end{array}$ & $\begin{array}{l}\Delta \log _{10} \\
\tau_{M}(\mathrm{~s}) \\
\end{array}$ & $\begin{array}{c}\mathbf{G}_{\text {im }} \\
(\mathrm{GPa}) \\
\end{array}$ & $\begin{array}{l}\Delta \log _{10} \\
\tau_{\mathrm{M}}(\mathrm{s}) \\
\end{array}$ & $\begin{array}{c}\mathbf{G}_{\text {im }} \\
(\mathbf{G P a}) \\
\end{array}$ & $\begin{array}{l}\Delta \log _{10} \\
\tau_{M}(\mathrm{~s}) \\
\end{array}$ & $\begin{array}{c}\mathbf{G}_{\text {im }} \\
(\mathbf{G P a}) \\
\end{array}$ \\
\hline 1.851 & 7.073 & 1.514 & 6.019 & 1.606 & 4.108 & 1.272 & 5.499 & 2.099 & 2.346 & 1.542 & 3.636 & 2.164 & 1.380 \\
\hline 1.903 & 7.012 & 1.575 & 5.714 & 1.663 & 3.886 & 1.401 & 5.081 & 2.109 & 2.329 & 1.552 & 3.601 & 2.245 & 1.271 \\
\hline 1.926 & 6.981 & 1.629 & 5.437 & 1.720 & 3.678 & 1.443 & 4.933 & 2.114 & 2.322 & 1.683 & 3.206 & 2.421 & 1.075 \\
\hline 1.927 & 6.980 & 1.637 & 5.394 & 1.726 & 3.659 & 1.450 & 4.906 & 2.137 & 2.284 & 1.765 & 2.992 & 2.464 & 1.035 \\
\hline 2.036 & 6.788 & 1.721 & 4.952 & 1.809 & 3.396 & 1.523 & 4.627 & 2.185 & 2.209 & 1.832 & 2.832 & 2.465 & 1.034 \\
\hline 2.104 & 6.635 & 1.739 & 4.857 & 1.826 & 3.345 & 1.635 & 4.156 & 2.411 & 1.850 & 1.853 & 2.784 & 2.629 & 0.905 \\
\hline 2.152 & 6.516 & 1.772 & 4.689 & 1.907 & 3.119 & 1.670 & 4.001 & 2.439 & 1.804 & 1.940 & 2.605 & 2.643 & 0.895 \\
\hline 2.169 & 6.471 & 1.815 & 4.478 & 1.964 & 2.975 & 1.704 & 3.851 & 2.497 & 1.707 & 1.984 & 2.520 & 2.765 & 0.820 \\
\hline 2.228 & 6.305 & 1.876 & 4.197 & 2.021 & 2.831 & 1.744 & 3.671 & 2.512 & 1.682 & 2.066 & 2.373 & 2.863 & 0.764 \\
\hline 2.240 & 6.269 & 1.930 & 3.977 & 2.110 & 2.620 & 1.799 & 3.423 & 2.550 & 1.615 & 2.230 & 2.105 & 2.930 & 0.726 \\
\hline 2.324 & 6.002 & 2.022 & 3.658 & 2.124 & 2.586 & 1.824 & 3.312 & 2.740 & 1.284 & 2.241 & 2.089 & 2.944 & 0.717 \\
\hline 2.337 & 5.959 & 2.035 & 3.618 & 2.224 & 2.357 & 1.936 & 2.836 & 2.798 & 1.186 & 2.285 & 2.021 & 3.066 & 0.641 \\
\hline 2.405 & 5.706 & 2.073 & 3.512 & 2.265 & 2.265 & 1.971 & 2.696 & 2.808 & 1.168 & 2.464 & 1.751 & 3.164 & 0.573 \\
\hline 2.470 & 5.437 & 2.137 & 3.360 & 2.358 & 2.061 & 2.005 & 2.566 & 2.813 & 1.162 & 2.531 & 1.645 & 3.245 & 0.515 \\
\hline 2.541 & 5.104 & 2.177 & 3.278 & 2.411 & 1.950 & 2.100 & 2.220 & 2.851 & 1.100 & 2.542 & 1.628 & 3.328 & 0.457 \\
\hline 2.625 & 4.670 & 2.261 & 3.137 & 2.419 & 1.932 & 2.142 & 2.082 & 3.109 & 0.773 & 2.584 & 1.559 & 3.464 & 0.371 \\
\hline 2.626 & 4.666 & 2.323 & 3.047 & 2.425 & 1.920 & 2.222 & 1.835 & 3.114 & 0.769 & 2.683 & 1.395 & 3.541 & 0.331 \\
\hline 2.735 & 4.061 & 2.328 & 3.040 & 2.525 & 1.715 & 2.272 & 1.695 & 3.137 & 0.747 & 2.765 & 1.264 & 3.629 & 0.292 \\
\hline 2.771 & 3.856 & 2.336 & 3.028 & 2.659 & 1.449 & 2.336 & 1.532 & 3.249 & 0.661 & 2.885 & 1.084 & 3.643 & 0.287 \\
\hline 2.883 & 3.251 & 2.438 & 2.877 & 2.663 & 1.442 & 2.401 & 1.383 & 3.325 & 0.616 & 2.940 & 1.012 & 3.765 & 0.250 \\
\hline 2.926 & 3.032 & 2.562 & 2.653 & 2.720 & 1.334 & 2.403 & 1.380 & 3.411 & 0.576 & 2.984 & 0.959 & 3.842 & 0.235 \\
\hline 2.927 & 3.029 & 2.575 & 2.625 & 2.809 & 1.178 & 2.443 & 1.297 & 3.439 & 0.566 & 3.066 & 0.874 & 3.930 & 0.226 \\
\hline 2.939 & 2.969 & 2.629 & 2.508 & 2.826 & 1.149 & 2.523 & 1.148 & 3.512 & 0.541 & 3.241 & 0.739 & 3.944 & 0.226 \\
\hline 3.036 & 2.541 & 2.721 & 2.290 & 2.964 & 0.941 & 2.637 & 0.973 & 3.550 & 0.530 & 3.283 & 0.715 & 4.066 & 0.228 \\
\hline 3.169 & 2.065 & 2.739 & 2.245 & 3.021 & 0.866 & 2.670 & 0.929 & 3.627 & 0.511 & 3.285 & 0.713 & 4.328 & 0.287 \\
\hline 3.184 & 2.020 & 2.876 & 1.917 & 3.025 & 0.861 & 2.704 & 0.888 & 3.808 & 0.476 & 3.446 & 0.635 & 4.464 & 0.346 \\
\hline 3.240 & 1.863 & 2.920 & 1.822 & 3.057 & 0.823 & 2.799 & 0.787 & 3.813 & 0.475 & 3.464 & 0.627 & 4.503 & 0.366 \\
\hline 3.324 & 1.665 & 2.930 & 1.801 & 3.110 & 0.764 & 2.824 & 0.764 & 3.851 & 0.468 & 3.584 & 0.571 & 4.541 & 0.386 \\
\hline 3.337 & 1.639 & 2.960 & 1.740 & 3.224 & 0.656 & 2.971 & 0.655 & 4.024 & 0.430 & 3.683 & 0.524 & 4.629 & 0.437 \\
\hline 3.470 & 1.399 & 3.022 & 1.627 & 3.326 & 0.577 & 2.999 & 0.639 & 4.109 & 0.407 & 3.747 & 0.494 & 4.765 & 0.513 \\
\hline 3.541 & 1.294 & 3.137 & 1.455 & 3.358 & 0.556 & 3.005 & 0.636 & 4.114 & 0.406 & 3.765 & 0.486 & 4.804 & 0.532 \\
\hline 3.554 & 1.277 & 3.221 & 1.359 & 3.411 & 0.522 & 3.035 & 0.620 & 4.141 & 0.397 & 3.885 & 0.432 & 4.842 & 0.547 \\
\hline 3.582 & 1.241 & 3.261 & 1.319 & 3.419 & 0.517 & 3.100 & 0.590 & 4.249 & 0.362 & 3.984 & 0.391 & 4.930 & 0.572 \\
\hline 3.625 & 1.187 & 3.323 & 1.264 & 3.525 & 0.459 & 3.222 & 0.546 & 4.325 & 0.336 & 4.066 & 0.362 & 5.202 & 0.519 \\
\hline 3.735 & 1.068 & 3.328 & 1.259 & 3.659 & 0.396 & 3.300 & 0.523 & 4.442 & 0.297 & 4.145 & 0.337 & 5.240 & 0.499 \\
\hline 3.855 & 0.958 & 3.438 & 1.165 & 3.720 & 0.371 & 3.336 & 0.513 & 4.512 & 0.275 & 4.283 & 0.303 & 5.328 & 0.445 \\
\hline 3.883 & 0.935 & 3.562 & 1.047 & 3.723 & 0.370 & 3.401 & 0.495 & 4.550 & 0.264 & 4.354 & 0.290 & 5.503 & 0.333 \\
\hline
\end{tabular}


Appendix 4d. Parameters of the lines fitted to the imaginary shear modulus data for Fe-bearing melts (G8-G14) - continuation...

\begin{tabular}{|c|c|c|c|c|c|c|c|c|c|c|c|c|c|}
\hline \multicolumn{14}{|c|}{ FITS TO THE IMAGINARY SHEAR MODULUS DATA } \\
\hline \multicolumn{2}{|c|}{ G8 } & \multicolumn{2}{|c|}{ G9 } & \multicolumn{2}{|c|}{ G10 } & \multicolumn{2}{|c|}{ G11 } & \multicolumn{2}{|c|}{ G12 } & \multicolumn{2}{|c|}{ G13 } & \multicolumn{2}{|c|}{ G14 } \\
\hline $\begin{array}{l}\Delta \log _{10} \\
\tau_{\mathrm{M}}(\mathrm{s}) \\
\end{array}$ & $\begin{array}{c}\mathbf{G}_{\text {im }} \\
(\mathbf{G P a}) \\
\end{array}$ & $\begin{array}{l}\Delta \log _{10} \\
\tau_{M}(\mathrm{~s}) \\
\end{array}$ & $\begin{array}{c}\mathbf{G}_{\text {im }} \\
(\mathrm{GPa})\end{array}$ & $\begin{array}{l}\Delta \log _{10} \\
\tau_{\mathrm{M}}(\mathrm{s}) \\
\end{array}$ & $\begin{array}{c}\mathbf{G}_{\text {im }} \\
(\mathbf{G P a}) \\
\end{array}$ & $\begin{array}{l}\Delta \log _{10} \\
\tau_{\mathrm{M}}(\mathrm{s}) \\
\end{array}$ & $\begin{array}{c}\mathbf{G}_{\text {im }} \\
(\mathrm{GPa}) \\
\end{array}$ & $\begin{array}{l}\Delta \log _{10} \\
\tau_{M}(\mathrm{~s}) \\
\end{array}$ & $\begin{array}{c}\mathbf{G}_{\text {im }} \\
(\mathrm{GPa}) \\
\end{array}$ & $\begin{array}{l}\Delta \log _{10} \\
\tau_{\mathrm{M}}(\mathrm{s}) \\
\end{array}$ & $\begin{array}{c}\mathbf{G}_{\text {im }} \\
(\mathbf{G P a}) \\
\end{array}$ & $\begin{array}{l}\Delta \log _{10} \\
\tau_{\mathrm{M}}(\mathrm{s}) \\
\end{array}$ & $\begin{array}{c}\mathbf{G}_{\text {im }} \\
(\mathbf{G P a}) \\
\end{array}$ \\
\hline 3.926 & 0.902 & 3.607 & 1.000 & 3.724 & 0.370 & 3.403 & 0.494 & 4.627 & 0.242 & 4.446 & 0.278 & 5.521 & 0.322 \\
\hline 3.939 & 0.892 & 3.619 & 0.987 & 3.809 & 0.338 & 3.523 & 0.458 & 4.813 & 0.200 & 4.464 & 0.276 & 5.541 & 0.311 \\
\hline 4.036 & 0.825 & 3.629 & 0.975 & 4.021 & 0.277 & 3.637 & 0.418 & 4.840 & 0.195 & 4.584 & 0.269 & 5.629 & 0.263 \\
\hline 4.184 & 0.735 & 3.721 & 0.870 & 4.024 & 0.277 & 3.695 & 0.396 & 4.851 & 0.193 & 4.655 & 0.269 & 5.804 & 0.185 \\
\hline 4.240 & 0.705 & 3.908 & 0.655 & 4.025 & 0.276 & 3.698 & 0.395 & 4.999 & 0.174 & 4.747 & 0.271 & 5.822 & 0.178 \\
\hline 4.253 & 0.699 & 3.920 & 0.643 & 4.057 & 0.269 & 3.704 & 0.393 & 5.024 & 0.172 & 4.765 & 0.272 & 5.842 & 0.171 \\
\hline 4.257 & 0.697 & 3.930 & 0.632 & 4.110 & 0.259 & 3.799 & 0.357 & 5.141 & 0.167 & 4.885 & 0.278 & 6.202 & 0.084 \\
\hline 4.324 & 0.665 & 3.960 & 0.601 & 4.326 & 0.229 & 3.996 & 0.296 & 5.249 & 0.168 & 5.053 & 0.289 & 6.220 & 0.081 \\
\hline 4.541 & 0.580 & 4.022 & 0.542 & 4.358 & 0.227 & 3.999 & 0.295 & 5.300 & 0.170 & 5.145 & 0.293 & 6.240 & 0.078 \\
\hline 4.554 & 0.575 & 4.221 & 0.393 & 4.419 & 0.224 & 4.005 & 0.294 & 5.325 & 0.172 & 5.283 & 0.295 & 6.503 & 0.049 \\
\hline 4.558 & 0.574 & 4.261 & 0.370 & 4.422 & 0.224 & 4.035 & 0.287 & 5.442 & 0.182 & 5.311 & 0.295 & 6.521 & 0.047 \\
\hline 4.582 & 0.566 & 4.306 & 0.348 & 4.454 & 0.223 & 4.100 & 0.275 & 5.550 & 0.194 & 5.354 & 0.295 & 6.541 & 0.046 \\
\hline 4.625 & 0.553 & 4.324 & 0.340 & 4.659 & 0.229 & 4.300 & 0.261 & 5.627 & 0.204 & 5.446 & 0.290 & 6.804 & 0.031 \\
\hline 4.855 & 0.492 & 4.328 & 0.338 & 4.720 & 0.236 & 4.336 & 0.262 & 5.698 & 0.212 & 5.584 & 0.276 & 6.822 & 0.030 \\
\hline 4.883 & 0.486 & 4.562 & 0.264 & 4.723 & 0.236 & 4.394 & 0.267 & 5.840 & 0.226 & 5.612 & 0.272 & 7.202 & 0.021 \\
\hline 4.939 & 0.474 & 4.607 & 0.255 & 4.724 & 0.236 & 4.403 & 0.267 & 5.904 & 0.229 & 5.655 & 0.266 & 7.220 & 0.020 \\
\hline 4.956 & 0.471 & 4.619 & 0.253 & 4.755 & 0.240 & 4.637 & 0.309 & 5.999 & 0.228 & 5.747 & 0.251 & 7.503 & 0.017 \\
\hline 4.993 & 0.463 & 4.625 & 0.252 & 5.024 & 0.301 & 4.695 & 0.322 & 6.024 & 0.226 & 6.010 & 0.213 & 7.521 & 0.017 \\
\hline 5.184 & 0.427 & 4.629 & 0.251 & 5.025 & 0.301 & 4.698 & 0.323 & 6.141 & 0.213 & 6.053 & 0.209 & 7.822 & 0.015 \\
\hline 5.240 & 0.417 & 4.908 & 0.228 & 5.057 & 0.311 & 4.704 & 0.324 & 6.205 & 0.203 & 6.145 & 0.203 & 8.220 & 0.014 \\
\hline 5.253 & 0.415 & 4.920 & 0.228 & 5.153 & 0.343 & 4.996 & 0.366 & 6.300 & 0.185 & 6.311 & 0.203 & 8.521 & 0.013 \\
\hline 5.257 & 0.414 & 4.960 & 0.227 & 5.221 & 0.366 & 4.999 & 0.366 & 6.325 & 0.180 & 6.322 & 0.203 & & \\
\hline 5.294 & 0.408 & 5.023 & 0.227 & 5.326 & 0.399 & 5.035 & 0.364 & 6.442 & 0.158 & 6.354 & 0.204 & & \\
\hline 5.554 & 0.363 & 5.073 & 0.227 & 5.358 & 0.408 & 5.300 & 0.314 & 6.603 & 0.135 & 6.446 & 0.210 & & \\
\hline 5.558 & 0.362 & 5.221 & 0.228 & 5.422 & 0.422 & 5.336 & 0.305 & 6.698 & 0.126 & 6.612 & 0.225 & & \\
\hline 5.582 & 0.358 & 5.261 & 0.229 & 5.454 & 0.428 & 5.394 & 0.289 & 6.840 & 0.119 & 6.623 & 0.226 & & \\
\hline 5.692 & 0.338 & 5.306 & 0.230 & 5.522 & 0.435 & 5.695 & 0.230 & 6.860 & 0.118 & 6.655 & 0.230 & & \\
\hline 5.765 & 0.324 & 5.324 & 0.230 & 5.723 & 0.424 & 5.698 & 0.230 & 6.904 & 0.118 & 7.010 & 0.276 & & \\
\hline 5.855 & 0.308 & 5.374 & 0.231 & 5.724 & 0.424 & 5.996 & 0.194 & 6.999 & 0.118 & 7.021 & 0.277 & & \\
\hline 5.883 & 0.303 & 5.607 & 0.232 & 5.755 & 0.418 & 5.999 & 0.193 & 7.141 & 0.122 & 7.053 & 0.281 & & \\
\hline 5.956 & 0.290 & 5.619 & 0.232 & 5.920 & 0.381 & 6.394 & 0.124 & 7.161 & 0.123 & 7.311 & 0.308 & & \\
\hline 5.993 & 0.283 & 5.625 & 0.232 & 6.024 & 0.352 & 6.695 & 0.087 & 7.205 & 0.124 & 7.322 & 0.309 & & \\
\hline 6.066 & 0.271 & 5.772 & 0.230 & 6.025 & 0.352 & & & 7.300 & 0.128 & 7.354 & 0.313 & & \\
\hline 6.253 & 0.239 & 5.856 & 0.228 & 6.153 & 0.315 & & & 7.559 & 0.134 & 7.392 & 0.316 & & \\
\hline 6.257 & 0.239 & 5.908 & 0.226 & 6.221 & 0.295 & & & 7.603 & 0.134 & 7.612 & 0.336 & & \\
\hline 6.294 & 0.233 & 5.920 & 0.226 & 6.422 & 0.241 & & & 7.698 & 0.133 & 7.623 & 0.337 & & \\
\hline 6.464 & 0.208 & 6.023 & 0.221 & 6.454 & 0.233 & & & 7.860 & 0.128 & 7.693 & 0.341 & & \\
\hline
\end{tabular}


Appendix 4d. Parameters of the lines fitted to the imaginary shear modulus data for Fe-bearing melts (G8-G14) - continuation...

\begin{tabular}{|c|c|c|c|c|c|c|c|c|c|c|c|c|c|}
\hline \multicolumn{14}{|c|}{ FITS TO THE IMAGINARY SHEAR MODULUS DATA } \\
\hline \multicolumn{2}{|c|}{ G8 } & \multicolumn{2}{|c|}{ G9 } & \multicolumn{2}{|c|}{ G10 } & \multicolumn{2}{|c|}{ G11 } & \multicolumn{2}{|c|}{ G12 } & \multicolumn{2}{|c|}{ G13 } & \multicolumn{2}{|c|}{ G14 } \\
\hline $\begin{array}{l}\Delta \log _{10} \\
\tau_{\mathrm{M}}(\mathrm{s})\end{array}$ & $\begin{array}{c}\mathbf{G}_{\text {im }} \\
\text { (GPa) }\end{array}$ & $\begin{array}{l}\Delta \log _{10} \\
\tau_{M}(\mathrm{~s})\end{array}$ & $\begin{array}{c}\mathbf{G}_{\text {im }} \\
(\mathrm{GPa})\end{array}$ & $\begin{array}{l}\Delta \log _{10} \\
\tau_{M}(\mathbf{s})\end{array}$ & $\begin{array}{c}\mathrm{G}_{\mathrm{im}} \\
(\mathrm{GPa})\end{array}$ & $\begin{array}{l}\Delta \log _{10} \\
\tau_{M}(\mathbf{s})\end{array}$ & $\begin{array}{c}\mathbf{G}_{\text {im }} \\
(\mathbf{G P a})\end{array}$ & $\begin{array}{l}\Delta \log _{10} \\
\tau_{M}(\mathrm{~s})\end{array}$ & $\begin{array}{c}G_{\text {im }} \\
\text { (GPa) }\end{array}$ & $\begin{array}{l}\Delta \log _{10} \\
\tau_{M}(\mathrm{~s})\end{array}$ & $\begin{array}{c}\mathbf{G}_{\text {im }} \\
(\mathrm{GPa})\end{array}$ & $\begin{array}{l}\Delta \log _{10} \\
\tau_{\mathrm{M}}(\mathrm{s})\end{array}$ & $\begin{array}{c}\mathbf{G}_{\text {im }} \\
\text { (GPa) }\end{array}$ \\
\hline 6.554 & 0.196 & 6.073 & 0.219 & 6.522 & 0.218 & & & 7.871 & 0.128 & 8.010 & 0.334 & & \\
\hline 6.558 & 0.195 & 6.157 & 0.214 & 6.723 & 0.177 & & & 7.904 & 0.127 & 8.021 & 0.333 & & \\
\hline 6.692 & 0.178 & 6.306 & 0.205 & 6.755 & 0.172 & & & 7.999 & 0.124 & 8.091 & 0.322 & & \\
\hline 6.765 & 0.170 & 6.324 & 0.204 & 6.920 & 0.148 & & & 8.161 & 0.121 & 8.311 & 0.273 & & \\
\hline 6.956 & 0.151 & 6.374 & 0.200 & 7.153 & 0.127 & & & 8.173 & 0.121 & 8.322 & 0.271 & & \\
\hline 6.993 & 0.148 & 6.555 & 0.189 & 7.221 & 0.123 & & & 8.205 & 0.121 & 8.392 & 0.256 & & \\
\hline 7.066 & 0.141 & 6.607 & 0.185 & 7.454 & 0.114 & & & 8.559 & 0.143 & 8.525 & 0.233 & & \\
\hline 7.257 & 0.128 & 6.625 & 0.184 & 7.522 & 0.112 & & & 8.570 & 0.144 & 8.623 & 0.222 & & \\
\hline 7.294 & 0.125 & 6.772 & 0.175 & 7.920 & 0.104 & & & 8.603 & 0.148 & 8.693 & 0.217 & & \\
\hline 7.464 & 0.117 & 6.856 & 0.171 & 8.221 & 0.098 & & & 8.860 & 0.177 & 8.826 & 0.209 & & \\
\hline 7.692 & 0.108 & 7.023 & 0.163 & & & & & 8.871 & 0.178 & 9.021 & 0.192 & & \\
\hline 7.765 & 0.105 & 7.073 & 0.161 & & & & & 8.904 & 0.180 & 9.091 & 0.182 & & \\
\hline 7.993 & 0.100 & 7.157 & 0.159 & & & & & 8.943 & 0.181 & 9.223 & 0.157 & & \\
\hline 8.066 & 0.098 & 7.324 & 0.154 & & & & & 9.161 & 0.165 & 9.322 & 0.136 & & \\
\hline 8.464 & 0.089 & 7.374 & 0.153 & & & & & 9.173 & 0.164 & 9.392 & 0.121 & & \\
\hline 8.765 & 0.078 & 7.555 & 0.149 & & & & & 9.244 & 0.151 & 9.525 & 0.094 & & \\
\hline $0.10 \mathrm{~J}$ & 0.070 & 7.772 & 0.142 & & & & & 9.559 & 0.089 & $\begin{array}{l}9.693 \\
9\end{array}$ & 0.066 & & \\
\hline & & 7.856 & 0.139 & & & & & 9.570 & 0.087 & $\begin{array}{l}9.727 \\
9.727\end{array}$ & $\begin{array}{l}0.060 \\
0.062\end{array}$ & & \\
\hline & & 8.073 & 0.131 & & & & & 9.642 & 0.076 & 9.826 & 0.050 & & \\
\hline & & 8.157 & 0.127 & & & & & 9.860 & 0.047 & 10.028 & 0.031 & & \\
\hline & & 8.555 & 0.107 & & & & & 9.871 & 0.046 & 10.091 & 0.027 & & \\
\hline & & 8.856 & 0.087 & & & & & 9.943 & 0.039 & 10.223 & 0.020 & & \\
\hline & & & & & & & & 10.080 & 0.029 & 10.392 & 0.014 & & \\
\hline & & & & & & & & 10.173 & 0.023 & 10.426 & 0.013 & & \\
\hline & & & & & & & & 10.244 & 0.020 & 10.525 & 0.010 & & \\
\hline & & & & & & & & 10.381 & 0.014 & 10.727 & 0.006 & & \\
\hline & & & & & & & & 10.570 & 0.009 & 10.826 & 0.005 & & \\
\hline & & & & & & & & 10.642 & 0.008 & 11.005 & 0.003 & & \\
\hline & & & & & & & & 10.779 & 0.006 & 11.028 & 0.003 & & \\
\hline & & & & & & & & 10.871 & 0.005 & 11.223 & 0.002 & & \\
\hline & & & & & & & & 10.943 & 0.004 & 11.306 & 0.002 & & \\
\hline & & & & & & & & 11.080 & 0.003 & 11.426 & 0.001 & & \\
\hline & & & & & & & & 11.244 & 0.002 & 11.525 & 0.001 & & \\
\hline & & & & & & & & 11.289 & 0.002 & 11.704 & 0.001 & & \\
\hline & & & & & & & & 11.381 & 0.001 & 11.727 & 0.001 & & \\
\hline & & & & & & & & $\begin{array}{l}11.590 \\
11.00\end{array}$ & 0.001 & $\begin{array}{l}12.005 \\
12028\end{array}$ & 0.000 & & \\
\hline
\end{tabular}


Appendix 4d. Parameters of the lines fitted to the imaginary shear modulus data for Fe-bearing melts (G8-G14) - continuation...

\begin{tabular}{|c|c|c|c|c|c|c|c|c|c|c|c|c|c|}
\hline \multicolumn{14}{|c|}{ FITS TO THE IMAGINARY SHEAR MODULUS DATA } \\
\hline \multicolumn{2}{|c|}{ G8 } & \multicolumn{2}{|c|}{ G9 } & \multicolumn{2}{|c|}{ G10 } & \multicolumn{2}{|c|}{ G11 } & \multicolumn{2}{|c|}{ G12 } & \multicolumn{2}{|c|}{ G13 } & \multicolumn{2}{|c|}{ G14 } \\
\hline $\begin{array}{l}\Delta \log _{10} \\
\tau_{\mathrm{M}}(\mathrm{s})\end{array}$ & $\begin{array}{c}\mathbf{G}_{\text {im }} \\
(\mathrm{GPa})\end{array}$ & $\begin{array}{l}\Delta \log _{10} \\
\tau_{M}(s)\end{array}$ & $\begin{array}{l}\mathbf{G}_{\mathrm{im}} \\
(\mathrm{GPa})\end{array}$ & $\begin{array}{l}\Delta \log _{10} \\
\tau_{M}(s)\end{array}$ & $\begin{array}{c}\mathbf{G}_{\text {im }} \\
(\mathrm{GPa})\end{array}$ & $\begin{array}{l}\Delta \log _{10} \\
\tau_{M}(s)\end{array}$ & $\begin{array}{l}\mathbf{G}_{\text {im }} \\
(\mathrm{GPa})\end{array}$ & $\begin{array}{l}\Delta \log _{10} \\
\tau_{M}(\mathrm{~s})\end{array}$ & $\begin{array}{l}\mathbf{G}_{\text {im }} \\
(\mathrm{GPa})\end{array}$ & $\begin{array}{l}\Delta \log _{10} \\
\tau_{M}(s)\end{array}$ & $\begin{array}{c}G_{\text {im }} \\
(G P a)\end{array}$ & $\begin{array}{l}\Delta \log _{10} \\
\tau_{M}(\mathrm{~s})\end{array}$ & $\begin{array}{c}\mathbf{G}_{\text {im }} \\
(\mathrm{GPa})\end{array}$ \\
\hline & & & & & & & & $\begin{array}{l}11.779 \\
11.943 \\
11.988 \\
12.080 \\
12.289 \\
12.381 \\
12.576 \\
12.590 \\
12.779 \\
12.877 \\
12.988 \\
13.080 \\
13.275 \\
13.289 \\
13.576 \\
13.590 \\
13.877 \\
13.988 \\
14.275 \\
14.289 \\
14.576 \\
14.877 \\
15.275 \\
15.576\end{array}$ & $\begin{array}{l}0.001 \\
0.000 \\
0.000 \\
0.000 \\
0.000 \\
0.000 \\
0.000 \\
0.000 \\
0.000 \\
0.000 \\
0.000 \\
0.000 \\
0.000 \\
0.000 \\
0.000 \\
0.000 \\
0.000 \\
0.000 \\
0.000 \\
0.000 \\
0.000 \\
0.000 \\
0.000 \\
0.000\end{array}$ & $\begin{array}{l}12.306 \\
12.367 \\
12.426 \\
12.668 \\
12.704 \\
12.727 \\
13.005 \\
13.306 \\
13.367 \\
13.668 \\
13.704 \\
13.820 \\
14.005 \\
14.066 \\
14.121 \\
14.367 \\
14.519 \\
14.668 \\
14.820 \\
15.066 \\
15.121 \\
15.367 \\
15.519 \\
15.820 \\
16.121 \\
16.519 \\
16.820 \\
\end{array}$ & $\begin{array}{l}0.000 \\
0.000 \\
0.000 \\
0.000 \\
0.000 \\
0.000 \\
0.000 \\
0.000 \\
0.000 \\
0.000 \\
0.000 \\
0.000 \\
0.000 \\
0.000 \\
0.000 \\
0.000 \\
0.000 \\
0.000 \\
0.000 \\
0.000 \\
0.000 \\
0.000 \\
0.000 \\
0.000 \\
0.000 \\
0.000 \\
0.000 \\
\end{array}$ & & \\
\hline
\end{tabular}


Appendix 5a. Parameters of the fits to Eq. 58 for the NS (G0) and Fe-free melts (G1-G7). The $G_{x}$ values are calculated separately from the imaginary and the real component of the data.

\begin{tabular}{|c|c|c|c|c|c|c|c|c|}
\hline \multirow{2}{*}{$\begin{array}{c}\log _{10} \\
\left(\omega \tau_{M}+x\right)\end{array}$} & \multicolumn{2}{|c|}{ G0 } & \multicolumn{2}{|c|}{ G1 } & \multicolumn{2}{|c|}{ G2 } & \multicolumn{2}{|c|}{ G3 } \\
\hline & G real & G im & G real & G im & G real & G im & G real & G im \\
\hline-2.0 & 0.0000 & 0.0000 & 0.0019 & 0.0002 & 0.1750 & 0.1246 & 0.0000 & 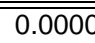 \\
\hline-1.5 & 0.0219 & 0.0000 & 0.1349 & 0.0312 & 0.1751 & 0.0056 & 0.0000 & 0.0000 \\
\hline-1.0 & 0.0001 & 0.0000 & 0.1625 & 0.0000 & 0.1553 & 0.1134 & 0.0391 & 0.0000 \\
\hline-0.5 & 0.3660 & 0.0000 & 0.5722 & 0.0036 & 0.0822 & 0.2377 & 0.0352 & 0.0561 \\
\hline 0.0 & 15.3951 & 14.9655 & 1.0856 & 2.2426 & 0.0725 & 0.2310 & 0.0015 & 0.2968 \\
\hline 0.5 & 9.3074 & 0.0301 & 15.0522 & 10.0915 & 14.6287 & 0.2967 & 8.1990 & 5.8657 \\
\hline 1.0 & 1.5469 & 0.0004 & 6.4520 & 5.0549 & 7.3647 & 4.0287 & 9.4410 & 7.5078 \\
\hline 1.5 & 0.9018 & 0.0000 & 3.2551 & 3.6187 & 5.9618 & 11.2787 & 10.6608 & 9.6254 \\
\hline 2.0 & 0.0018 & 0.0004 & 1.7552 & 0.8130 & 3.3260 & 3.9675 & 3.8472 & 1.4609 \\
\hline 2.5 & 0.0001 & 0.0001 & 1.2422 & 0.7270 & 1.4730 & 3.5155 & 0.8425 & 1.5879 \\
\hline 3.0 & 0.0012 & 0.0000 & 0.3270 & 0.0996 & 0.0010 & 0.0941 & 0.0065 & 0.7968 \\
\hline 3.5 & 0.0010 & 0.0057 & 0.2548 & 0.1563 & 0.0020 & 0.4230 & 0.2604 & 0.0375 \\
\hline 4.0 & 0.0018 & 0.0038 & 0.1844 & 0.0773 & 0.0110 & 0.3699 & 0.4156 & 0.2175 \\
\hline 4.5 & 0.0004 & 0.0004 & 0.0959 & 0.0011 & 0.0051 & 0.1326 & 0.4077 & 0.3276 \\
\hline 5.0 & 0.0081 & 0.0000 & 1.8824 & 0.0000 & 0.0000 & 0.0523 & 0.3669 & 0.4712 \\
\hline 5.5 & 0.0000 & 0.0000 & 0.5231 & 1.8272 & 0.0001 & 0.0044 & 0.3936 & 0.0000 \\
\hline 6.0 & 0.0000 & 0.0001 & 0.3658 & 0.4107 & 0.0185 & 1.1911 & 0.3743 & 0.0000 \\
\hline 6.5 & 0.0000 & 0.0000 & 0.0361 & 0.0110 & 0.2058 & 1.7713 & 0.3075 & 0.0000 \\
\hline 7.0 & 0.0097 & 0.0001 & 0.0230 & 0.0086 & 1.0386 & 0.6120 & 0.1509 & 0.3080 \\
\hline 7.5 & 0.0000 & 0.0002 & 0.0068 & 0.0333 & 0.0004 & 0.0069 & 1.2116 & 0.9406 \\
\hline 8.0 & 0.0001 & 0.0000 & 0.0002 & 0.0283 & 0.0033 & 0.0050 & 0.4487 & 0.2569 \\
\hline 8.5 & 0.0000 & 0.0001 & 0.0096 & 0.0217 & 0.0075 & 0.0053 & 0.0464 & 0.0000 \\
\hline 9.0 & 0.0499 & 0.0000 & 0.0224 & 0.0435 & 0.0018 & 0.0069 & 0.0365 & 0.0000 \\
\hline
\end{tabular}

\begin{tabular}{|c|c|c|c|c|c|c|c|c|}
\hline \multirow{2}{*}{$\begin{array}{c}\log _{10} \\
\left(\omega \tau_{M}+x\right)\end{array}$} & \multicolumn{2}{|c|}{ G4 } & \multicolumn{2}{|c|}{ G5 } & \multicolumn{2}{|c|}{$\mathbf{G 6}$} & \multicolumn{2}{|c|}{ G7 } \\
\hline & G real & G im & G real & G im & G real & G im & G real & G im \\
\hline $\begin{array}{l}-2.0 \\
\end{array}$ & 0.0000 & 0.0943 & 0.0000 & 0.0331 & 0.0004 & 0.0000 & 0.0000 & 0.0000 \\
\hline-1.5 & 0.0001 & 0.0396 & 0.0041 & 0.0170 & 0.0016 & 0.0000 & 0.0000 & 0.0000 \\
\hline-1.0 & 0.0487 & 0.0394 & 0.0000 & 0.0142 & 0.0000 & 0.0042 & 0.000 & 0.0014 \\
\hline-0.5 & 0.0006 & 0.0520 & 0.0000 & 0.0141 & 0.0361 & 0.0000 & 0.4900 & 0.0008 \\
\hline 0.0 & 1.5280 & 0.0834 & 4.1363 & 0.0423 & 4.4974 & 5.7770 & 17.7325 & 16.1418 \\
\hline 0.5 & 5.9260 & 7.2443 & 19.7777 & 14.1438 & 19.5208 & 6.0992 & 16.8805 & 11.3698 \\
\hline 1.0 & 7.6788 & 11.4305 & 4.8209 & 4.0414 & 10.4056 & 2.2264 & 3.9630 & 0.1729 \\
\hline 1.5 & 7.9675 & 2.3323 & 4.4279 & 3.7268 & 4.1285 & 1.6094 & 0.9825 & 0.0003 \\
\hline 2.0 & 1.6608 & 0.4935 & 0.6727 & 0.0106 & 0.6088 & 1.2668 & 1.8693 & 0.0086 \\
\hline 2.5 & 0.9852 & 0.0224 & 0.6900 & 0.0117 & 0.7708 & 0.0000 & 1.9549 & 0.0000 \\
\hline 3.0 & 0.8287 & 0.0214 & 1.1252 & 0.0122 & 1.7880 & 0.0001 & 0.0000 & 0.0005 \\
\hline 3.5 & 0.5856 & 0.0218 & 0.0000 & 0.0122 & 0.0277 & 0.0002 & 0.0000 & 0.0002 \\
\hline 4.0 & 0.0629 & 0.0213 & 0.0052 & 0.0118 & 0.0463 & 0.0010 & 0.0000 & 0.0015 \\
\hline 4.5 & 0.0000 & 0.0182 & 0.0001 & 0.0117 & 0.0658 & 0.0002 & 0.0000 & 0.0004 \\
\hline 5.0 & 0.0395 & 0.0146 & 0.0016 & 0.0115 & 0.0790 & 0.0000 & 0.0000 & 0.0006 \\
\hline 5.5 & 0.2874 & 0.0130 & 0.0092 & 0.0114 & 0.0887 & 0.0041 & & 0.0028 \\
\hline 6.0 & 0.1781 & 0.0120 & 0.2608 & 0.0110 & 0.2051 & 0.0060 & & \\
\hline 6.5 & 0.2851 & 0.0096 & 0.0853 & 0.0105 & 0.4789 & 0.0000 & & \\
\hline 7.0 & 0.2216 & 0.0083 & 0.5321 & 0.0100 & 0.3994 & 0.0000 & & \\
\hline 7.5 & 0.7514 & 1.2934 & 0.9496 & 0.9754 & 0.9981 & 0.8812 & & \\
\hline 8.0 & 1.0168 & 0.0095 & 0.0000 & 2.7436 & 0.1060 & 0.5487 & & \\
\hline 8.5 & 0.0854 & 0.0098 & 0.0037 & 0.0101 & 0.1696 & 0.0021 & & \\
\hline 9.0 & 0.0018 & 0.0116 & 0.0001 & 0.0105 & 0.0731 & 0.0005 & & \\
\hline
\end{tabular}


Appendix 5b. Parameters of the fits to Eq. 58 for the Fe-bearing melts (G8-G14). The $G_{x}$ values are calculated separately from the imaginary and the real component of the data.

\begin{tabular}{|c|c|c|c|c|c|c|c|c|}
\hline \multirow{2}{*}{$\begin{array}{c}\log _{10} \\
\left(\omega \tau_{M}+x\right)\end{array}$} & \multicolumn{2}{|c|}{ G8 } & \multicolumn{2}{|c|}{ G9 } & \multicolumn{2}{|c|}{ G10 } & \multicolumn{2}{|c|}{ G11 } \\
\hline & G real & G im & G real & G im & G real & G im & G real & G im \\
\hline-2.0 & 0.0000 & 0.0004 & 0.0000 & 0.0096 & 0.0000 & 0.0003 & 0.0000 & 0.0000 \\
\hline-1.5 & 0.0000 & 0.0004 & 0.0000 & 0.0009 & 0.0000 & 0.0008 & 0.0000 & 0.0000 \\
\hline-1.0 & 0.0000 & 0.0010 & 0.0000 & 0.0030 & 0.0000 & 0.1964 & 0.0000 & 0.0000 \\
\hline-0.5 & 0.0000 & 0.4334 & 0.0000 & 0.0028 & 2.2171 & 0.0001 & 0.0000 & 0.0937 \\
\hline 0.0 & 5.2465 & 4.4479 & 0.0000 & 0.0000 & 1.4041 & 0.3566 & 6.9409 & 0.8966 \\
\hline 0.5 & 16.1477 & 8.5137 & 3.4302 & 0.0312 & 15.8547 & 14.4501 & 12.2113 & 12.9970 \\
\hline 1.0 & 3.0832 & 4.9116 & 6.9371 & 10.4640 & 5.5866 & 1.3858 & 4.3732 & 3.7738 \\
\hline 1.5 & 2.6781 & 0.3499 & 9.7484 & 4.5439 & 3.5716 & 1.3829 & 3.7152 & 1.4656 \\
\hline 2.0 & 3.5174 & 1.1559 & 3.1225 & 3.3974 & 4.0870 & 0.4867 & 2.7744 & 1.4317 \\
\hline 2.5 & 0.0000 & 0.9578 & 1.8179 & 1.0503 & 0.0000 & 0.3755 & 0.0000 & 0.2065 \\
\hline 3.0 & 0.0000 & 0.0001 & 1.2603 & 1.9413 & 0.0000 & 0.3531 & 0.0000 & 0.0000 \\
\hline 3.5 & 0.0000 & 0.0000 & 0.0000 & 0.0391 & 0.0000 & 0.1636 & 0.0000 & 0.0000 \\
\hline 4.0 & 0.0000 & 0.8452 & 0.0000 & 0.0280 & 2.3880 & 0.0720 & 0.0000 & 0.0000 \\
\hline 4.5 & 1.9993 & 0.0000 & 0.0000 & 0.0251 & 0.0060 & 0.0096 & 2.1744 & 0.9251 \\
\hline 5.0 & 0.0263 & 0.0001 & 1.5419 & 0.0148 & 0.3084 & 0.7122 & 0.1752 & 0.7976 \\
\hline 5.5 & 0.1986 & 0.0003 & 0.0862 & 1.4871 & 0.0000 & 0.0146 & 0.0000 & 0.0984 \\
\hline 6.0 & 0.0000 & 0.0004 & 0.9836 & 0.2736 & 0.0000 & 0.0012 & 0.0000 & 0.0000 \\
\hline 6.5 & 0.0000 & 0.0006 & 0.0000 & 0.0004 & 0.0000 & 0.0388 & 0.0000 & 0.0000 \\
\hline 7.0 & 0.0000 & 0.0006 & 0.0000 & 0.0159 & 0.0000 & 0.0486 & 0.0000 & 0.0000 \\
\hline 7.5 & 0.0000 & 0.0025 & 0.0001 & 0.0568 & 0.0000 & 0.0267 & 0.0000 & 0.0001 \\
\hline 8.0 & 0.0000 & 0.0220 & 0.0006 & 0.0692 & 0.0002 & 0.0001 & 0.0000 & 0.0015 \\
\hline 8.5 & 0.0000 & 0.2151 & 0.0021 & 0.0634 & 0.0157 & 0.0008 & 0.0000 & 0.0153 \\
\hline 9.0 & 0.0017 & 0.0000 & 0.0150 & 0.0585 & 0.0000 & 0.0000 & 0.0000 & 0.0000 \\
\hline
\end{tabular}

\begin{tabular}{|c|c|c|c|c|c|c|}
\hline \multirow{2}{*}{$\begin{array}{c}\log _{10} \\
\left(\omega \tau_{M}+x\right)\end{array}$} & \multicolumn{2}{|c|}{ G12 } & \multicolumn{2}{|c|}{ G13 } & \multicolumn{2}{|c|}{ G14 } \\
\hline & G real & G im & G real & G im & G real & G im \\
\hline-2.0 & 0.1072 & 0.0095 & 0.0094 & 0.0000 & 0.0013 & 0.0000 \\
\hline-1.5 & 0.1304 & 0.0233 & 0.0094 & 0.0001 & 0.0014 & 0.0001 \\
\hline-1.0 & 0.1927 & 0.0764 & 0.0093 & 0.0020 & 0.0019 & 0.0001 \\
\hline-0.5 & 0.3242 & 0.0631 & 0.0083 & 0.0660 & 0.0044 & 0.0001 \\
\hline 0.0 & 0.3922 & 0.0016 & 0.0938 & 0.1117 & 6.7304 & 4.8103 \\
\hline 0.5 & 9.0289 & 7.0788 & 9.1963 & 9.7856 & 8.5225 & 6.5394 \\
\hline 1.0 & 10.6936 & 6.5564 & 13.0917 & 5.1587 & 5.0040 & 3.2280 \\
\hline 1.5 & 9.5671 & 0.9890 & 1.7607 & 2.1221 & 3.7534 & 1.3111 \\
\hline 2.0 & 1.2907 & 1.8048 & 1.1934 & 1.7142 & 1.7408 & 0.9825 \\
\hline 2.5 & 3.0537 & 1.4779 & 1.0198 & 0.8163 & 1.6519 & 0.3950 \\
\hline 3.0 & 0.7542 & 0.0896 & 1.5969 & 0.2861 & 0.3570 & 0.7156 \\
\hline 3.5 & 0.6931 & 0.2601 & 0.9414 & 0.1834 & 0.1780 & 0.0099 \\
\hline 4.0 & 0.2658 & 0.4572 & 0.0198 & 0.1615 & 0.8879 & 0.0100 \\
\hline 4.5 & 0.2987 & 0.1348 & 0.0202 & 0.0744 & 0.0121 & 0.0102 \\
\hline 5.0 & 0.1812 & 0.0261 & 0.0197 & 0.0930 & 0.0020 & 1.1174 \\
\hline 5.5 & 0.0472 & 0.0805 & 0.0147 & 0.0650 & 0.1904 & 0.0099 \\
\hline 6.0 & 0.0955 & 0.3614 & 0.0086 & 0.0000 & 0.5020 & 0.0097 \\
\hline 6.5 & 0.0069 & 0.0000 & 1.0308 & 0.0145 & 0.0095 & 0.0100 \\
\hline 7.0 & 0.0880 & 0.0497 & 0.2020 & 0.4551 & 0.0109 & 0.0105 \\
\hline 7.5 & 0.1792 & 0.1476 & 0.4399 & 1.1050 & 0.0129 & 0.0100 \\
\hline 8.0 & 0.1481 & 0.0789 & 0.0196 & 0.5373 & 0.0145 & 0.0106 \\
\hline 8.5 & 0.0550 & 0.0002 & 0.0015 & 0.0258 & 0.0194 & 0.0101 \\
\hline 9.0 & 0.0570 & 0.3341 & 0.0008 & 0.0001 & 0.0940 & 0.0101 \\
\hline
\end{tabular}




\section{LEBENSLAUF}

Persönliche Daten

Name:

Katarzyna Falenty

Geburtstag:

22.09.1980

Geburtsort:

Wołomin, Polen

Staatsangehörigkeit:

polnisch

\section{Schulausbildung}

09/1987-06/1990

Grundschule, Bosewo Stare, gm. Długosiodło, Polen

09/1990-06/1995

Grundschule Nummer 4, Obrońców Westerplatte, Wyszków, Polen

09/1995-06/1999

Allgemeinbildende Oberschule, Cyprian Kamil Norwid, (Ausbildungsprofil Biologie-Chemie), Wyszków, Polen Reifeprüfung: 09/06/1999

\section{Universitätsausbildung}

$10 / 1999-06 / 2002$

Warschauer Universität, Fakultät für Geologie, Abteilung für Geochemie, Mineralogie und Petrologie, Warschau, Polen Abschluss mit Bachelor Titel (BSc - Bachelor of Science)

$10 / 2001-02 / 2002$

Georg - August - Universität Göttingen, Deutschland, Socrates-Erasmus Stipendium (das Aktionsprogramm der Europäischen Union für die grenzüberschreitende Zusammenarbeit in verschiedenen Bereichen der allgemeinen Bildung)

$10 / 2002-06 / 2004$

Warschauer Universität, Fakultät für Geologie, Abteilung für Geochemie, Mineralogie und Petrologie, Warschau, Polen Abschluss mit Magister Titel (MSc - Master of Science)

Diplom in Geochemie, Mineralogie und Petrologie

\section{Berufspraxis}

$11 / 2004-11 / 2007$

Wissenschaftliche Mitarbeiterin

Universität Göttingen, Fakultät für Geowissenschaften und Geographie, Abteilung für Experimentelle und Angewandte Mineralogie, Göttingen, Deutschland

14/11/2007 Ende der Anstellung als Wissenschaftliche Mitarbeiterin 
